\title{
NREL Combined Experimental Final Report-Phase II
}

C.P. Butterfield

W.P. Musial

G.N. Scott

D.A. Simms

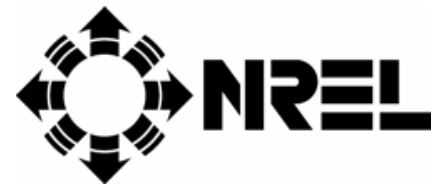

National Renewable Energy Laboratory 1617 Cole Boulevard

Golden, Colorado 80401-3393

A national laboratory of the U.S. Department of Energy Managed by Midwest Research Institute for the U.S. Department of Energy under contract No. DE-AC36-83CH10093 


\section{NREL Combined Experimental Final Report-Phase II}

C.P. Butterfield

W.P. Musial

G.N. Scott

D.A. Simms

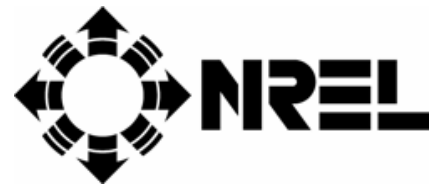

National Renewable Energy Laboratory 1617 Cole Boulevard Golden, Colorado 80401-3393

A national laboratory of the U.S. Department of Energy Managed by Midwest Research Institute for the U.S. Department of Energy under contract No. DE-AC36-83CH10093

Prepared under Task No. AS575440

August 1992 


\section{NOTICE}

This report was prepared as an account of work sponsored by an agency of the United States government. Neither the United States government nor any agency thereof, nor any of their employees, makes any warranty, express or implied, or assumes any legal liability or responsibility for the accuracy, completeness, or usefulness of any information, apparatus, product, or process disclosed, or represents that its use would not infringe privately owned rights. Reference herein to any specific commercial product, process, or service by trade name, trademark, manufacturer, or otherwise does not necessarily constitute or imply its endorsement, recommendation, or favoring by the United States government or any agency thereof. The views and opinions of authors expressed herein do not necessarily state or reflect those of the United States government or any agency thereof. 


\section{Acknowledgments}

The authors wish to acknowledge the Department of Energy for the support of this work through contract number DE-AC02-83CH10093. Other contributors include Al Eggers, Craig Hansen, Bob Akins, Bob Thresher, Dan Reda (Sandia National Laboratories), Mike Jenks, Dave Jager, Bob Keller and Diane PipanLogan. 


\subsection{Table of Contents}

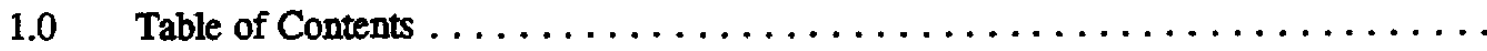

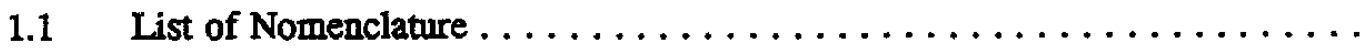

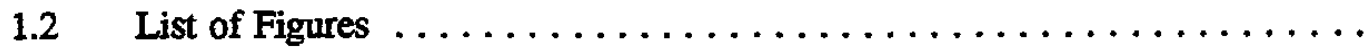

1.3 List of Tables

2.0 Introduction

3.0 Quasi-Steady Aerodynamic Measurements

3.1 Introduction

3.2 Data Processing

3.3 Results

3.3.1 Force Coefficients

3.3.2 Airfoil Pressure Distributions

3.3.3 Spanwise Pressure Distributions

3.4 Conclusions

4.0 Unsteady Aerodynamic Loads

4.1 Introduction

4.2 Data Preparation

4.2.1 Test Cases

4.2.2 Pressure Coefficient Normalization

4.3 Azimuth-Averaged Results

4.4 Dynamic Stall Results

4.5 Transient Pressure Distributions

4.6 Conclusions

5.0 Flow Visualization Tests

5.1 Liquid Crystal Tests

5.2 Correlations of Pressure Distribution with Flow Patterns

5.2.1 Introduction

5.2.2 Flow Visualization Test Setup

5.2.3 Data Processing

5.2.4 Pressure Distributions

5.3 Conclusions

6.0 Structural Loads

6.1 Introduction

6.2 Air Load Estimating Tool (ALEST)

6.3 Blade-Load Data Base

6.4 Conclusions 


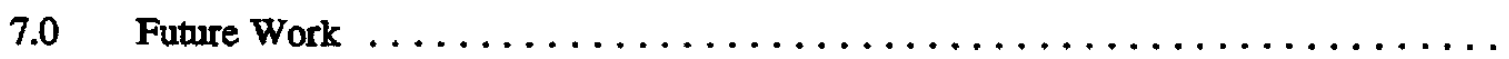

8.0 Conclusions

9.0 References

10.0 Appendices 


\subsection{List of Nomenclature}




\subsection{List of Figures}

Figure 3.1. Airfoil pressure distribution integration $\ldots \ldots \ldots \ldots \ldots \ldots \ldots$

Figure 3.2. Relationships of force coefficients to airfoil sections $\ldots \ldots \ldots \ldots \ldots$

Figure 3.3. Normal force comparisons at $80 \%$ blade span $\ldots \ldots \ldots \ldots \ldots \ldots$

Figure 3.4. Normal force comparisons throughout blade span $\ldots \ldots \ldots \ldots \ldots \ldots$

Figure 3.5. Tangent coefficient comparisons throughout blade span $\ldots \ldots \ldots \ldots \ldots$

Figure 3.6. Torque and thrust coefficient comparisons for rotating-blade

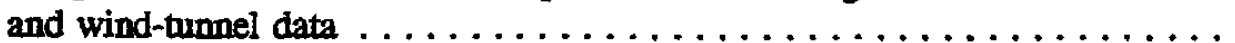

Figure 3.7. Rotating blade normal and tangent force coefficient comparisons with wind-tunnel data

Figure 3.8. Life coefficient comparisons throughout blade span

Figure 3.9. Pressure drag coefficient comparisons throughout blade span

Figure 3.10. Pressure distributions comparison at $63 \%$ blade span and 2.5 deg AOA .....

Figure 3.11. Pressure distribution comparisons at $63 \%$ blade span and 9.5 deg AOA ....

Figure 3.12. Pressure distributions at $63 \%$ blade span for post-stall AOAs $\ldots \ldots \ldots \ldots$

Figure 3.13. Post-stall pressure distributions at $80 \%$ blade span $\ldots \ldots \ldots \ldots \ldots$

Figure 3.14. Post-stall pressure distributions at $30 \%$ blade span $\ldots \ldots \ldots \ldots \ldots \ldots$

Figure 3.15. Bin averaged spanwise pressure coefficients for the $4 \%$ chord

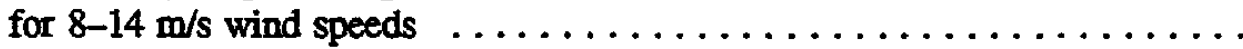

Figure 3.16. Bin averaged spanwise pressure coefficients for the $4 \%$ chord . for $14.5-19.2 \mathrm{~m} / \mathrm{s}$ wind speeds

Figure 3.17. Bin averaged spanwise pressure coefficients for the $36 \%$ chord for $8.8-14.5 \mathrm{~m} / \mathrm{s}$ wind speeds

Figure 3.18. Bin averaged spanwise pressure coefficients for the $36 \%$ chord for $14.5-19.2 \mathrm{~m} / \mathrm{s}$ wind speeds

Figure 4.1. Dynamic-pressure variations during yaw

Figure 4.2. Effects of time-varying dynamic-pressure normalization 
Figure 4.3. Azimuth-averaged AOA and lift coefficient at $80 \%$ span for $30^{\circ}$ yaw case

Figure 4.4. Azimuth-averaged AOA and lift coefficient at 63\% span for $30^{\circ}$ yaw case

Figure 4.5. Azimuth-averaged AOA and lift coefficient at 47\% span for $30^{\circ}$ yaw case

Figure 4.6. Azimuth-averaged AOA and lift coefficient at $30 \%$ span for $30^{\circ}$ yaw case

Figure 4.7. Azimuth-averaged AOA and lift coefficient at the $80 \%$ span for no yaw case

Figure 4.8. Azimuth-averaged AOA and lift coefficient at $63 \%$ span for no yaw case

Figure 4.9. Azimuth-averaged AOA and lift coefficient at $47 \%$ span for no yaw case

Figure 4.10. Azimuth-averaged AOA and lift coefficient at $30 \%$ span for no yaw case

Figure 4.11. Dynamic stall at $80 \%$ span

Figure 4.12. Dynamic stall at $63 \%$ span compared to wind-tunnel dynamic stall

Figure 4.13. Dynamic stall at $47 \%$ span

Figure 4.14. Dynamic stall at $30 \%$ span

Figure 4.15. Drag dynamic stall at $80 \%$ span

Figure 4.16. Drag dynamic stall at $63 \%$ span

Figure 4.17. Drag dynamic stall at $47 \%$ span

Figure 4.18." . Drag dynamic stall at $30 \%$ span

Figure 4.19. Pitching moment dynamic stall at 63\% span

Figure 4.20. Chordwise surface-pressure distribution vs. azimuth angle. $V=14.7 \mathrm{~m} / \mathrm{sec}$, $0^{\circ}$ yaw, $30 \%$ span. One cycle of data.

Figure 4.21. Measured and geometric local AOA vs. azimuth angle

Figure 4.22. Theoretical geometric AOA vs. azimuth angle. $30^{\circ}$ yaw, $\mathrm{V}=16.7 \mathrm{~m} / \mathrm{sec} \ldots$ 
Figure 4.23. Chordwise surface-pressure distribution vs. azimuth angle. $30^{\circ}$ yaw, $V=14 \mathrm{~m} / \mathrm{sec}, 30 \%$ span. Bin-averaged data

Figure 4.24. Chordwise surface-pressure distribution vs. azimuth angle. $30^{\circ}$ yaw, $V=16.7 \mathrm{~m} / \mathrm{sec}, 30 \%$ span. One cycle of data.

Figure 4.25. Chordwise surface-pressure distribution vs. azimuth angle. $-33^{\circ}$ yaw, $V=13.3 \mathrm{~m} / \mathrm{sec}, 30 \%$ span. One cycle of data.

Figure 4.26 Chordwise surface-pressure distribution vs. azimuth angle. $-33^{\circ}$ yaw, $\mathrm{V}=13.3 \mathrm{~m} / \mathrm{sec}, 63 \%$ span. One cycle of data $\ldots \ldots \ldots \ldots \ldots \ldots$

Figure 4.27 Spanwise pressure distribution vs. azimuth angle. $7.7 \mathrm{~m} / \mathrm{s}$ wind speed, $4 \%$ chord, tip-velocity normalization, bin-averaged data.

Figure 4.28. Spanwise pressure distribution vs. azimuth angle. $18.7 \mathrm{~m} / \mathrm{s}$ wind speed, $4 \%$ chord, tip-velocity normalization, bin-averaged data.

Figure 4.29. Spanwise pressure distribution vs. azimuth angle. $18 \mathrm{~m} / \mathrm{s}$ wind speed, $4 \%$ chord, tip-velocity normalization, bin-averaged data, $-26.5^{\circ}$ yaw.

Figure 4.30. Spanwise pressure distribution vs. azimuth angle. $14 \mathrm{~m} / \mathrm{s}$ wind speed, $4 \%$ chord, tip-velocity normalization, bin-averaged data, $30^{\circ}$ yaw.

Figure 4.31. Spanwise pressure distribution vs. azimuth angle. $16.7 \mathrm{~m} / \mathrm{s}$ wind speed, $4 \%$ chord, tip-velocity normalization, bin-averaged data, $30^{\circ}$ yaw.

Figure 5.1. Wind turbine with video cameras

Figure 5.2. . Video signal path

Figure 5.3. View of blade at low AOA

Figure 5.4. View of blade at medium AOA

Figure 5.5. View of blade at high AOA

Figure 5.6. Comparison of wind-tunnel and rotating-blade pressures

Figure 5.7. Comparison of lift coefficients from wind tunnel and wind turbine

Figure 5.8. Comparison of pressure drag coefficients from wind tunnel and wind turbine

Figure 6.1. Sensitivity to input bending moment

Figure 6.2. Sensitivity to precone angle

Figure 6.3. Sensitivity to blade mass distribution 
Figure 6.4. Blade airload estimates

Figure 6.5. ALEST analysis procedure

Figure 6.6. Comparison of ??? 


\subsection{List of Tables}

Table 4.1.

Table 5.1. Angles of Attack Probe

Table 6.1. Case Study Parameter Statistics

Table 6.2. Input Bending Moment Channels

Table 6.3. Blade Mass Distribution 


\subsection{Introduction}

Wind turbine operating experience has shown that current analysis techniques are inadequate when used to predict peak power and loads on a fixed-pitch wind turbine. Butterfield et al. (1992), Viterna and Corrigan (1981), and Tangler (1983) all show evidence of higher-than-predicted power levels on stallcontrolled wind turbines. Because performance and loads are the most important design information needed to achieve more reliable and inexpensive wind turbines, it is important to understand the cause of the discrepancy. The primary question is how the performance of the airfoil in the wind tunnel differs from the performance on an operating horizontal-axis wind turbine (HAWT). The National Renewable Energy Laboratory (NREL) conducted a comprehensive test program, the Combined Experiment, that focused on answering this question and understanding the basic fluid mechanics of rotating HAWT stall aerodynamics in both steady and unsteady flow regimes.

The basic approach was to instrument a wind-turbine rotor, using an airfoil that was well documented by wind-tunnel tests, and measure operating pressure distributions on the rotating blade. Using the integrated values of the pressure data, airfoil performance coefficients were obtained, and comparisons were made between the rotating-blade data and the wind-tunnel data. Care was taken to minimize the aerodynamic and geometric differences between the rotating-blade and the wind-tunnel models. Blades and wind-tunnel models were made in the same molds, and the same instruments were used for both the rotating-blade and the wind-tunnel tests.

This is the second of two reports describing the Combined Experiment Program and results. This Phase II report concentrates on the results derived from aerodynamic pressure measurements, wind-turbine load measurements, and flow-visualization studies. Average and unsteady aerodynamic measurements are presented for yawed and unyawed operation. These reports disseminate basic aerodynamic data for code validation and wind turbine-design information, and will hopefully assist other researchers using the Combined Experiment data. These reports provide a comprehensive description of results to date and a description of how the experiment operates.

The Combined Experiment was planned and carried out over a period of 4 years. It was the most comprehensive wind-turbine test program ever attempted. The test program was divided into two phases, the first beginning in the spring of 1987. Many configurations of instrumentation were considered during both phases of testing. The most significant configuration change between Phase I and Phase II was the extent of the pressure measurements. The instrumented blade for Phase I had only one spanwise radial station of pressure taps (32 taps) at $80 \%$ radius, while the Phase II blade had four radial stations of pressure taps at radial positions ranging from $30 \%$ radius to $80 \%$, as well as six intermediate radial stations of taps located between the primary stations. Appendix A describes the test blade layout with pressure tap locations and strain gage locations. A more detailed description of this and the other instrumentation changes that were made between Phases I and II is given by Butterfield et al. (1992) in the Phase I report.

The Phase I report also covers the test set-up, instrumentation, wind-tunnel tests, and airfoil-roughness testing. However, most of the data came from the Phase II data sets and are presented in this report. This report covers:

- Bin-averaged aerodynamic coefficients data integrated from pressure distributions

- Unsteady aerodynamic data

- Flow-visualization-test results

- Bin-averaged blade-load data. 


\subsection{Quasi-Steady Aerodynamic Measurements}

\subsection{Introduction}

The aircraft industry has historically avoided operating in aerodynamic stall. As a result there has been reiatively little effort expended to investigate steady stall on poststall airfoil performance. Unilike airfoils on conventional straight-wing aircraft and helicopters, wind turbines must be designed to operate in stall and to withstand the forces associated with this operating condition. In moderate to high winds, a fixedpitch wind turbine continuously experiences stall over large portions of its blades. Peak power production and blade loads occur during high-wind operating conditions, when stall dominates the applied airloads. Therefore, it is critically important for wind turbine designers to understand and predict stall behavior.

This section presents the results of the quasi-steady airfoil measurements from the Combined Experiment's rotor. Because of the untwisted geometry of the rotor's blades, the root sections stall at relatively low wind speeds and operate in stall most of the time. This allowed researchers to observe the progression of stall over the blade for a range of wind speeds under normal operating conditions.

The objective of this part of the analysis was to document the average airfoil performance on the rotating wind turbine blade and compare it to wind-tunnel results. This section discusses some of these comparisons and reviews the data-processing techniques. Detailed descriptions of the data-processing procedures are included in Butterfield et al. (1992). Appendix B contains the complete catalog of results from the bin-averaging data analyses, including angle of attack (AOA) and local total pressure (dynamic pressure) measurements correlated with wind speed. This section discusses only the highlights of the comparisons.

\subsection{Data Processing}

After the raw data were recorded, calibration coefficients were applied to the data. During testing, pressure instrumentation calibrations were performed every 5 minutes. This enabled calibration coefficients to be updated frequently enough to reduce the total measurement uncertainty to less than $3 \%$ of the local dynamic pressure. The procedure was laborious but ensured accurate engineering data for later processing.

The reference pressures for each transducer located in the blade were transferred from the hub to the transducer through a tube as described in Butterfield et al. (1991). The effects of centrifugal force on air in the tube were corrected per Equation 3.1, which is very similar to the procedure described by Hurst and Owen (1988), Equation 3.2. Hurst's equation assumed that the transducer was located at the axis of rotation and that a long tube was run from the transducer down the blade to the surface pressure tap. Equation 3.2 includes compressibility effects that are negligible and not included in Equation 3.1.

In this test program, two centrifugal-force corrections were needed. The first corrected the reference tube pressures from the axis of rotation to the transducer, and the second corrected the pressures in the tubes leading from the transducer to the blade surface.

$$
P_{\text {ref }}=P_{\text {arm }}+\frac{1}{2} \rho(r \omega)^{2}
$$




$$
P_{\text {surtace }}=P_{\text {meas }} \frac{\omega^{2} r^{2}}{2 K T}
$$

where

$P_{\text {ref }} \quad=$ reference pressure at transducer

$P_{\text {am }}=$ atmospheric pressure

$P_{\text {surface }}=$ actual surface pressure

$P_{\text {meas }}=$ measured surface pressure

$\omega \quad=$ rotor speed

$\mathrm{K} \quad=$ gas constant

$\mathrm{T} \quad=$ temperature

I $\quad=$ radius to reference port or surface pressure tap

$\rho \quad=$ air density.

To obtain normalized pressure coefficients $\left(C_{p}\right)$, dimensional pressure data were divided by local dynamic pressure as shown in Equation 3.3. Dynamic pressure was established in two ways. First, atmospheric pressure was subtracted from measured total pressure to get a local, measured dynamic pressure $\left(Q_{\text {meas }}\right)$ using Equation 3.4. The second method derived the local value of dynamic pressure $\left(Q_{\text {der }}\right)$ by using Equation 3.5 and the disk-averaged wind speed (measured from the vertical-plane array of anemometers one diameter upwind of the rotor), the rotor angular speed, and the radius to the pressure tap.

$$
\begin{gathered}
C_{p}=\frac{P_{\text {surface }}-P_{\text {atm }}}{Q_{\text {meas }}} \\
Q_{\text {meas }}=P_{\text {tot }}-P_{\text {atm }} \\
Q_{\text {der }}=\frac{1}{2} \rho\left(V^{2}+(r \omega)^{2}\right)
\end{gathered}
$$

where

$Q_{\text {meas }}=$ measured dynamic pressure

$Q_{\text {der }} \quad=$ derived dynamic pressure

$\mathrm{V}=$ disk-averaged wind speed

$\mathrm{C}_{\mathrm{p}} \quad=$ normalized pressure coefficient

$\mathrm{P}_{\text {tot }}^{\mathrm{p}} \quad=$ measured total pressure.

Both methods gave similar results that agreed with wind-tunnel data at low AOAs. At high AOAs (greater than $25 \mathrm{deg}$ ) on the $30 \%$ blade-span pressure distribution, the $Q_{\text {meas }}$ method gave values of pressure coefficient $\left(C_{p}\right)$ greater than 1 at the stagnation point. This indicates that the value of $Q$, measured at the total-pressure probe, was lower than the leading-edge stagnation pressure. The stagnation pressure should 
always be equal to the dynamic pressure. Because of this problem, all the pressure data presented in this section were normalized by the calculated dynamic pressure $\left(Q_{d z}\right)$.

All the pressure data were digitized at a $520-\mathrm{Hz}$ sample rate. Data were later block-averaged by a factor of 52 to obtain a final 10-Hz sample rate. These data were then sorted into bins using the measured angle of attack as the independent variable. Analog filters were used to eliminate biasing of the data. These filters were four-pole Butterworth type, set at a 100- $\mathrm{Hz}$ roll-off frequency.

\subsection{Results}

\subsubsection{Force Coefficients}

One of the main objectives of the data processing was to transform the large number of measured pressure distributions into a smaller number of performance coefficients to make them more useful for application in aerodynamic analysis and code validation. Expression in terms of performance coefficients also makes it possible to easily quantify the comparisons with wind-tunnel data. The raw pressure distribution data, which provide a more fundamental picture of aerodynamic performance characteristics, are presented in Section 3.3.2.

The pressure distributions for both rotating-blade and wind-tunnel data were integrated to compute normal force coefficients $\left(C_{N}\right)$ and the tangent force coefficients $\left(C_{T}\right)$. These are the fundamental coefficients that are commonly used to describe airfoil performance from pressure measurements because their derivation is independent of the AOA measurements. They represent the forces acting tangent and parallel to the airfoil chord, respectively. The integration procedure is shown in Figure 3.1, where the pressures are first projected onto the chord line and integrated to determine the $\mathrm{C}_{\mathrm{N}}$ values, and then projected onto an axis orthogonal to the chord and integrated to compute the $C_{T}$ values. This procedure is described in detail by Pope (1966). Equations 3.6 and 3.7 give the integration procedure used to determine $C_{N}$ and $C_{T}$.

Figure 3.1. Airfoll pressure distribution integration

$$
\begin{aligned}
& C_{N}=\frac{1}{\frac{1}{2} c \rho V_{t}^{2}} \sum P_{i}\left(\frac{x_{i+1}-x_{i-1}}{2}\right) \\
& C_{T}=\frac{1}{\frac{1}{2} c \rho V_{z}^{2}} \sum P_{i}\left(\frac{y_{i+1}-y_{i-1}}{2}\right)
\end{aligned}
$$

All other airfoil performance coefficients, such as $\mathrm{C}_{1}, \mathrm{C}_{\mathrm{d}}, \mathrm{C}_{\text {Torque, }}$ and $\mathrm{C}_{\mathrm{Thros}}$, can be computed using the $C_{N}$ and $C_{T}$ values in conjunction with their reference angles. Figure 3.2 shows the relationship of each of the force coefficients to an airfoil section on a rotating wind turbine blade. For the Combined Experiment rotor, the pitch angle $(\phi)$ is the same over the entire blade span because the blades are untwisted.

Figure 3.2. Relationships of force coefficients to airfoil sections 


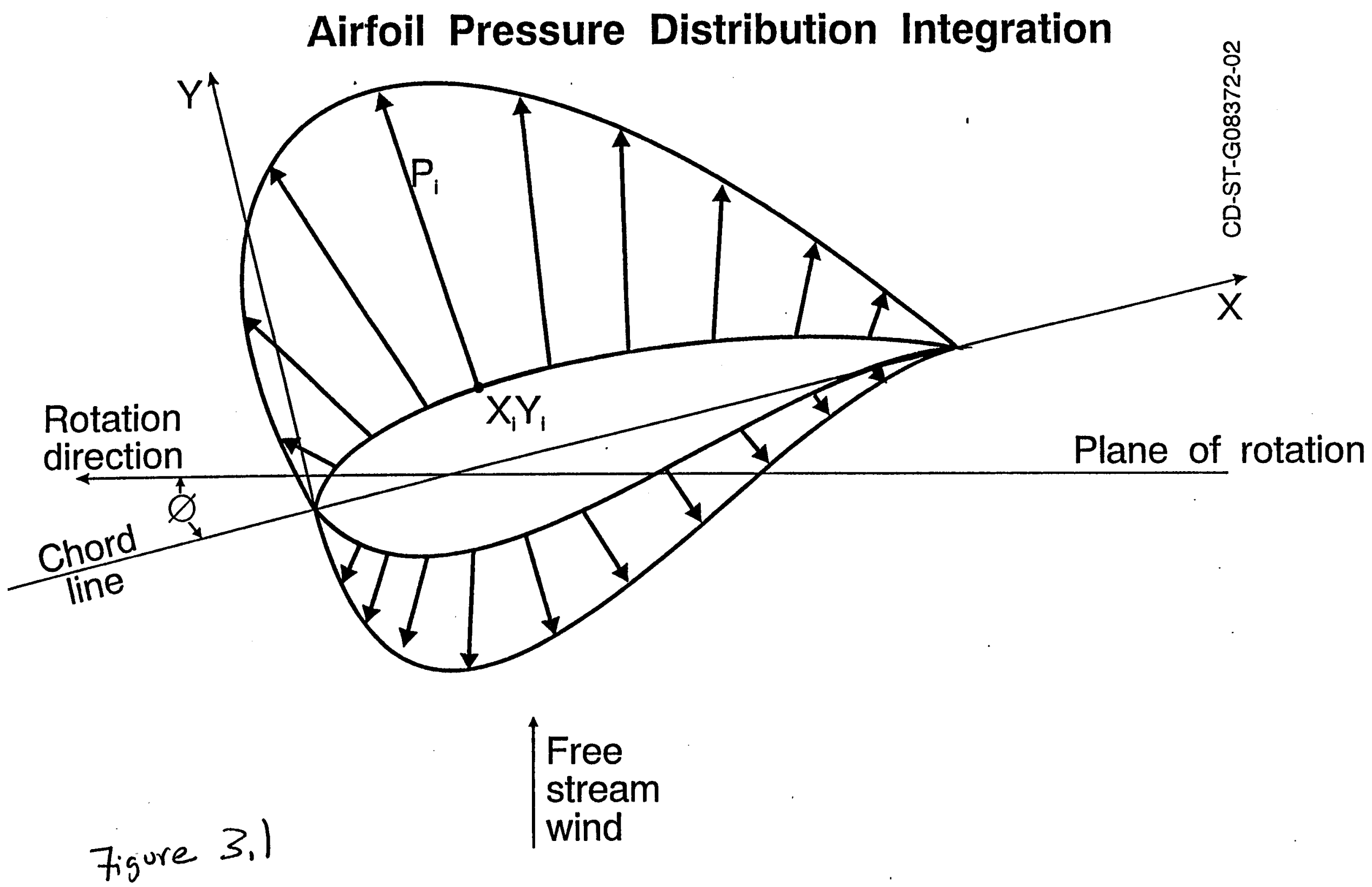




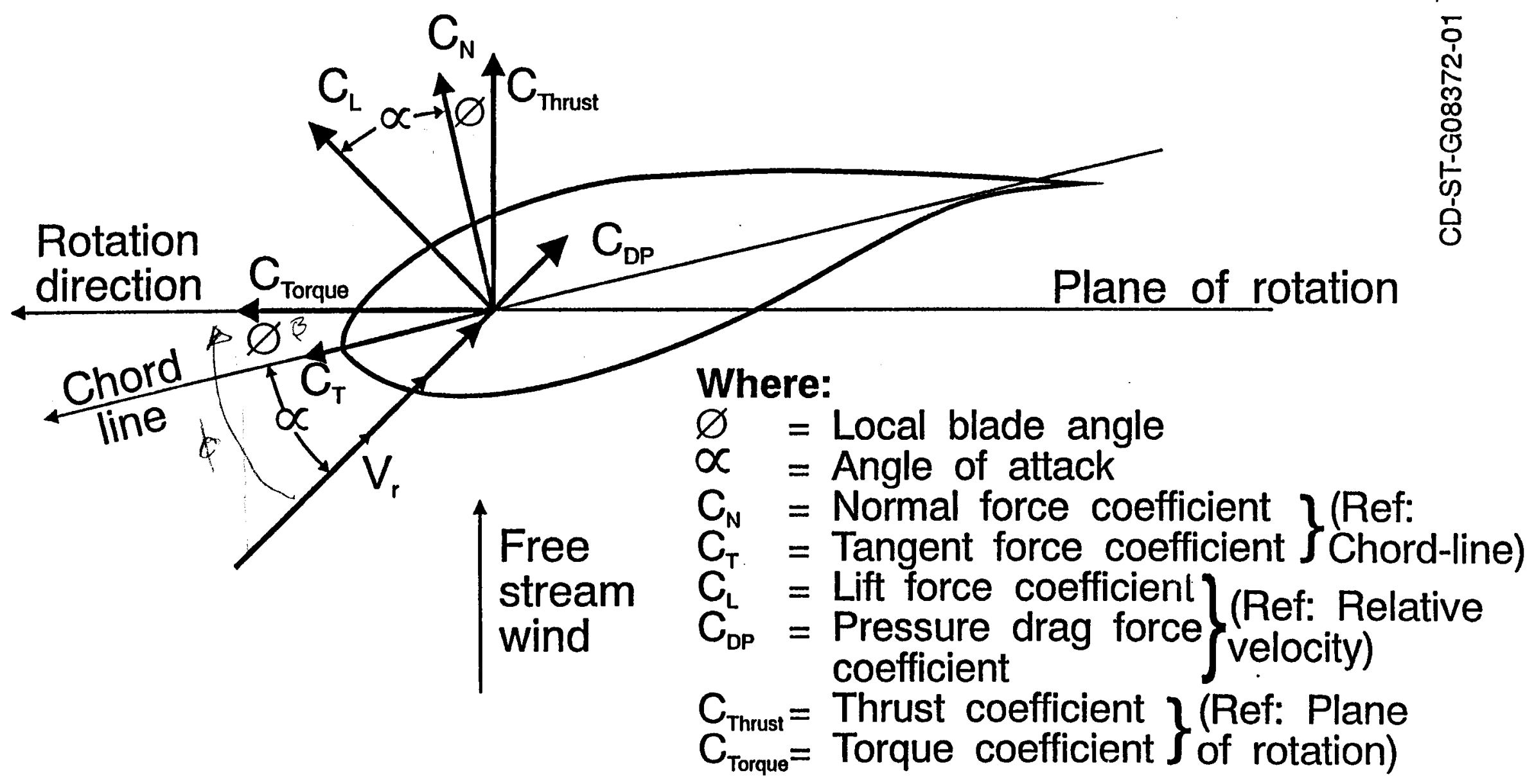

- Figure 3.2 
The $C_{N}$ and $C_{T}$ curves are presented in Figures 3.3, 3.4, and 3.5. Some general trends prevail throughout the data. First, it is clear from all of the data that effects of rotation on airfoil performance are strictly a stall and poststall phenomenon. At low AOAs the correlation with wind-tunnel data is good in each case. The discrepancies generally begin to take place at the normal static wind-tunnel stall angle. Second, the rotating-blade coefficients can usually be characterized by gradual stall behavior whereas the wind-tunnel coefficients show abrupt stall. This gradual stall characteristic tends to cause all of the rotating blade force coefficients to be higher than those in the wind tunnel at high AOAs. Another trend that was consistently observed was that the rotating-blade effects become more pronounced at inboard blade stations, particularly at the $30 \%$ station.

\section{Figure 3.3. Normal force comparisons at $80 \%$ blade span}

\section{Figure 3.4. Normal force comparisons throughout blade span}

\section{Figure 3.5. Tangent coefficient comparisons throughout blade span}

Figure 3.3 shows normal force coefficients $\left(C_{N}\right)$ for both the Colorado State University (CSU) wind tunnel and the $80 \%$ blade span on the wind turbine. (A complete catalog of the CSU wind-tunnel test results is presented in Butterfield et al. (1992).) Beyond $15 \mathrm{deg}$, stall causes normal force coefficients to fall abruptly for the wind-tunnel data, whereas the rotating-blade data drop very gradually with increasing AOA. This is consistent with results from Phase I testing as described by Butterfield et al. (1992).

Figure 3.4 shows the $\mathrm{C}_{\mathrm{N}}$ versus $\mathrm{AOA}$ curves at the $30 \%, 47 \%, 63 \%$, and $80 \%$ blade span locations. All curves agree at low AOA. At the normal stall angle, the two mid-span stations (47\% and 63\%) show an increase of $10 \%$ in $\mathrm{C}_{\mathrm{N}(\max )}$ and a levelling of values for higher AOAs. The $80 \%$ station $\mathrm{C}_{\mathrm{N}(\max )}$ agrees with the wind-tunnel data but shows the same flattening at higher AOAs. At the $30 \%$ span the results are very different. The magnitude of $C_{N}$ continues to increase with increasing AOA. A maximum value of 2.0 was recorded at an $A O A$ of $30 \mathrm{deg}$, which is more than twice as large as the maximum value measured in the wind tunnel.

Tangent force coefficients are compared in Figure 3.5. Wind-tunnel data again show good agreement with rotating-blade data for low AOAs. The wind-tunnel data stall sharply, whereas the rotating-blade data show a more gradual drop-off after stall. This discrepancy can have a large effect on rotor torque because for relatively small blade pitch angles the tangent forces are closely aligned with the plane of rotation.

The $\mathrm{C}_{\mathrm{N}}$ and $\mathrm{C}_{\mathrm{T}}$ values can be related to wind turbine performance parameters such as $\mathrm{C}_{\text {Torgue }}$ and $\mathrm{C}_{\text {Thrust }}$ by Equations 3.8 and 3.9 .

$$
\begin{gathered}
C_{\text {Torque }}=C_{N} \sin (\phi)+C_{T} \cos (\phi) \\
C_{\text {Thrast }}=C_{N} \cos (\phi)-C_{T} \sin (\phi)
\end{gathered}
$$

By these equations, these coefficients are geometrically related to the $C_{N}$ and $C_{T}$ values by the pitch angle of the airfoil. For a stall-controlled wind turbine, the pitch is fixed, and this angle will not change. For the Combined Experiment rotor, which has no twist in the blade, this angle is a constant for the entire span for all wind speeds.

Figure 3.6 shows the $C_{\text {Torque }}$ and $C_{\text {Thrast }}$ versus AOA curves for the $63 \%$ blade span. These curves are compared to the wind-tunnel data. The effect of rotation on wind turbine performance can be most easily seen from these data because the force coefficients are aligned with the in-plane and out-of-plane forces, rotor torque and thrust. After stall is reached, there is a tremendous discrepancy between both the torque 


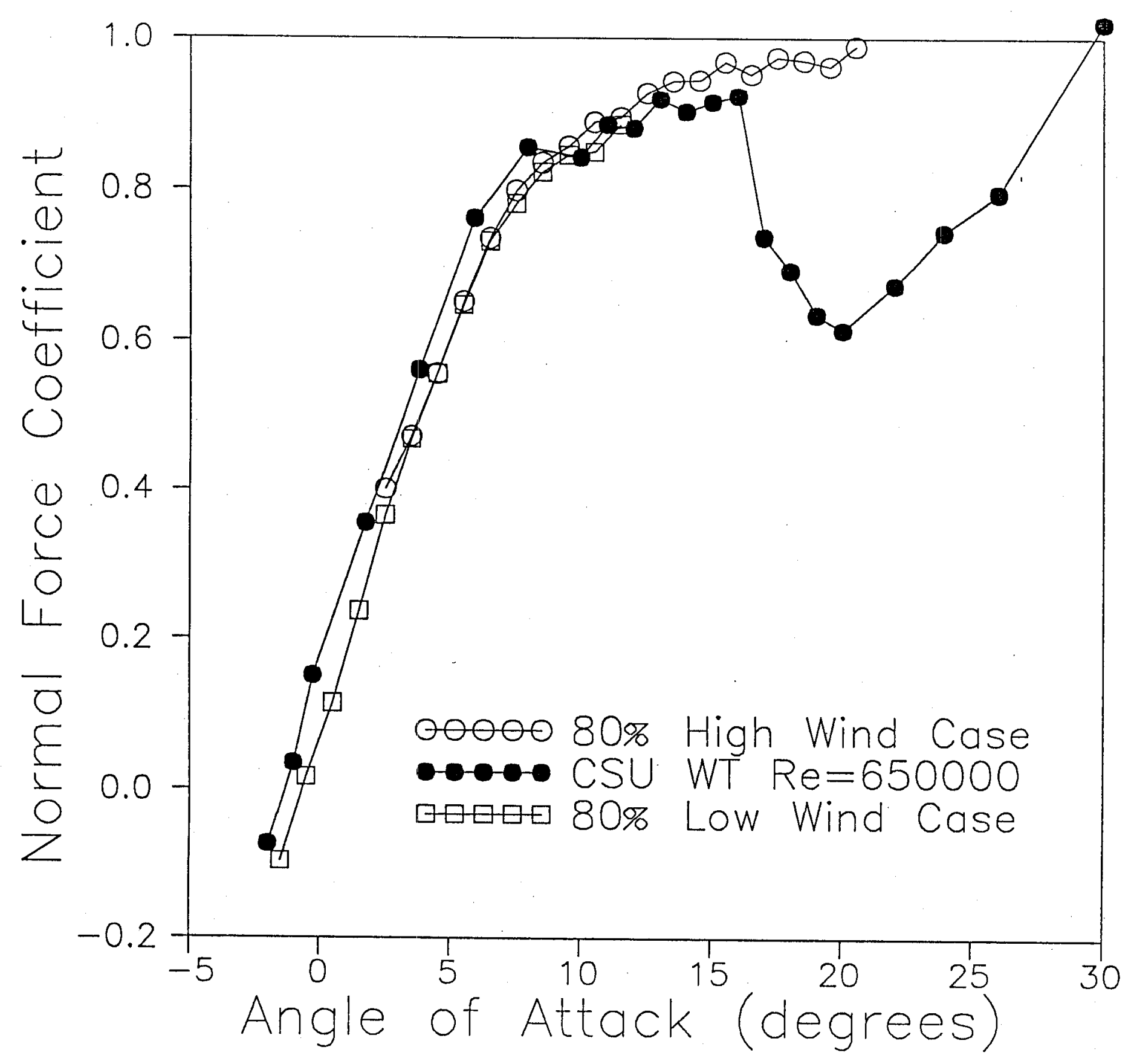

7 igure
3.3 


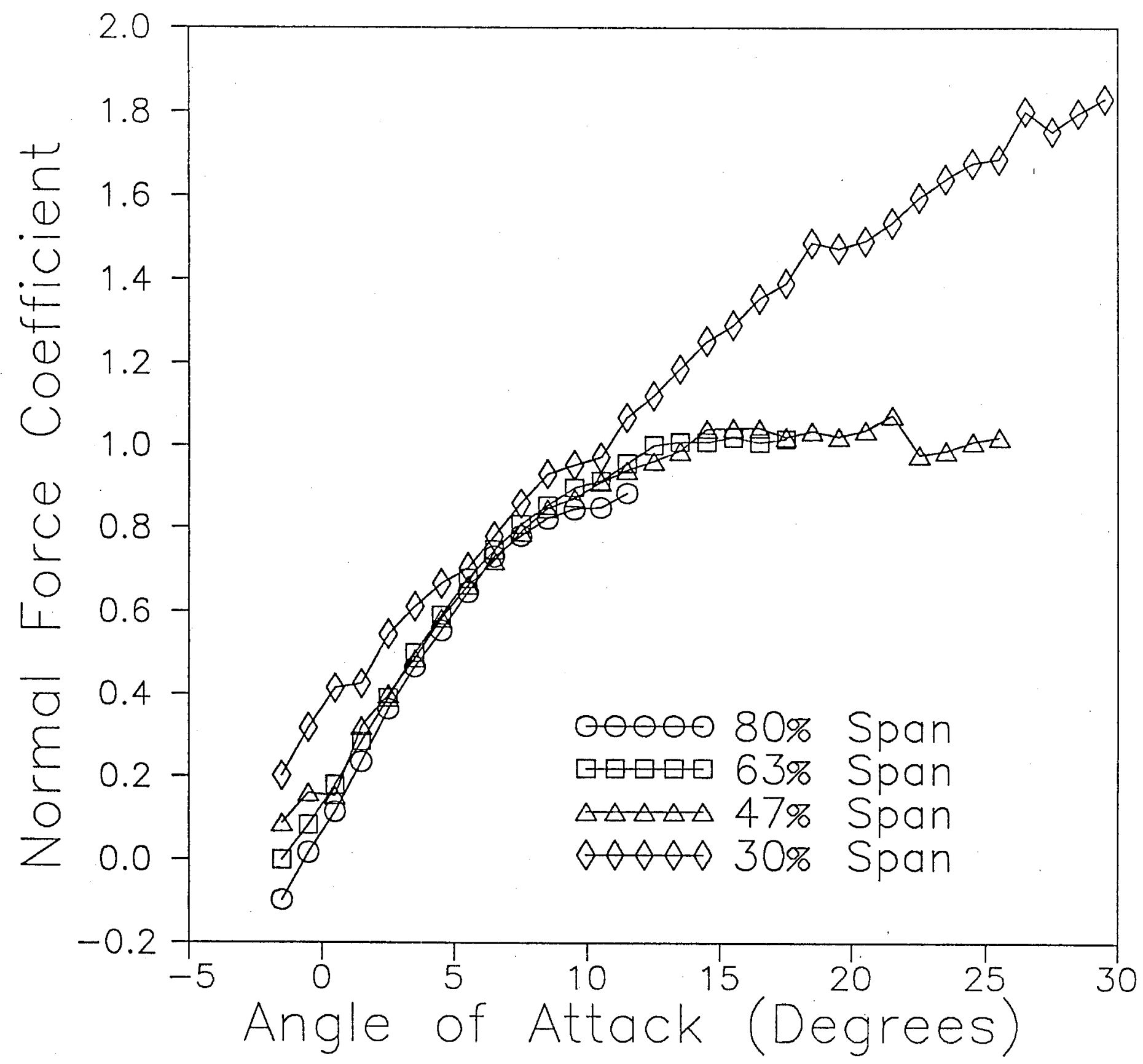

$7 i g u r e$
3.4 


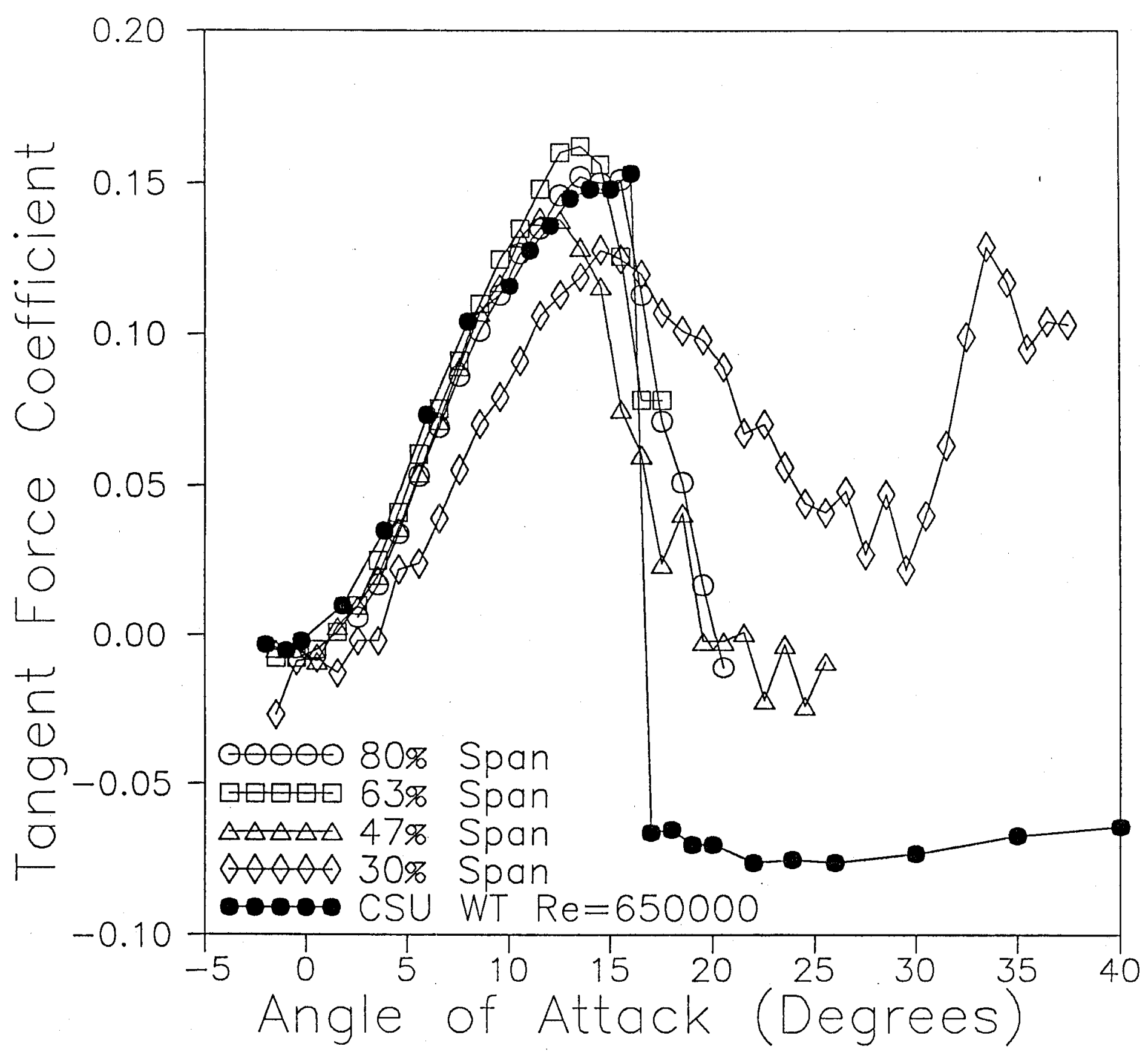

7 igure
3.5

$=.2 .5$ 
and the thrust coefficients at $63 \%$ span. Torque coefficients are more than three times the wind-tunnel results, which would translate into higher power and increased service loads on the gearbox and drivetrain. Similarly, the thrust coefficients are up to twice the wind-tunnel values. These loads are transmitted directly into the blades and wind turbine supporting structure. In terms of wind turbine design, discrepancies such as these would be unacceptable for predicting the structural fatigue life of key wind turbine components.

Figure 3.6. Torque and thrust coefficient comparisons for rotating-blade and wind-tunnel data

Figure 3.7 shows a comparison of normal and tangent coefficients for the rotating-blade and wind-tunnel data cases. The discrepancy near stall is obvious.

Figure 3.7. Rotating blade normal and tangent force coefficient comparisons with wind-tunnel data

Aerodynamic performance is also evaluated by the lift and drag coefficients, $C_{1}$ and $C_{d}$, respectively. At low AOAs, $C_{1}$ is approximately equal to $C_{N}$. As the AOA increases the deviations are greater. These relationships are given in equations (3.10) and (3.11). These measurements give a more intuitive picture of the airfoil performance, because most airfoils are documented by measuring lift and drag.

$$
\begin{gathered}
\mathrm{C}_{\mathrm{l}}=\mathrm{C}_{\mathrm{N}} \cos (\alpha)+\mathrm{C}_{\mathrm{T}} \sin (\alpha) \\
\mathrm{C}_{\mathrm{Dp}}=\mathrm{C}_{\mathrm{N}} \sin (\alpha)-\mathrm{C}_{\mathrm{T}} \cos (\alpha)
\end{gathered}
$$

Lift coefficients, shown in Figure 3.8, have characteristics very similar to those of the normal-force coefficients shown in Figure 3.4. In a side-by-side comparison, the two curves are difficult to distinguish.

Figure 3.8. Lift coefficient comparisons throughout blade span

Pressure-drag data are shown in Figure 3.9. Below stall, the wind turbine blade data show the usual agreement with the wind-tunnel data. Beyond stall, the wind turbine drag values are greater than windtunnel values. This is a surprising result, when one considers that the tangent forces were greater than the wind-tunnel data. However, Equation (3.11) shows that the drag term can be dominated by $\mathrm{C}_{\mathrm{N}}$ if AOAs are large enough. Because $\mathrm{C}_{\mathrm{N}}$ is typically large compared to $\mathrm{C}_{\mathrm{T}}$, this is not particularly surprising.

Figure 3.9. Pressure drag coefficient comparisons throughout blade span

By understanding the effect of blade rotation and stall on these coefficients, improved methods for predicting wind turbine performance may result.

\subsubsection{Airfoil Pressure Distributions}

This section describes highlights of the bin-averaged pressure distribution results. The complete catalog of the pressure distributions is presented in Appendices C and D. These pressure distributions are correlated with two different measured parameters to provide two independent means for validating the data measurements, and to provide a separate perspective from which the data can be examined. First, in Appendix $\mathrm{C}$ measured $\mathrm{AOA}$ was used to correlate the pressure data. This method gives a convenient format for comparing the behavior of the four spanwise stations at the same AOA. However, since the AOA measurements are made directly on the rotor, they are subject to the effects of wake-induced velocities that could have influenced the data. 
Thrust and Force Coef. Comparison for the $\$ 809$ Airfoil

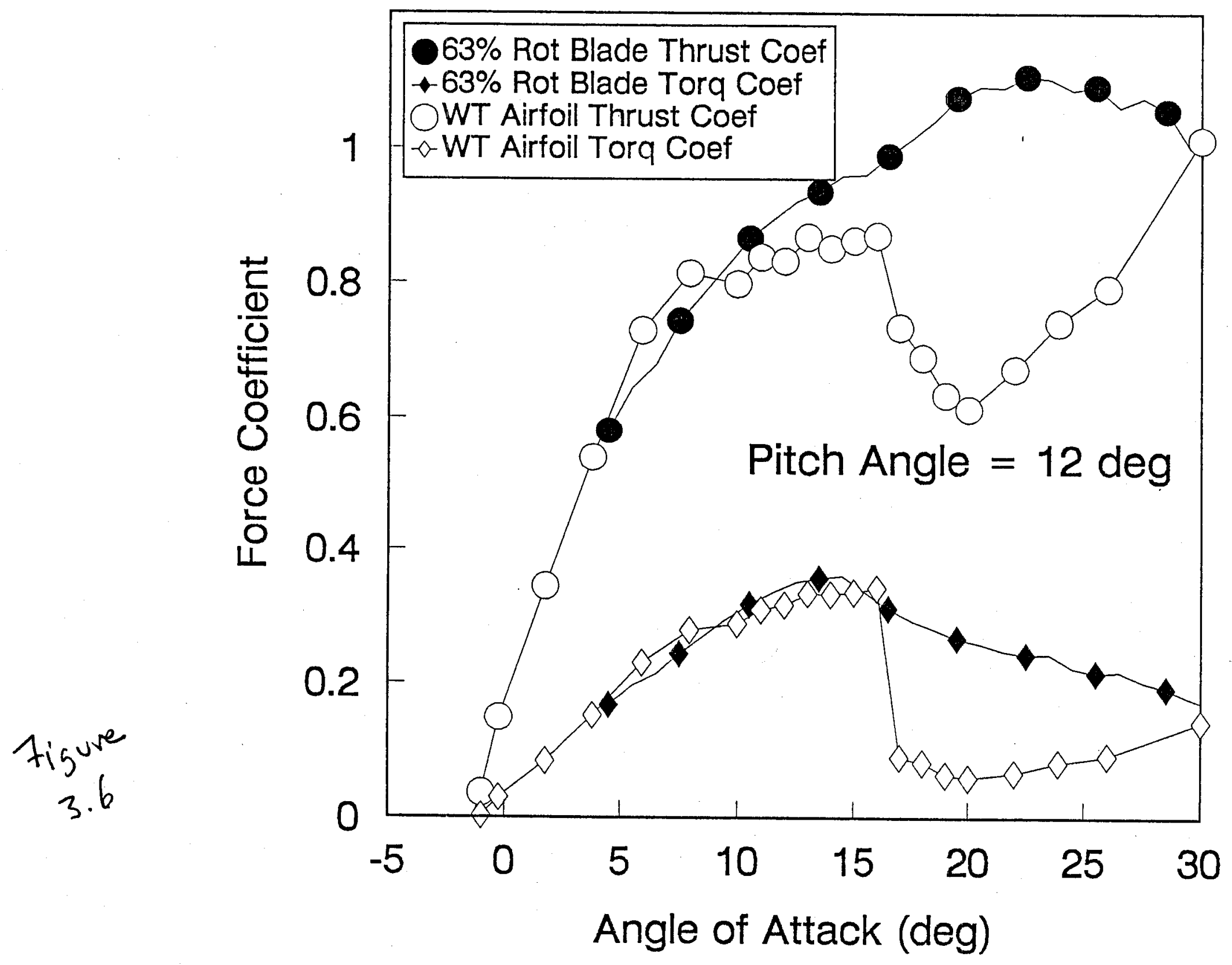


Wind Tunnel and Wind Turbine Comparison For the $\$ 809$ Airfoil

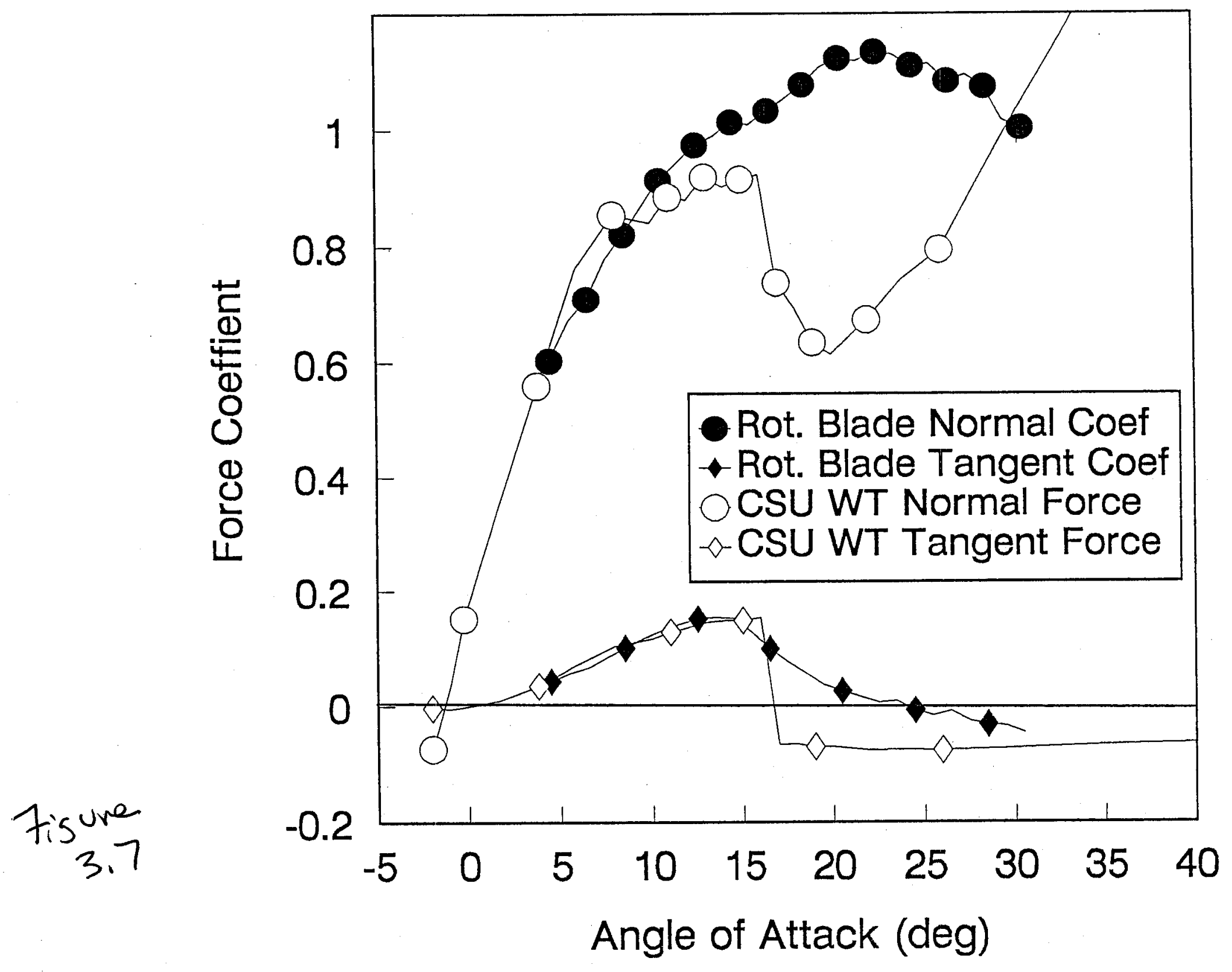




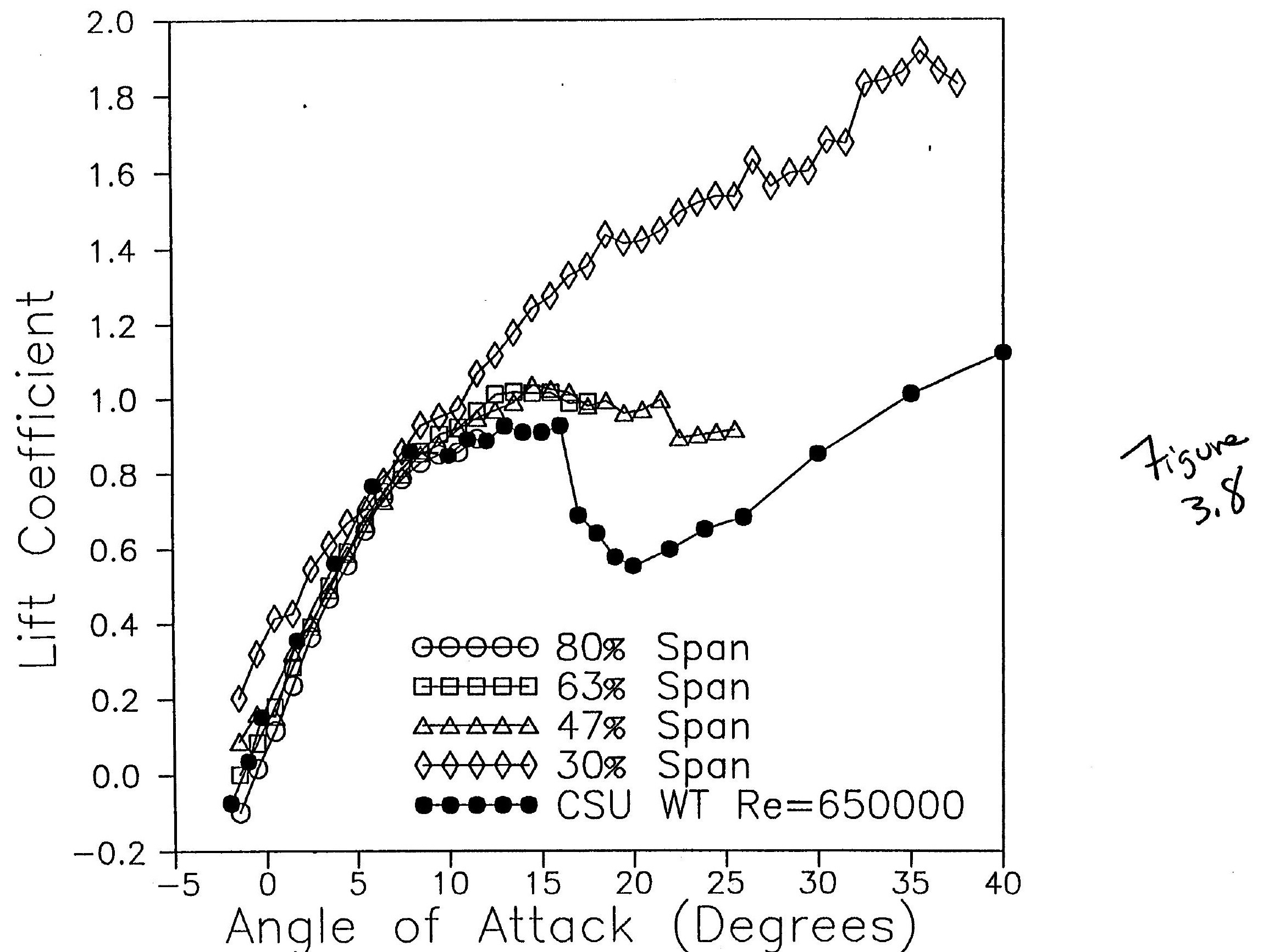




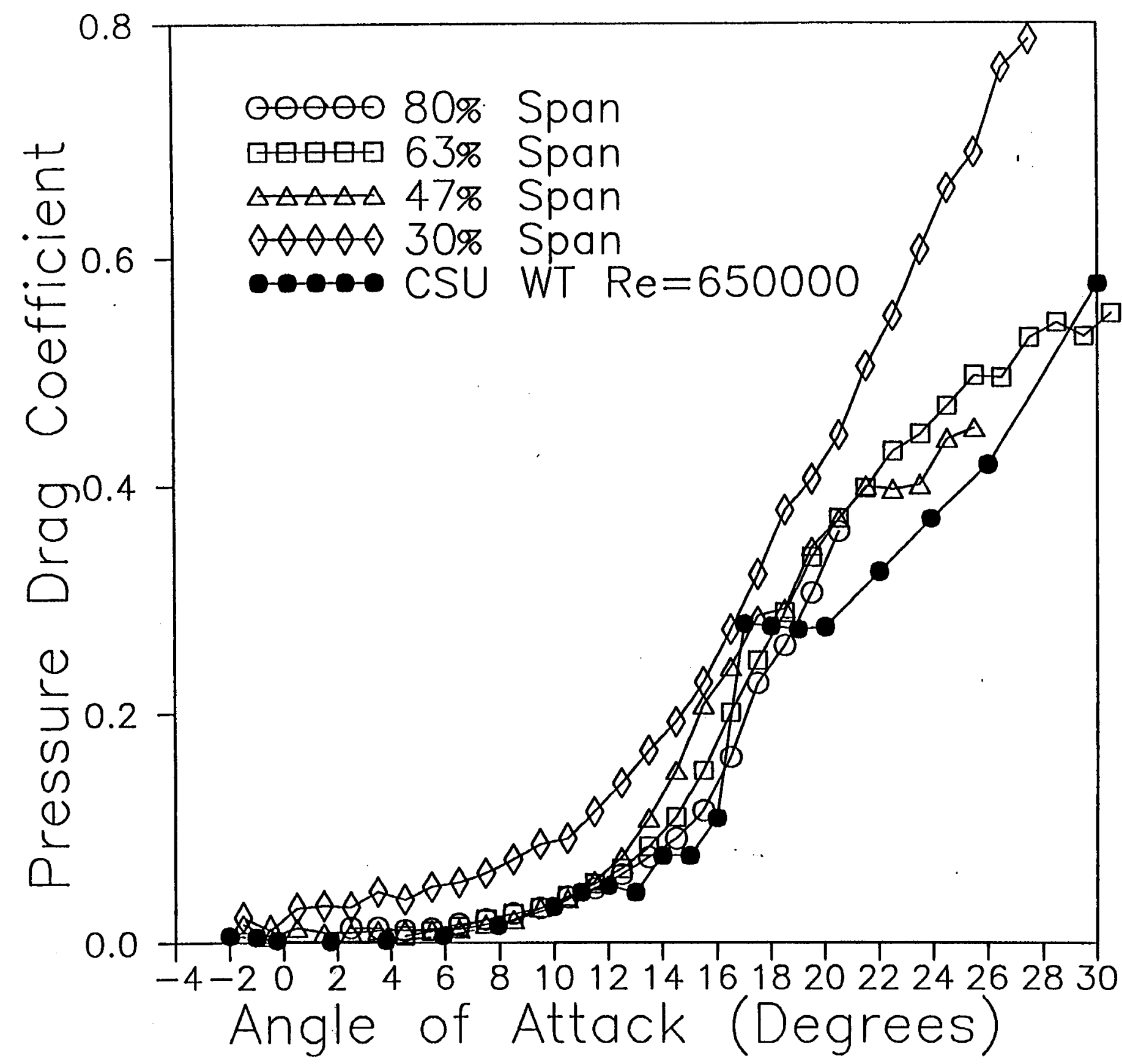

7igure 3.9 
In Appendix D, measured wind speed is used to correlate the data. In this appendix, two different data sets were used to obtain the complete range of wind speeds. The wind-speed correlation is presented as a means of relating the pressure measurements to a measured quantity that is not influenced by rotorinduced effects. These plots also provide a means for comparing the behavior of each of the spanwise distributions at the same point in time.

In the body of this report, only the pressure distribution comparisons based on AOA are discussed. Figure 3.10 shows one such comparison at $63 \%$ span and a low AOA of $2.5 \mathrm{deg}$. The good correlation at this low angle helps to confirm the integrity of the data-acquisition system and data-reduction techniques. The close agreement indicates that the airfoil is behaving in a two-dimensional manner for this attached flow condition.

Figure 3.10. Pressure distributions comparison at $63 \%$ blade span and 2.5 deg AOA

Figure 3.11 shows the same $63 \%$ span station at $9.5 \mathrm{deg}$ AOA. At this angle the suction peaks on the pressure distribution are fully developed. These data show good correlation with the wind-tunnel suction peaks as well as between the high-pressure-side (bottom) pressure coefficients. The flat regions behind $60 \%$ chord on the low-pressure side of the pressure distributions indicate that flow separation has occurred in both the wind tunnel and on the rotating airfoil. The pressure gradient that begins about mid-chord indicates the point of flow attachment. The wind-turbine data show this attachment point at $65 \%$ chord whereas the wind-tunnel data show attachment at $55 \%$ chord. The difference in integrated area under the pressure distribution curves is $5 \%$ to $10 \%$ higher for the wind turbine for this range of AOA.

Figure 3.11. Pressure distribution comparisons at $63 \%$ blade span and 9.5 deg AOA

This discrepancy was consistently observed between the two data sets. As the AOA rises and stall separation progresses forward from the trailing edge, the point of separation is generally further aft in the wind-turbine data at the same AOA.

Figure 3.12 shows a wind-nunnel pressure distribution at $18.5 \mathrm{deg} \mathrm{AOA}$ and a family of wind-turbine curves for the $63 \%$ span ranging from $18.5 \mathrm{deg}$ to $21.5 \mathrm{deg}$ AOA. The wind-tunnel data are flat across the entire low-pressure side of the airfoil, incicating leading-edge separation. In contrast, the wind-turbine data clearly show that the suction peaks and pressure gradients are sustained to AOAs higher than $23.5 \mathrm{deg}$.

Figure 3.12. Pressure distributions at $63 \%$ blade span for post-stall AOAs

Figure 3.13 shows data for similar AOAs at the $80 \%$ span. The suction peak appears to be more pronounced at this station, and the pressure gradient following the peak is well defined. In both the $80 \%$ and the $63 \%$ cases, the existence of suction peaks and higher negative pressures along the low-pressure side of the airfoil results in the higher-than-expected normal force coefficients shown in Figures 3.3 and 3.4.

Figure 3.13. Post-stall pressure distributions at $80 \%$ blade span

Results from the $47 \%$ span pressure measurements are very similar to those for the $63 \%$ span. These results are shown in their complete form in Appendix $\mathrm{C}$.

Figure 3.14 shows results from the $30 \%$ span measurements. At this inboard location the same behavior is continued, with good correlation at low angles (Appendix C) and delayed stall at high angles. In this 

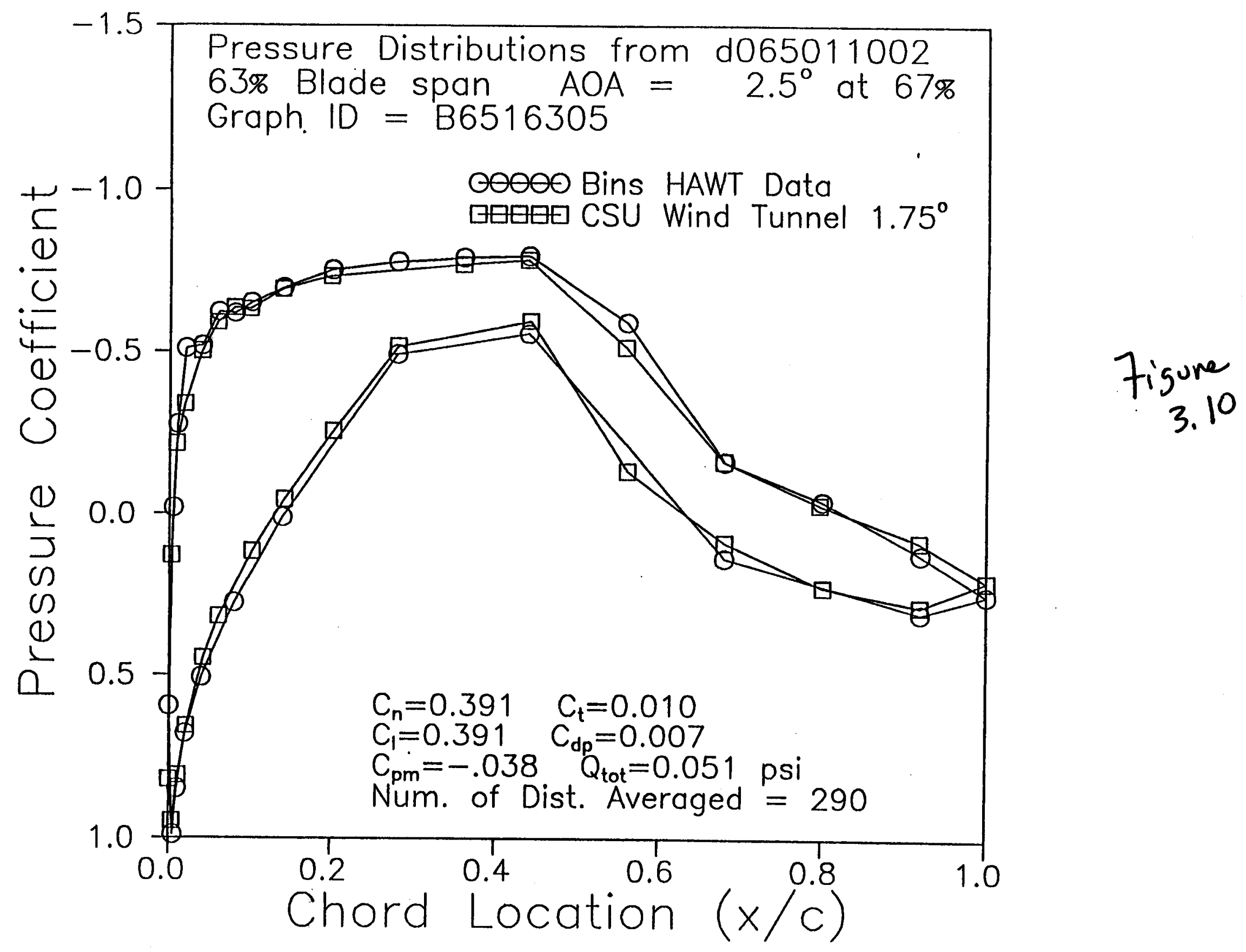

7.. $? \ln$ 


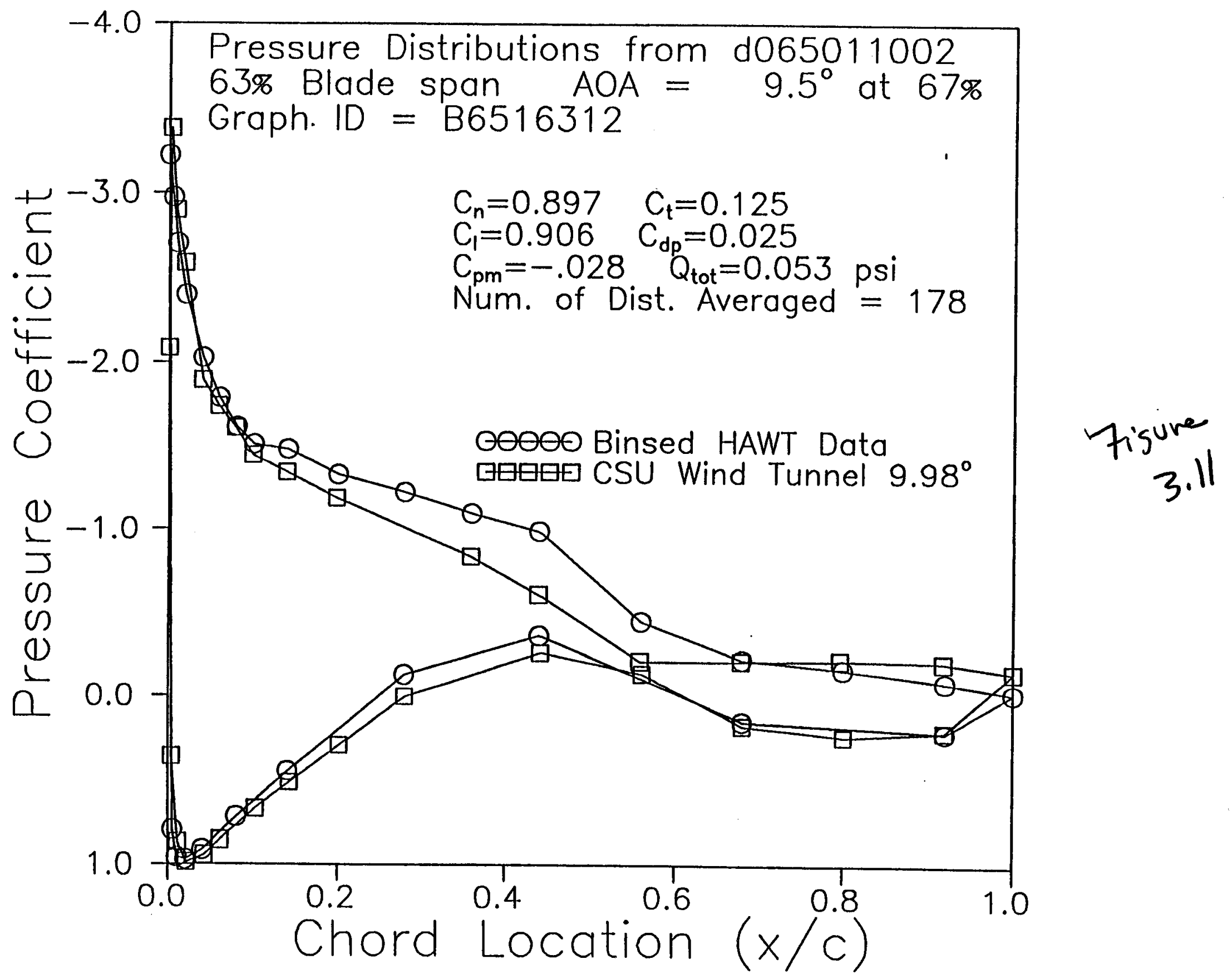




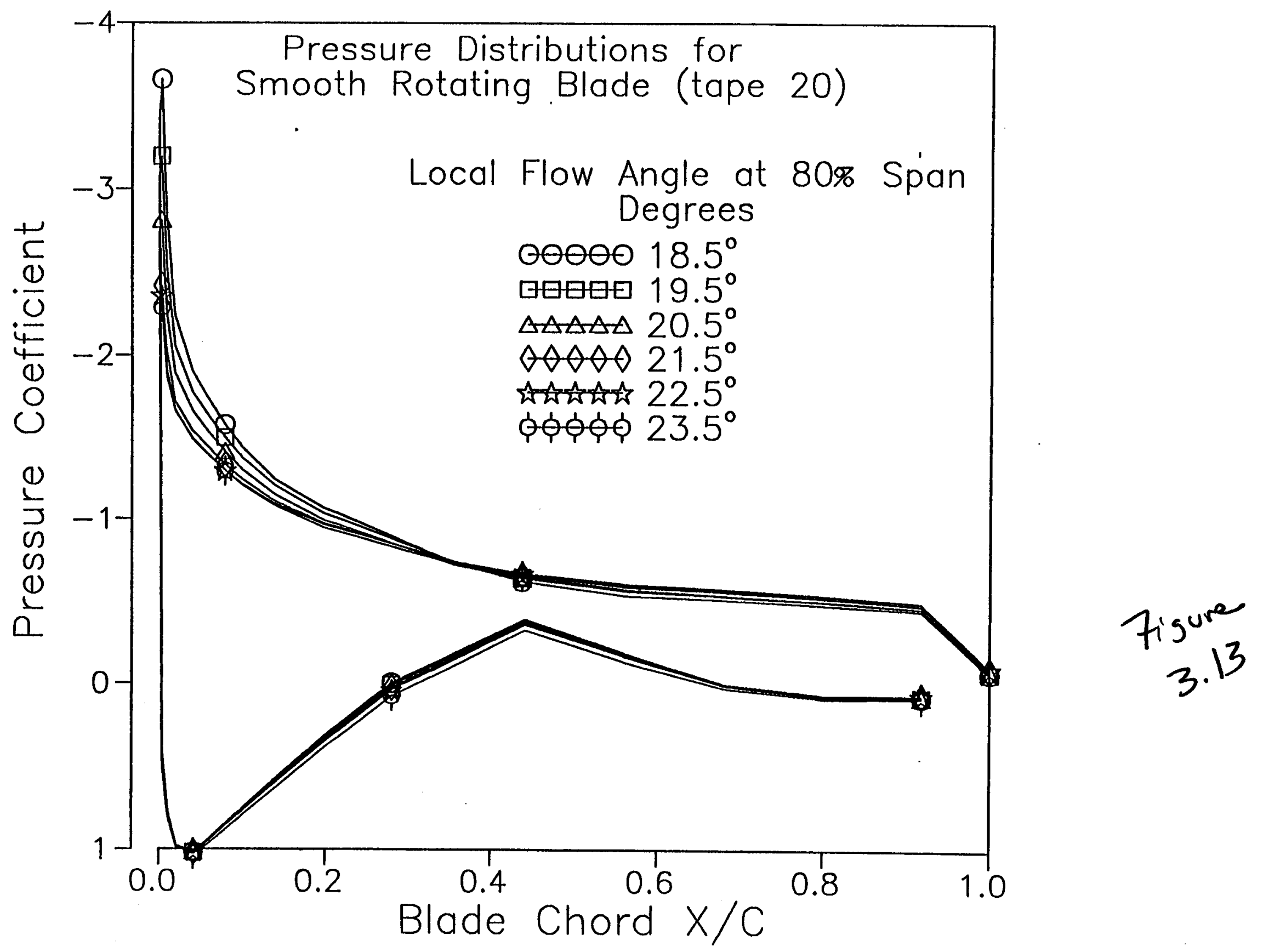


case the low-pressure-side pressure coefficients are more negative than at the outboard stations. The larger area under the rotating-blade pressure curve results in higher values of $C_{N}$ and $C_{L}$ at high AOAs.

\section{Figure 3.14. Post-stall pressure distributions at $\mathbf{3 0 \%}$ blade span}

The existence of suction peaks and pressure gradients on the low-pressure side of the high-AOA wind turbine pressure distributions leads the authors to the conclusion that stall was delayed on the rotating airfoils. This is reasonable because these characteristics are commonly used to identify attached flow in wind-tunnel pressure measurements. Conversely, their disappearance is used to determine stall. Therefore, it would follow that the pressure gradients such as those shown in Figure 3.14 indicate attached flow in spite of the high AOAs. However, by using the flow-visualization studies that were conducted concurrently with the pressure measurements, it was demonstrated that the flow is actually separated over most of the airfoil surface. This was accomplished using video data of tufts time-correlated with pressure distributions. These flow-visualization measurements are discussed in detail in Section 5.2, and by Scott et al. (1991). They have led to the surprising conclusion that the pressure gradients measured on the wind turbine exist in the presence of separated flow.

The higher suction pressures on the wind turbine explain why the integrated force coefficients are higher but do not explain what is causing the pressure distributions to be consistently different from wind-tunnel data measured on the same airfoil. There are some possible explanations, but they are very speculative. One possibility is that the chordwise pressure gradients may be caused by spanwise flow in the separation region. Another is that vorticity in the separation bubble may be influencing the surface pressures. Future tests and data analysis will be focused on the answers to these questions.

\subsubsection{Spanwise Pressure Distributions}

This section presents the results from pressure measurements that were made along the blade span. Pressure taps were located on the low-pressure surface at both the $4 \%$ chord and the $36 \%$ chord locations at 10 spanwise locations. The taps at each of the primary stations were supplemented by two additional pressure taps in between primary stations. The $4 \%$ chordwise position was chosen because it is close to the location of the suction peak on the airfoil. The $36 \%$ chordwise position gave another cross-section of the pressure distributions at a point behind the pressure peak, but on a significant part of the pressure distributions. The blade layout and pressure-tap locations are described in Appendix $A$ and in more detail in Butterfield et al. (1992).

In this section the pressure coefficients $\left(C_{p}\right)$ were normalized by the local dynamic pressure as described in Equation 3.11 of Section 3.2. These spanwise distributions are bin-averaged and are presented as a function of wind speed. No comparisons with predictions are made.

These two rows of pressure taps made it possible to measure spanwise pressure gradients in addition to the chordwise distributions shown in Section 3.3.2. The pressure measurements that were made between the chordwise distributions provide the resolution necessary for examining the spanwise pressure gradients.

Figures 3.15 through 3.18 show the spanwise pressure distributions for the $4 \%$ and the $36 \%$ chordwise stations. Each plot shows a family of spanwise pressure distributions for a range of wind speeds.

Figure 3.15. Bin-averaged spanwise pressure coefficients for the $4 \%$ chord for $8-14 \mathrm{~m} / \mathrm{s}$ wind speeds

Figure 3.16. Bin-averaged spanwise pressure coefficients for the $4 \%$ chord for $14.5-19.2 \mathrm{~m} / \mathrm{s}$ wind speeds 


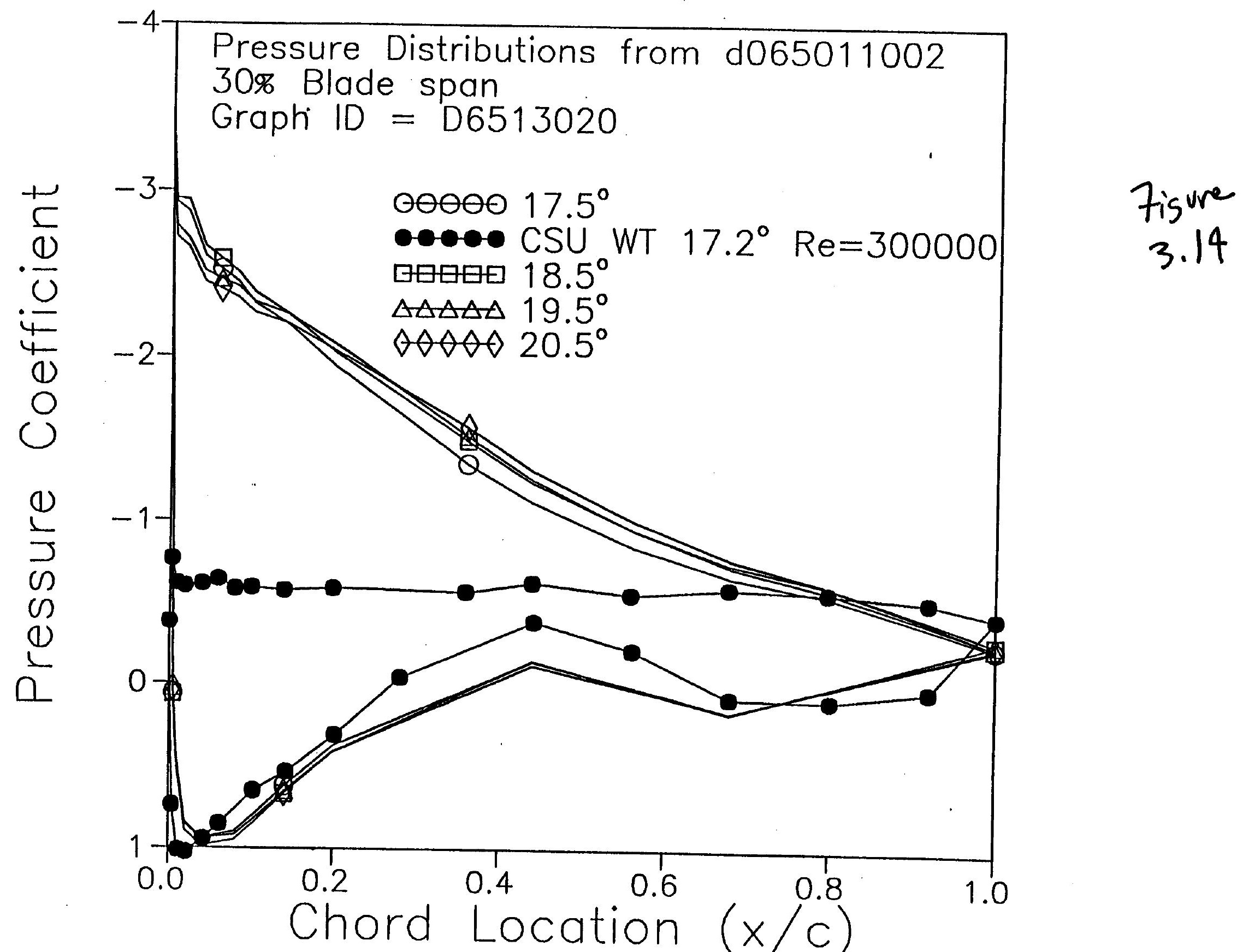


Tape 68 , Data

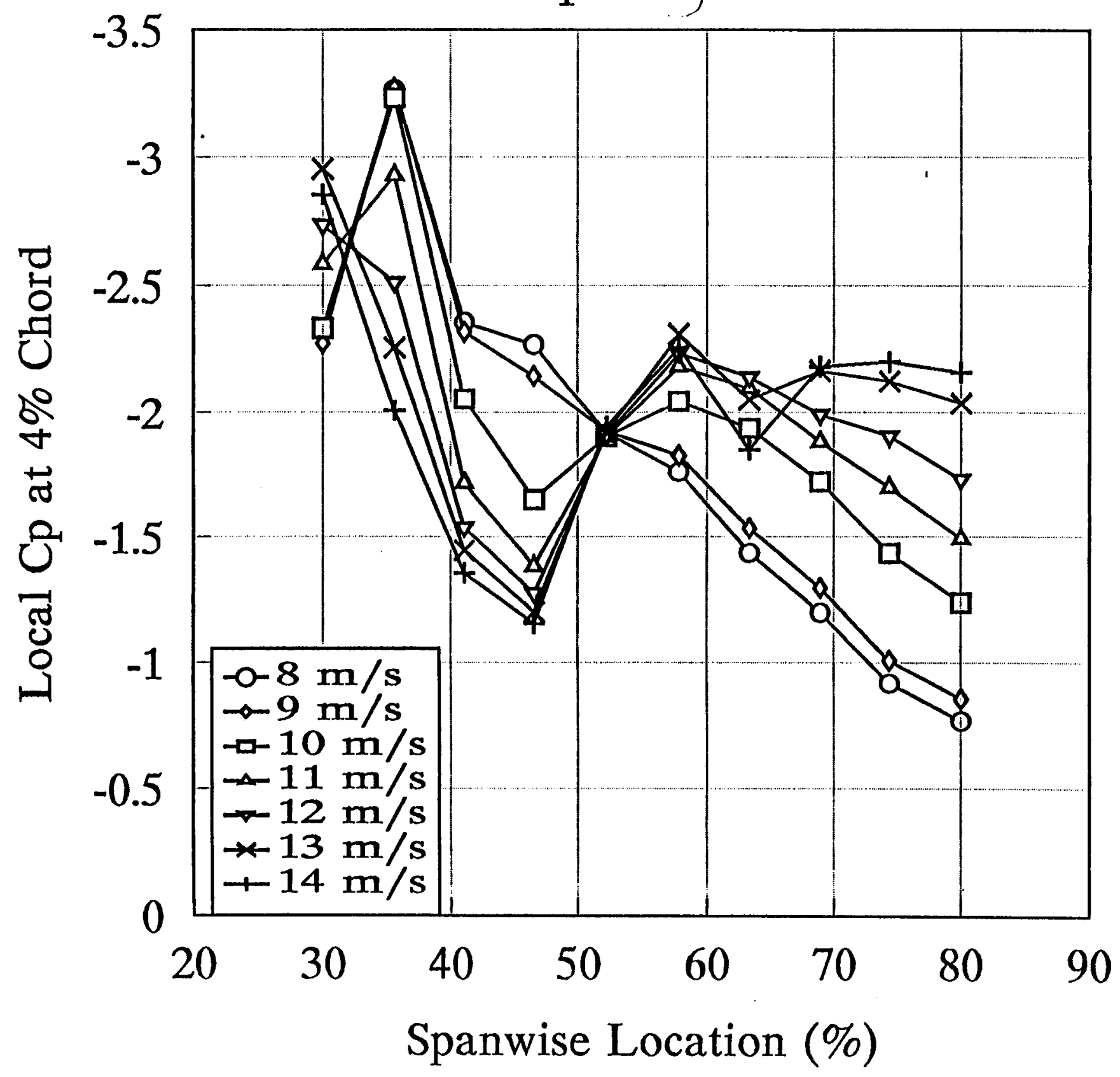

7isure 


\section{Tape 68 Data}

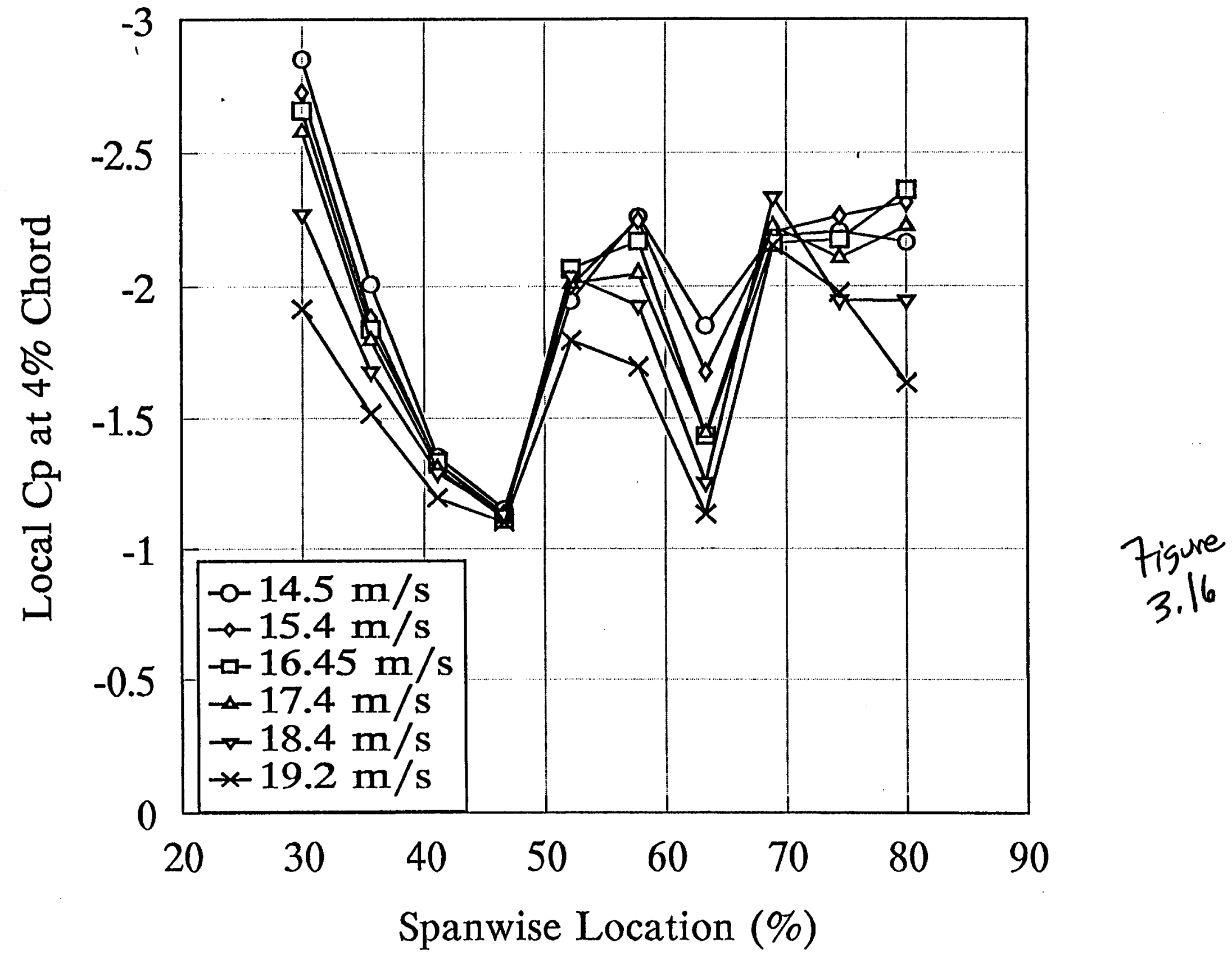


Figure 3.17. Bin-averaged spanwise pressure coefficients for the $36 \%$ chord for $8.8-14.5 \mathrm{~m} / \mathrm{s}$ wind speeds

Figure 3.18. Bin-averaged spanwise pressure coefficients for the $36 \%$ chord for $14.5-19.2 \mathrm{~m} / \mathrm{s}$ wind speeds

The first curve in Figure 3.15 shows pressure coefficients decreasing from the $30 \%$ span to the $80 \%$ span at the $4 \%$ chord and $8 \mathrm{~m} / \mathrm{s}$ wind speed. At this low wind speed the inboard stations are operating at high AOA and the outboard stations are operating at low AOA. Because of this AOA distribution, the suction pressures at $4 \%$ chord are high inboard and steadily decrease toward outboard stations, as expected. However, as wind speed and mean AOA increase, conventional theory would predict stall separation at inboard stations to result in suction pressures less than 1 because of leading-edge stall, and outboard suction pressures to dramatically increase where the flow becomes reattached. However, for the high-windspeed cases, shown in Figure 3.16, inboard suction pressures are significantly greater than expected, reaching values over 2.5. This is not surprising because the data from Section 3.3.2 showed that the pressure peaks were sustained at high AOAs for the inboard station.

Further outboard, near the 47\% span, these curves exhibit irregular behavior, with a precipitous drop followed by a rise near midspan. As wind speed continues to increase, a second drop in pressures develops at the $63 \%$ span, followed by a rise near the far out-board stations.

Figures 3.15 and 3.16 show high inboard suction pressure and unexpected drops in the spanwise pressure coefficients at $47 \%$ and $63 \%$ span, but for high winds only. The cause of this behavior is unknown.

Figures 3.17 and 3.18 show similar trends in the $36 \%$ chord spanwise distributions. Again, it is expected that pressure coefficients would increase at outboard stations and decrease at inboard stations in high winds. This trend can be seen, but the erratic curves are unexpected. Local stall separation could explain a single dip in spanwise pressure coefficient curve. Only one such decrease would be expected, and its location should progress outboard as wind speed increases.

Similar irregularities can be seen in the unsteady instantaneous spanwise pressure distributions presented in Section 4.6. In the unsteady case, one would expect to see a spanwise progression of stall boundaries, yet unexpected dips in the curves are evident again. It is possible that instrumentation error could cause inconsistent behavior, but the dips only appear at high wind speeds. If the instrumentation were the cause, then the dips would be expected to exist at all wind speeds. Future tests that focus on the spanwise pressure distributions could help determine the source of this odd behavior.

\subsection{Conclusions}

The data show the averaged behavior of rotating aiffoils located at $30 \%, 47 \%, 63 \%$, and $80 \%$ spanwise blade stations on a stall-controlled wind turbine operating over a range of conditions. Several conclusions can be drawn from these data:

- Rotating airfoils behave as they would in the wind tunnel for angles of attack below stall.

- Wind-turbine airfoils operating in quasi-steady conditions show a delay in stall due to a combination of suction-peak persistence and high negative pressures on the suction side of the airfoil.

- Delayed stall observed on rotating airfoils has resulted in high normal forces and high tangent forces in the poststall region that are uncorrelated with wind-tunnel stall measurements. 
Tape 68 Data

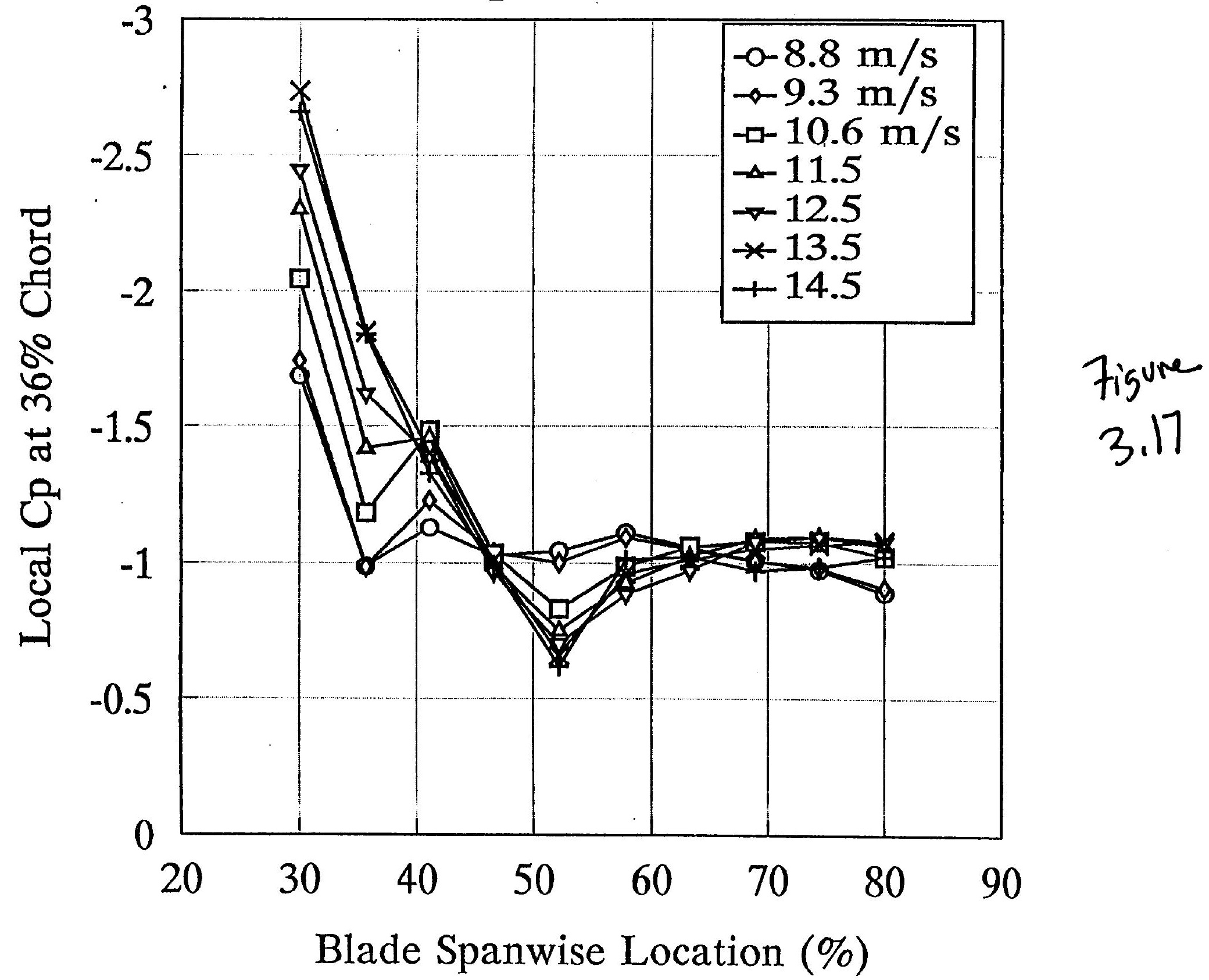




\section{Tape 68 Data}

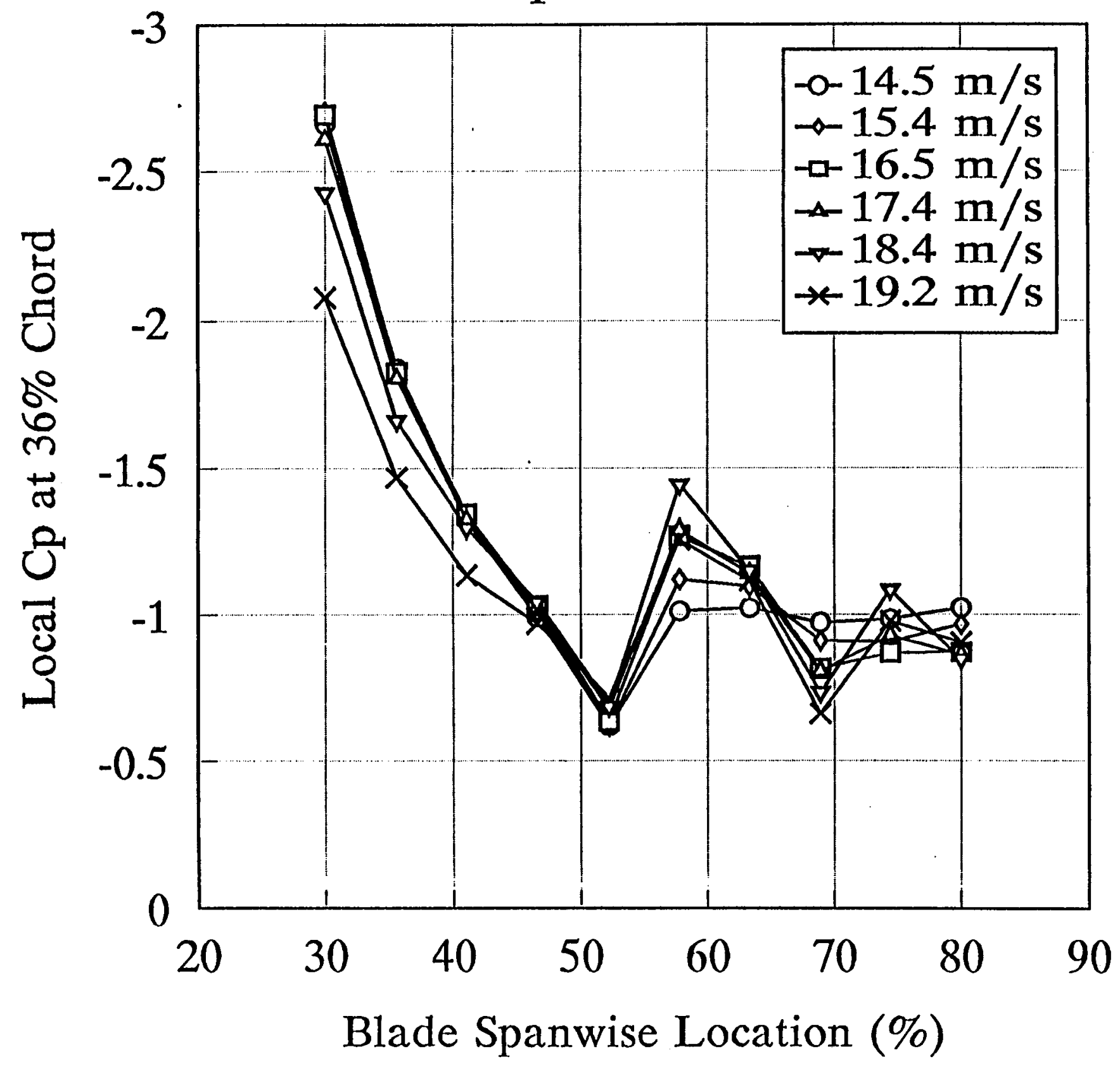

7igure

3.18 
- The high suction-pressure peaks and pressure gradients measured on the wind turbine during delayed stall at high AOAs exist in the presence of separated flow.

- The performance deviations on wind-turbine airfoil force coefficients caused by delayed stall are more pronounced at inboard blade stations. While outboard stations experienced only a delayed stall effect, maximum values of the inboard force coefficients were increased to over twice the values measured in the wind tunnel. Some of the behavior of spanwise pressures was erratic and inexplicable.

- The airfoil behavior on a fixed-pitch wind-turbine blade is modified significantly enough by stall effects that wind-tunnel stall data may not be accurate for design purposes.

- The causes of this behavior are not understood yet, and the current Combined Experiment test configuration is not equipped to identify the causes. It is not known what effects blade planform (twist and taper) will have on the airfoil performance. Future work must be conducted to understand the complexities of this problem. 


\subsection{Unsteady Aerodynamic Loads}

\subsection{Introduction}

Wind turbines commonly operate in steady stall as a means of regulating peak power and loads. It was shown in Chapter 3.0 of this report and previously by others (Butterfield et al, 1991; Musial and Butterfield, 1990; and Viterna and Corrigan, 1981) that airfoils do not stall on rotating wings as they do in wind-tunnel tests under quasi-steady conditions. The helicopter industry has ignored steady stall because helicopters avoid operating conditions that would result in large-scale steady stall. However, they have invested significant research time into understanding dynamic stall, a condition that helicopters cannot avoid.

Wind turbines are also subjected to dynamic loading. Wind shear and turbulence cause time-varying inflow that results in unsteady airloads. Tower shadow, wakes from upwind turbines, and yaw errors also introduce unsteady inflow to wind-turbine rotors. Wind-turbine designers must predict these loads in order to adequately design blades, hubs, and the remaining support structure to achieve a 30-year life. Structural analysts have not been able to predict mean or dynamic loads accurately enough to confidently determine the fatigue life of major wind-turbine components.

Part of the problem is due to the uncertainty in the stochastic wind environment. Another important factor is the lack of basic knowledge of rotary-wing airfoil stall performance. Through an earlier effort, Butterfield (1989) found that dynamic stall can exist on wind-turbine blades during normal operating conditions. There is also mounting evidence that this may contribute to higher dynamic loads. Wright and Butterfield (1991) and Hansen and Butterfield (1990) showed improved correlations with measured dynamic loads and yaw loads when dynamic stall was introduced into their analyses. Semi-empirical dynamic-stall models used by the helicopter industry may be applicable to wind turbines for predicting these dynamic loads. However, they will require validation using measurements from operating wind turbines. The dynamic-stall data collected during the Combined Experiment have provided researchers with an opportunity to perform this validation and adapt these models for wind turbines. Some of the work being conducted at the University of Utah is focused on this code development.

This chapter gives a complete summary of the work performed by Butterfield (1989) along with the most recent empirical findings. Dynamic-stall predictions are not discussed. Dynamic-stall measurements are described at four blade spanwise stations of a rotating wind-turbine blade. Loads are correlated with the measured airloads. Finally, dynamic stall is related to increased yaw moments.

\subsection{Data Preparation}

\subsubsection{Test Cases}

The sign convention used for this analysis assumes that the yaw error is the compass angle of the turbine minus the wind direction. It is important to note that the rotor's direction of rotation has a major influence over dynamic-stall response caused by yawed flow. For this turbine, the rotor spins clockwise when viewed from a downwind location. The instrumented blade is pointing up when in the zero-degree azimuth position.

Two yaw cases were chosen for analysis. The first was at 30 deg yaw error and spanned 20 seconds at wind speeds of $13.5 \mathrm{~m} / \mathrm{s}$. The second case was at zero yaw error and spanned 10 seconds at wind speeds of $15 \mathrm{~m} / \mathrm{s}$. These two cases are compared in Table 4.1 and were chosen to illustrate the conditions that cause dynamic stall. 
Table 4.1

\begin{tabular}{lcc}
\hline & $3^{\circ}$ Yaw Case & Zero Yaw Case \\
\hline VPA Ave. Wind Speed (m/s) & 13.67 & 15.39 \\
Ave. Turb. Intensity & 0.14 & 0.06 \\
Shear (m/s) & 1.24 & 0.72 \\
Pitch Angle (degree) & 11.3 & 11.54 \\
Pitch Std. Dev. (degree) & 0.33 & 0.21. \\
Rotor Torq. (N-m) & 1392 & 1976 \\
\hline
\end{tabular}

The 30 deg yaw error case is typical of conditions that normally occur for yaw-driven and free-yaw HAWTs. Yaw-driven wind turbines typically respond to wind-direction changes at low yaw rates (1-5 deg per second) to limit gyroscopic bending moments on the main-shaft. Wind direction can change at much higher rates and result in yaw errors of 30-40 deg while the turbine's yaw system is catching up with wind direction. In free-yaw wind turbines, tracking problems, slow yaw response, and dynamic yaw overshoot can lead to the same high yaw errors experienced by yaw-driven machines. In either case, the yawed flow case represents a realistic operating condition that may be typical for many other wind-turbine configurations.

\subsubsection{Pressure Coefficient Normalization}

All the pressure data were digitally sampled at a $520 \mathrm{~Hz}$ during testing. During post-processing, data were filtered at $10 \mathrm{~Hz}$ and block-averaged by a factor of 10 to obtain a final $10 \mathrm{~Hz}$ bandwidth and $52 \mathrm{~Hz}$ sample rate. These rates are different from those in Section 3.0. These data were then sorted into bins using the measured AOA as the independent variable. This general approach was used for results presented in Figures 4.3 through 4.19.

Three different methods can be used to determine the dynamic pressure needed to normalize measured pressure distributions. The first two methods are described in Equations 3.4 and 3.5 of Section 3.0 . Equation 3.4 shows the empirical method. It depends solely on measured quantities and therefore reflects all the flow asymmetries in dynamic pressure. The second method uses Equation 3.5 and yields a constant value for a given wind speed. It does not correct for wind shear, tower shadow, or yawed flow conditions, all of which are common for wind-turbine rotors. The third method is an analytical approach that was developed to correct for the effects of yawed flow by allowing the dynamic pressures to vary azimuthally.

This third method and its effect on unsteady data are illustrated in Figure 4.1. This figure shows measured and predicted dynamic pressures during $30 \mathrm{deg}$ yawed operation at the $67 \%$ span. The effects of the tower shadow are visible on the measured dynamic pressure ( $Q$ ) curve at approximately 180 deg. Equations 4.1 and 4.2 were used to calculate the predicted dynamic pressure curve in Figure 4.1. The resulting values include the vector sum of the free-stream velocity, and the in-plane blade-velocity component, which take into account the effects of the advancing and retreating blade in yawed flow. A comparison of these two curves shows very good correlation between the measured data and the data normalized by the third method. This agreement provides a good validation of the measured data.

Figure 4.1. Dynamic-pressure variations during yaw 
30 Yaw Case

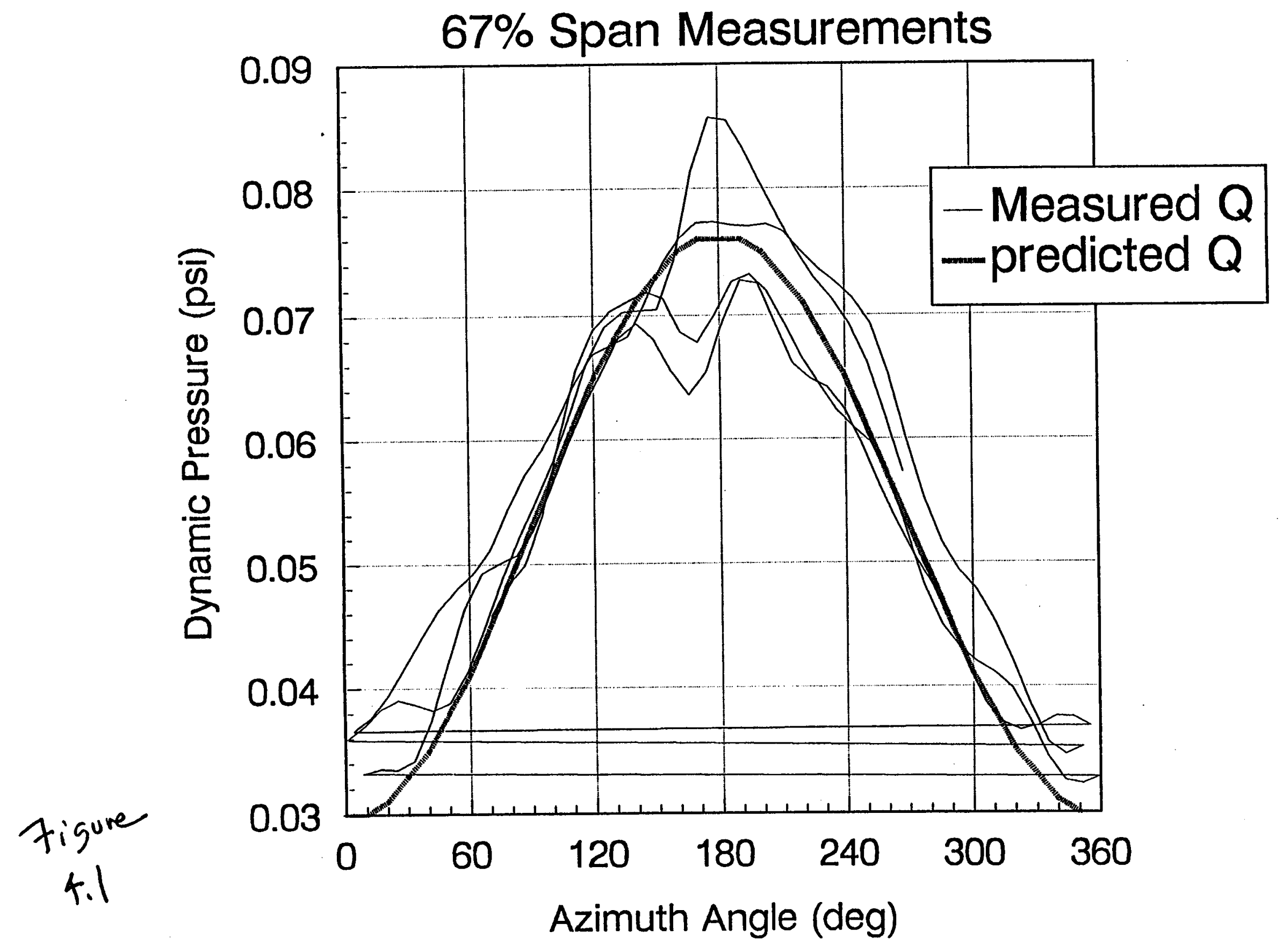

$[\quad, \quad 7$ is 4.1 


$$
\begin{gathered}
Q=\frac{1}{2} \rho\left(V^{2}+(\pi \Omega)^{2}-2 V r \Omega \sin \phi\right) \\
\phi=\phi_{0} \sin \Psi
\end{gathered}
$$

where:

$$
\begin{aligned}
& \phi_{0}=\text { Yaw Angle } \\
& \psi=\text { Azimuth Angle } \\
& V=\text { Wind Speed } \\
& \rho=\text { Air Density } \\
& \text { I = Local Blade Radius } \\
& \mathrm{Q}=\text { Dynamic Pressure. }
\end{aligned}
$$

Figure 4.2 shows the lift coefficient during dynamic stall with constant (uncorrected) $Q$, which was calculated per Equation 3.5, and azimuthally varying (corrected) Q, which was calculated using Equations 4.1 and 4.2. This shows that the effects of dynamic stall can vary depending on the normalization used.

\section{Figure 4.2. Effects of time-varying dynamic-pressure normalization}

There is no clear consensus among aerodynamicists about which normalization should be used on rotating airfoils. The measured data from Equation 3.4 might seem to provide the most realistic normalization; but because the measurements are made directly in front of the rotating blade, they may be subject to wakeinduced velocities that could affect the measurement accuracy. Therefore this technique is met with some skepticism. The corrected, azimuthally varying method is also subject to measurement errors caused by the calculation of yaw error, which combines the yaw angle measurement made on the turbine with the instantaneous wind direction measurement made on the Vertical Plane Array (VPA). Spatial separation between the turbine and the VPA will introduce an unknown amount of error. In spite of this, the corrected method has merit and is probably a valid technique, because it correlated well with the measured data. However, the current accepted practice is to use the constant-normalization method described by Equation 3.5. Therefore, in this report, all chordwise pressure-distribution data are normalized by local dynamic pressure based on the free-stream velocity using Equation 3.5. In future analyses of these data it may be insightful to use the corrected normalization.

For spanwise airloads, it is appropriate to normalize by the dynamic pressure calculated at the blade tip using the constant $C_{p}$ method This technique provides a more representative full-blade load distribution of the dimensional pressures, because the pressures are all nomalized by the same constant. Normalization of each spanwise location with its unique local dynamic pressure would make station-to-station comparisons difficult. Therefore, all spanwise pressure distributions presented in Section 4.5 are normalized by blade-tip dynamic pressure.

\subsection{Azimuth-Averaged Results}

Figures 4.3 through 4.6 illustrate angle of attack (A.OA) and lift coefficient $\left(C_{\mathfrak{L}}\right)$ variations azimuthaveraged over 25 revolutions for the $30 \mathrm{deg}$ yawed case at $80 \%, 63 \%, 47 \%$, and $30 \%$ blade spans. For reference, the wind-tunnel static stall $C_{L(\operatorname{mx})}$, equal to 0.95 , is provided on each curve. If the airfoil did not stall and the lift curve remained linear even at high values of $A O A$, then $C_{L}$ would follow the cosine 
30 Yaw Case

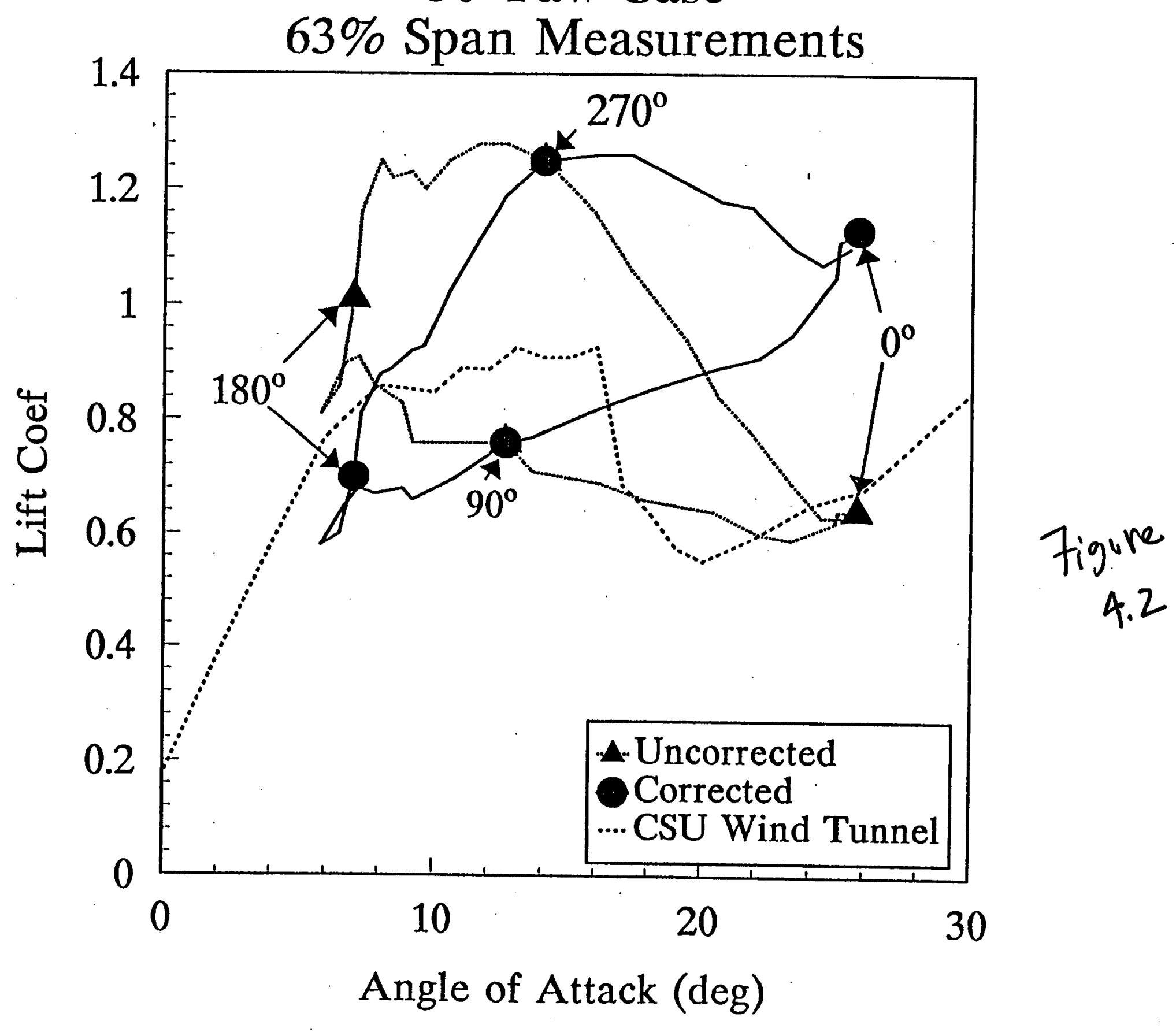


shape of the AOA curves shown in these figures. This is clearly not the case with these data. The inboard stations reach the highest values of $\mathrm{C}_{\mathrm{L}(\max )}$ while the blade is rising (advancing), at azimuth angles of $270 \mathrm{deg}$. Minimum values of $\mathrm{C}_{\mathrm{L}}$ occur after the airfoil has stalled (begun retreating), when the blade azimuth angle is between 0 and $90 \mathrm{deg}$ on the downwind side of the blade. The large differences in lift that exist from the upwind side of the yawed rotor (200 to 300 deg azimuth angle) to the downwind side of the rotor ( 0 to 100 deg azimuth angle) are caused by dynamic stall on the airfoils. This is evident because peak $C_{1}$ values exceed even the higher steady-stall performance described in Chapter 3.0. These dynamic loads are responsible for high yaw moments and increased low-speed shaft (LSS) cyclic loads.

Figure 4.3. Azimuth-averaged AOA and lift coefficient at $80 \%$ span for $30^{\circ}$ yaw case

Figure 4.4. Azimuth-averaged AOA and lift coefficient at $63 \%$ span for $30^{\circ}$ yaw case

Figure 4.5. Azimuth-averaged AOA and lift coefficient at $47 \%$ span for $30^{\circ}$ yaw case

Figure 4.6. Azimuth-averaged AOA and lift coefficient at $30 \%$ span for $30^{\circ}$ yaw case

The tower shadow can be seen in some of the curves as a dip just before an azimuth angle of 180 deg is reached. In zero-yaw flow this dip would be at exactly $180 \mathrm{deg}$. It is important to note that the magnitude of the tower-shadow effect is dwarfed by the effects of yaw.

These figures show that peak-to-peak values of AOA range from $11 \mathrm{deg}$ at the $80 \%$ span to 26 deg at the $30 \%$ span. Because 30 deg yaw errors are not unusual, most horizontal-axis rotors experience AOA cyclic amplitudes of this magnitude, which are large enough to cause dynamic stall. Both fixed-pitch and pitchcontrol rotors would be affected by this.

Figures 4.7 through 4.10 show similar plots of $C_{L}$ and AOA variations for the zero-yaw error case. As can be seen, AOA variations are small compared to the yawed case. In this case, asymmetrical inflow is due only to a wind shear of $0.7 \mathrm{~m} / \mathrm{s}$ across the rotor and to the tower shadow. Unlike the yawed case, tower shadow is the major contributor to inflow disturbances. This is obvious from the rapid change in $A O A$ and $C_{L}$ at a 180 deg azimuth angle. This disturbance is large enough to cause dynamic stall, as is evident from the sudden rise in $C_{L}$ above the static stall $C_{L(\max )}$ of 0.95 .

Figure 4.7. Azimuth-averaged AOA and lift coefficient at $80 \%$ span for no yaw case

Figure 4.8. Azimuth-averaged AOA and lift coefficient at $63 \%$ span for no yaw case

Figure 4.9. Azimuth-averaged AOA and lift coefficient at $47 \%$ span for no yaw case

Figure 4.10. Azimuth-averaged AOA and lift coefficient at $30 \%$ span for no yaw case

\subsection{Dynamic Stall Results}

Figures 4.11 through 4.14 show azimuth-averaged $C_{L}$ vs. AOA curves compared to lift curves measured in the CSU wind-tunnel tests (Butterfield, Scott, and Musial, 1990). The dynamic-stall behavior is evident in the large hysteresis loops that surround the static curves. At the $80 \%$ span the rotating blade $\mathrm{C}_{\mathrm{L}(\max )}$ values do not significantly exceed static values. However, at inboard stations the effect on $\mathrm{C}_{1(\max )}$ becomes more pronounced. At spanwise stations $63 \%, 47 \%$, and $30 \%$, shown in Figures 4.12 through $4.14, C_{L(\max )}$ values exceed static values by $32 \%$ to $110 \%$. The labeled symbols indicate blade azimuth angle. The variation in lift at aximuth angles of $90 \mathrm{deg}$ and $270 \mathrm{deg}$, discussed in Section 4.3, is again obvious in these curves. As the hysteresis loops grow larger, the differences increase and the resulting yaw moments 


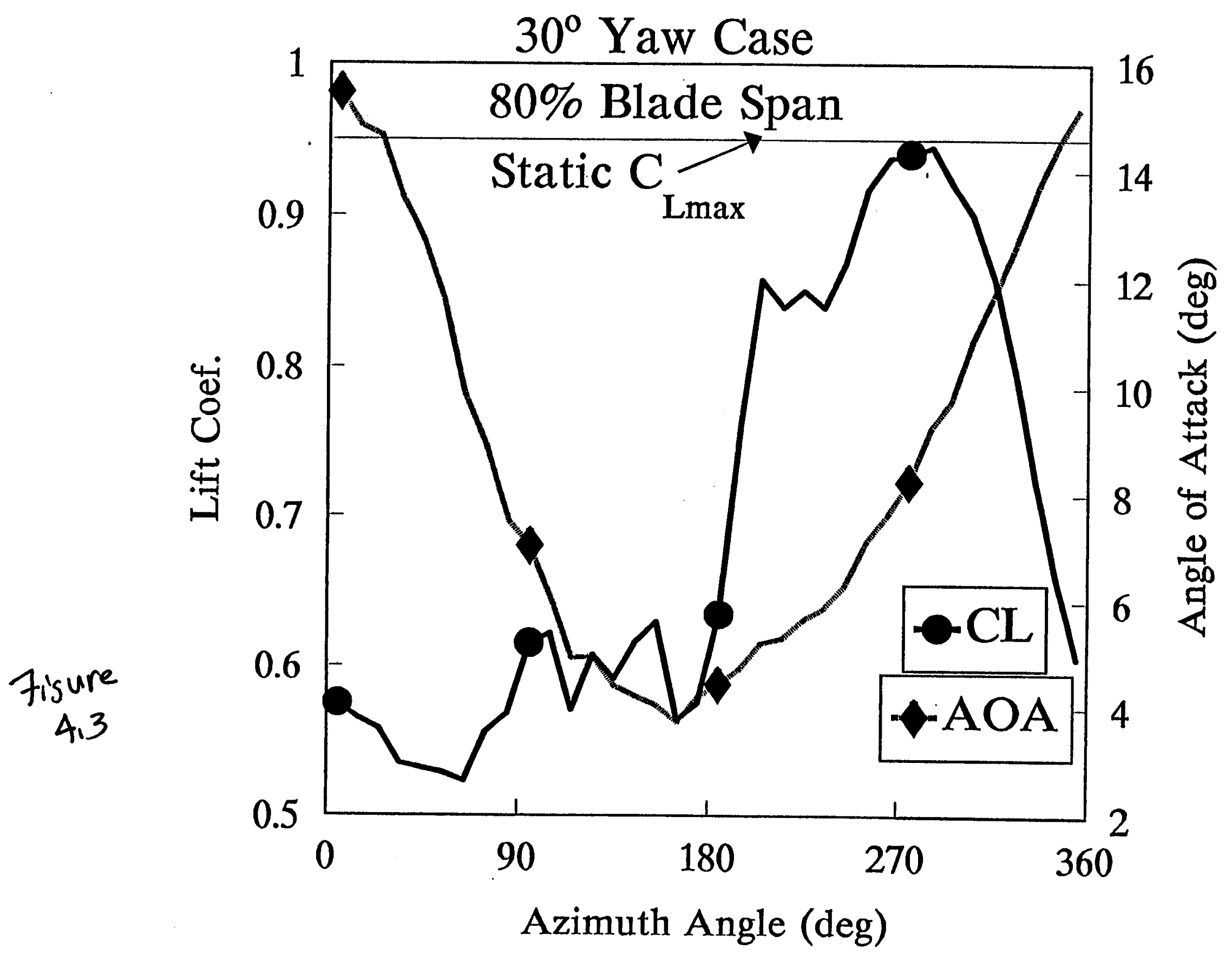

我福 


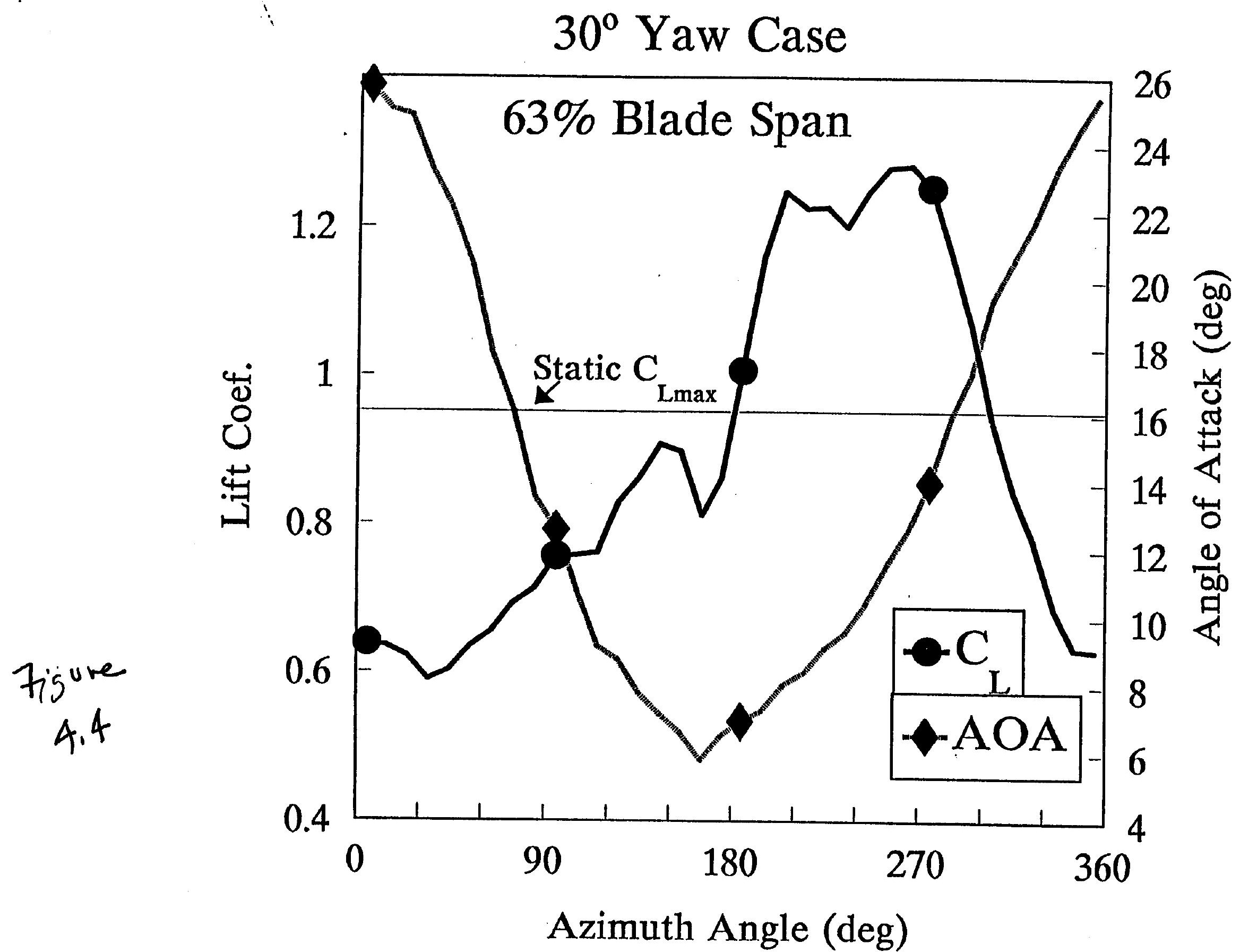




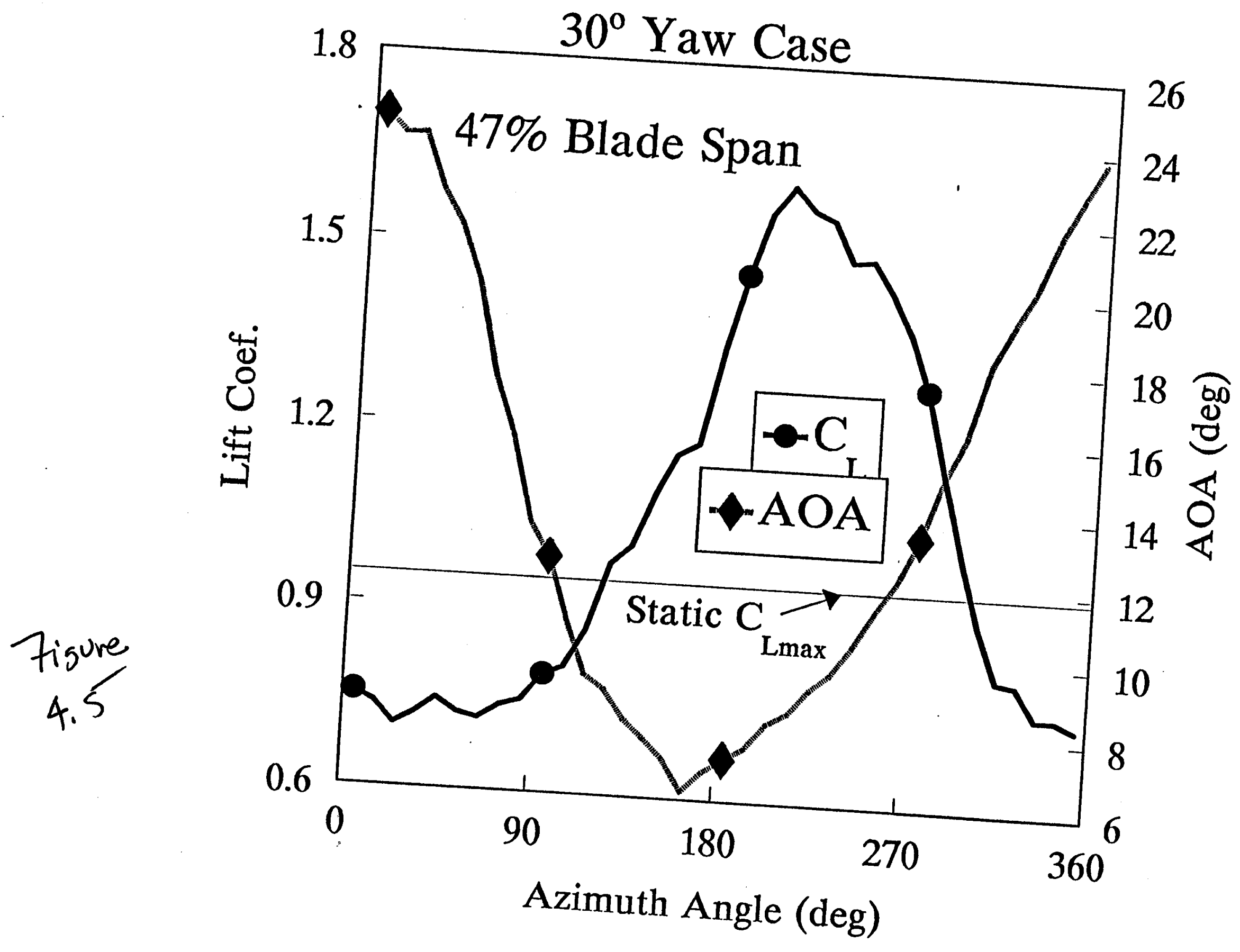




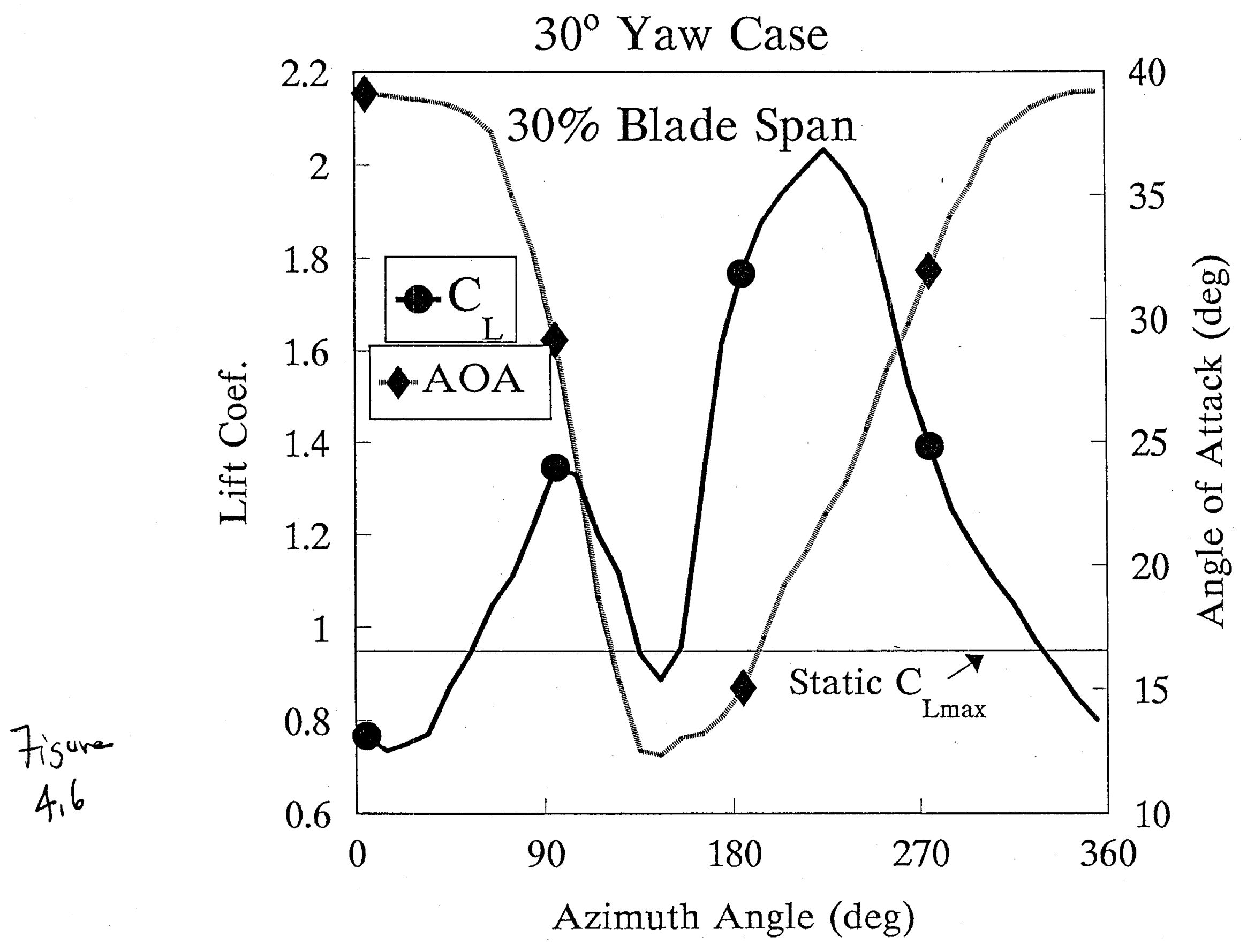

$1,7.54 .6$ 


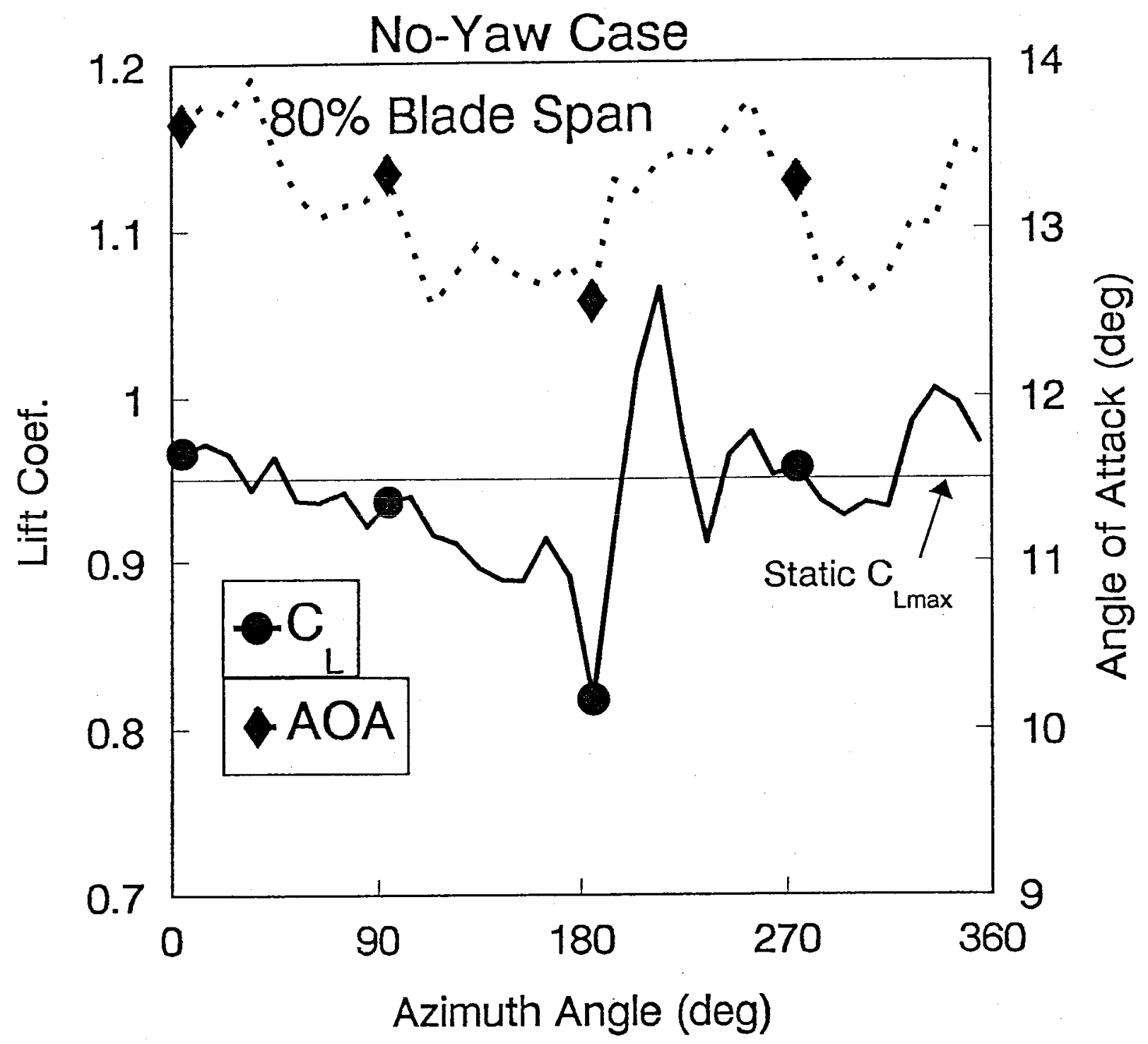

7isure 4.7 

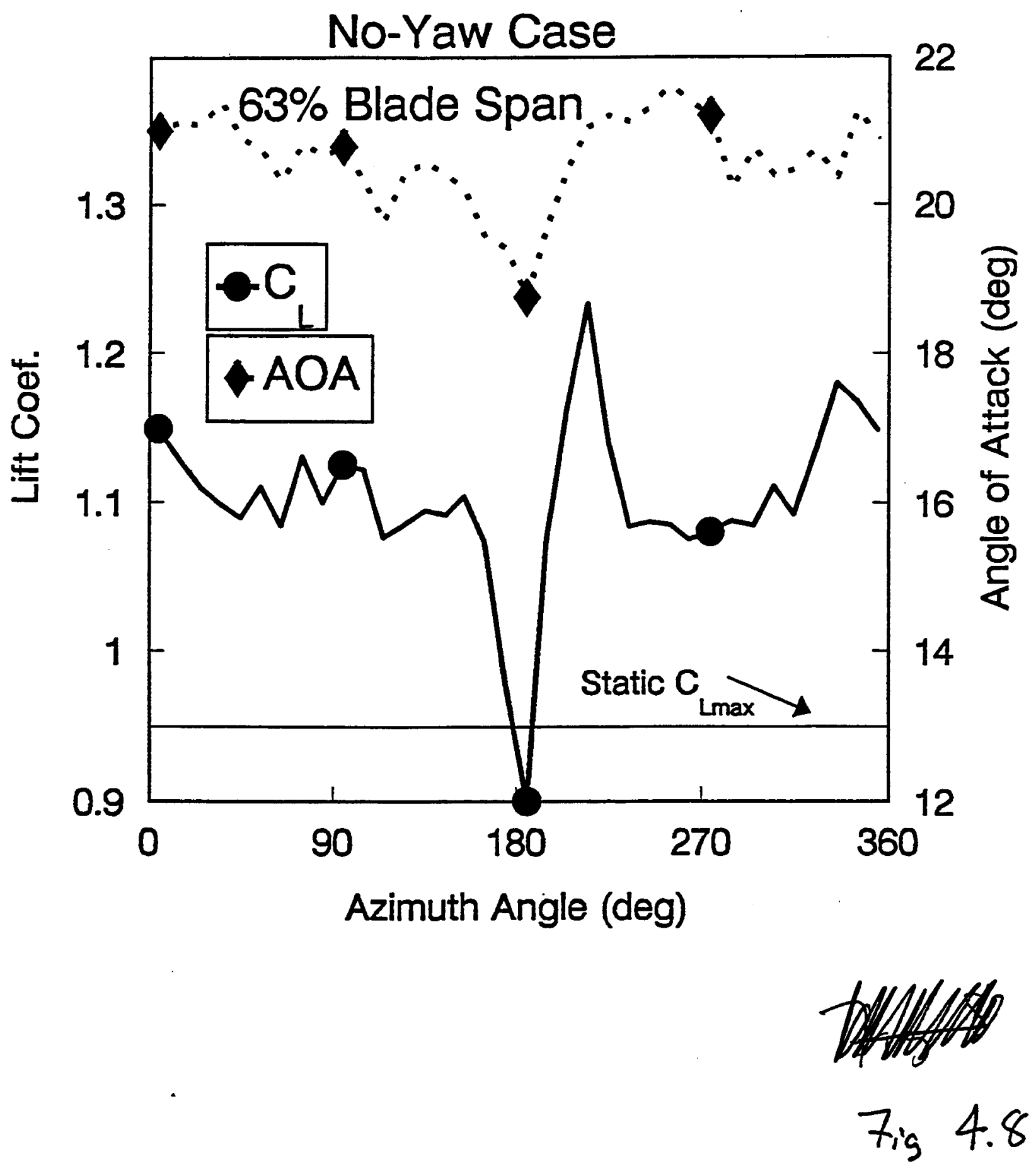


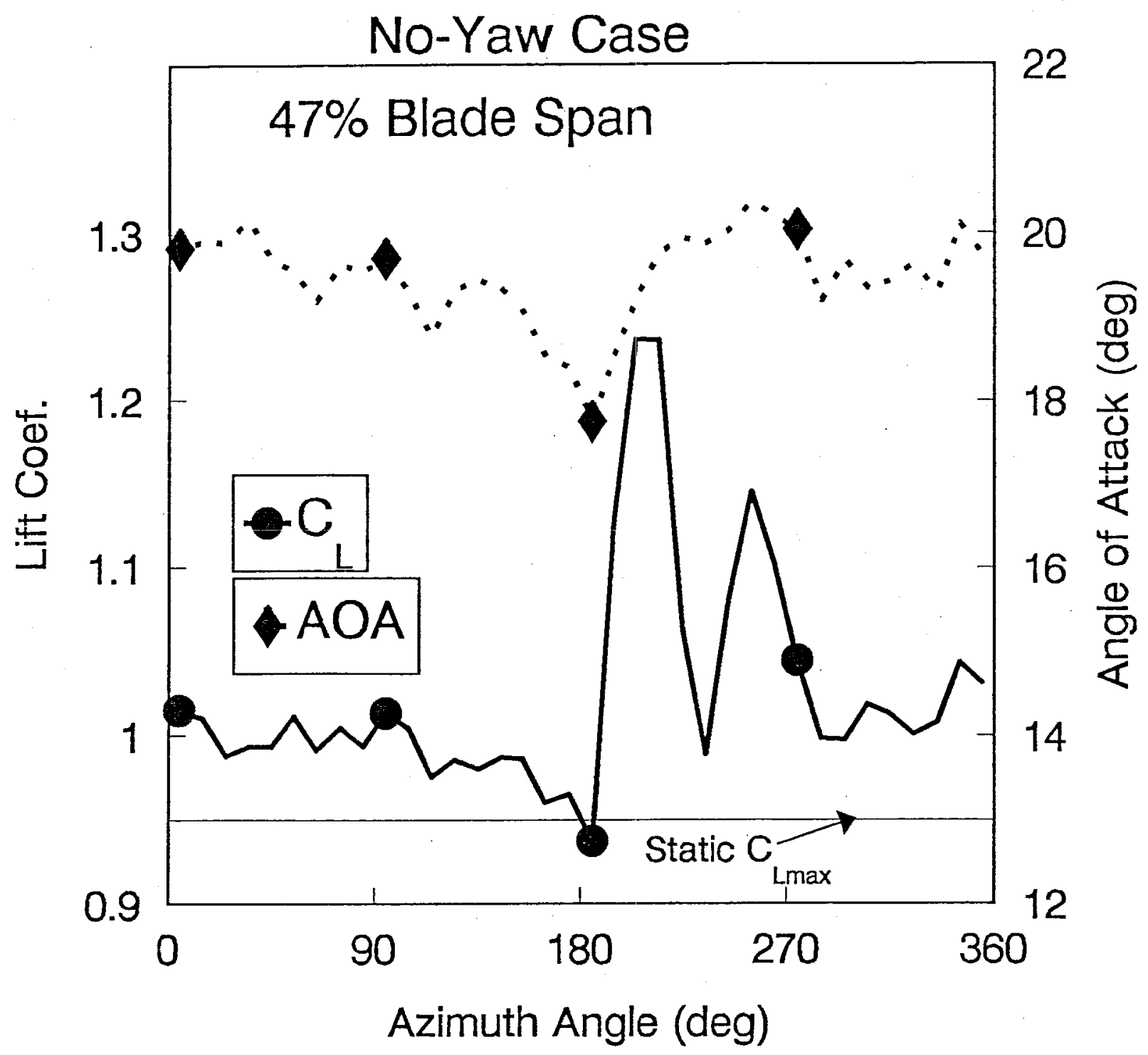

7 is 4.9 


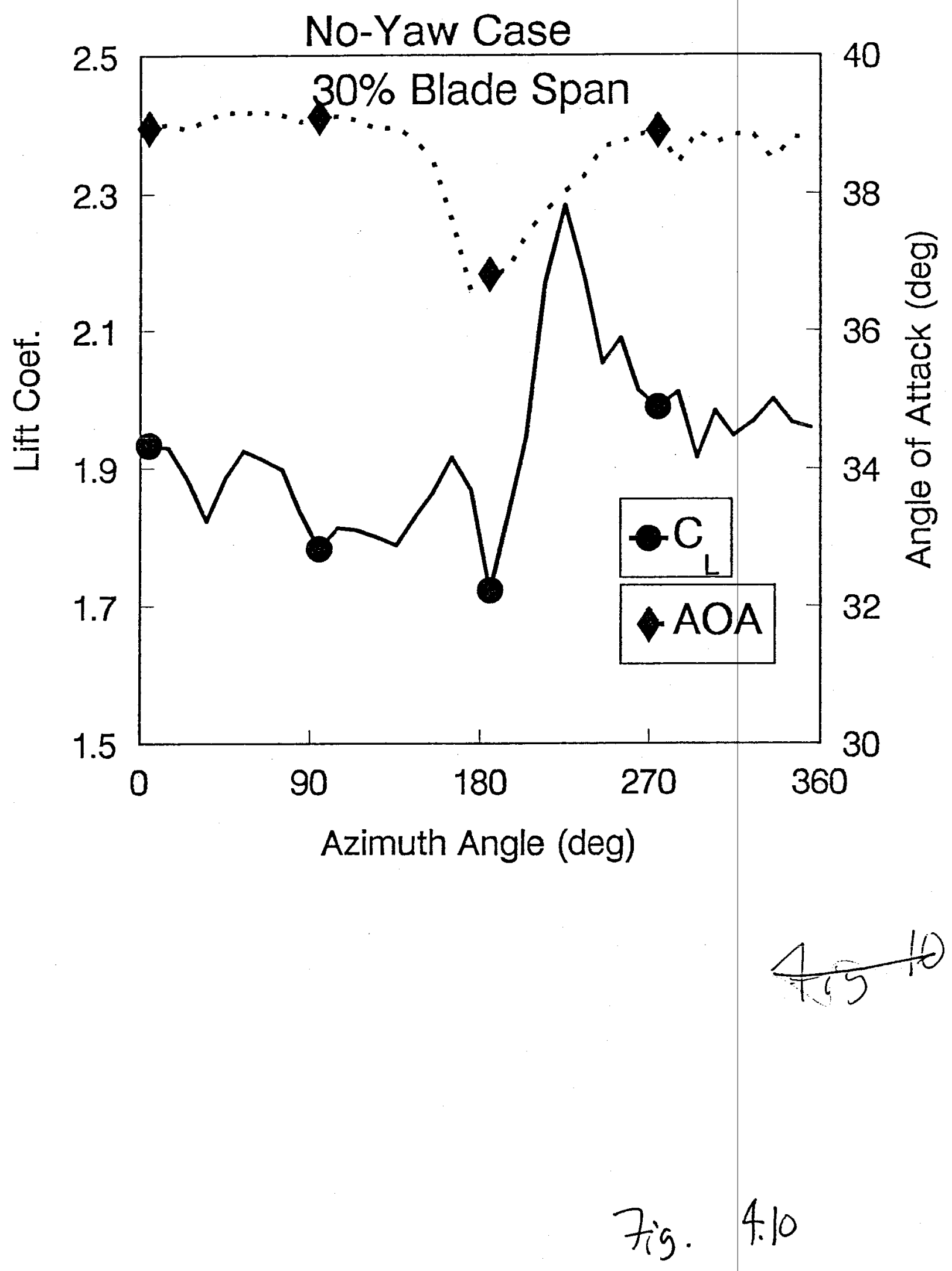


increase. Hansen and Butterfield (1990) showed that predicted yaw moments double when dynamic stall is introduced into YAWDYN, a structural dynamics model.

Figure 4.11. Dynamic stall at $80 \%$ span

Figure 4.12. Dynamic stall at $63 \%$ span compared with wind-tunnel dynamic stall

Figure 4.13. Dynamic stall at $47 \%$ span

Figure 4.14. Dynamic stall at $30 \%$ span

Drag is also subject to the effects of dynamic stall. Figures 4.15 through 4.18 compare dynamic stall measurements for the $30 \mathrm{deg}$ yaw error case with data from the CSU wind tunnel. The $80 \%$ span is affected minimally, but inboard stations experience nearly double the drag from one side of the rotor to the other. This is illustrated for the $47 \%$ span in Figure 4.17 , which shows a $C_{D p}$ of 0.15 at 270 deg azimuth angle and 0.07 at $90 \mathrm{deg}$, an increase of more than a factor of two.

Figure 4.15. Drag dynamic stall at $80 \%$ span

Figure 4.16. Drag dynamic stall at $63 \%$ span

Figure 4.17. Drag dynamic stall at $47 \%$ span

Figure 4.18. Drag dynamic stall at $30 \%$ span

Pitching-moment coefficients also experience dynamic stall. Figure 4.19 shows $C_{p_{m}}$ hysteresis loops measured at the $63 \%$ blade span. These moments can have a significant effect on blade pitch angle if there is flexibility in the pitch system of pitch-control rotors. This can also cause increased fatigue loads on pitch linkages and pitch actuators.

Figure 4.19. Pitching moment dynamic stall at $63 \%$ span

\subsection{Transient Pressure Distributions}

Figure 4.20 shows the suction-side pressure distributions of a rotating turbine blade with zero yaw taken at $30 \%$ span at a wind velocity of $14.7 \mathrm{~m} / \mathrm{sec}$ over a single rotational cycle. At 0 deg azimuth, the turbine blade is pointing up, and at $180 \mathrm{deg}$ the blade is straight down behind the tower. Three-dimensional surface plots illustrate the chordwise pressure distribution and its variation throughout the rotation cycle. Chordwise pressure distributions are shown for every $0.82 \mathrm{deg}$ of rotation. The axis of this plot showing "azimuth angle" can also be converted to "time" with 0.833 second passing for each $360 \mathrm{deg}$. This figure shows a shift in the suction pressure maxima toward the trailing edge as blade advances through the rotation cycle. Robinson (1985) used flow visualization in conjunction with surface-pressure data to correlate dynamic-stall vortex formation with unsteady surface-pressure signatures. He observed a temporal shift in maximum suction pressure toward the trailing edge, which corresponded to passage of a dynamic-stall vortex. Current results seem consistent with this observed vortex formation and with convection over the suction surface of wind-turbine blades. The transient pressure signature becomes more obvious for a yawed HAWT, as will be demonstrated later.

Figure 4.20. Chordwise surface-pressure distribution vs. azimuth angle. $V=14.7 \mathrm{~m} / \mathrm{s}, 0^{\circ}$ yaw, $30 \%$ span. One cycle of data. 


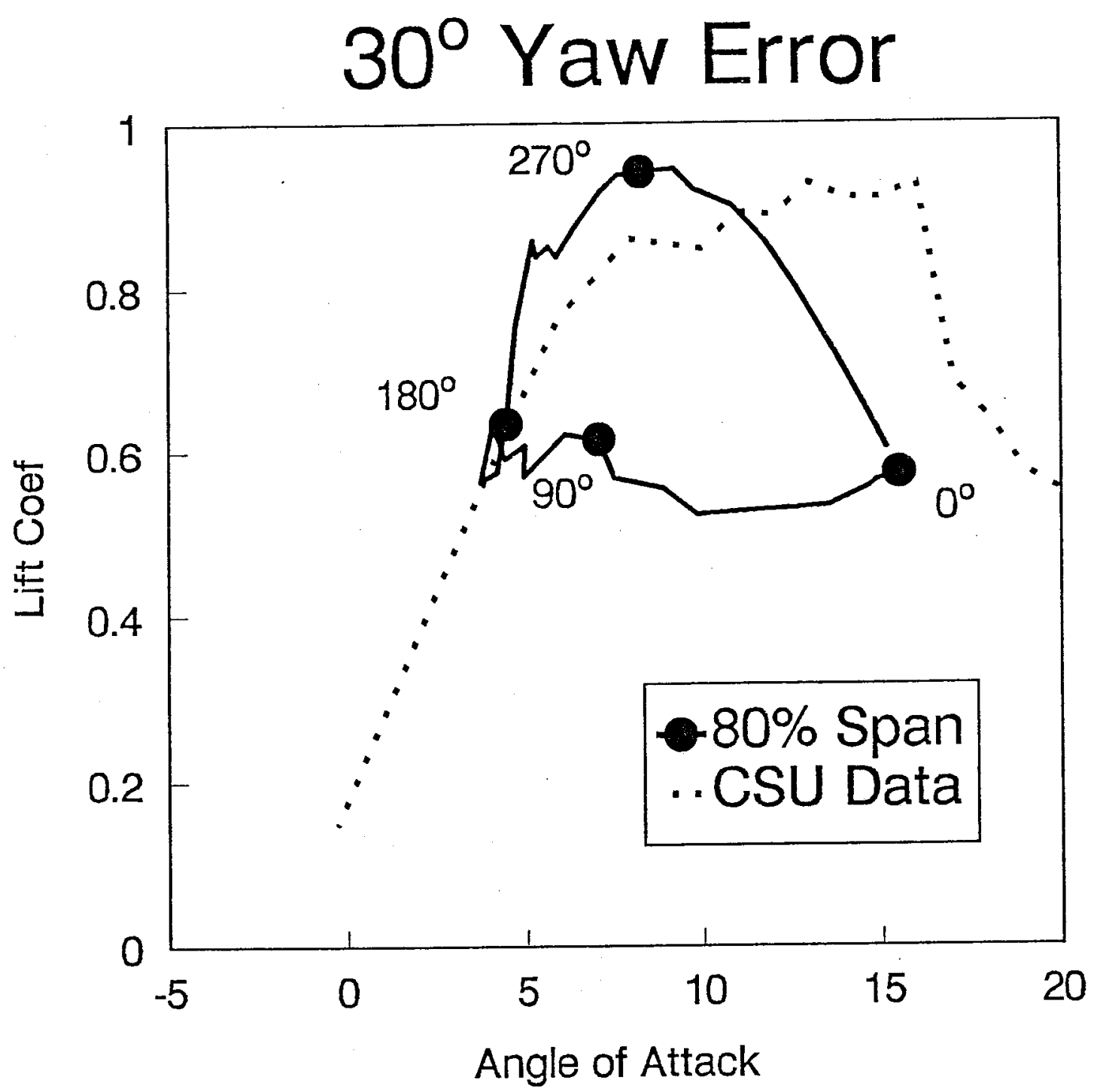

Fis 11

7 is 4.11 


\section{$30^{\circ}$ Yaw Error}

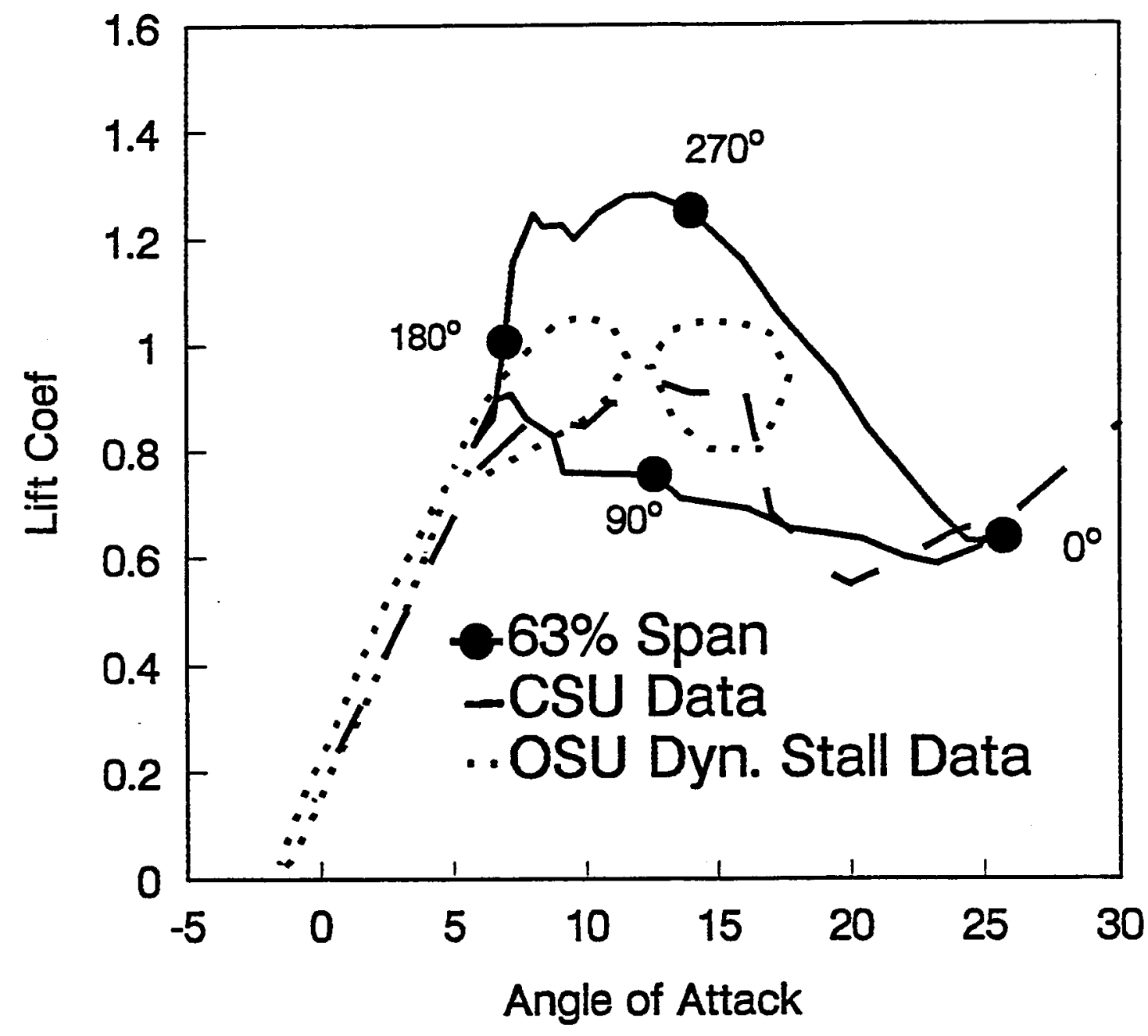

सig 12

7is 4.12 


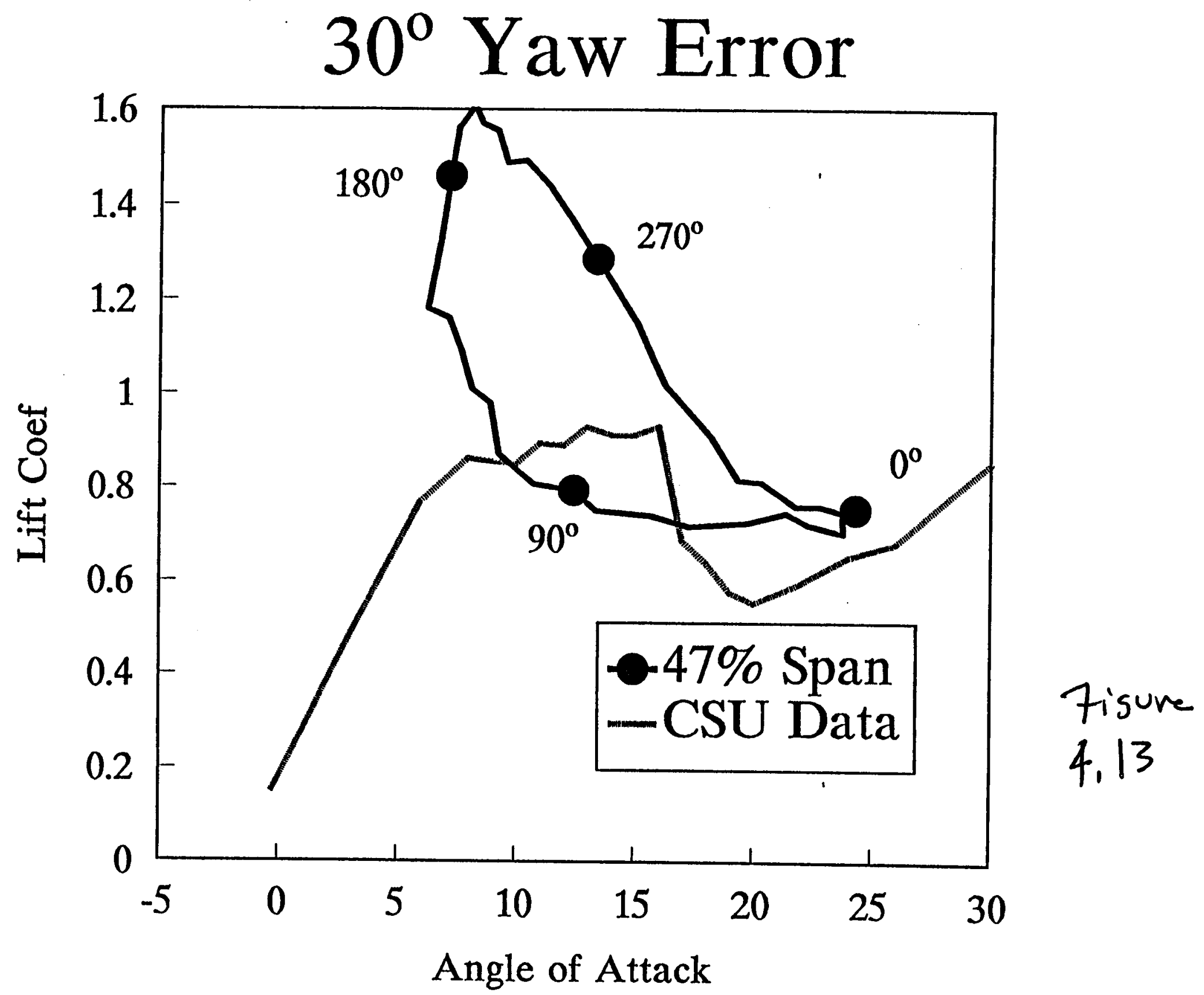

D 4211 


\section{$30^{\circ}$ Yaw Error}

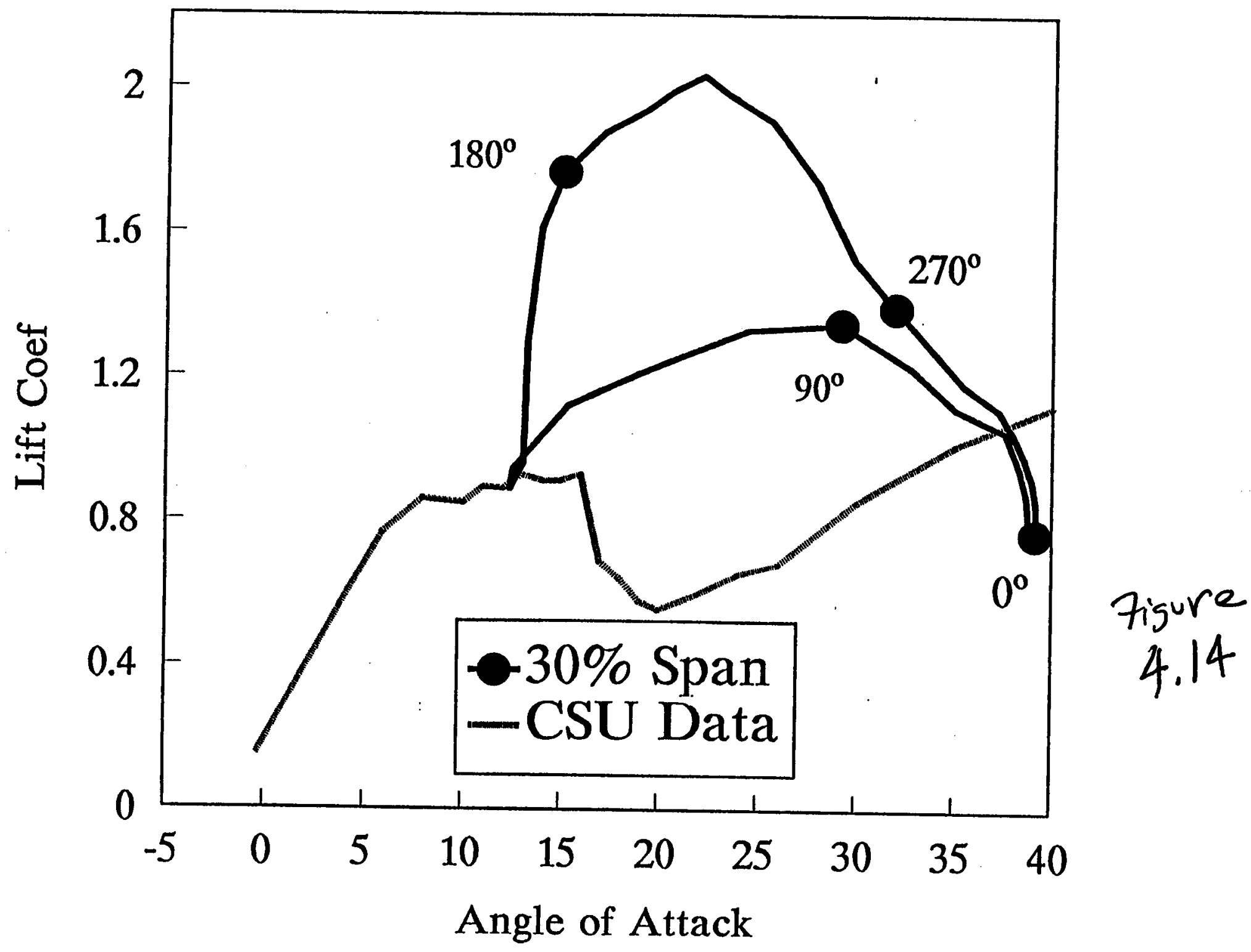


$30^{\circ}$ Yaw Error

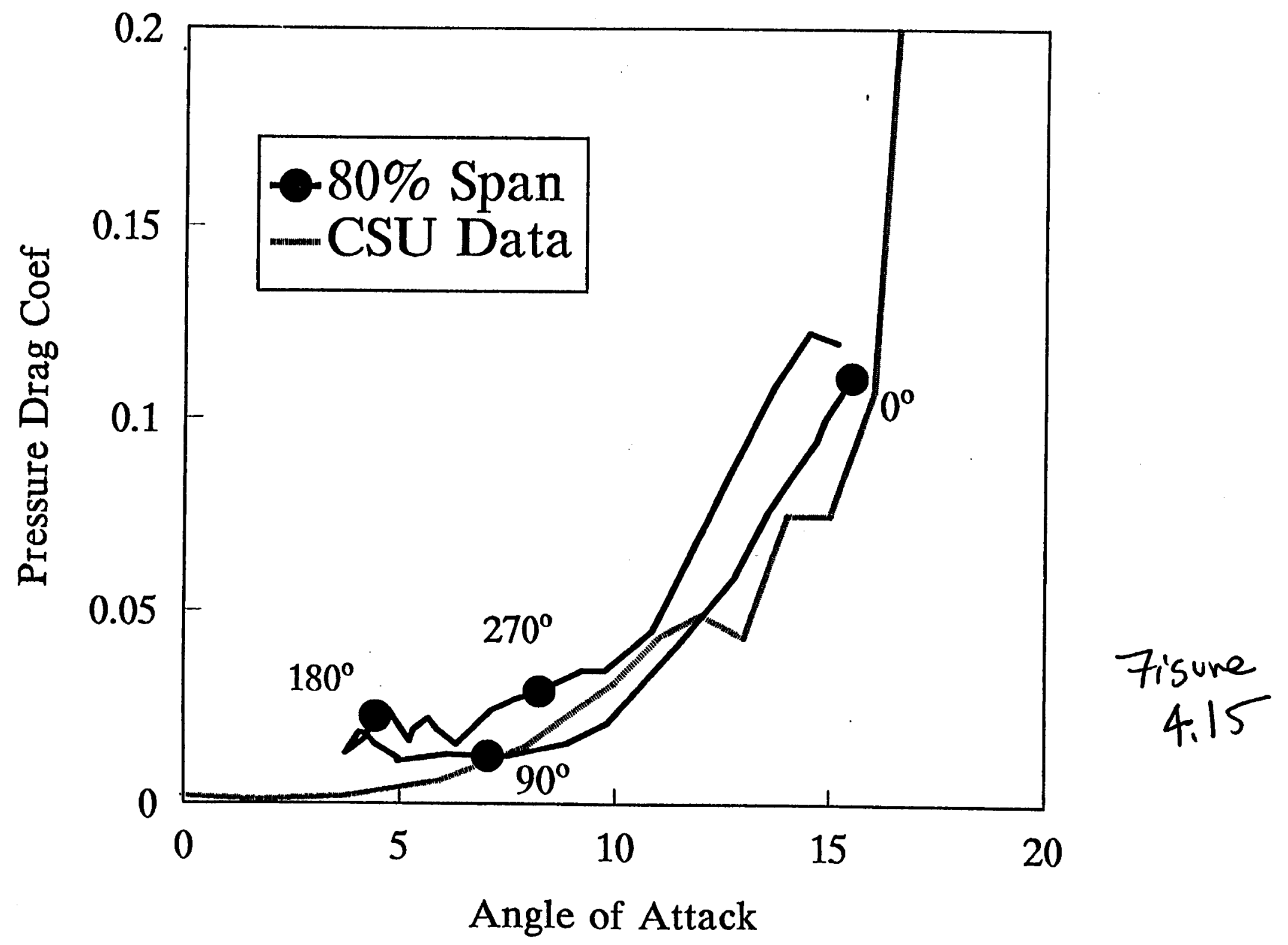




\section{$30^{\circ}$ Yaw Error}

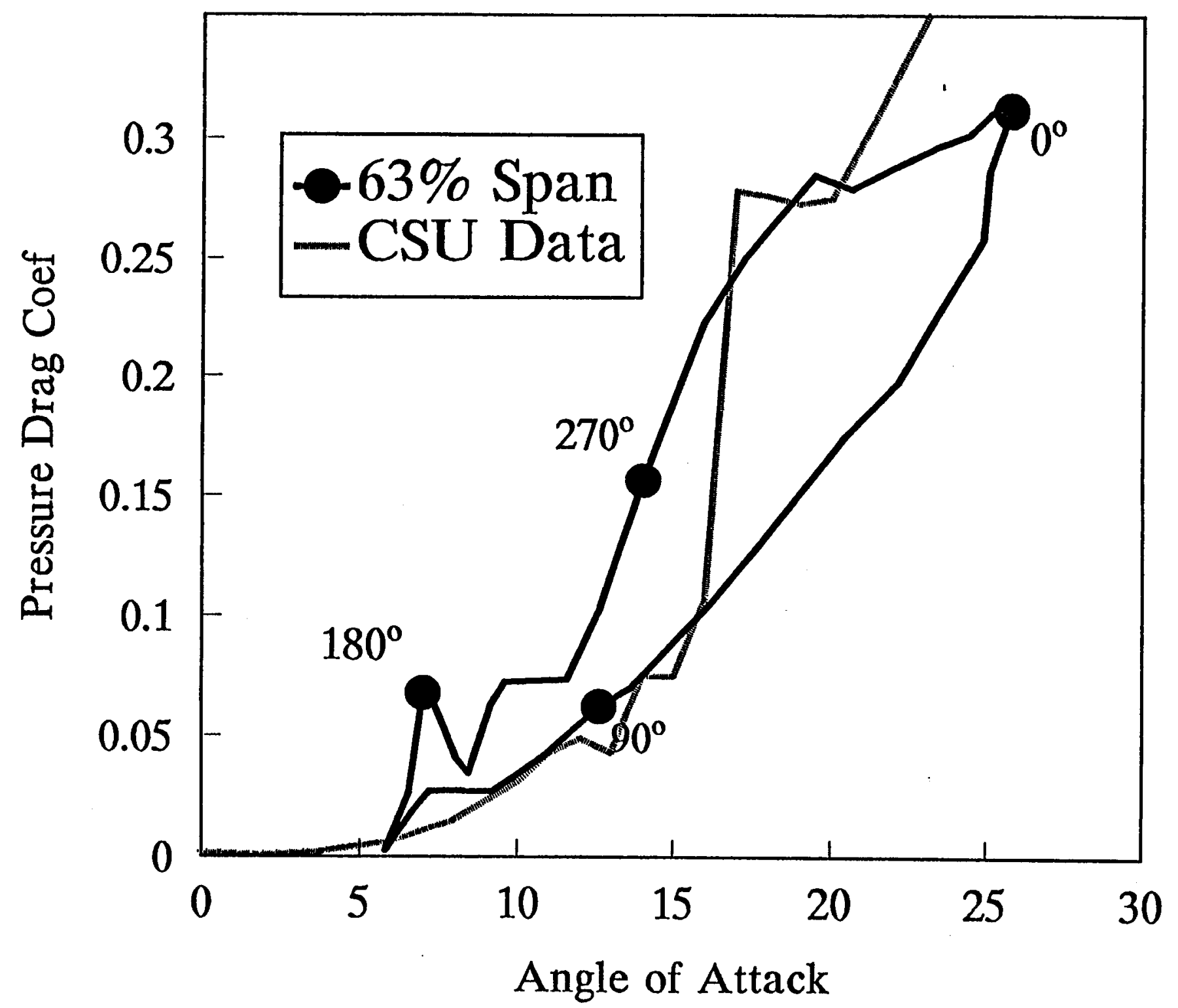

Figure 4.16 


\section{$30^{\circ}$ Yaw Error}

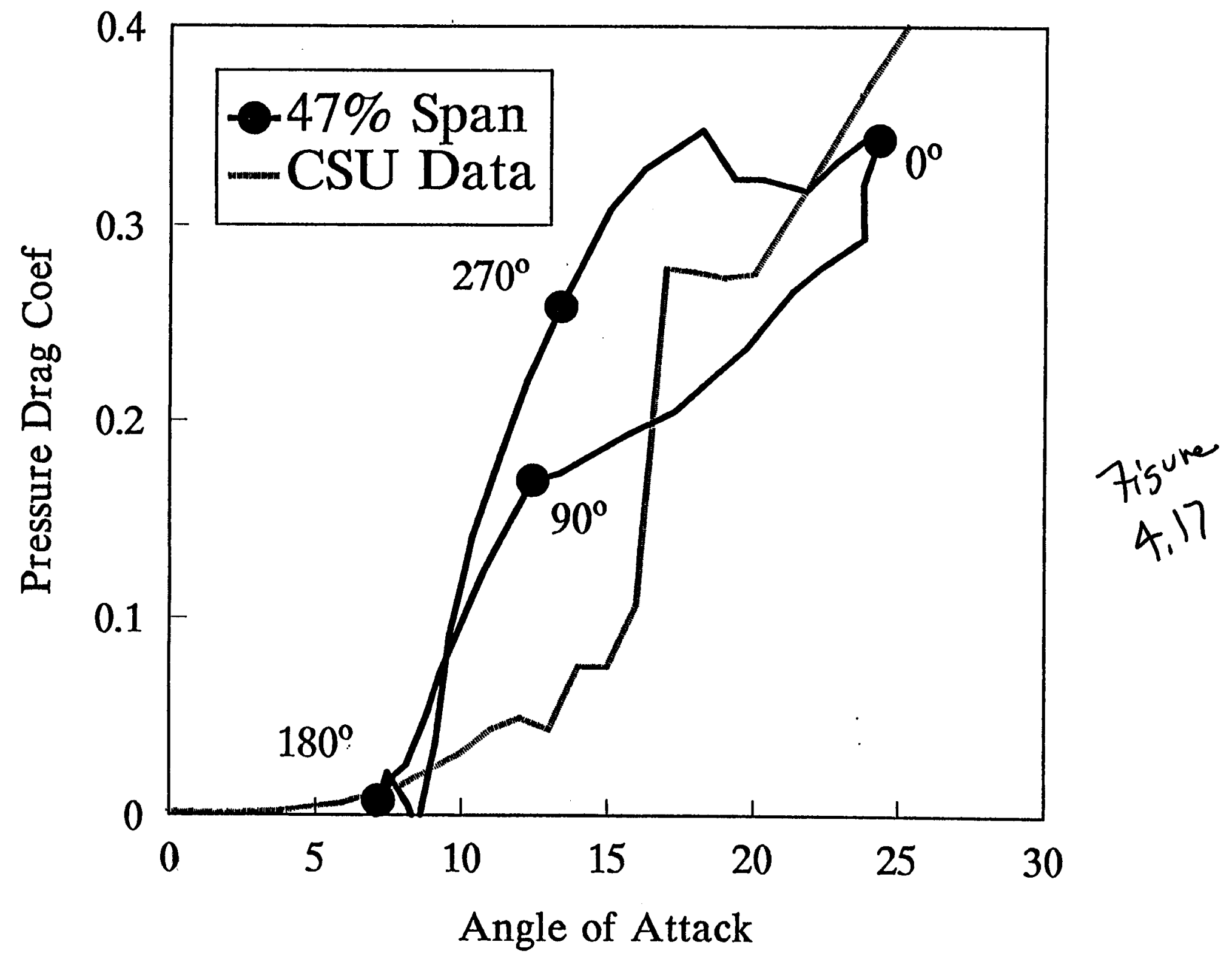

$7 / 14$ 


\section{$30^{\circ}$ Yaw Error}

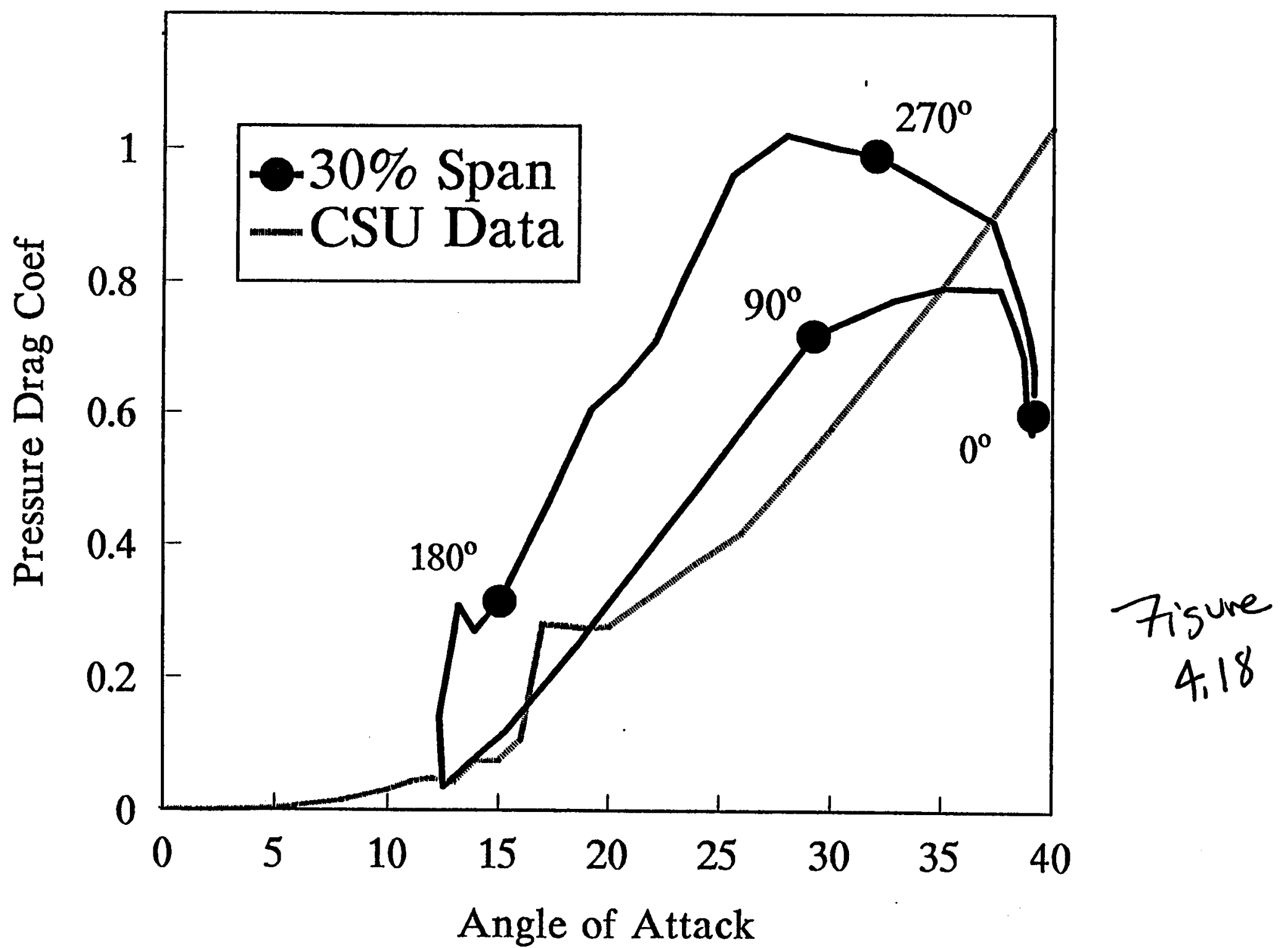




\section{$30^{\circ}$ Yaw Error}

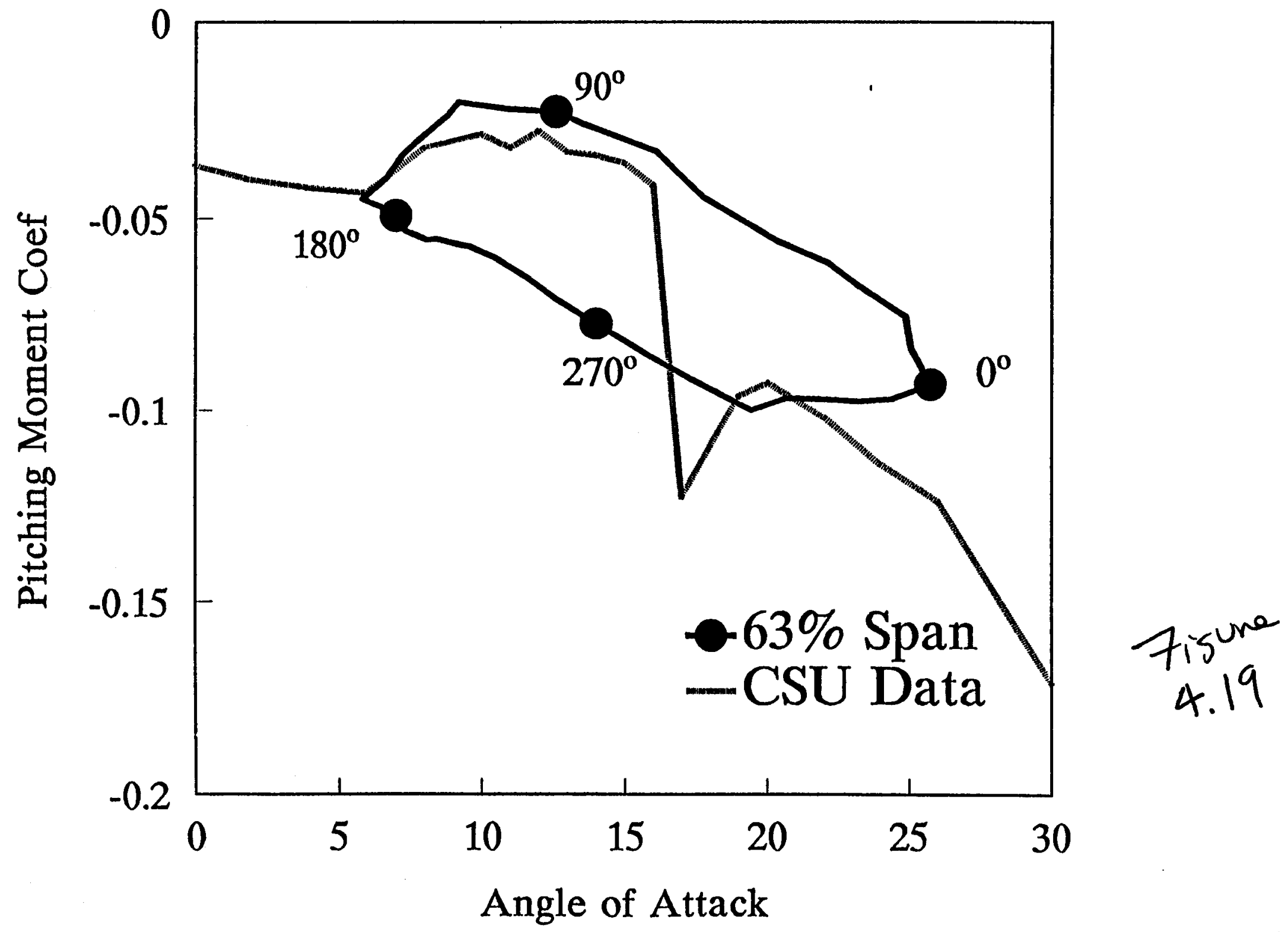

749. 

$30 \%$ SPAN LOCATION

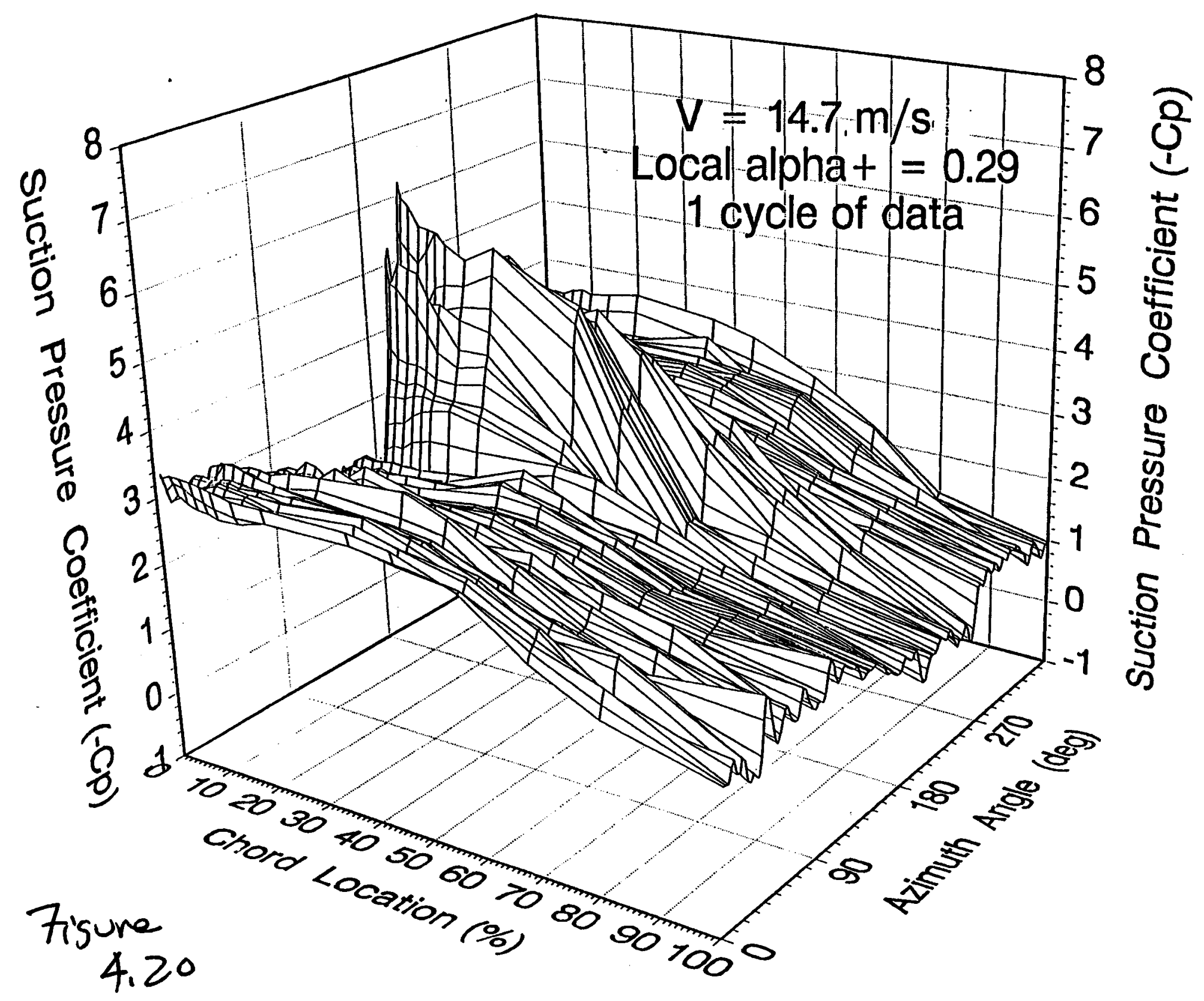


Figure 4.21 shows a comparison of measured and theoretical AOAs. The theoretical model includes the vector addition of free-stream velocity, local in-plane velocity, the effect of the yawed turbine, and a simple tower-shadow model. The agreement is generally good, but there are some discrepancies. At $180 \mathrm{deg}$, the frequency response of the transducer is not fast enough to characterize rapid changes in flow angles due to the tower shadow. At high AOA, there is a discrepancy that may be caused by induced velocity effects or flow irregularities that are not included in the theoretical-model curve. This comparison demonstrates that the measured AOA yields reasonable results and has sufficient frequency response to give accurate dynamic-stall characterization for yawed flow conditions. However, the flow-angle sensor cannot accurately respond to the impulsive nature of the tower shadow.

Figure 4.21. Measured and geometric local AOA vs. azimuth angle

Figure 4.22 shows the theoretical AOA variation of a yawed downwind HAWT for a yaw angle of 30 deg and wind velocity of $16.7 \mathrm{~m} / \mathrm{sec}$. Figure 4.23 shows a bin-averaged upper-surface pressure distribution at $30 \%$ span for $30 \mathrm{deg}$ yaw. This may be contrasted with single-revolution data shown in Figure 4.24 for similar test conditions. In both cases, two leading-edge suction-pressure peaks were seen over the averaged rotational cycle. Data selected for the single-revolution show that the suction pressure peaks can be larger and more transient in nature. Convection of a vortex structure may be inferred by the temporal shift in maximum suction pressure away from the leading edge, which is consistent with results seen for pitching airfoils.

Figure 4.22. Theoretical geometric AOA vs. azimuth angle. $30^{\circ}$ yaw, V $=16.7 \mathrm{~m} / \mathrm{s}$

Figure 4.23. Chordwise surface-pressure distribution vs. azimuth angle. $30^{\circ}$ yaw, $V=14 \mathrm{~m} / \mathrm{s}, 30 \%$ span. Bin-averaged data.

Figure 4.24. Chordwise surface-pressure distribution vs. azimuth angle. $30^{\circ}$ yaw, $\mathrm{V}=16.7 \mathrm{~m} / \mathrm{s}, 30 \%$ span. One cycle of data.

Figure 4.25 shows the upper-surface pressure distribution for a turbine blade operating at -33 deg yaw over one revolution at the $30 \%$ span. The suction-pressure peaks are approximately 180 deg out of phase with respect to the positive yaw case. Four discrete suction-pressure peaks were observed, implying formation of a number of vortices throughout the rotation cycle. Convection of these vortices toward the trailing edge may be inferred at both $75 \mathrm{deg}$ and $270 \mathrm{deg}$ aximuth.

Figure 4.25. Chordwise surface-pressure distribution vs. azimuth angle. $-33^{\circ}$ yaw, $V=13.3 \mathrm{~m} / \mathrm{s}$, $30 \%$ span. One cycle of data.

The upper-surface pressure distribution at 63\% span is shown in Figure 4.26. A suction-pressure spike was observed at approximately $120 \mathrm{deg}$ azimuth, and a smaller spike was seen at $210 \mathrm{deg}$. The suctionpressure peak persists over a larger portion of the cycle ( $90 \mathrm{deg}-120 \mathrm{deg}$ ), and convection of this vortex can be inferred in the suction-pressure ridge. This convection extends only back to $50 \%$ chord, in contrast to the situration at $30 \%$ span, shown in Figure 4.25 , where well-defined vortex convection is seen all the way to the trailing edge.

Figure 4.26 Chordwise surface-pressure distribution vs. azimuth angle. $-33^{\circ}$ yaw, $\mathrm{V}=13.3 \mathrm{~m} / \mathrm{s}$, $63 \%$ span. One cycle of data.

Figures 4.27 and 4.28 show bin-averaged spanwise pressure distributions taken at the $4 \%$ chord location for zero yaw error. All spanwise pressures were normalized with respect to the tip velocity using Equation 3.5 where $R=5.05 \mathrm{~m}$. As can be seen in Figure 4.27, for the low wind velocity case of 
EXPERIMENTAL AND THEORETICAL AOA

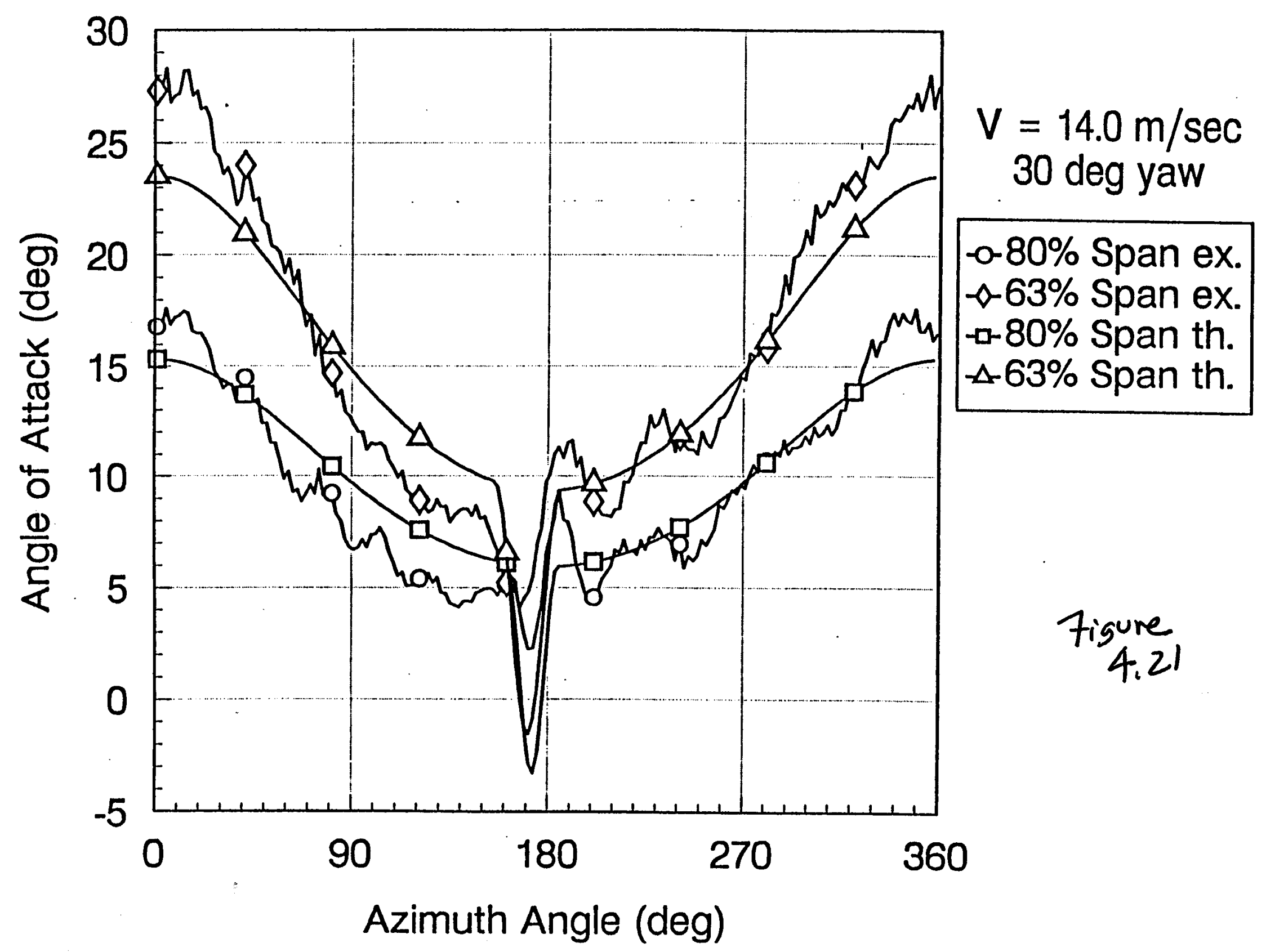




\section{ANGLE OF ATTACK vs. AZIMUTH ANGLE 30 deg yaw, $V=16.7 \mathrm{~m} / \mathrm{s}$}

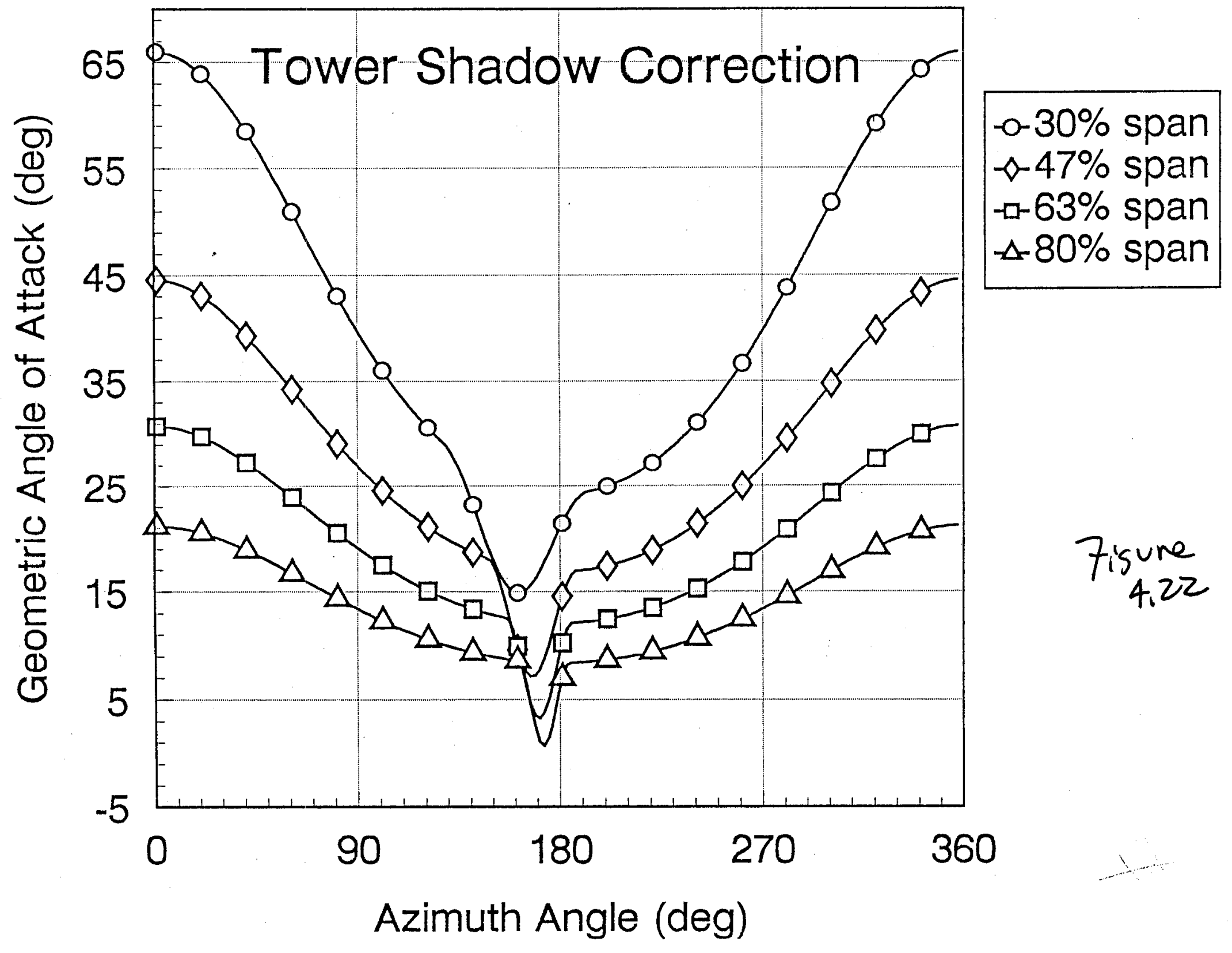




\section{UPPER SURFACE PRESSURE DISTRIBUTION $30 \%$ SPAN LOCATION}

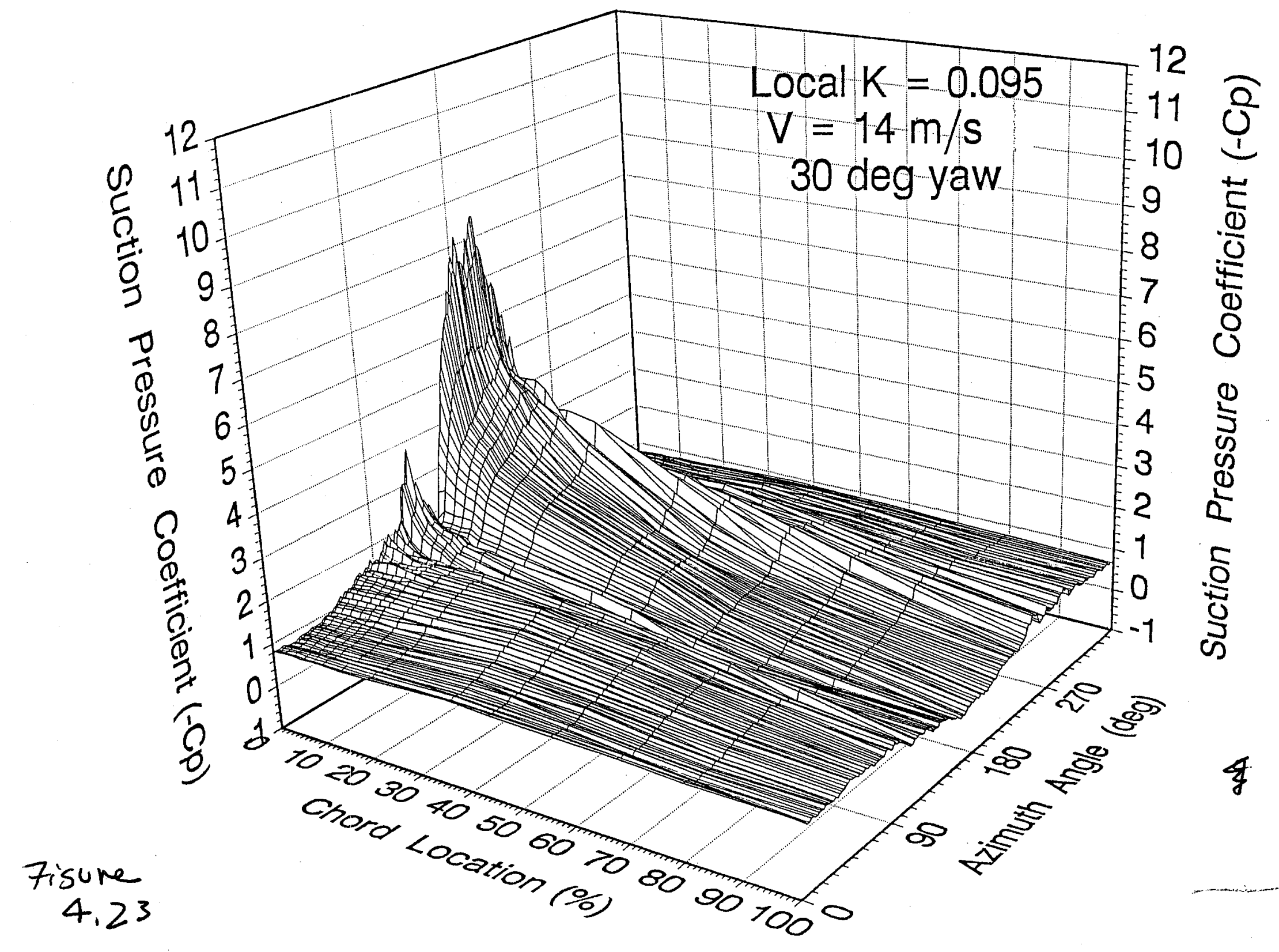




\section{UPPER SURFACE PRESSURE DISTRIBUTION $30 \%$ SPAN LOCATION}

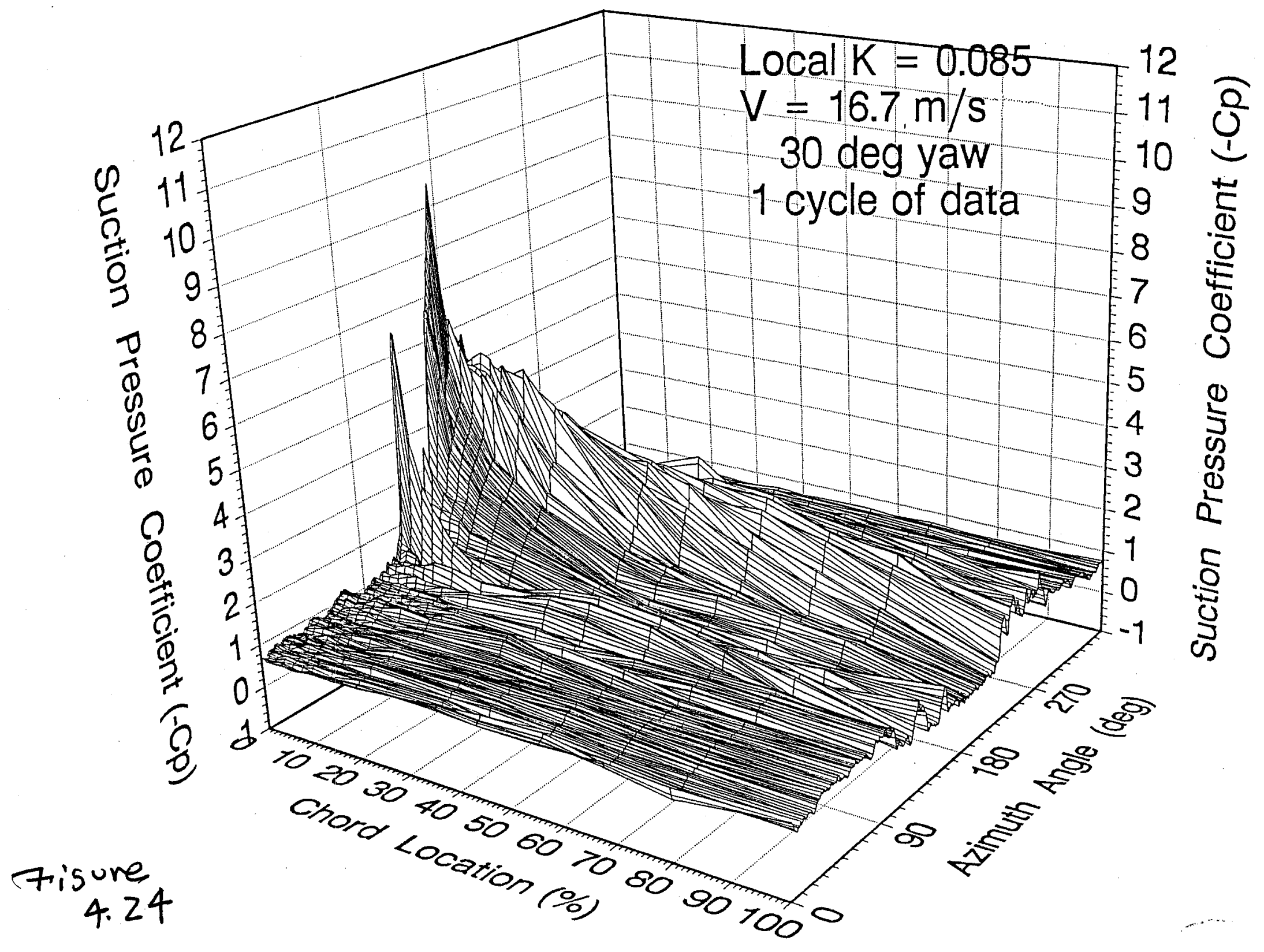


UPPER SURFACE PRESSURE DISTRIBUTION

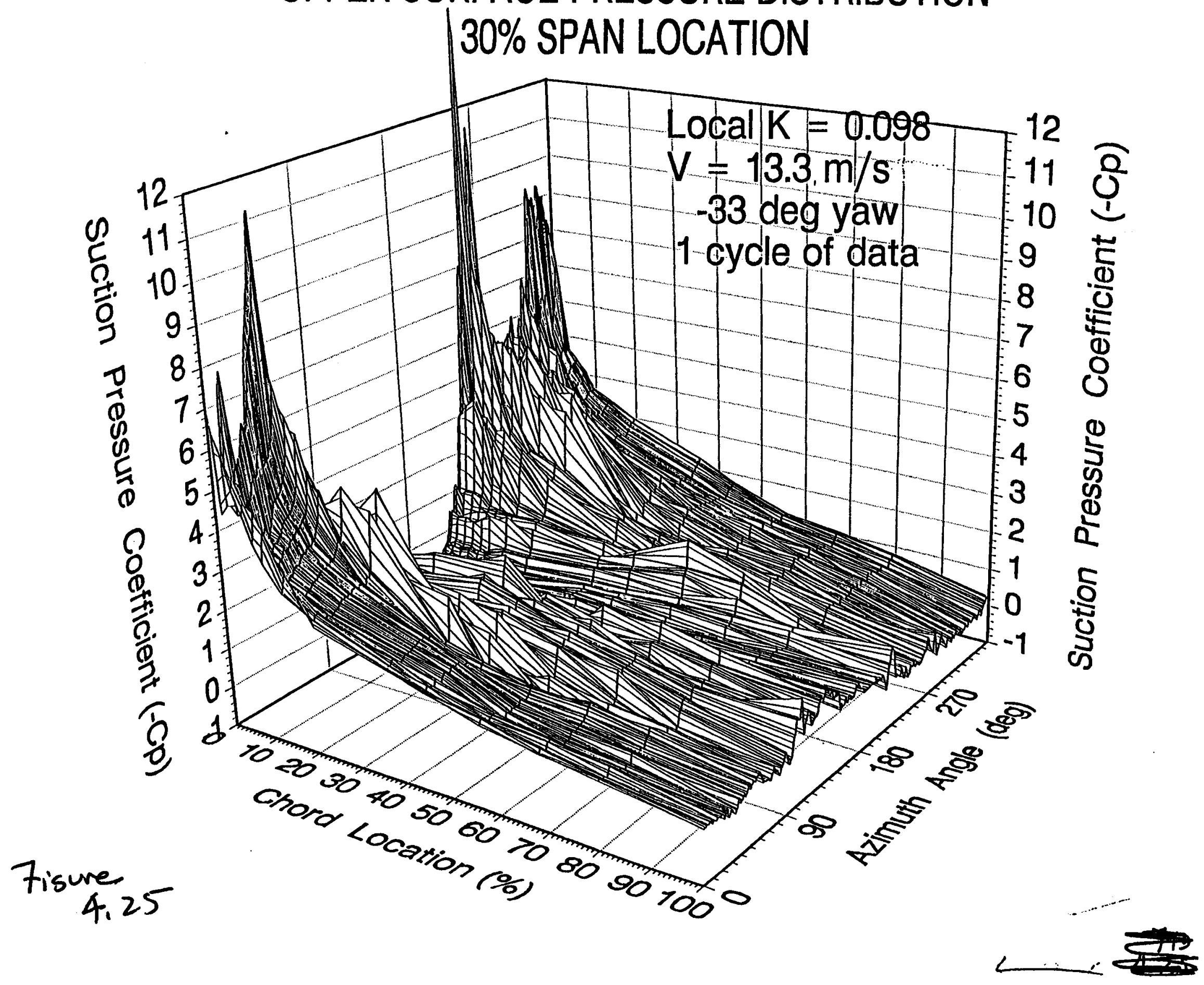




\section{UPPER SURFACE PRESSURE DISTRIBUTION 63\% SPAN LOCATION}

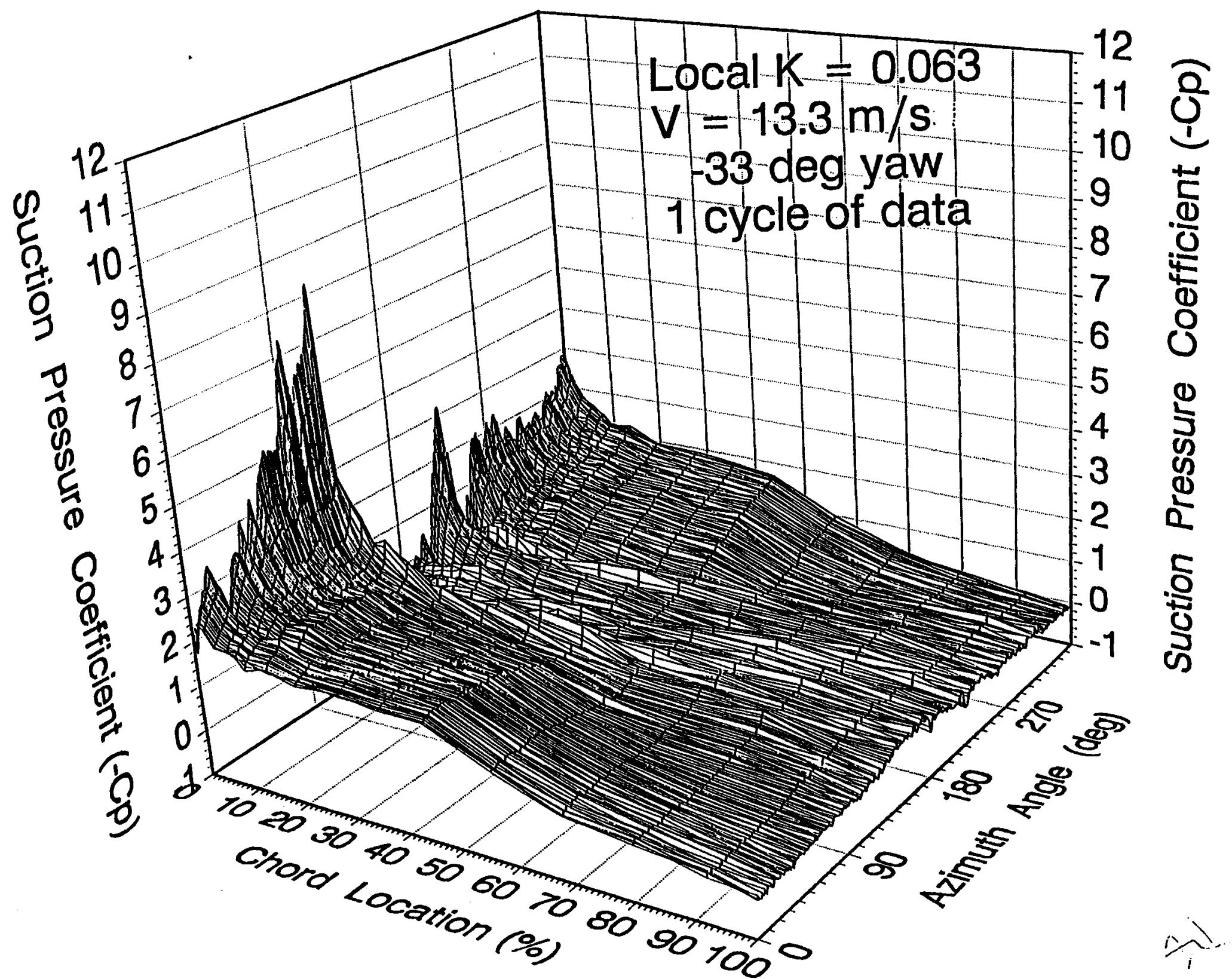


$7.7 \mathrm{~m} / \mathrm{sec}$, surface pressure remains approximately constant along the span. The dip at $180 \mathrm{deg}$ is due to the tower shadow. A suction-pressure maximum appears at approximately $47 \%$ span, with suction pressure decreasing modestly inboard and outboard. This may be contrasted with the high wind velocity case of $18.7 \mathrm{~m} / \mathrm{sec}$ seen in Figure 4.28 . For this case, suction pressure decreases slightly from $30 \%$ to 47\% span, and little evidence of a tower-shadow-induced vortex can be seen. From $50 \%$ span outboard, however, overall suction pressure increases appreciably. In addition, a tower-shadow-induced vortex signature becomes better defined at 180 deg azimuth for outboard stations.

Figure 4.27. Spanwise pressure distribution vs. azimuth angle. $7.7 \mathrm{~m} / \mathrm{s}$ wind speed, $4 \%$ chord, tipvelocity normalization, bin-averaged data.

Figure 4.28. Spanwise pressure distribution vs. azimuth angle. $18.7 \mathrm{~m} / \mathrm{s}$ wind speed, $4 \%$ chord, tipvelocity normalization, bin-averaged data.

Figures 4.29 and 4.30 show surface plots of bin-averaged surface-pressure distributions taken at $4 \%$ chord for $-26.5 \mathrm{deg}$ and $30 \mathrm{deg}$ yaw cases. Again, all surface-pressure data were normalized with respect to the tip velocity. Figure 4.29 shows the -26.5 deg yaw case. In general, maximum suction pressures occurred as the blade reached maximum height ( $0 \mathrm{deg}$ or $360 \mathrm{deg}$ azimuth), and minimum pressures were encountered at $180 \mathrm{deg}$. These data exhibit some interesting characteristics. From $30 \%$ to $47 \%$ span, suction pressure decreased appreciably. From $47 \%$ to $63 \%$ span, suction pressure increased, and from $63 \%$ to $80 \%$ span, it leveled off. In addition, at $30 \%$ span, there was no evidence of a tower-induced vortex (which would likely occur near $180 \mathrm{deg}$ aximuth), but this vortex did appear in the pressure signature near $63 \%$ span and further outboard.

Figure 4.29. Spanwise pressure distribution vs. azimuth angle. $18 \mathrm{~m} / \mathrm{s}$ wind speed, $4 \%$ chord, tipvelocity normalization, bin-averaged data, $-26.5^{\circ}$ yaw.

Figure 4.30. Spanwise pressure distribution vs. azimuth angle. $14 \mathrm{~m} / \mathrm{s}$ wind speed, $4 \%$ chord, tipvelocity normalization, bin-averaged data, $30^{\circ}$ yaw.

Figure 4.30 shows a comparable surface plot of spanwise pressure distributions for the 30 deg yaw case. As expected, maximum suction pressures occurred approximately 180 deg out-of-phase with respect to the negative yaw case. There is no evidence of a tower-induced vortex in the pressure profile except at $80 \%$ span. A pressure ridge does persist at approximately 200 deg azimuth along the entire span, possibly indicating uniform vortex initiation. This suction-pressure peak persists over a relatively short azimuth range at $30 \%$ span, but persists longer at outboard stations. At $80 \%$ span, the suction-pressure maxima persist over $45 \mathrm{deg}$ of the rotation cycle.

Figure 4.31 is identical to Figure 4.30 except that single-revolution instead of bin-averaged results are presented. In general, the characteristics are identical to the bin-averaged results with a few exceptions. The overall suction pressures are higher for the single-revolution case selected. Turbulence levels, indicated by random pressure fluctuations, were more apparent for the single-revolution case. This effect was greater at outboard stations.

Figure 4.31. Spanwise pressure distribution vs. azimuth angle. $16.7 \mathrm{~m} / \mathrm{s}$ wind speed, $4 \%$ chord, tipvelocity normalization, bin-averaged data, $30^{\circ}$ yaw.

\subsection{Conclusions}

The influences of unsteady flow were examined on the Combined Experiment wind-turbine rotor. The effects of yawed flow and tower shadow were investigated extensively. Some general conclusions are: 


\section{SPANWISE PRESSURE DISTRIBUTION $4 \%$ CHORD LOCATION}

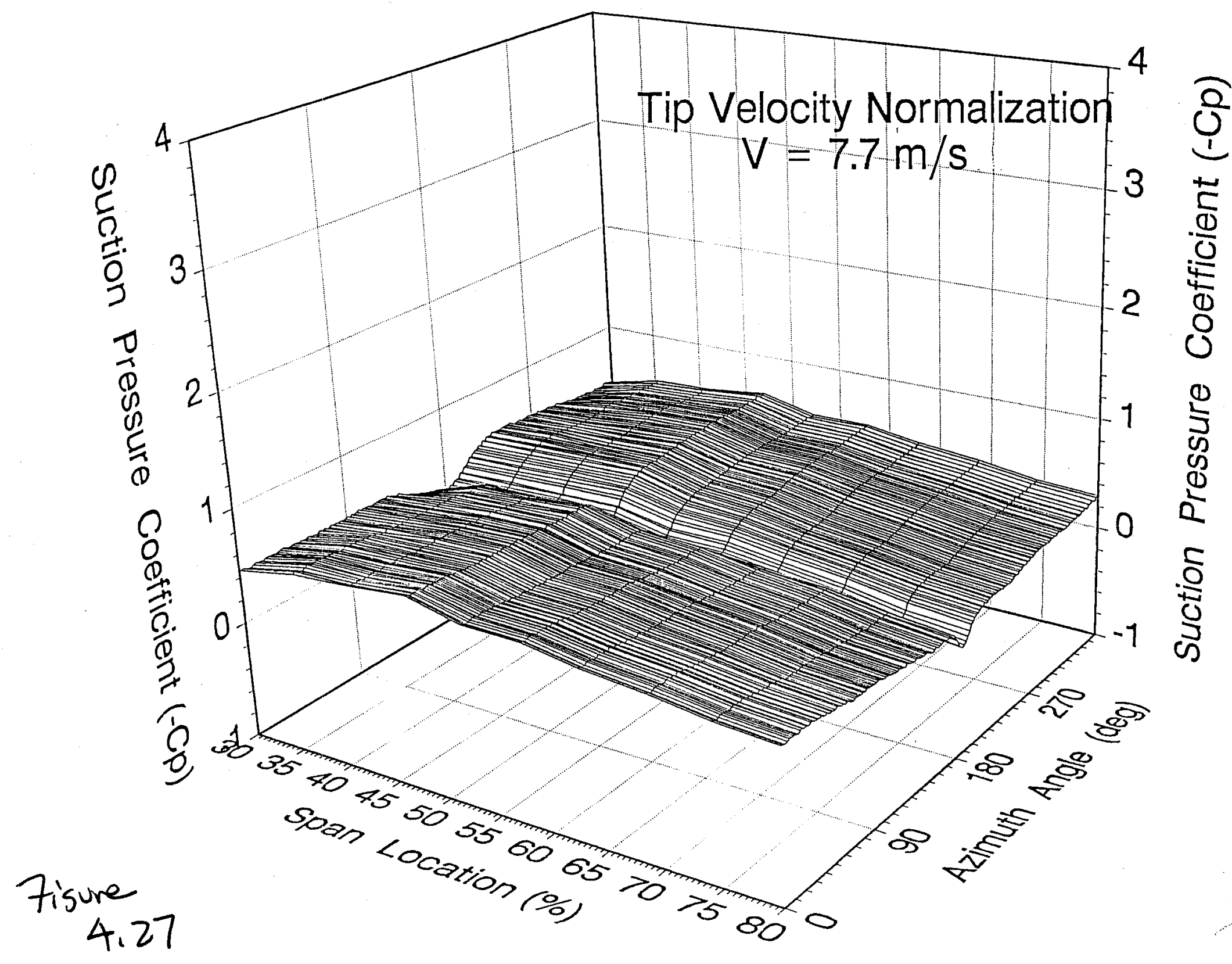

$$
4.27
$$



$4 \%$ CHORD LOCATION

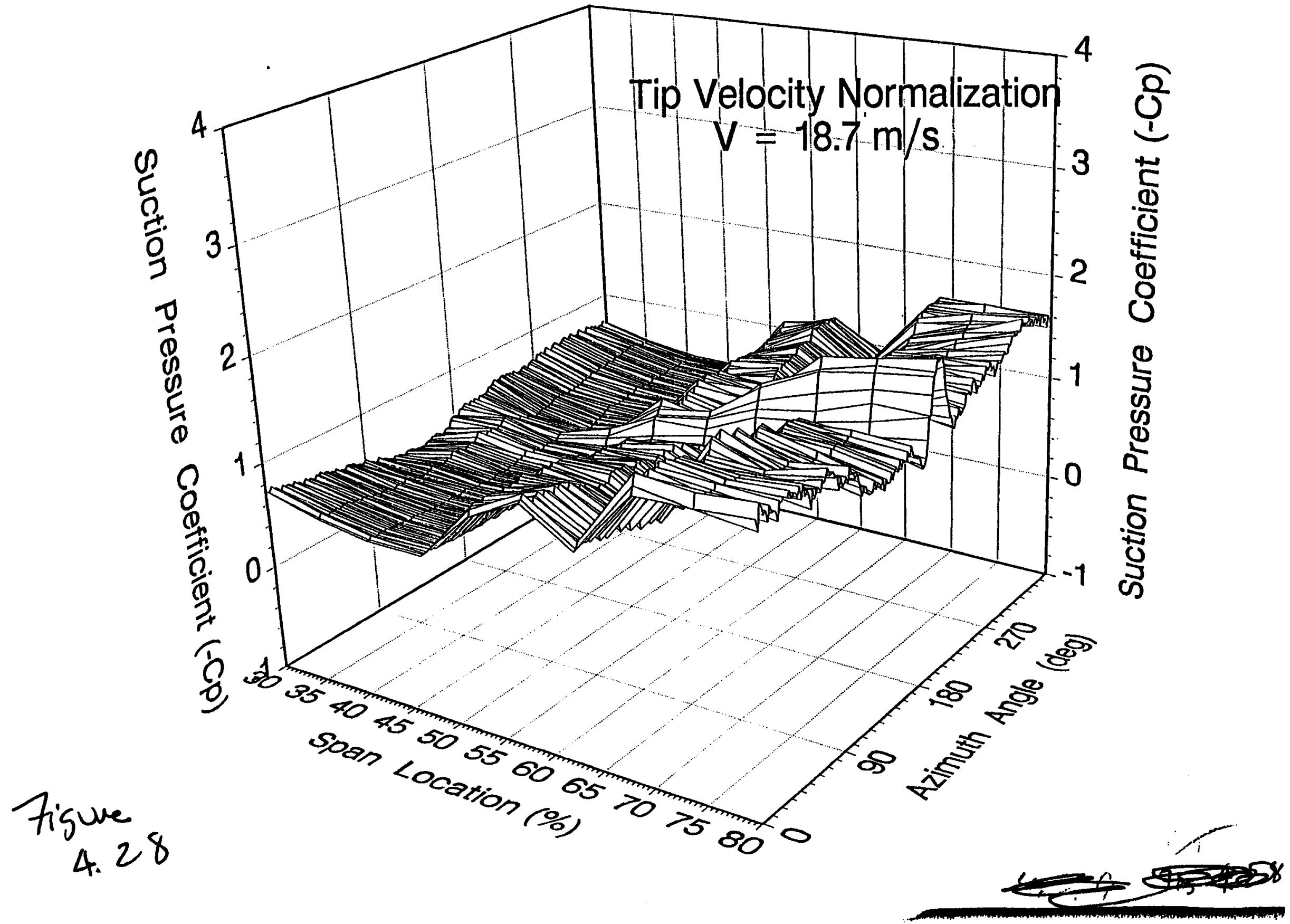




\section{SPANWISE PRESSURE DISTRIBUTION $4 \%$ CHORD LOCATION}

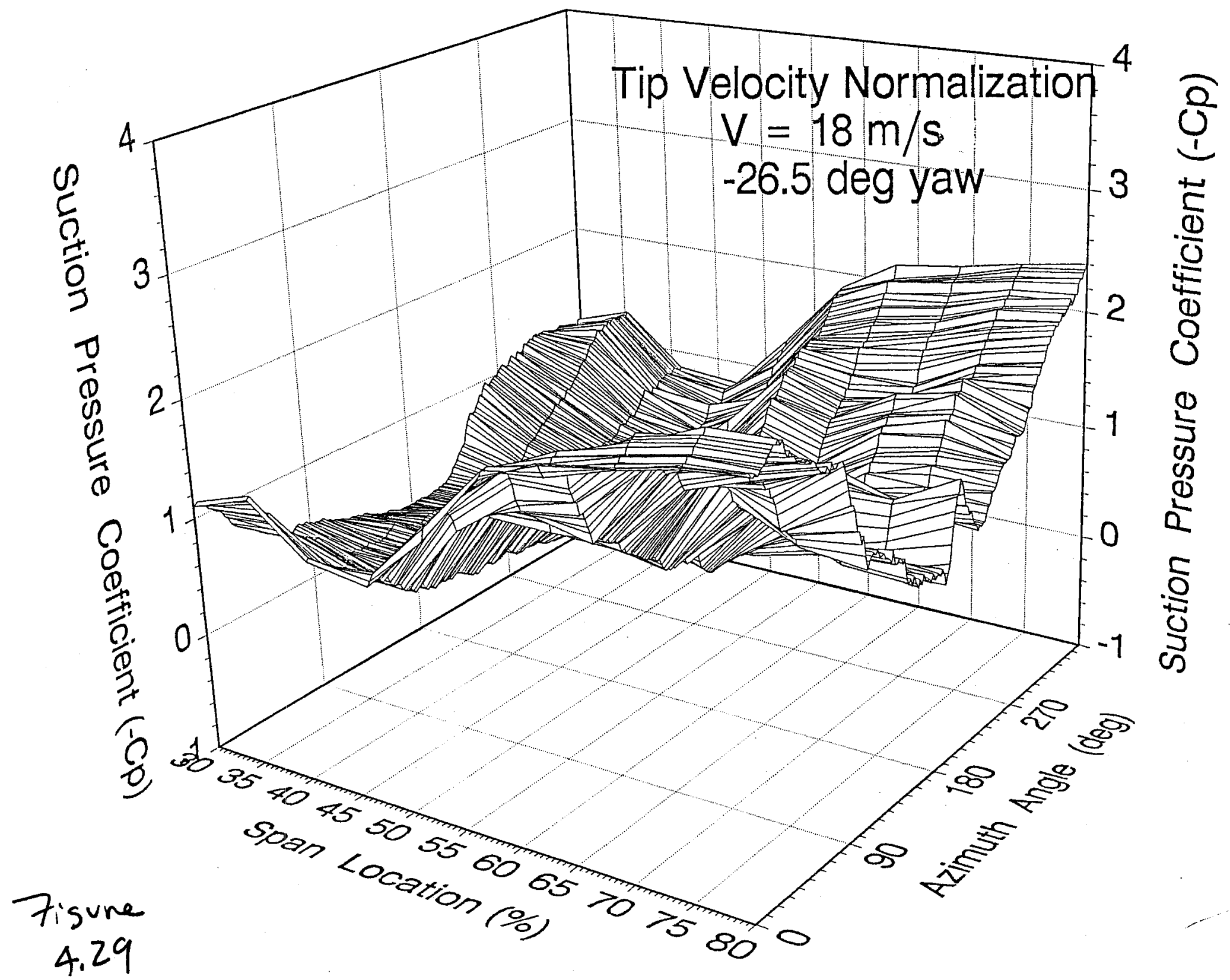

迹 
SPANWISE PRESSURE DISTRIBUTION $4 \%$ CHORD LOCATION

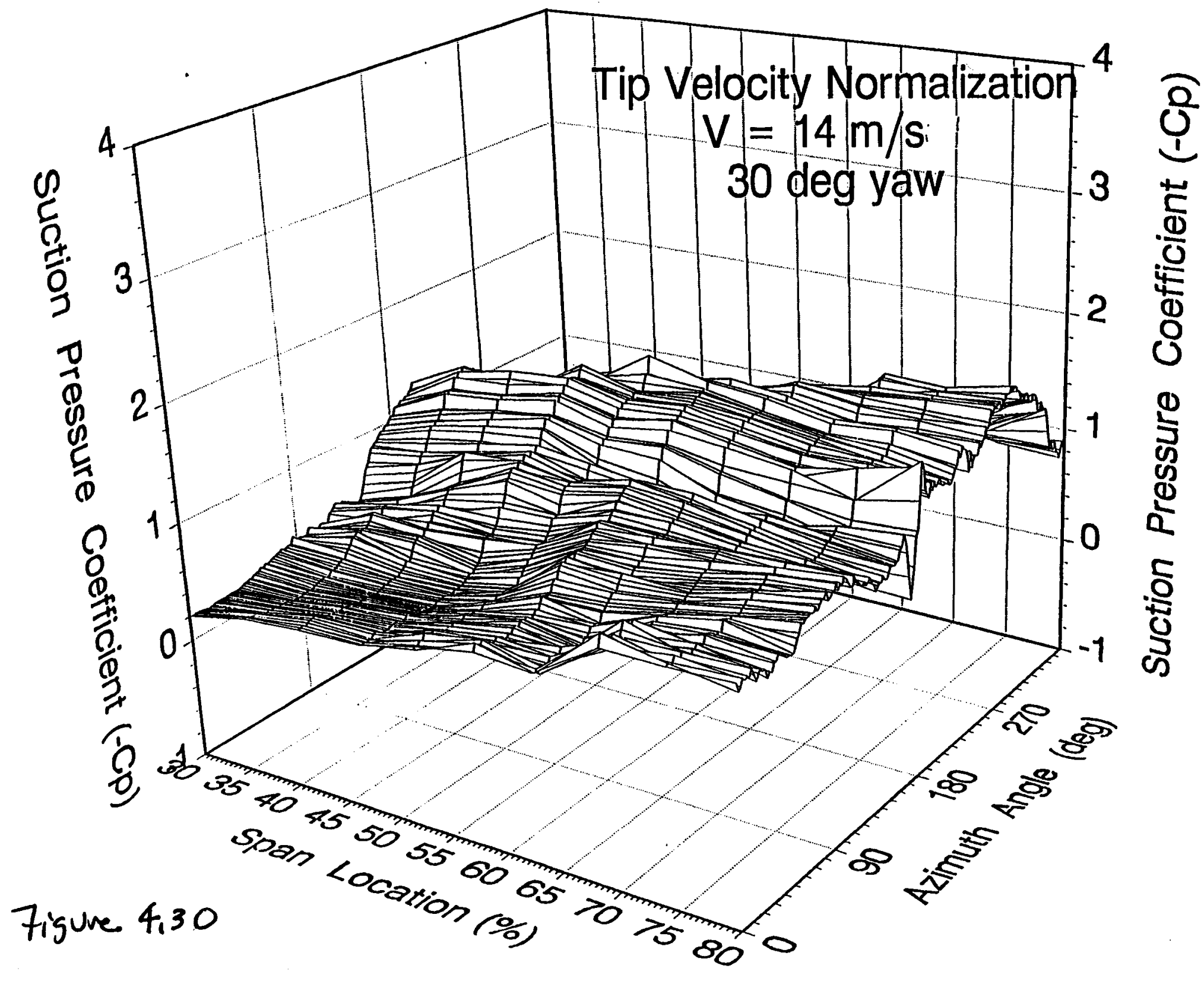

Figure 4,30 


\section{SPANWISE PRESSURE DISTRIBUTION $4 \%$ CHORD LOCATION}

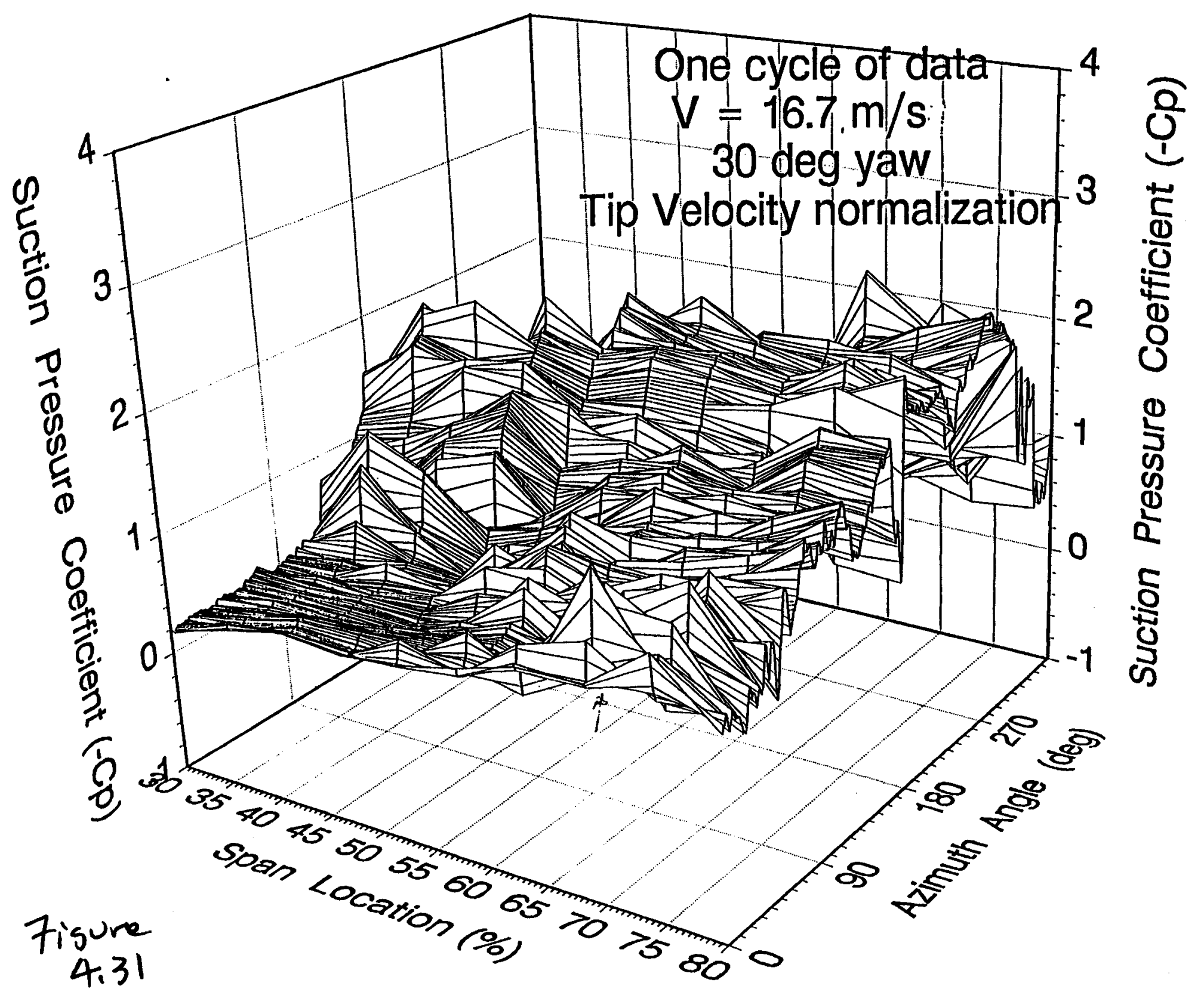


- Dynamic stall was found to exist on a HAWT operating at 30 deg yaw angle.

- Dynamic stall was also found for zero yaw error when tower shadow, wind shear, or inflow turbulence caused large AOA excursions.

- Increased aerodynamic loads due to dynamic stall cause increased structural loading.

- Dynamic stall forces caused by tower shadow are shorter in duration and lower in magnitude than those caused by yawed flow.

- Dynamic stall should be included in structural dynamic rotor models in order to accurately predict service loads. The data presented in this report plus future data will provide the basic information needed to develop dynamic-stall models.

- Surface plots of suction-pressure distributions on a rotating airfoil demonstrated the presence of discrete vortex structures.

- Temporal shifts in maximum suction pressures toward the trailing edge suggested the convection of these vortex structures, which was consistent with pressure signatures produced by vortex formations on pitching airfoils.

- Future analysis is required to more accurately predict the formation of vortex structures on rotating wind turbines. This will allow for better modeling of dynamic stall, structural loading, and rotor performance. 


\subsection{Flow-Visualization Tests}

Flow visualization was used to establish a correlation with the surface-flow patterns and pressure data. Section 5.1 describes a liquid-crystal surface-shear-stress coating that was used to examine the boundarylayer characteristics. Section 5.2 describes the correlation of pressure distributions with video-taped flow patterns using tufts. This technique was used to prove that separated flow conditions coexist with highnegative-pressure coefficients on the inboard sections of the rotating blade. In general the video correlation technique is useful for a variety of data-analysis tasks, but in this case it is used to correlate surface-flow patterns with surface-pressure distributions.

\subsection{Liquid Crystal Tests}

A diagnostic technique capable of "visualizing" the instantaneous surface-shear-stress pattern in dynamic flow fields, in a continuous and reversible manner, would be a valuable research tool. The potential of liquid crystals to meet this objective has been under investigation at Sandia National Laboratories. A description of the technique and results obtained to date, from both the laboratory and an operating vertical-axis wind turbine, are given in Reda (1988a, b).

A cooperative research effort was jointly conducted by Sandia National Laboratories and the National Renewable Energy Laboratory to investigate the feasibility of applying the liquid-crystal technique to horizontal-axis wind turbines operating in field environments.

Initial experiments were run under light-wind conditions while the machine was motor-driven. A portion of the blade surface near the pressure taps at $80 \%$ span was coated with the liquid-crystal solution. Under these conditions, the airfoil AOA was essentially equal to the airfoil pitch setting. Both thermochromic (shear/temperature-dependent) and shear-sensitive-only liquid-crystal mixtures were employed. Liquidcrystal response was recorded by a boom-mounted (downwind) video camera. Test conditions and test results are summarized in a 10-minute color video, obtainable upon request from the authors.

The technical feasibility and viability of the liquid-crystal technique in WECS field environments were further demonstrated. Results for the SERI-S809 airfoil showed the existence of an adverse-pressuregradient-induced (contour-generated) laminar separation bubble near the airfoil mid-chord at low AOAs. For AOAs near $10 \mathrm{deg}$, this bubble quickly moved to the airfoil leading edge. In both cases, transition to turbulence occurred in the shear layer above the local reverse-flow region, resulting in a high-shearstress turbulent reattachment zone immediately downstream of the bubble. At low AOAs, the turbulent boundary layer remained attached to the airfoil surface all the way to the trailing edge. However, at $10 \mathrm{deg}$ AOA, turbulent-boundary-layer separation occurred at the $60 \%$ to $70 \%$ chord location. Higher AOAs could not be tested on the wind turbine but were tested in the wind tunnel at Ohio State University.

Wind-tunnel tests demonstrated that the boundary-layer behavior was very similar to the boundary-layer behavior in the rotating-blade case at low AOAs. The chordwise transition locations were not measurably different, and the transition AOAs appeared to be the same. The laminar separation bubble occurred at the same chordwise location, and moved forward toward the leading edge at AOAs larger than 8 deg.

The airfoil was dynamically pitched to induce unsteady flow to see if the resulting unsteady-pressure distributions would affect the boundary-layer-transition locations. The blades were tested at reduced frequencies $\left(\mathrm{C} \omega / V_{\mathrm{r}}\right.$, where $\mathrm{C}$ is chord length, $\omega$ is cyclic frequency, and $V_{\mathrm{r}}$ is relative velocity) up to 0.05 , which corresponds to the once-per revolution sinusoidal pitch variation that the turbine experiences during yawed operation). Transition locations occurred at the same chordwise locations when the unsteady AOA 
corresponded to the static AOA. Reda and Butterfield (1989) examined this unsteady behavior in detail on a similar symmetrical airfoil.

Dynamic stall is known to exist at this reduced frequency for large AOA amplitudes. The liquid crystals did not show a distinct color change at the turbulent separation boundary. This characteristic made it difficult to quantify the unsteady turbulent separation location. Therefore, the effects of dynamic stall may have been present but unnoticed in the liquid-crystal results.

\subsection{Correlations of Pressure Distribution with Flow Patterns}

\subsubsection{Introduction}

In the past, the research community has not been able to predict dynamic loads on wind-turbine blades resulting from complex inflow. This prediction is particularly difficult during high-wind-speed operation, when stall occurs throughout the blade span (Viterna and Corrigan, 1981; Madsen et al., 1988). Flow visualization has been used to determine if stall separation is occurring as expected. Correlating these flow-visualization data with operating data such as wind speeds and power levels has not been done previously because of the difficulty in establishing a link between video data and recorded engineering data.

This link has been established as part of Phase II of the Combined Experiment. Correlating the flow visualization with the recorded time-series data gave researchers a new tool for validating and interpreting unsteady-flow phenomena.

This work has been described in more detail in the paper by Scott et al. (1991).

\subsubsection{Flow Visualization Test Setup}

Two rotor-mounted black-and-white high-resolution video cameras spin with the hub and give stationary (in the rotating coordinate frame) images of the downwind side of the blade. One camera is mounted on the blade, giving a low-angle (oblique) view of the entire blade, and the other is mounted at the end of a rigid boom extending $3 \mathrm{~m}$ downwind. The blade-mounted camera can be remotely zoomed, although it is typically set so that the low-angle view of the blade fills the bottom half of the video frame. The second (boom-mounted) camera can be panned to view almost any part of the blade. The video signals from the cameras and all the pressure data are passed through slip rings to cables to the recorders. Figure 5.1 shows the turbine setup.

\section{Figure 5.1. Wind turbine with video cameras}

To visualize the air flow over the blade, half-inch-long tufts of white thread (polyester \#60) are attached to the blade. When flow is attached, the boundary layer is thin, and the tufts remain close to the blade and align themselves with steady streamlines. When the flow becomes separated, the tufts' movement becomes random as the tufts are caught up in the recirculating turbulence. This difference in behavior makes it easy to see the boundary between attached and separated flow on the video images.

Additional video equipment consists of a video mixer and a Super-VHS video cassette recorder. The Super-VHS recorder, when used with the high-resolution cameras, gives about a $25 \%$ improvement in horizontal resolution of the video image over a standard VHS image. The mixer allows a combination of the two camera signals to be recorded on a single video tape. Because the view of the blade from the blade-mounted camera fills less than half the video frame, the upper half of the frame is usually filled with 


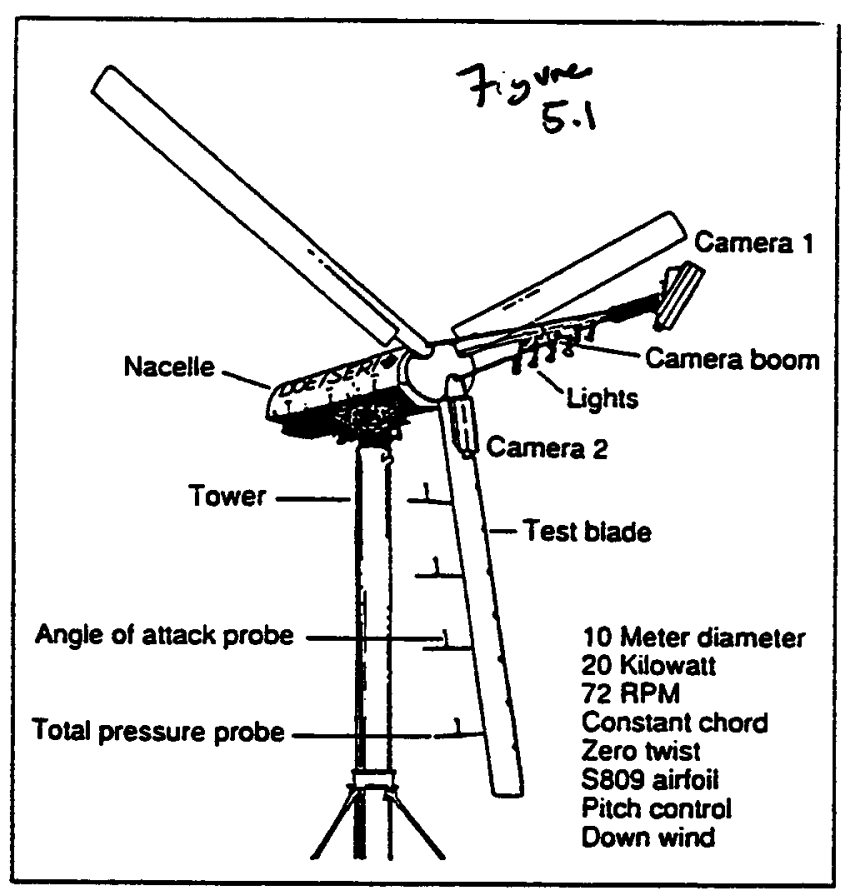

zisure 5.1 
part of the view from the boom-mounted camera. Figures in this report show only the view from the blade-mounted camera. Figure 5.2 shows the video signal path.

\section{Figure 5.2. Video signal path}

A good video image of the tufts on the blade requires high contrast between the white tufts and the flat black blade. The best way to achieve this is to record all video data at night. During recording, the blade is illuminated by $12100-\mathrm{W}$ tungsten/halogen spotlights that are mounted on the boom and that rotate with the blade.

The master system clock is a Datum 9100 IRIG-B Time Code Generator. The same IRIG-B time code that is recorded on tape with the pulse code modulated (PCM) data streams is fed to a Datum Video Time Inserter, which overlays a block containing the time code on the video image.

The final product of the video system is a Super-VHS tape showing views of the blade. This tape is then taken to the NREL office for further processing. The video system in the NREL office consists of a 25-MHz 386-based PC with a Truevision Targa-16 video board and a BCD-4000 VCR controller that controls two Panasonic editing VCRs. Software on the system allows the researchers to grab still images from video tape, modify them to improve visibility, overlay other graphics on top of the video image, and produce an animated sequence of still frames on video tape.

\subsubsection{Data Processing}

\section{Video Data}

Standard NTSC video is recorded at a rate of 30 frames per second, while the PCM data stream from the turbine has a sample rate of $520.8 \mathrm{~Hz}$. To correlate the video data with the PCM data, a program was written that reads a list of frame time codes, picks the closest data record from a disk file of PCM data, and writes the time code and the upper-surface pressure record to another file. This file is then transferred to the PC for use by the pressure overlay program.

Picking the closest record for a given time code is somewhat arbitrary, because a video frame actually requires $1 / 30$ th of a second to generate, a time period that spans 17 PCM data records. The procedure does guarantee that the data record was recorded at some point during the frame generation, and that PCM data records are separated by $1 / 30$ th of a second, plus or minus one PCM data-sample interval $(0.33 \pm$ $0.00192 \mathrm{sec}$ ).

\section{Video Image Processing}

When a segment of interest has been chosen, it must be saved as a series of individual frames. First a channel of a proprietary frame code is added to the video tape. This frame-code information is required by the VCR controller. Next, a table is prepared listing frame code versus inter-range instrumentation group (IRIG) time code for each frame in the segment. An automated frame-grabbing program grabs each frame from the video tape and field copies it. Because of the interlaced nature of NTSC video, adjacent horizontal lines are actually recorded $1 / 60$ th of a second apart, a time long enough to blur a moving object. Field copying replaces each even-numbered line with another copy of the previous odd-numbered line, reducing the vertical resolution by a factor of 2 , but greatly reducing inter-field jitter. The resulting cleaned-up frame is then saved as an image file on the hard disk of the PC. 


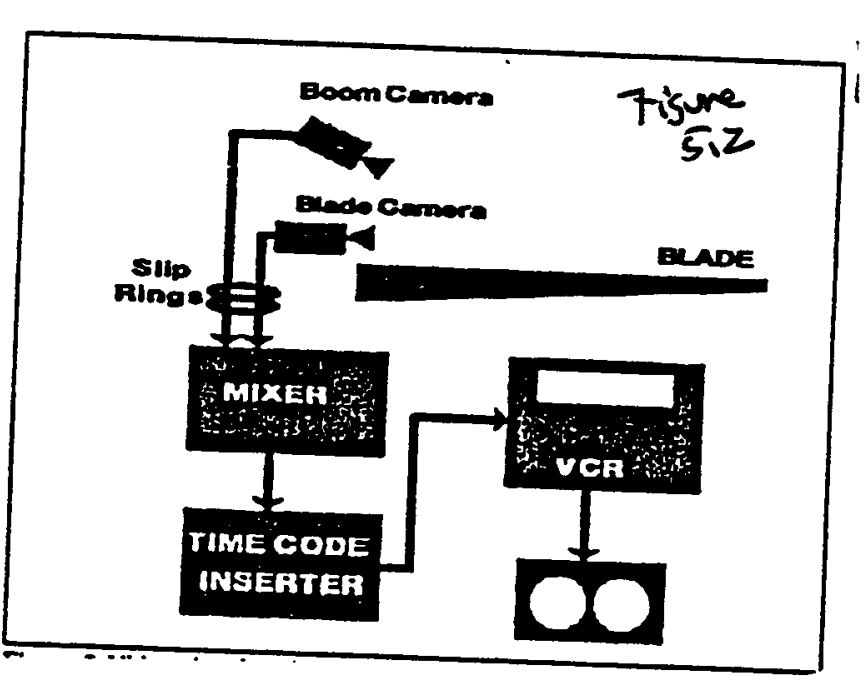

Figure 5.2 
The pressure overlay program loads each frame image file, finds the proper pressure record in the data file, draws the pressure graphs on the blade image, and stores the result as another PC image file. The animation program writes a specified number of frames of each image file to video tape. For example, if 10 frames of each image file are written to tape, the resulting animated sequence runs at one-tenth the original speed, allowing rapid transitions in flow on the blade to be observed. Single frames can also be studied to map boundaries between attached and separated flow.

Figure 5.3 shows an example of a pressure distribution superimposed on a video frame. The video frame shows tufts attached to the rotating wind-turbine blade. Angle-of-attack probes can be seen extending from the leading (right-hand) edge of the blade. This display allows the researcher to correlate the separation boundary displayed by the tufts with pressure distributions measured by transducers mounted inside the blade.

\section{Figure 5.3. View of blade at low AOA}

A variety of data-display programs are available on the UNIX system. These programs give a more detailed view of pressure profiles, and also show wind speeds, time histories of attack angle, pitch angle, and other variables from the PCM data stream. It is very informative to simultaneously study both the video and the output of these graphics programs. The VCR is advanced a frame at a time, and the time code displayed on each video frame is noted. The graphics program can then be set to display the corresponding PCM data record.

The resolution of the camera and video tape is not sufficient to resolve individual tufts near the blade tip. Thus, it is very difficult to map the boundary between separated and attached flow at positions farther out than about $65 \%$ span. Perhaps in the future, we can use the high-angle (boom-mounted) camera to map this transition.

\subsubsection{Pressure Distributions}

Figures 5.3, 5.4, and 5.5 show low-angle views of the blade and tufts at low, medium, and high angles of attack (AOAs). Table 5.1 gives the AOA at each probe for each figure. Superimposed on each figure are the graphs of the four chordwise pressure distributions. The bottom edge of each figure is located at approximately $32 \%$ span.

Table 5.1. Angles of Attack

\begin{tabular}{ccccc}
\hline Figure & $34 \%$ & $51 \%$ & $67 \%$ & $86 \%$ \\
\hline 5.3 & $13.3^{\circ}$ & $8.5^{\circ}$ & $3.7^{\circ}$ & $0.9^{\circ}$ \\
5.4 & $23.2^{\circ}$ & $14.1^{\circ}$ & $5.0^{\circ}$ & $2.1^{\circ}$ \\
5.5 & $28.9^{\circ}$ & $19.5^{\circ}$ & $11.0^{\circ}$ & $6.7^{\circ}$ \\
\hline
\end{tabular}

In Figure 5.3, the low-attack-angle case, there is no stall, and the flow is attached everywhere. The outboard pressure profiles show the characteristic rounded distribution that is seen in the wind tunnel at low attack angles, while the inboard (30\%) profile has a strong suction peak. A strong suction peak is characteristic of high AOA prestall operation. 

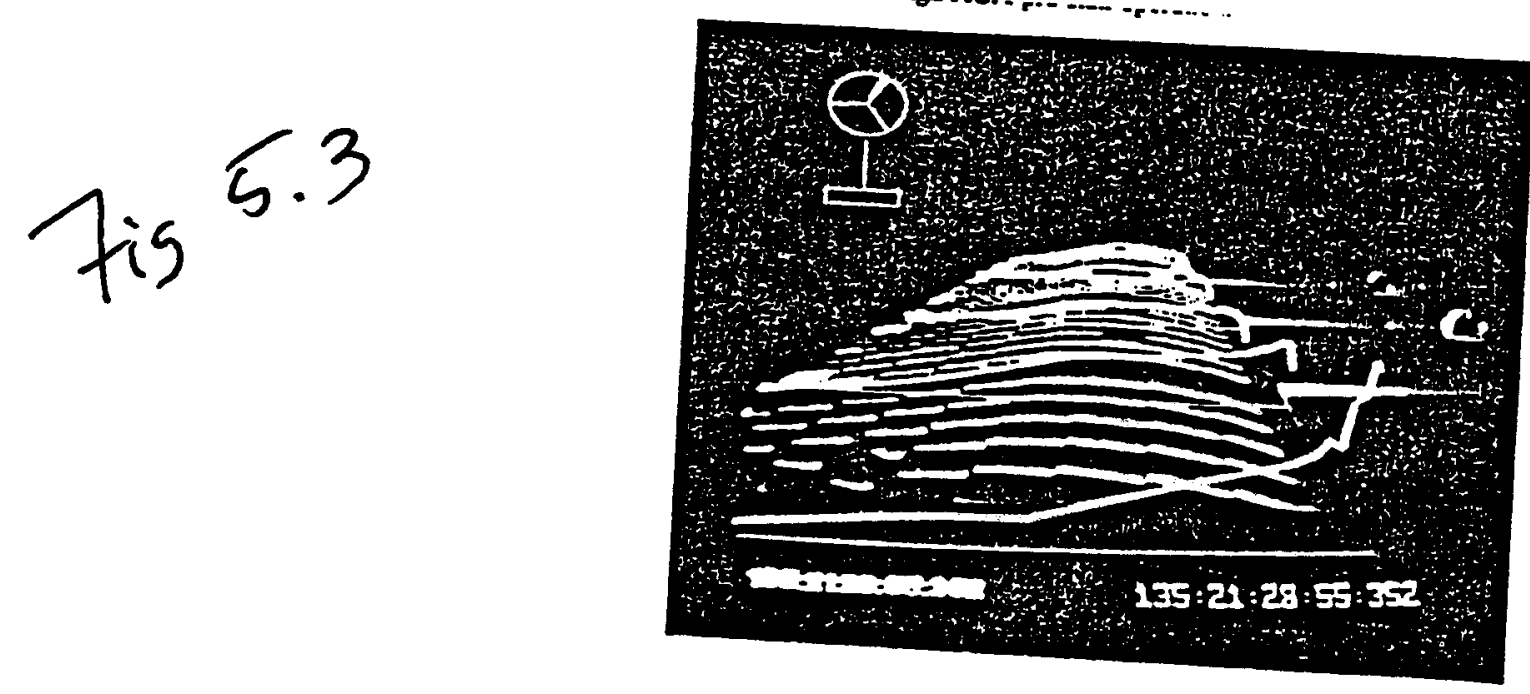

7 is 5.4

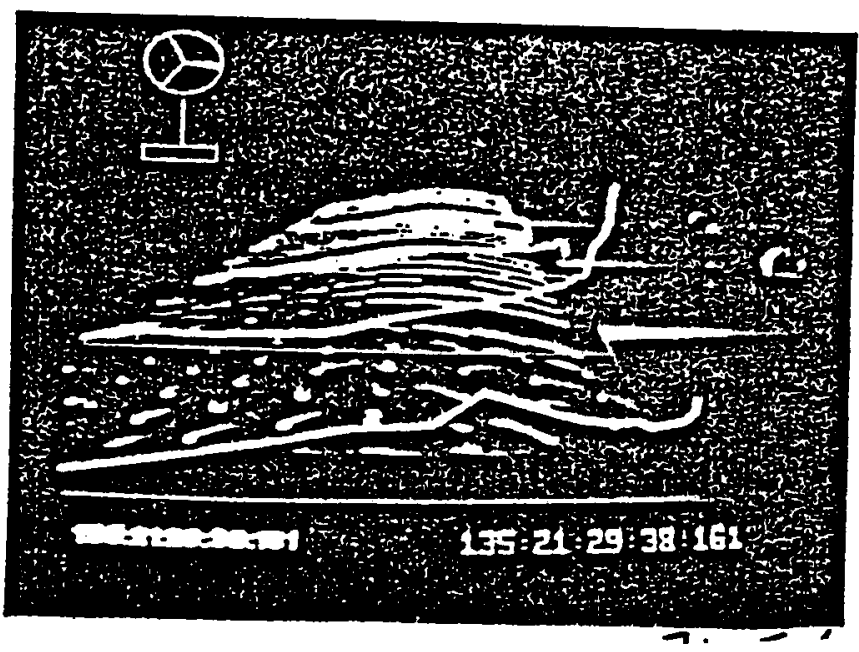

7 is 5.5

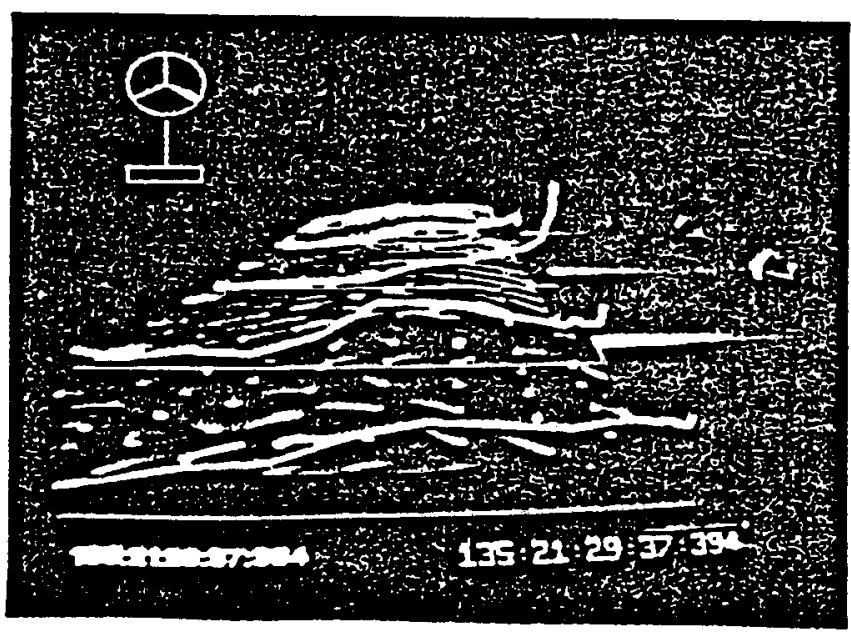


Figure 5.4, the moderate-attack-angle case, shows separated flow from about $40 \%$ inboard. The inboard profile has lost its suction peak, a characteristic of leading-edge stall, and is approaching a flat profile. The leading-edge suction peak has reappeared at the $47 \%$ profile.

\section{Figure 5.4. View of blade at medium AOA}

In the high-attack-angle case of Figure 5.5, the two inboard profiles are stalled, and the suction peak has moved out to the $67 \%$ profile. Flow at the inboard stations is definitely separated, but the pressure gradient is non-zero.

\section{Figure 5.5. View of blade at high AOA}

The pressure measurements made on the rotating blade are significantly different from those made in the wind tunnel. Figure 5.6 shows a comparison of a wind-tunnel test at an AOA of $26.2 \mathrm{deg}$, and a sample from a wind-turbine test at an AOA of $26.5 \mathrm{deg}$ at the $30 \%$ blade span. Both upper- and lower-surface pressure coefficients are shown for each case. The rotating-blade tests show suction peaks with values up to about -3.0; the wind-tunnel data shov a flat pressure distribution at the same AOA. This flat distribution is typical of a fully stalled two-dirnensional aiffoil. Local pressure coefficients on the rotating blade were measured at up to three times those in the wind tunnel.

\section{Figure 5.6. Comparison of wind-tunnel and rotating-blade pressures}

Figure 5.7 compares lift coefficients $\left(C_{L}\right)$ measured in the wind tunnel with data measured from the wind turbine. The wind tunnel shows a rapid drop in $C_{L}$ at an $A O A$ of $18 \mathrm{deg}$. This drop is caused by leading-edge stall (the loss of the leading-edge suction peak). The wind-turbine data show no drop because the suction peak and pressure gradients persist at high AOAs, as shown in Figure 5.6.

\section{Figure 5.7. Comparison of lift coefficients from wind tunnel and wind turbine}

Figure 5.8 shows the corresponding pressure drag coefficient $\left(C_{D_{p}}\right)$ curves. This comparison shows that the wind-turbine drag is greater than that in the wind tunnel at all AOAs. If the delayed stall (high lift coefficients) shown in Figure 5.7 was caused by extended attached flow (less separation), one would expect lower pressure drag from the wind-turbine data. Instead the pressure drag is higher than the windtunnel results, implying more separated flow. This lift results from high negative values of $C_{p}$ in regions of separated flow.

\section{Figure 5.8. Comparison of pressure drag coefficients from wind tunnel and wind turbine}

Performance analyses of stall-controlled wind turbines depend on accurate inputs of $C_{L}$ and $C_{D p}$ to predict the peak performances. The differences shown in Figures 5.7 and 5.8 explain why analyses based on wind-tunnel data often underpredict wind-turbine performance.

\subsection{Conclusions}

A method of correlating video flow-visualization data and standard time-series pressure data recorded on an operating wind turbine has been shown. A key to this correlation is to use a single clock signal and to simultaneously record it on the video tape using a time code inserter and on the PCM data stream.

Significant differences between the wind-turbine-blade field results and wind-tunnel tests have been observed. Beyond stall, leading suction peaks persist to high angles of attack. At inboard stations on the blade, non-zero pressure gradients exist in regions of separated flow. These gradients do not occur in the 
7 is 5.6

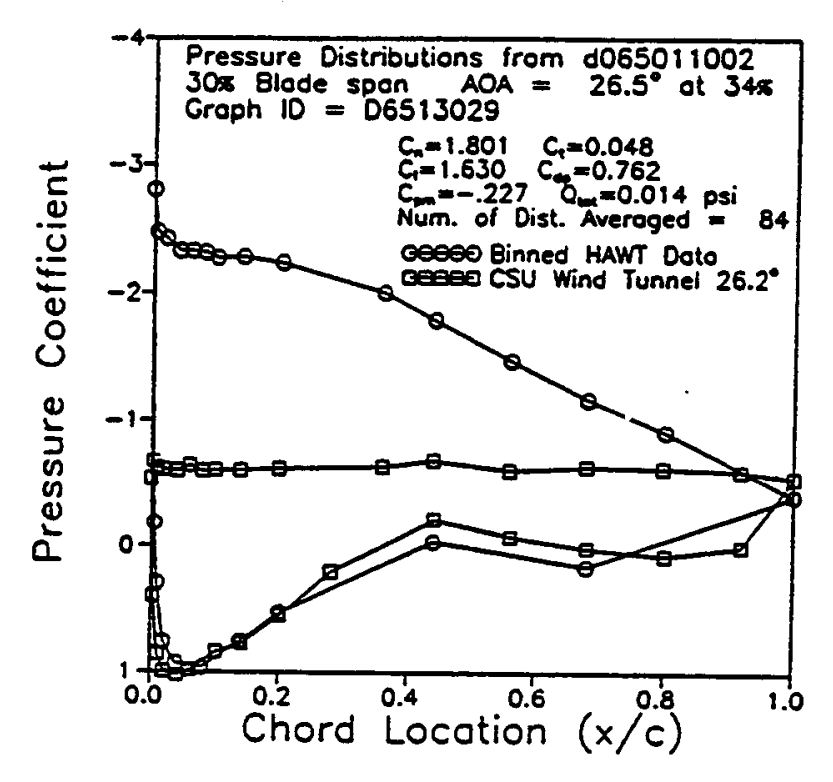

Figue 5.7

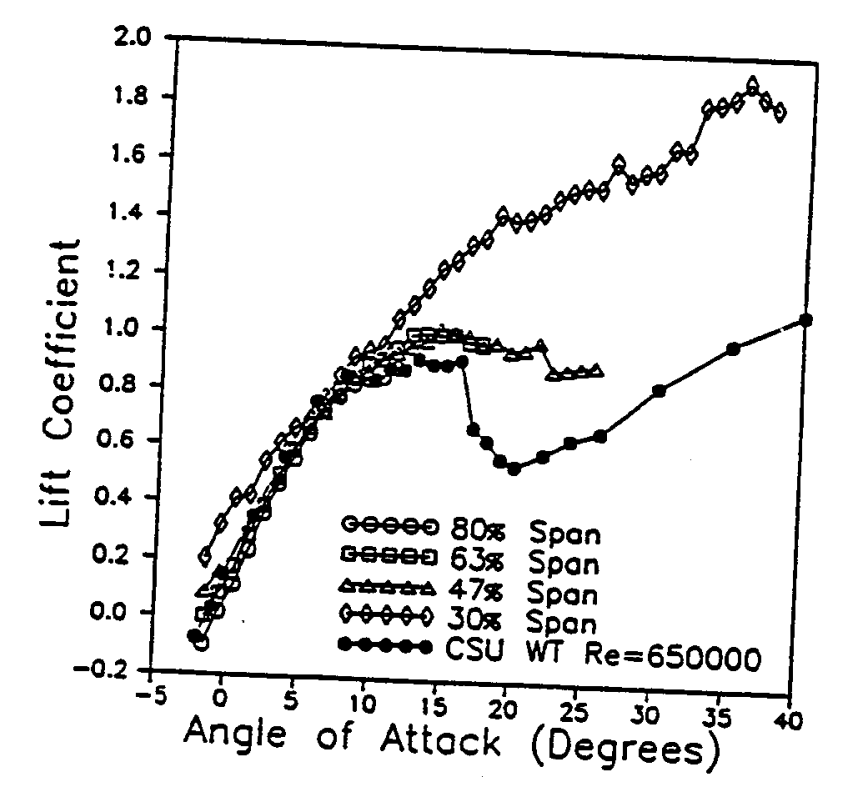

Fisure 5.8

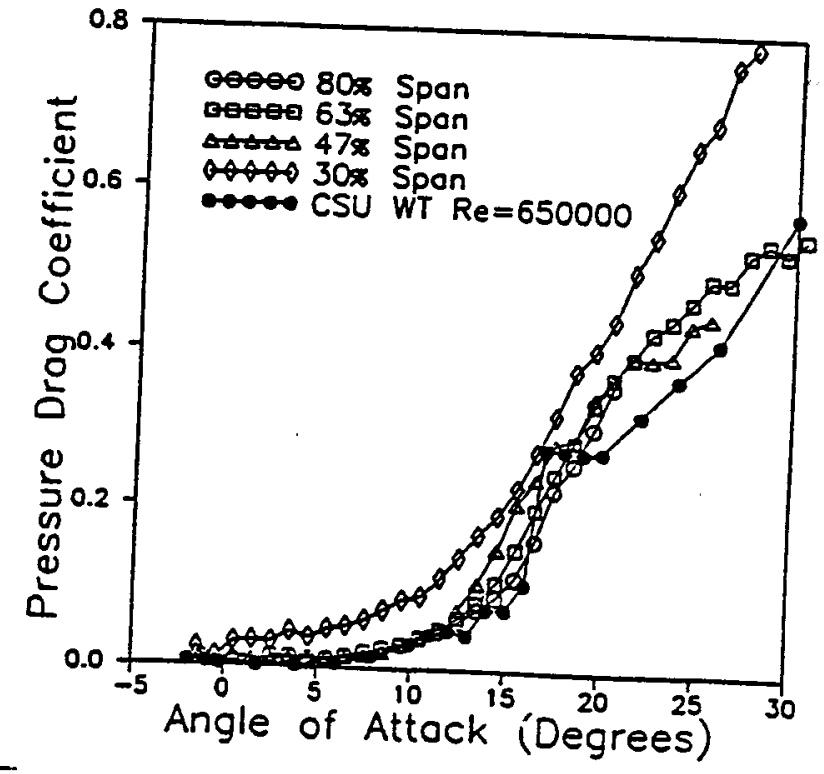


wind tunnel. These results help explain why performance analyses typically under predict the peak performance on stall-control wind turbines. 


\title{
6.0 Structural Loads
}

\subsection{Introduction}

\begin{abstract}
Structural loads are caused by airloads, whifh,in turn, are caused by inflow conditions. Understanding the connection between structural loads and the inflow was one of the major reasons for conducting this experiment. Various techniques for establishing the correlation between airloads and structural loads were investigated.
\end{abstract}

There was a second motivation for investigating structural loads. During the test plan, design attempts were made to include redundant measurements or means to check primary measurements. Blade bending moment distribution measurements were made in order to confirm aerodynamic load distributions. They were also made so that blade motions or aeroelastic interactions could be isolated from the natural aerodynamic forces caused by inflow alone. Steady load distributions are affected by both airloads and centrifugal forces. Dynamic loading is cyclic in nature and involves blade flapwise motions. It is more difficult to use dynamic blade loads to estimate unsteady aerodynamic loads.

Section 6.2 describes a technique that uses dynamic blade loads to estimate unsteady aerodynamic loads. This technique was used to estimate airioads that were then compared to integrated airloads measured by pressure taps. Although the greatest degree of confirmation of the pressure measurements was gained through comparisons with wind tunnel data, this method provided another piece of confidence-building information. It was also useful to develop the technique and validate it for later use on turbines that cannot be instrumented with pressure taps.

Section 6.3 describes "steady" or bin-averaged load data that can be used for comparison with steady prediction codes.

Structural loads are the important end result of aerodynamic applied loads. It is important to determine the transfer function that relates aerodynamic loads to structural loads, so that the wind turbine designer can determine the frequency band of aerodynamic loads with which he should be concerned. Steady structural loads are important as well. Section 6.2 describes a technique adapted from the helicopter industry, which uses measured structural loads plus an analytical dynamics model (transfer function) to estimate airloads acting on the rotor. This technique was validated by comparing its predictions to the loads measured on the Combined Experiment turbine. A good correlation with measured airloads is demonstrated for a variety of operating conditions.

Section 6.3 briefly describes the bin-averaged load data base presented in Appendix E.

\subsection{Air Load Estimating Tool (ALEST)}

Two methods can be used to determine aerodynamic loads on a rotating wind-turbine blade. The first is to make direct pressure measurements on the blade surface. This is a difficult process requiring costly pressure instrumentation. The second method uses measured flap bending moments in conjunction with analytical techniques to estimate airloads. Estimating airloads using flap bending moments is much simpler and less costly because measurements can be made with conventional strain gages and equipment. This section presents results of airload estimates obtained using both methods under a variety of operating conditions. Insights on the limitations and usefulness of the bending-moment technique are also included.

Surface pressures provide a direct measurement of spanwise blade aerodynamic load distribution. Air-load distribution can also be estimated using strain-gage bending-moment measurements (DuWaldt and Statler, 
1966). The Combined Experiment data set provides an excellent resource for comparing airloads obtained using either method. Because accurate pressure measurements from a rotating blade are difficult and expensive to obtain, a simpler strain-gage method could be a valuable tool in better understanding aerodynamic behavior. Blade airload data are especially useful for validating wind-turbine analytical tools. To explore this further, we have evaluated a technique in which flapwise bending-moment data are used in conjunction with known blade mass and stiffness properties to estimate airloads. This technique was originally developed for use on helicopter rotors as a computer code called ALEST (Air Load ESTimation) (Bousman, 1987).

In ALEST, free vibration modes of the blade are used in a single-rotating-blade model to determine modal displacements based on measured bending-moment distributions. An empirical least-squares method is used to determine modal amplitudes. This information is used in the blade model to estimate aerodynamic loads. The airloads are a function of the mode shapes, modal amplitudes, mass distribution, and frequency information. ALEST solves the dynamic uncoupled flap equation of motion to determine resultant aerodynamic forces.

The modification of ALEST for wind turbine use required adding terms to include gravity loading affected by precone angle and blade pitch. A set of test conditions was devised to verify modifications made to ALEST. First, the code was checked by comparing estimated airloads and bending moments with those obtained from simple beam theory using an analytical continuous beam model. Estimated results from ALEST were identical to those from theory (Schnepp, et al., 1992). Second, the modifications to ALEST were checked by comparisons with the Force and Loads Analysis Program (FLAP) (Wright and Thresher, 1987). FLAP uses wind inflow characteristics and machine properties in conjunction with known blade aerodynamic data to calculate blade loads. Bending moments calculated by FLAP were input directly into ALEST. Comparisons between ALEST and FLAP showed good correlation (Simms et al., 1990).

In this section, measured airloads are compared to those determined from ALEST using corresponding measured bending-moment data. The second phase of the Combined Experiment provided measured airload distributions from pressure data at four span locations: $30 \%, 47 \%, 63 \%$, and $80 \%$. At each of these locations, approximately 28 pressure taps were distributed around the blade chord. Tap distribution was concentrated more toward the leading-edge suction side of the blade to better characterize the active peak-pressure region. Each individual pressure measurement was normalized into a pressure coefficient by dividing by the total pressure, $\mathrm{P}_{\text {toos }}$, which was obtained using:

$$
P_{t o t}=\frac{1}{2} \rho\left(V_{w}^{2}+(r \omega)^{2}\right)
$$

where $\rho$ is the air density, $\omega$ is the blade rotation speed, $r$ is the radius, and $V_{w}$ is the hub-height wind velocity.

The aerodynamic normal force coefficient, $\mathrm{C}_{\mathrm{N}}$, is determined by integrating the blade-normal component of each pressure coefficient over all pressure coefficients around each chord section. Spanwise airloads, $F_{s}$, are then obtained at each span location using:

$$
F_{s}=C_{N} \cdot P_{\text {tot }} \cdot c
$$


where $C_{N}$ is the normal force coefficient, $P_{t o t}$ is the total pressure, and $c$ is the blade chord length $\left(0.457 \mathrm{~m}_{\mathrm{s}}\right.$ or $18 \mathrm{in}$.). Because spanwise airloads are calculated at a particular span location, they are in units of force per length of span. They can be multiplied by span length to determine overall blade load in the blade-normal direction.

Strain-gage bridges configured to measure flapwise bending moments were located at the blade root and at the $20 \%, 40 \%, 50 \%, 70 \%$, and $90 \%$ span locations. These were used to provide bending-moment inputs to ALEST. Because ALEST requires inputs expressed in terms of harmonic content, strain-gage data were azimuth-averaged over the number of blade cycles shown in Table 6.1 into 5 deg bins and then converted into an eighth-order Fourier expansion. Because the blade rotates at $1.2 \mathrm{~Hz}$, this provides bandwidth close to $10 \mathrm{~Hz}$. The original pressure data were sampled with $100 \mathrm{~Hz}$ bandwidth, and were digitally low-pass filtered to $10 \mathrm{~Hz}$ for these comparisons. Higher-bandwidth data could be used in ALEST; however, this would require characterizing higher frequency response with increasing numbers of harmonic terms.

Table 6.1. Case Study Parameter Statistics

\begin{tabular}{lccc}
\hline \multicolumn{1}{c}{ Parameter } & Low-Wind & High-Yaw & High-Wind \\
\hline Time duration (sec) & 30 & 20 & 60 \\
\# of blade cycles & 36 & 24 & 72 \\
Wind speed (m/s) & 8.3 & 13.7 & 18.3 \\
Wind direction (deg) & 279 & 276 & 288 \\
Yaw angle (deg) & 287 & 306 & 283 \\
Pitch angle (deg) & 12.1 & 11.3 & 12.4 \\
\hline
\end{tabular}

Three test cases were chosen: low wind, high yaw, and high wind. Minimal yaw error existed in both low-wind and high-wind cases. In the high-yaw case, dynamic stall causes large cyclic aerodynamic load variations. In all cases, there was no significant yaw motion. ALEST was not set up to model yaw motion effects. Statistics summarizing the three cases are shown in Table 6.1.

ALEST also required blade natural frequency flap mode shapes. These were obtained from blade mass and stiffness distribution characteristics and were estimated using a program called TMQ. TMQ uses the transmission matrix method (Murthy, 1976) to provide flap natural frequency mode shapes, slopes, bending moments, and shear. The first three flapwise modes for the Combined Experiment blade occurred at approximately 5,20 , and $50 \mathrm{~Hz}$. Only the first mode shape data were used in these comparisons. An alternative approach would be to conduct a modal test to directly measure blade natural frequencies and mode shapes. The measured results could be input to ALEST instead of using TMQ. Two other operational parameters, precone angle and mean pitch angle, are the final required inputs to ALEST.

Comparisons of blade airload distributions obtained using ALEST versus direct blade-measured airloads were performed on data from three test cases. Results are summarized in Figures 6.1 through 6.4. In Figures 6.1 through 6.3, each plot shows an airload distribution over the full (azimuth-averaged) blade cycle. Zero degrees corresponds to the instrumented blade pointing straight up, and $180 \mathrm{deg}$ is in the tower shadow. Airload distributions from the $30 \%, 47 \%, 63 \%$, and $80 \%$ span locations are shown in the four rows in each figure. Data from each of the test cases are shown in the three columns. In each plot, ALEST results are shown as a dotted line, and direct pressure measurements are a solid line. 
Figure 6.1. Sensitivity to input bending moment

Figure 6.2. Sensitivity to precone angle

Figure 6.3. Sensitivity to blade mass distribution

Airloads estimated by ALEST show good agreement with those observed in both the high-wind and highyaw cases. Mean values are close, and harmonic content is similar. ALEST works best when predicting outboard airloads in higher winds. In the low-wind case, however, ALEST does not work well, especially inboard. This is because the aerodynamic forces under these conditions are extremely small compared to the other gravitational, inertial, and centrifugal forces. The code can not extract small differences from large load values with enough precision to provide accurate estimates for this case.

Sensitivity studies were performed to show how variations in input parameters affect resulting airload estimation. Shaded regions in Figures 6.1 through 6.3 show these results. The sensitivity studies also give an idea as to the level of accuracy required of the measured input parameters.

Figure 6.1 shows the sensitivity to input bending moment. The upper boundary of the shaded region was obtained by adding a $5 \%$ full-scale bias error with a $\pm 5 \%$ of measured-value random error to the input data. Similarly, the lower boundary was obtained by subtracting a $5 \%$ bias error, and then adding the oscillating 5\% random error. This was done to strain-gage data at the six spanwise locations as summarized in Table 6.2.

Table 6.2. Input Bending Moment Channels

\begin{tabular}{cccc}
\hline $\begin{array}{c}\text { Location } \\
\text { (\% of Span) }\end{array}$ & $\begin{array}{c}\text { Full-Scale } \\
\text { Measurement Range } \\
(\mathbf{N}-\mathbf{m})\end{array}$ & $\begin{array}{c}\text { Bias Error } \\
(\mathbf{N}-\mathbf{m})\end{array}$ & $\begin{array}{c}\text { Random Error } \\
(\%)\end{array}$ \\
\hline 0 (Root) & \pm 3200 & \pm 160 & +5 \\
20 & \pm 3000 & \pm 150 & -5 \\
40 & \pm 2300 & \pm 115 & +5 \\
50 & \pm 1400 & \pm 70 & -5 \\
70 & \pm 800 & \pm 40 & +5 \\
90 & \pm 300 & \pm 15 & -5 \\
\hline
\end{tabular}

Figure 6.2 shows the sensitivity to a $\pm 10 \%$ variation in precone angle. The measured precone angle is $3.5 \mathrm{deg}$. The upper and lower limits of the shaded regions correspond to precone angles of $3.85 \mathrm{deg}$ and $3.15 \mathrm{deg}$, respectively. Figure 6.3 shows the sensitivity to blade-mass distribution. Blade mass (and stiffness) are input in segments as shown in Table 6.3. The blade-mass input values were varied by $\pm 10 \%$ to simulate random measurement uncertainty. Similar parameter variations were performed on the input stiffness distribution values, but results are not shown because effects were minimal.

The sensitivity studies show that measurement error does not adversely affect results. All cases show similar error band width, with measurement errors having increased impact outboard. This is somewhat unfortunate because outboard airloads are usually of greatest interest. It is also unfortunate because outboard bending-moment measurements are most susceptible to error from low strain-gage signal level and high gain. This means that care must be taken to ensure accurate measurements outboard. Overall, 

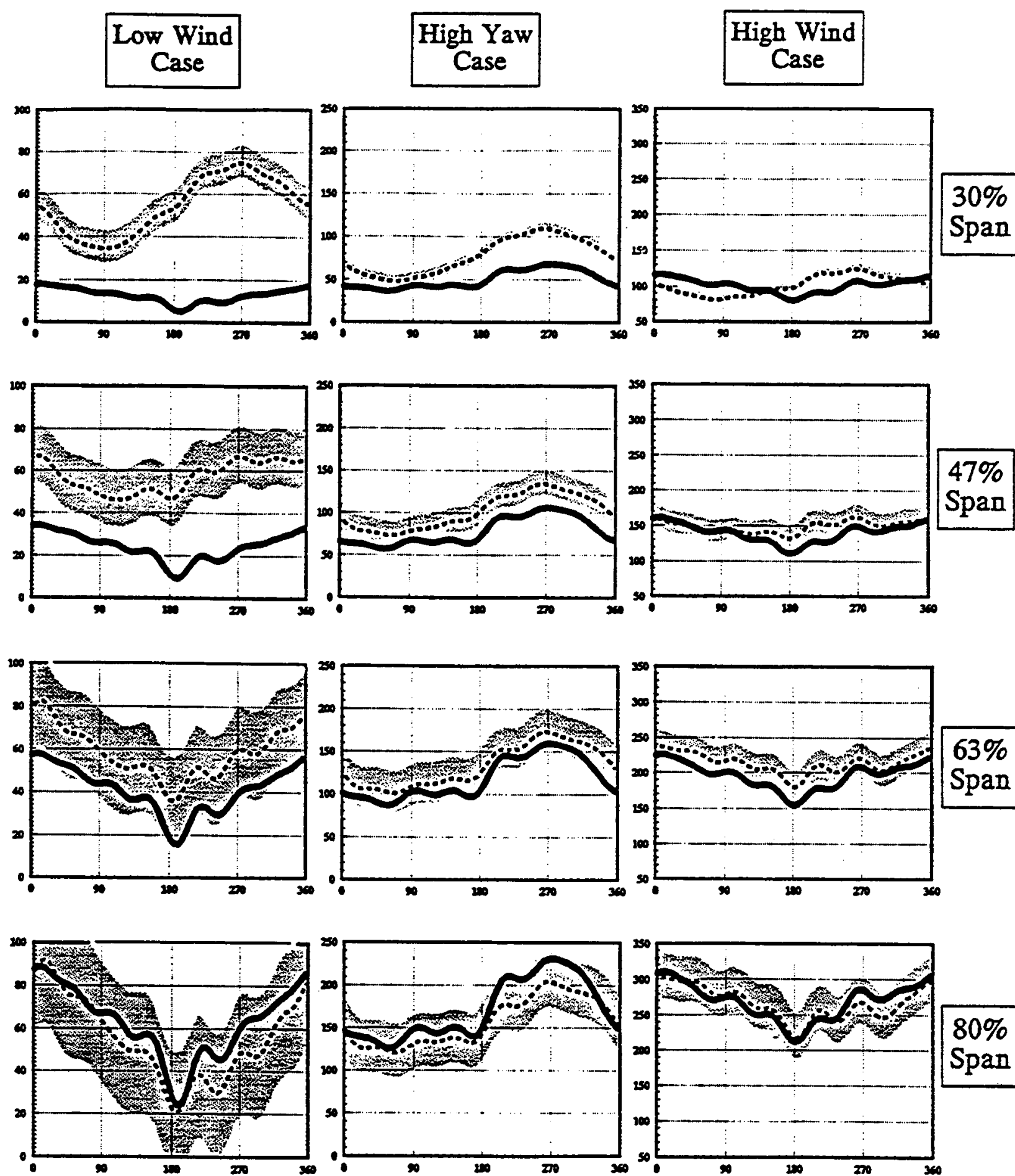

$80 \%$

Span

\section{Blade Position (deg)}

isure 6.1 $\quad$-ALEST +-10\% Parameter Variation

ALEST Sensitivity to Bending Moments

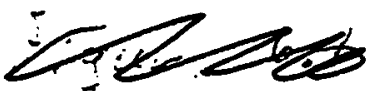




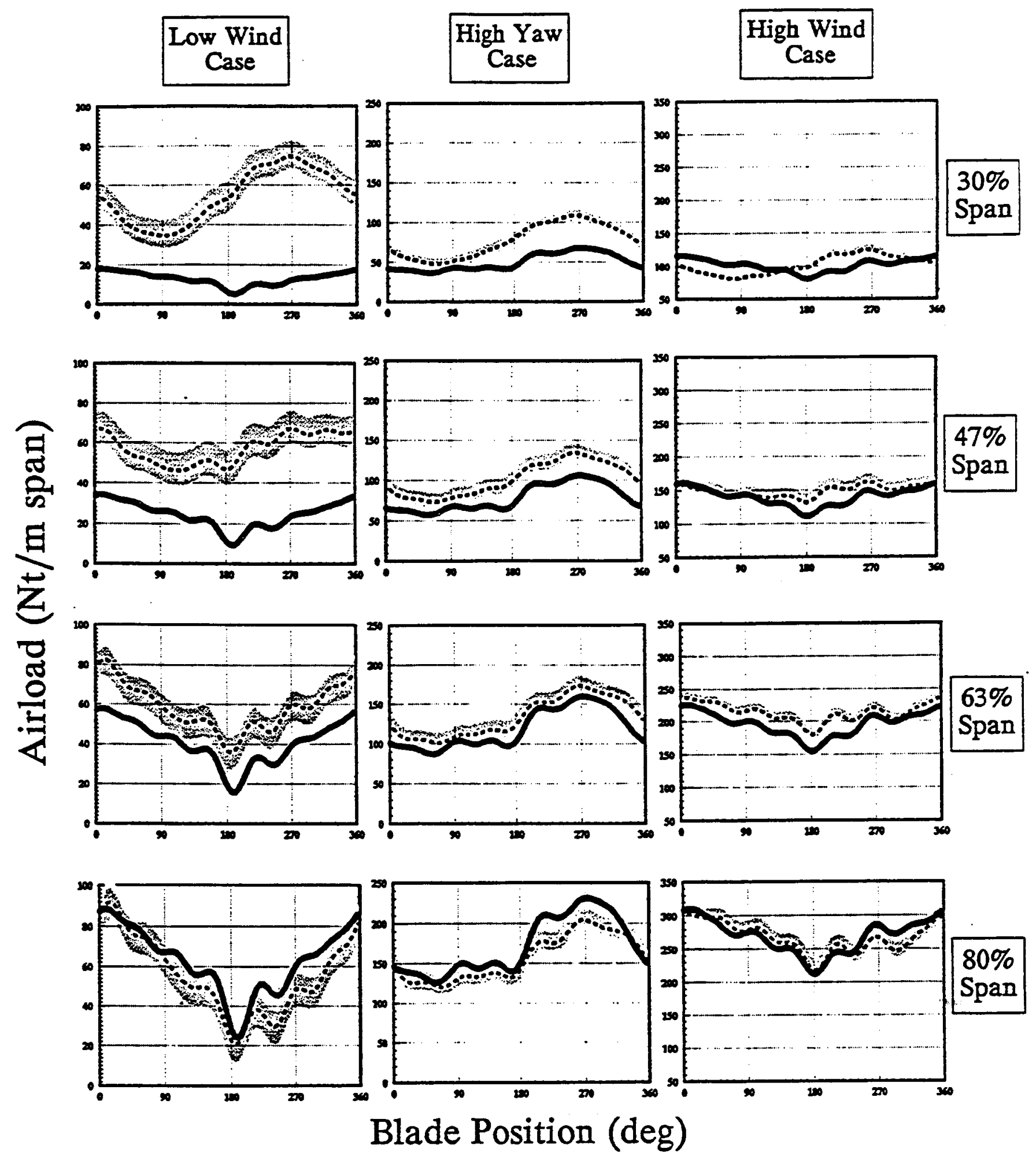

Figure $6.2 \quad$-ALEST +-10\% Parameter Variation

ALEST Sensitivity to Precone Angle 


\section{Low Wind Case}
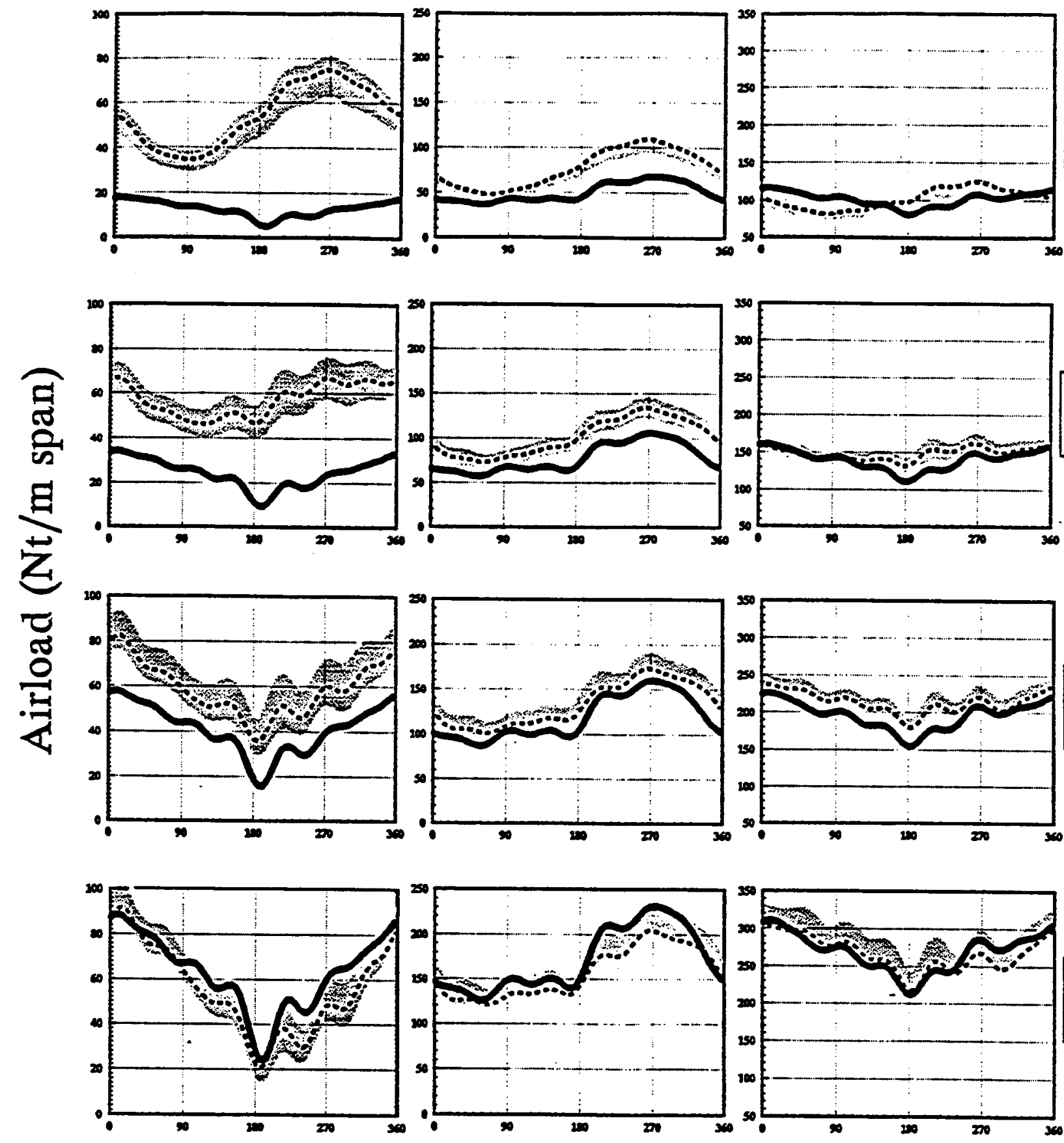

Blade Position (deg)
$30 \%$

Span

Span

$63 \%$

Span

$80 \%$

Span

Fisure 6.3

ALEST +-10\% Parameter Variation
- Direct Pressure Measurements

ALEST Sensitivity to Blade Mass Distribution 
the technique is shown to be robust in predicting airloads and tolerating typical measurement uncertainty levels. Furthermore, the relative effects of measurement error become less significant as the blade is subjected to higher loading conditions.

Figure 6.4 shows full spanwise blade airload estimates from ALEST averaged over a complete blade cycle for each test case. The averaged measured pressures are also indicated with triangular symbols. This is probably the most useful form of result that could be used to verify airload inputs to a blade performance model such as PROP. Cyclic data, as shown in Figures 6.1 through 6.3, would be useful in verifying airloads in analytical tools such as FLAP.

Figure 6.4. Blade airload estimates

Table 6.3. Blade Mass Distribution

\begin{tabular}{cccc}
\hline $\begin{array}{c}\text { Location } \\
\text { (\% of Span) }\end{array}$ & $\begin{array}{c}\text { Mass } \\
(\mathrm{kg} / \mathrm{m})\end{array}$ & $\begin{array}{c}\text { Stiffness } \\
\left(\mathrm{N}-\mathrm{m}^{2}\right)\end{array}$ & $\begin{array}{c}\text { Random Error } \\
(\%)\end{array}$ \\
\hline 0 (Root) & 17.98 & $4.75 \times 10^{5}$ & +5 \\
10 & 17.98 & $4.34 \times 10^{5}$ & -5 \\
20 & 17.00 & $3.55 \times 10^{5}$ & +5 \\
30 & 12.14 & $2.36 \times 10^{5}$ & -5 \\
40 & 9.715 & $1.16 \times 10^{5}$ & +5 \\
50 & 8.750 & $1.07 \times 10^{5}$ & -5 \\
60 & 8.018 & $9.50 \times 10^{4}$ & +5 \\
70 & 7.286 & $7.85 \times 10^{4}$ & -5 \\
80 & 6.572 & $5.37 \times 10^{4}$ & +5 \\
90 & 5.590 & $7.02 \times 10^{4}$ & -5 \\
100 (Tip) & 5.590 & $4.13 \times 10^{4}$ & +5 \\
\hline
\end{tabular}

Figure 6.5 summarizes the ALEST analysis procedure, showing how flap bending moments, blade properties, and machine operating conditions are used to produce an airload distribution. Figure 6.6 is a schematic representation of the data-processing procedure. It shows an example in which the $80 \%$ span pressure-measurement data are used to calculate airload at one span location. Figure 6.6 also shows how this airload value compares to the corresponding ALEST airload distribution. It is important to note that results are produced in terms of rotational harmonics, which enable comparisons to be made over a full blade cycle.

Figure 6.5. ALEST analysis procedure

Figure 6.6. Comparison of estimated airloads with measured airloads

\subsection{Blade-Load Data Base}

Blade loads were measured at 10 spanwise locations on the Combined Experiment turbine in order to correlate structural-load distributions with aerodynamic-load distributions. Appendix E contains plots of bin-averaged blade bending moments vs. wind speed for all the spanwise locations. Rotor torque and power measurements are also compared with predictions from PROPPC, an aerodynamic loads and 


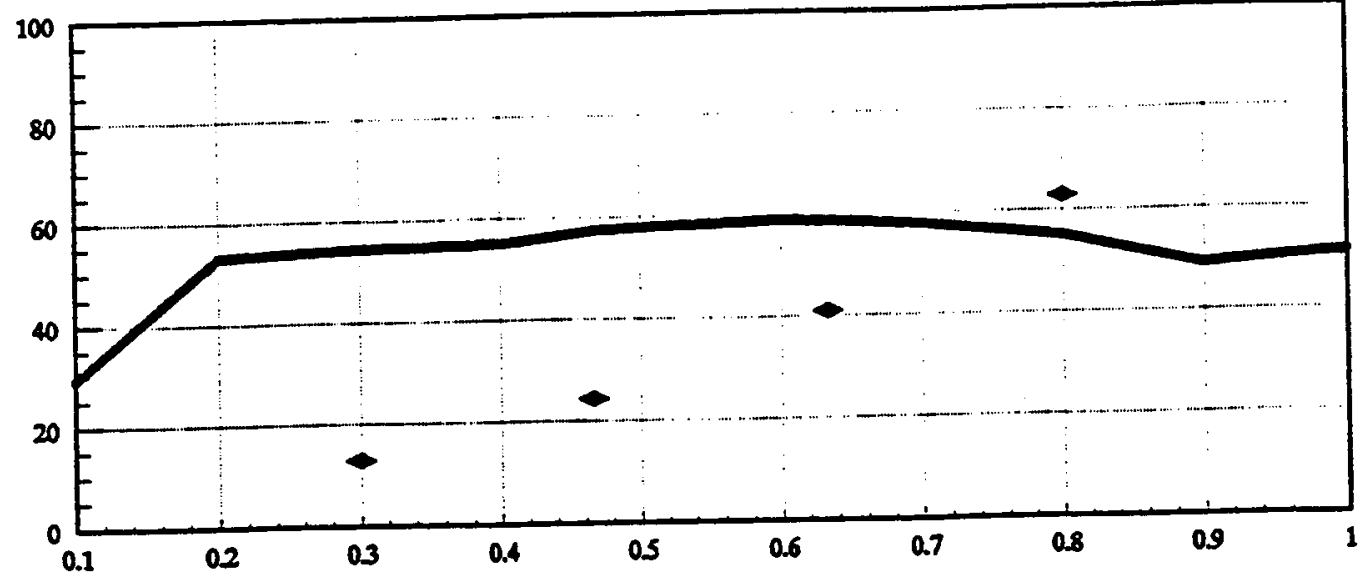

Low Wind

Case

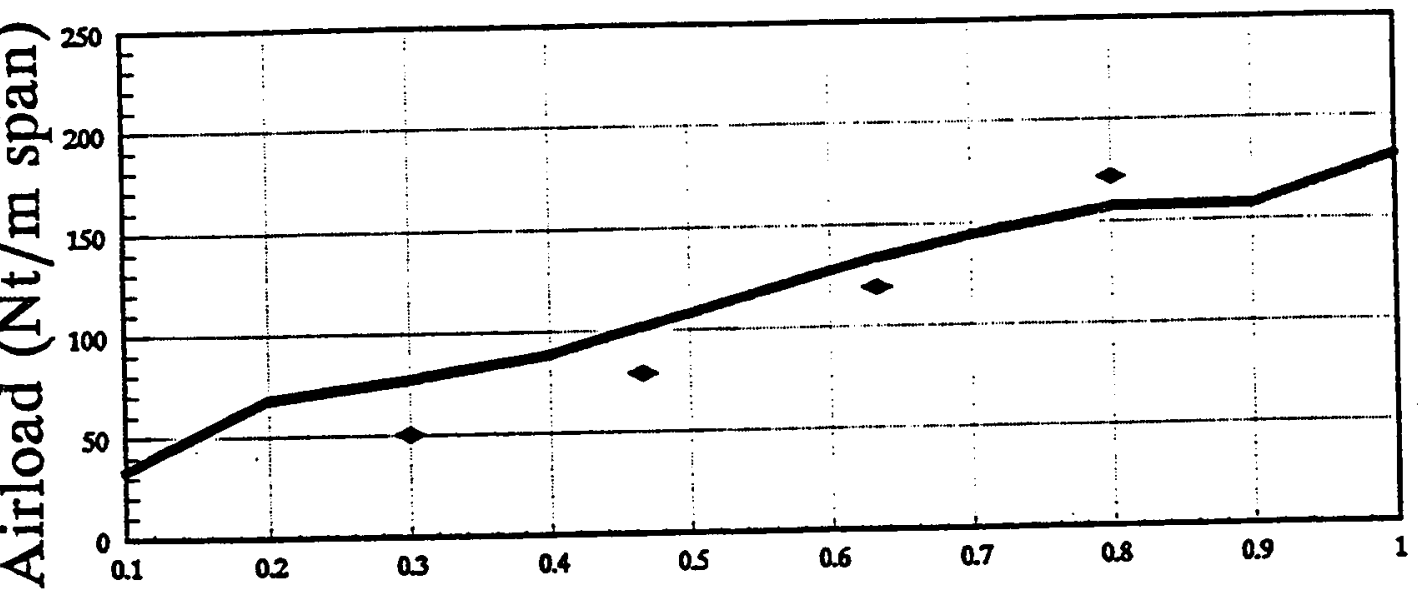

High Yaw Case

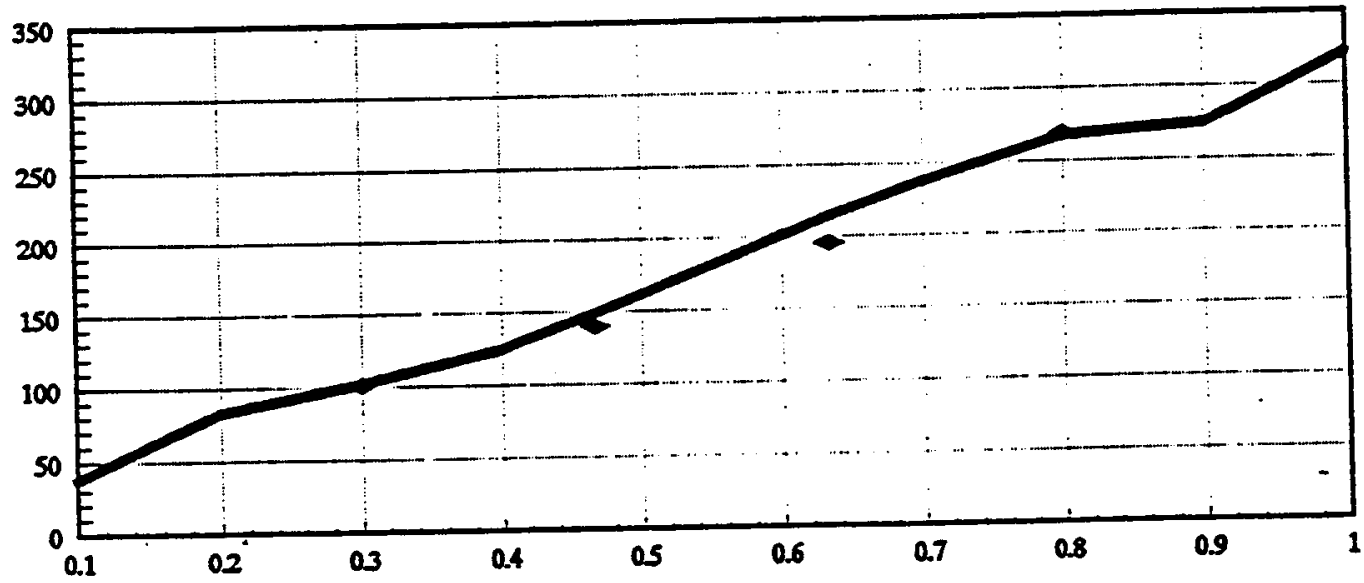

High Wind Case

Span Location (\% of Span)

Figure 6.4 -ALEST Average Airload Distribution 


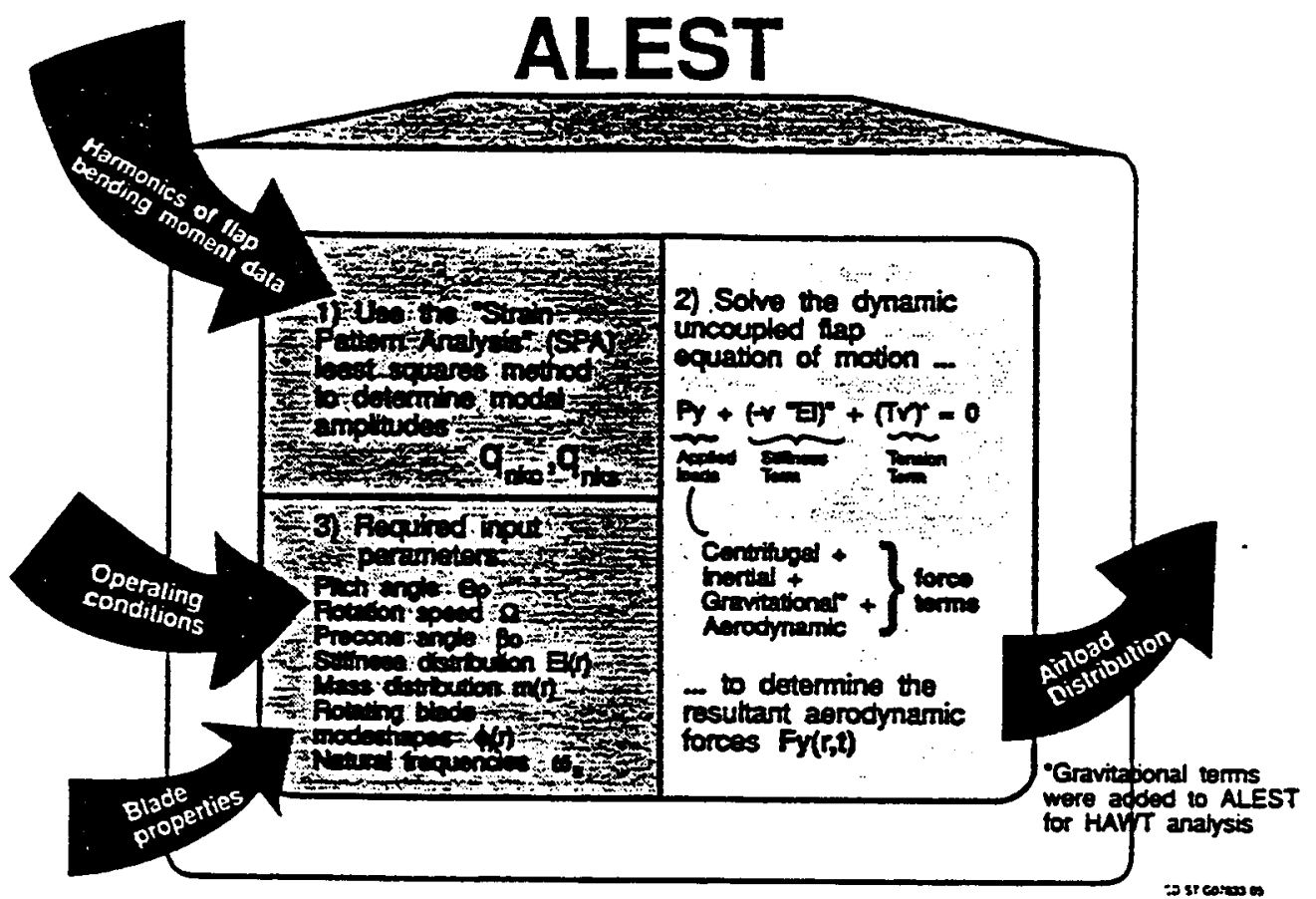

7.gure 6.5 ALEST Analysis Procedure

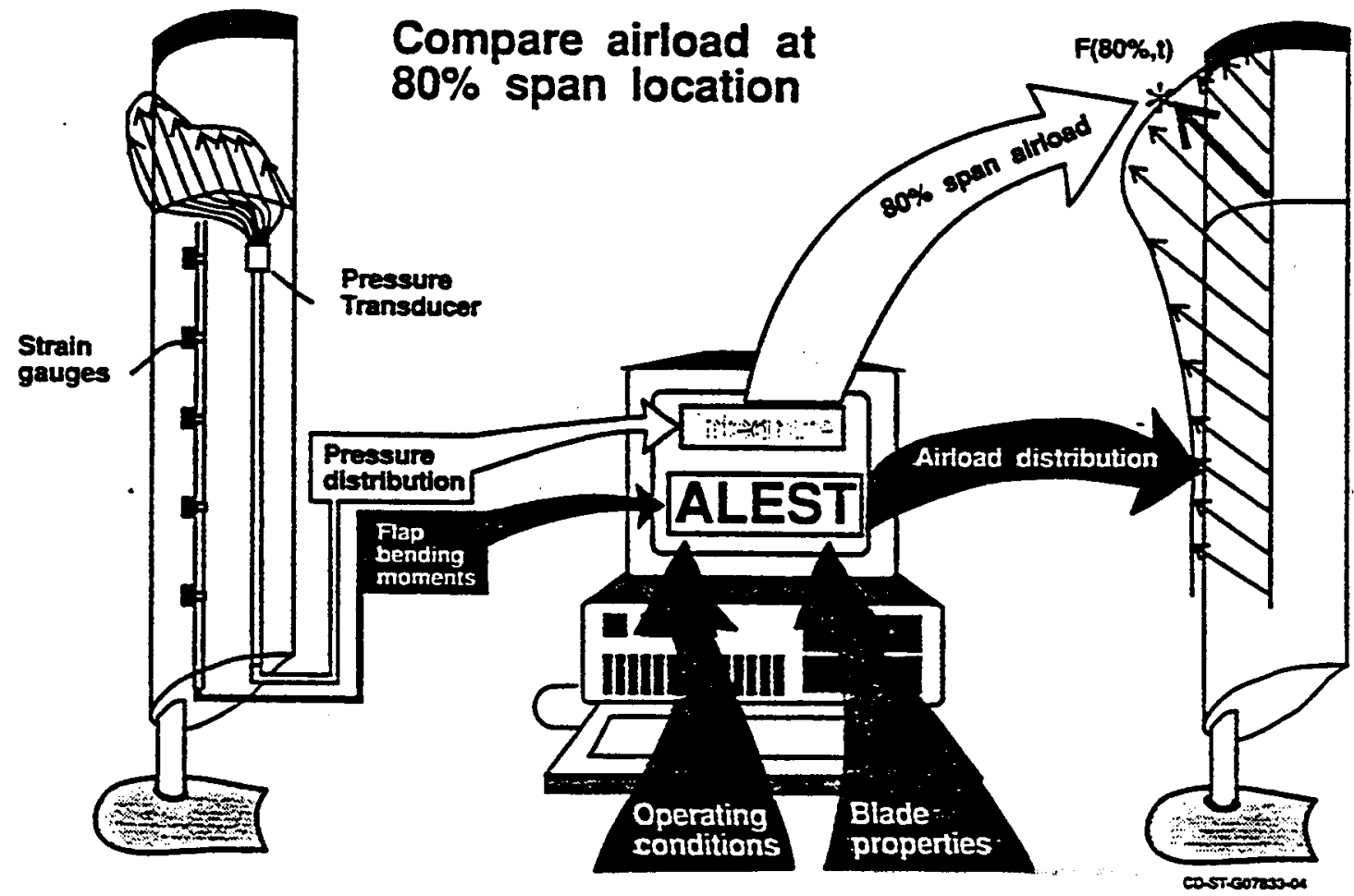

7igure 6.6 Data Processing Procedure 
performance program. Angle of attack and local total pressure (dynamic pressure) data are correlated with wind speed. A measured generator efficiency curve is also presented. To date, very little effort has been expended on making analytical comparisons, but it was felt that the existing data should be presented to enable other researchers to make their own comparisons.

\subsection{Conclusions}

ALEST requires measured strain-gage flap bending moments, blade properties, and machine operating parameters as input to estimate blade aerodynamic loads. It works well during higher-wind loading conditions, even if input parameters have high measurement uncertainty. ALEST accurately depicts both mean and cyclic airload content under these conditions.

The ALEST technique becomes less reliable as airloads decrease. The point at which this occurs cannot easily be determined. It depends on blade configuration, aerodynamic loads relative to other loads, mode shape, measured bending-moment accuracy, and deflection.

ALEST could be used to show the impact of aerodynamic phenomena, such as unsteady effects and delayed stall, because these are most likely to occur at higher wind conditions. An example is seen in the high-yaw case presented, where dynamic stall is occurring as seen in the measured airloads, and is corroborated in the ALEST results. This lends support to the hypothesis that aerodynamically induced loads can be transmitted into a machine and adversely affect structural response.

The technique works well on the particular blade configuration presented here because of the simple blade planform and zero twist, and because the blade does not have any edgewise or torsional mode frequencies near the first flap mode frequency that might introduce false flap bending-moment signals. Other blade configurations would have to be carefully considered as to their feasibility for this technique.

ALEST is probably most suited for use as a research tool to provide airload estimations for verifying analytical models such as FLAP or PROP. It might also be used to study effects of relative changes made to a blade configuration.

The code is undergoing refinement by incorporating a weighting matrix technique to be used to equalize strain distribution and thus improve mode-shape curve fitting. This should provide better airload estimation under low-load conditions. However, it requires a more in-depth knowledge of the ALEST analysis technique. 


\subsection{Future Work}

Blade geometry appears to affect airfoil performance. To understand the effect of blade twist and taper on airfoil stall performance, a tapered and twisted blade will be developed and tested. Results will be compared to those from the existing blade, which has no twist or taper.

The flow conditions adjacent to the blade but off the surface (outer flow condition) may reveal the cause of airfoil performance abnormality. These flow states can be tested by observing smoke flow patterns as the blade is rotating through the smoke. Video cameras will be used to record these smoke patterns. Video image processing will be used to correlate the patterns with pressure distributions and other operating conditions. This information will be used to improve stall models for wind turbines. 


\subsection{Conclusions}

Never before, in wind turbine research has such a complete investigation of wind turbine aerodynamics been conducted. The results have led to new insights in both steady and unsteady aerodynamic behavior. Designers have always used wind tunnel data to describe airfoil performance on wind turbine blades and assumed that adjacent spanwise sections can be treated as though they were independent. The test results presented in this report show that under low wind speed conditions, many of these assumptions were true. But under high wind speed conditions, they were not true.

Under high wind conditions, steady and unsteady stall behavior of airfoils does not correlate well with wind tunnel data. Unfortunately, these conditions occur at maximum load operating conditions and prevent the designer from making accurate predictions of maximum load or performance predictions. The data presented in this report helps the designer know what the origins of the inaccuracies are and help him estimate their magnitude. This report also presents a comprehensive body of wind turbine aerodynamic data that can be used for code validation.

The data presented demonstrate that airfoils on wind turbines behave as they would in the wind tunnel for low to moderate AOAs. Near stall, the wind-turbine airfoils show a delay in stall due to a combination of suction peak persistence and high negative pressures on the suction side of the airfoil. This results in high normal forces and high tangent forces in the post-stall region. Because of this modified airfoil behavior, wind-tunnel stall data may not be accurate for stall-control wind-turbine design purposes. However, it is not clear what causes this behavior or what effect blade planform (twist and taper) will have on the airfoil performance.

Dynamic stall was shown to exist on a HAWT operating at 30 deg yaw angle. Dynamic stall also occurs for low-yaw-error operation when tower shadow, wind shear, or inflow turbulence cause large AOA excursions. These increased aerodynamic loads cause increased structural loading. It was shown that dynamic stall forces caused by tower shadow are shorter in duration and lower in magnitude than those caused by yawed flow. It is important to model dynamic stall for yawed rotors for this reason. The data presented in this report plus future data will provide the basic information needed to develop dynamic-stall models.

We have shown how video flow-visualization data can be correlated with standard time-series pressure data recorded on an operating wind turbine. A key to this correlation is to use a single clock signal and to record it on the video tape using a time-code inserter as well as recording it as another item in the PCM data stream.

We have observed significant differences between the wind-turbine-blade field-test results and wind-tunnel tests. Beyond stall, leading suction peaks persist to high AOAs. At inboard stations on the blade, nonzero pressure gradients exist in regions of separated flow. These gradients do not occur in the wind tunnel. These results help explain why performance analyses typically under-predict the peak performance on stall-control wind turbines.

Many of the specific results presented in this report have led researchers to a better understanding of airfoil performance on rotating wings as well as a realization that airfoil may be affected by overall blade geometry. The variation of airfoil performance with spanwise location suggests that optimum blade geometries developed based on wind tunnel data may not be true optimums. Different geometries may produce better performing blades, and still others may produce blades that stall in a more progressive manner, reducing dynamic loading. The concept of controlling stall progression to achieve a balance 
between high performance and low dynamic loads has been suggested as a result of examining stall behavior measured on this test turbine.

Dynamic stall measurements have proven that this phenomenon does occur on wind turbines operating in yawed conditions, and it can significantly increase yaw loads and dynamic loads. These results have convinced rsearchers and designers that dynamic stall must be introduced into structural dynamics codes in order to improve the accuracy of the codes' fatigue life predictions. 


\subsection{References}

Bousman, W.G., 1987, Estimation of Blade Airloads from Rotor Blade Bending Moments, NASA Technical Memorandum 100020, USAAVSCOM Technical Report 87-A-8.

Butterfield, C.P., 1989, Three Dimensional Airfoil Performance Measurements on a Rotating Wing, SERI/TP-217-3505, Golden, CO: National Renewable Energy Laboratory.

Butterfield, C.P., D.A. Simms, W.P. Musial, and G.N. Scott, 1990, Spanwise Aerodynamic Loads on a Rotating Blade, SERI/TP-257-3983, presented at Windpower '90, Golden, CO: National Renewable Energy Laboratory.

Butterfield, C.P., D.A. Simms, W.D. Musial, and G.N. Scott, 1992, NREL Combined Experiment-Phase I Final Report, NREL/TP-257-4655, Golden, CO: National Renewable Energy Laboratory.

Butterfield, C.P., G.N. Scott, and W.P. Musial, 1990, Comparison of Wind Tunnel Airfoil Performance Data with Wind Turbine Blade Data, SERITP-254-3799, Golden, CO: National Renewable Energy Laboratory.

DuWaldt, F.A., and I.C. Statler, 1966, Derivation of Rotor Blade Generalized Air Loads from Measured Flapwise Bending Moment and Measured Pressure Distributions, USAAVLABS TR 66-13,

Hansen, A.C., and C.P. Butterfield, 1990, "Yaw Loads and Motions of a Horizontal Axis Wind Turbine," ASME Journal of Solar Energy, Vol. 112.

Hurst, D.W., and D.T. Owen, Nacelle Installation Effects on Propeller Blade Surface Pressure Distributions, 0096-736X/88/9606-0940, Southampton, England: Society of Automotive Engineers.

Madsen, H.A., F. Rasmussen, and T.F. Pedersen, 1988, Aerodynamics of a Full-Scale HAWT Blade, presented at European Wind Energy Conference, Conference Proceedings.

Murthy, V.R., 1976, "Dynamic Characteristics of Rotor Blades," Journal of Sound and Vibrations, Vol. 49, No. 4, pp. 483-500.

Musial, W., and C.P. Butterfield, 1990, A Comparison of Two- and Three-Dimensional S809 Airfoil Properties for Rough and Smooth HAWT Operation, SERI/TP-257-3603, Golden, CO: NationaI Renewable Energy Laboratory.

Pope, A., and J.J. Harper, 1966, Low-Speed Wind Tunnel Testing, Wiley \& Sons, NY.

Reda, D.C., 1988a, "Liquid Crystals for Unsteady Surface Shear Stress Visualization," ABBE-3841CP, Proceedings, First National Fluid Dynamics Congress, Cincinnati, OH: July 1988.

Reda, D.C., 1988b, "Liquid Crystals for Surface Shear Stress Visualization on Wind Turbine Airfoils," Proceedings, Windpower '88, Honolulu, HI, Sept. 1988.

Reda, D.C., and C.P. Butterfield, 1989, "Visualization of Boundary Layer Transition Zone Movement on Oscillating Airfoils with Shear-Sensitive Liquid Crystal Coatings," Proceedings, Symposium on Flow Visualization, ASME Winter Annual Meeting, San Francisco, CA, Dec. 10-15. 
Robinson, M.C., 1985, Development of Vorticity and Vortices from Forced Unsteady Flow Separation, Ph.D. Thesis, Boulder, CO: University of Colorado at Boulder.

Schnepp, R.R., A.C. Hansen, and A.D. Wright, 1992, A Method for Determining Aerodynamic Loads on $a$ Wind Turbine from Blade Flap Bending Moments, Proceedings of the 11th ASME Wind Energy Symposium, Houston, TX.

Scott, G., C.P. Butterfield, D. Simms, and W. Musial, 1991, Correlation of Flow Visualization and Pressure Distribution Data on a Wind Turbine Blade, 10th ASME-ETCE Conference Proceedings, Houston, TX.

Simms, D.A., C.P. Butterfield, and A.D. Wright, 1990, "A Procedure for Estimating Aerodynamic Loads on Horizontal Axis Wind Turbines Using Measured Bending Moments," Proceedings of the Ninth ASME Wind Energy Symposium, New Orleans, LA.

Tangler, J.L., 1983, Horizontal-Axis Wind System Rotor Performance Model Comparison-A Compendium, RFP-3508, NTIS.

Viterna, L.A, and R.D. Corrigan, 1981, Fixed Pitch Rotor Performance of Large Horizontal Axis Wind Turbines, DOE/NASA Workshop on Large Horizontal Axis Wind Turbines, Cleveland, OH, July 28-30.

Wright, A., and C.P. Butterfield, 1991, The NREL Teetering Hub Rotor Code: Final Results and Conclusions, NREL/TP-257-4517, presented at Windpower '91, Golden, CO: National Renewable Energy Laboratory.

Wright, A.D., and R.W. Thresher, 1987, "Accurate Rotor Loads Prediction Using the FLAP Dynamics Code," SERITP-217-3315, Windpower '87 Proceedings, Alexandria, VA: American Wind Energy Association. 
Appendix A

Blade Layouts 


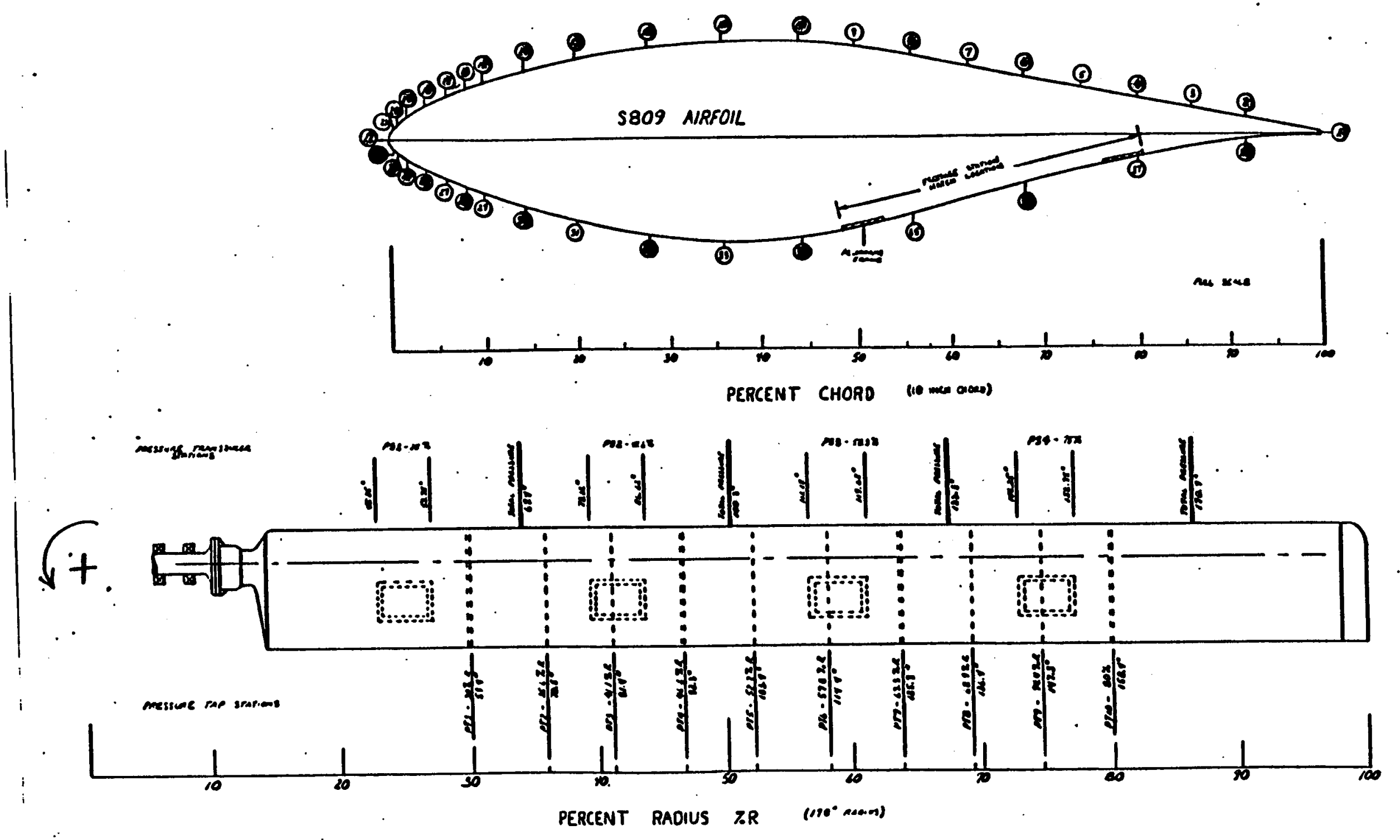




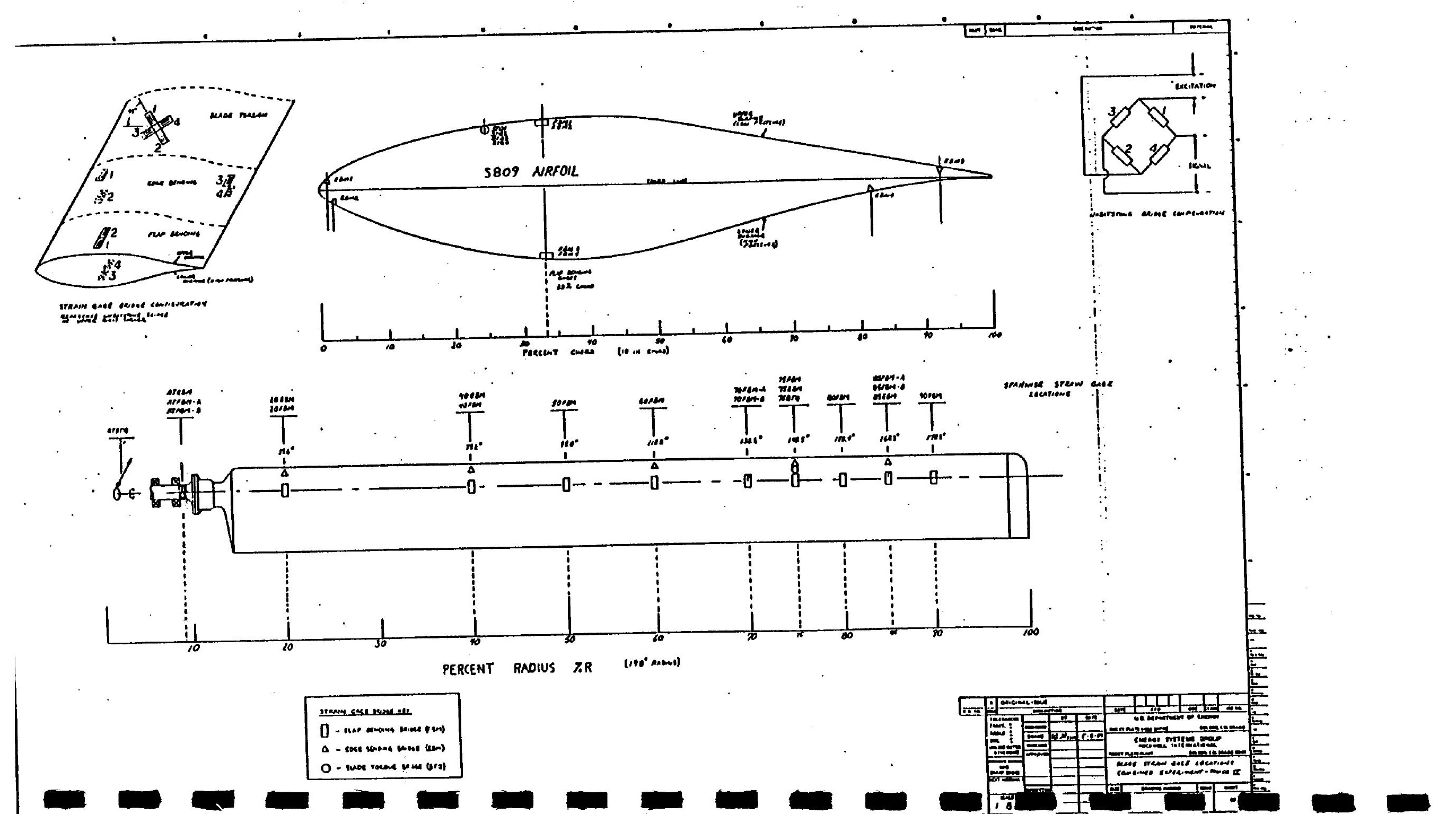


Appendix B

Bin-Averaged Aerodynamic Coefficients 
Tape 65 Data

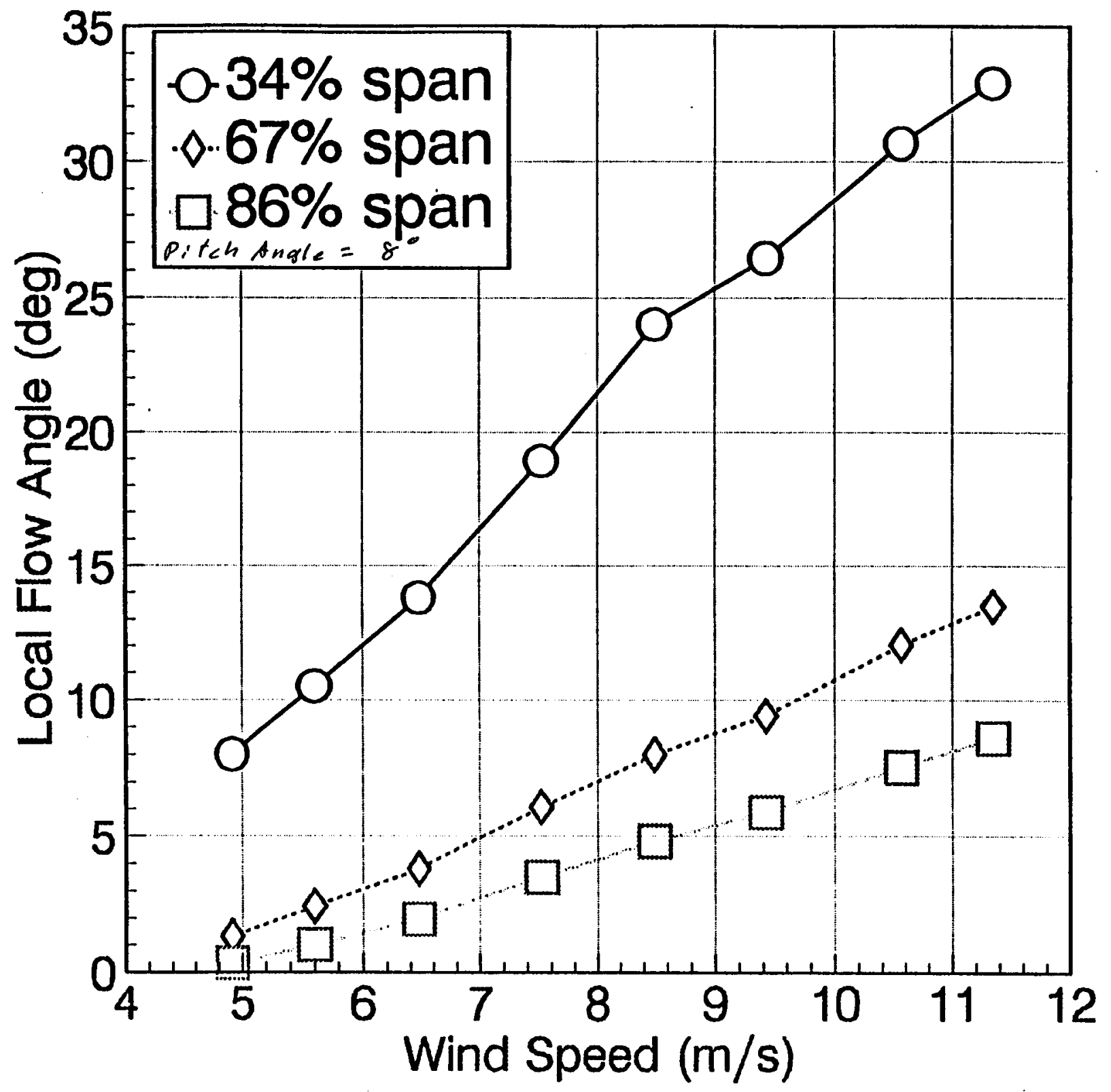




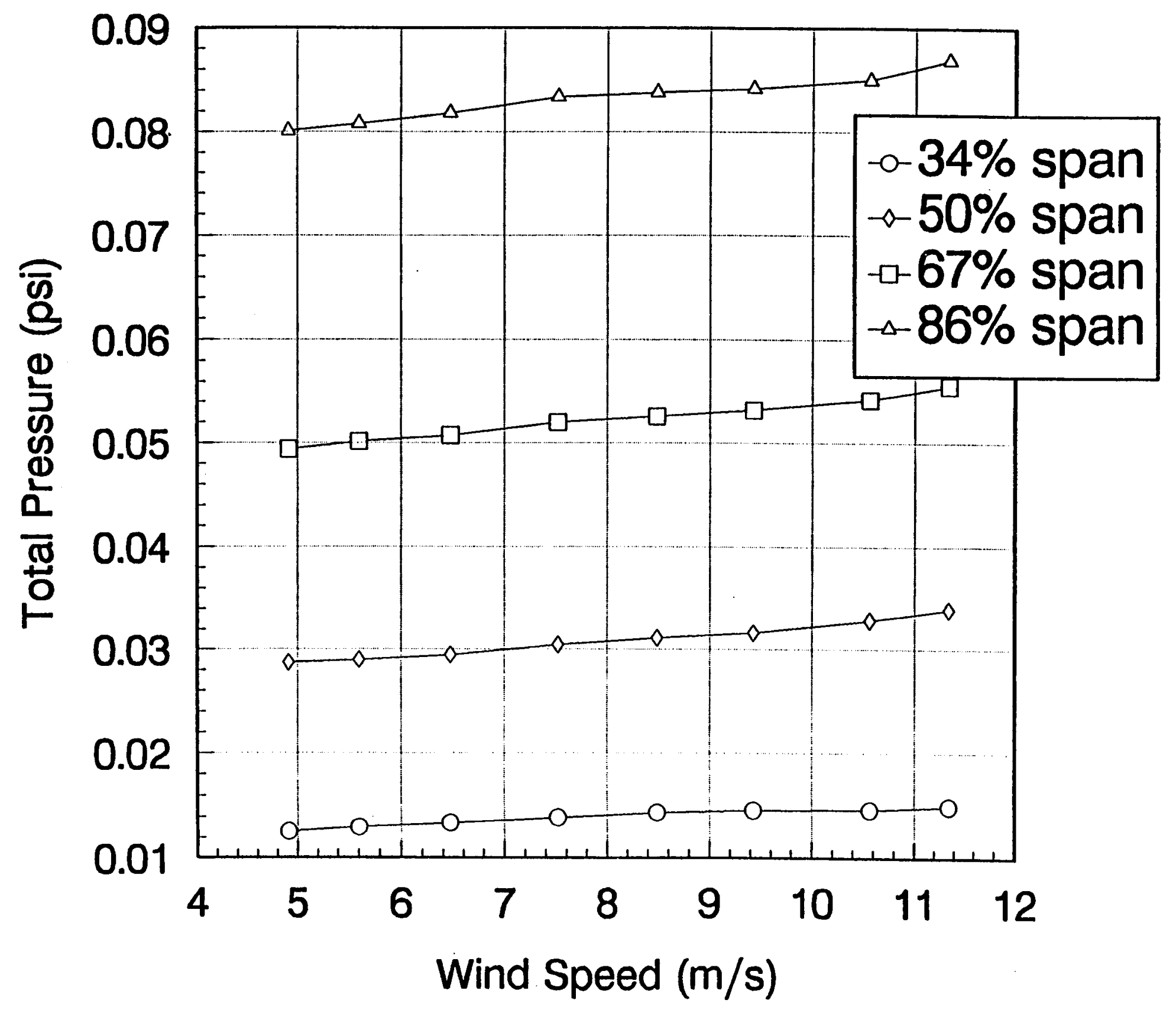


Tape 65 bata

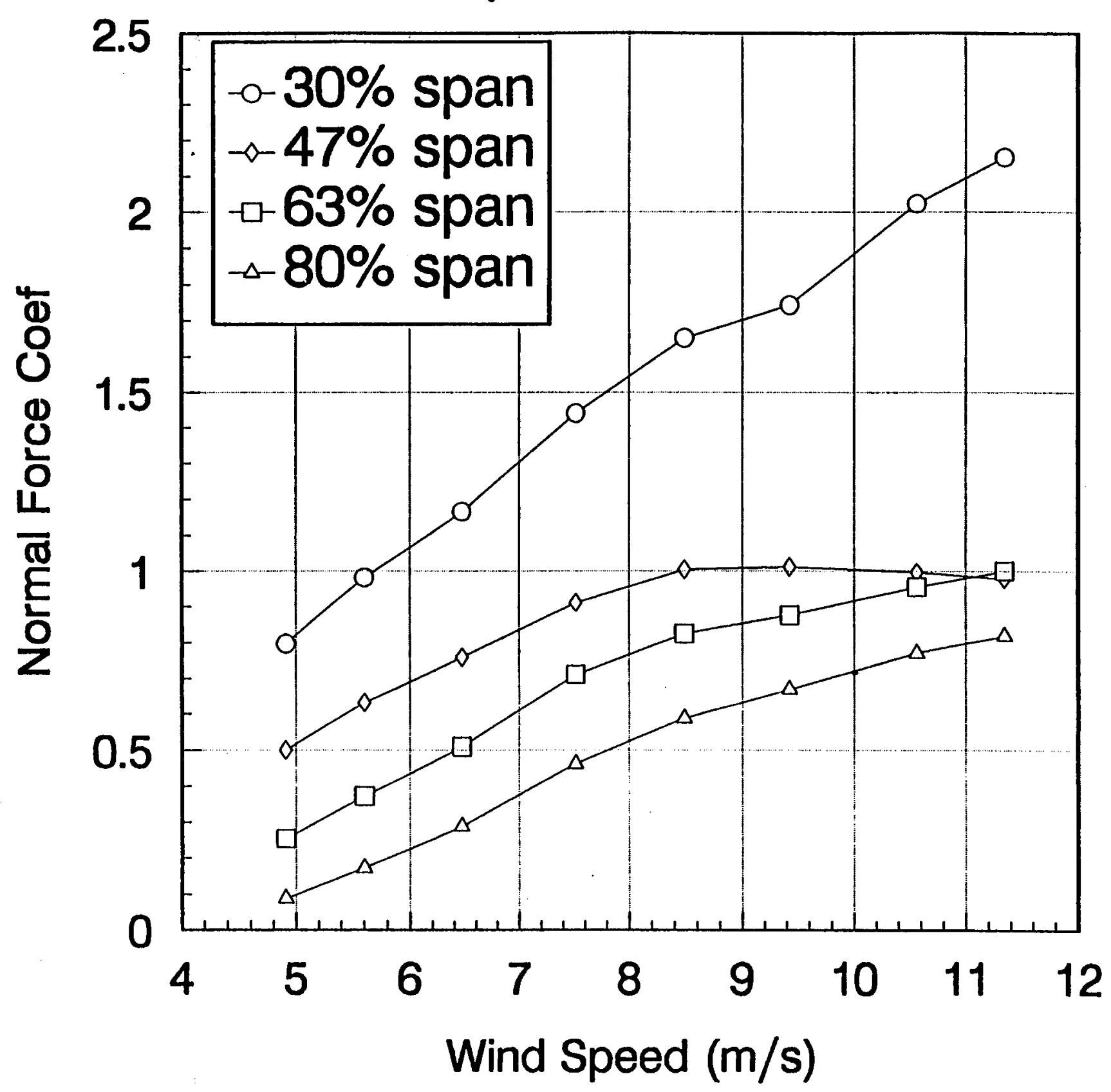




\section{Tape 68 Data}

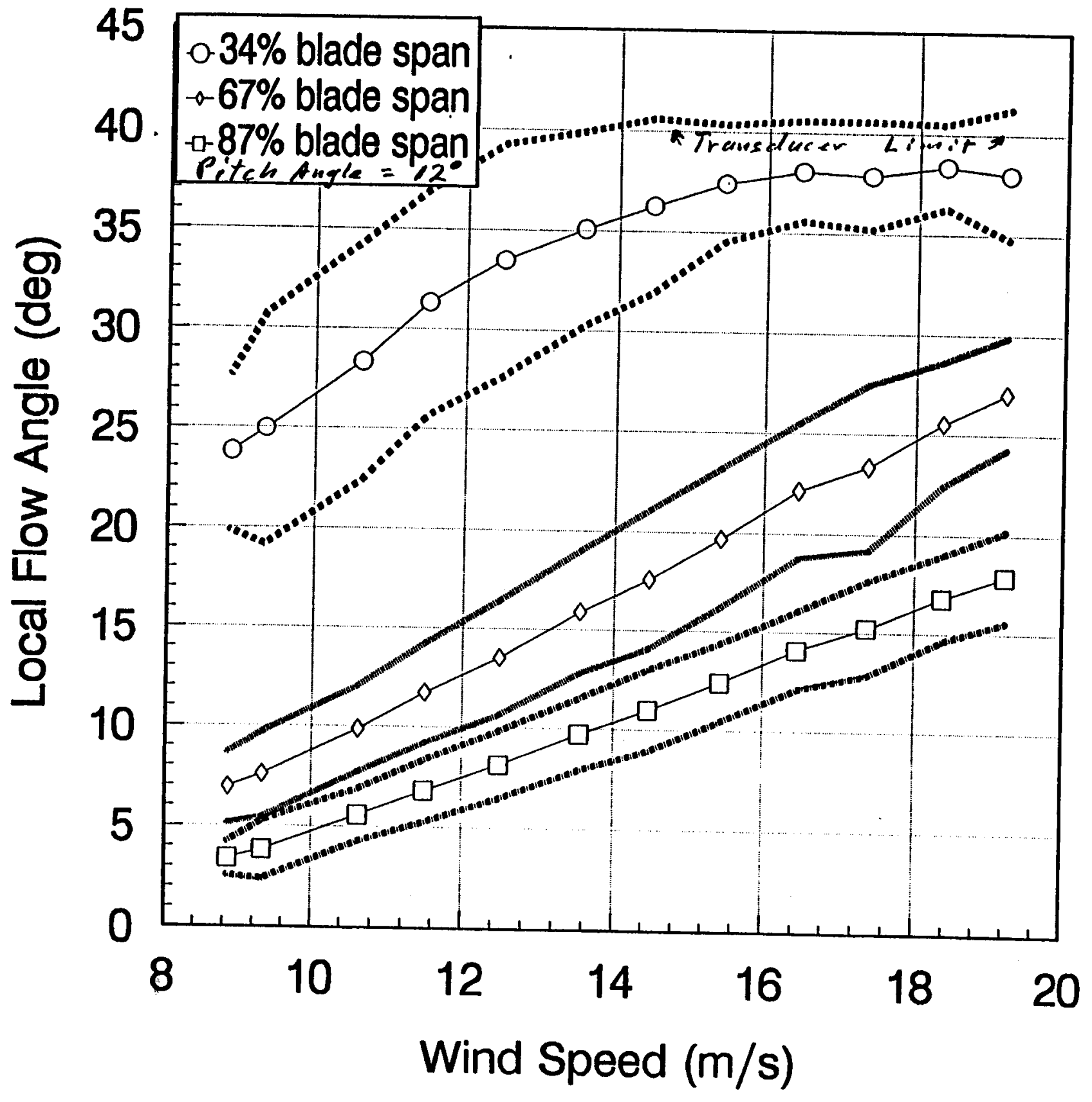


Tape 65 Data

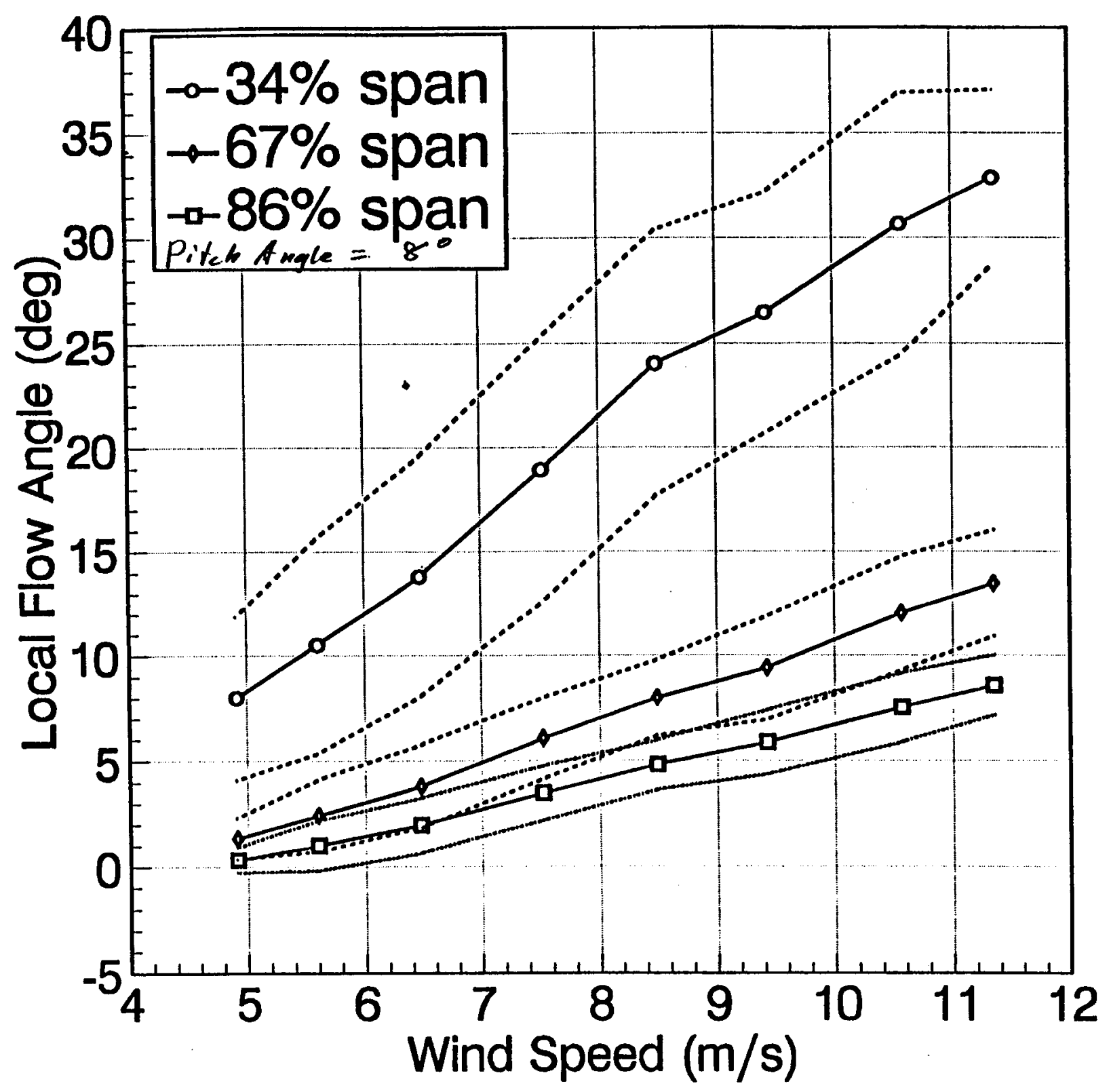




\section{Tape 68 Data}

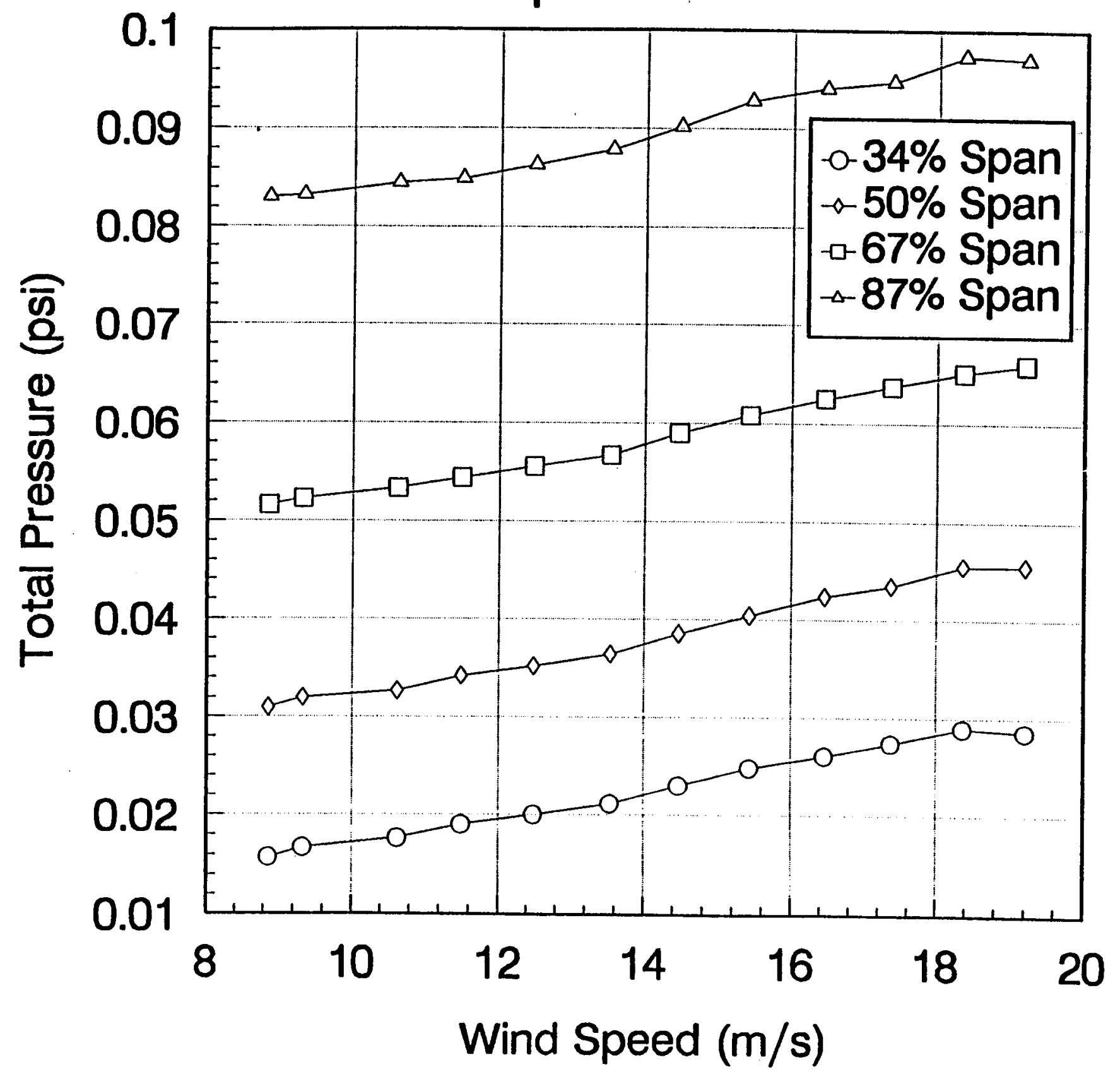


Tape 68 Data

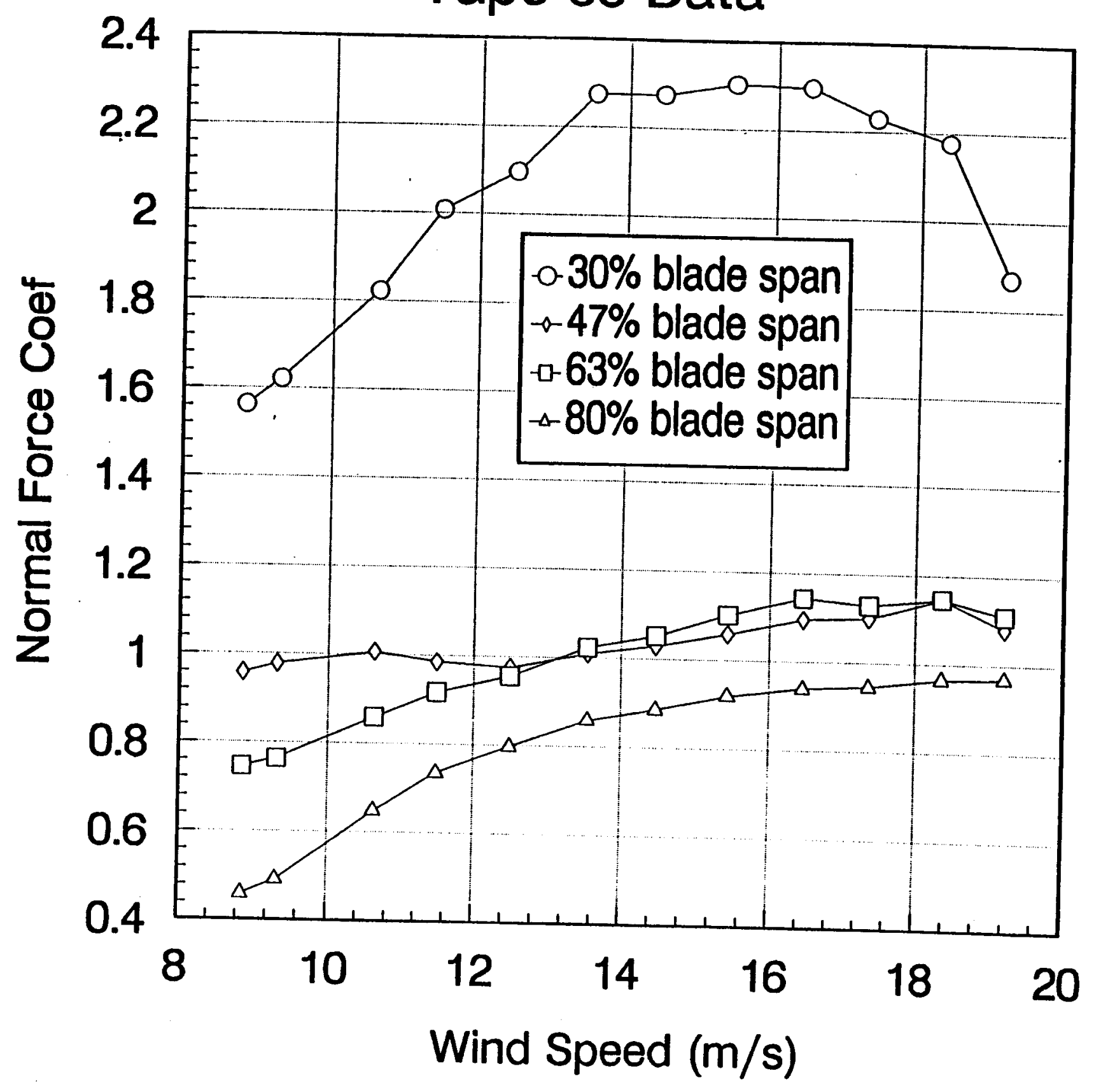


AWEA-90' Report Data files from the SERI Combined Experiment

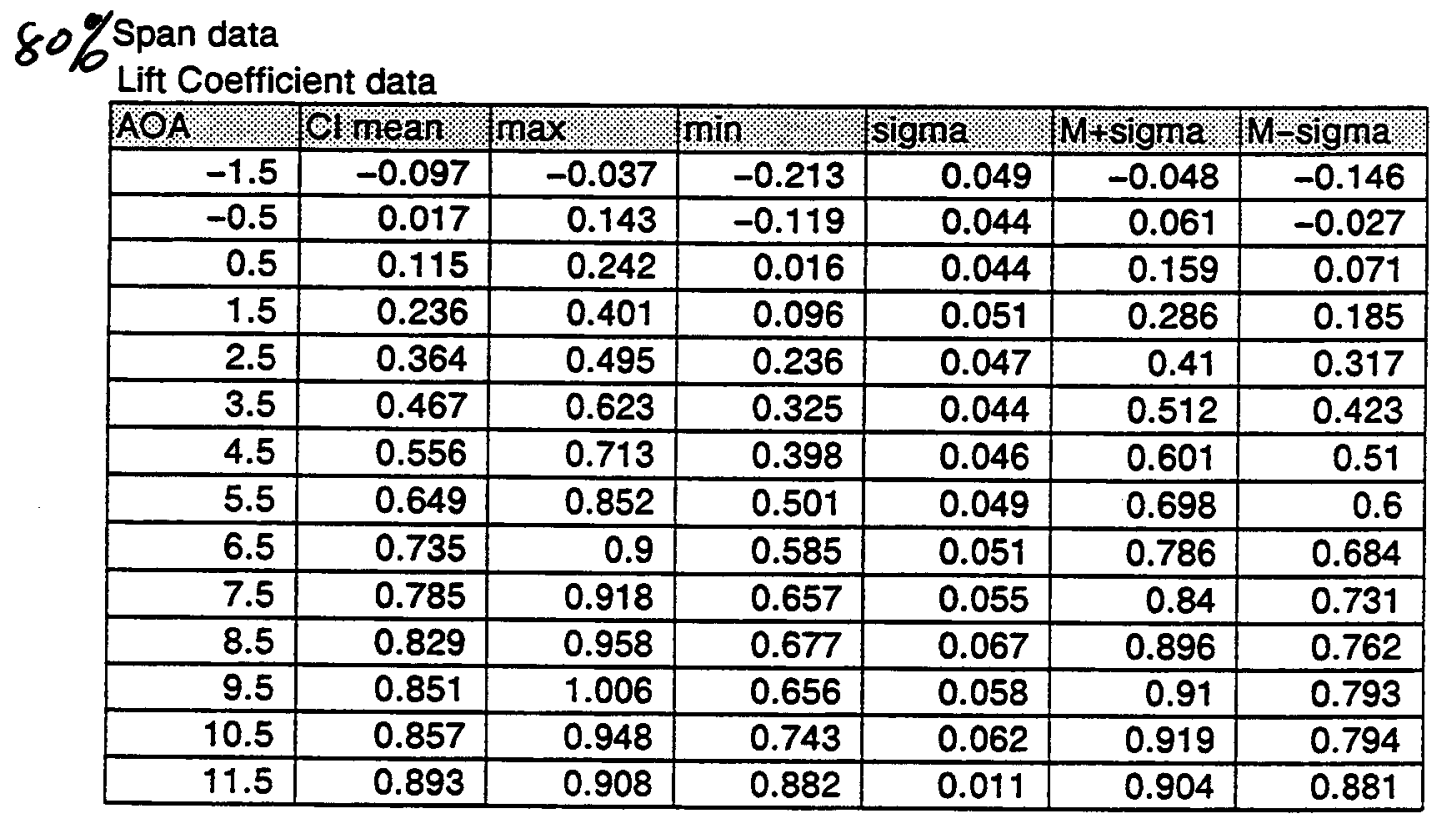


$80 \%$ Span

Drag Coefficient Data

\begin{tabular}{|r|r|r|r|r|r|r|}
\hline $\mathrm{AOA}$ & Comean & Max & & & \\
\hline-1.5 & 0.012 & 0.015 & 0.009 & 0.002 & 0.014 & 0.01 \\
\hline-0.5 & 0.011 & 0.018 & 0.006 & 0.002 & 0.013 & 0.009 \\
\hline 0.5 & 0.011 & 0.018 & 0.002 & 0.002 & 0.013 & 0.009 \\
\hline 1.5 & 0.011 & 0.017 & 0.004 & 0.002 & 0.013 & 0.009 \\
\hline 2.5 & 0.012 & 0.018 & -0.004 & 0.002 & 0.014 & 0.01 \\
\hline 3.5 & 0.012 & 0.022 & -0.005 & 0.003 & 0.015 & 0.009 \\
\hline 4.5 & 0.01 & 0.026 & -0.004 & 0.004 & 0.014 & 0.006 \\
\hline 5.5 & 0.009 & 0.026 & 0 & 0.003 & 0.012 & 0.006 \\
\hline 6.5 & 0.012 & 0.024 & 0.005 & 0.003 & 0.015 & 0.009 \\
\hline 7.5 & 0.015 & 0.025 & 0.006 & 0.004 & 0.019 & 0.011 \\
\hline 8.5 & 0.019 & 0.031 & 0.006 & 0.004 & 0.024 & 0.015 \\
\hline 9.5 & 0.025 & 0.037 & 0.014 & 0.005 & 0.029 & 0.02 \\
\hline 10.5 & 0.03 & 0.037 & 0.025 & 0.004 & 0.034 & 0.026 \\
\hline 11.5 & 0.045 & 0.048 & 0.041 & 0.003 & 0.048 & 0.042 \\
\hline
\end{tabular}


63\% Span

Drag Coefficient Data

\begin{tabular}{|c|c|c|c|c|c|c|}
\hline$A \odot A$ & Codmean & Max: & Min & Sigma & M+sigma & M-sigma. \\
\hline-1.5 & 0.01 & 0.015 & 0.004 & 0.003 & 0.013 & 0.006 \\
\hline-0.5 & 0.009 & 0.012 & -0.001 & 0.003 & 0.011 & 0.006 \\
\hline 0.5 & 0.008 & 0.016 & -0.001 & 0.002 & 0.01 & 0.005 \\
\hline 1.5 & 0.007 & 0.014 & -0.008 & 0.003 & 0.01 & 0.004 \\
\hline 2.5 & 0.007 & 0.018 & -0.008 & 0.003 & 0.01 & 0.004 \\
\hline 3.5 & 0.006 & 0.016 & -0.005 & 0.004 & 0.01 & 0.002 \\
\hline 4.5 & 0.006 & 0.043 & -0.007 & 0.004 & 0.011 & 0.002 \\
\hline 5.5 & 0.007 & 0.019 & -0.006 & 0.004 & 0.01 & 0.003 \\
\hline 6.5 & 0.011 & 0.029 & -0.004 & 0.005 & 0.016 & 0.006 \\
\hline 7.5 & 0.016 & 0.037 & 0 & 0.005 & 0.021 & 0.011 \\
\hline 8.5 & 0.018 & 0.047 & 0.004 & 0.006 & 0.024 & 0.012 \\
\hline 9.5 & 0.025 & 0.044 & 0.011 & 0.006 & 0.031 & 0.019 \\
\hline 10.5 & 0.034 & 0.063 & 0.016 & 0.008 & 0.041 & 0.026 \\
\hline 11.5 & 0.046 & 0.064 & 0.033 & 0.008 & 0.053 & 0.038 \\
\hline 12.5 & 0.063 & 0.112 & 0.042 & 0.014 & 0.077 & 0.048 \\
\hline 13.5 & 0.08 & 0.187 & 0.056 & 0.024 & 0.104 & 0.057 \\
\hline 14.5 & 0.101 & 0.168 & 0.073 & 0.026 & 0.127 & 0.075 \\
\hline 15.5 & 0.15 & 0.227 & 0.092 & 0.043 & 0.193 & 0.107 \\
\hline 16.5 & 0.211 & 0.277 & 0.141 & 0.037 & 0.248 & 0.174 \\
\hline 17.5 & 0.233 & 0.286 & 0.171 & 0.041 & 0.274 & 0.192 \\
\hline
\end{tabular}


AWEA-90' Report Data files from the SERI Combined Experiment

$47 \%$ Span data

Lift Coefficient data

\begin{tabular}{|c|c|c|c|c|c|c|}
\hline $18 \mathrm{~A}$ & Shnean & max & $\mathrm{min}$ & Signa. & $\mathrm{M}+$ sigma & $\mathrm{M}-\mathrm{sigma}$ \\
\hline-1.5 & 0.088 & 0.088 & 0.088 & 0 & 0.088 & 0.088 \\
\hline-0.5 & 0.161 & 0.257 & 0.078 & 0.074 & 0.235 & 0.087 \\
\hline 0.5 & 0.152 & 0.296 & 0.06 & 0.078 & 0.231 & 0.074 \\
\hline 1.5 & 0.322 & 0.713 & 0.103 & 0.114 & 0.436 & 0.208 \\
\hline 2.5 & 0.401 & 0.686 & 0.171 & 0.084 & 0.484 & 0.317 \\
\hline 3.5 & 0.487 & 0.701 & 0.329 & 0.08 & 0.567 & 0.407 \\
\hline 4.5 & 0.585 & 0.782 & 0.307 & 0.088 & 0.673 & 0.497 \\
\hline 5.5 & 0.666 & 0.956 & 0.487 & 0.092 & 0.758 & 0.574 \\
\hline 6.5 & 0.725 & 0.946 & 0.377 & 0.101 & 0.826 & 0.624 \\
\hline 7.5 & 0.795 & 1.052 & 0.376 & 0.112 & 0.907 & 0.684 \\
\hline 8.5 & 0.852 & 1.106 & 0.543 & 0.09 & 0.942 & 0.761 \\
\hline 9.5 & 0.878 & 1.158 & 0.419 & 0.106 & 0.984 & 0.772 \\
\hline 10.5 & 0.919 & 1.225 & 0.659 & 0.107 & 1.026 & 0.813 \\
\hline 11.5 & 0.948 & 1.259 & 0.583 & 0.106 & 1.054 & 0.842 \\
\hline 12.5 & 0.969 & 1.291 & 0.682 & 0.105 & 1.074 & 0.864 \\
\hline 13.5 & 0.99 & 1.311 & 0.627 & 0.113 & 1.102 & 0.877 \\
\hline 14.5 & 1.035 & 1.415 & 0.605 & 0.127 & 1.162 & 0.909 \\
\hline 15.5 & 1.024 & 1.439 & 0.705 & 0.114 & 1.138 & 0.91 \\
\hline 16.5 & 1.015 & 1.256 & 0.682 & 0.13 & 1.145 & 0.885 \\
\hline 17.5 & 0.98 & 1.293 & 0.68 & 0.144 & 1.125 & 0.836 \\
\hline 18.5 & 0.993 & 1.421 & 0.673 & 0.171 & 1.164 & 0.822 \\
\hline 19.5 & 0.961 & 1.273 & 0.723 & 0.157 & 1.118 & 0.804 \\
\hline 20.5 & 0.969 & 1.264 & 0.731 & 0.129 & 1.098 & 0.84 \\
\hline 21.5 & 0.996 & 1.395 & 0.701 & 0.177 & 1.173 & 0.818 \\
\hline 22.5 & 0.894 & 1.381 & 0.726 & 0.142 & 1.036 & 0.753 \\
\hline 23.5 & 0.902 & 1.227 & 0.729 & 0.127 & 1.029 & 0.775 \\
\hline 24.5 & 0.909 & 1.152 & 0.765 & 0.116 & 1.025 & 0.793 \\
\hline 25.5 & 0.916 & 1.207 & 0.742 & 0.144 & 1.06 & 0.772 \\
\hline
\end{tabular}


AWEA-90' Report Data files from the SERI Combined Experiment

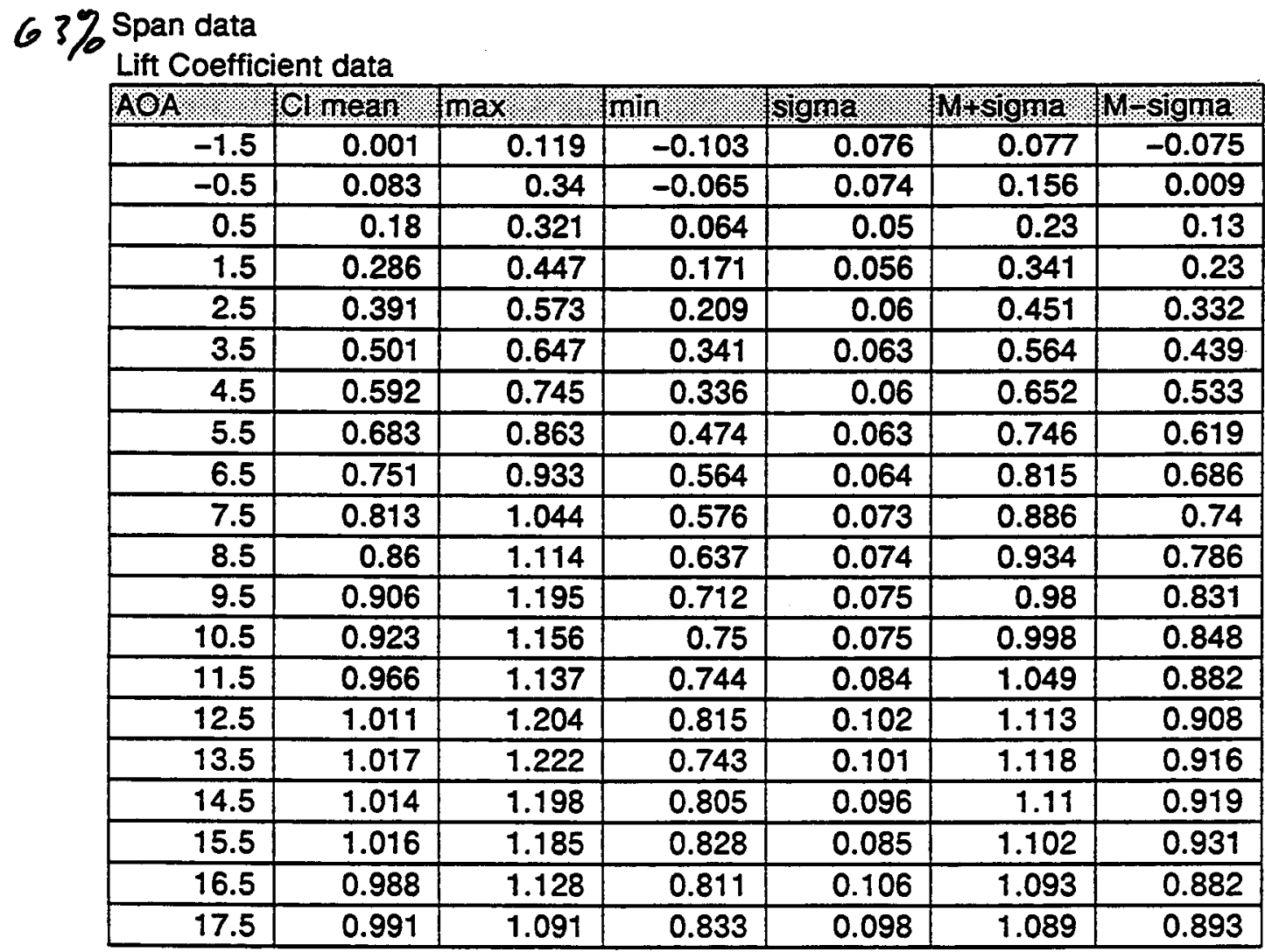


47\% Span

Drag Coefficient Data

\begin{tabular}{|c|c|c|c|c|c|c|}
\hline $\mathrm{AOA}$ & Comean & Max & Min & Sigma & $\mathrm{M}+$ sigma & $\mathrm{M}-$ sigma \\
\hline-1.5 & 0.016 & 0.016 & 0.016 & 0 & 0.016 & 0.016 \\
\hline-0.5 & 0.005 & 0.009 & 0.002 & 0.003 & 0.008 & 0.003 \\
\hline 0.5 & 0.013 & 0.021 & 0.008 & 0.004 & 0.017 & 0.009 \\
\hline 1.5 & 0.009 & 0.02 & -0.025 & 0.007 & 0.016 & 0.002 \\
\hline 2.5 & 0.009 & 0.019 & -0.021 & 0.007 & 0.015 & 0.002 \\
\hline 3.5 & 0.011 & 0.032 & -0.012 & 0.006 & 0.017 & 0.005 \\
\hline 4.5 & 0.011 & 0.034 & -0.024 & 0.007 & 0.018 & 0.004 \\
\hline 5.5 & 0.011 & 0.038 & -0.017 & 0.008 & 0.019 & 0.002 \\
\hline 6.5 & 0.012 & 0.04 & -0.013 & 0.007 & 0.019 & 0.005 \\
\hline 7.5 & 0.016 & 0.041 & -0.014 & 0.007 & 0.023 & 0.009 \\
\hline 8.5 & 0.02 & 0.049 & 0.002 & 0.008 & 0.029 & 0.012 \\
\hline 9.5 & 0.03 & 0.174 & 0.009 & 0.016 & 0.046 & 0.015 \\
\hline 10.5 & 0.038 & 0.127 & 0.017 & 0.014 & 0.052 & 0.024 \\
\hline 11.5 & 0.053 & 0.162 & 0.025 & 0.02 & 0.073 & 0.033 \\
\hline 12.5 & 0.074 & 0.209 & 0.037 & 0.029 & 0.104 & 0.045 \\
\hline 13.5 & 0.107 & 0.237 & 0.053 & 0.039 & 0.145 & 0.068 \\
\hline 14.5 & 0.149 & 0.272 & 0.072 & 0.052 & 0.202 & 0.097 \\
\hline 15.5 & 0.207 & 0.323 & 0.078 & 0.057 & 0.264 & 0.15 \\
\hline 16.5 & 0.24 & 0.374 & 0.106 & 0.067 & 0.307 & 0.174 \\
\hline 17.5 & 0.285 & 0.444 & 0.146 & 0.058 & 0.344 & 0.227 \\
\hline 18.5 & 0.291 & 0.427 & 0.173 & 0.056 & 0.347 & 0.235 \\
\hline 19.5 & 0.345 & 0.451 & 0.225 & 0.056 & 0.401 & 0.289 \\
\hline 20.5 & 0.37 & 0.463 & 0.248 & 0.051 & 0.421 & 0.319 \\
\hline 21.5 & 0.399 & 0.573 & 0.265 & 0.076 & 0.475 & 0.323 \\
\hline 22.5 & 0.396 & 0.476 & 0.318 & 0.042 & 0.438 & 0.354 \\
\hline 23.5 & 0.4 & 0.524 & 0.344 & 0.046 & 0.447 & 0.354 \\
\hline 24.5 & 0.44 & 0.594 & 0.348 & 0.061 & 0.501 & 0.379 \\
\hline 25.5 & 0.449 & 0.535 & 0.394 & 0.05 & 0.499 & 0.399 \\
\hline
\end{tabular}


AWEA-90' Report Data files from the SERI Combined Experiment

$30 \%$ Span data

Lift Coefficient data

\begin{tabular}{|c|c|c|c|c|c|c|}
\hline $\mathrm{AOA}$ & Q Inean & max & 1101\% & Sigma & M + sigma & $\mathrm{M}=$ signa \\
\hline-1.5 & 0.202 & 0.202 & 0.202 & 0 & 0.202 & 0.202 \\
\hline-0.5 & 0.319 & 0.413 & 0.19 & 0.096 & 0.415 & 0.224 \\
\hline 0.5 & 0.413 & 0.748 & 0.143 & 0.178 & 0.591 & 0.236 \\
\hline 1.5 & 0.425 & 0.719 & 0.197 & 0.14 & 0.565 & 0.285 \\
\hline 2.5 & 0.545 & 0.994 & 0.17 & 0.192 & 0.736 & 0.353 \\
\hline 3.5 & 0.61 & 0.973 & 0.354 & 0.165 & 0.775 & 0.445 \\
\hline 4.5 & 0.667 & 0.994 & 0.402 & 0.157 & 0.824 & 0.51 \\
\hline 5.5 & 0.703 & 1.049 & 0.384 & 0.169 & 0.872 & 0.534 \\
\hline 6.5 & 0.778 & 1.224 & 0.314 & 0.15 & 0.928 & 0.628 \\
\hline 7.5 & 0.859 & 1.247 & 0.523 & 0.125 & 0.983 & 0.734 \\
\hline 8.5 & 0.929 & 1.286 & 0.488 & 0.17 & 1.099 & 0.759 \\
\hline 9.5 & 0.951 & 1.472 & 0.58 & 0.171 & 1.122 & 0.781 \\
\hline 10.5 & 0.971 & 1.469 & 0.461 & 0.191 & 1.162 & 0.779 \\
\hline 11.5 & 1.066 & 1.562 & 0.564 & 0.199 & 1.265 & 0.867 \\
\hline 12.5 & 1.115 & 1.585 & 0.609 & 0.167 & 1.282 & 0.949 \\
\hline 13.5 & 1.177 & 1.945 & 0.671 & 0.195 & 1.371 & 0.982 \\
\hline 14.5 & 1.243 & 1.628 & 0.746 & 0.174 & 1.417 & 1.069 \\
\hline 15.5 & 1.275 & 1.622 & 0.606 & 0.182 & 1.457 & 1.093 \\
\hline 16.5 & 1.33 & 1.665 & 0.749 & 0.153 & 1.484 & 1.177 \\
\hline 17.5 & 1.354 & 1.865 & 0.813 & 0.201 & 1.555 & 1.153 \\
\hline 18.5 & 1.438 & 1.821 & 0.985 & 0.174 & 1.612 & 1.264 \\
\hline 19.5 & 1.416 & 1.861 & 0.852 & 0.194 & 1.61 & 1.222 \\
\hline 20.5 & 1.423 & 2.09 & 0.767 & 0.219 & 1.642 & 1.203 \\
\hline 21.5 & 1.448 & 1.811 & 0.744 & 0.181 & 1.629 & 1.266 \\
\hline 22.5 & 1.495 & 2.181 & 1.004 & 0.195 & 1.691 & 1.3 \\
\hline 23.5 & 1.521 & 2.097 & 0.873 & 0.195 & 1.716 & 1.326 \\
\hline 24.5 & 1.538 & 2.108 & 1.007 & 0.214 & 1.752 & 1.325 \\
\hline 25.5 & 1.536 & 2.007 & 0.9 & 0.213 & 1.749 & 1.323 \\
\hline 26.5 & 1.63 & 2.46 & 1.106 & 0.229 & 1.859 & 1.4 \\
\hline 27.5 & 1.563 & 2.129 & 1.008 & 0.23 & 1.793 & 1.333 \\
\hline 28.5 & 1.598 & 2.375 & 1.13 & 0.242 & 1.839 & 1.356 \\
\hline 29.5 & 1.602 & 2.398 & 1.081 & 0.255 & 1.858 & 1.347 \\
\hline 30.5 & 1.682 & 2.584 & 1.182 & 0.319 & 2.001 & 1.364 \\
\hline 31.5 & 1.675 & 2.062 & 1.039 & 0.257 & 1.932 & 1.419 \\
\hline 32.5 & 1.831 & 2.398 & 1.301 & 0.271 & 2.102 & 1.56 \\
\hline 33.5 & 1.839 & 2.254 & 1.218 & 0.272 & 2.111 & 1.568 \\
\hline 34.5 & 1.859 & 2.45 & 1.205 & 0.307 & 2.166 & 1.552 \\
\hline 35.5 & 1.916 & 2.244 & 1.586 & 0.184 & 2.099 & 1.732 \\
\hline 36.5 & 1.865 & 2.347 & 1.338 & 0.283 & 2.149 & 1.582 \\
\hline 37.5 & 1.83 & 2.023 & 1.547 & 0.149 & 1.98 & 1.681 \\
\hline
\end{tabular}


$30 \%$ Span

Drag Coefficient Data

\begin{tabular}{|c|c|c|c|c|c|c|}
\hline $\mathrm{AOA}$ & Codmean & Max & Min & Sigma & M + sigma & Ml-sigma \\
\hline-1.5 & 0.022 & 0.022 & 0.022 & 0 & 0.022 & 0.022 \\
\hline-0.5 & 0.011 & 0.029 & -0.003 & 0.013 & 0.024 & -0.002 \\
\hline 0.5 & 0.03 & 0.061 & -0.011 & 0.017 & 0.046 & 0.013 \\
\hline 1.5 & 0.032 & 0.046 & 0.019 & 0.009 & 0.041 & 0.023 \\
\hline 2.5 & 0.031 & 0.085 & -0.048 & 0.028 & 0.059 & 0.003 \\
\hline 3.5 & 0.044 & 0.078 & 0.002 & 0.018 & 0.061 & 0.026 \\
\hline 4.5 & 0.037 & 0.07 & -0.021 & 0.022 & 0.059 & 0.015 \\
\hline 5.5 & 0.048 & 0.092 & 0.018 & 0.019 & 0.067 & 0.029 \\
\hline 6.5 & 0.052 & 0.09 & -0.004 & 0.019 & 0.071 & 0.034 \\
\hline 7.5 & 0.06 & 0.161 & 0.017 & 0.023 & 0.083 & 0.037 \\
\hline 8.5 & 0.072 & 0.206 & 0.01 & 0.028 & 0.1 & 0.045 \\
\hline 9.5 & 0.085 & 0.206 & 0.035 & 0.029 & 0.113 & 0.056 \\
\hline 10.5 & 0.09 & 0.232 & 0.027 & 0.027 & 0.117 & 0.062 \\
\hline 11.5 & 0.113 & 0.309 & 0.04 & 0.037 & 0.15 & 0.076 \\
\hline 12.5 & 0.138 & 0.34 & 0.057 & 0.04 & 0.178 & 0.098 \\
\hline 13.5 & 0.167 & 0.382 & 0.07 & 0.054 & 0.221 & 0.113 \\
\hline 14.5 & 0.192 & 0.42 & 0.081 & 0.049 & 0.241 & 0.142 \\
\hline 15.5 & 0.227 & 0.447 & 0.104 & 0.053 & 0.28 & 0.175 \\
\hline 16.5 & 0.273 & 0.442 & 0.138 & 0.054 & 0.328 & 0.219 \\
\hline 17.5 & 0.321 & 0.542 & 0.138 & 0.074 & 0.395 & 0.246 \\
\hline 18.5 & 0.378 & 0.55 & 0.216 & 0.078 & 0.456 & 0.3 \\
\hline 19.5 & 0.405 & 0.533 & 0.21 & 0.074 & 0.48 & 0.331 \\
\hline 20.5 & 0.443 & 0.687 & 0.238 & 0.1 & 0.543 & 0.343 \\
\hline 21.5 & 0.504 & 0.673 & 0.22 & 0.1 & 0.604 & 0.404 \\
\hline 22.5 & 0.548 & 0.923 & 0.32 & 0.107 & 0.655 & 0.441 \\
\hline 23.5 & 0.606 & 0.868 & 0.367 & 0.105 & 0.71 & 0.501 \\
\hline 24.5 & 0.659 & 1.034 & 0.38 & 0.116 & 0.776 & 0.543 \\
\hline 25.5 & 0.69 & 0.879 & 0.303 & 0.119 & 0.809 & 0.57 \\
\hline 26.5 & 0.762 & 1.116 & 0.41 & 0.131 & 0.892 & 0.631 \\
\hline 27.5 & 0.786 & 1.12 & 0.445 & 0.118 & 0.904 & 0.668 \\
\hline 28.5 & 0.817 & 1.086 & 0.466 & 0.148 & 0.964 & 0.669 \\
\hline 29.5 & 0.883 & 1.27 & 0.607 & 0.136 & 1.019 & 0.747 \\
\hline 30.5 & 0.947 & 1.33 & 0.569 & 0.161 & 1.108 & 0.786 \\
\hline 31.5 & 0.951 & 1.28 & 0.504 & 0.182 & 1.133 & 0.769 \\
\hline 32.5 & 1.051 & 1.327 & 0.684 & 0.164 & 1.215 & 0.887 \\
\hline 33.5 & 1.068 & 1.333 & 0.776 & 0.185 & 1.253 & 0.884 \\
\hline 34.5 & 1.137 & 1.508 & 0.806 & 0.175 & 1.312 & 0.963 \\
\hline 35.5 & 1.253 & 1.427 & 0.955 & 0.107 & 1.36 & 1.145 \\
\hline 36.5 & 1.25 & 1.563 & 0.853 & 0.18 & 1.429 & 1.07 \\
\hline 37.5 & 1.277 & 1.451 & 0.982 & 0.137 & 1.414 & 1.139 \\
\hline
\end{tabular}


0

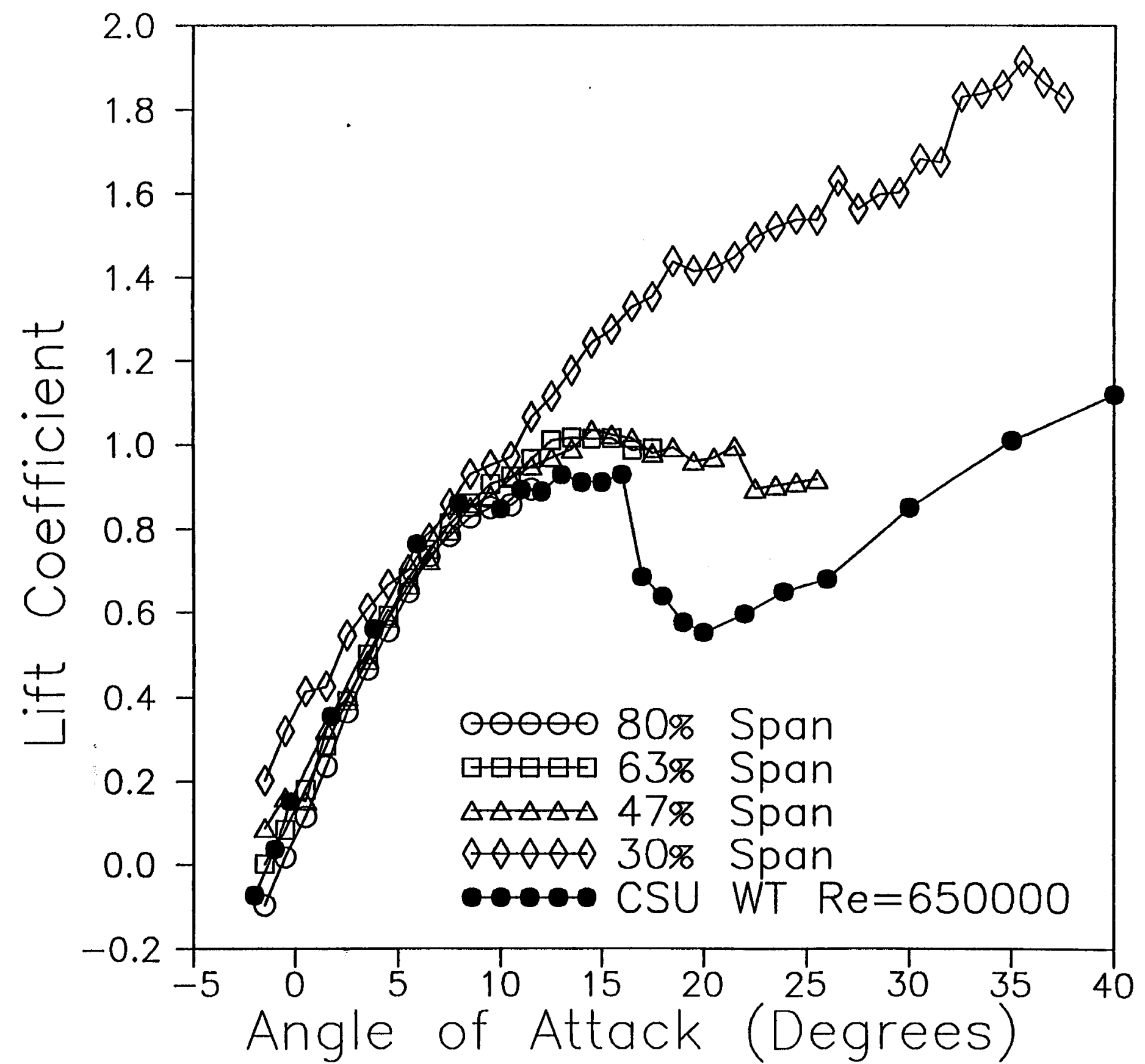




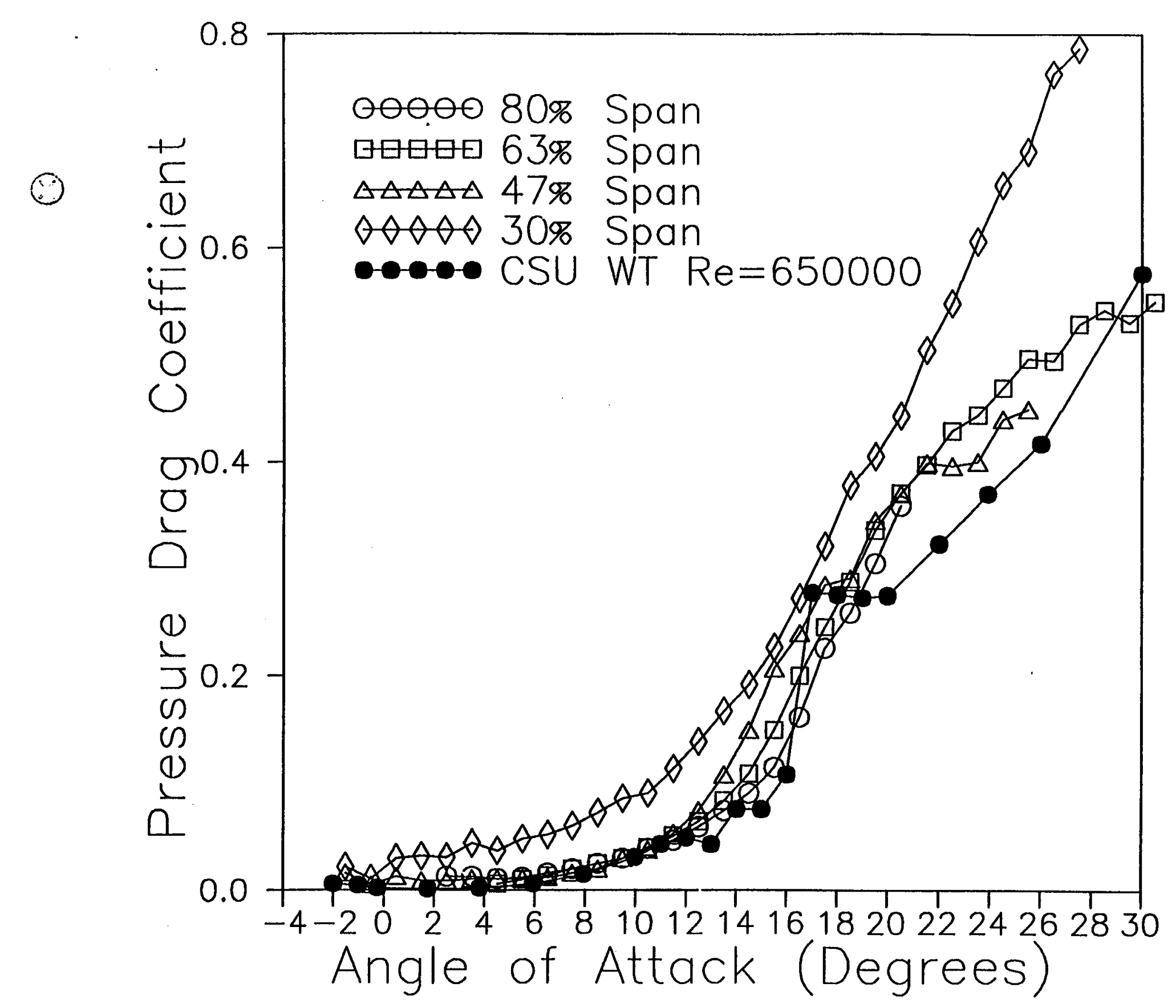




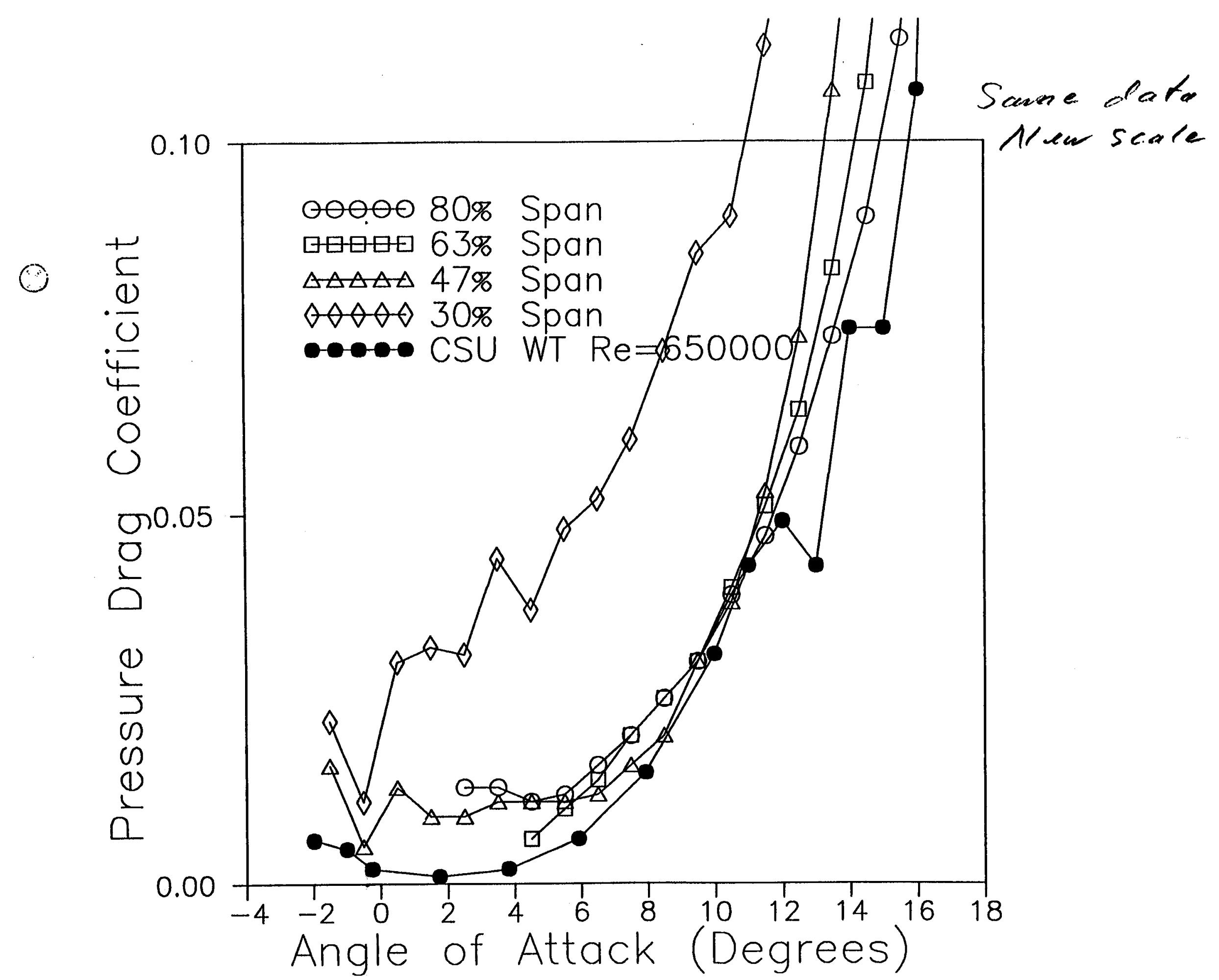




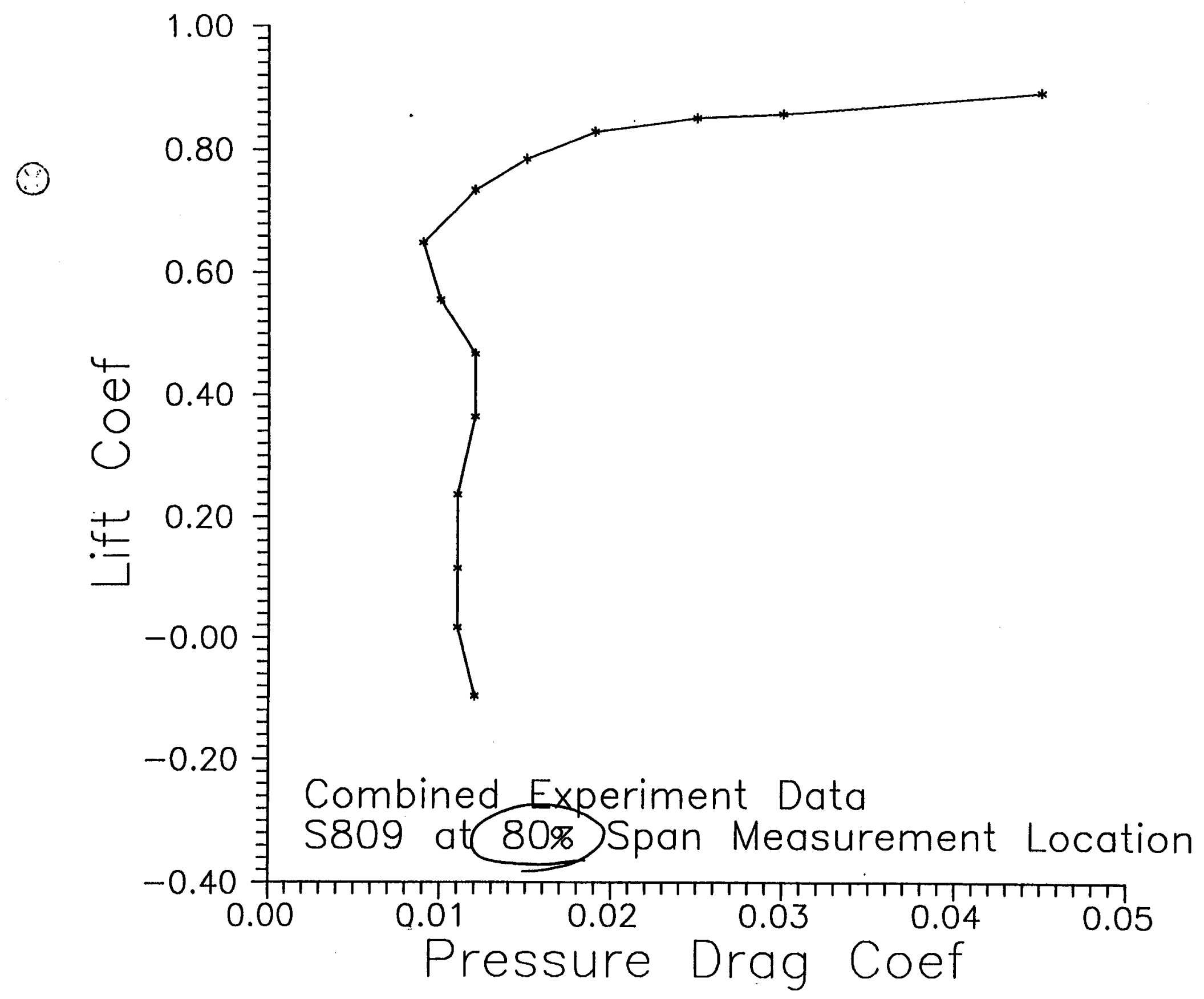




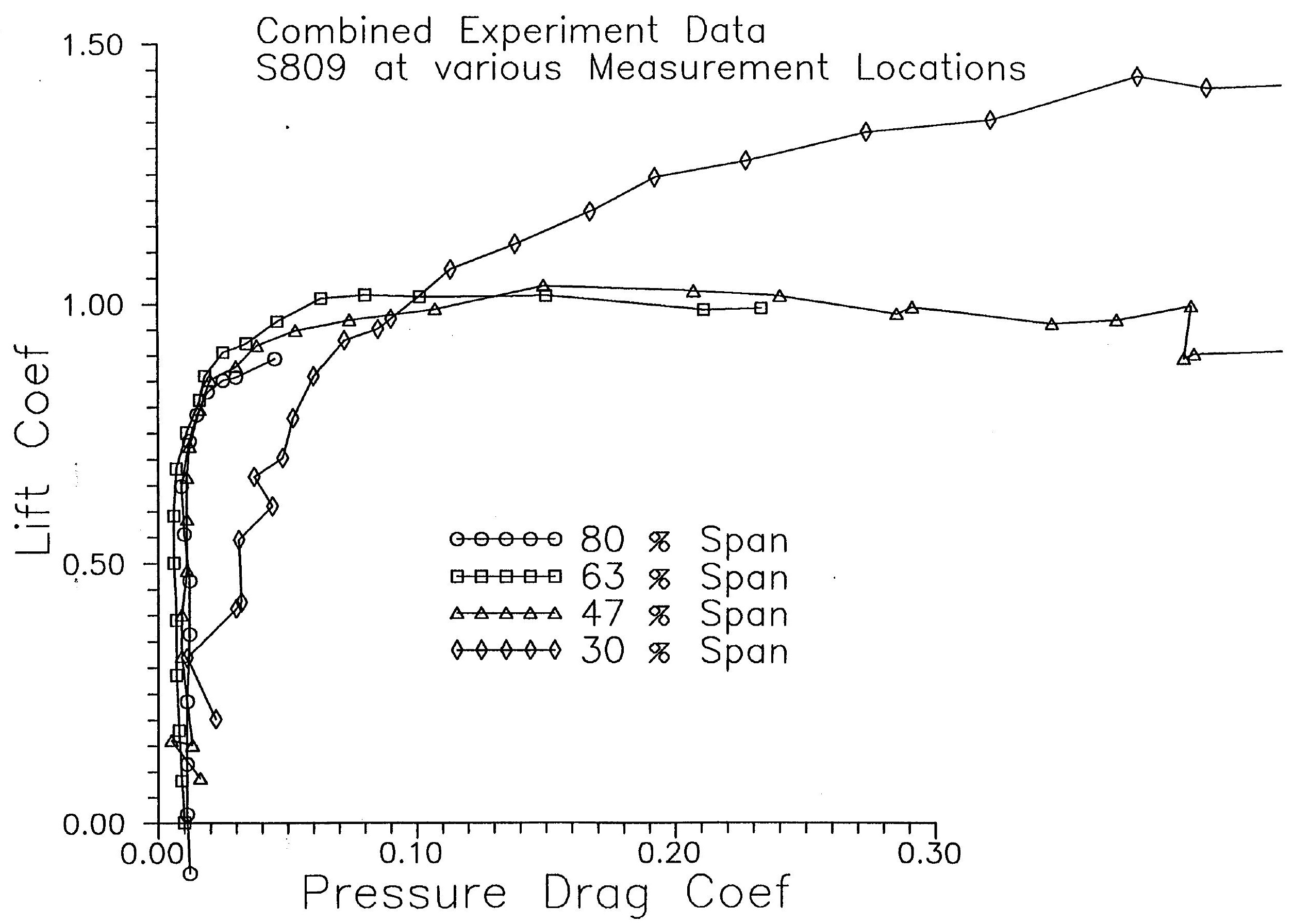




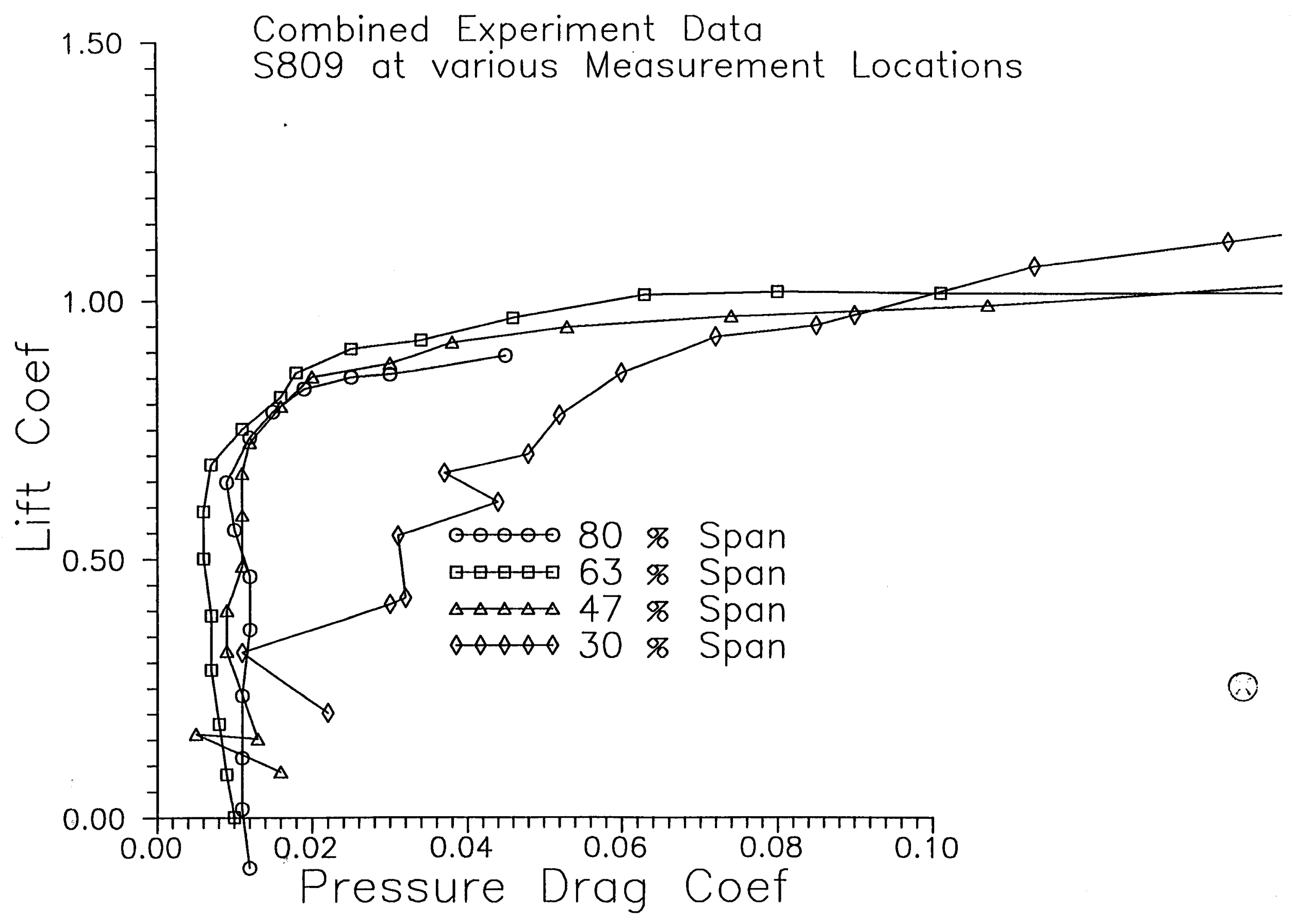




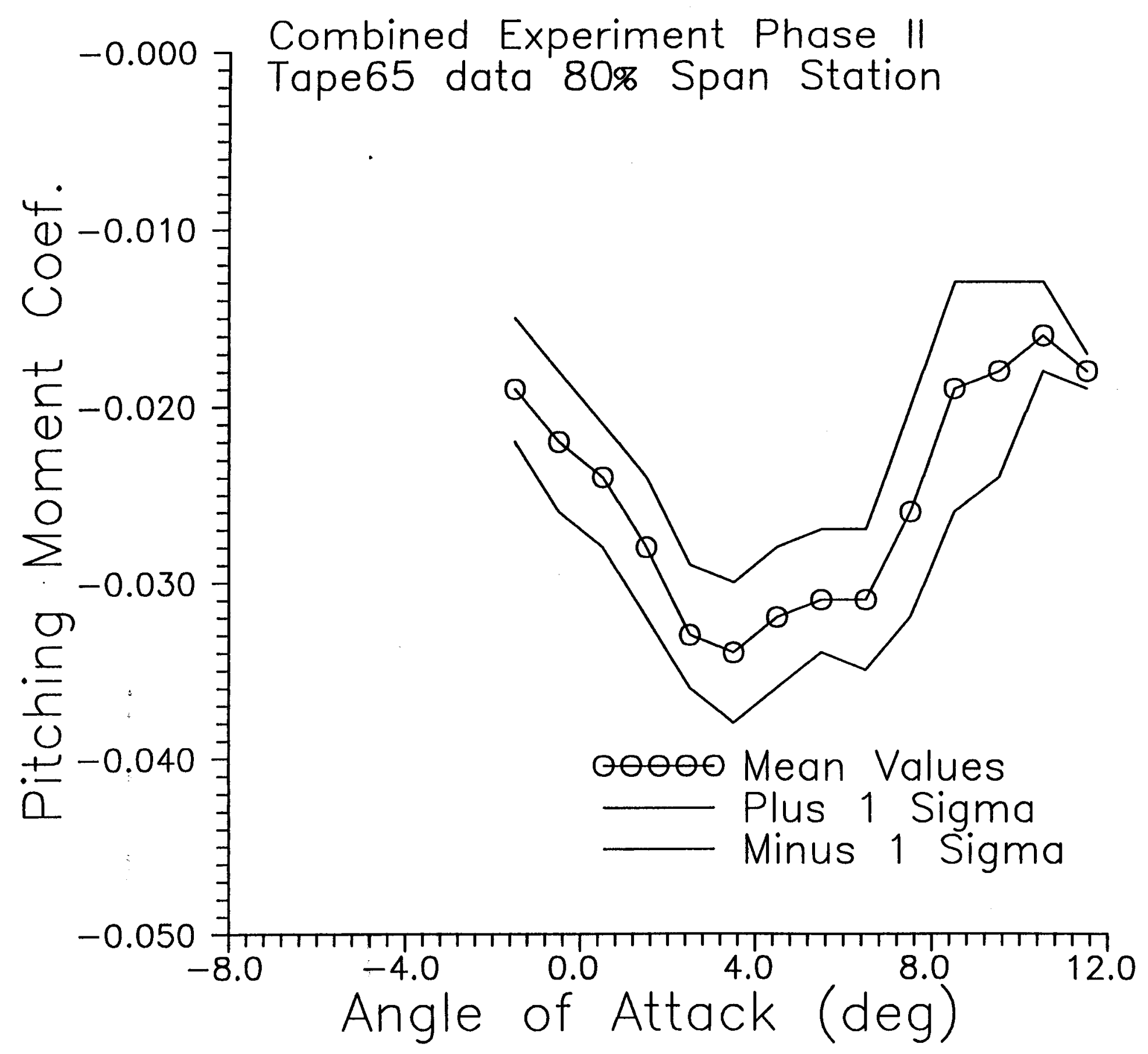




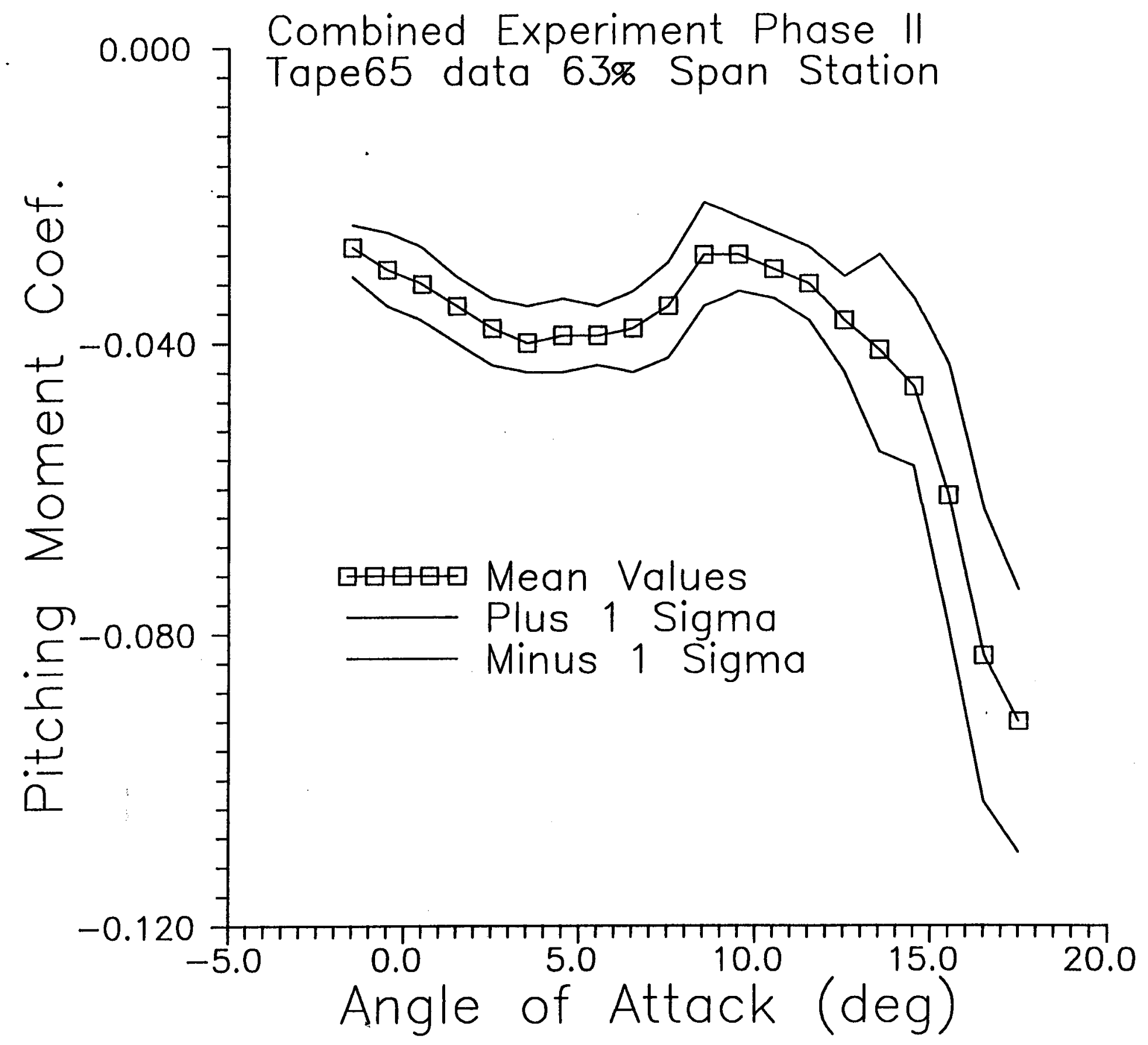




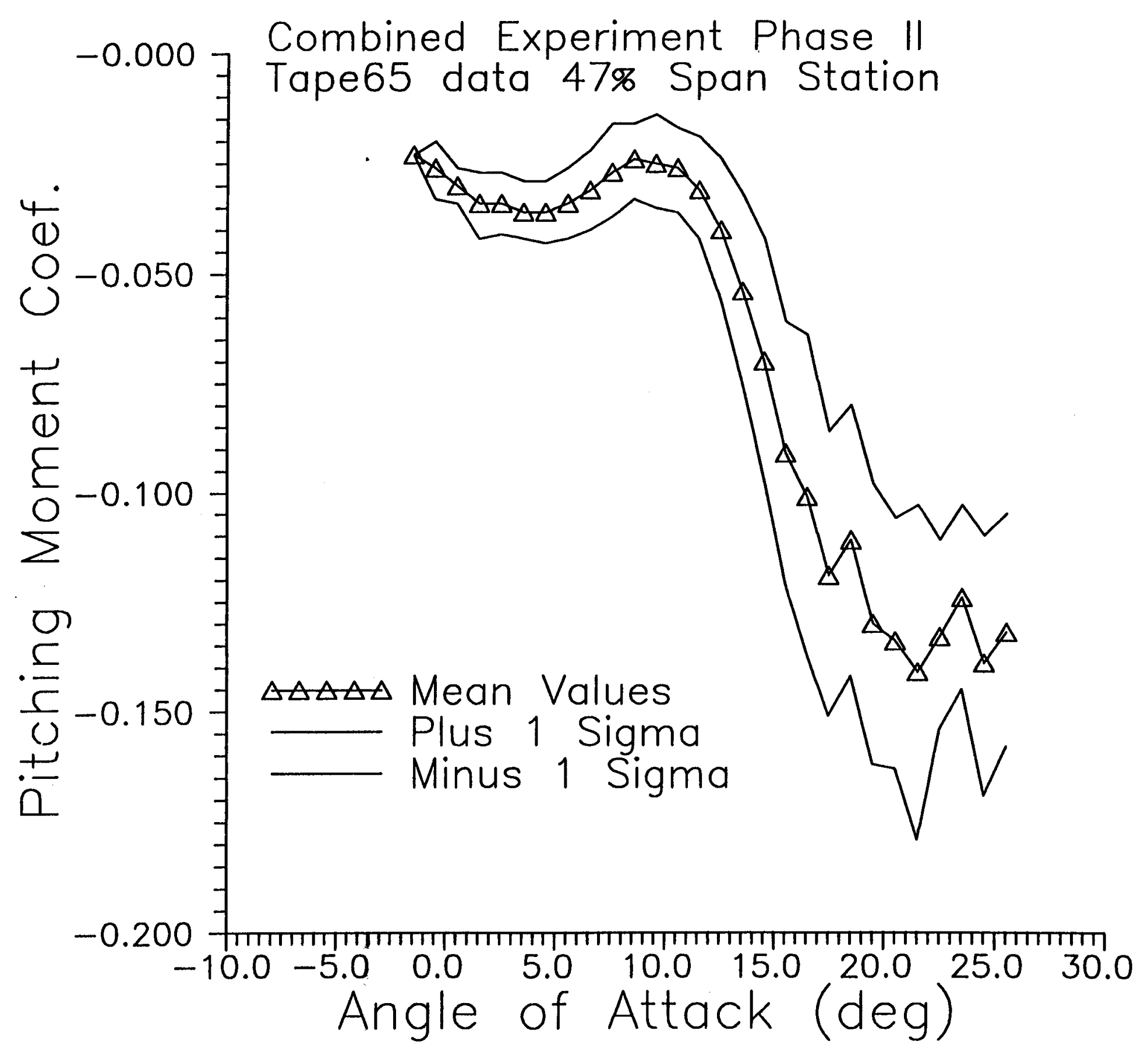




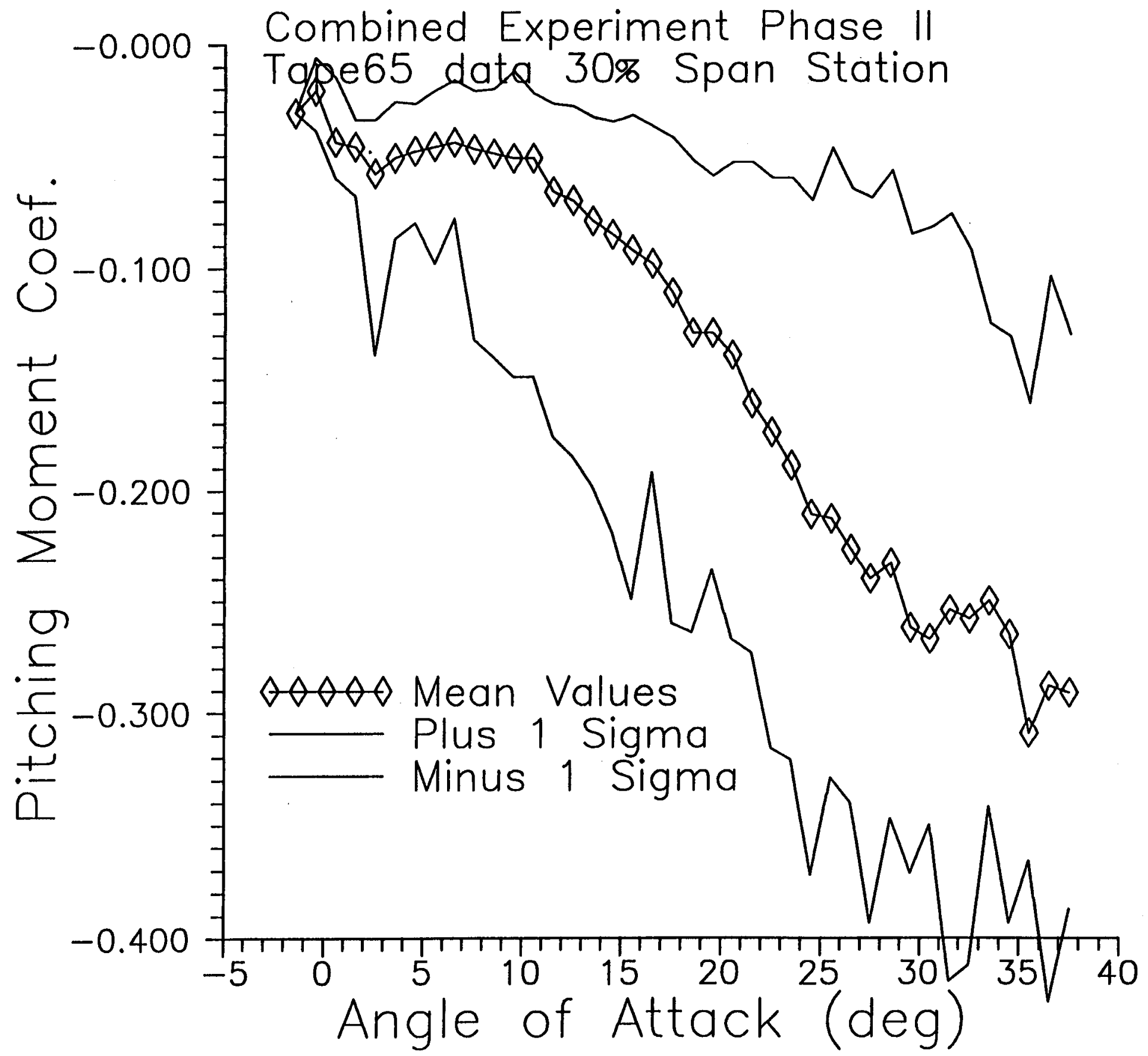




\section{Tape 68 Data}

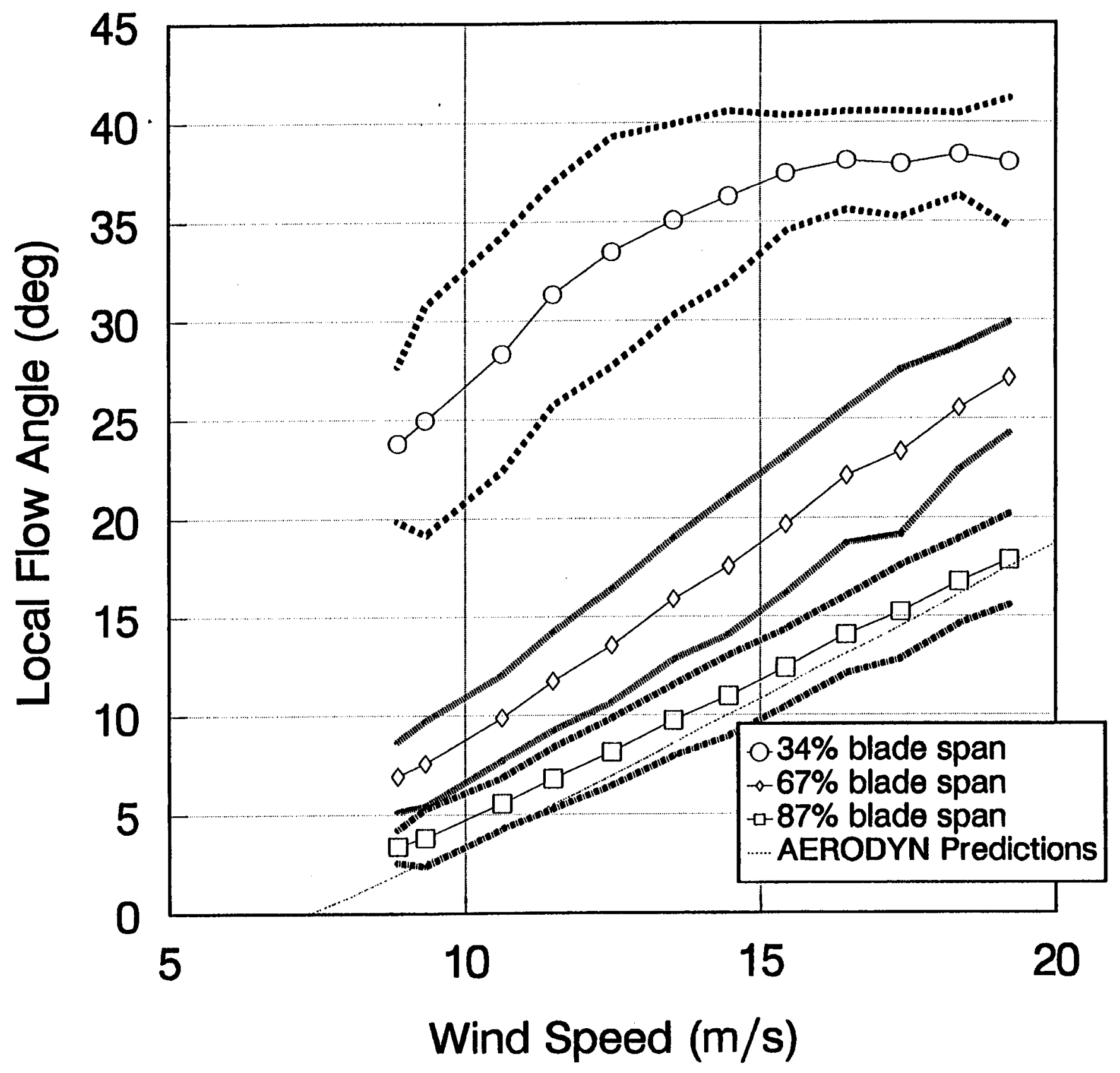




\section{Tape 68 Data}

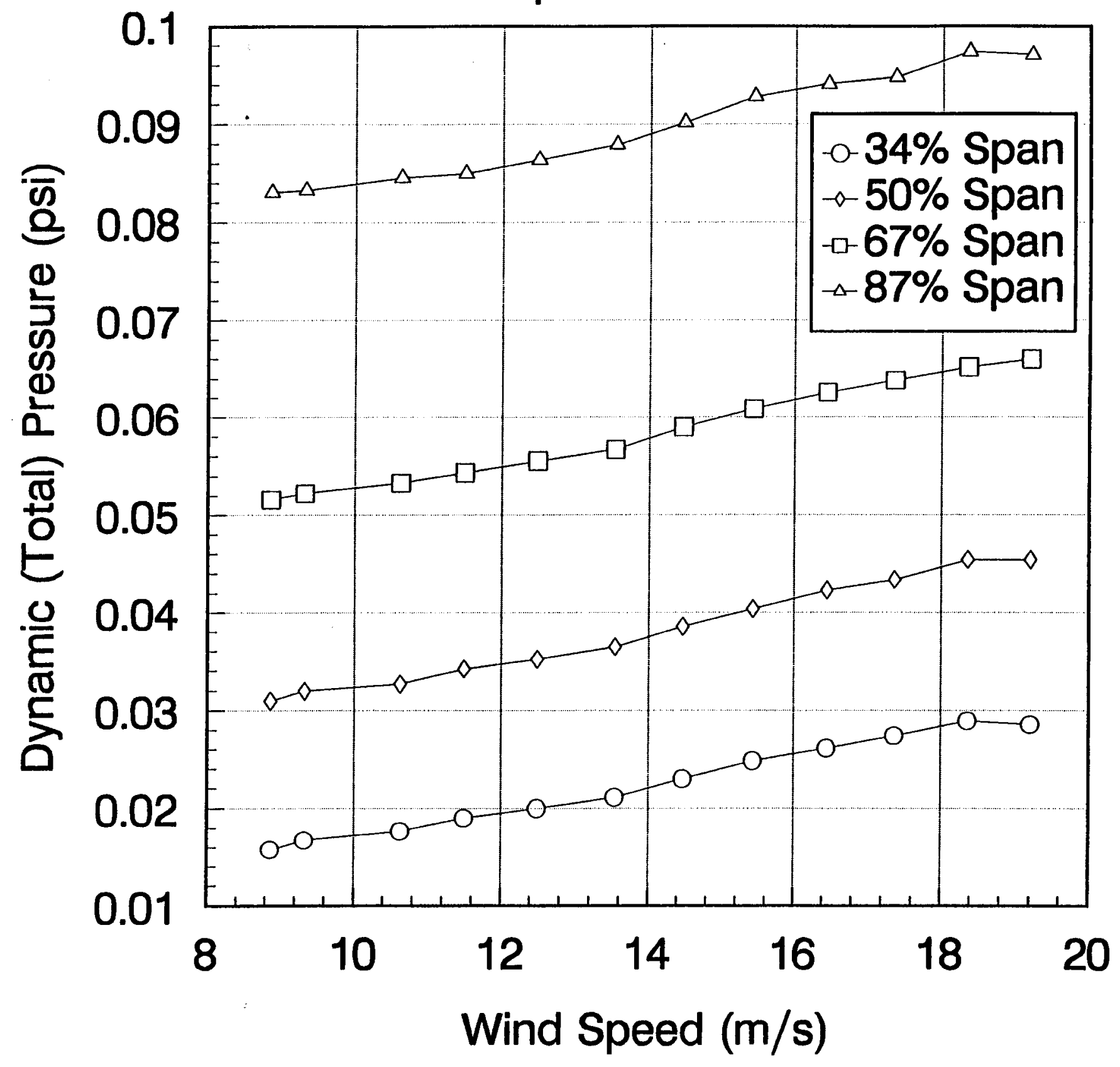




\section{Appendix C Bin-Averaged Pressure Distributions}

C.1 Moderate Wind Speed Case

C.1.1 Statistics for All Channels for Tape 65, Case 2

C.1.2 Pressure Distributions for $80 \%$ Span

C.1.3 Pressure Distributions for 63\% Span

C.1.4 Pressure Distributions for $47 \%$ Span

C.1.5 Pressure Distributions for $30 \%$ Span

C.2 High Wind Speed Case

C.2.1 Statistics for All Channels for Tape 68, Case 1

C.2.2 Pressure Distributions for $80 \%$ Span

C.2.3 Pressure Distributions for 63\% Span

C.2.4 Pressure Distributions for 30\% Span 
Appendix C.1

Moderate Wind Speed Case 


\section{Appendix C.1.1}

Statistics for All Channels for Tape 65, Case 2 


\section{COMBINED EXPERIMENT PHASE II - PROCESSED DATA FILE SUMHARY}

Analog tape 65. Tape file I0 11, Processed via pass4 version 2.2
Raw binary integer data read from file: /usr50/dave/p2/t065011.dat
Corresponding header read from file:

Processed EU data written to file: /usr50/dave/p2/d065019.dat corresponding header written to file: /usr50/dave/p2/0065011. hdr This sumary for printing written to file: /usr50/dave/p2/d065011.prt Data processed on: Mon Jut 16 21:37:05 1990

This data set contains:

- 1.92 msec data averaged by a factor of 1 to $0.0019 \mathrm{sec}$.

- 299.4394 seconds of data, from 115:09:31:14:706 to 115:09:36:14:145.

- 236 channels of engineering unit data values comprised of:

190 original measured data channels (pressures converted to coefficients)

5 channels of time (day, hour, minute, second, mill isecond)

41 channels of new derived paraweters

- 155958 data scans at $520.8333 \mathrm{~Hz}$.

- Corrected (Linear smoothed) azimuth data for exactly 358 blade cycles.

All pressure coefficients Cp are derived from measured pressures Pmeas which have been corrected for centrifugal forces Pcent due to rotation, and have been normalized using estimated induced total pressure aind:

$$
C p=(\text { Pweas + Pcent }) / \text { Qind }
$$

- Centrifugat force correction Peent is calculated using:

where:

$$
\text { Pcent }=a / 2 * r * 2 * \text { onega**2 }
$$

$r$ is the radius at which the pressure tap is located

onega is the blade rotation speed $(72.0 \mathrm{rpm})$

$a$ is the air density ( 0.0019 slugs/ft3) determined from:

$$
\begin{aligned}
& a=\text { Patm } /(822=(\text { Tair }+459.6)) \\
& \text { where: }
\end{aligned}
$$

Pata is atmospheric pressure $(805.2 \mathrm{mb}$ from channel 234)

Tair is temperature ( 57.5 deg F from channel 231)

centrifugal forces at pressure distribution locations:

Pcent $=0.0092$ psi at $r=59.4$ inches

Pcent $=0.0221$ psi at $r=92.3$ inches

Pcent $=0.0408$ psi at $r=125.3$ inches

Pcent $=0.0652$ psi at $r=158.4$ inches

Centrigugal forces at interwediate tap locations:

Pcent $=0.0129$ psi at $r=70.5$ inches

Pcent $=0.0172$ psi at $r=81.4$ inches

Pcent $=0.0278$ psi at $r=103.4$ inches

Pcent $=0.0340$ psi at $r=114.4$ inches

Pcent $=0.0483$ psi at $r=136.4$ inches

Pcent $=0.0563$ psi at $r=147.3$ inches

- Induced total pressure aind is deterwined using:

where:

$$
\text { Gind }=a / 2 *\left((\text { onega } * r)=2+V_{w i n d} * \star 2\right)
$$

Radius $r$ and rotation speed onega were described above.

Vwind is the instantaneous hub-height wind speed from channel 71

Total probe pressure values Ptp were obtained by correcting weasured total probe pressures Pweas for centrifugal forces using:

$$
\text { Ptp }=\text { Pmess + Pcent }
$$

Centrifugal forces at total probe locations:

$$
\text { Pcent }=0.0118 \text { psi at } r=67.4 \text { inches }
$$

Pcent $=0.0261$ psi at $r=100.3$ inches

Pcent $=0.0461$ psi at $r=133.3$ inches

Pcent $=0.0758$ psi at $r=170.9$ inches

Channel Sumary: 
* Tag

$1901:$

2 902:

$3903:$

$4904:$

$5905:$

6 906:

$7907:$

8 908:

9 909:

$10910:$

11 911:

12 912:

13 913:

14 914:

15 915:

16 916:

17 917:

18 918:

19 919:

20 920:

21 921:

22 922:

23923

24 924:

25 925:

26 926:

27 927:

28 928:

29 929:

30 930:

31 931:

32932

33 933:

34 934:

35 935:

36 936:

$37937:$

38 938:

$39201:$

40 202:

41 203:

42 204:

43 205:

44 206:

45 207:

46 208:

47 209:

48 210:

49 211:

50 212:

51 213:

52 214:

53 215:

54 216:

55 301:

56 302:

57 303:

58 304:

59 305:

60 306:

61 307:

62 308:

63 310:

64 311:

65 314:

66 315:

67 316:

68 401:

69 402:

70 403:

71 404:

72405 :
Channel Mane

Pressure "11 StapT5, 36\% chord, upper Pressure $\# 18$ StaPT5, 4X chord, upper Pressure 11 stapT7, $100 \%$ chord, trailing Pressure \#2 StaPT7, 92X chord, upper Pressure 14 StaPT7, 80\% chord, upper Pressure "16 StaPT7. 68\% chord, upper Pressure "\$8 StaPT7, $56 x$ chord, upper Pressure \$10 StaPT7, 44\% chord, upper Pressure "\#11 StaPT7, 36\% chord, upper Pressure \#12 StaPT7, 28X chord, upper Pressure 13 StaPT7, 20\% chord, upper Pressure "14 StaPT7, 14\% chord, upper Pressure 115 StaPT7, 10\% chord, upper Pressure "16 StaPT7, 8\% chord, upper Pressure 117 stapT7, 6\% chord, upper Pressure 118 StapT7, 4X chord, upper Pressure 119 StaPT, $2 x$ chord, upper Pressure 120 StaPT7, 12 chord, upper Pressure "21 staPT7, 0.5\% chord, upper Pressure 12Z StaPT7, 0\% chord, leading Pressure "23 StaPT7. 0.5\% chord, lower Pressure "24 StaPT7, 1X chord, Lover Pressure 625 StaPT7, 2\% chord, Laver Pressure 526 staPT7, 4\% chord, Lower Pressure 128 5tapT7. 8X chord. Lower Pressure \#30 5taPT7, $14 \%$ chord, Lower Pressure \$32 StaPT7, 28\% chord, Lower Pressure \$34 StaPT7, 46x chord, lower Pressure "136 StaPT7, 68x chord, Lower Pressure \#38 stapT, 92\% chord, lower Pressure \$18 StaPT6, 4X chord, upper Pressure "18 StaPT8, 4X chord, upper Pressure "11 stapT6, $36 \%$ chord, upper Total Pressure Probe, $67.3 \%$ span Angle-of-Attack, $86 \%$ span

Angle-of-Attack, $67.3 \mathrm{x}$ span

Angle-of-Attack, $50.6 x$ span

Angle-of-Attack, 34X span

VPA Prop Vane Speed US-1 (12:00)

VPA Prop Vane speed US-2 $(1: 30)$

VPA.Prop Vane Speed US-3 ( 3:00)

VPA Prop Vane speed uS-4 $(4: 30)$

VPA Prop Vane Speed ws-5 $(6: 00)$

VPA Prop Vane speed us-6 $(7: 30)$

VPA Prop Vane Speed us-7 (9:00)

VPA Prop Vane speed us-8 (10:30)

VPA Prop Vane Speed us-9 thub Height

VPA Prop Vane Direction uD-9 Hub Height

VPA Bi-Vane Speed us-12 (3:00 a100\%)

VPA Bi-Vane Direction ND-12 (3:00 a100\%)

VPA Bi-Vane Angle HA-12 (3:00 a100z)

VPA Bi-Vane Speed US-13 (9:00 a100\%)

VPA Bi-Vane Direction wo-13 (9:00 2100\%)

VPA 8i-Vane Angle HA-13 (9:00 a100\%)

VPA Prop Vane speed us-10 (12:00 240\%)

VPA Prop Vane Speed us-11 (6:00 240\%)

Low Speed Shaft Azimuth Angle

Yaw Moment

Tower Bending about East-West Axis (X)

Tower Bending about North-South Axis ( $Y$ )

Yaw Angle

Generator Power

TSI (SOUth) $X$-Fil $X$ (NOT WORKING)

TSI (South) $X$-Fil $Y$ (nOT WORKIHG)

Sonic Anewometer Channel $A$ (NOT WORKING) Sonic Anemoneter Channel B (NOT WORKING) Sonic Anewometer Channel $C$ (nOT WORKING) Root Flap Bending "RTFBK- $A$ " Root Flop Bending 'RTFBH-B'

Root Flap Bending 'RTFBli-2" (Blade 2)

Root Flap Bending "RTFBH-3" (Blade 3)

$20 \%$ flap Bending "20FEn"
Units Mean std Min Max

[coef] -9.138E-01 (2.091E-01) - $1.563 E+00-6.712 E-02$

[coef] $-1.486 E+00(6.234 E-01)-4.715 E+00 \quad 7.219 E-01$

[coef] 1.611E-01 (1.531E-01) -7.400E+00 1.662E+00

[coef] $5.237 E-02$ (1.333E-01) -8.053E-01 3.671E-01

[coef] -9.572E-02 (1.130E-01) -1.164E+C0 3.512E-01

[coef] -2.128E-01 (1.116E-01) -1.111E+01 1.531E+00

[coef] -5.303E-01 (1.313E-01) -1.258E+00 1.258E-01

[coef] -9.075E-01 (1.698E-01) $-2.433 E+00-1.647 E-01$

[coef] -9.430E-01 (2.015E-01) -1.632E+00 2.456E+01

[coef] $-9.862 E-01(2.386 E-01)-1.810 E+001.797 E+01$

[coef] $-1.020 E+00(2.848 E-01)-7.651 E+00-1.123 E-01$

[coef] $-1.045 E+00$ (3.677E-01) -1.022E+01 5.078E-02

[coef] $-1.058 E+00(4.193 E-01)-1.058 E+01 \quad 2.419 E-01$

[coef] $-1.080 E+00(4.765 E-01)-3.158 E+00 \quad 1.466 E+01$

[coef] $-1.153 E+00(5.468 E-01)-8.658 E+00 \quad 4.088 E-01$

[coef] $-1.162 E+00(6.685 E-01)-1.195 E+01 \quad 6.906 E-01$

[coef] $-1.359 E+00(8.871 E-01)-9.939 E+00 \quad 8.726 E-01$

[coef] $-1.354 E+00(1.128 E+\infty 0)-5.348 E+00$ 1.317E+01

[coef] $-1.319 E+00(1.363 E+\infty 0)-1.117 E+01 \quad 1.379 E+\infty 0$

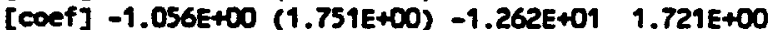

[coef] 9.180E-01 (2.014E-01) -5.947E-01 6.023E+00

[coef] 9.085E-01 (1.712E-01) -5.520E-01 6.227E+00

[coef] 8.197E-01 (2.011E-01) -1.420E+01 1.386E+00

[coef] 6.970E-01 (2.283E-01) -1.219E+01 1.255E+00

[coef] 4.797E-01 (2.375E-01) -5.240E-01 1.390E+01

[coef] 2.161E-01 (2.309E-01) -1.332E+C0 1.316E+01

[coef] -3.213E-01 (2.082E-01) -1.216E+01 1.313E+00

[coef] $-4.587 E-01$ (1.473E-01) -1.293E+01 3.268E-02

[coef] 1.482E-01 (7.850E-02) -3.737E-01 6.002E-01

[coef] 2.752E-01 (9.859E-02) -1.514E+01 5.819E-01

[coef] $-1.411 E+00$ (6.801E-01) $-3.058 E+01 \quad 4.874 E-01$

[coef] -9.983E-01 (6.103E-01) -2.814E+00 1.015E+00

[coef] -9.619E-01 (1.939E-01) -2.068E+00 1.551E-01

[psi ] 5.166E-02 (5.641E-03) -6.112E-01 8.361E-02

[Degr] $3.245 E+00(2.510 E+00)-5.831 E+001.628 E+01$

[Degr] $5.692 E+00(3.630 E+00)-2.741 E+01 \quad 2.537 E+01$

[Degr] 1.042E+01 $(5.504 E+00)-1.152 E+01 \quad 3.883 E+01$

[Degr] 1.801E+01 $(8.807 E+\infty 0)-1.283 E+01 \quad 3.966 E+01$

$[\omega / \mathrm{s}] \quad 7.632 E+\infty 0(1.667 E+\infty 0) \quad 4.139 E+\infty 01.225 E+01$

$[\omega / \mathrm{s}] \quad 7.217 E+\infty 0(1.600 E+\infty) \quad 4.702 E+\infty 01.192 E+01$

$[\omega / s] \quad 7.393 E+00(1.615 E+\infty 0) \quad 4.346 E+00 \quad 1.205 E+01$

$[\mathrm{w} / \mathrm{s}] \quad 7.347 \mathrm{E}+00(1.627 \mathrm{E}+\infty 0) \quad 4.278 E+\infty 0 \quad 1.241 E+01$

$[\omega / \mathrm{s}] \quad 7.281 E+\infty 0(1.593 E+\infty 0) \quad 4.105 E+\infty 01.247 E+01$

[W/s ] $7.197 \mathrm{E}+00(1.623 \mathrm{E}+00) \quad 4.179 \mathrm{E}+00$ 1.221E+01

[E/s ] $7.313 E+00(1.649 E+00) \quad 4.157 E+00 \quad 1.200 E+01$

$[=/ s] \quad 7.470 E+00(1.645 E+\infty 0) \quad 4.256 E+\infty 01.224 E+01$

$[\mathrm{E} / \mathrm{s}] \quad 7.525 E+00(1.655 E+00) \quad 4.425 E+00 \quad 1.266 E+01$

[degr] $2.570 E+02(9.066 E+00) \quad 2.268 E+02 \quad 2.891 E+02$

$[\omega / s] \quad 7.374 E+\infty 0(1.576 E+\infty 0) \quad 4.377 E+\infty 001.203 E+01$

[deg ] 2.585E+02 (8.698E+00) 2.287E+02 2.870E+02

[deg ] $-4.098 E+00(6.060 E+00)-2.019 E+011.558 E+01$

$[\omega / \mathrm{s}] \quad 7.180 E+00(1.614 E+\infty 0) \quad 3.707 E+001.206 E+01$

[deg $] 2.570 E+02(9.969 E+00) \quad 2.246 E+02 \quad 2.831 E+02$

[deg ] $-5.491 E+00(6.405 E+00)-2.639 E+01 \quad 1.430 E+01$

$[\mathrm{w} / \mathrm{s}] 7.628 \mathrm{E}+00(1.693 \mathrm{E}+00) 4.547 \mathrm{E}+00 \quad 1.242 \mathrm{E}+01$

$[\mathrm{E} / \mathrm{s}] \quad 7.414 \mathrm{E}+00(1.645 \mathrm{E}+00) \quad 4.137 \mathrm{E}+001.249 \mathrm{E}+01$

[Degr] 1.800E+02 (1.039E+02) 2.371E-03 3.600E+02

[N-M ] 1.215E+02 (8.393E+00) $1.006 E+02 \quad 1.390 E+02$

[N-H ] -1.962E+04 (1.914E+03) -2.478E+04 -1.331E+04

$[N-H]-8.399 E+03(1.427 E+03)-1.469 E+04-4.001 E+03$

[Degr] 2.584E+02 (1.011E+01) 2.228E+02 2.895E+02

[KW $] \quad 1.740 E+00(2.616 E+00)-3.287 E+00 \quad 9.297 E+00$

[volt] $2.018 E+\infty 0(3.195 E-04) \quad 2.018 E+\infty 0 \quad 2.018 E+\infty 0$

[volt] $2.039 E+00(1.467 E-04) \quad 2.039 E+\infty 02.039 E+\infty 0$

$[\mathrm{w} / \mathrm{s}] \quad 4.880 \mathrm{E}-01$ (1.955E-02) $3.894 \mathrm{E}-01 \quad 5.810 \mathrm{E}-01$

$[\mathrm{m} / \mathrm{s}] \quad 4.560 \mathrm{E}-01 \quad(1.828 \mathrm{E}-02) \quad 3.451 \mathrm{E}-01 \quad 5.396 \mathrm{E}-01$

$[\mathrm{w} / \mathrm{s}] \quad 6.272 E-01$ (1.527E-02) $5.355 E-01 \quad 7.212 E-01$

[NT-N] $-2.341 E+02(4.573 E+02)-1.681 E+03 \quad 1.533 E+03$

$[N T-M]-2.366 E+02(4.570 E+02)-1.684 E+03 \quad 1.531 E+03$

[NT-H] $-2.911 E+02(4.353 E+02)-1.758 E+03 \quad 2.009 E+03$

[NT-H] -1.833E+02 (4.355E+02) -1.706E+03 1.291E+03

[NT-H] -9.991E+01 (3.605E+02) -1.221E+03 2.714E+03 
73 406: 40\% Flap Bending -40FBm-

74 407: 50\% Flap Bending "50FBr"

75 409: 70\% Flap Bending -70FBn-

76 413: 90\% Flap Bending -90FBM.

77 414: Root Edge Bending "RTEBM-A"

78 415: $20 \%$ Edge Bending "ZOEEAr"

79 416: 50\% Edge Bending -50EBn"

80 417: 70X Blade Torque "70Te"

81 419: Root Torque (Link) "RTtR"

82 420: S0X Torsion -50Te-

83 421: $X-X$ LSS Bending "LSSX-X"

84 422: $Y-Y$ LSS Bending "LSSY-Y"

85 423: LSS Torque "LSSTE-A"

86 424: LSS Torque "LSSTa-B"

87 427:

88 428:

89 429:

90 430:

91 431:

92 432:

93 433:

94 434:

95 435:

96 436:

97 437:

98 438:

99 439:

100 440:

101 441:

102 442:

103 443:

104 464:

105 445:

106 446:

107 447:

108 448:

109 449:

110 450:

111 451:

112 452:

113 453:

114 454:

115 455:

116 456:

117 457:

118 458:

119 459:

120 461:

121 804:

122 805:

123 806:

124807 :

125 808:

126 809:

127 810:

128 811:

129 812:

130 813:

131 814:

132 815:

133 816:

134 817:

135 818:

136 819:

137 820:

$138821:$

139822 :

140823 :

141824 :

142825

143 826:

144 827:

145 828:
Pressure 11 5taPT10, $100 \%$ chord, trailing Pressure "12 stapT10, $92 x$ chord, upper Pressure $\$ 4$ StaPT10, $80 x$ chord, upper Pressure "6 StaPT10, 68X chord, upper Pressure $\$ 8$ StaPTI0, 562 chord, upper Pressure $\$ 10$ stapT10, $44 \%$ chord, upper Pressure \#11 Stapt10, 36X chord, upper Pressure \#12 StaPT10, 28\% chord, upper Pressure 113 StapT10, $20 \%$ chord, upper Pressure \#14 StaPT10, $14 \%$ chord, upper Pressure "15 StapT10, 10\% chord, upper Pressure "16 5taPT10, 8\% chord, upper Pressure $\$ 17$ StapT10, 6\% chord, upper Pressure \#18 StaPT10, $4 X$ chord, upper Pressure 119 StaPT10, 2X chord, upper Pressure \#20 stapT10, 1X chord, upper Pressure \$21 stapt10, 0.5\% chord, upper Pressure $\$ 22$ StaPT10, 0z chord, leading Pressure 23 stapt10, 0.5x chord, lower Pressure 24 StaPT10, 12 chord, lover Pressure "25 StaPT10, 2\% chord, Lower Pressure \#26 StaPTIO, 47 chord, lower Pressure \#28 StaPT10, 8\% chord, Lower Pressure $\$ 30$ StaPT10, 16\% chord, Laver Pressure \$32 StaPT10, 28\% chord, Lover Pressure \$34 StaPT10, $44 \%$ chord, lower Pressure 136 stapT10, 68\% chord, Lower Pressure 338 StaPT10, 92\% chord, Lower Pressure "18 StaPT9, 4X chord, upper Pressure "111 5taPT9, 36\% chord, upper Pressure 111 StapT8. $36 \%$ chord, upper Total Pressure Probe, 86x span absolute Reference Pressure Pitch Angle

Pressure $\# 1$ StapTI. $100 \%$ chord, trailing Pressure $\$ 4$ StapT1, 80X chord, upper Pressure 66 staPT1, $68 \%$ chord, upper Pressure $\$ 8$ stapT1, $56 \%$ chord, upper Pressure "10 StaPTI, 44X chord, upper Pressure "11 StaPT1. 36X chord, upper Pressure \#13 StaPT1, 20\% chord, upper Pressure 114 StaPT1, $14 x$ chord, upper Pressure "15 5taPT1, $10 \%$ chord, upper Pressure \$16 StaPT1, 82 chord, upper Pressure \#17 StaPT1, 6X chord, upper Pressure \#18 StaPT1. 4X chord, upper Pressure "19 StaPTI, 27 chord, upper Pressure 121 StaPT1, 0.5\% chord, upper Pressure $\$ 22$ StaPT1, OX chord, leading Pressure \#23 stapt1, 0.5x chord, lower Pressure "24 StaPT1, 1X chord, lower Pressure 25 stapt1, $2 \%$ chord, lower Pressure \$26 StaPT1, 4X chord, Lower Pressure "28 StapTI, 8x chord, lower Pressure "30 stapT1. $14 x$ chord, lover Pressure 131 StaPTI, 20\% chord, lower Pressure \$34 StaPT7, 44\% chord, lower Pressure $\$ 36$ StapT1, 68\% chord, Lower Pressure 138 stapt1, 92\% chord, Lower
[NT-N] -4.850E+01 (2.176E+02) -7.306E+02 1.972E+03 [NT-H] $-2.830 E+01(1.562 E+02)-5.073 E+02 \quad 6.054 E+02$ $[N T-N]-5.374 E+00(5.547 E+01)-1.830 E+02 \quad 2.557 E+02$ [NT-H] 5.396E+00 (6.274E+00) -1.671E+01 8.490E+01 [NT-H] 2.265E+02 (7.102E+02) -1.162E+03 2.750E+03 [NT-N] $3.043 E+02(4.805 E+02)-6.966 E+02 \quad 1.662 E+03$ [NT-H] 1.212E+02 (1.862E+02) $-2.968 E+02 \quad 1.218 E+03$ [NT-K] 6.746E-03 (1.631E+00) $0.000 E+00$ 5.260E+02 [NT-H] $-8.983 E+00$ (1.359E+01) -4.954E+01 5.567E+01 [NT-M] -2.259E+01 (1.179E+01) -5.910E+01 2.781E+02 [NT-H] $-7.936 E+01(4.412 E+03)-8.818 E+03 \quad 8.071 E+03$ [NT-H] 1.469E+02 (3.043E+03) $-5.602 E+03 \quad 6.178 E+03$ [NT-M] $4.368 E+02(3.736 E+02)-2.902 E+02 \quad 2.726 E+03$ [Degr] 4.221E+02 (3.663E+02) -3.345E+03 2.467E+03 [coef] 2.091E-01 (8.136E-02) $-3.985 E+00$ 4.380E-01 [coef] 1.019E-01 (7.769E-02) $-5.978 E+00 \quad 9.564 E+00$ [coef] $-2.241 E-02(7.405 E-02)-5.485 E+001.952 E+00$ [coef] $-1.610 E-01(8.789 E-02)-6.456 E+00 \quad 5.310 E-01$ [coef] $-6.063 E-01(1.346 E-01)-4.876 E+\infty 01.141 E+01$ [coef] -8.080E-01 (1.600E-01) $-4.974 E+00-2.544 E-01$ [coef] $-8.242 E-01(1.779 E-01)-5.228 E+00-2.687 E-01$ [coef] -8.199E-01 (2.080E-01) -5.108E+00 -2.030E-01 [coef] $-7.874 E-01$ (2.538E-01) $-5.375 E+001.094 E+01$ [coef] $-7.855 E-01$ (3.031E-01) $-5.151 E+00 \quad 7.206 E-02$ [coef] -7.625E-01 (3.586E-01) $-4.908 E+00 \quad 2.475 E-01$ [coef] -7.967E-01 (3.947E-01) $-5.272 E+00 \quad 2.543 E-01$ [coef] $-7.588 E-01$ (4.505E-01) $-5.021 E+00$ 1.092E+01 [coef] -7.287E-01 (5.360E-01) $-5.193 E+00 \quad 7.087 E-01$ [coef] $-6.616 E-01(6.754 E-01)-5.369 E+001.084 E+01$ [coef] $-4.430 E-01(7.887 E-01)-4.848 E+00 \quad 1.148 E+01$ [coef] -3.195E-01 (9.404E-01) $-5.828 E+00 \quad 1.517 E+00$ [coef] 2.047E-01 (9.652E-01) $-4.697 E+00 \quad 1.629 E+\infty 0$ [coef] 8.611E-01 (2.123E-01) $-4.480 E+00 \quad 1.425 E+00$ [coef] 8.026E-01 (2.355E-01) $-3.869 E+00 \quad 1.410 E+00$ [coef] $6.669 E-01(2.505 E-01)-6.879 E+001.233 E+00$ [coef] $5.338 E-01(2.449 E-01)-4.288 E+\infty 0 \quad 2.536 E+00$ [coef] 2.828E-01 (2.272E-01) $-4.957 E+\infty 0$ 1.156E+00 [coef] 1.581E-02 (2.123E-01) $-6.467 E+00 \quad 6.810 E-01$ [coef] -4.365E-01 (1.827E-01) -5.676E+00 1.679E-01 [coef] $-6.048 E-01(1.409 E-01)-6.028 E+00-8.027 E-02$ [coef] $8.245 E-02(6.758 E-02)-6.489 E+00 \quad 3.128 E-01$ [coef] 3.097E-01 (7.460E-02) $-6.317 E+00$ 5.576E-01 [coef] $-8.130 E-01$ (5.814E-01) $-6.397 \varepsilon+00 \quad 6.456 E-01$ [coef] -9.128E-01 (1.916E-01) -8.374E+00 -2.721E-01 [coef] -8.882E-01 (1.964E-01) -7.834E+00 -2.717E-01 [PSI ] 8.277E-02 (7.208E-03) -2.253E-01 1.196E-01 [a ] 8.060E+02 (7.063E-01) 8.050E+02 $9.574 E+02$ [Degr] $8.607 E+00(5.613 E-01) \quad 7.012 E+00 \quad 1.509 E+01$ [coef] $-2.083 E-01$ (2.967E-01) $-2.210 E+00 \quad 6.377 E-01$ [coef] -5.583E-01 (4.095E-01) -2.892E+00 4.680E-01 [coef] -7.099E-01 (4.995E-01) $-3.432 E+00$ 4.166E-01 [coef] $-9.673 E-01$ (5.539E-01) $-3.987 E+00 \quad 2.690 E-01$ [coef] $-1.286 E+00(5.633 E-01)-4.104 E+00 \quad 1.473 E-01$ [coef] $-1.486 E+\infty 0$ (5.871E-01) $-4.725 E+\infty 0$ 1.617E-02 [coef] -1.808E+00 (6.229E-01) -5.247E+00 3.994E-01 [coef] $-1.956 E+00$ (6.462E-01) -5.902E+00 $1.966 E-02$ [coef] $-2.021 E+00(6.841 E-01)-6.066 E+00 \quad 1.817 E-01$ [coef] -2.132E+00 (7.028E-01) -6.503E+00 2.802E-01 [coef] $-2.190 E+00$ (7.425E-01) $-7.488 E+00$ 4.783E-01 [coef] $-2.302 E+00(8.173 E-01)-8.157 E+00 \quad 7.904 E-01$ [coef] $-2.563 E+00(1.057 E+\infty)-9.858 E+\infty 01.103 E+\infty 0$ [coef] $-2.655 E+\infty 0(1.279 E+\infty)-1.374 E+01 \quad 1.547 E+\infty$ [coef] $-2.738 E+00(1.538 E+\infty)-1.500 E+012.004 E+\infty 0$ [coef] 1.410E-01 (5.358E-01) -9.851E+CO 2.054E+CO [coef] 5.156E-01 (3.948E-01) -3.796E+01 1.989E+00 [coef] 8.340E-01 (2.482E-01) -8.470E-01 8.374E+00 [coef] 9.035E-01 (2.191E-01) $-2.638 E+01 \quad 1.861 E+00$

[coef] 8.532E-01 (2.205E-01) -2.077E+01 1.665E+00

[coef] 5.782E-01 (2.532E-01) -2.040E+01 1.388E+00

[coef] 3.324E-01 (2.686E-01) -1.566E+01 1.047E+00

[coef] -1.581E-01 (2.353E-01) -2.013E+01 2.546E+01

[coef] 1.722E-01 (1.553E-01) -1.533E+01 2.450E+01

[coef] 1.546E-01 (1.777E-01) -1.174E+CO 2.189E+01 
146 829: Pressure "18 StaPT2, 4X chord, upper 147 830: Pressure 111 StaPT2, 36x chord, upper 168 831: Pressure 111 StaPT3, 36x chord, upper 169 832: Total Pressure Probe, 34x span 150833 : 151 834: 152 835: 153836 : 154 837: 155 838: 156 839: 157 840: 158 841: 159 842: 160 843: 161 844: 162 845: 163 846: 164 847: 165 848: 166 849: 167 850: 168 851: 169 852: 170 853: 171 854: 172 855: 173 856: 174 857: 175 858: 176 859: 177 860: 178 861: 179 862: 180 105: 181 106: 182 107: 183 108: 184 109: 185 110: 186 119: 187 112:

188 113:

189 114:

190 116:

$191701:$

192702 :

$193703:$

$194704:$

$195705:$

196 706:

$197707:$

$198708:$

$199709:$

200 710:

201 711:

202 712:

203 713:

204 714:

205 715:

206 716:

$207717:$

208718 :

209 719:

210720 :

211721 :

212722 :

$213723:$

214724 :

215725

216726 :

217 727:

218728 :

\section{Pressure 11 StaPT4, 100X chord, trailing}

Pressure 12 StaPT4, $92 X$ chord, upper Pressure 14 StaPT4, 80x chord, upper Pressure 16 StaPTh, $68 \%$ chord, upper Pressure $\$ 8$ StaPT4. $56 \%$ chord, upper Pressure "10 Stapt6, $44 X$ chord, upper Pressure \#11 StaPT6, $36 \%$ chord, upper Pressure 112 stapT4, $28 x$ chord, upper Pressure "13 StaPT4, $20 X$ chord, upper Pressure 114 StaPT4, 16\% chord, upper Pressure 15 StaPT4, 10\% chord, upper Pressure "16 StaPT4, 8x chord, upper Pressure 117 StaPT4, 6X chord, upper Pressure "18 StaPT6, 4\% chord, upper Pressure \#19 stapT4, $2 \%$ chord, upper Pressure 20 StaPTh, 1\% chord, upper Pressure 121 StaPT4, $0.5 \%$ chord, upper Pressure "22 5taPT4, ox chord, leading Pressure 23 StaPT4, 0.5\% chord, Lover Pressure "25 StaPT4, $2 x$ chord, lower Pressure "26 StaPTh, 4X chord, lower Pressure "27 StaPT4, 6x chord, lower Pressure 28 StaPT4, 8x chord, Lover Pressure "30 StaPT4, 14x chord, tower Pressure \$32 staPT4, 28x chord, Lower Pressure 134 StaPT4, 44X chord, Lower Pressure $\$ 36$ stapt4, 68X chord, lower Pressure $\$ 38$ stapT4. $92 \%$ chord, Lower Pressure 18 stapT3, 4\% chord, upper Total Pressure Probe, 50.6\% span 5 in wo

5 in US

10 N WD

10 i us

$20 \mathrm{ND}$

20 in us

50 W

$50 \mathrm{n}$ US

5 in Air Temperature

Delta Temperature T5O - TOS

Baro Pressure

Time

Time

Time

Time

Time

Lift CL(80x) Staptio

Lift $\mathrm{Cl}(63 \mathrm{x})$ StapT

Lift CL(47\%) stapT4

Lift CL(30\%) stapT1

Drag Cd(80\%) Staptio

Drag co(63\%) StapT

Drag co(47x) stapt6

Drag' $\operatorname{cd}(30 \%)$ Stapt1

Nortal Force Cn(80x) Stapt10

Normal Force $\operatorname{cn}(63 x)$ stapT

Normal Force Cn(47X) StaPT4

Normal force Cn(30\%) StaPTI

Tangent Force Ct(80z) StaPT10

Tangent Force Ct(63x) StaPT7

Tangent Force $\operatorname{ct}(47 \mathrm{7})$ Stapt4

Tangent force ct(30x) stapt

Hormal force Squared Cn2(80\%) stapt10 Normal force Squared Cn2(637) stapt7 Normal force Squared Cn2(47X) StapT4 Normal force Squared Cn2(30\%) StapTI Pitch Moment CDm(80\%) staptio Pitch Homent Cpa(63\%) StaPT7 Pitch Howent cpm(47\%) StaPT4 [coef] $-2.505 E+00(9.015 E+\infty 0)-7.887 E+\infty 0$ [coef] $-1.048 E+00(3.746 E-01)-4.740 E+00$ [coef] $-1.133 E+00$ (3.167E-01) -7.640E+00 [psi ] 1.376E-02 (3.334E-03) -4.168E-01 [coef] -1.088E-01 (2.854E-01) $-5.548 E+00$ [coef] $-1.647 E-01(2.806 E-01)-7.046 E+00$ [coef] -2.629E-01 (2.732E-01) -1.516E+01 [coef] $-3.673 E-01$ (2.555E-01) $-2.395 E+\infty 0$ [coef] $-5.870 E-01$ (2.285E-01) $-2.198 E+00$ [coef] -8.963E-01 (2.162E-01) -2.285E+00 [coef] -9.981E-01 (2.231E-01) -3.307E+00 [coef] $-1.058 E+00(2.508 E-01)-2.465 E+00$ [coef] -1.206E+00 (2.953E-01) -2.971E+00 [coef] $-1.297 E+00$ (3.486E-01) $-8.644 E+00$ [coef] $-1.390 E+00(4.140 E-01)-1.569 E+01$ [coef] $-1.666 E+00(4.690 E-01)-6.115 E+00$ [coef] $-1.549 E+00(5.367 E-01)-8.067 E+00$ [coef] -1.726E+00 (7.015E-01) -8.121E+00 [coef] $-1.943 E+00(9.364 E-01)-5.864 E+\infty 0$ [coef] $-2.068 E+00(1.146 E+00)-7.983 E+00$

[coef] -2.177E+00 (1.401E+00) -1.005E+01 [coef] $-2.151 E+00(1.736 E+00)-1.217 E+01$ [coef] 5.742E-01 (3.904E-01) -6.153E+00 [coef] $9.127 \mathrm{E}-01$ (1.562E-01) $-8.264 \mathrm{E}+00$ [coef] 8.660E-01 (1.848E-01) -7.830E+00 [coef] 7.743E-01 (2.039E-01) -7.399E+00 [coef] 6.745E-01 (2.098E-01) -8.517E+00 [coef] 4.091E-01 (2.202E-01) -8.865E+00 [coef] -1.570E-01 (2.161E-01) -8.216E+00 [coef] -3.848E-01 (1.597E-01) -1.451E+01 [coef] 1.314E-01 (1.029E-01) $-7.479 E+\infty 0$ [coef] 1.583E-01 (1.449E-01) -8.182E+00 [coef] $-1.914 E+00(6.568 E-01)-6.301 E+00$ tpsi ] 3.028E-02 (4.387E-03) -3.929E-01 [Deg] 3.300E+02 (4.855E+00) 3.211E+02 $[\mathrm{E} / \mathrm{s}] \mathrm{s} .393 E+00(1.291 E+\infty 0) \quad 2.309 E+\infty 0$ [Deg j $3.597 E+02$ (5.856E-02) 3.583E+02 $[\mathrm{d} / \mathrm{s}] \quad 9.317 \mathrm{E}-02(1.165 \mathrm{E}+00)-5.833 \mathrm{E}-02$ [Deg ] 2.543E+02 (7.879E+00) 2.189E+02 $[\mathrm{W} / \mathrm{s}] \quad 6.583 \mathrm{E}+00(1.435 \mathrm{E}+00) \quad 3.069 \mathrm{E}+00$ [Deg ] $2.552 E+02(8.790 E+00) 2.342 E+02$ $[\mathrm{W} / \mathrm{s}] 7.347 \mathrm{E}+00(1.440 \mathrm{E}+00) \quad 4.556 \mathrm{E}+00$ [Deg ] 1.419E+01 (6.019E-01) 1.382E+01 [Deg ] -1.225E+00 (5.321E-02) -3.324E+00 [mb ] 8.052E+02 (1.269E-01) 8.050E+02 [Day ] 1.150E+02 (0.000E+00) 1.150E+02 [Hour] $9.000 E+\infty 0(0.000 E+\infty) \quad 9.000 E+\infty 0$ [Minu] 3.325E+01 $(1.676 E+\infty) 3.100 E+01$ [Seco] 2.953E+01 (1.732E+01) 0.000E+00 [Mill] 4.999E+02 (2.885E+02) 2.400E-01 [coef] 4.119E-01 (2.376E-01) -3.631E+00 [coef] 6.368E-01 (2.563E-01) -3.171E+00 [coef] 8.318E-01 (2.316E-01) $-5.466 E+00$ [coef] 1.293E+00 (3.904E-01) $-9.978 E+00$ [coef] $1.172 E-02$ (8.986E-03) -5.696E-01 [coef] 1.659E-02 (2.976E-02) -8.402E-02 [coef] $8.752 E-02$ (1.219E-01) -1.272E+00 [coef] 3.999E-01 (3.491E-01) -3.223E+C0 [coef] 4.111E-01 (2.363E-01) -3.636E+00 [coef] $6.318 E-01$ (2.537E-01) $-3.763 E+\infty 0$ [coef] $8.373 E-01(2.369 E-01)-5.574 E+00$ [coef] $1.370 E+00$ (4.665E-01) -1.004E+01 [coef] 2.122E-02 (3.337E-02) -3.633E-01 [coef] $6.111 E-02$ (5.112E-02) -3.689E-01 [coef] 8.109E-02 (6.545E-02) $-4.754 E-01$ [coef] $7.897 E-02$ (7.489E-02) -2.116E-01 [coef] 2.248E-01 (2.132E-01) 2.351E-11 [coef] 4.635E-01 (3.137E-01) 1.349E-10 [coef] 7.572E-01 (4.231E-01) 2.945E-08 [coef] 2.094E+00 (1.462E+00) 1.754E-0? [coef] -2.929E-02 (1.068E-02) -9.984E-01 [coef] -3.619E-02 (1.320E-02) -5.760E-01 [coef] $-4.942 E-02(4.210 E-02)-1.876 E+00$
2.205E+01 $1.709 E+01$ $1.046 E-01$ $2.399 E-01$ $3.233 E+\infty 0$ 1.061E+01 $1.038 E+01$ $1.045 E+01$ $4.573 E+00$ $4.302 E+00$ $7.763 E+00$ $1.675 E+01$ $1.214 E+01$ $9.579 E+00$ $8.362 E+00$ $8.536 E+00$ 2.734E-01 $9.066 \mathrm{E}+00$ 1.217E+01 $9.205 E+00$ 1.367E+01 1.060E+01 $1.090 E+01$ $7.887 E+00$ $9.136 E+00$ $1.137 E+01$ $9.122 E+00$ 1.064E+01 $9.801 E+00$ $1.015 E+01$ $1.106 E+01$ $9.647 E+00$ $1.503 E+01$ 2.423E-01

$3.435 E+02$ $9.109 E+00$ $3.597 E+02$ 5.314E+01 $2.763 \mathrm{E}+02$ $1.777 \mathrm{E}+01$ $3.423 E+02$ 1. 100E+01 $4.083 E+01$ $-1.171 E+00$ 8.054E+02 $1.150 E+02$ $9.000 E+00$ $3.600 E+01$ 5.900E+01 9.999E+02 $4.278 E+00$ $1.498 E+00$ $7.495 E+00$ 1.384E+01 3.744E-01 $2.060 E+00$ $1.363 E+00$ $1.906 E+00$ $4.293 E+00$ $1.500 E+00$ $7.617 \varepsilon+\infty 0$

$1.369 E+01$ 5.420E-01

2.746E-01 6.552E-01 $3.821 E+00$ $1.843 E+01$ $1.416 E+01$ 5.801E+01 1.874E+02 $1.089 E+00$ $2.180 E+00$ $2.189 E+00$ 
219 729: Pitch Homent Cpa(30\%) StaPT1

220 730: Induced Total Pressure(86\%)

221 731: Induced Total Pressure(67x)

222 732: Induced Total Pressure(51x)

223 733: Induced Total Pressure(36\%)

- 224 734: Disc Average wind Speed

225 735: Richardson from North Met

226 736: Aoot-20x Force

227 737: 20x-40x force

228 738: 40x-50x force

229 739: $50 x-60 \%$ force

230 740: $60 \%-70 \%$ Force

231 741: 70\%-75x force

232 742: 75x-80x Force

233 743: 80x-85x force

234 746: 85\%-90\% force

235 745: 90x-100x Force

236 746: 80x Pressure force [coef] - $-1.318 E-01 \quad(9.629 E-02)-5.337 E+\infty 0$ 1.110E-01

[psi ] 8.005E-02 (1.909E-03) 7.723E-02 8.683E-02

[psi ] 5.034E-02 (1.909E-03) 4.753E-02 $5.713 E-02$

[Dsi ] 3.033E-02 (1.909E-03) 2.751E-02 3.712E-02

[psi ] 1.600E-02 (1.909E-03) 1.318E-02 2.279E-02

$[\mathrm{m} / \mathrm{s}] \quad 7.356 \mathrm{E}+00(1.568 E+00) \quad 4.824 E+001.178 E+01$

[ ] $-4.955 E+00(4.089 E-01)-5.000 E+00-3.620 E-04$

[NT/M] 1.908E+00 (3.069E+03) -1.713E+06 1.319E+04

[NT/N] -1.987E+02 (2.958E+03) -2.337E+04 1.691E+04

[NT/M] 3.452E+02 (5.847E+03) $-3.112 E+04 \quad 2.945 E+04$

$[N T / M]-2.681 E+02(4.979 E+03)-1.611 E+04 \quad 2.398 E+04$

[NT/M] -5.230E+01 (2.992E+03) -1.701E+04 9.540E+03

$[N T / M] \quad 4.114 E+02(2.819 E+03)-8.747 E+03 \quad 1.943 E+04$

[HT/M] -6.159E+02 (7.164E+02) -9.693E+03 1.908E+03

[NT/M] 6.159E+02 (7.164E+02) -1.908E+03 9.693E+03

[NT/M] $-4.106 E+02(4.776 E+02)-6.462 E+03 \quad 1.272 E+03$

[NT/M] 5.132E+01 (5.970E+01) -1.590E+02 8.078E+02

[NT/M] 1.081E+02 (6.459E+01) -8.219E+02 2.467E+03

$$
\text { Wind jporser inent }
$$

$$
\begin{aligned}
& \text { Eis } \\
& d \theta 65.2 \div 0 \mathrm{~V} \\
& 6=\% \\
& \text { Nos } 263 \mathrm{~V} \\
& 47 \% \\
& \text { dog52420 } \\
& \because=0 \\
& \text { d } 0=53=0 \%
\end{aligned}
$$


Appendix C.1.2

Pressure Distributions for $80 \%$ Span 


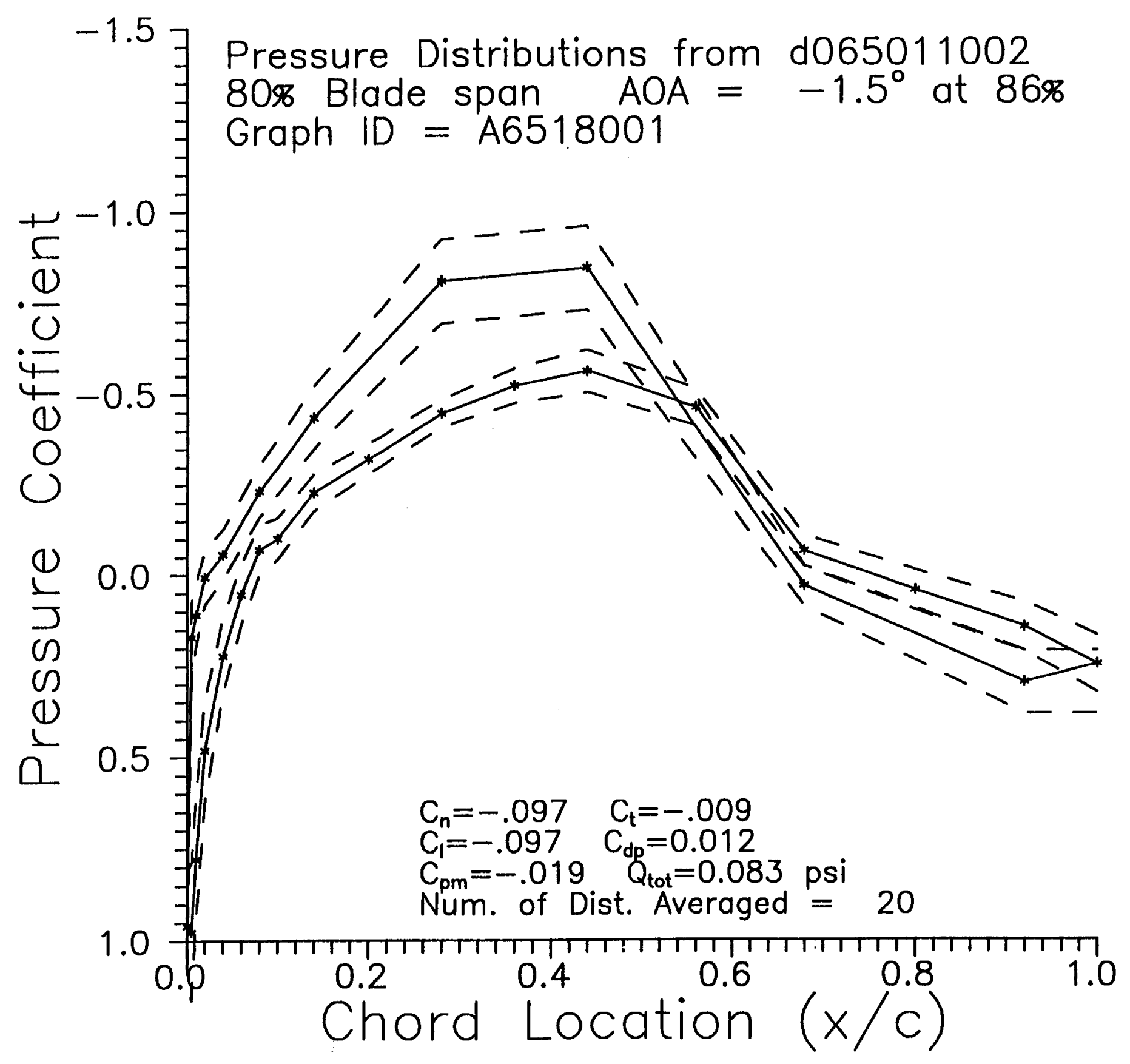




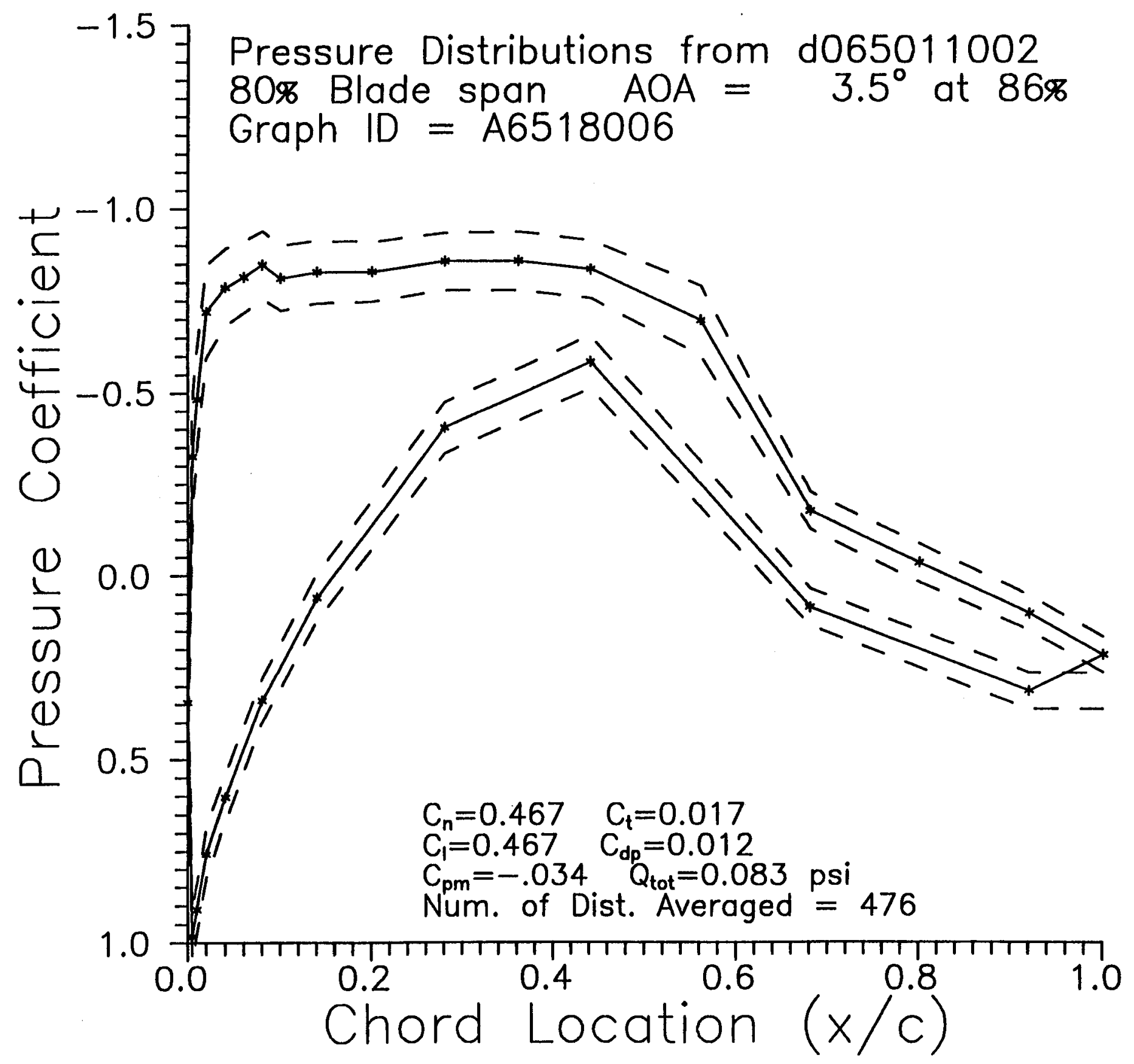




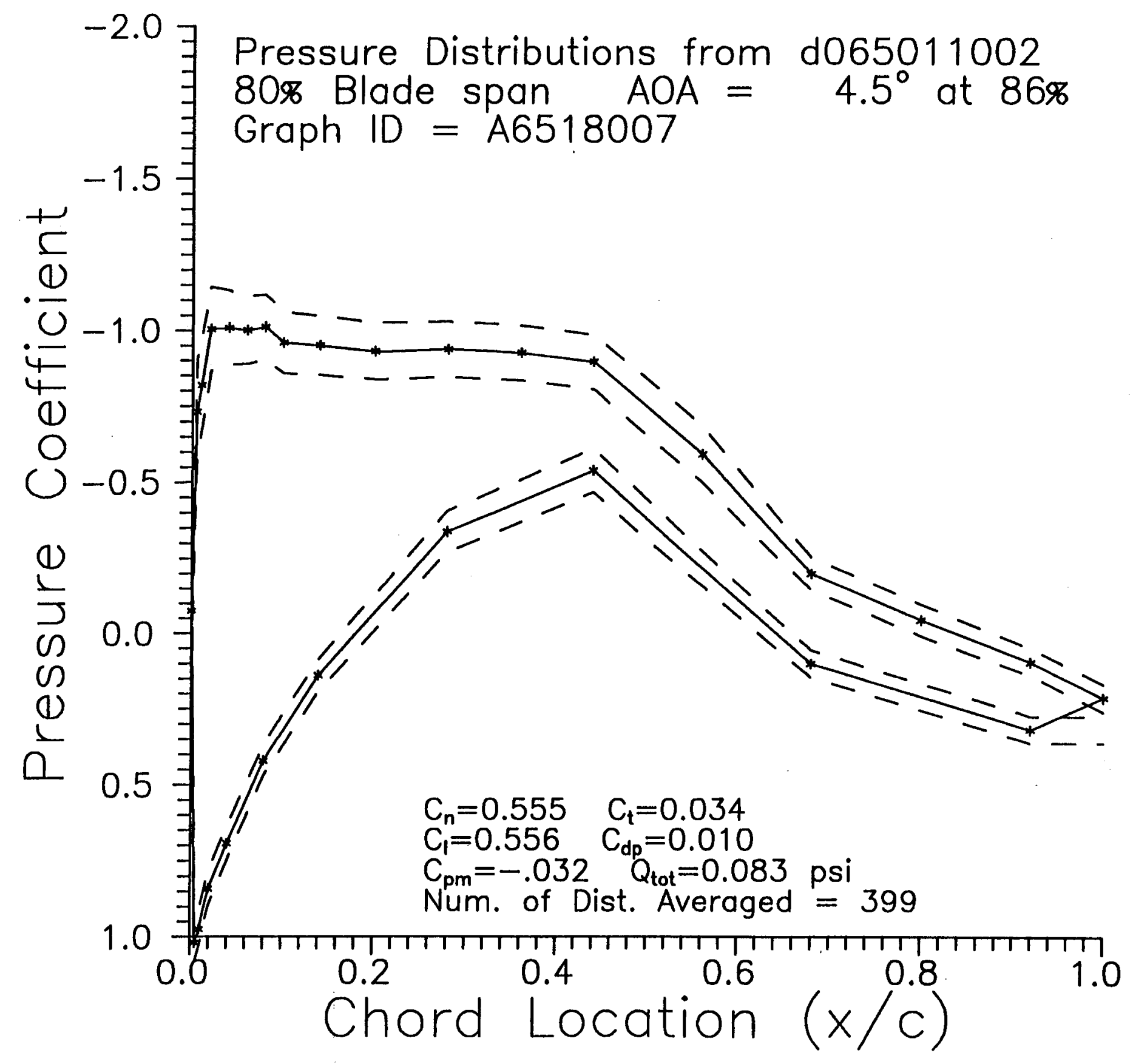




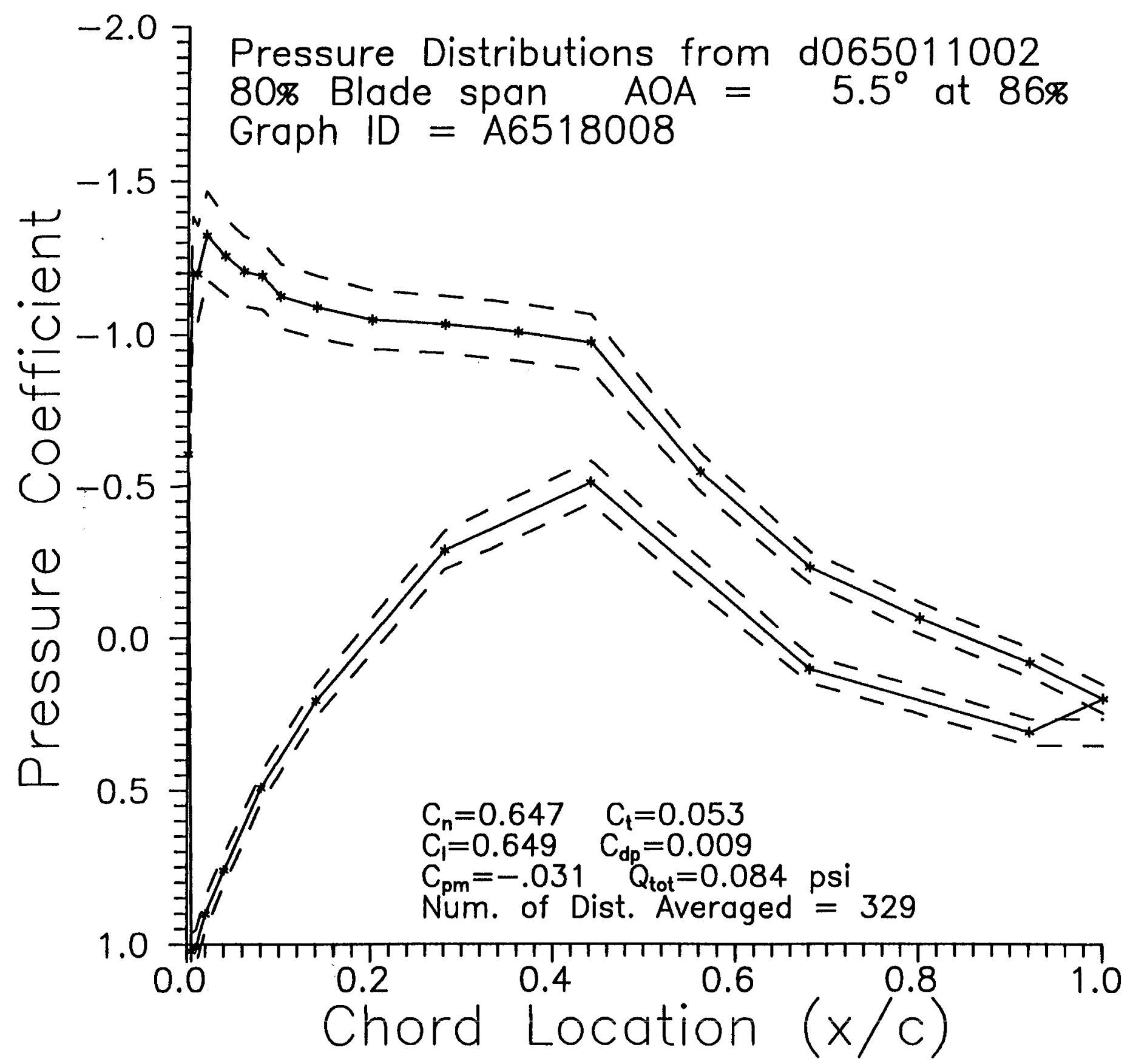




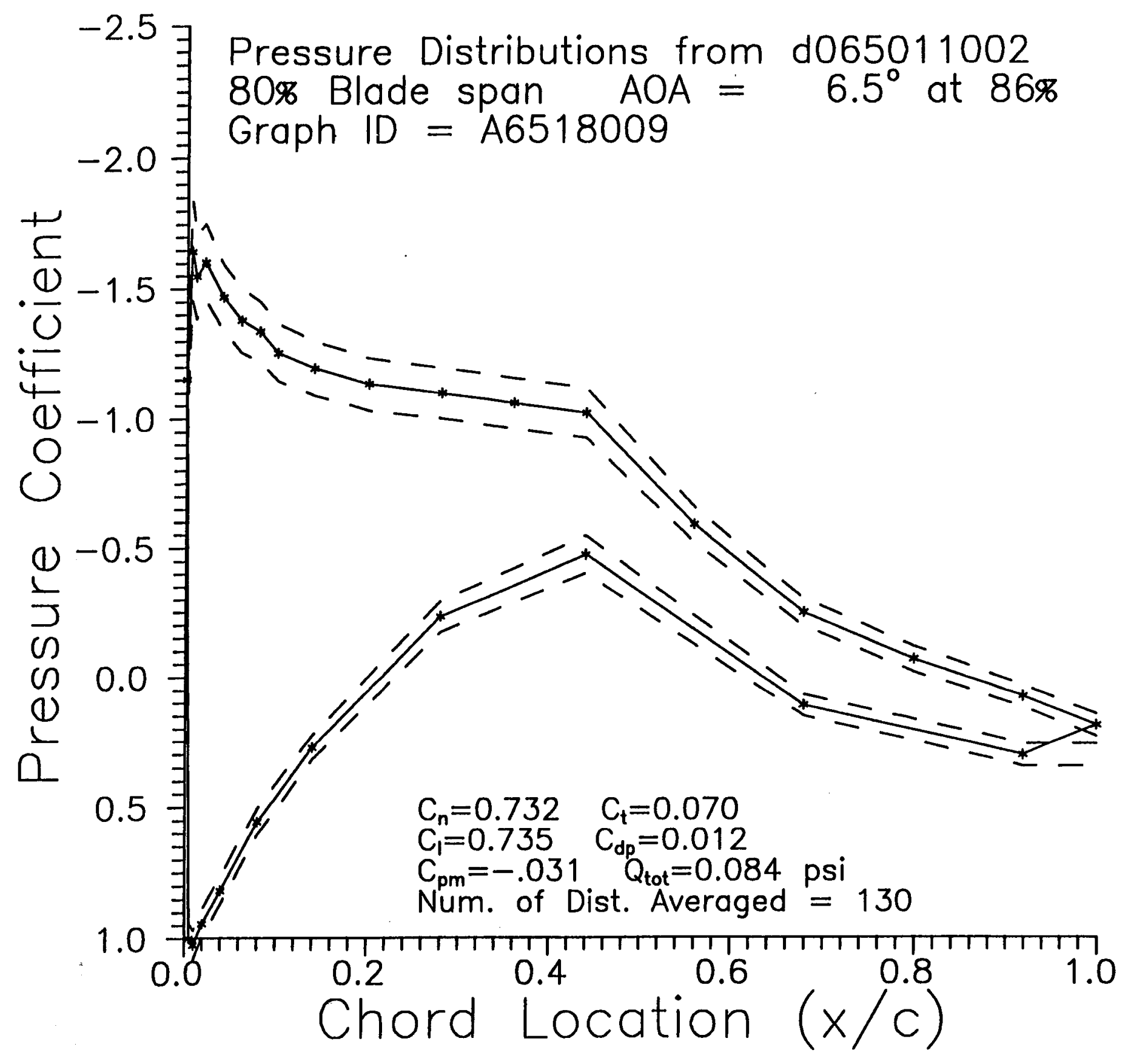




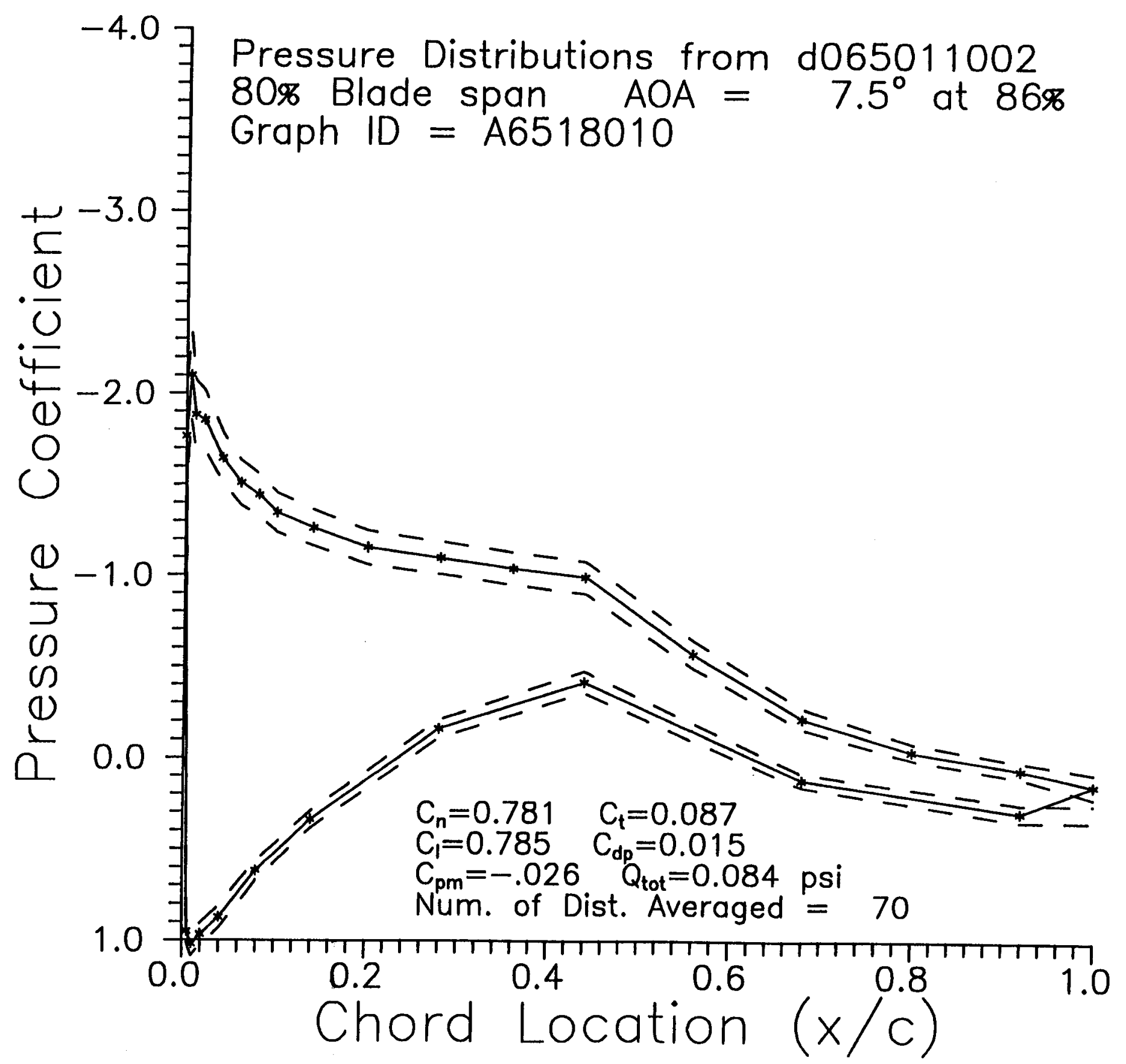




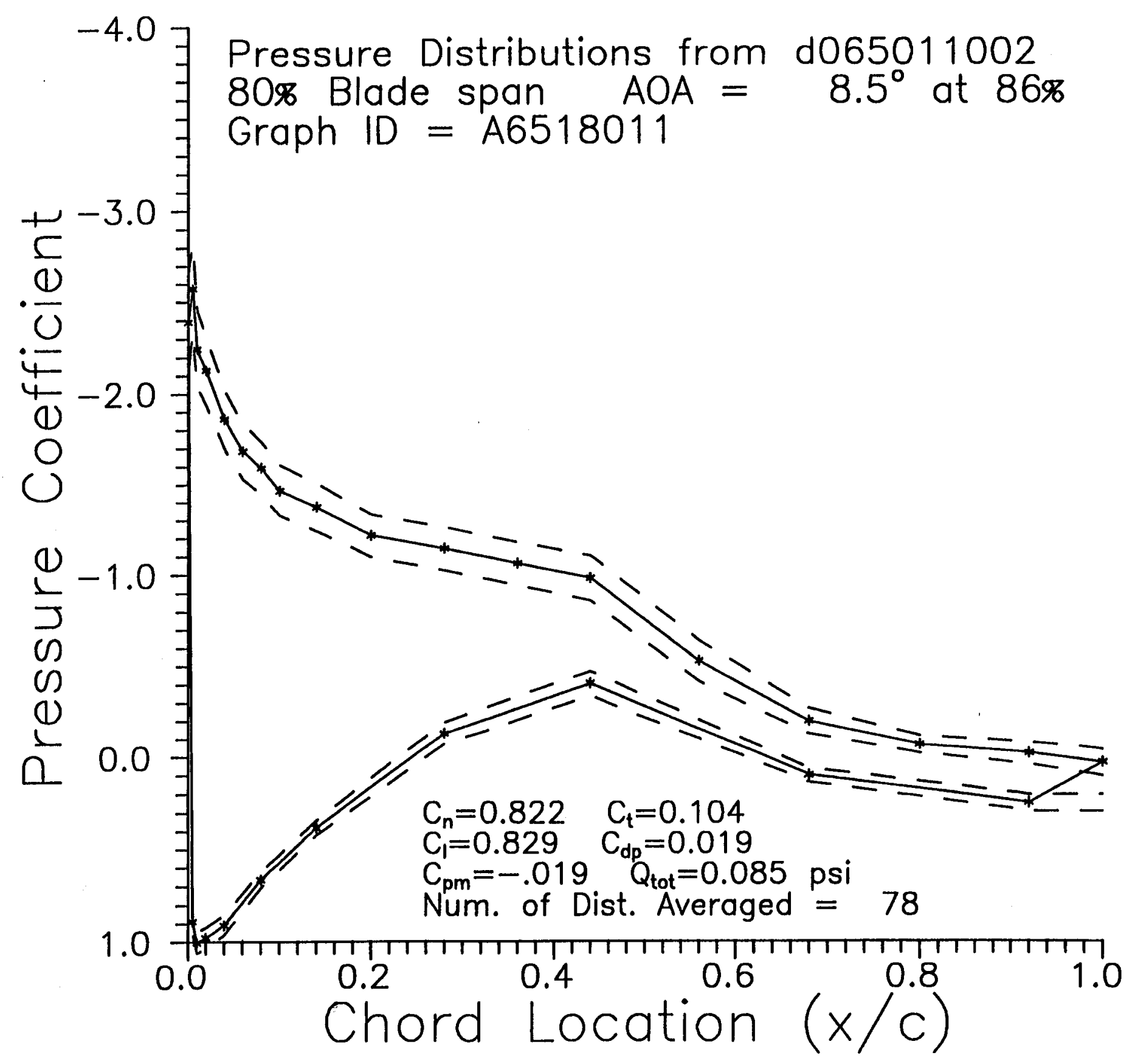




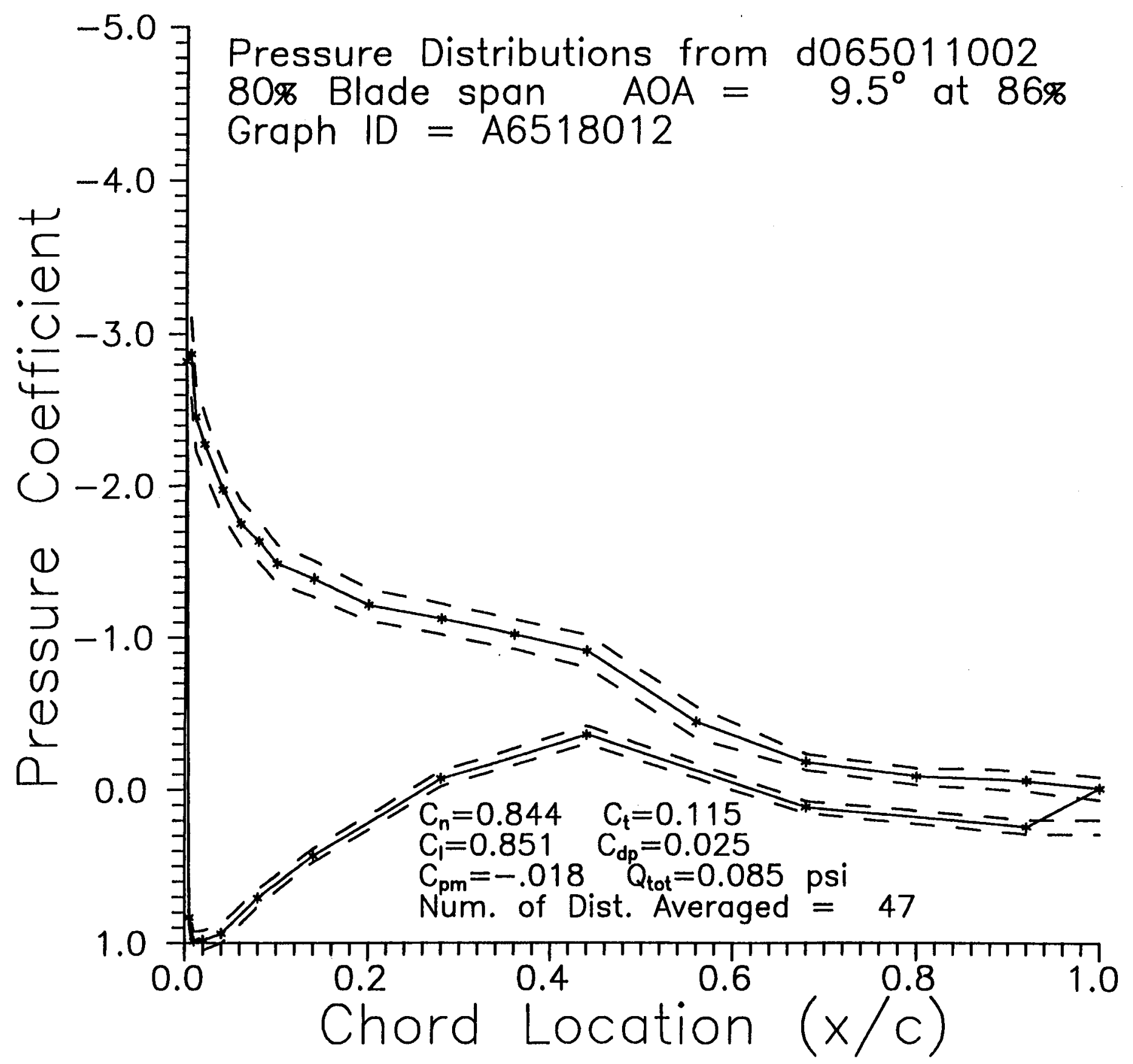




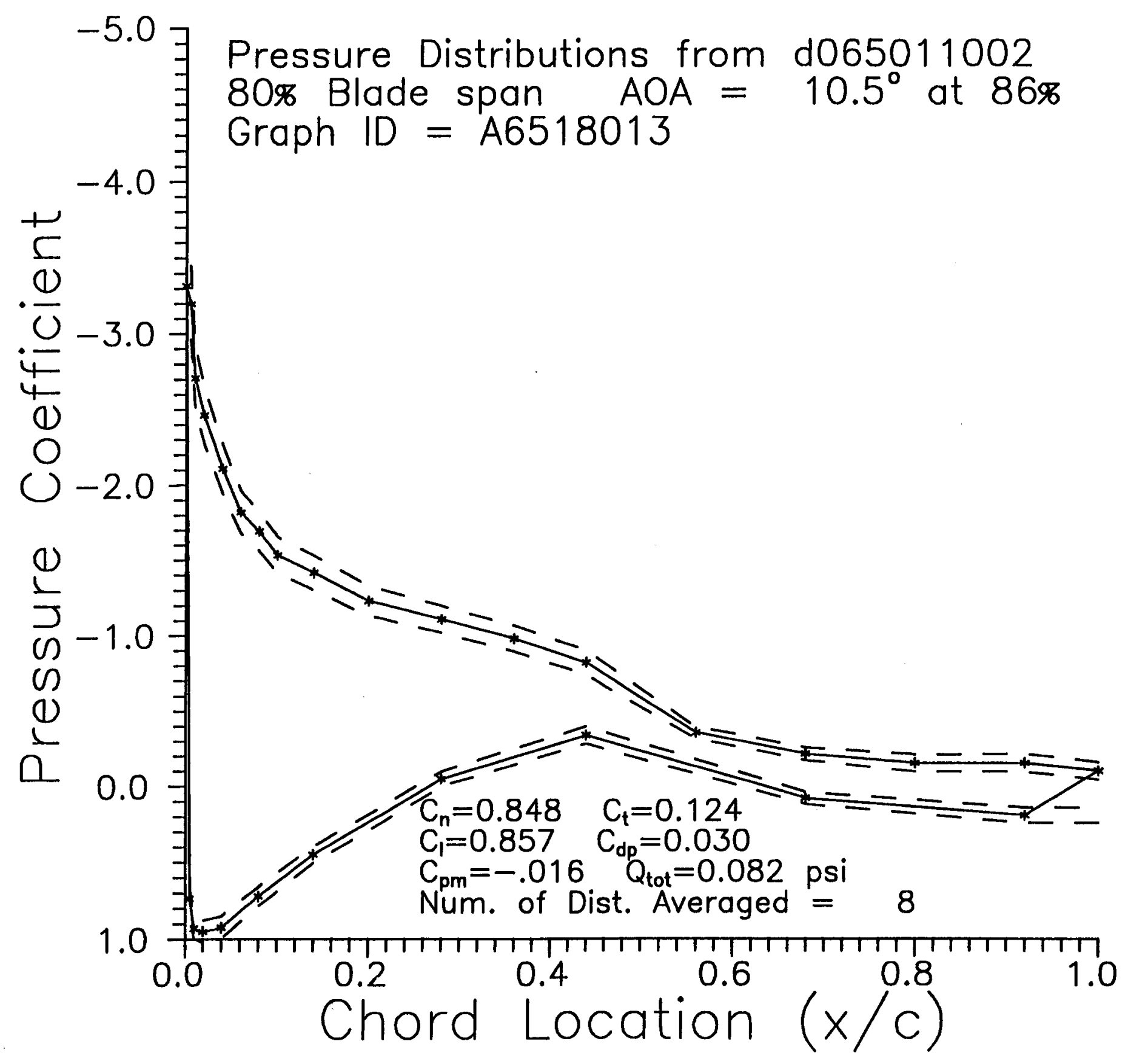




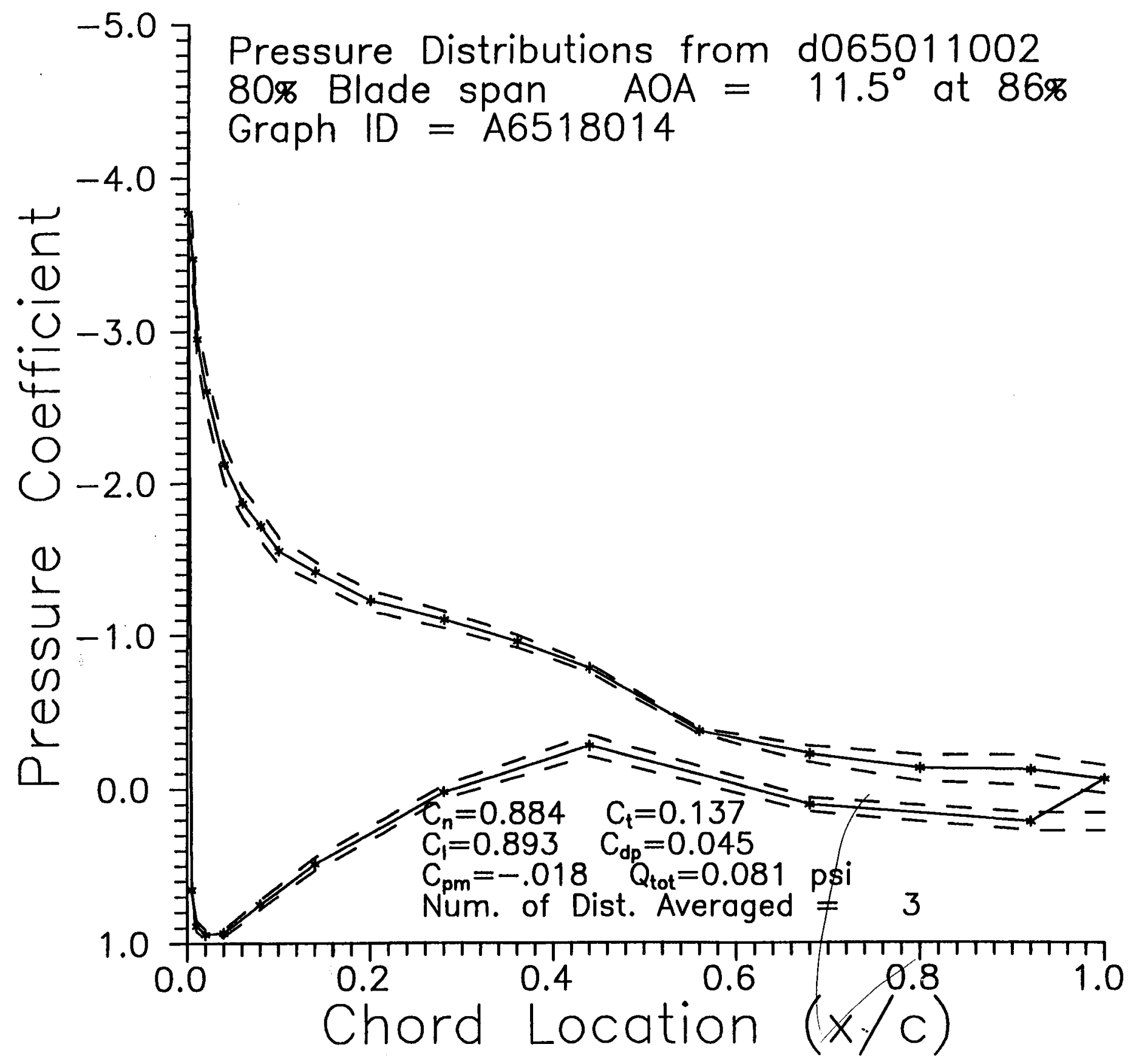


Appendix C.1.3

Pressure Distributions for $63 \%$ Span 


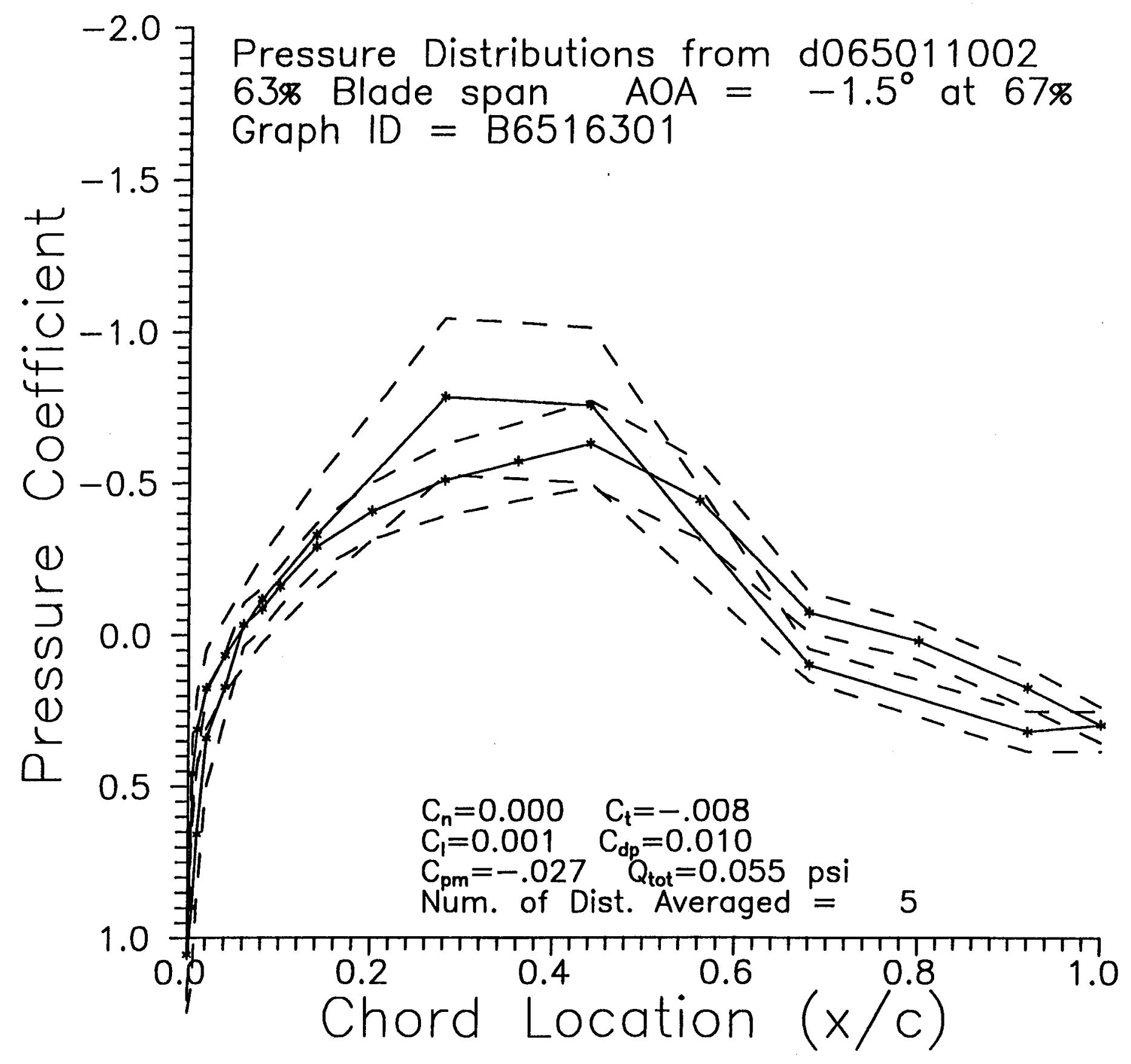




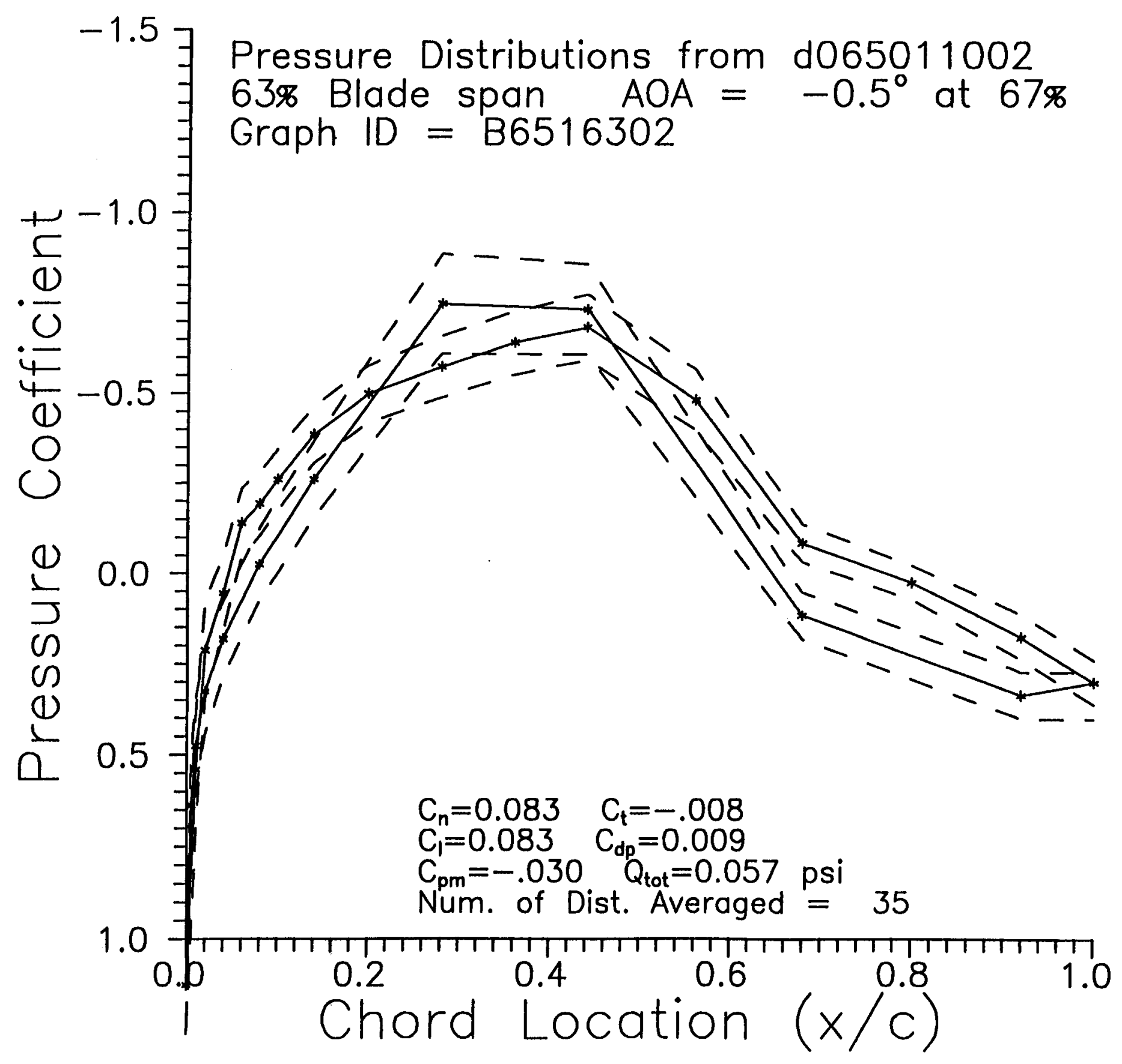




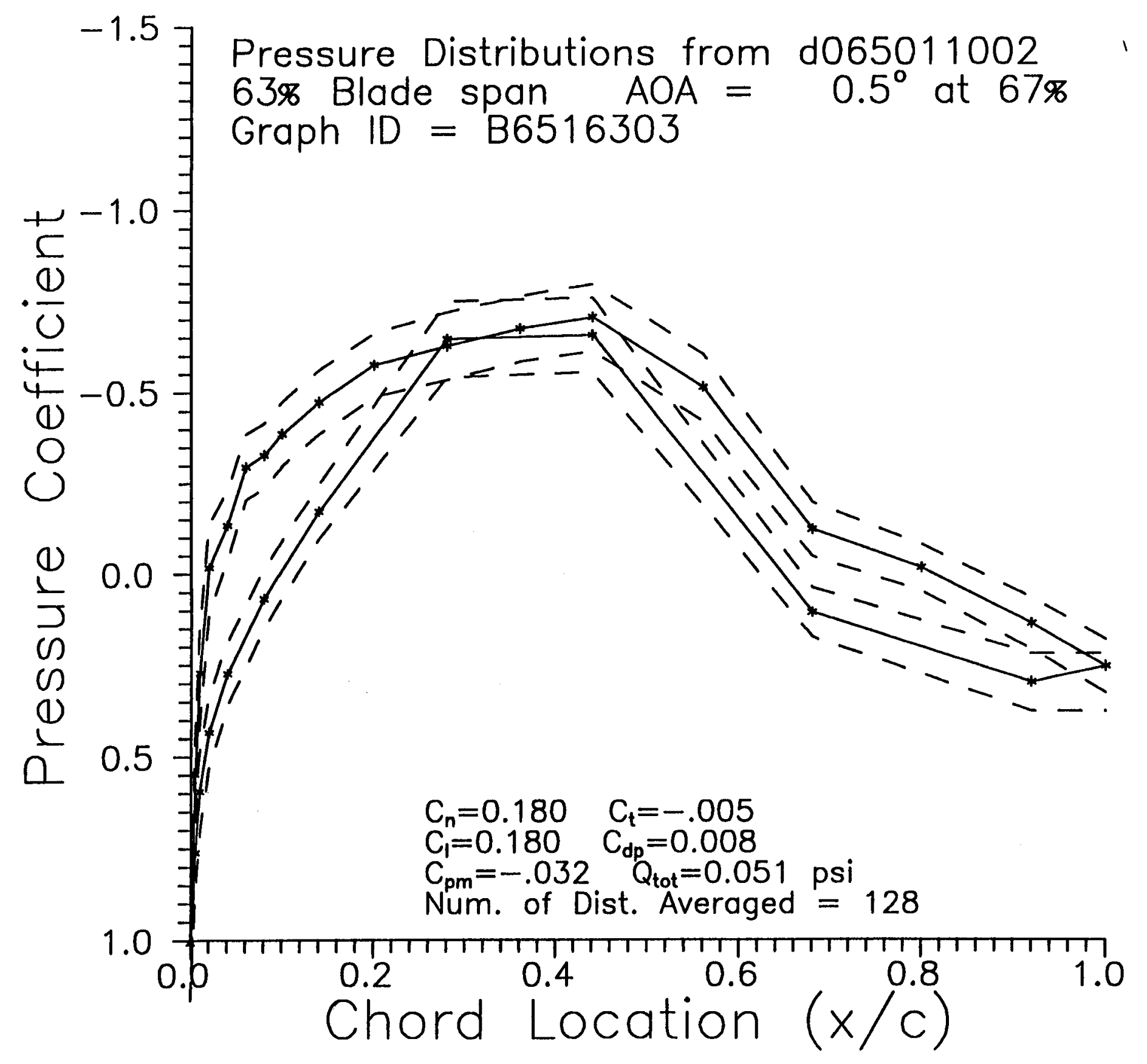




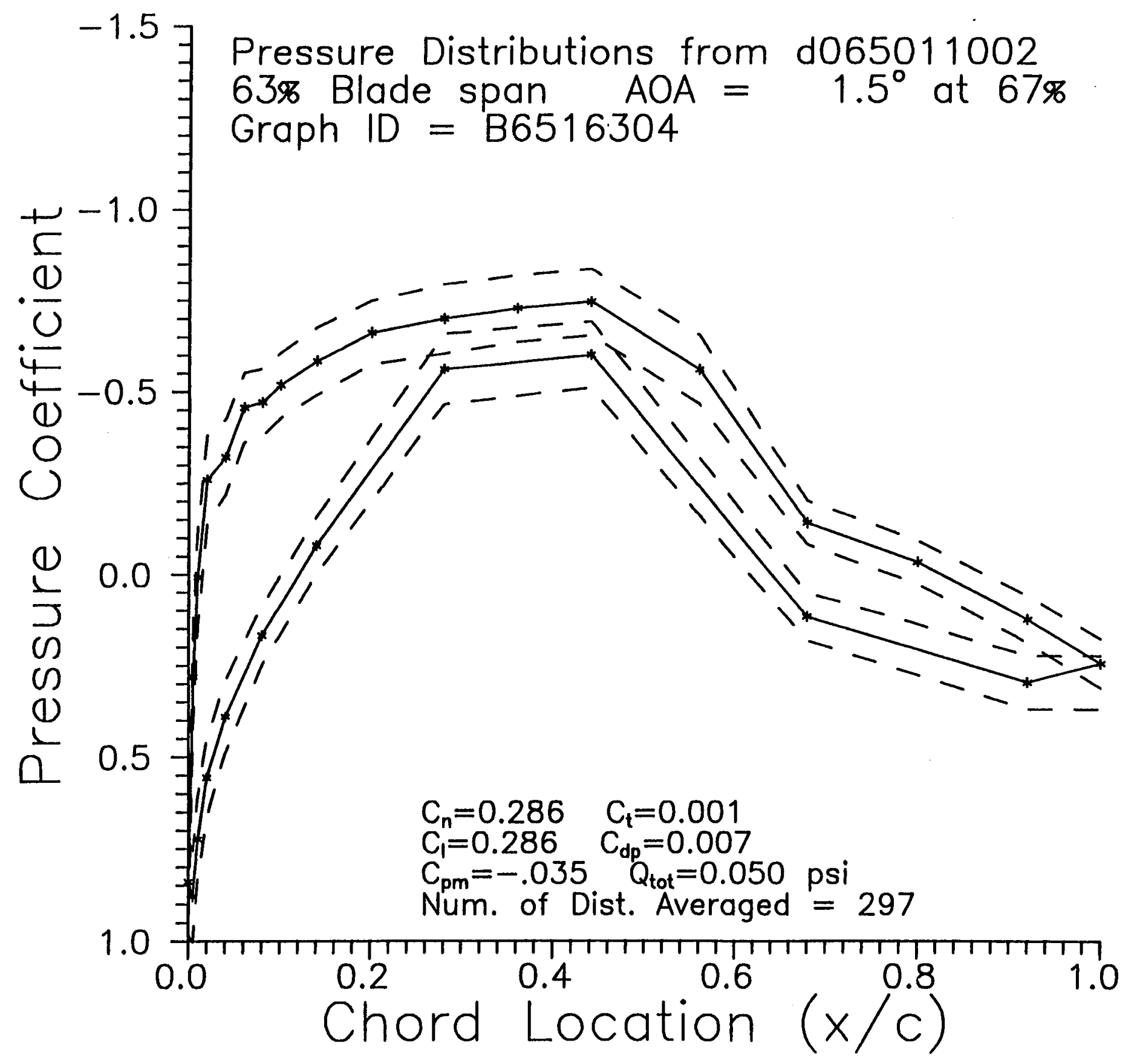




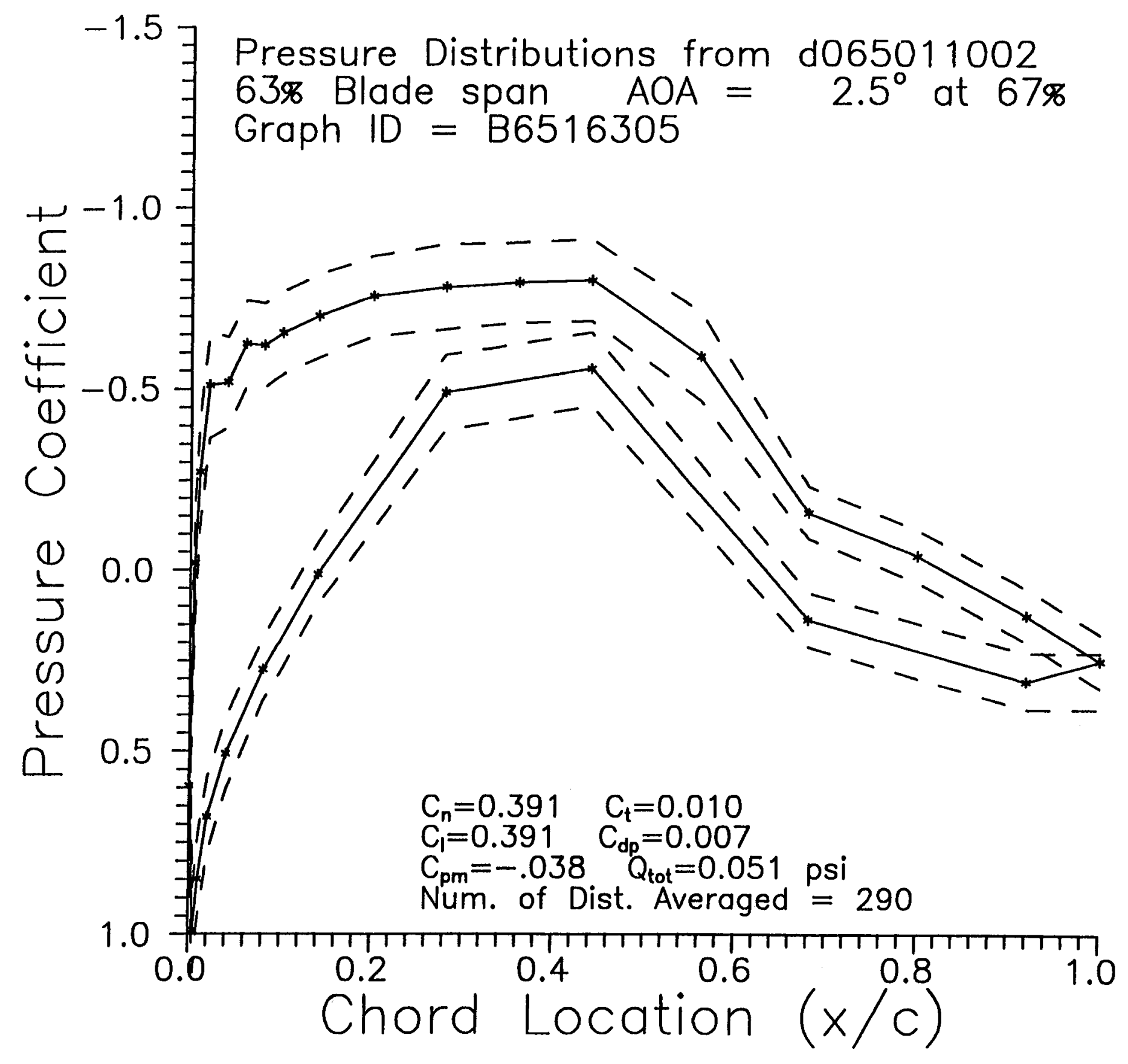




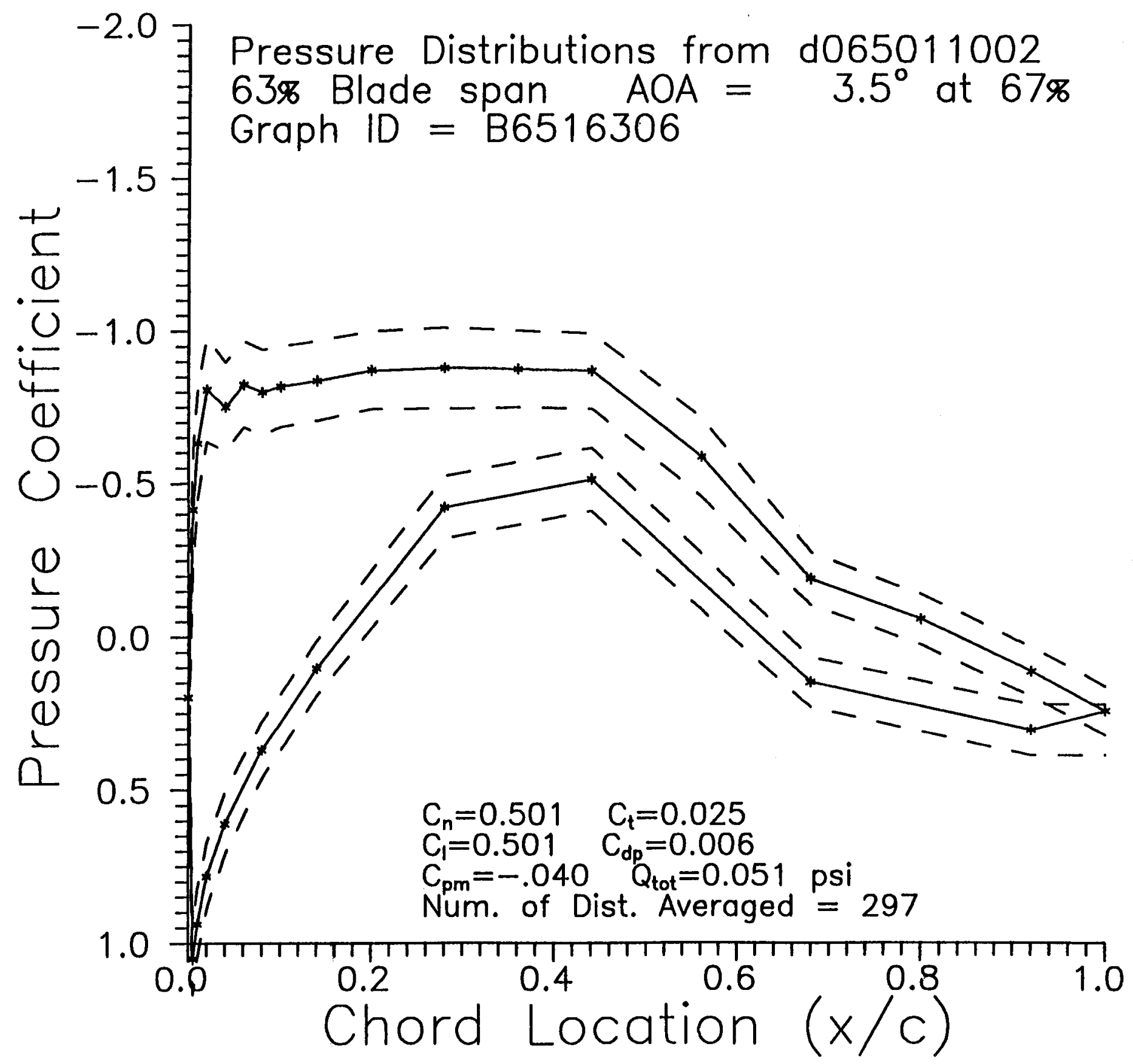




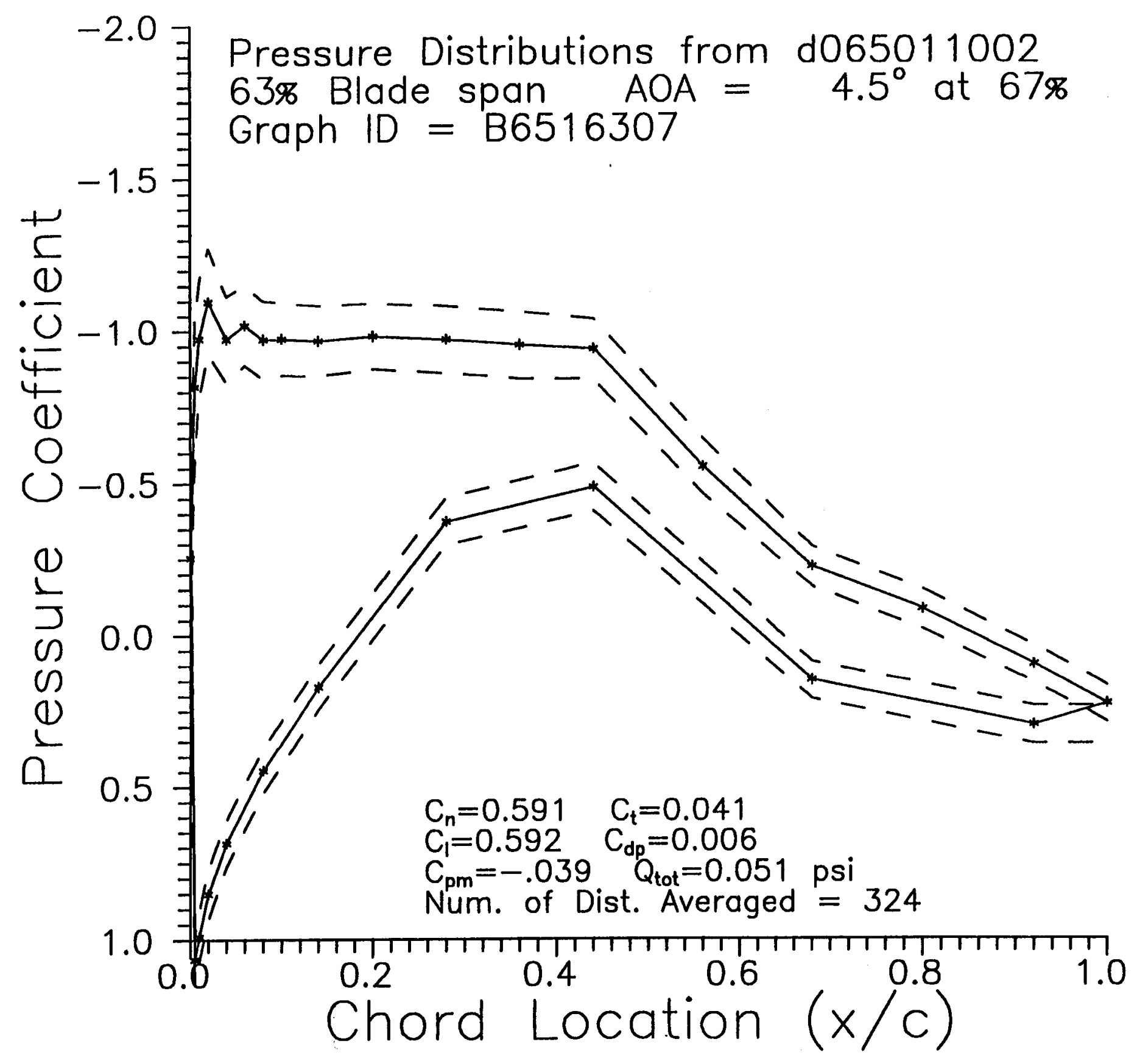




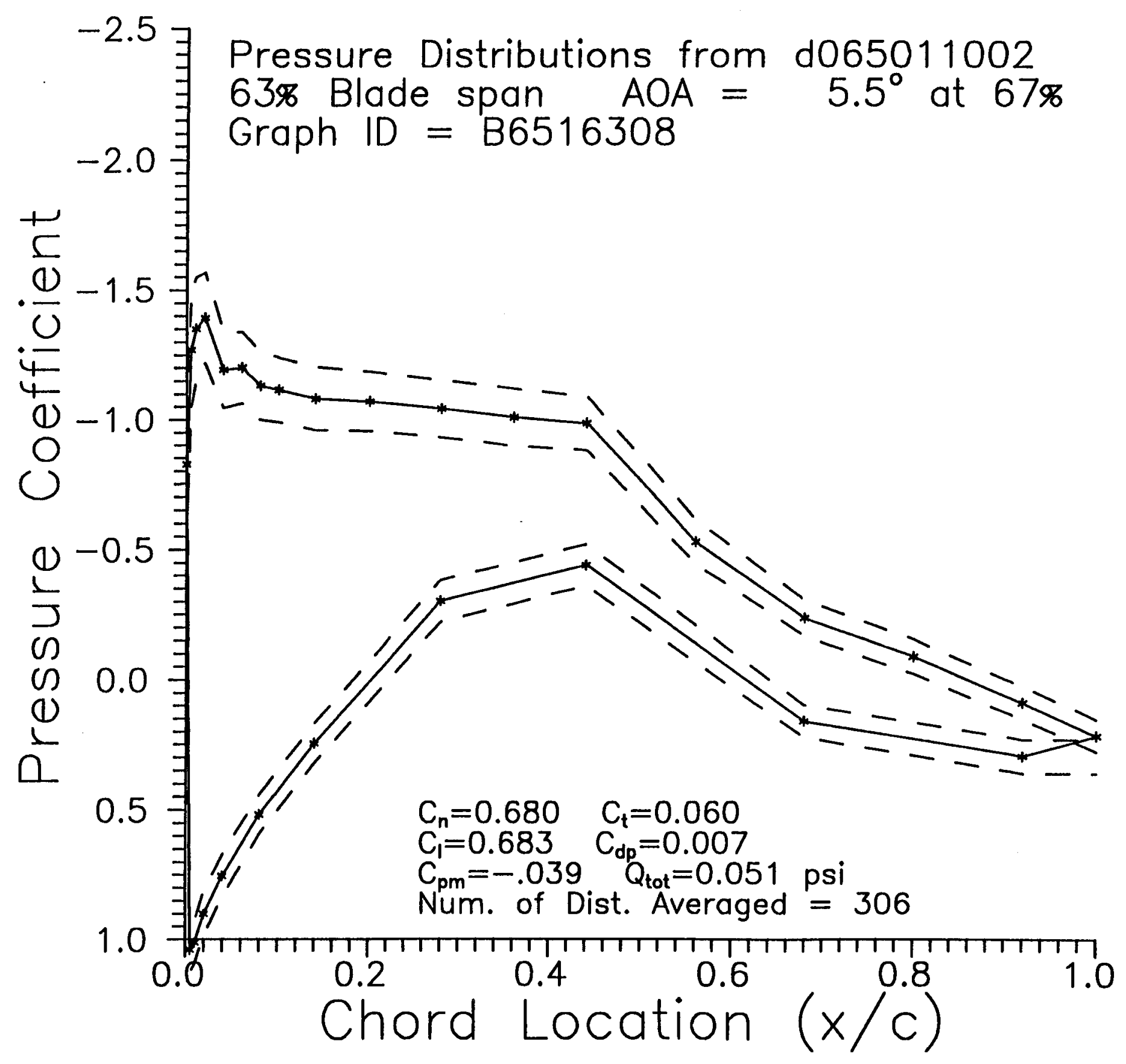




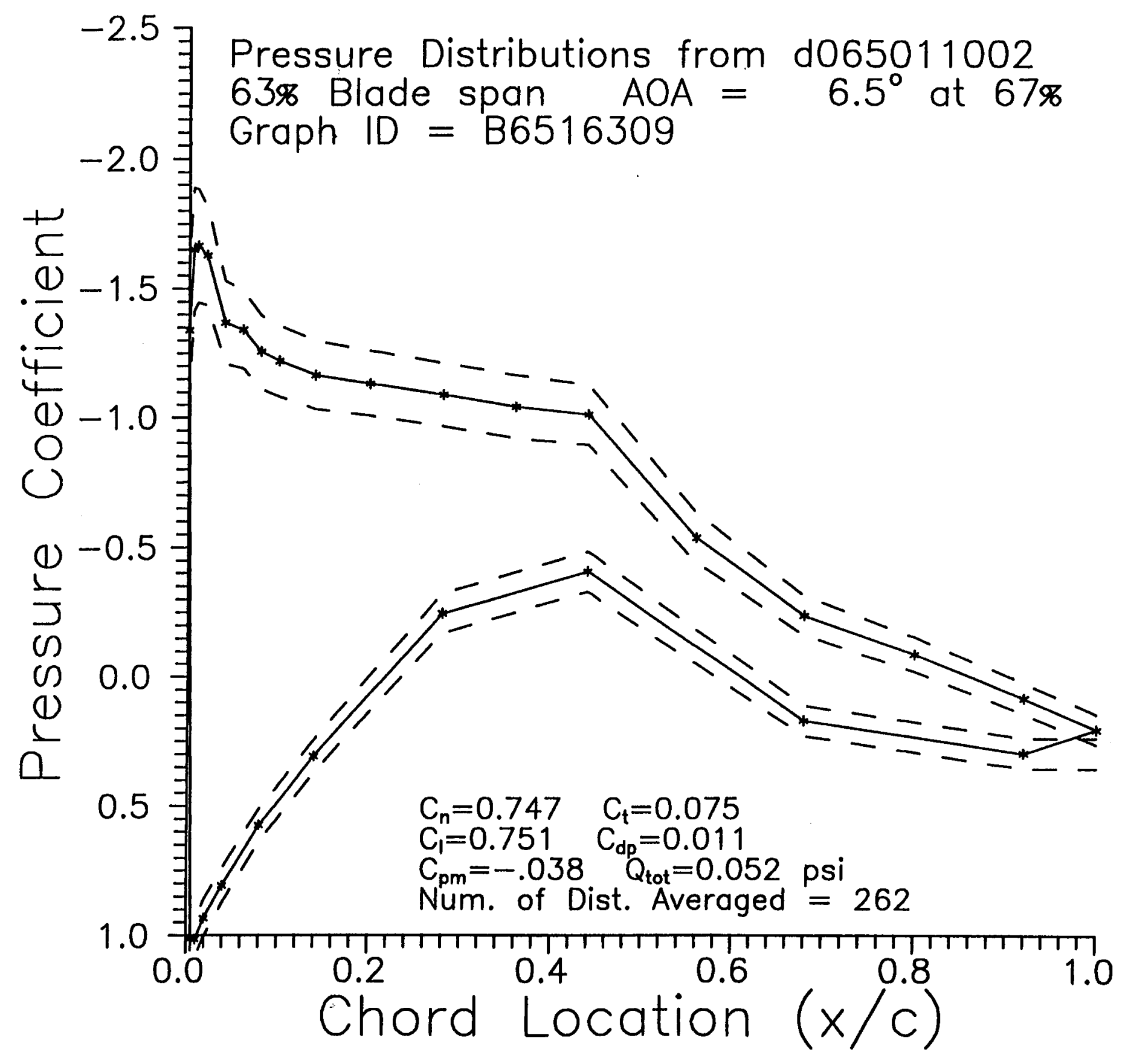




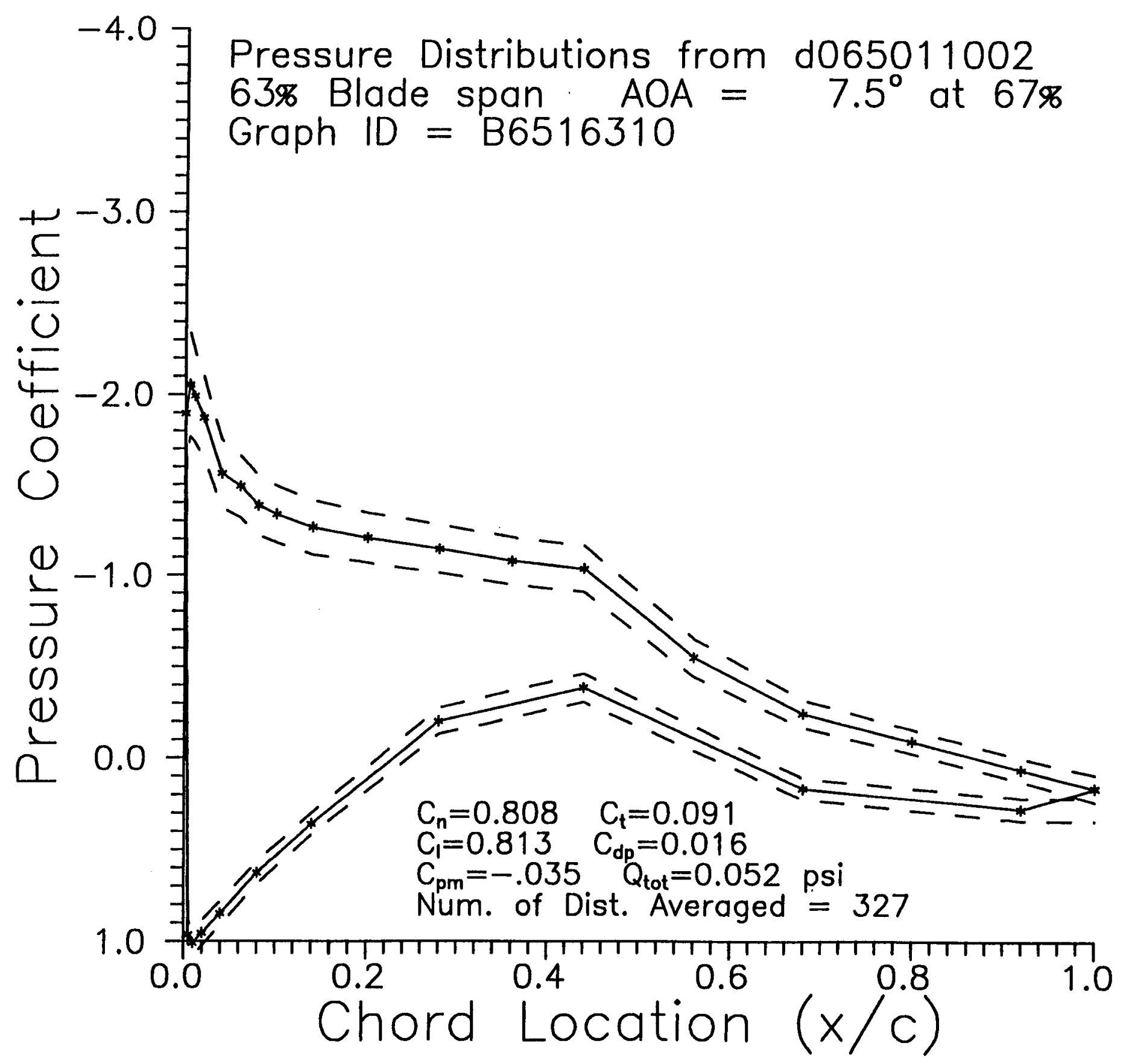




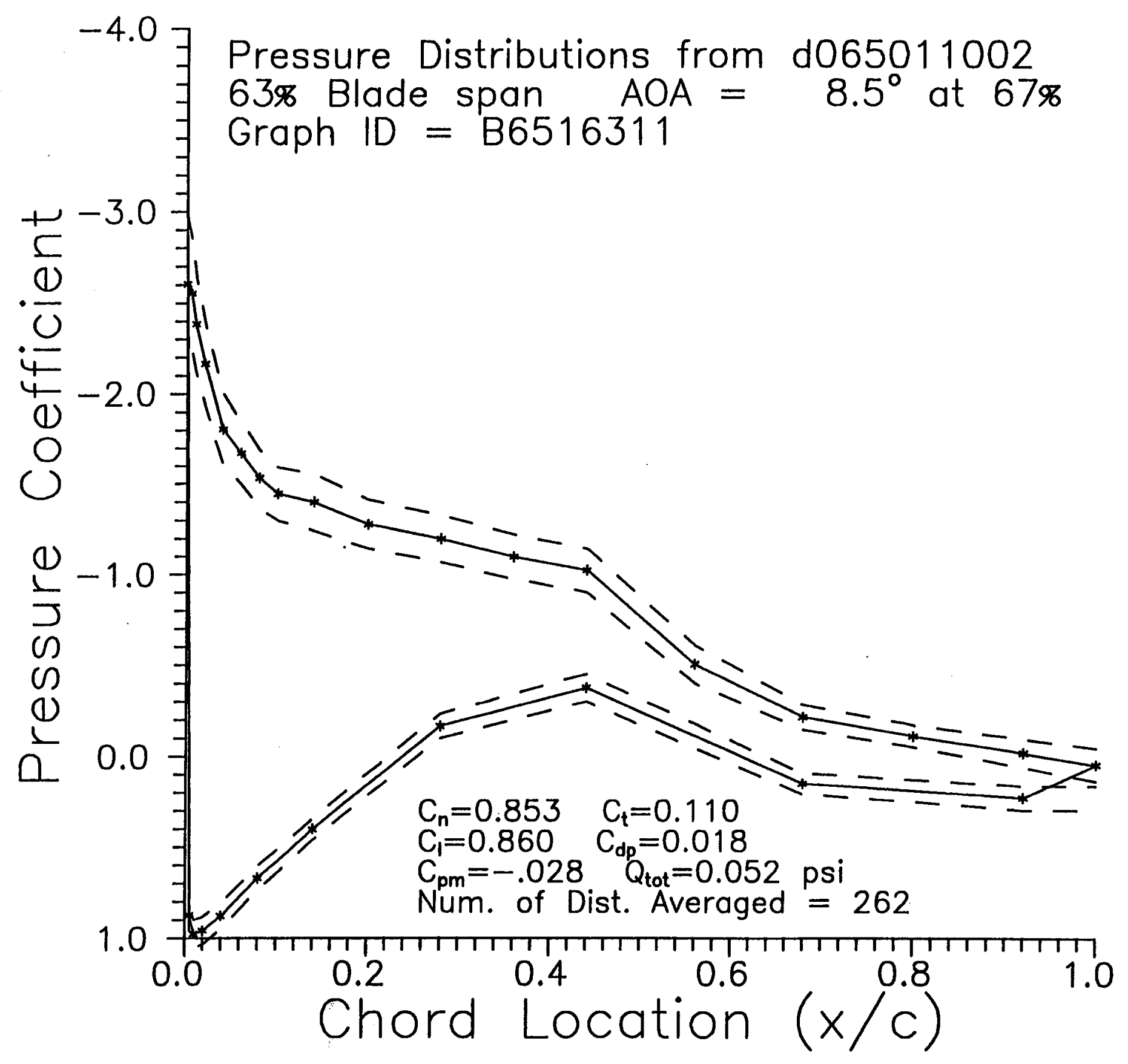




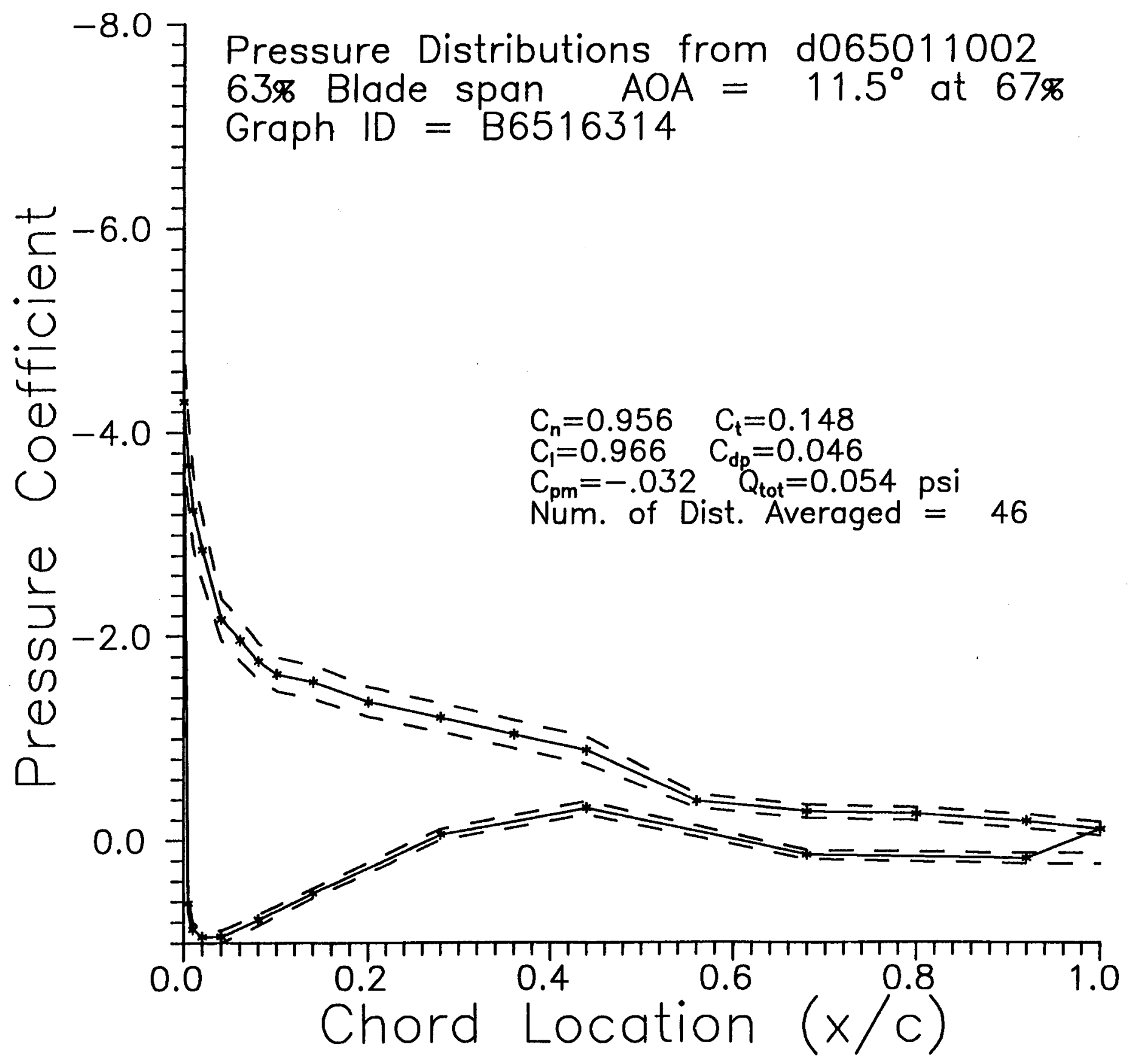




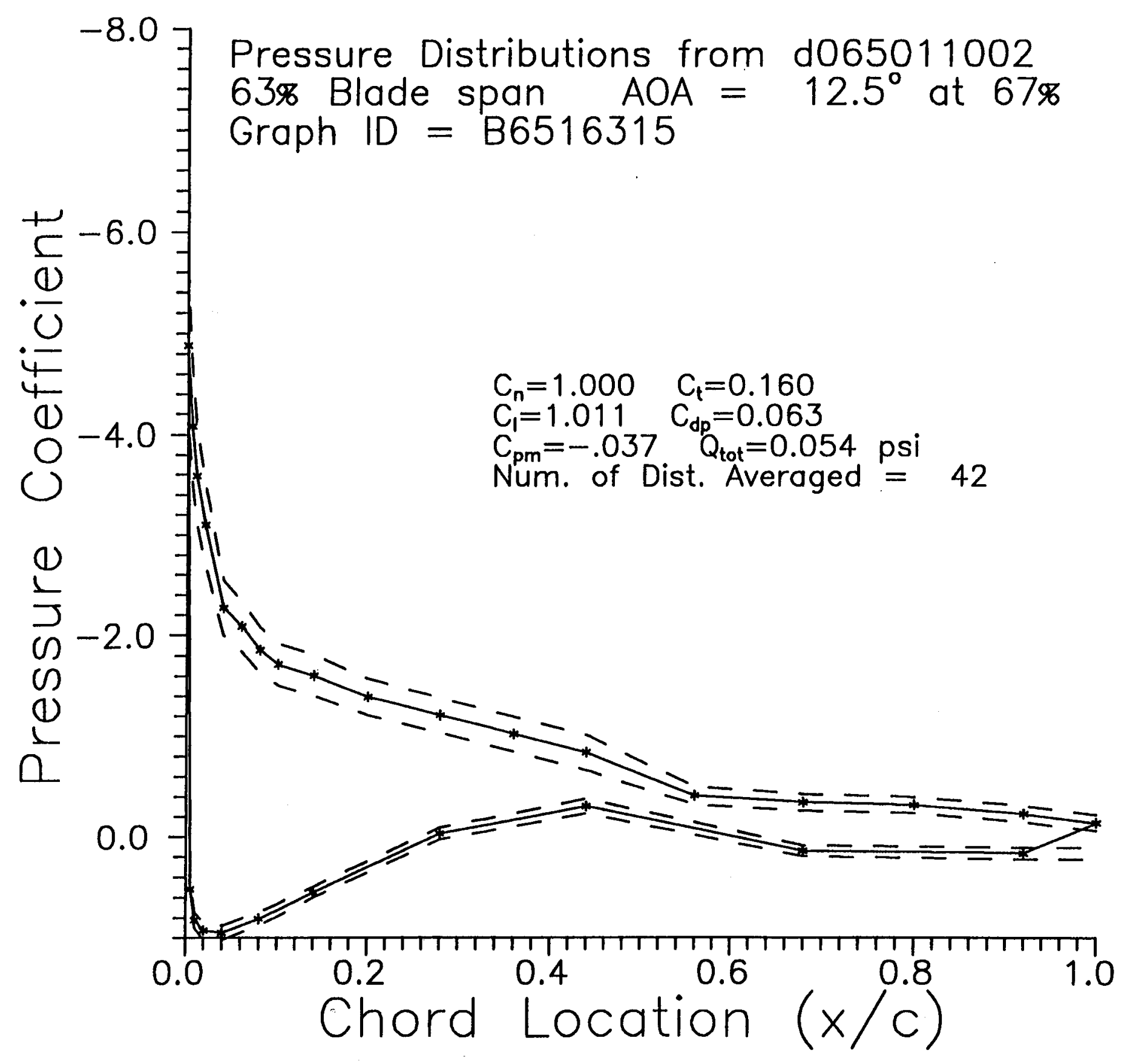




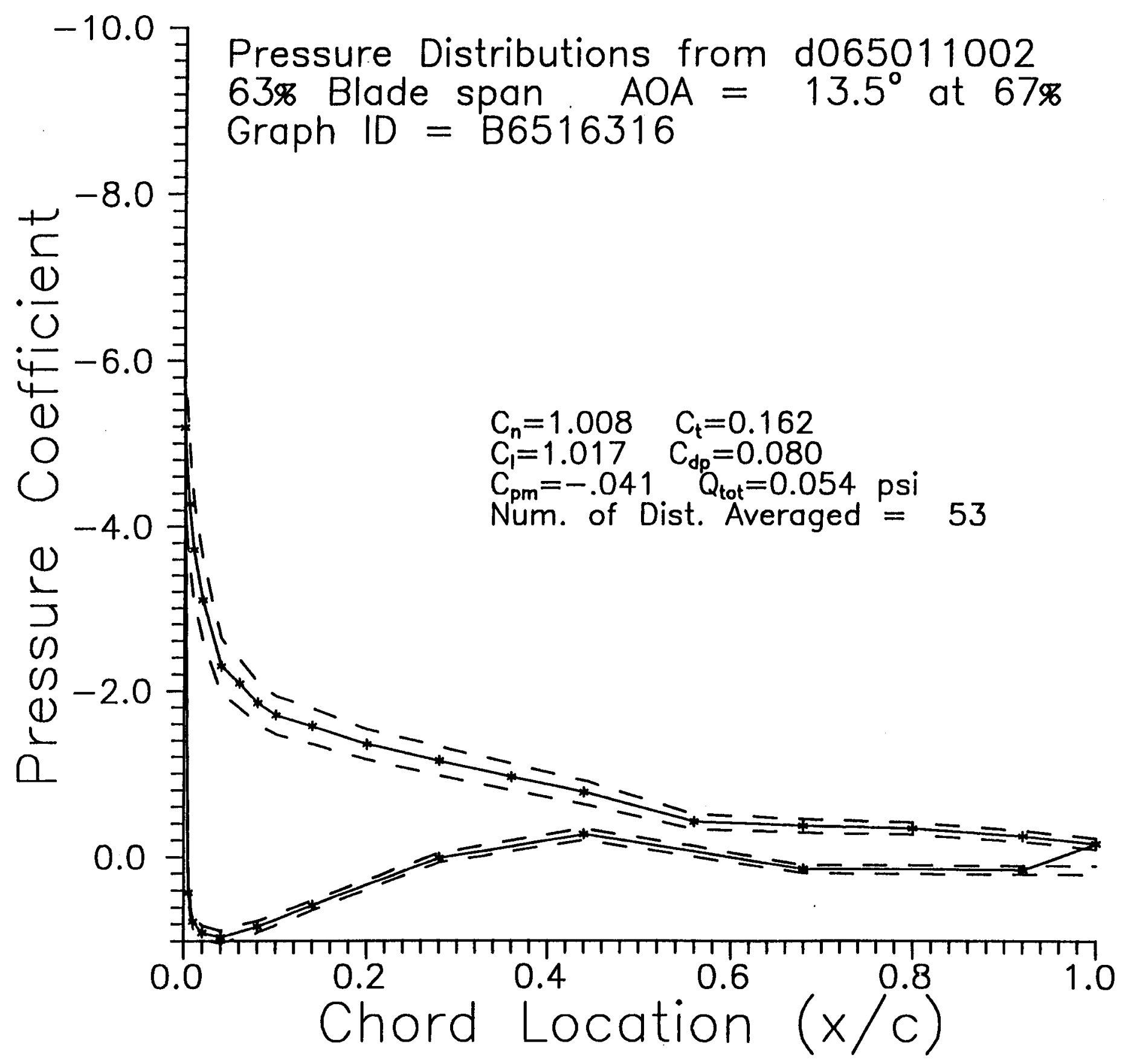




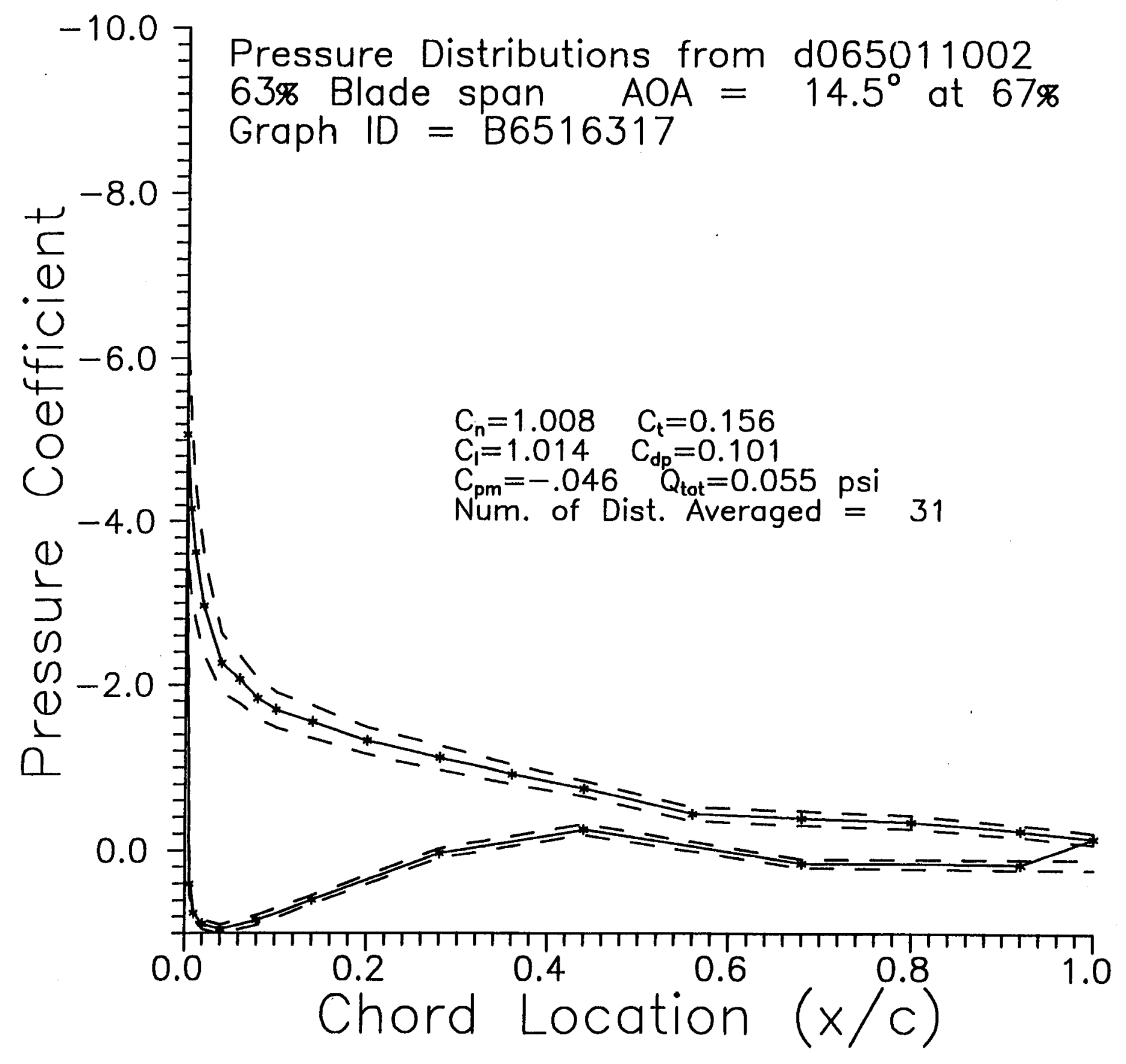




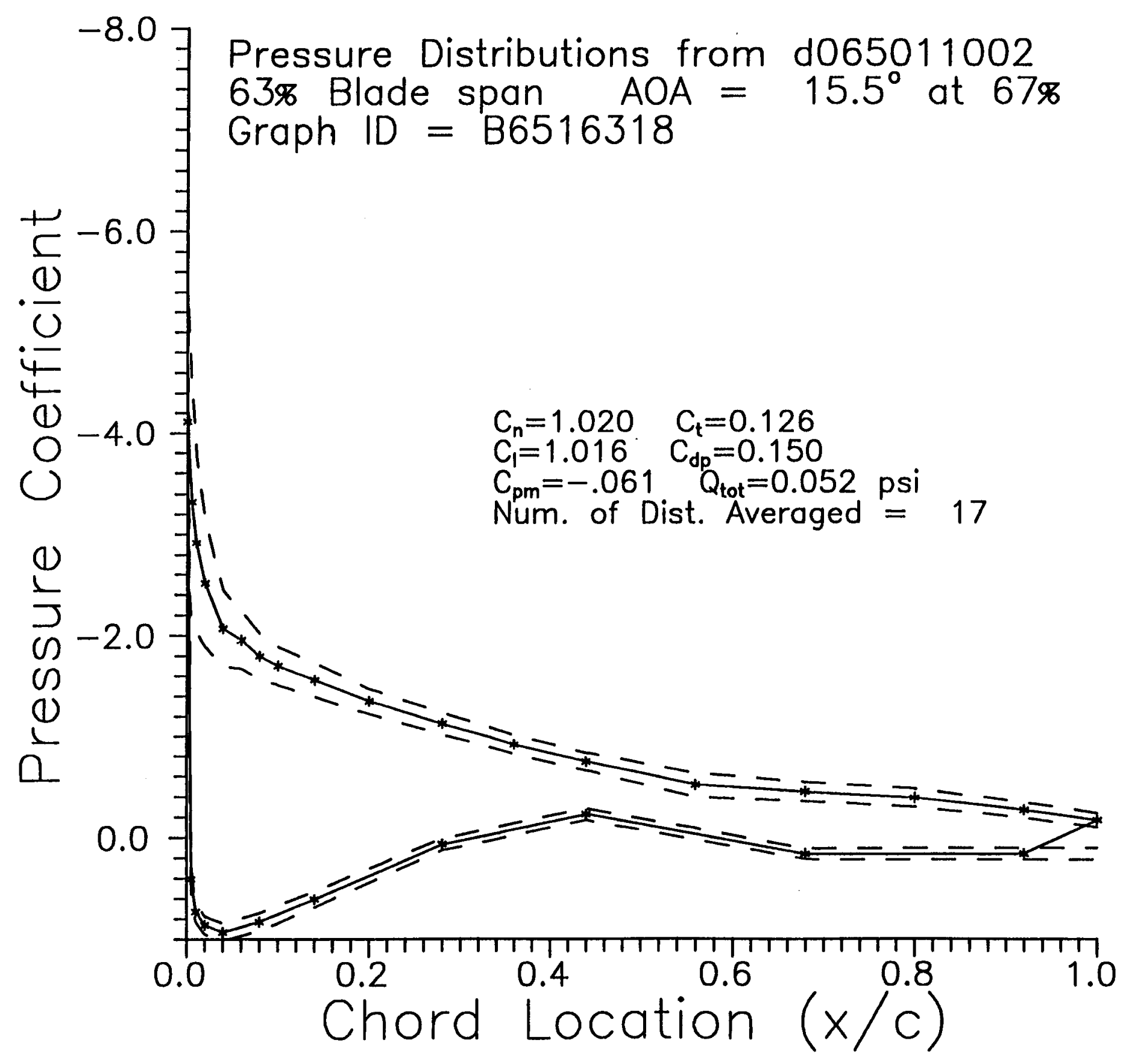




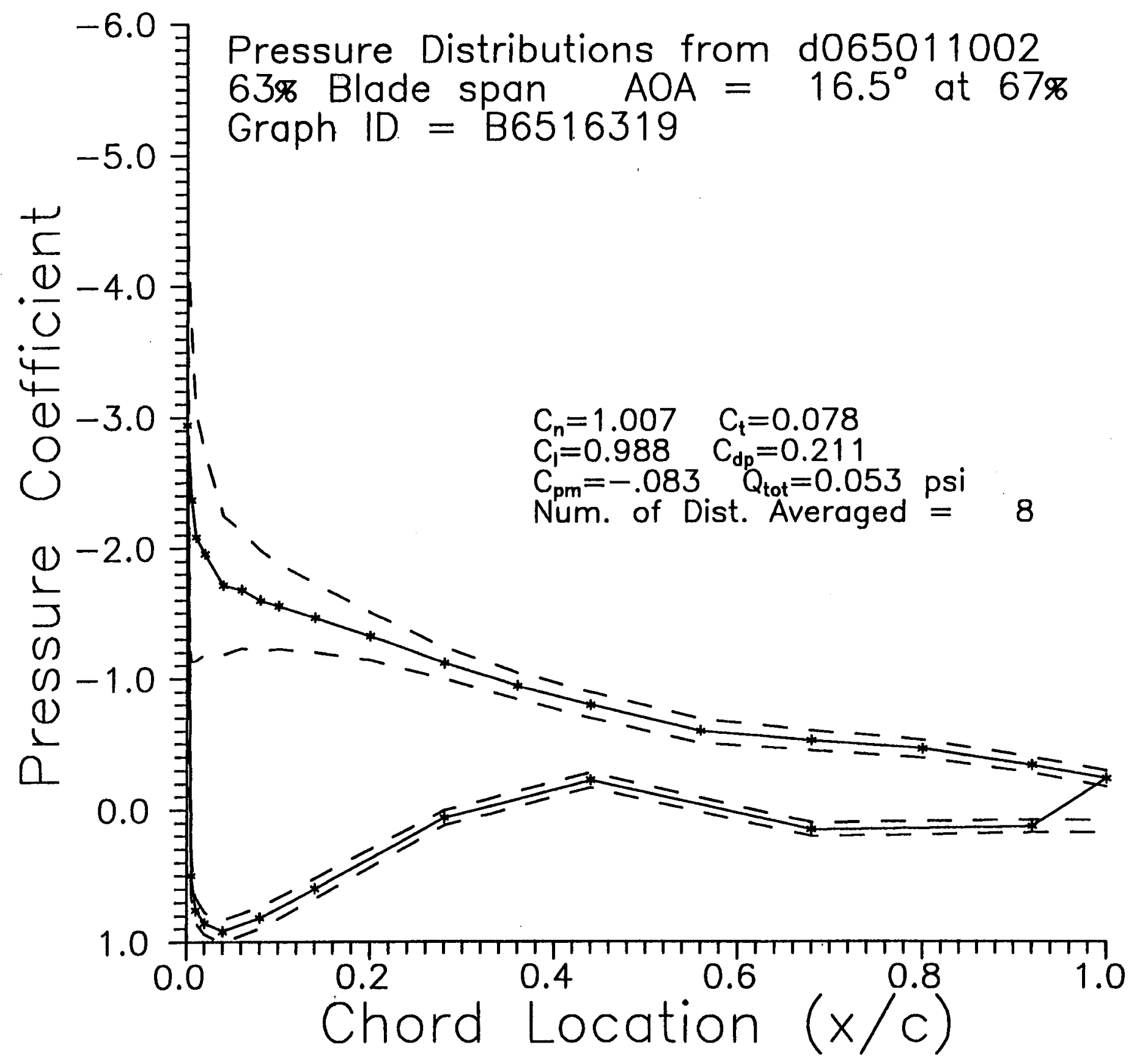




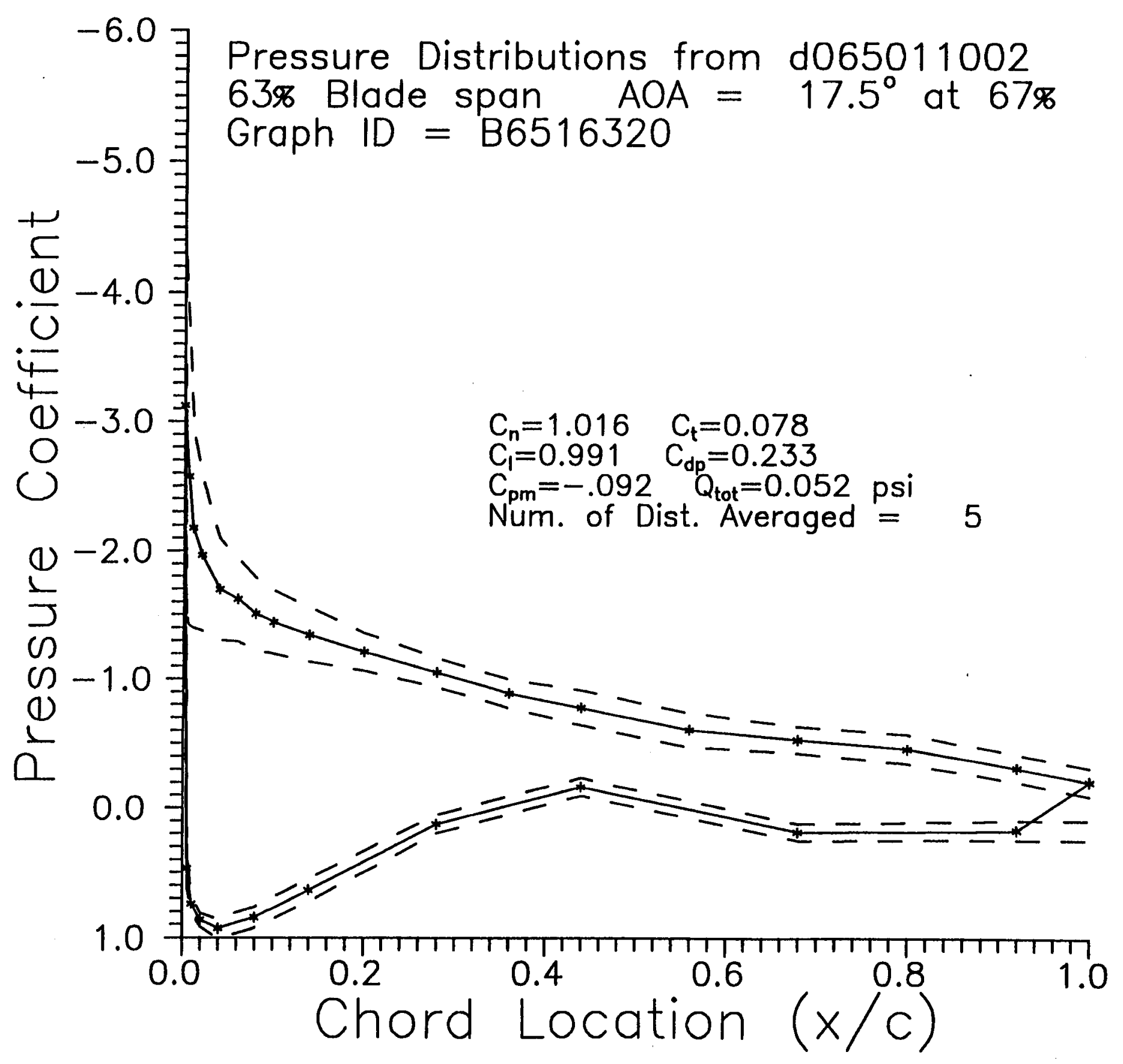


Appendix C.1.4

Pressure Distributions for 47\% Span 


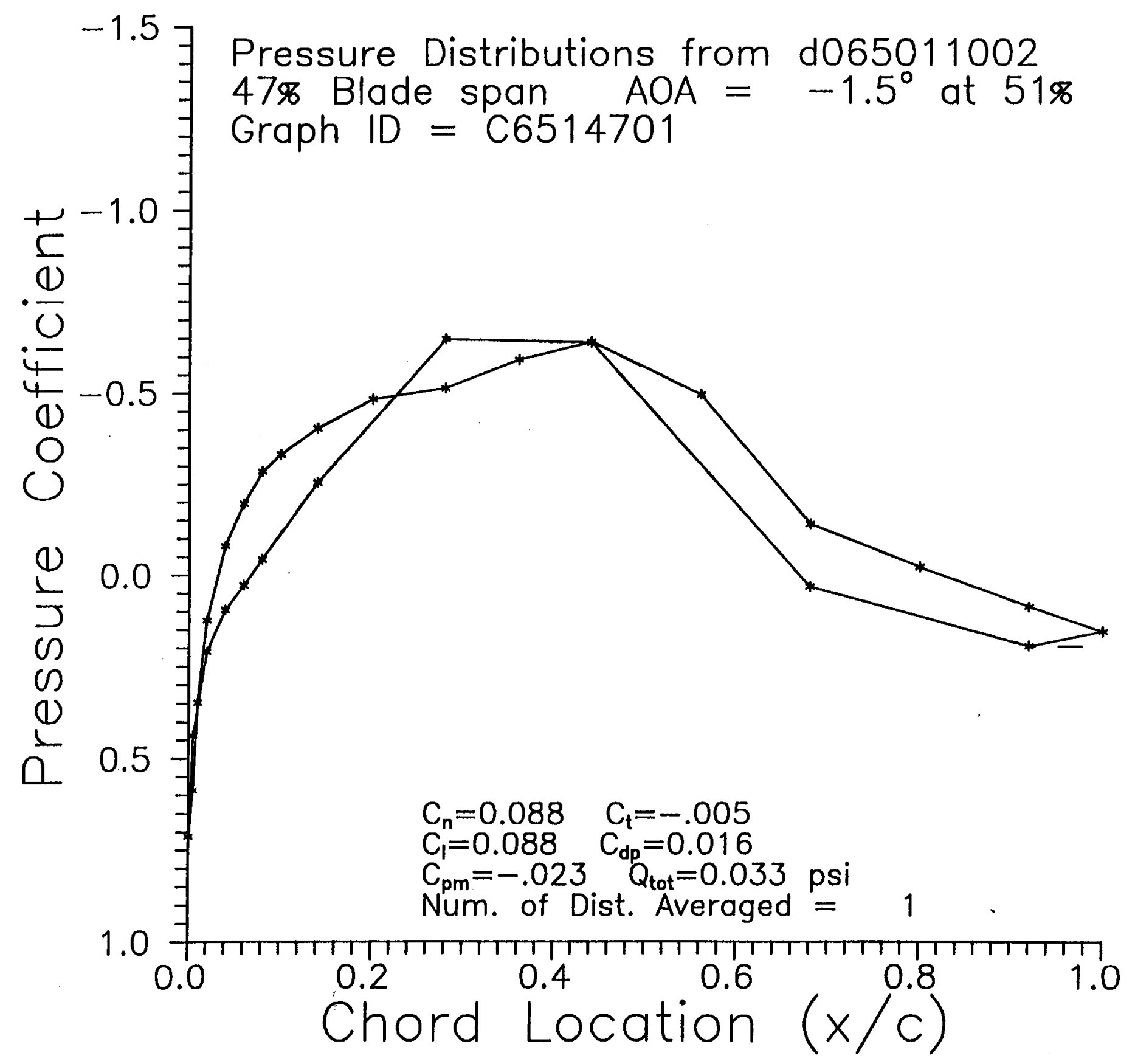




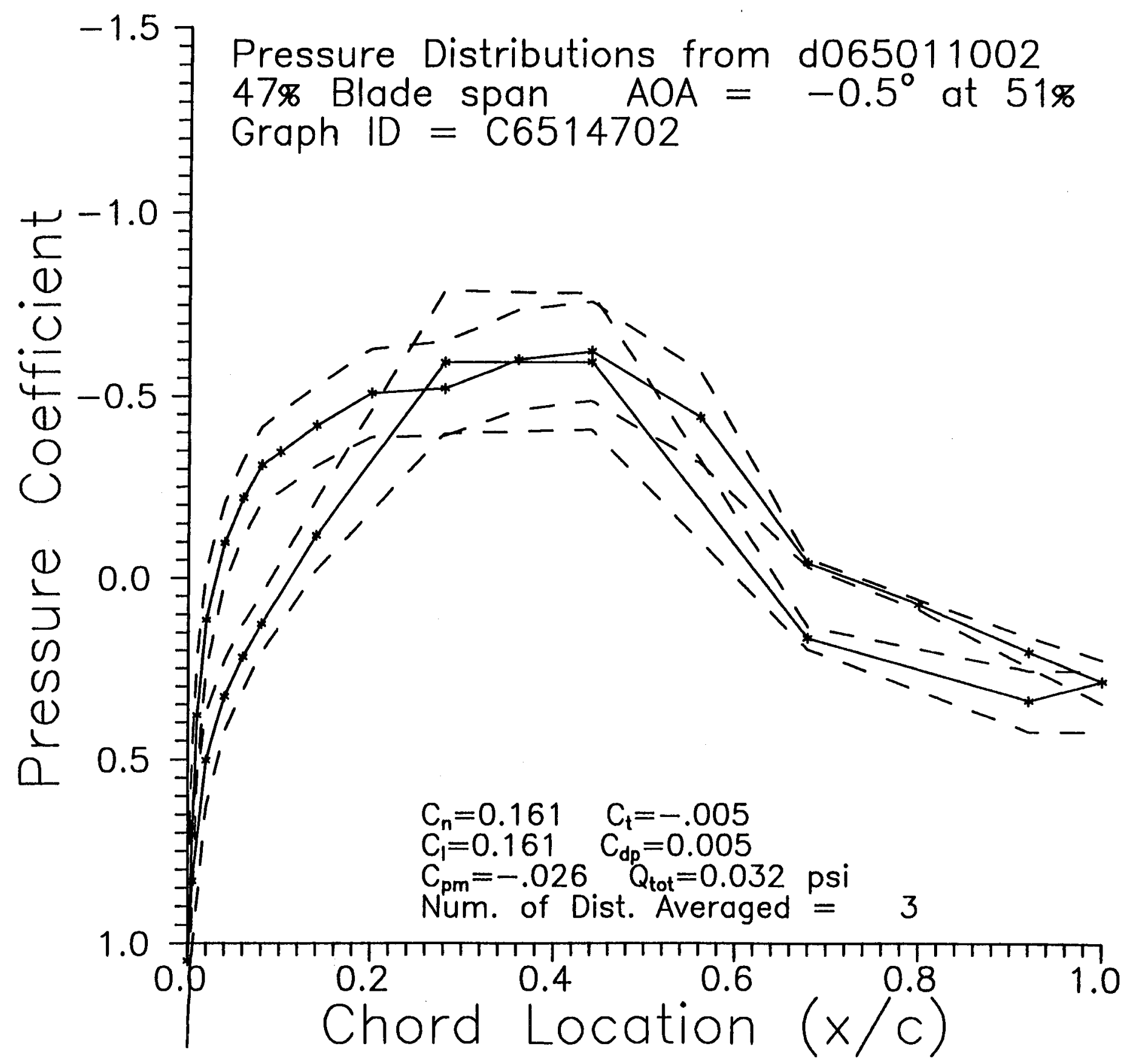




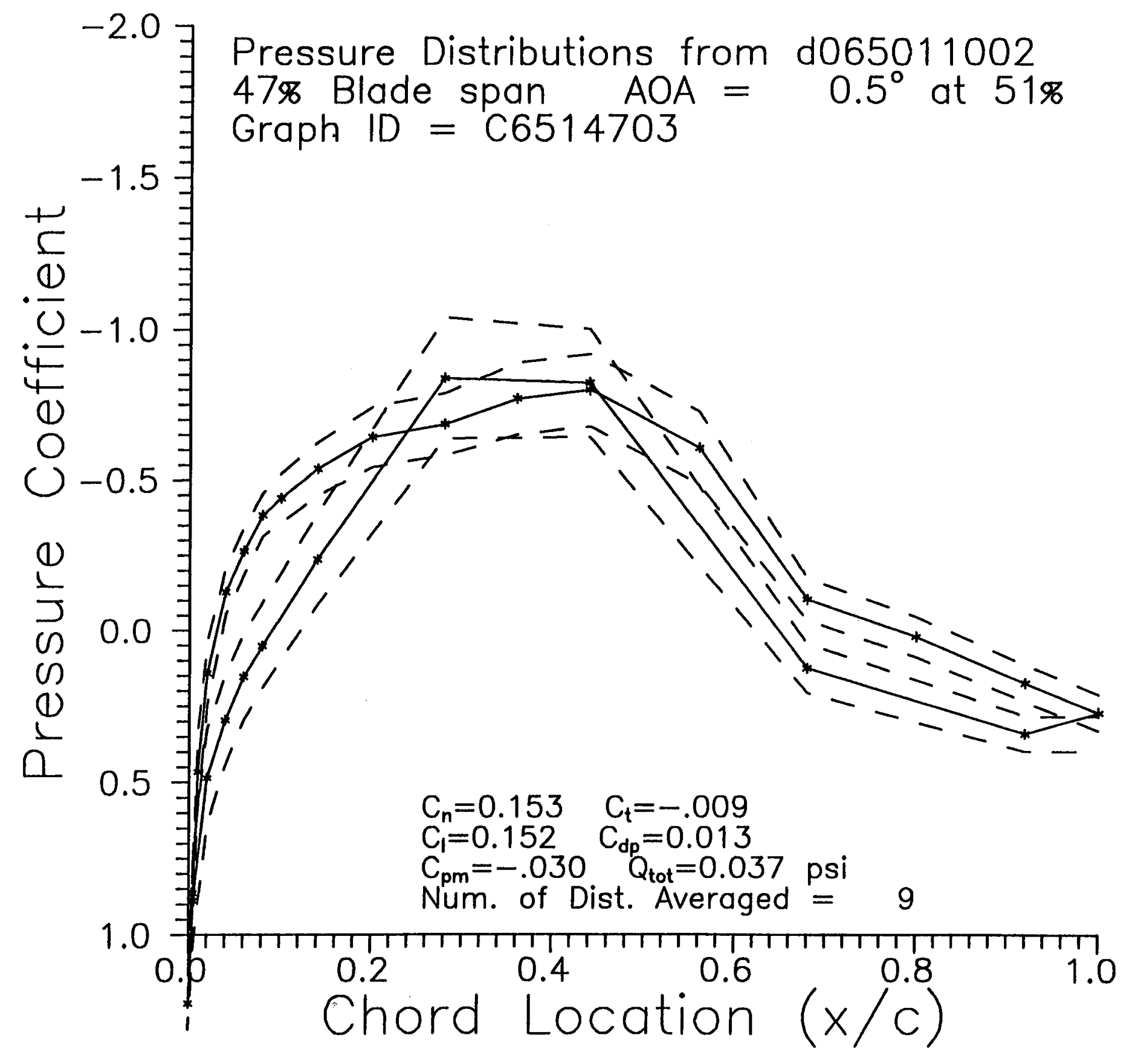




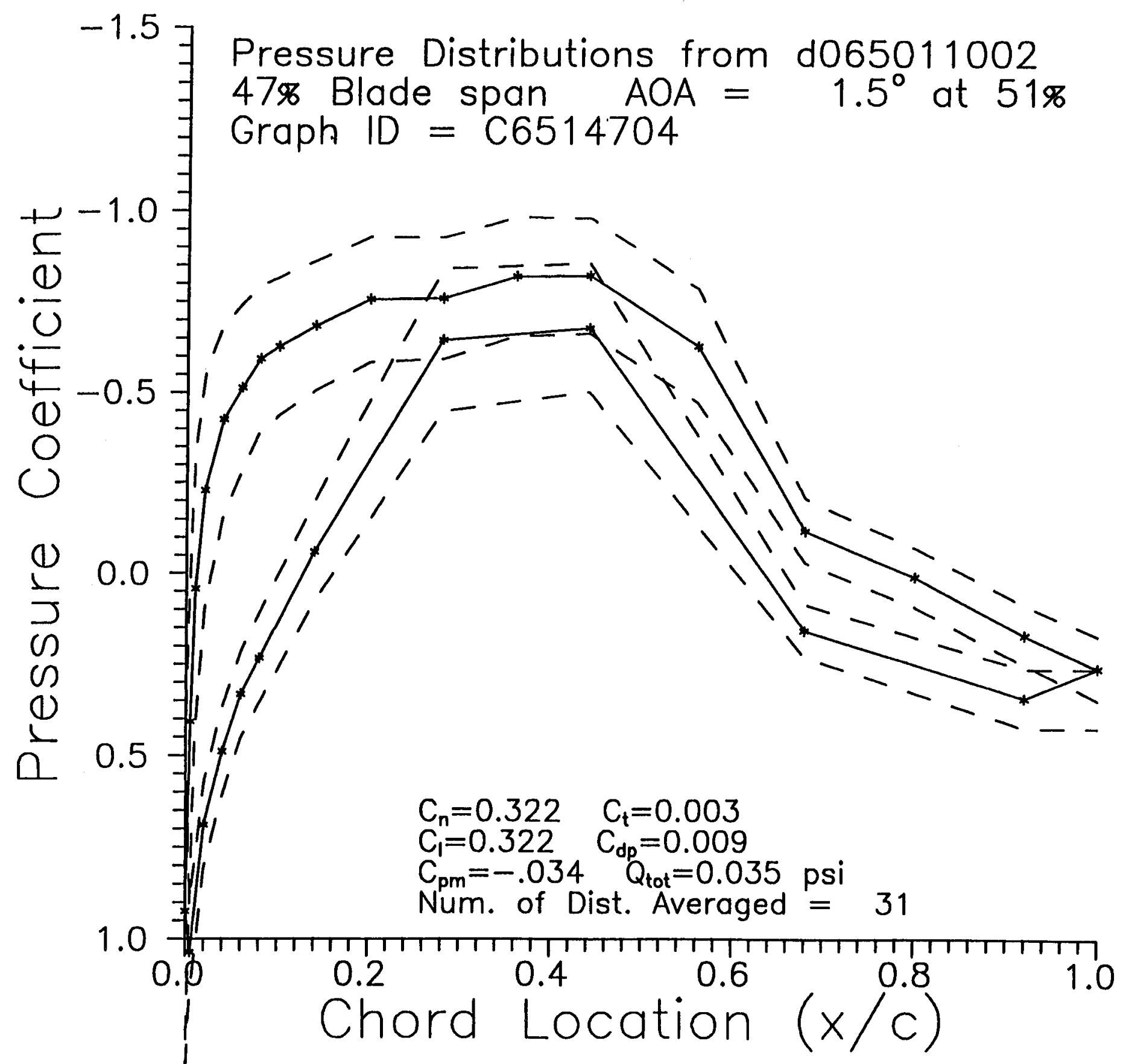




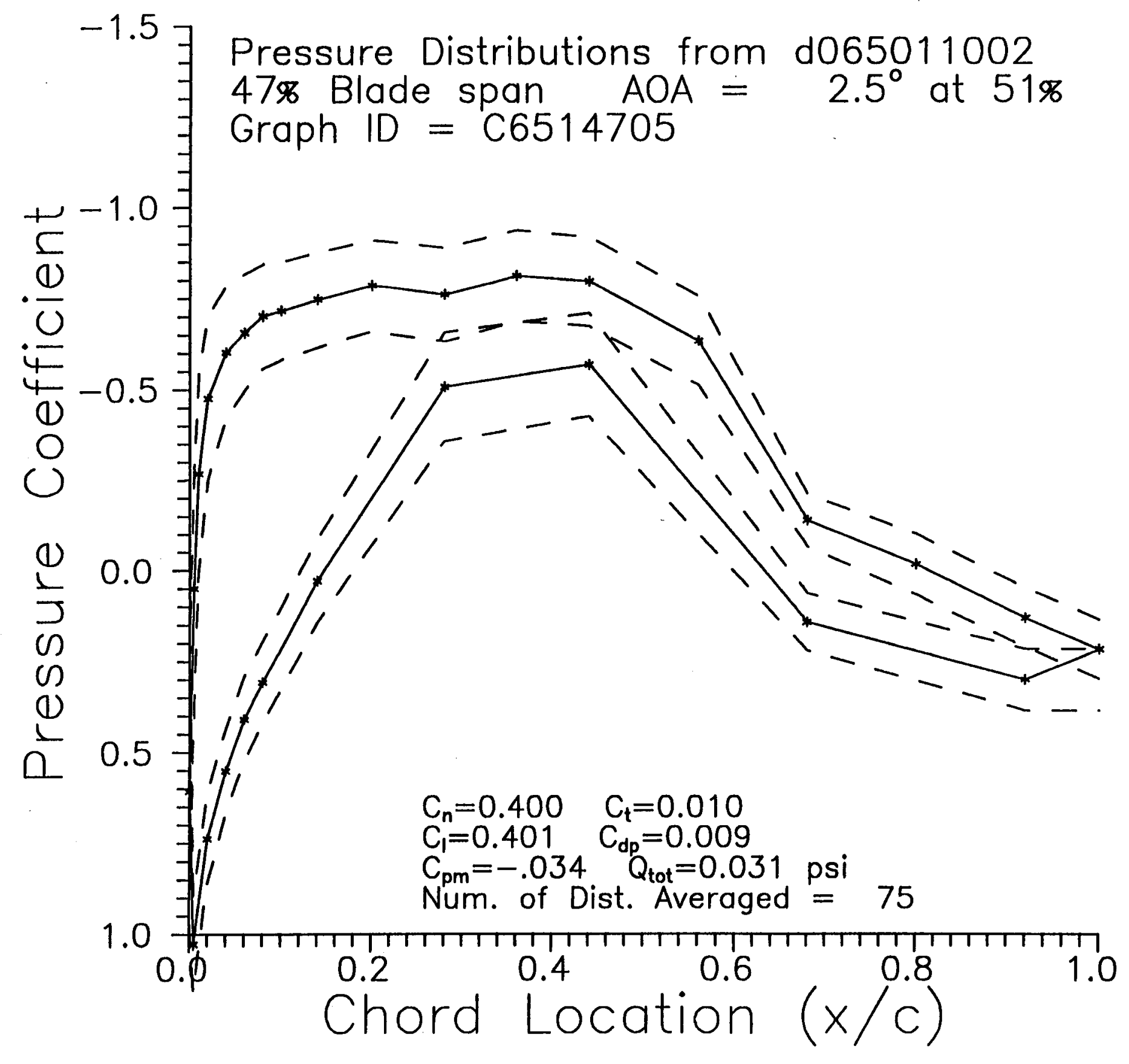




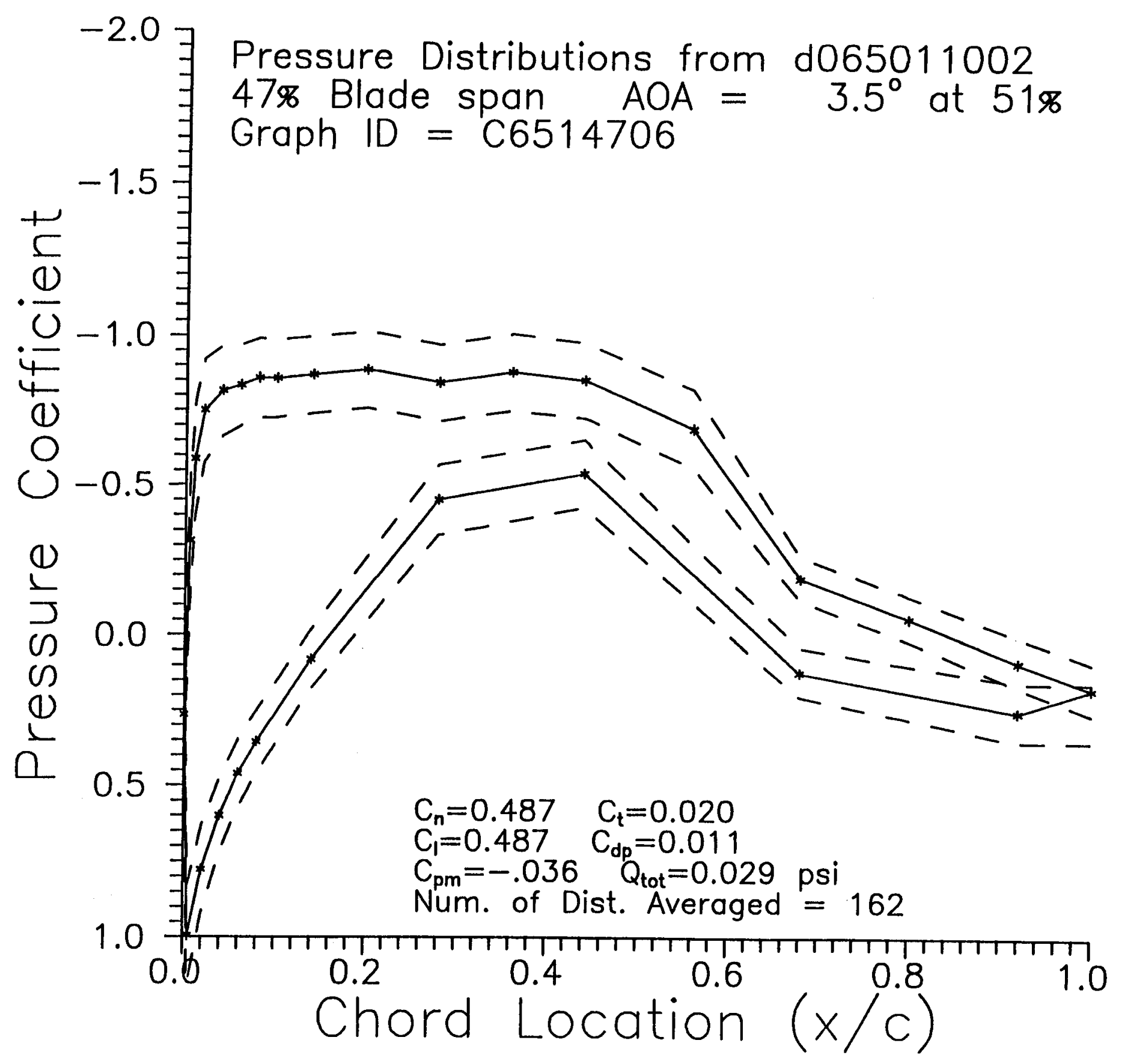




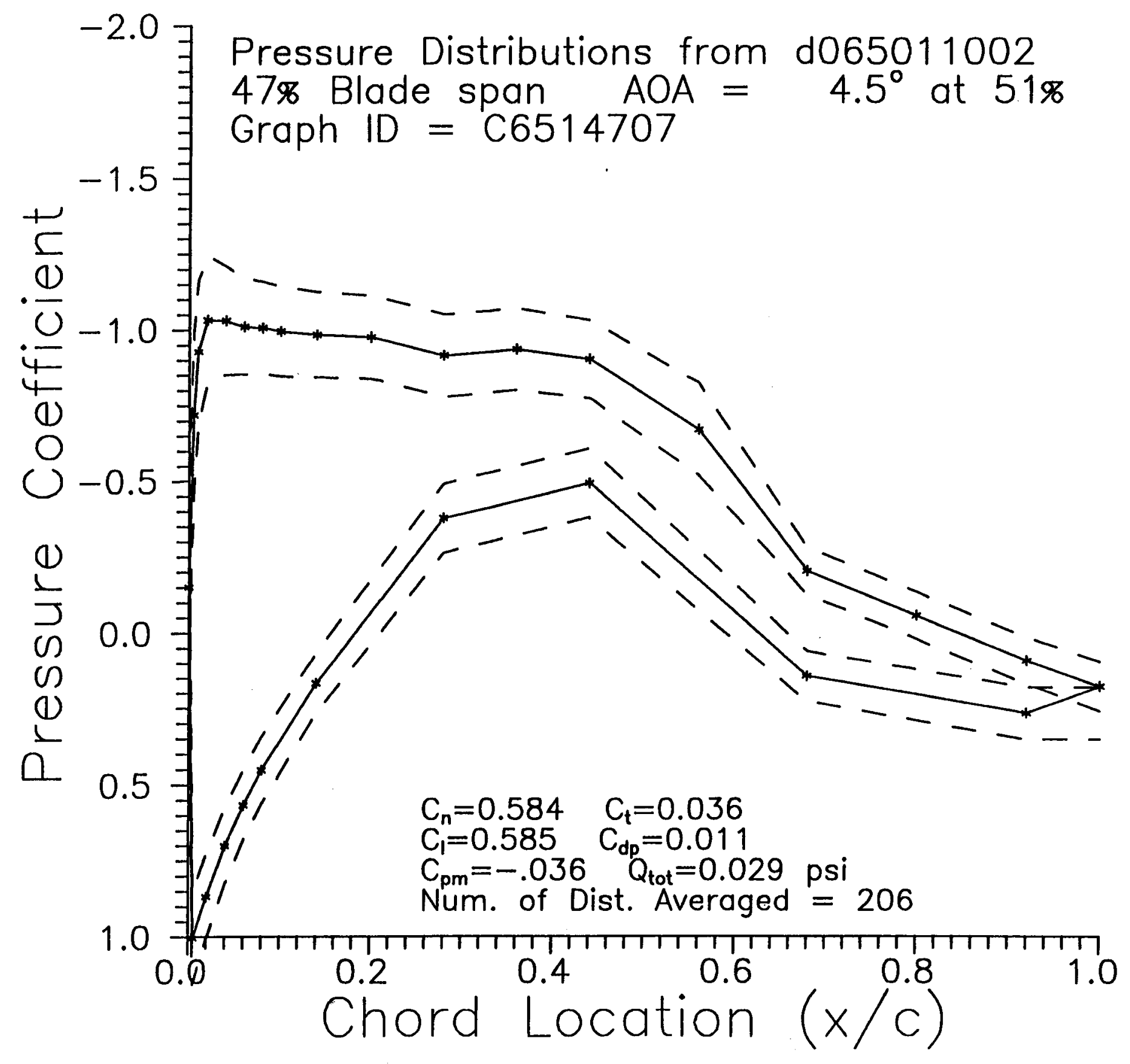




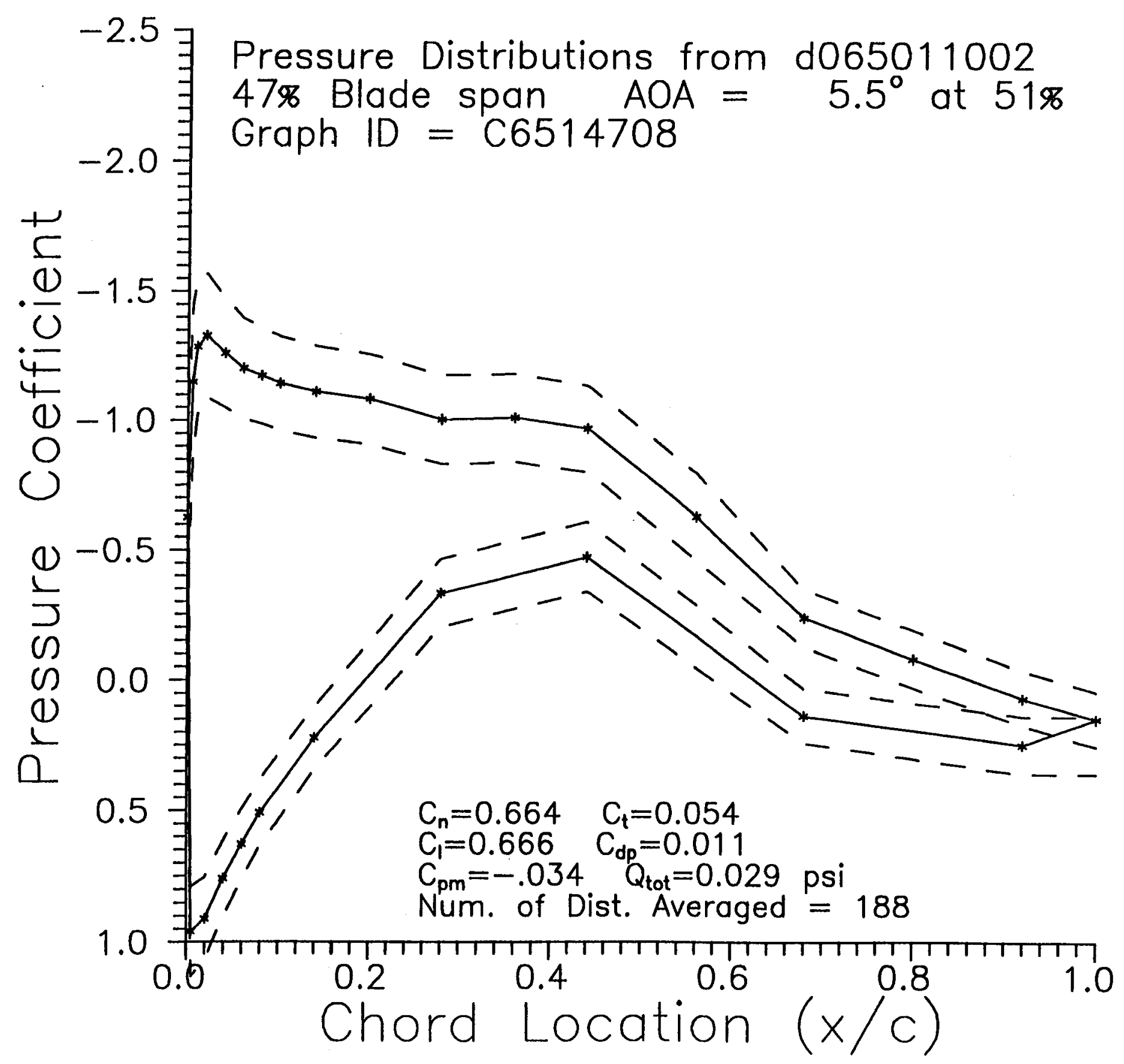




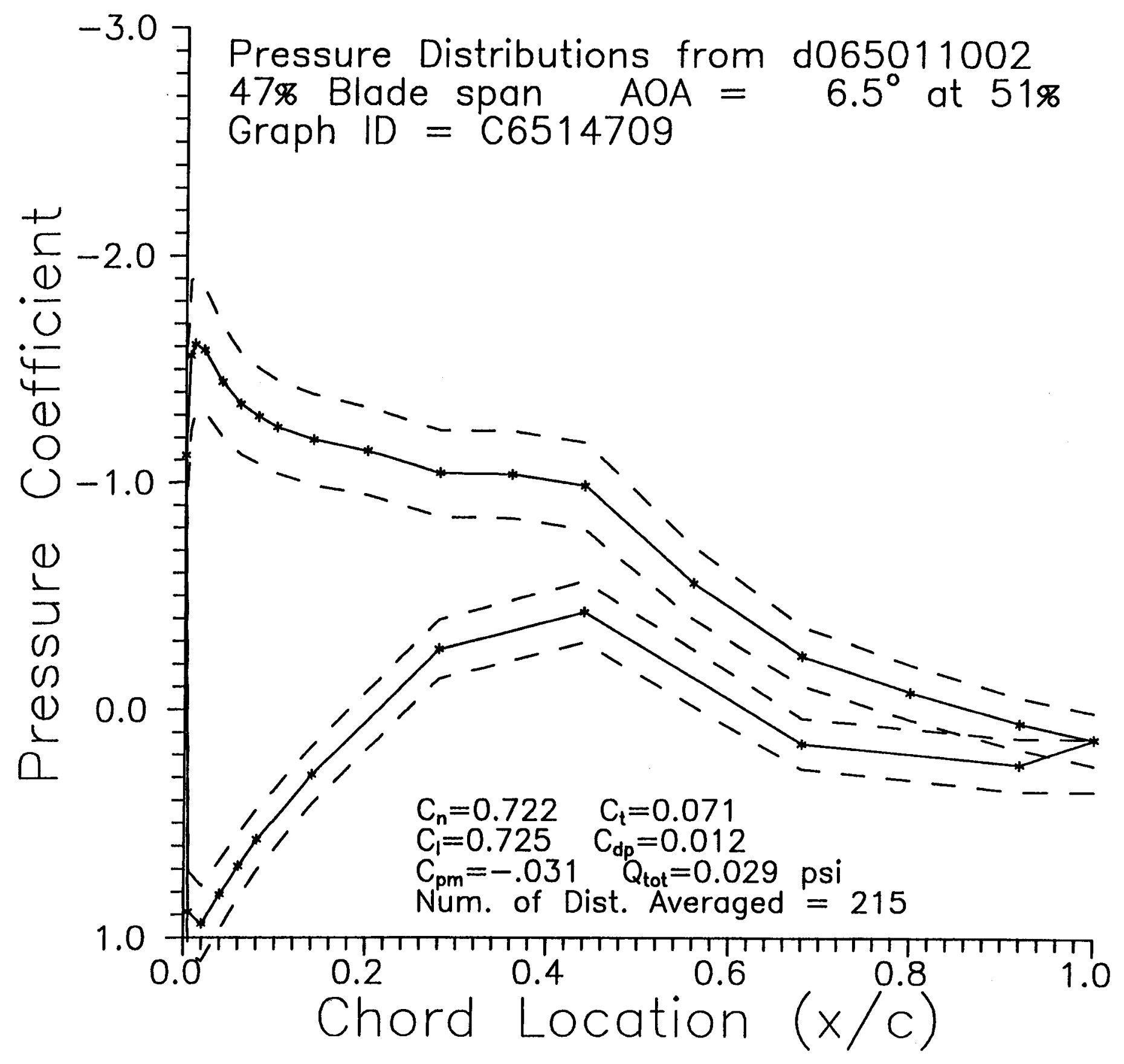




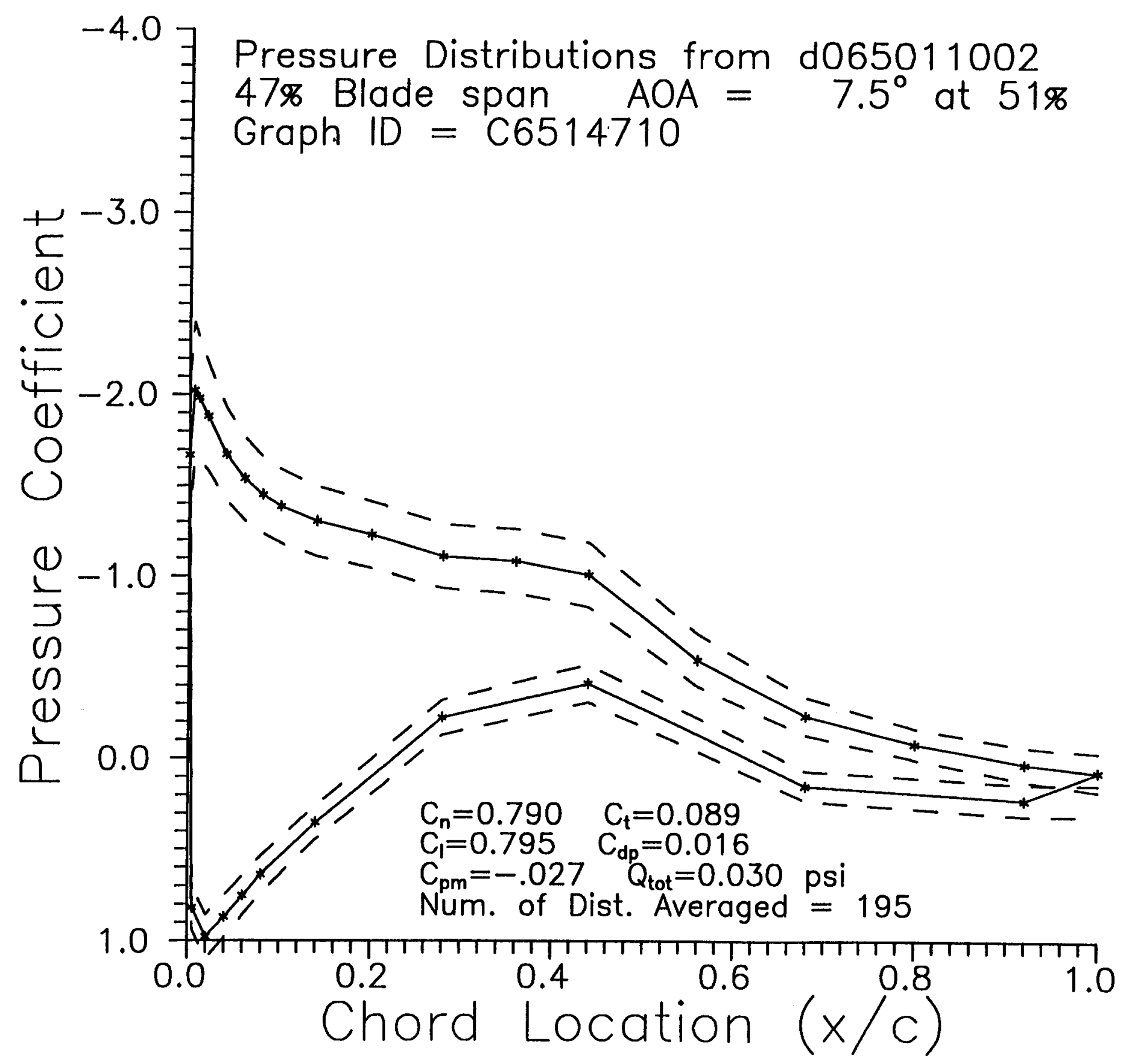




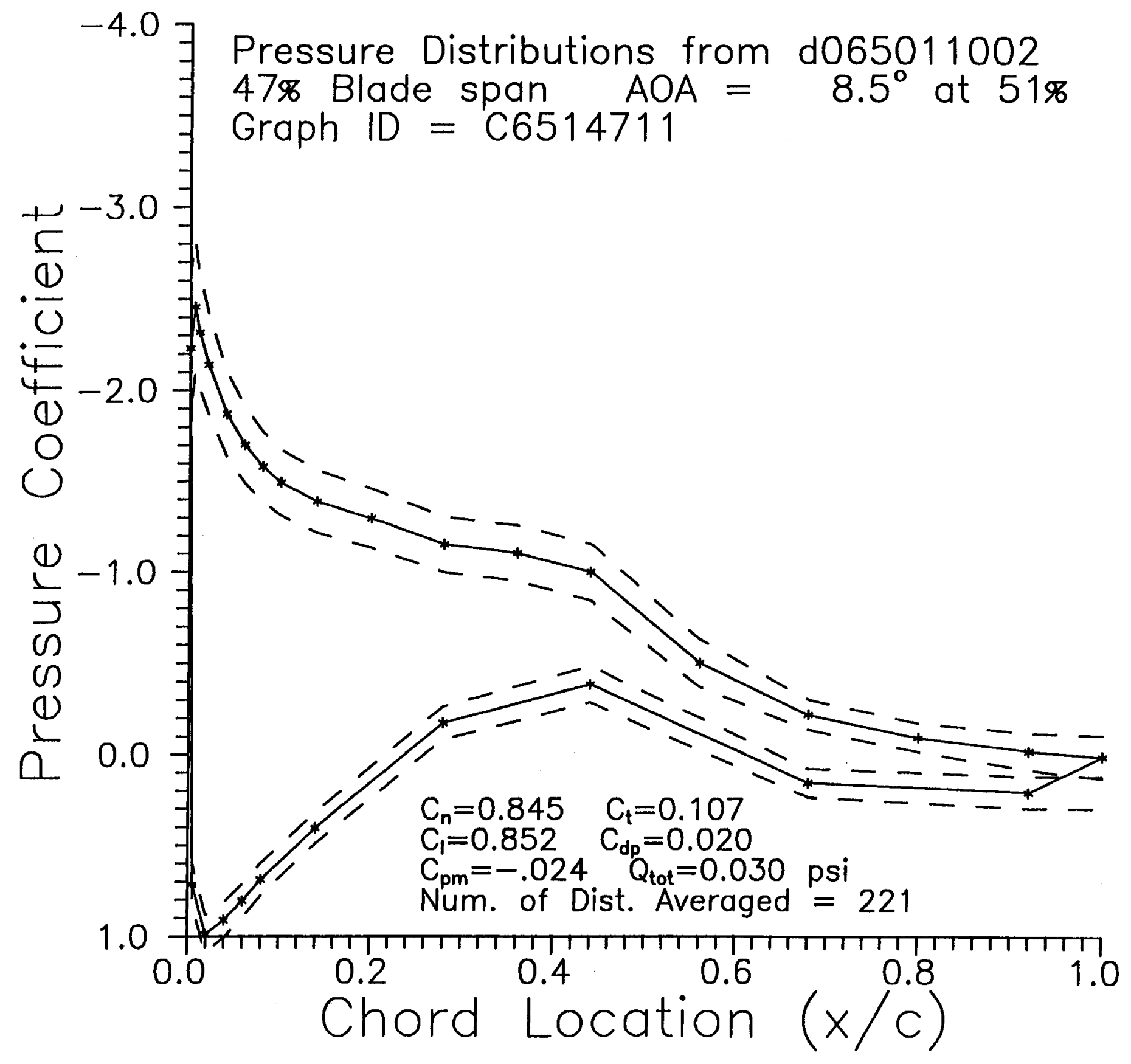




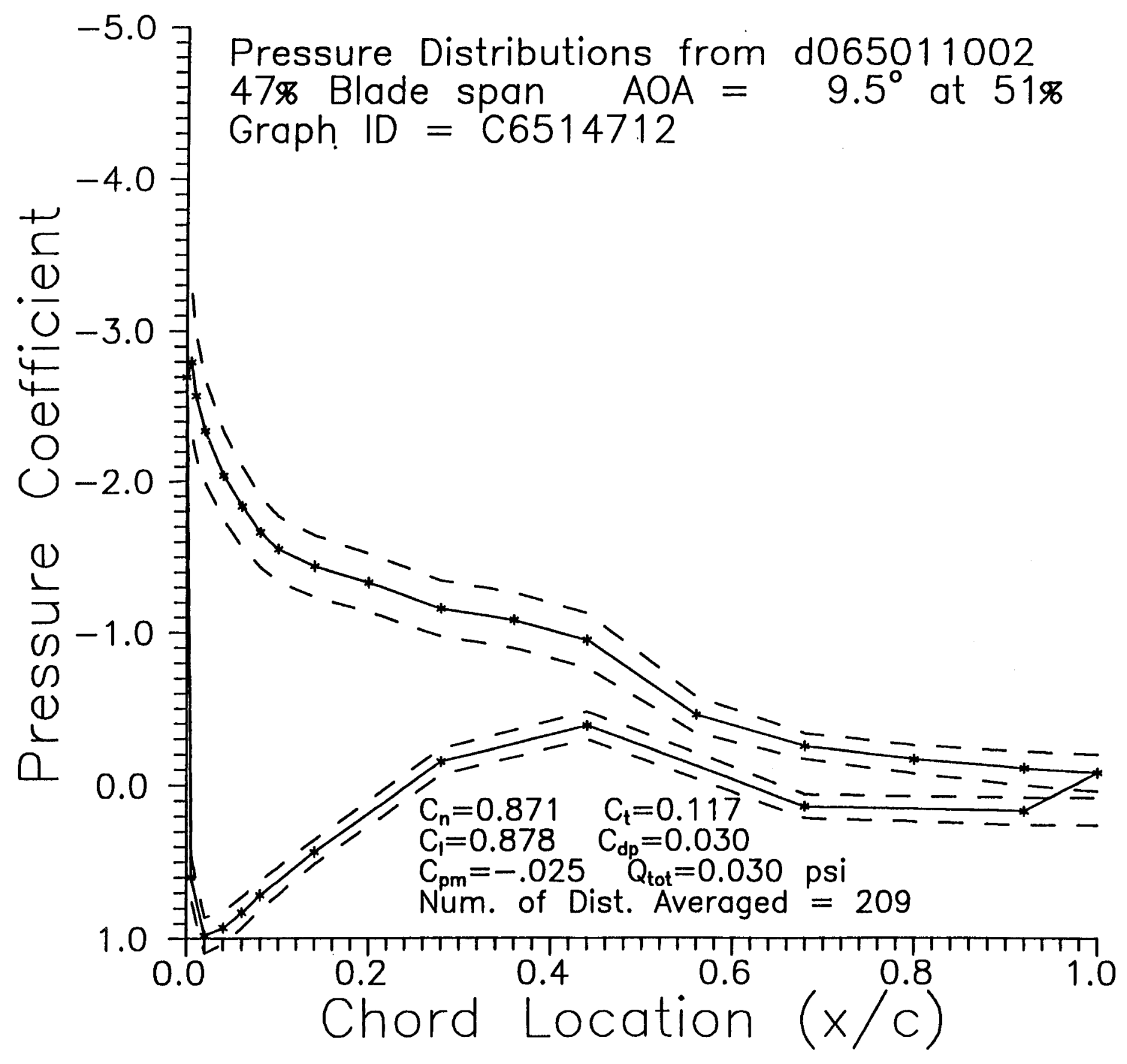




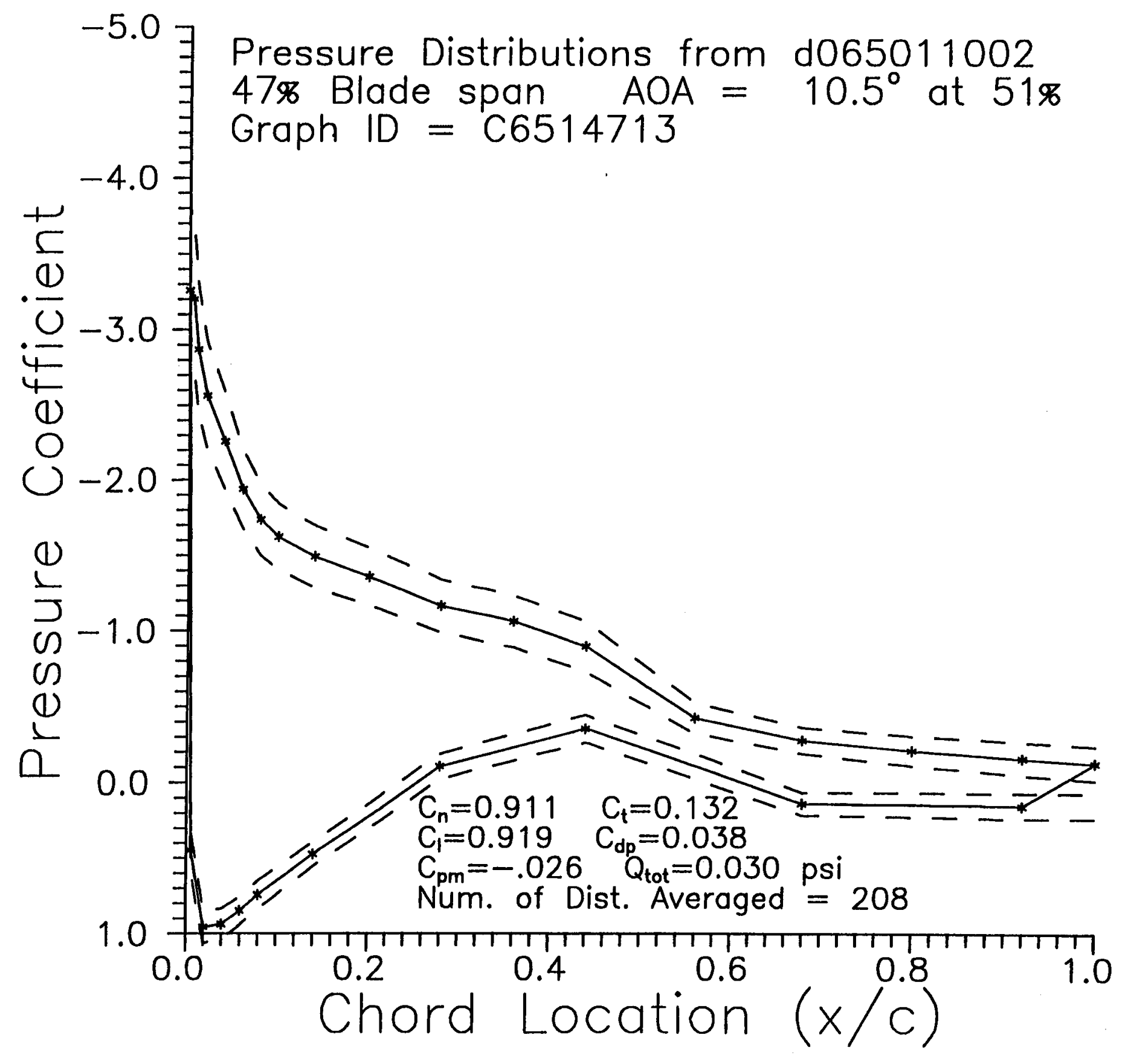




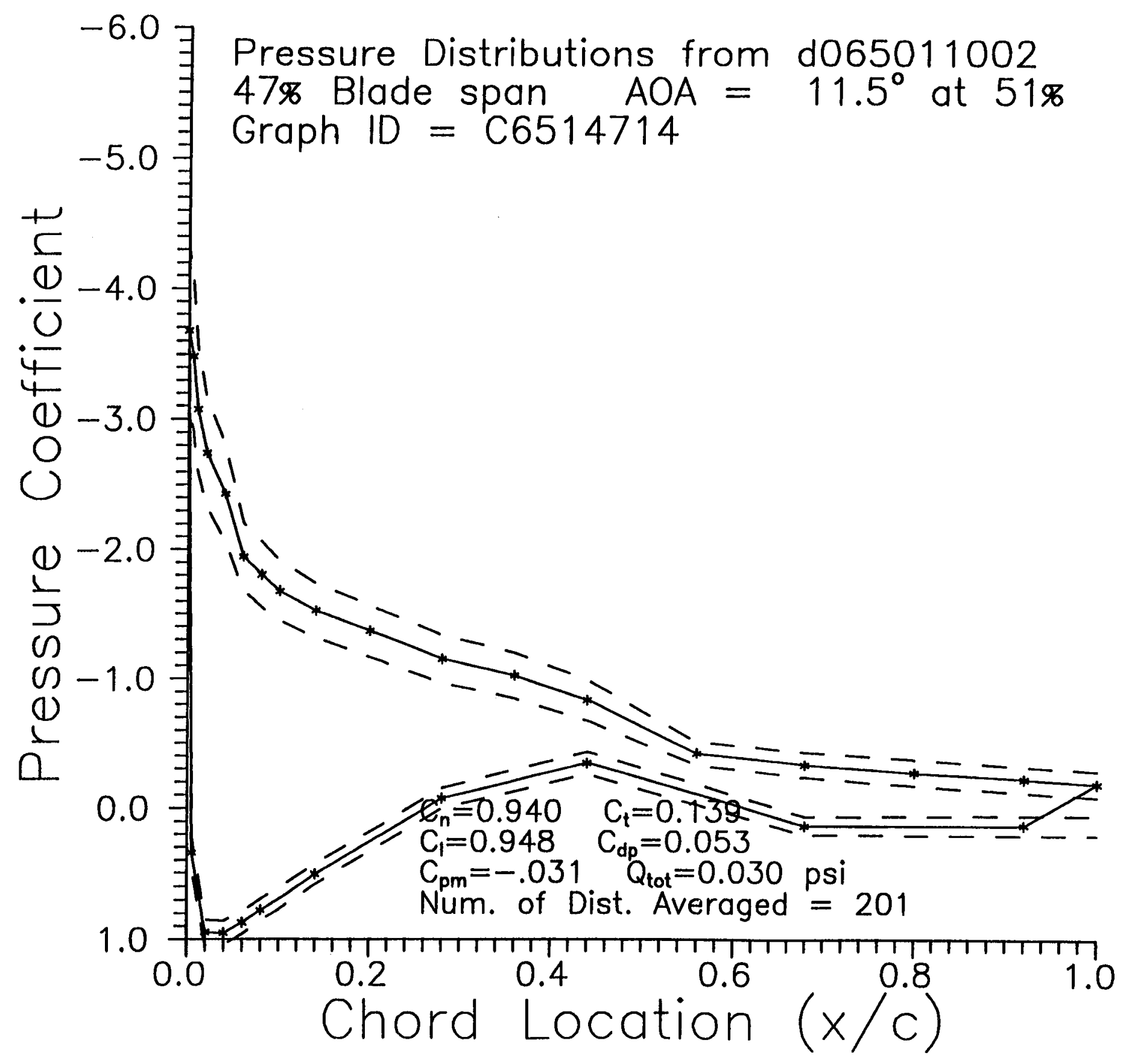




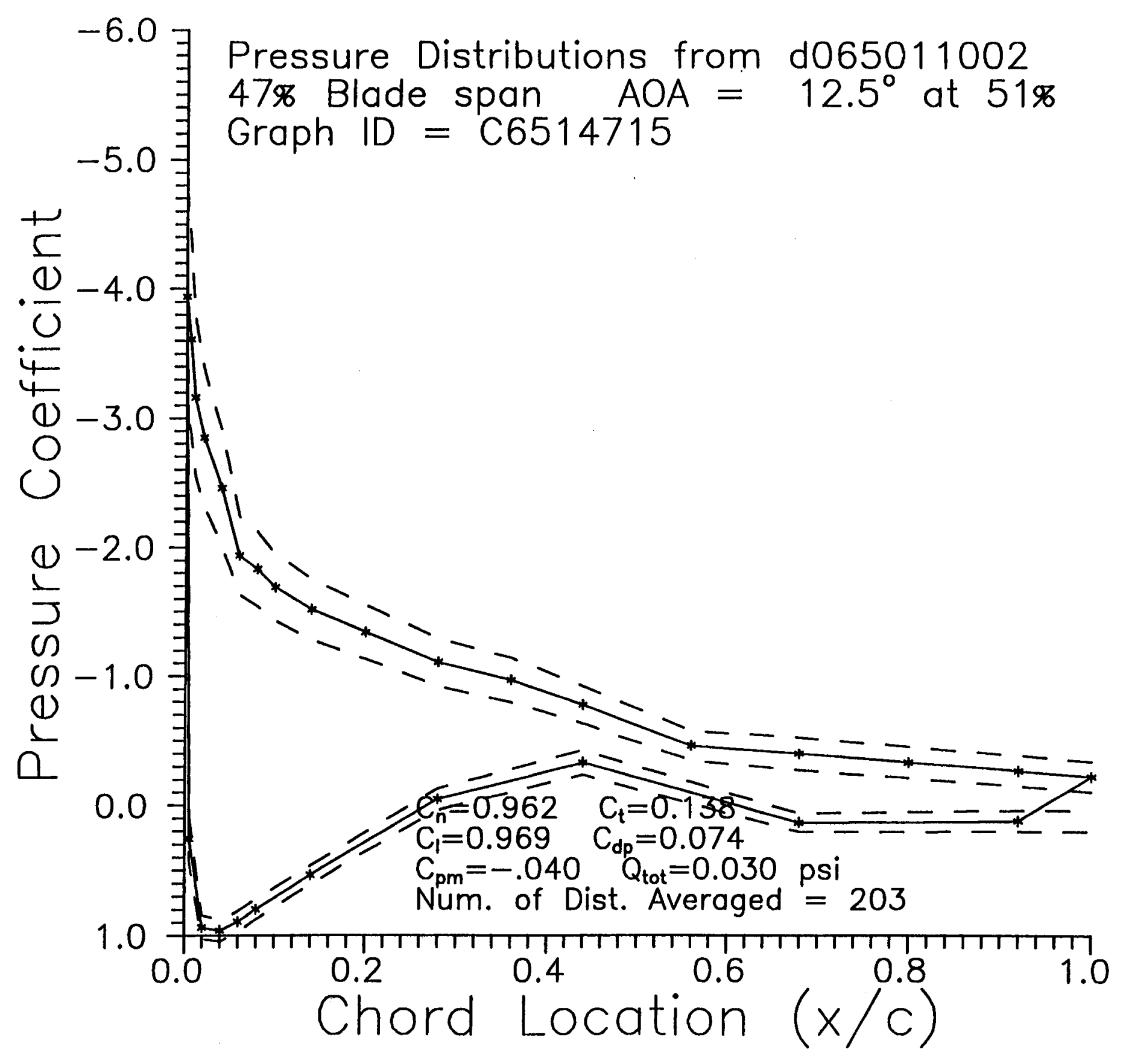




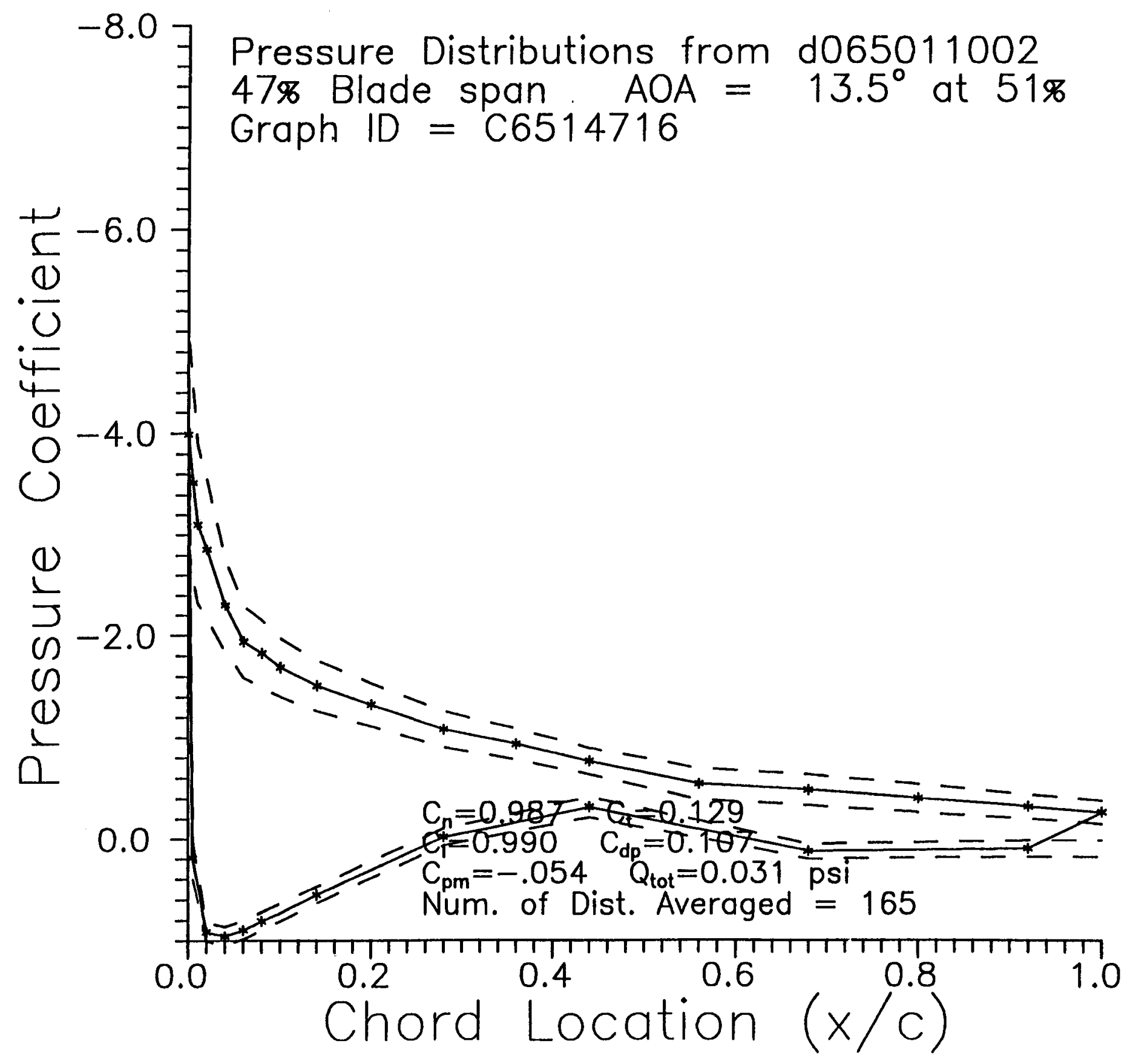




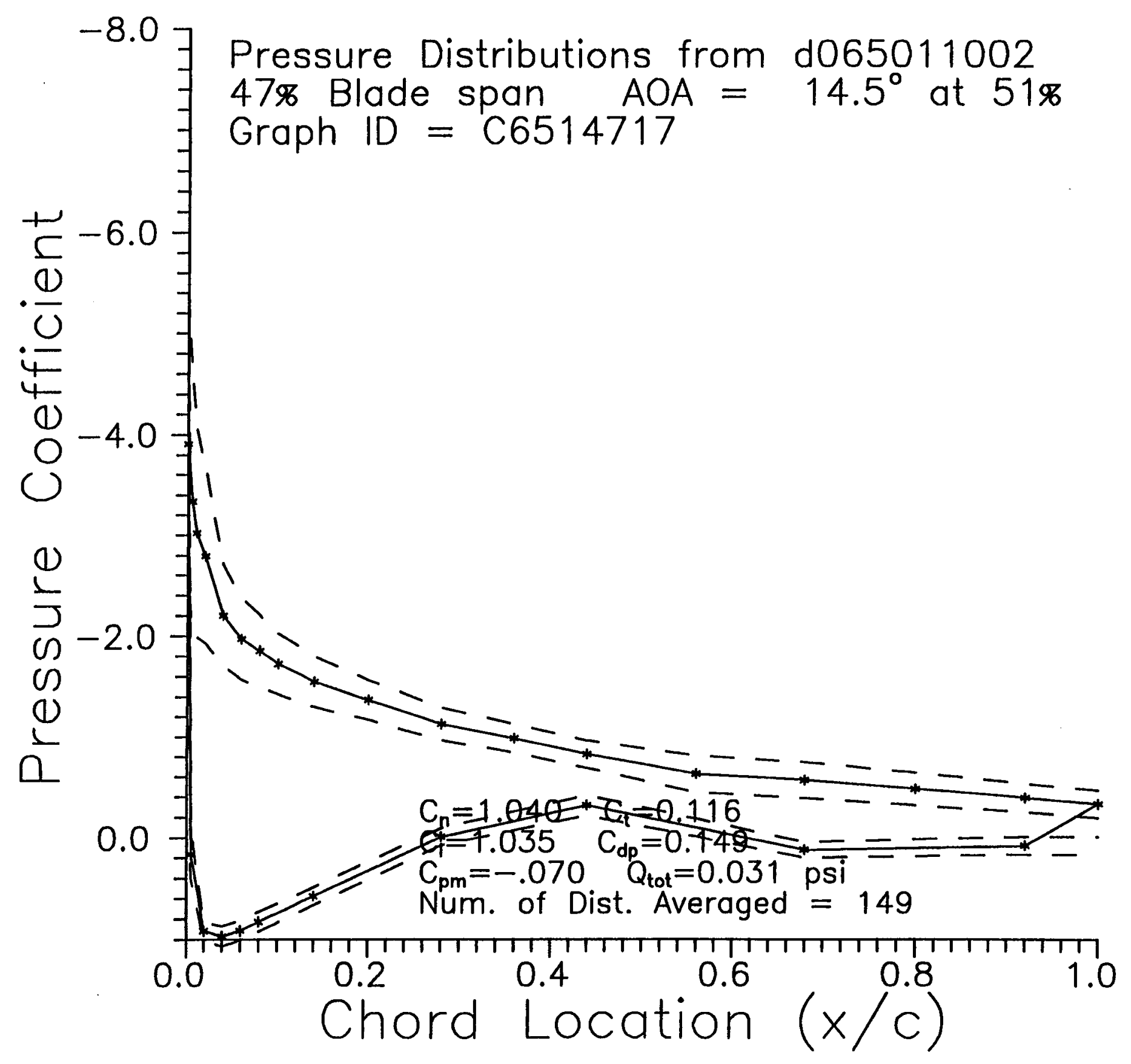




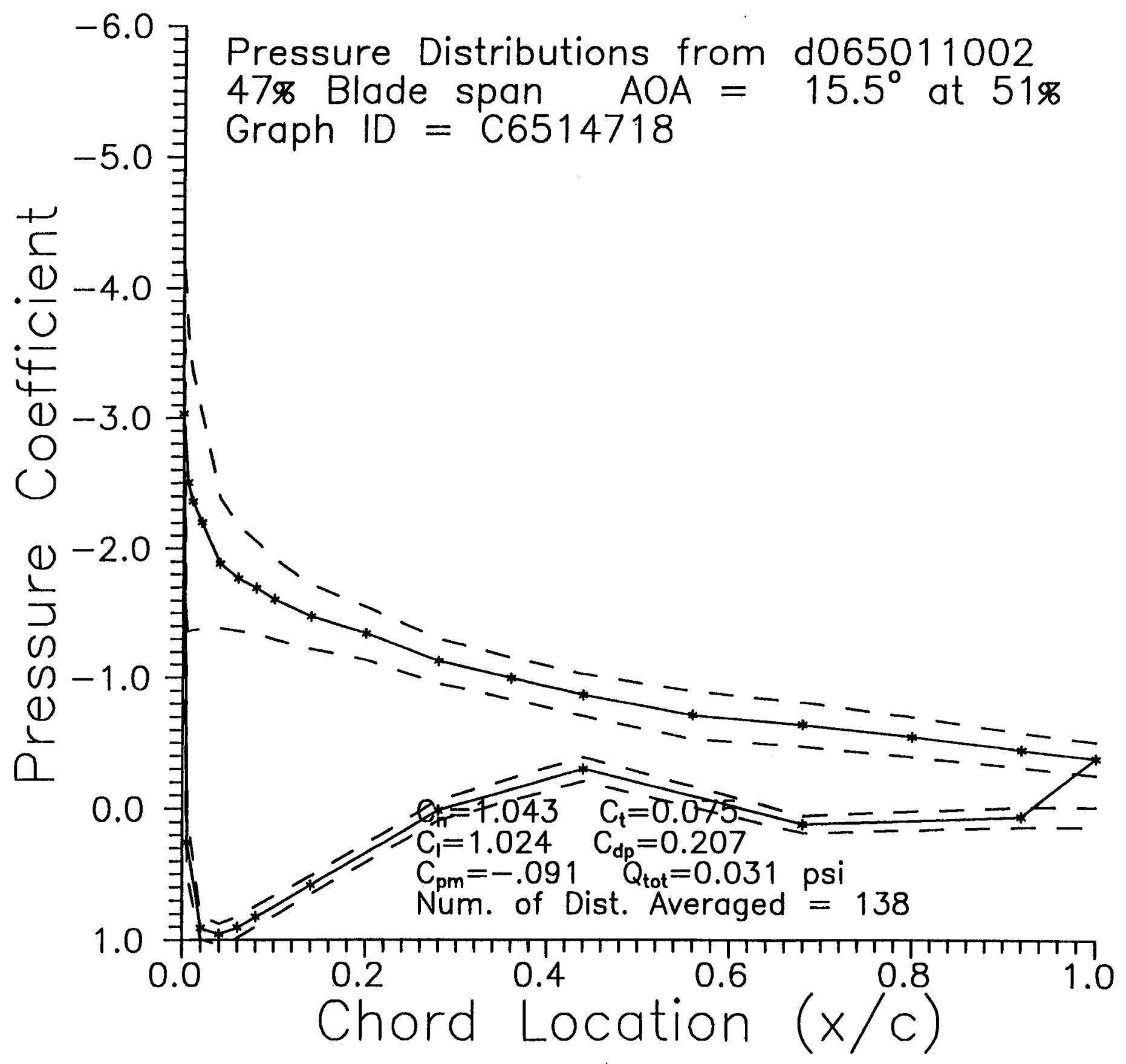




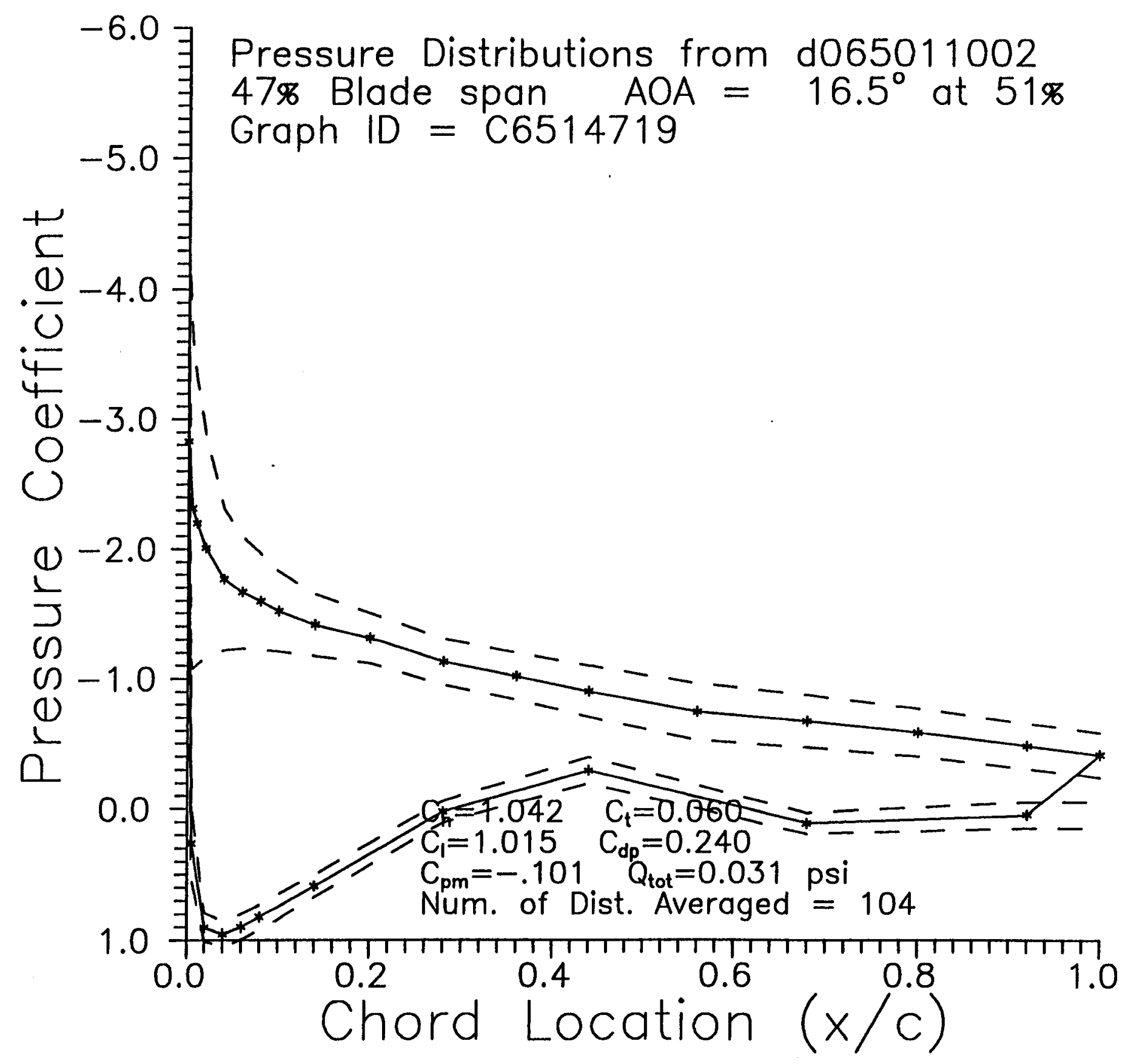




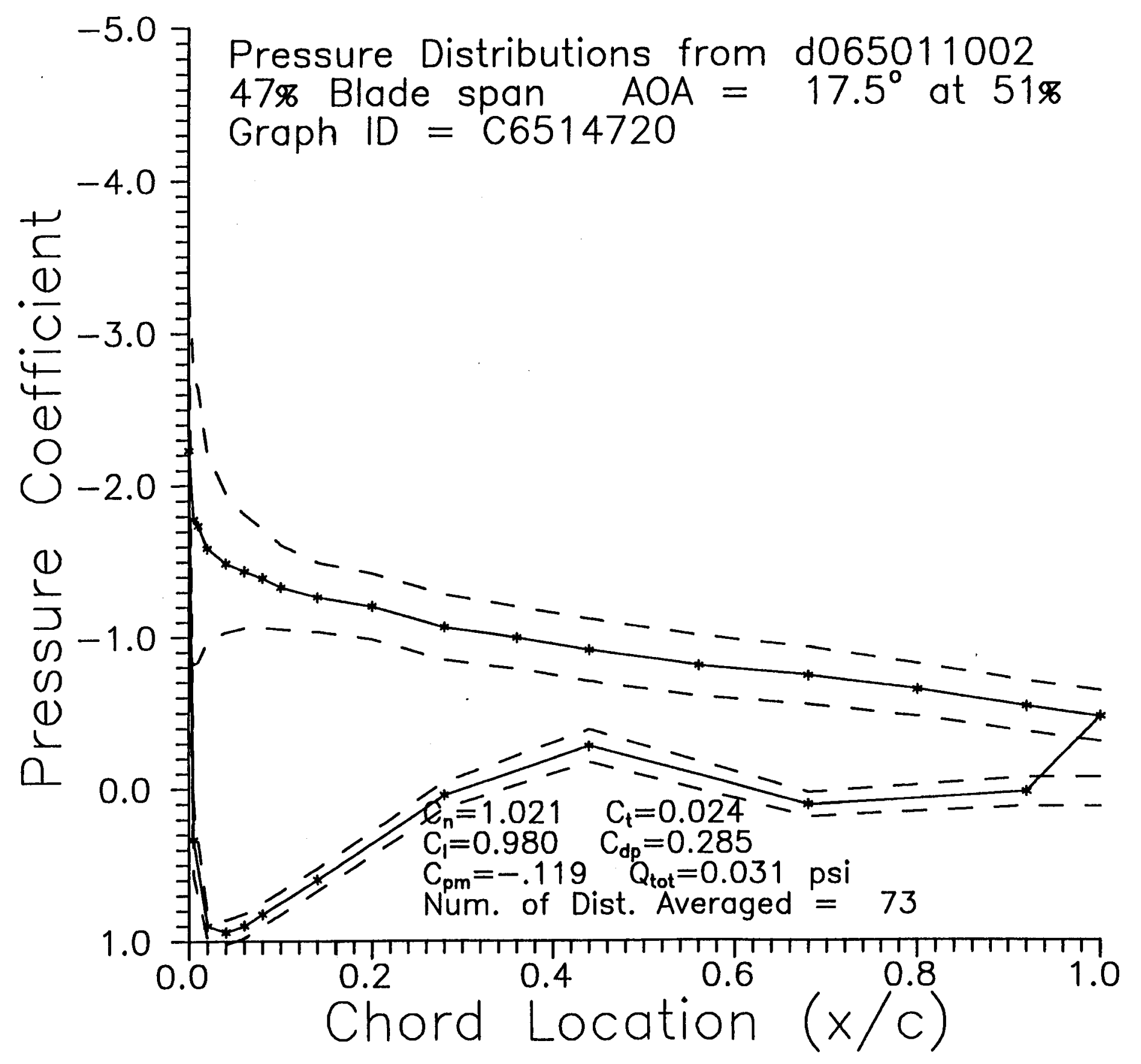




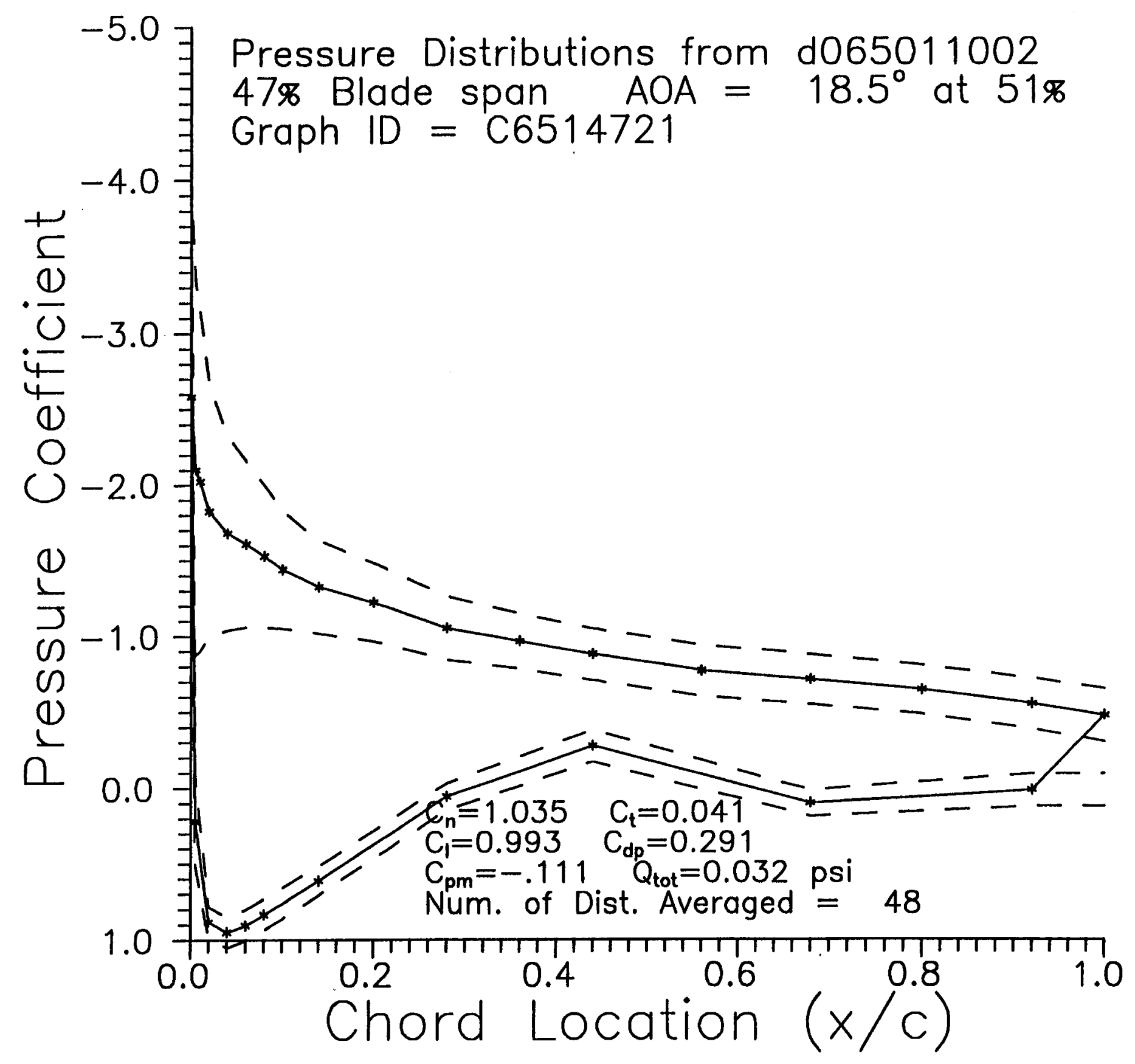




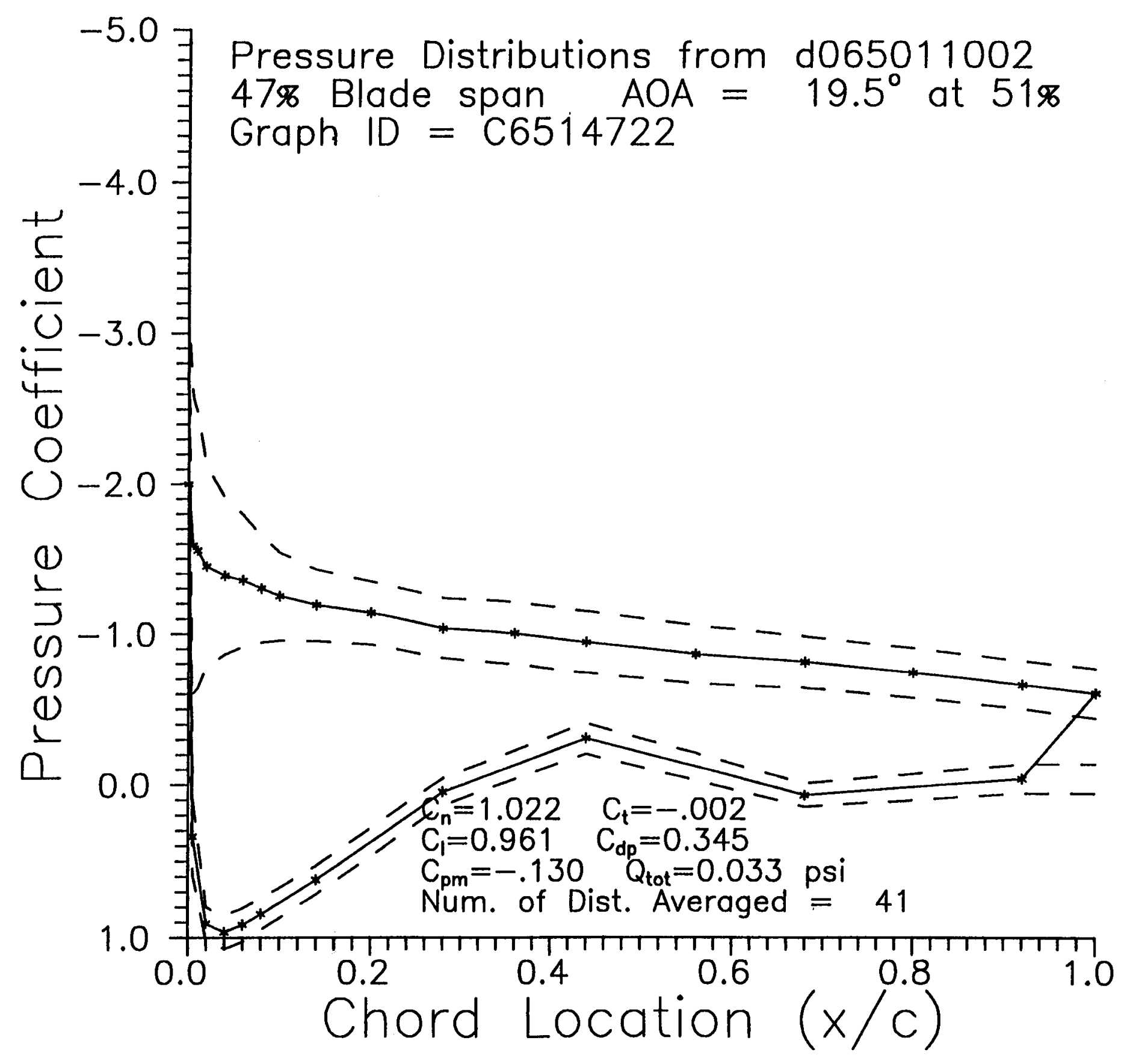




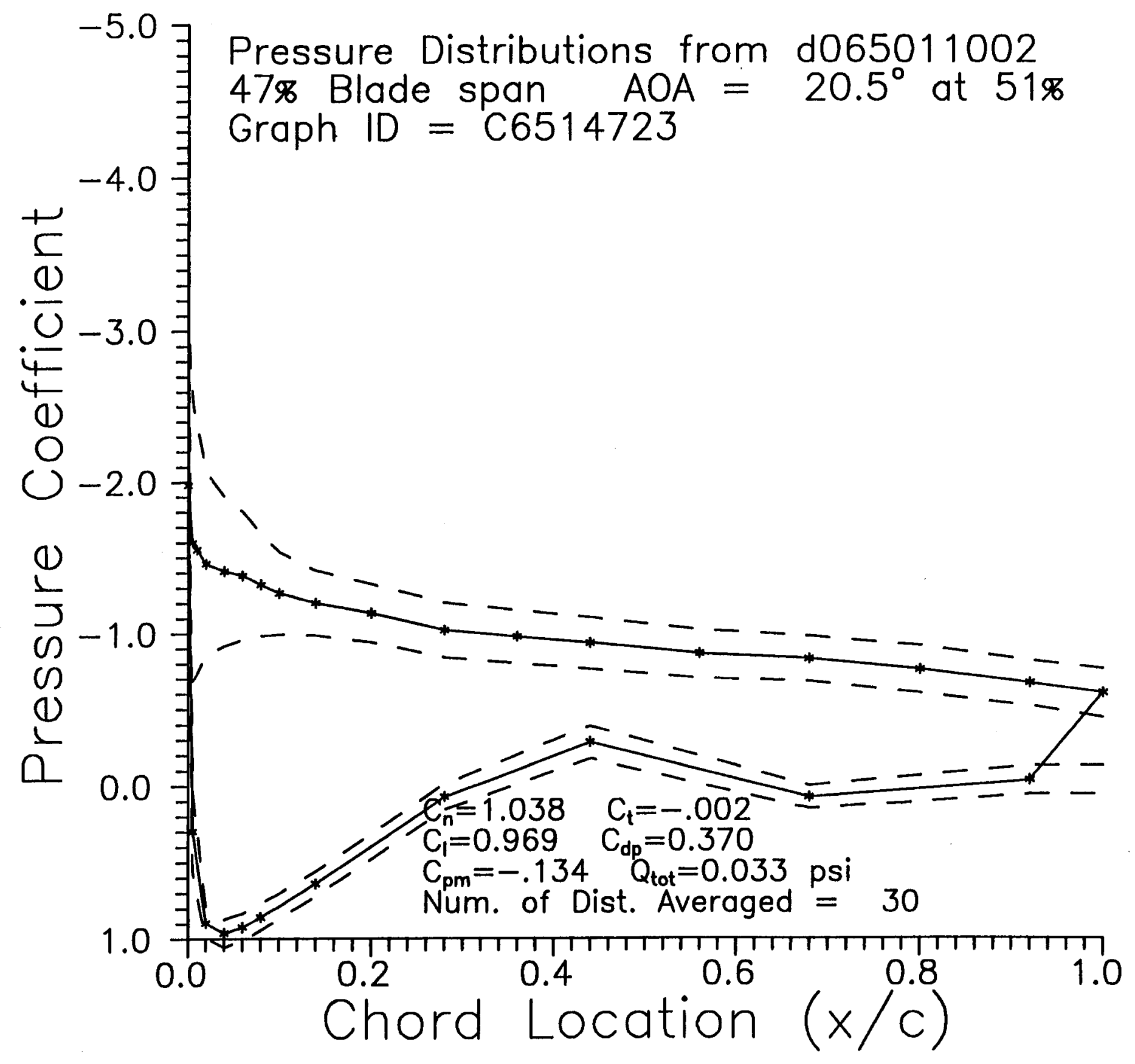




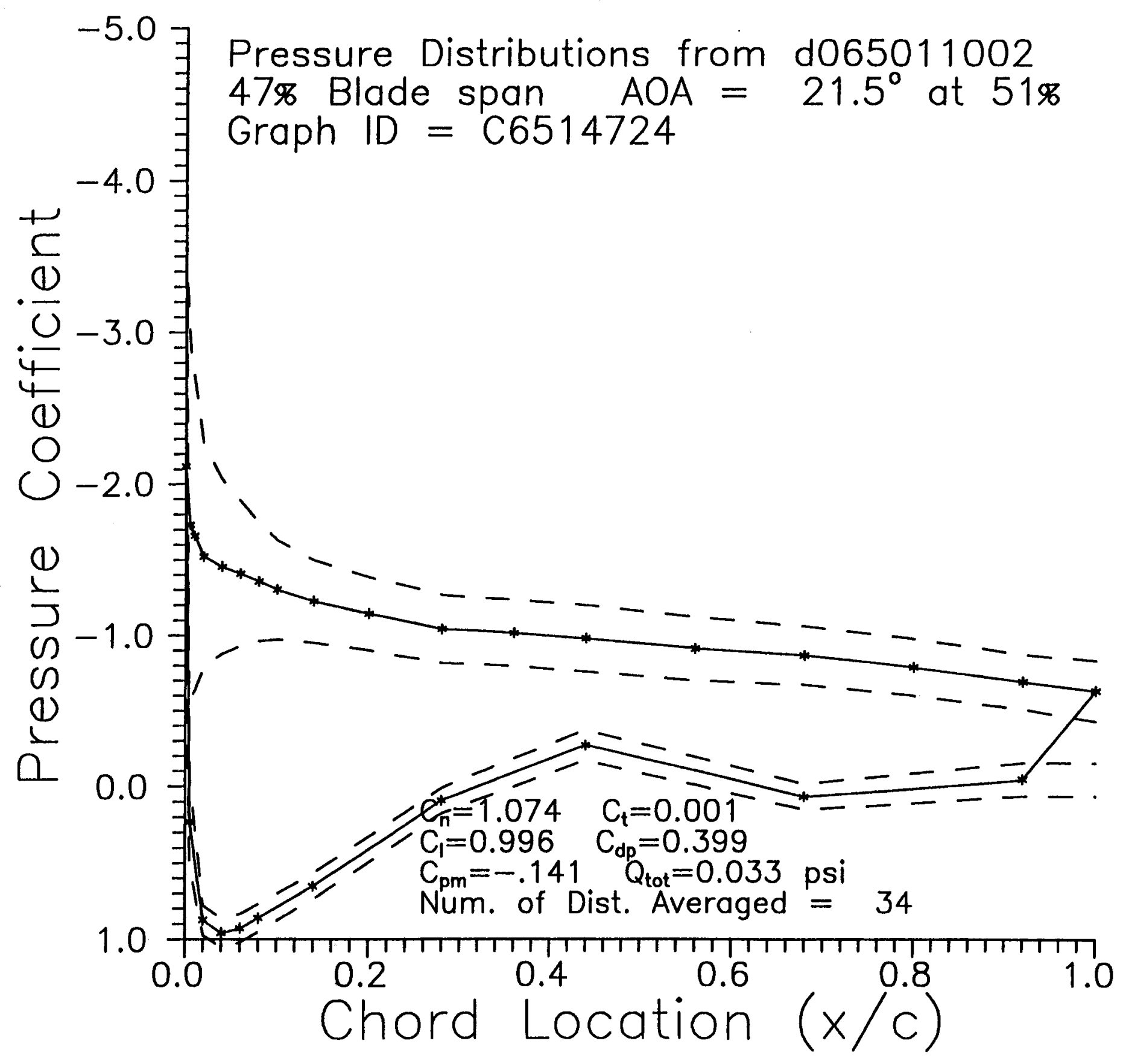




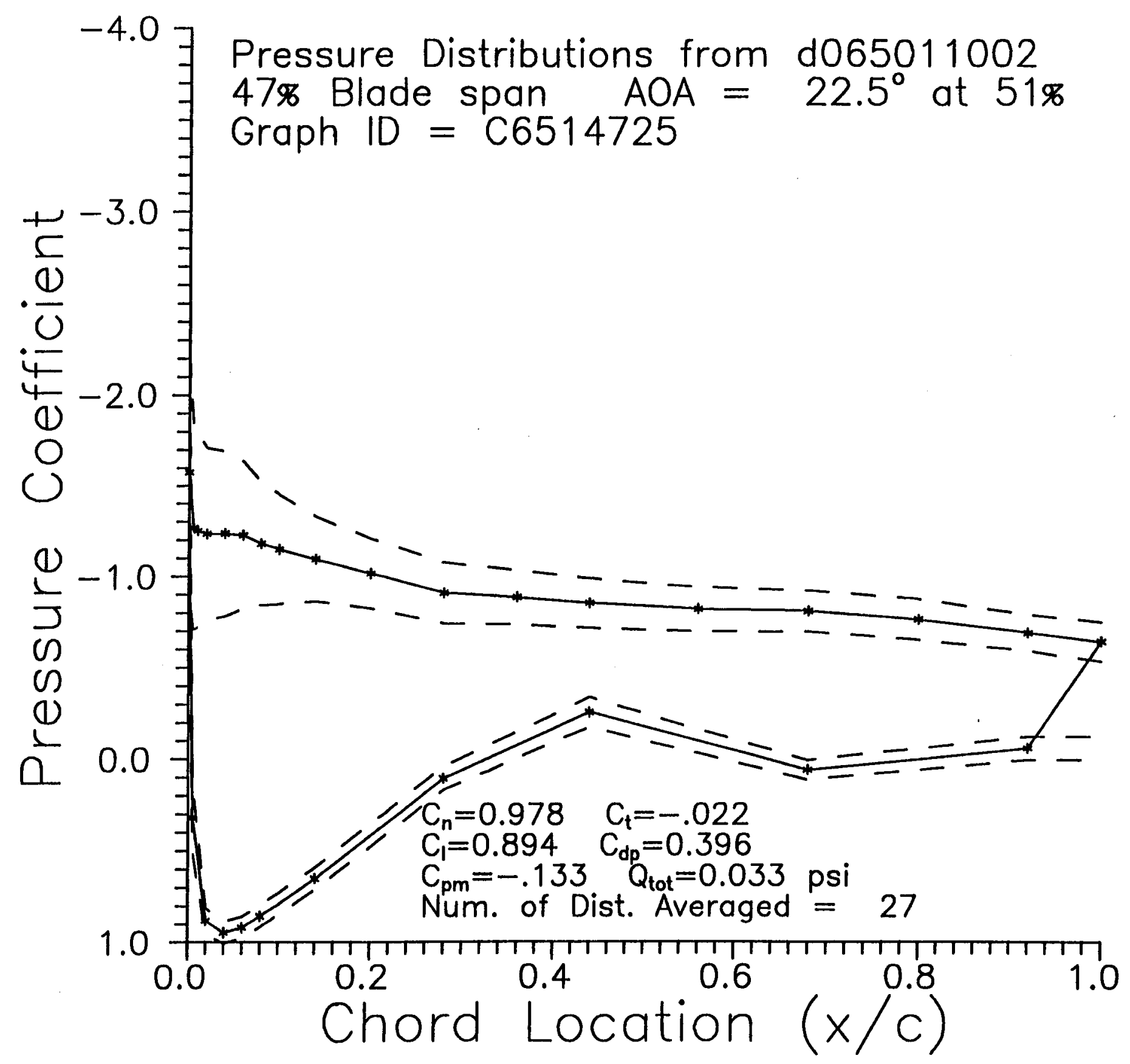




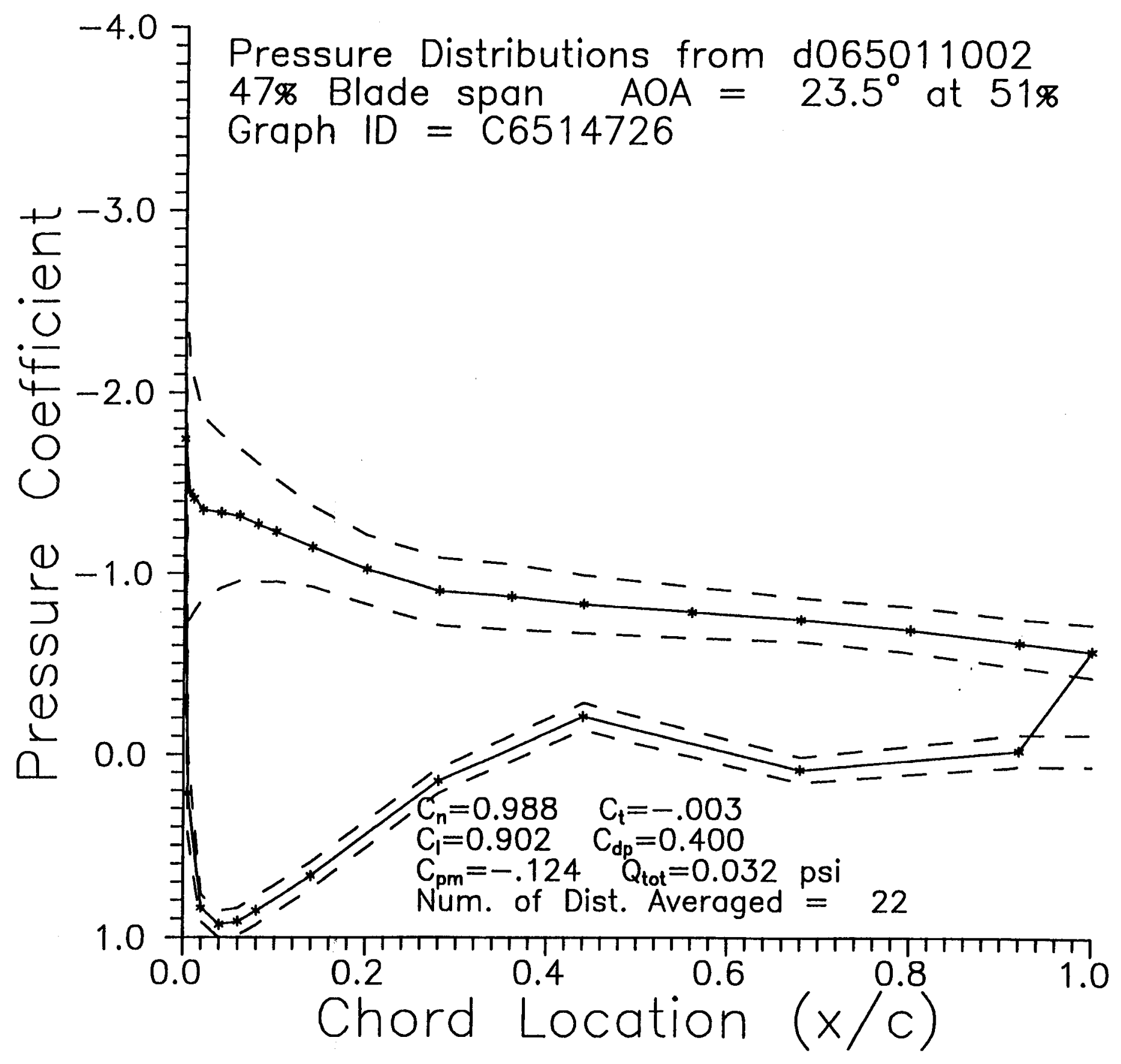




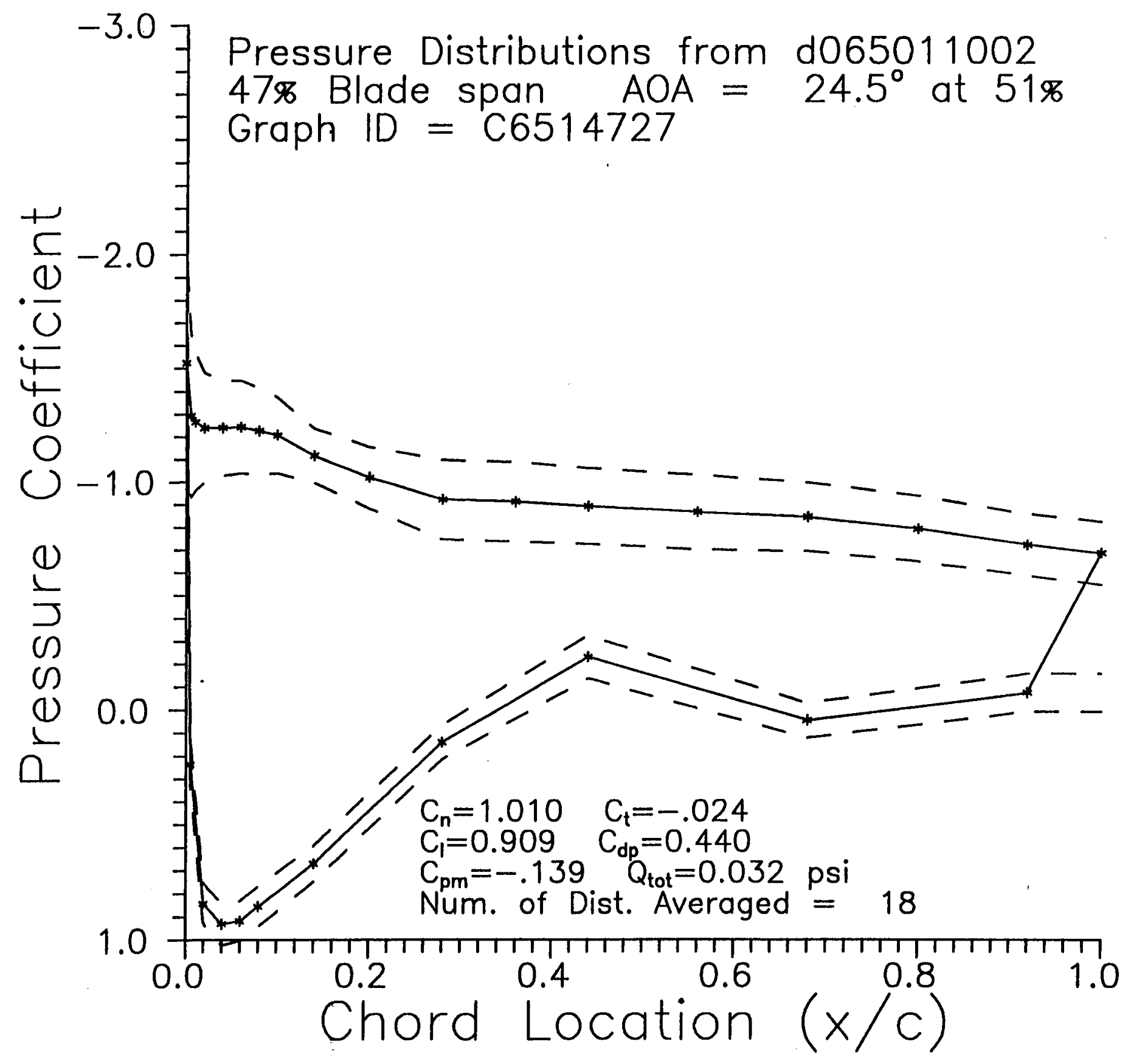




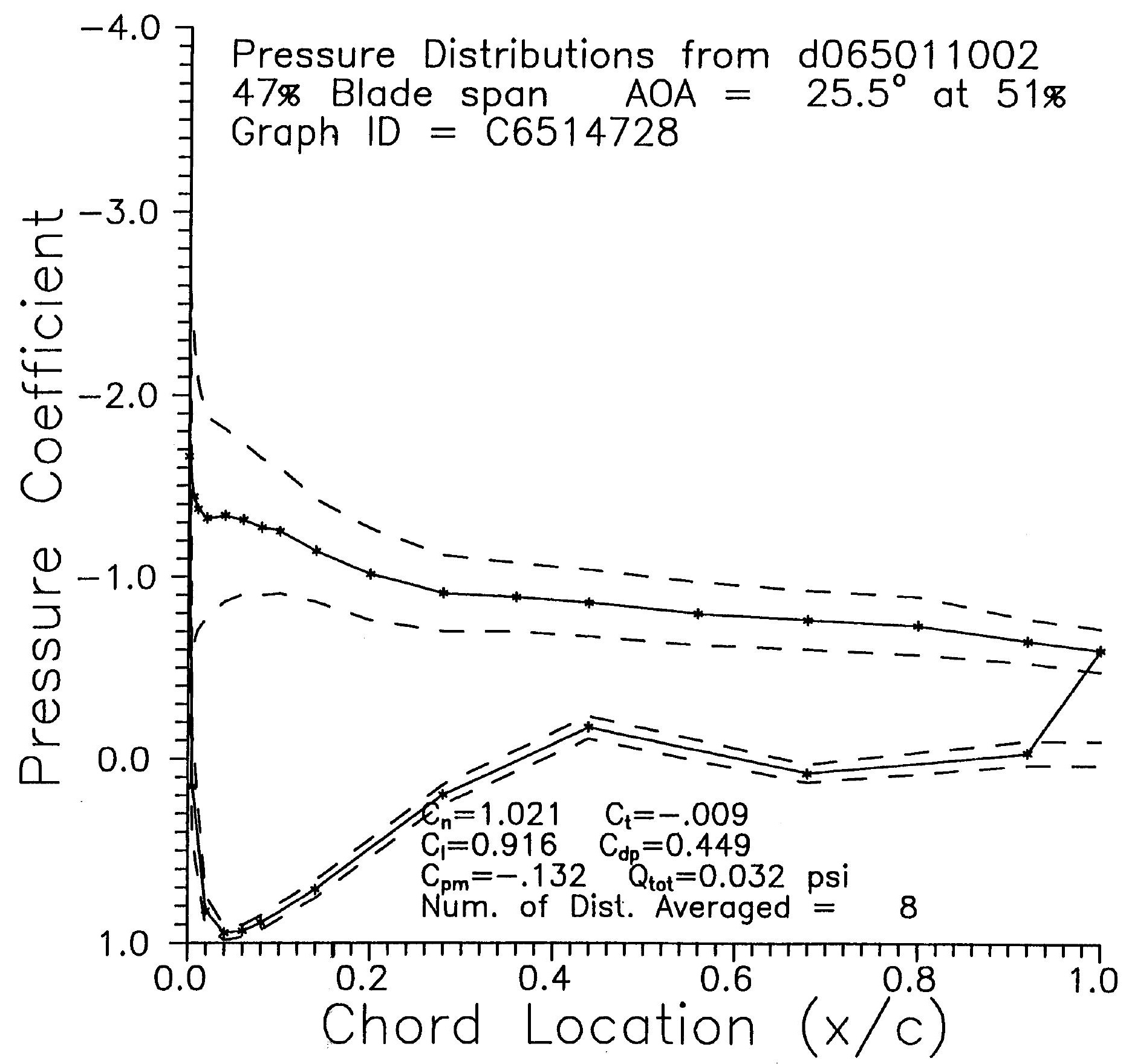


Appendix C.1.5

Pressure Distributions for $30 \%$ Span 


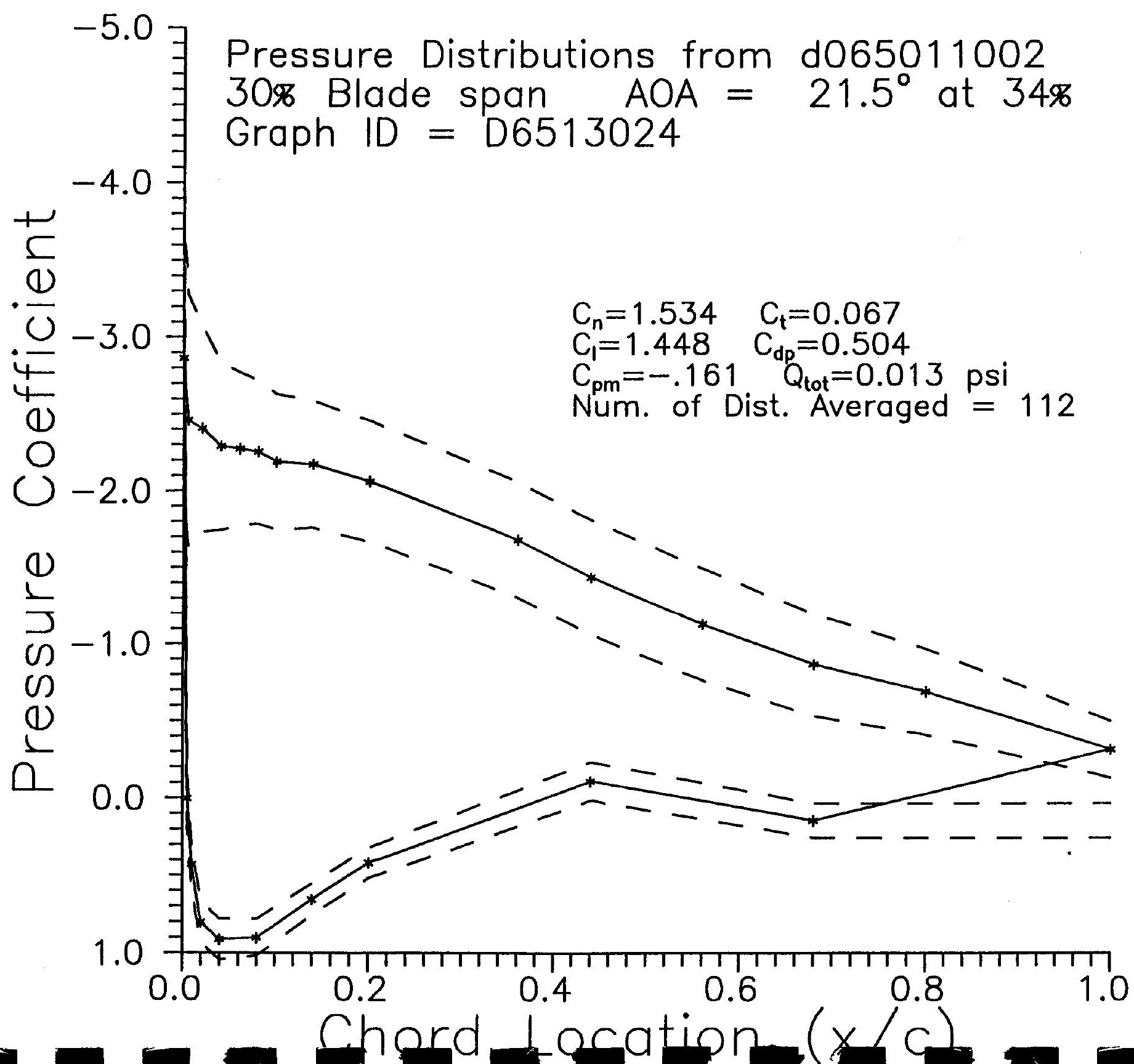




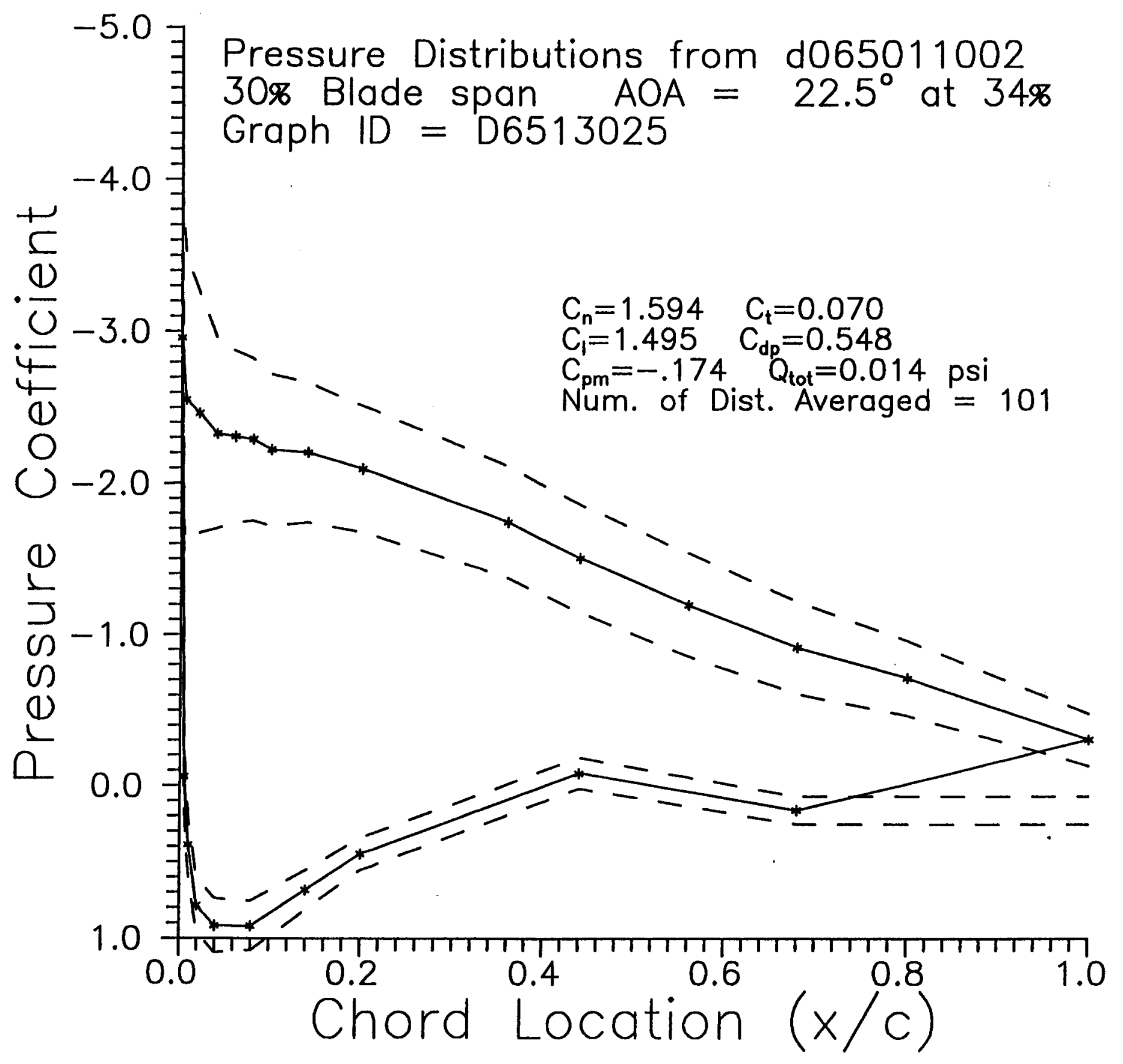




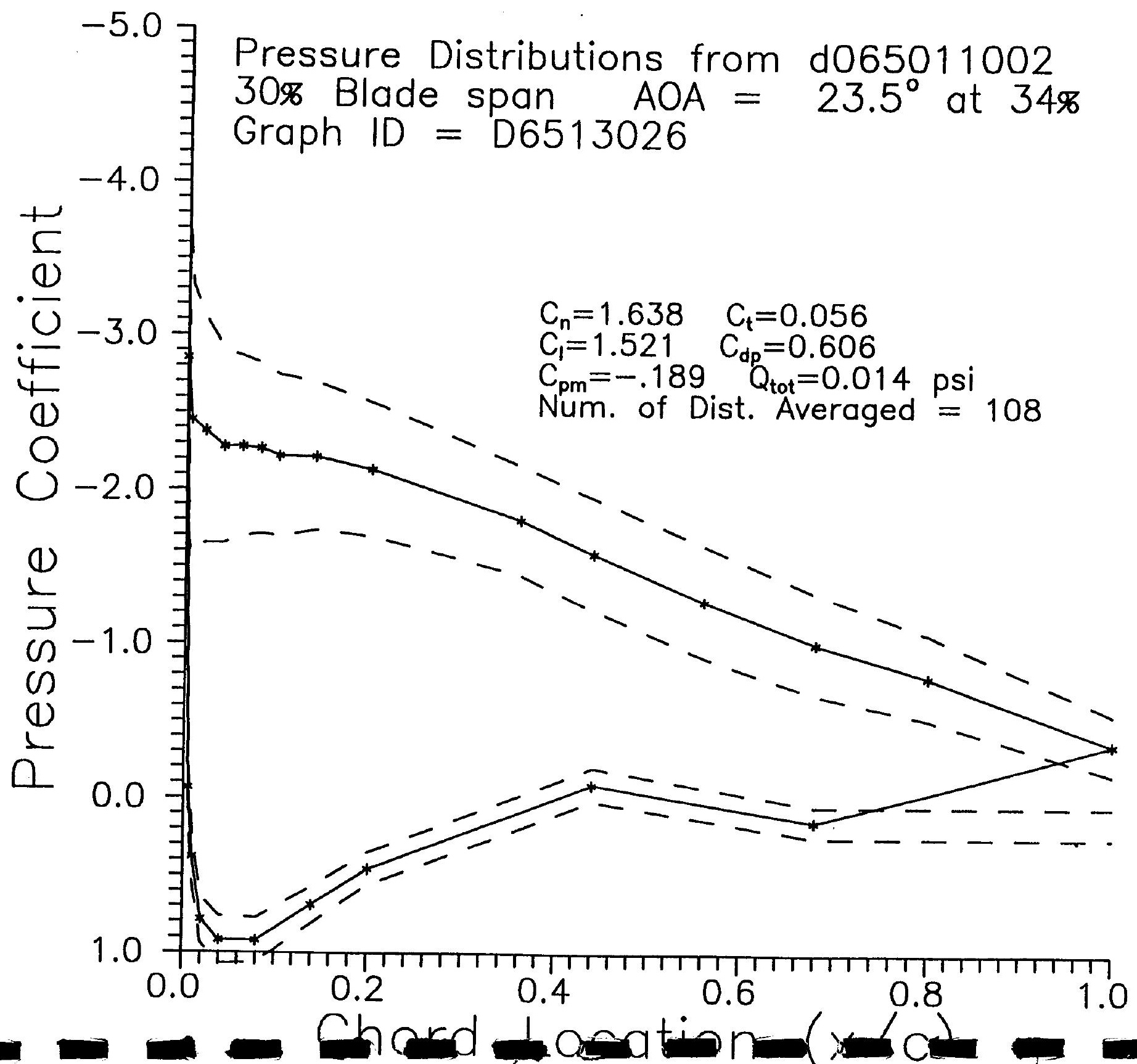




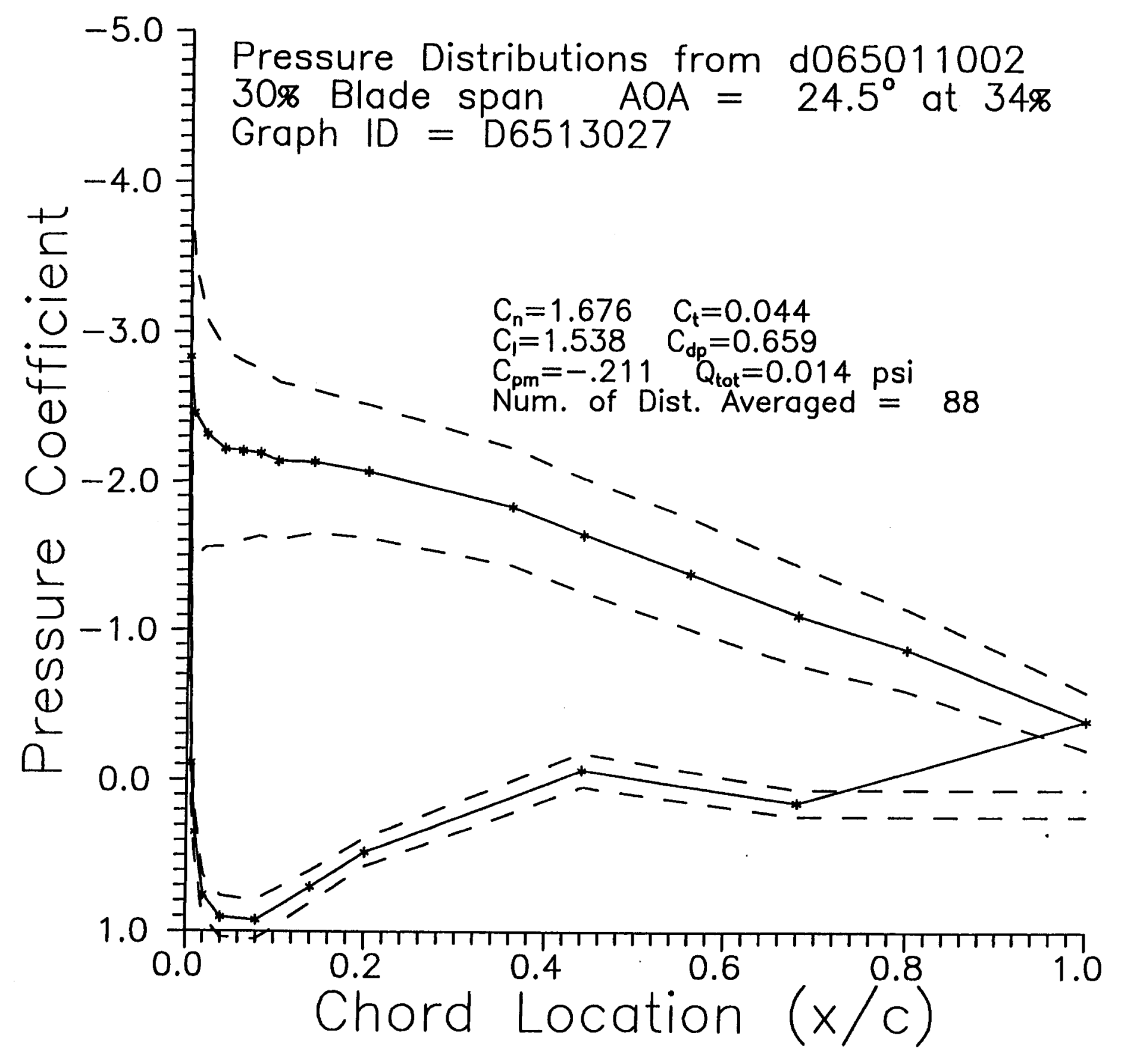




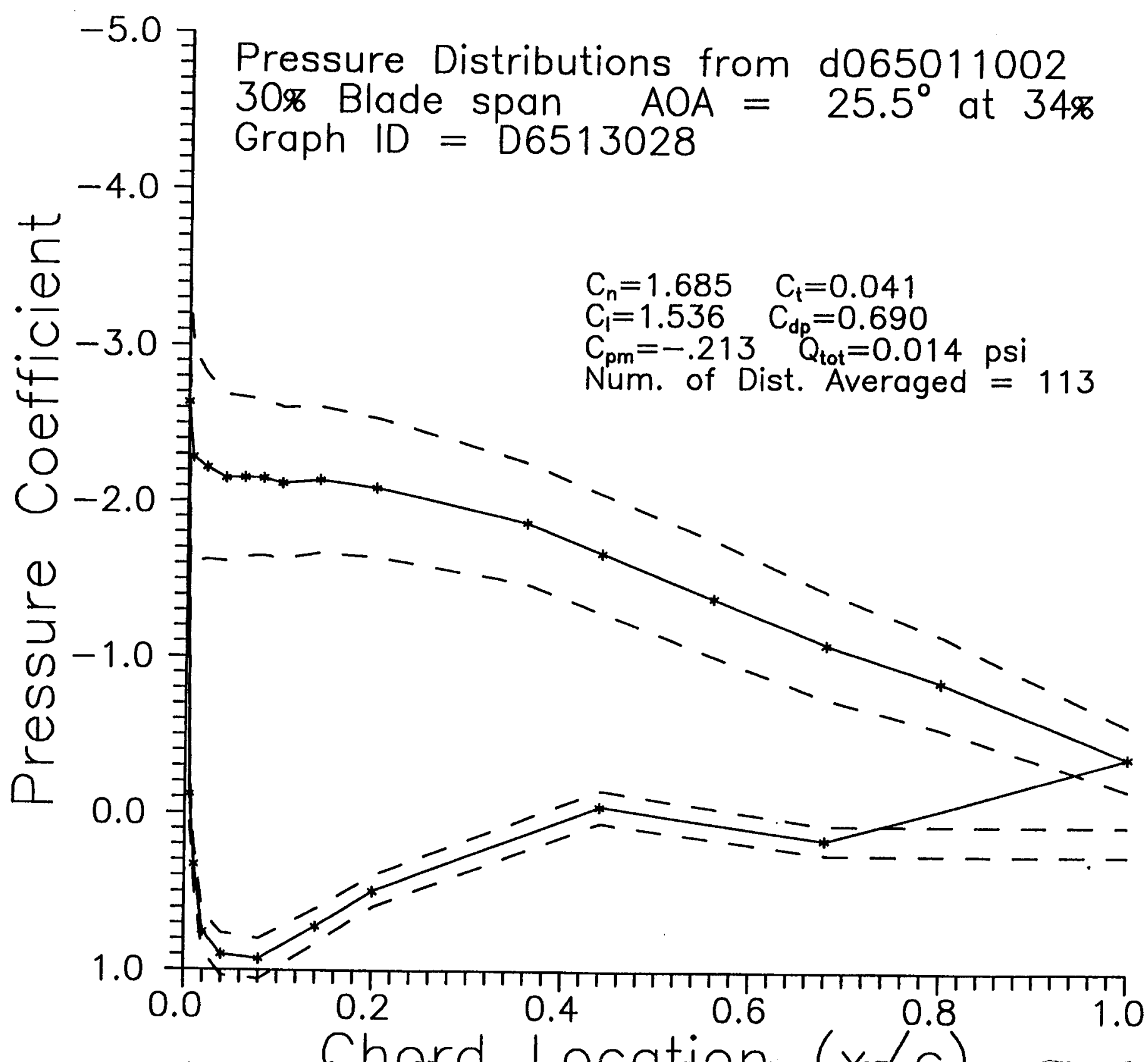




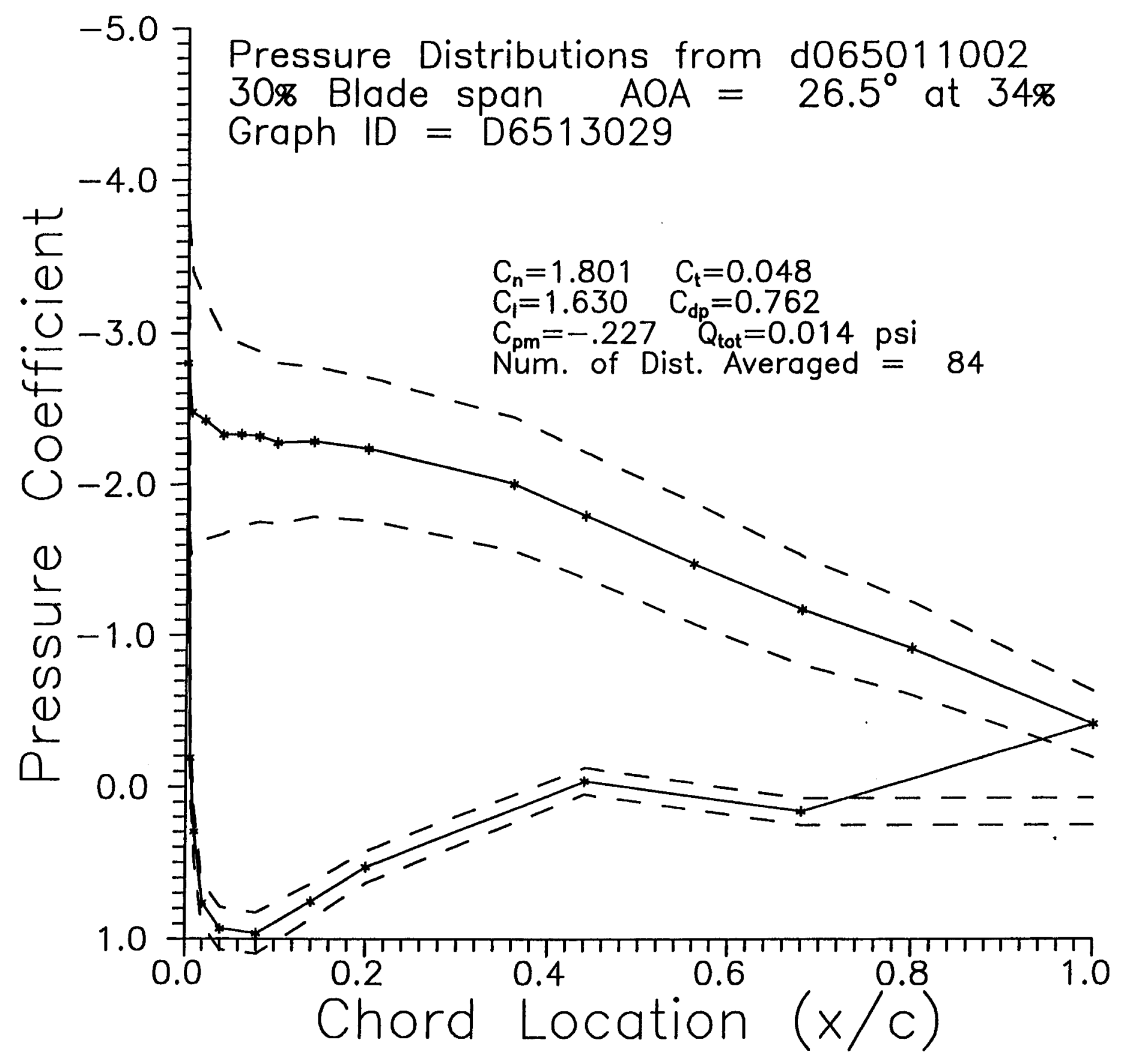




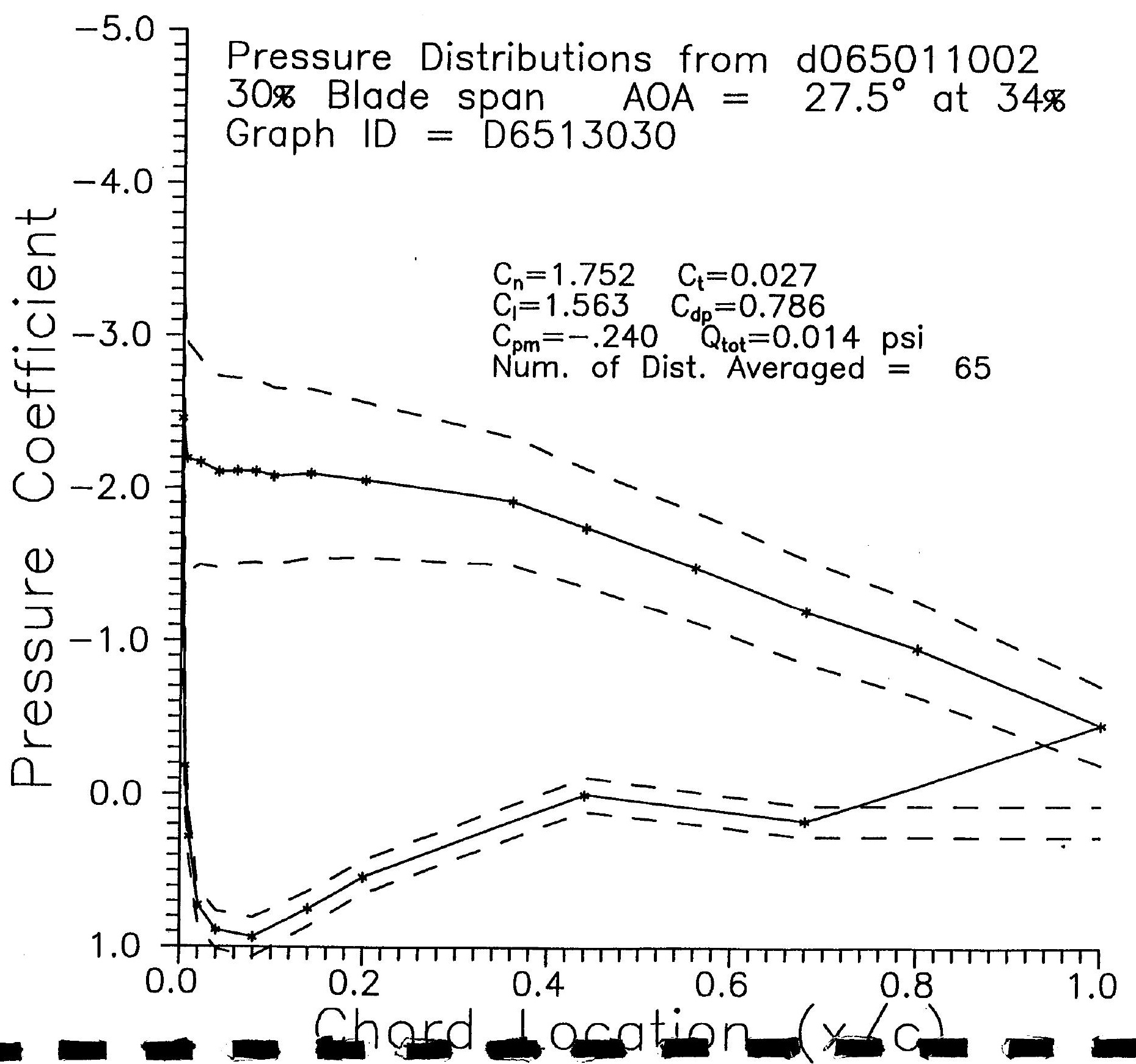




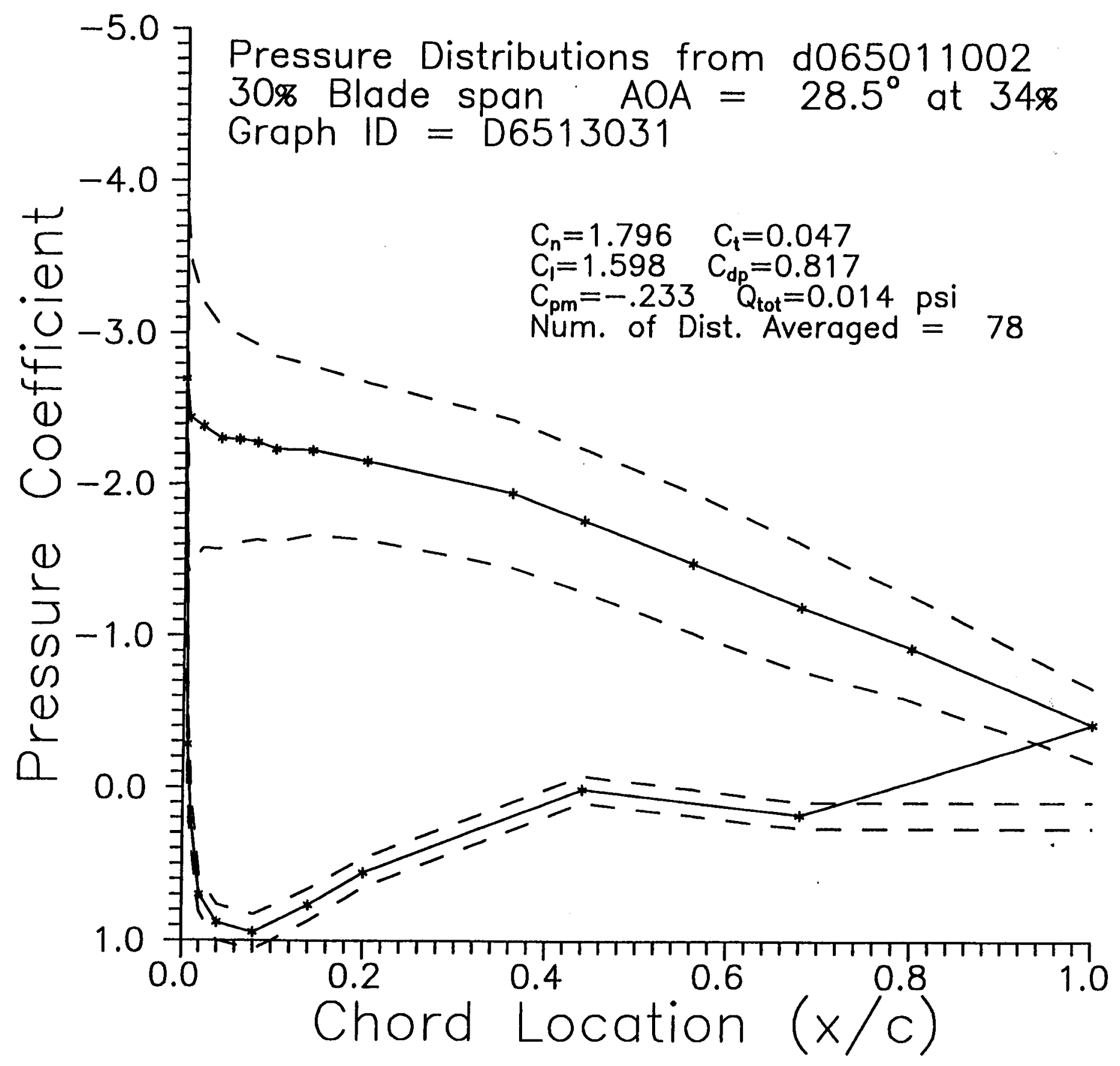




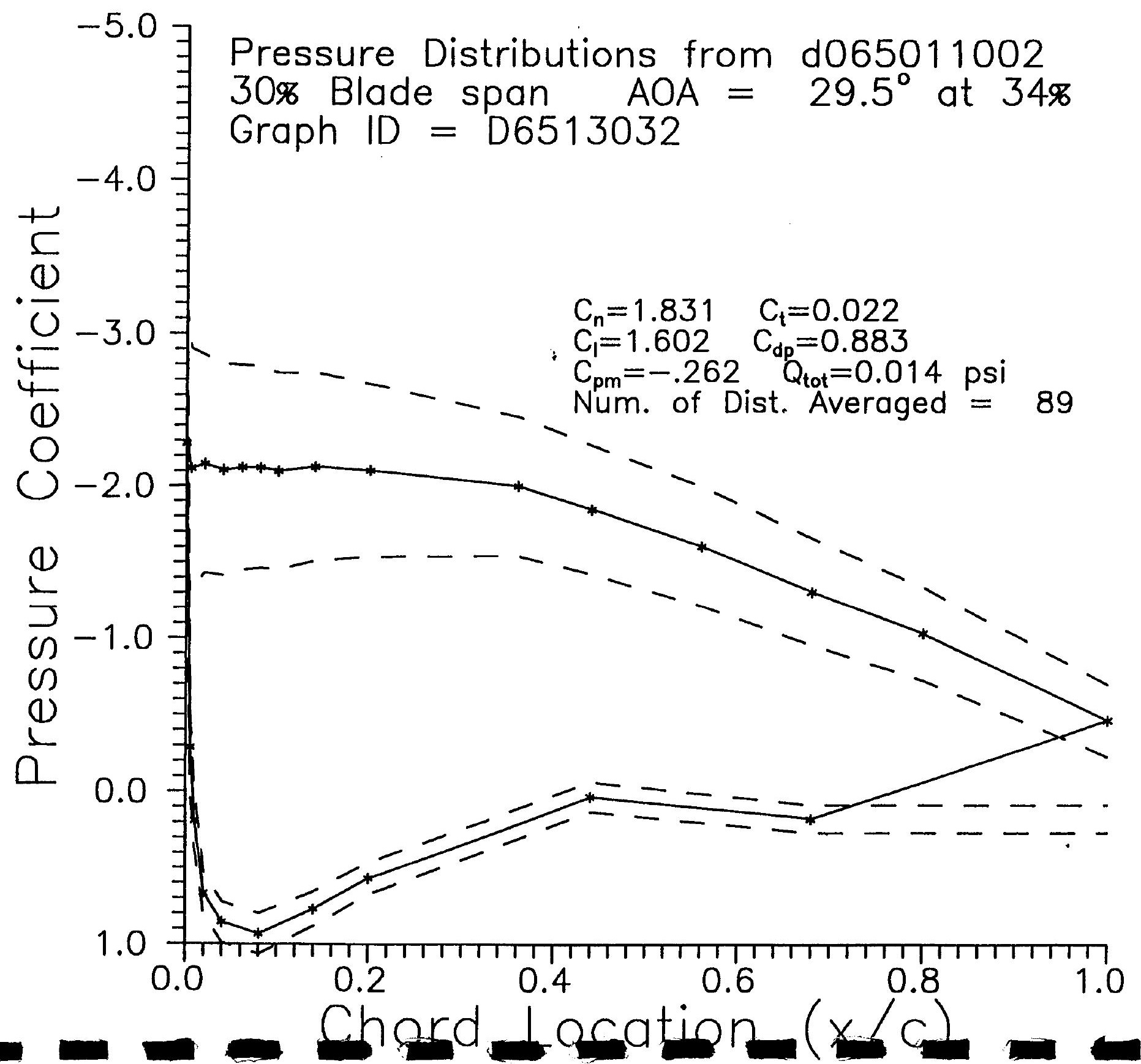




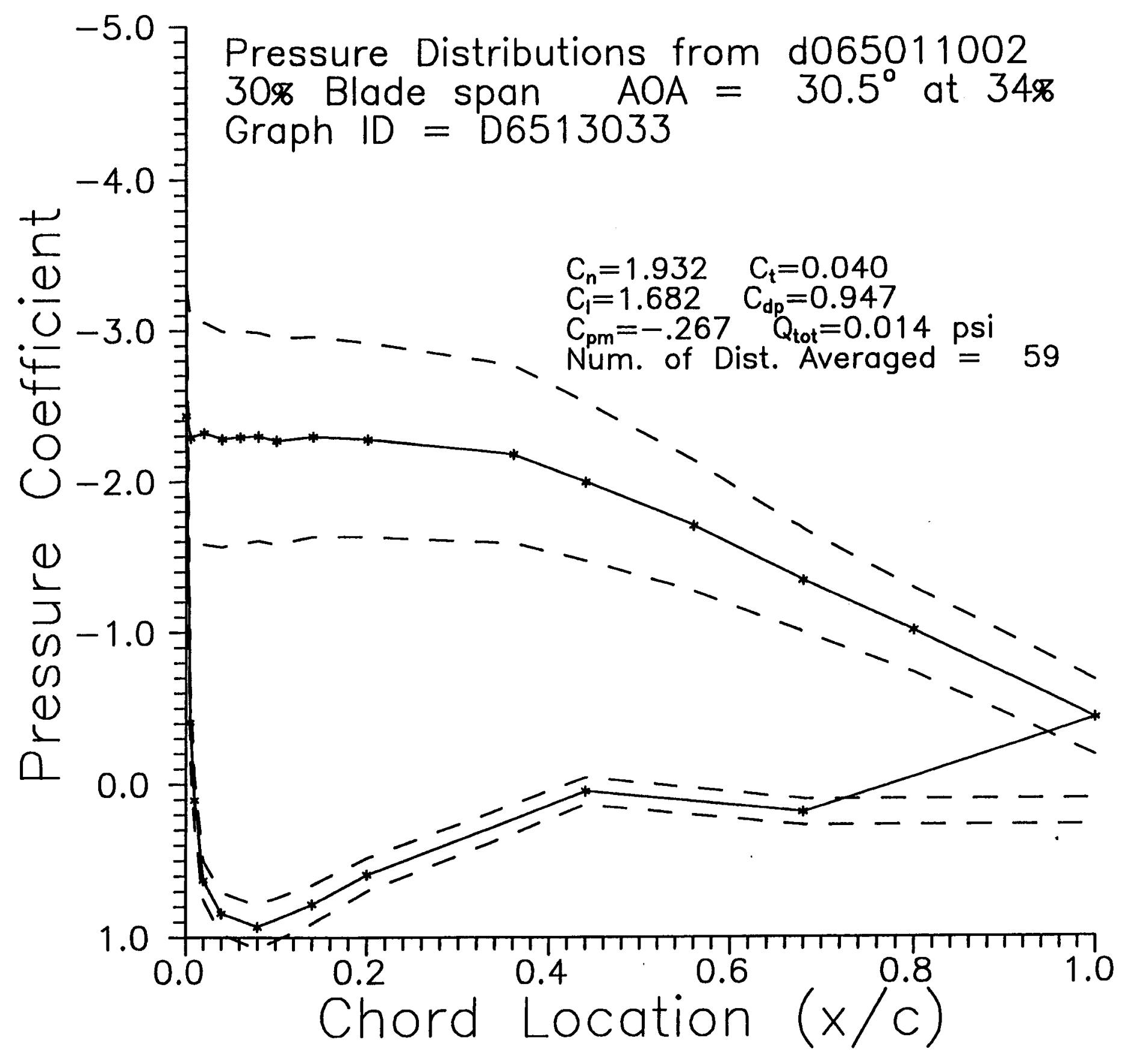




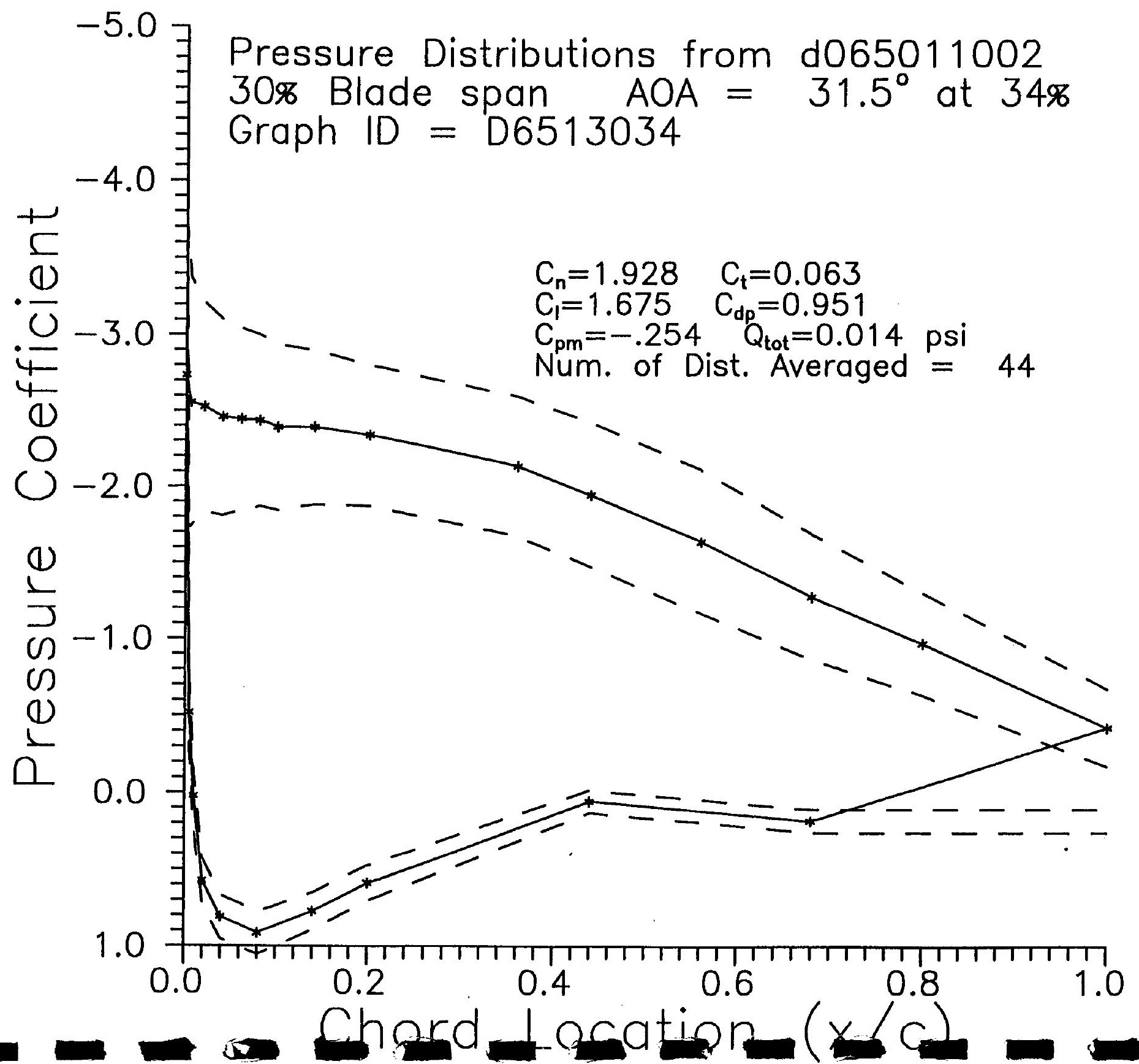




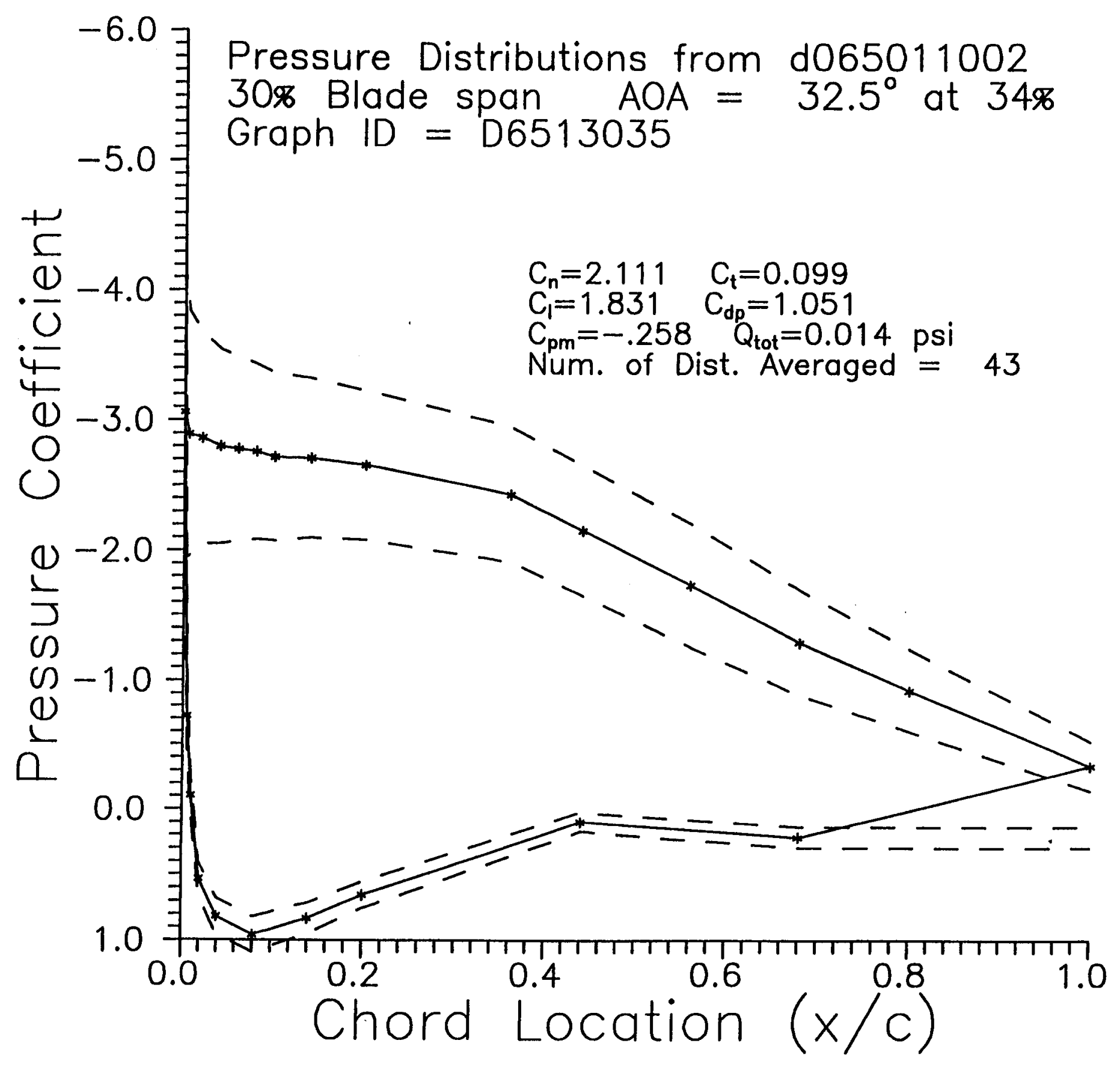




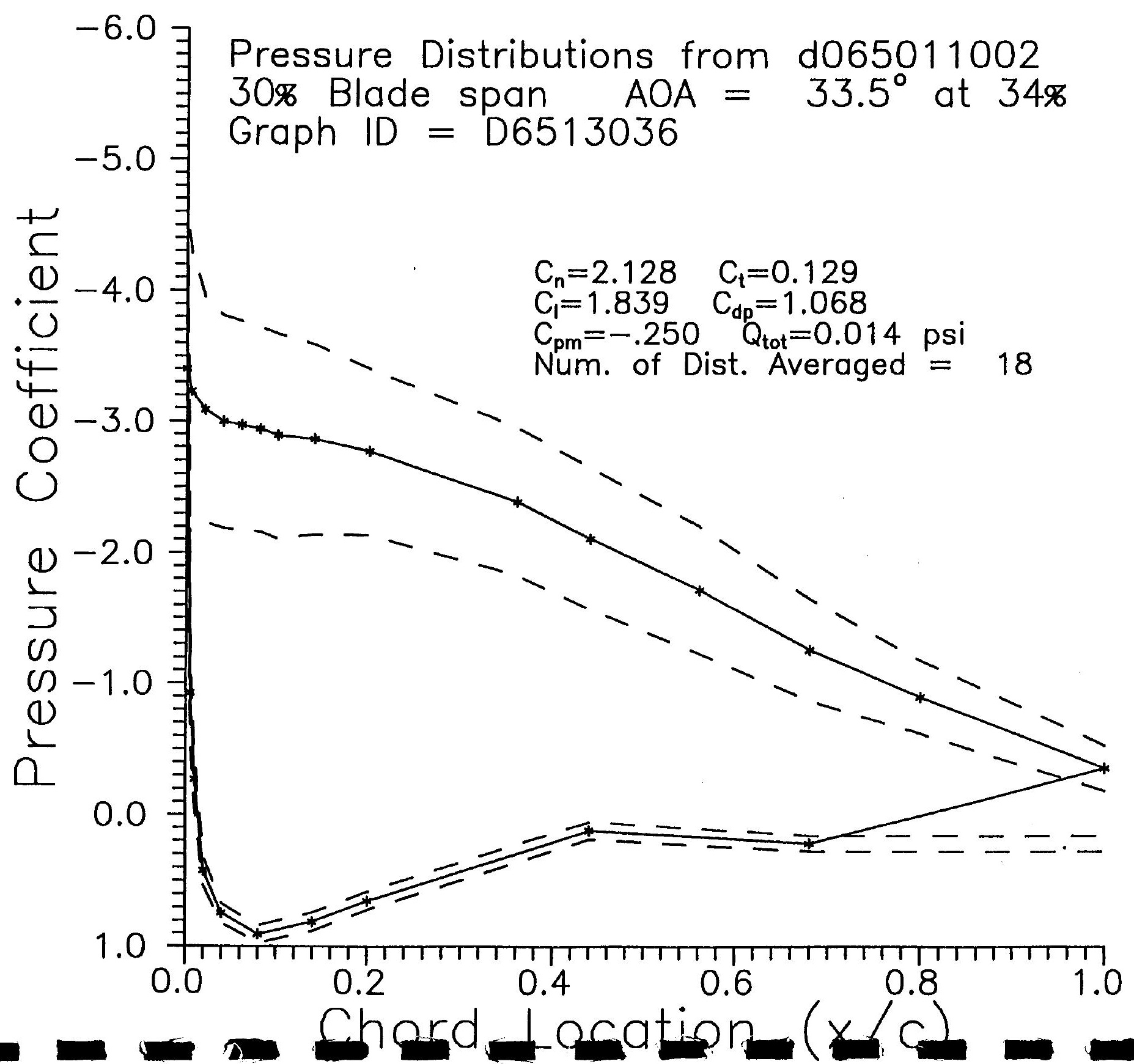




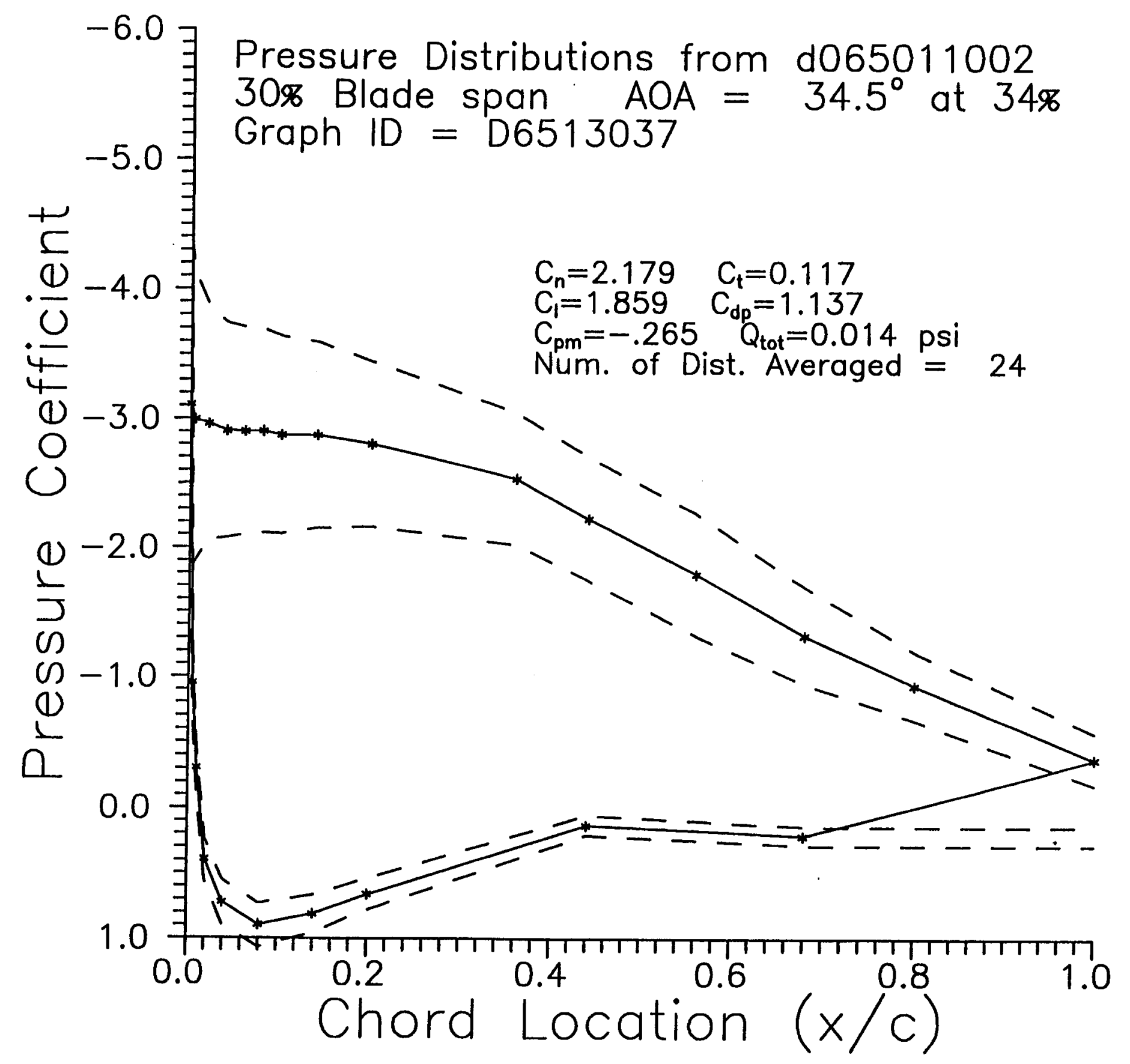




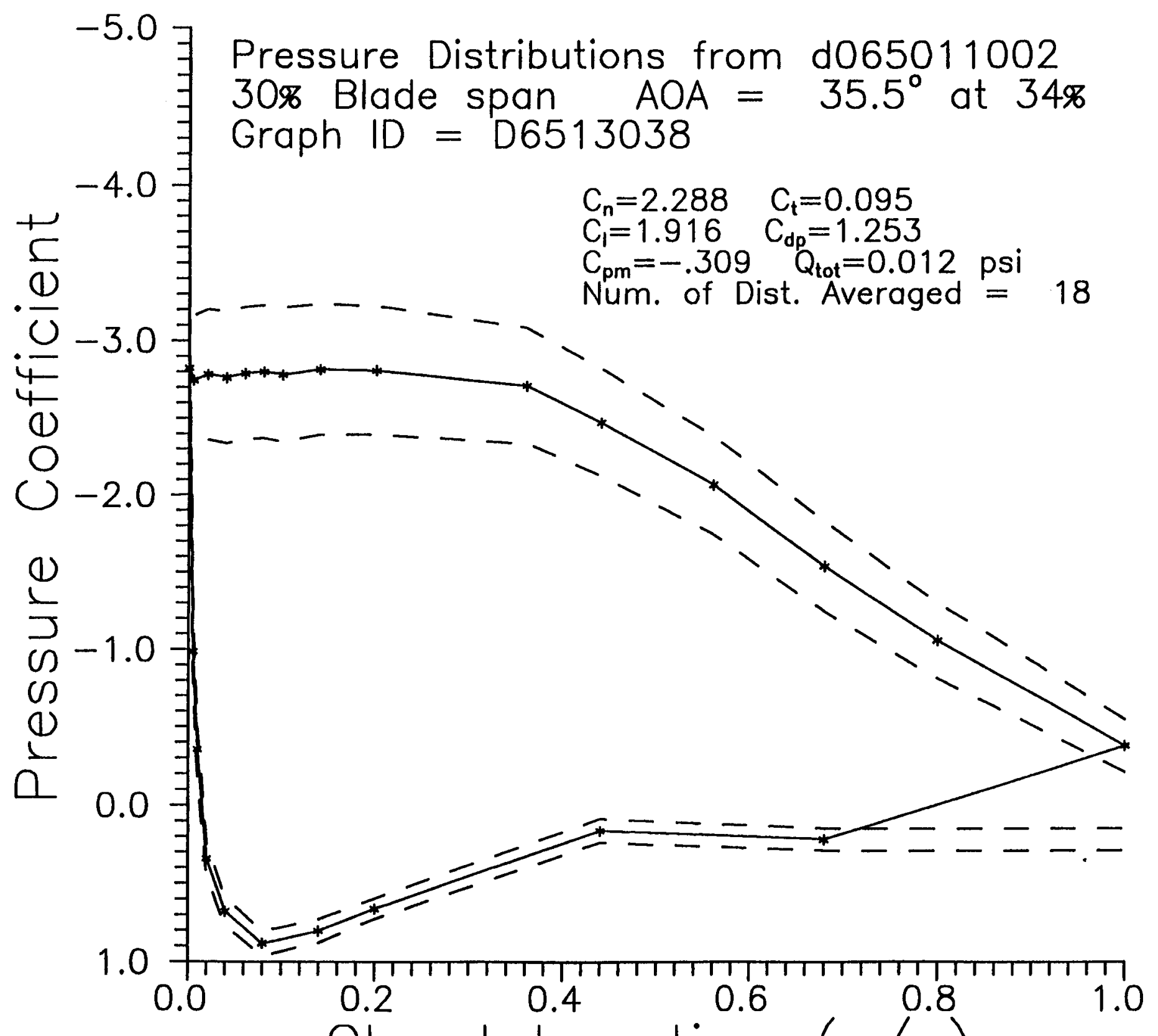

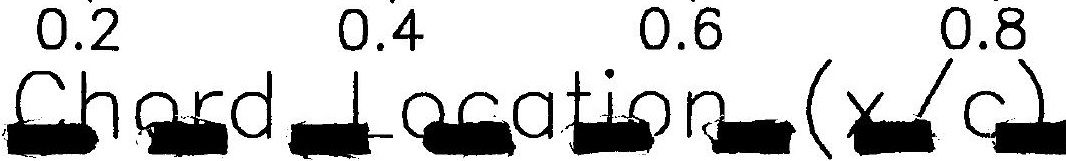




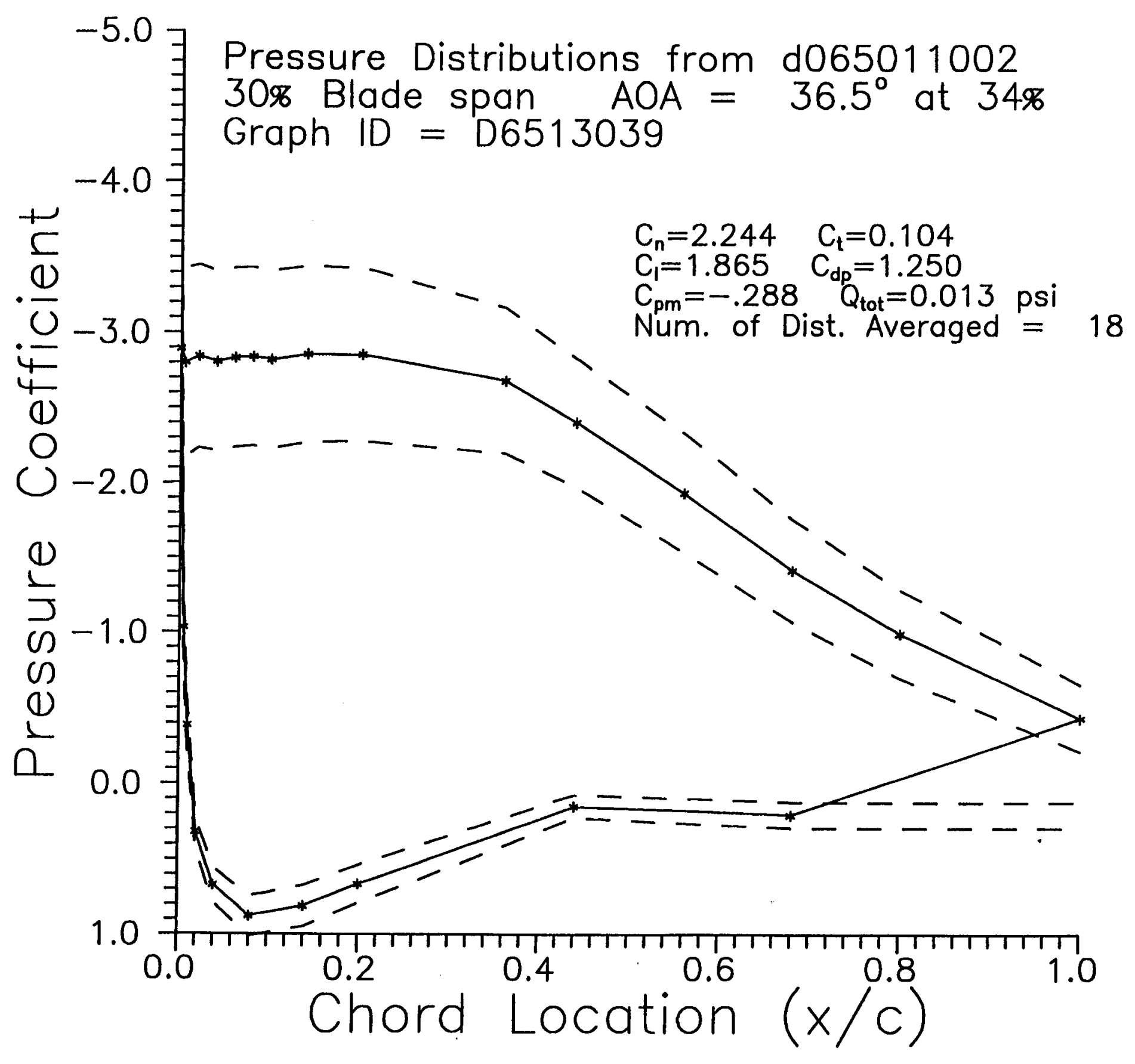




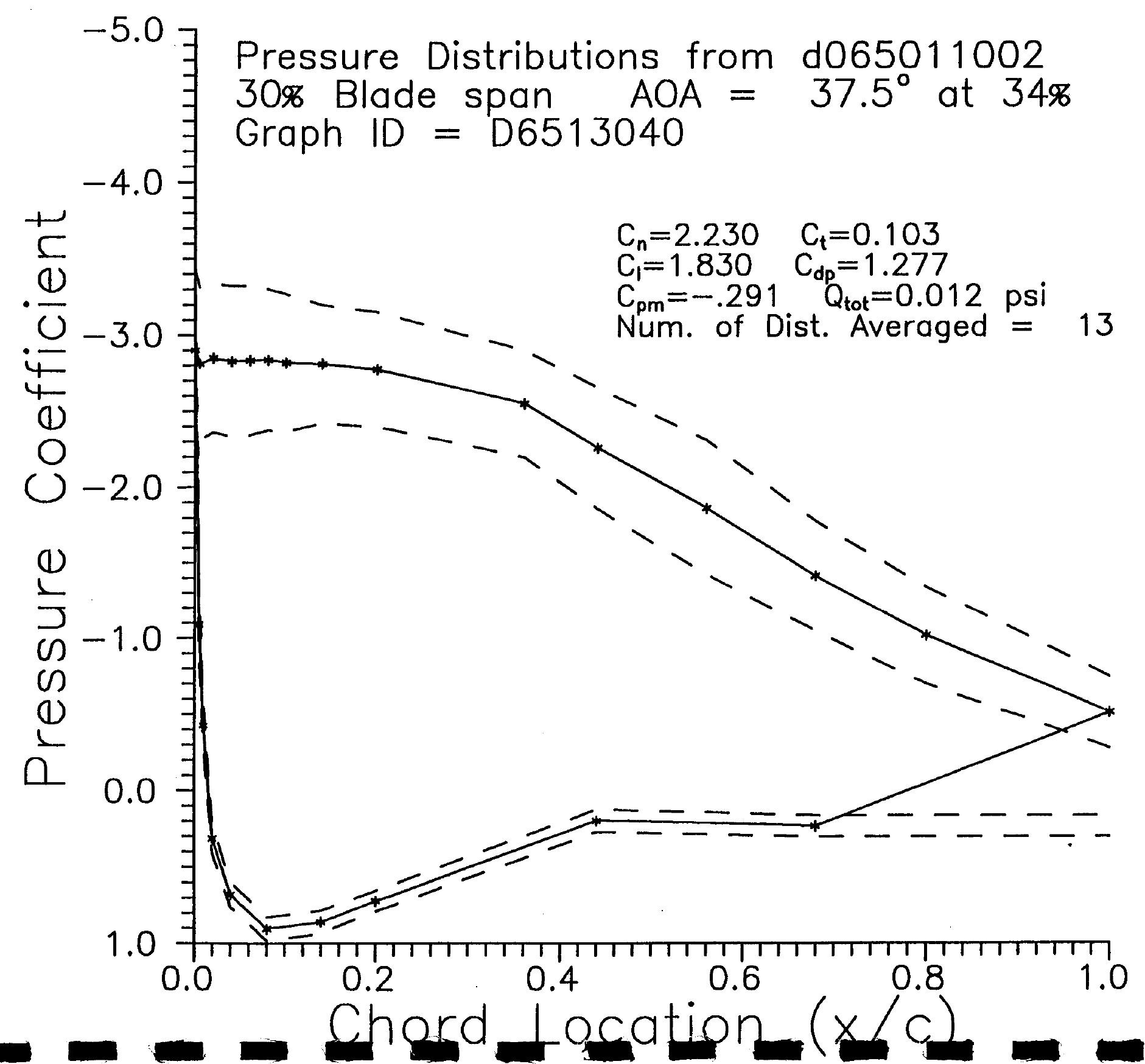




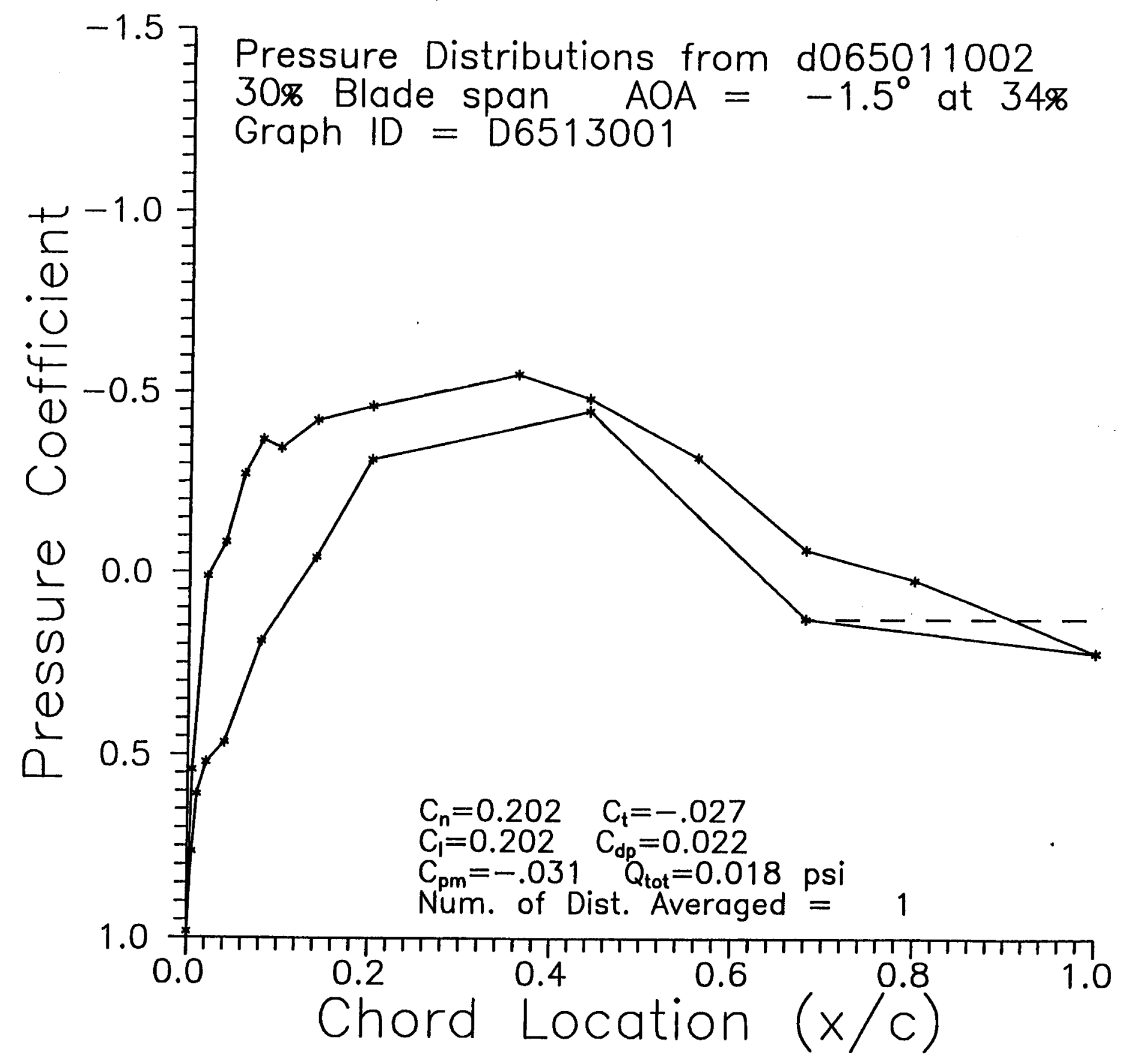




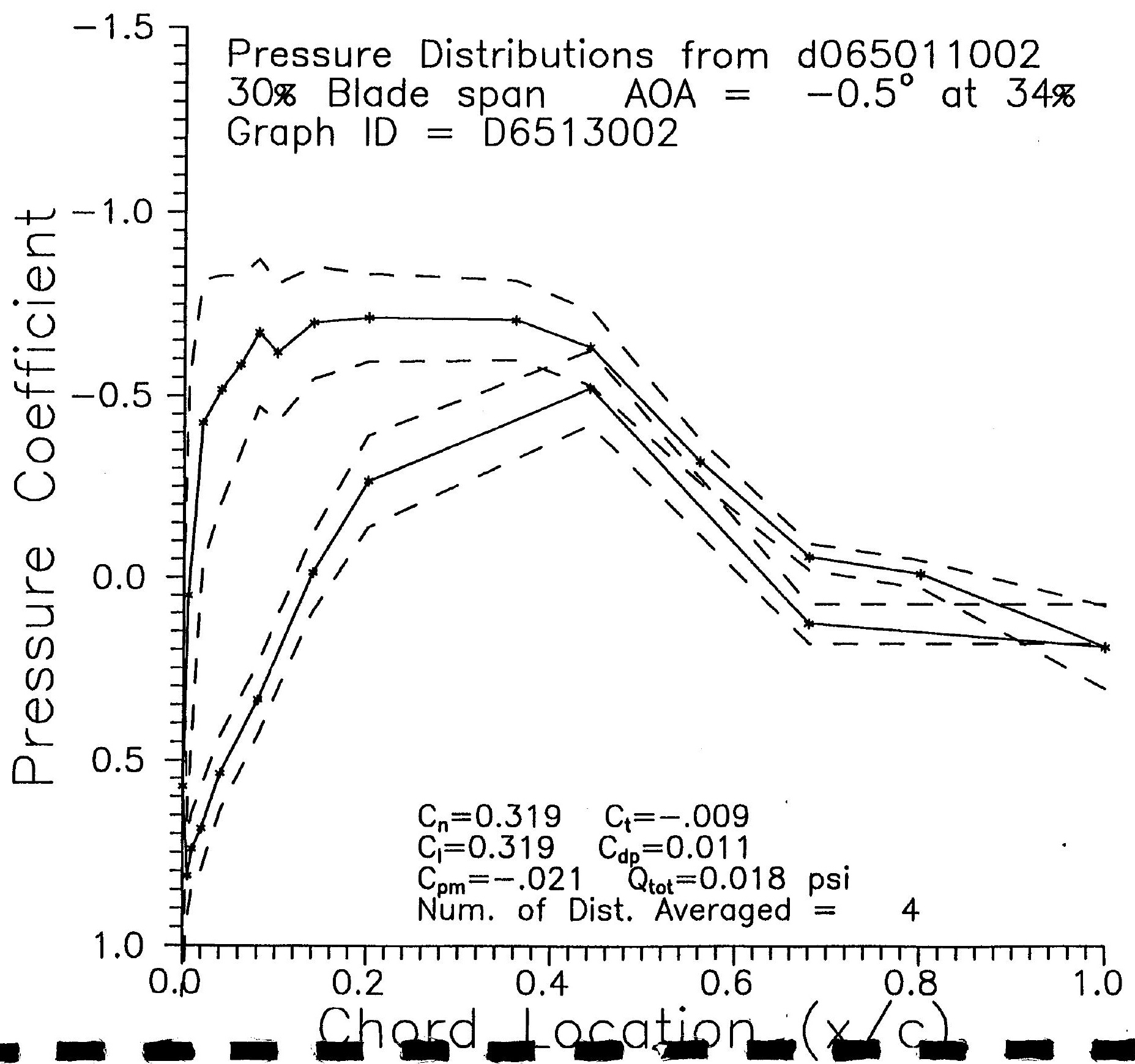




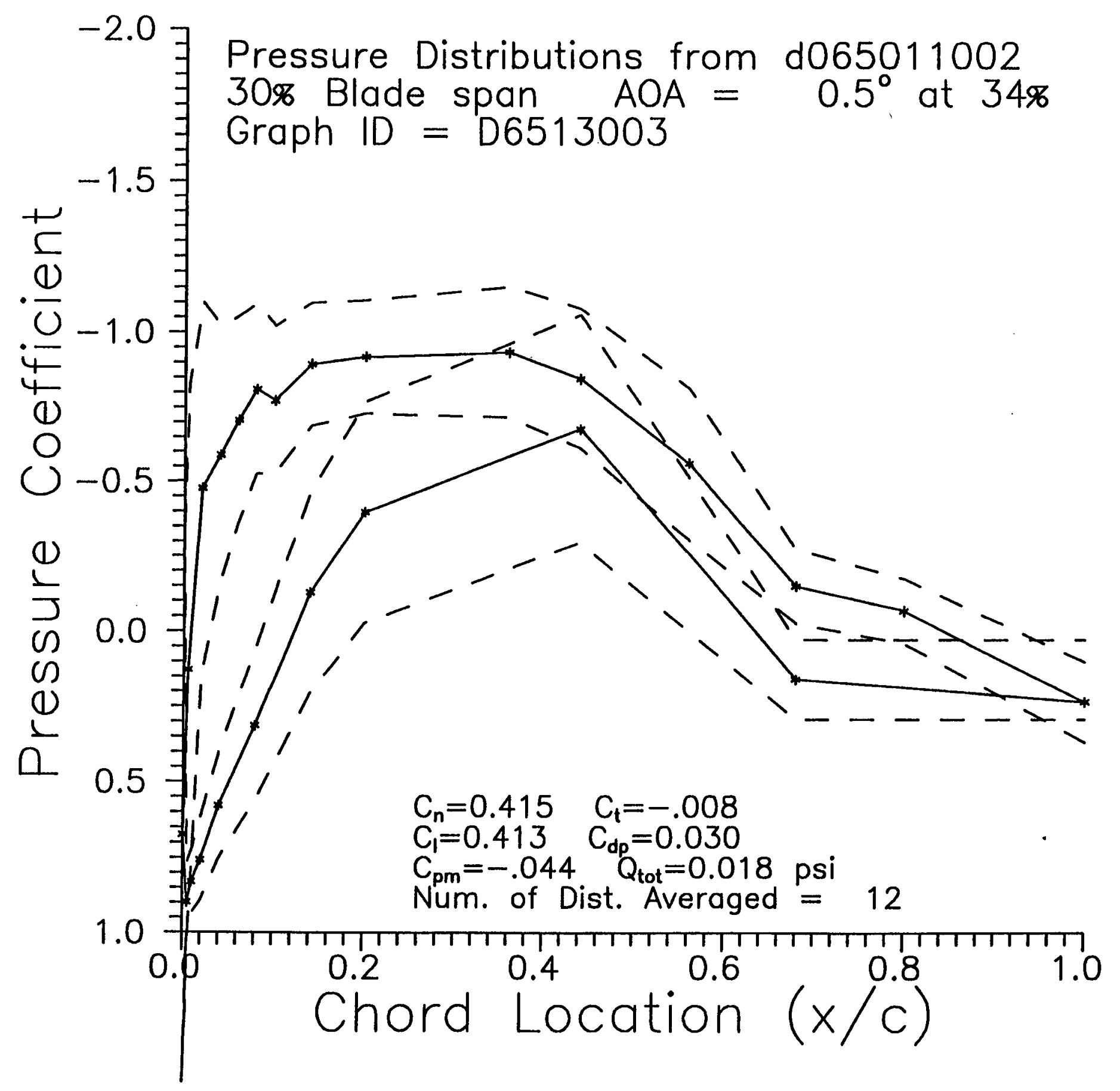




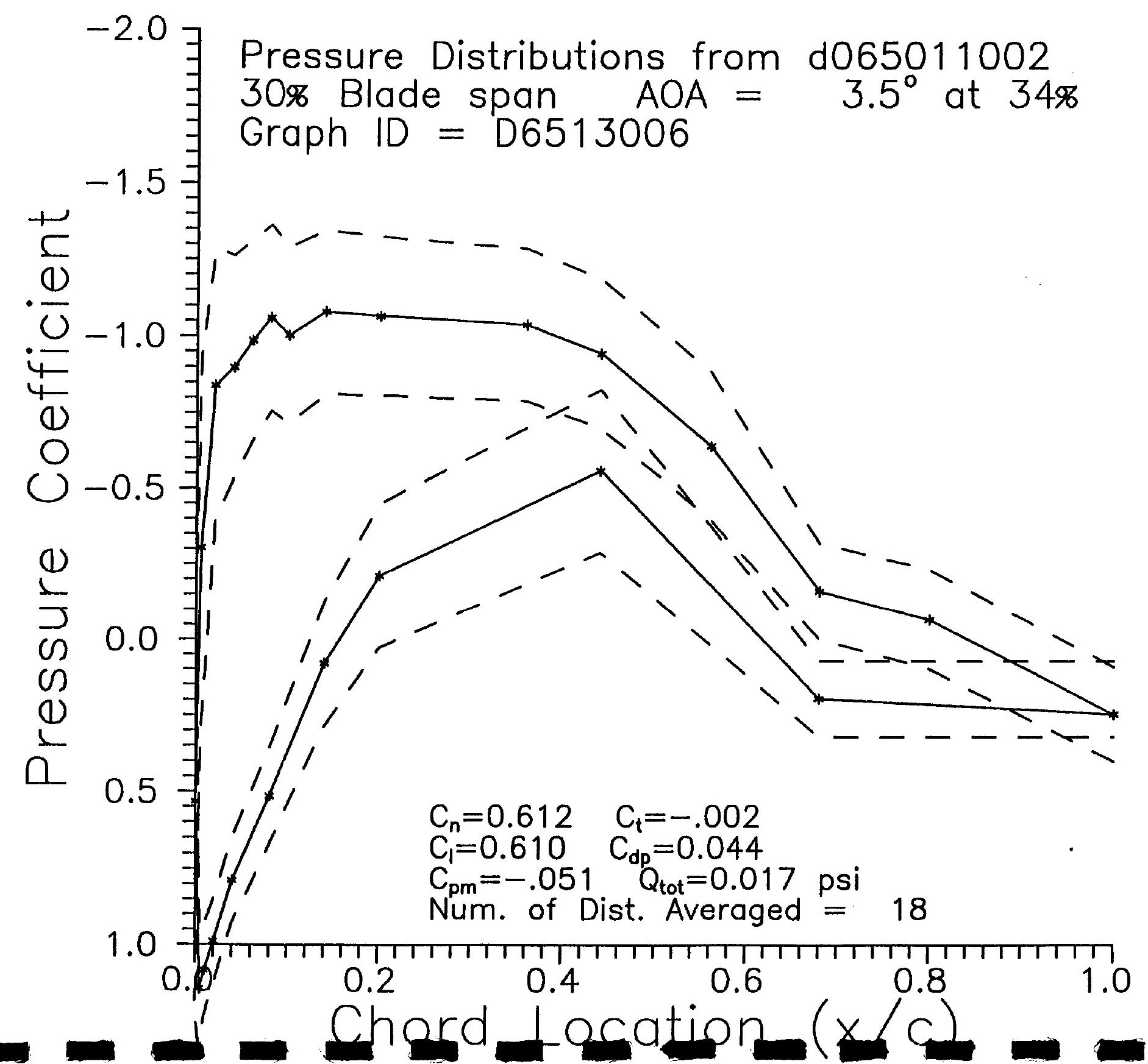




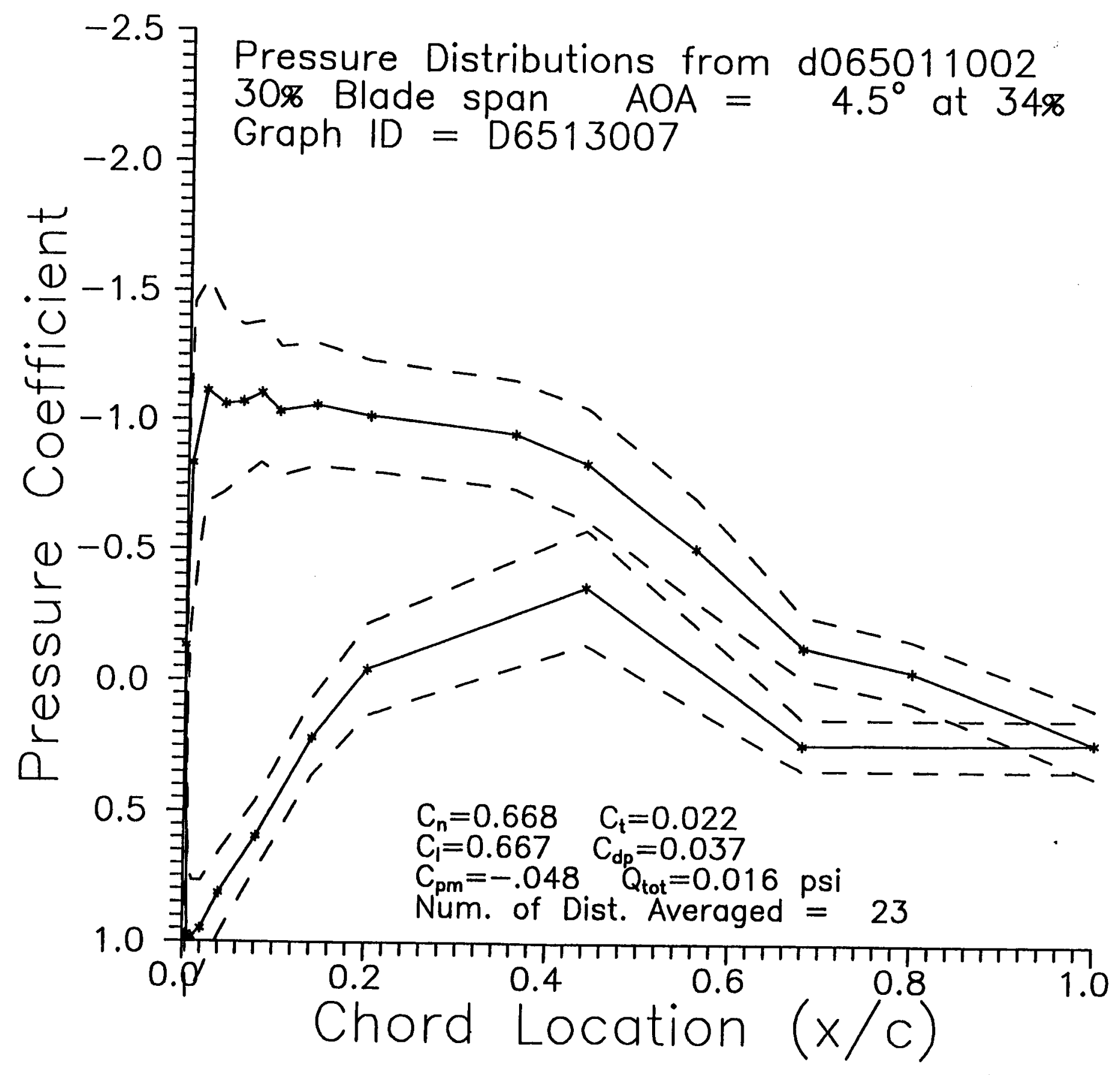




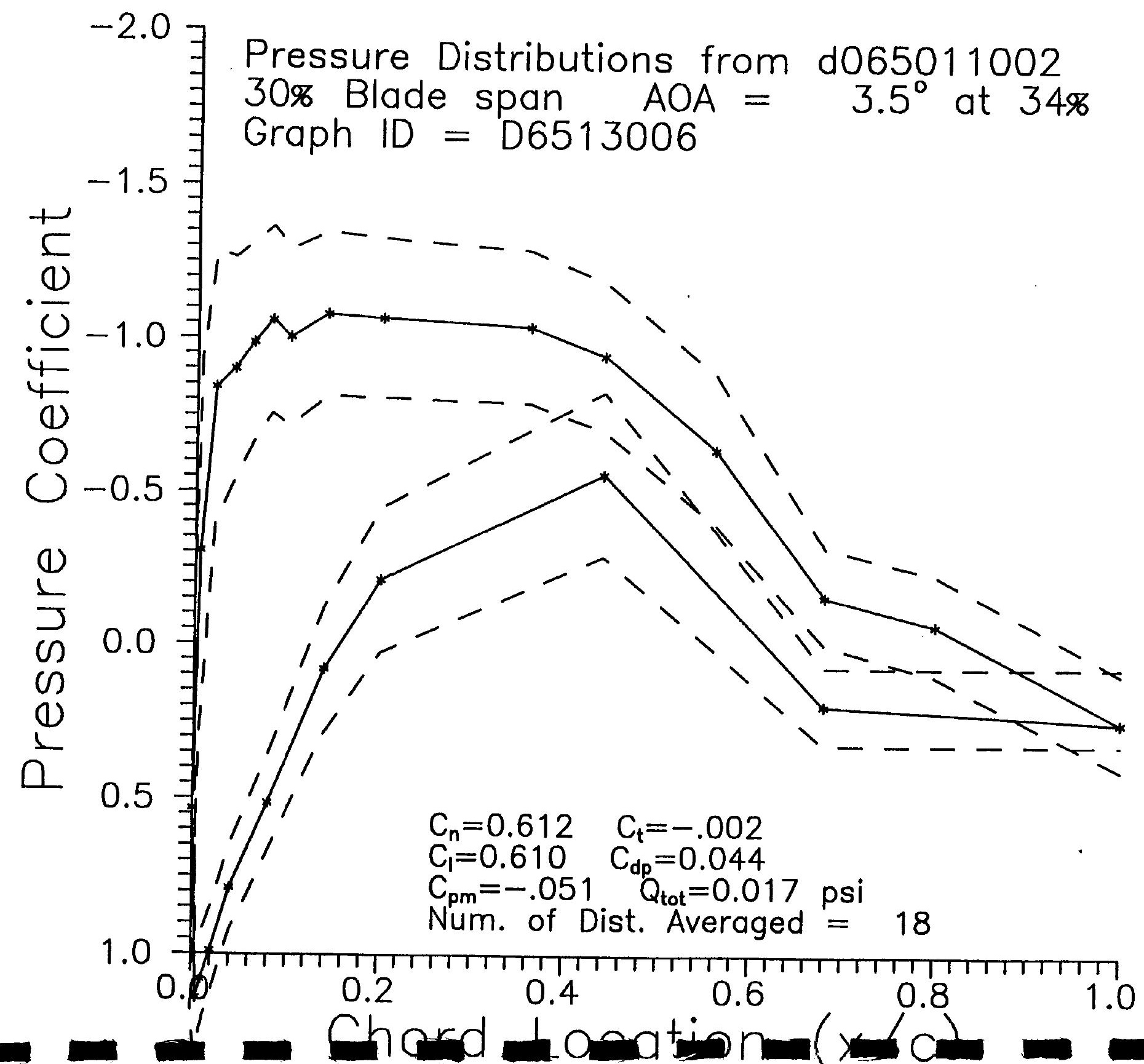




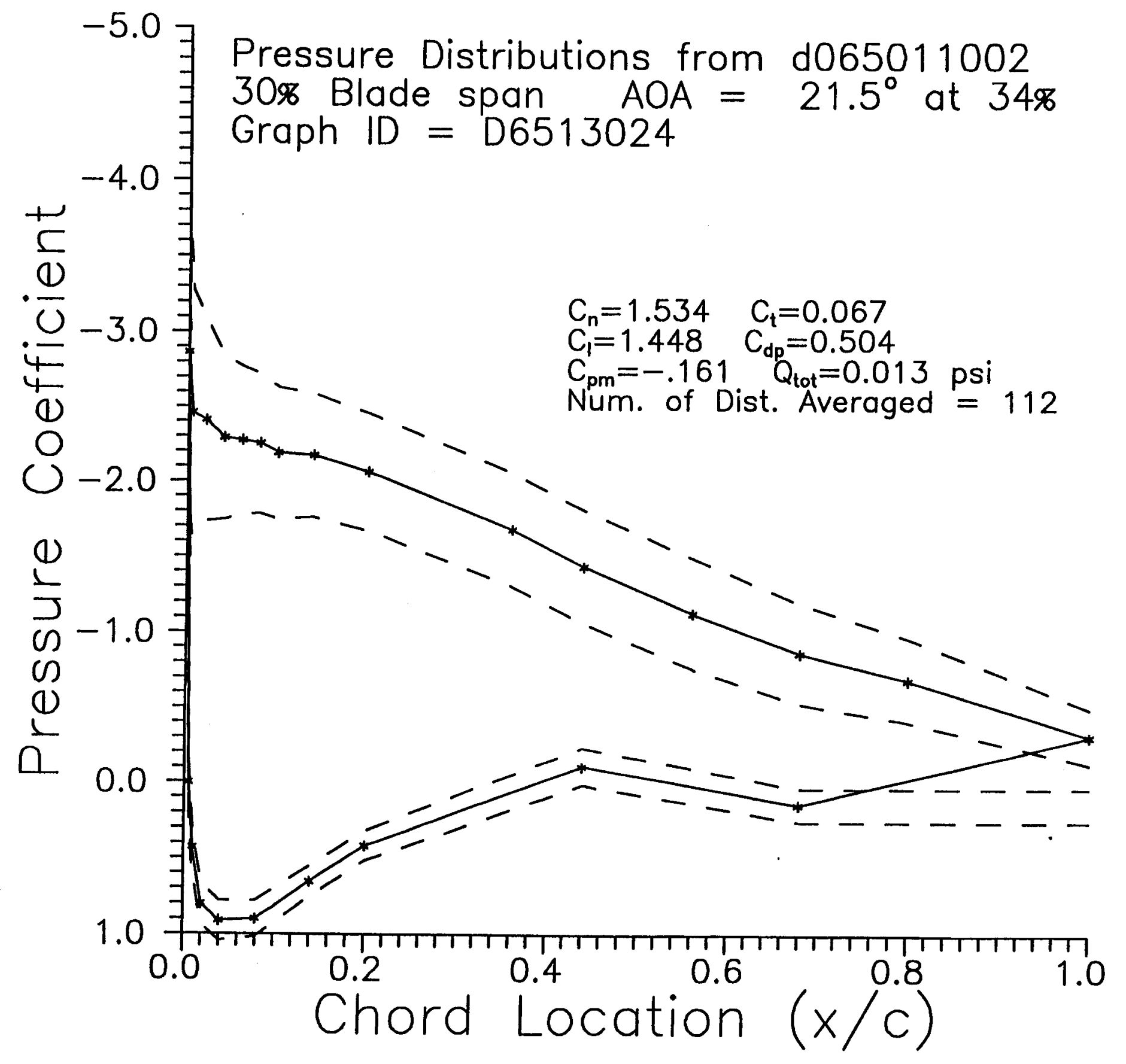




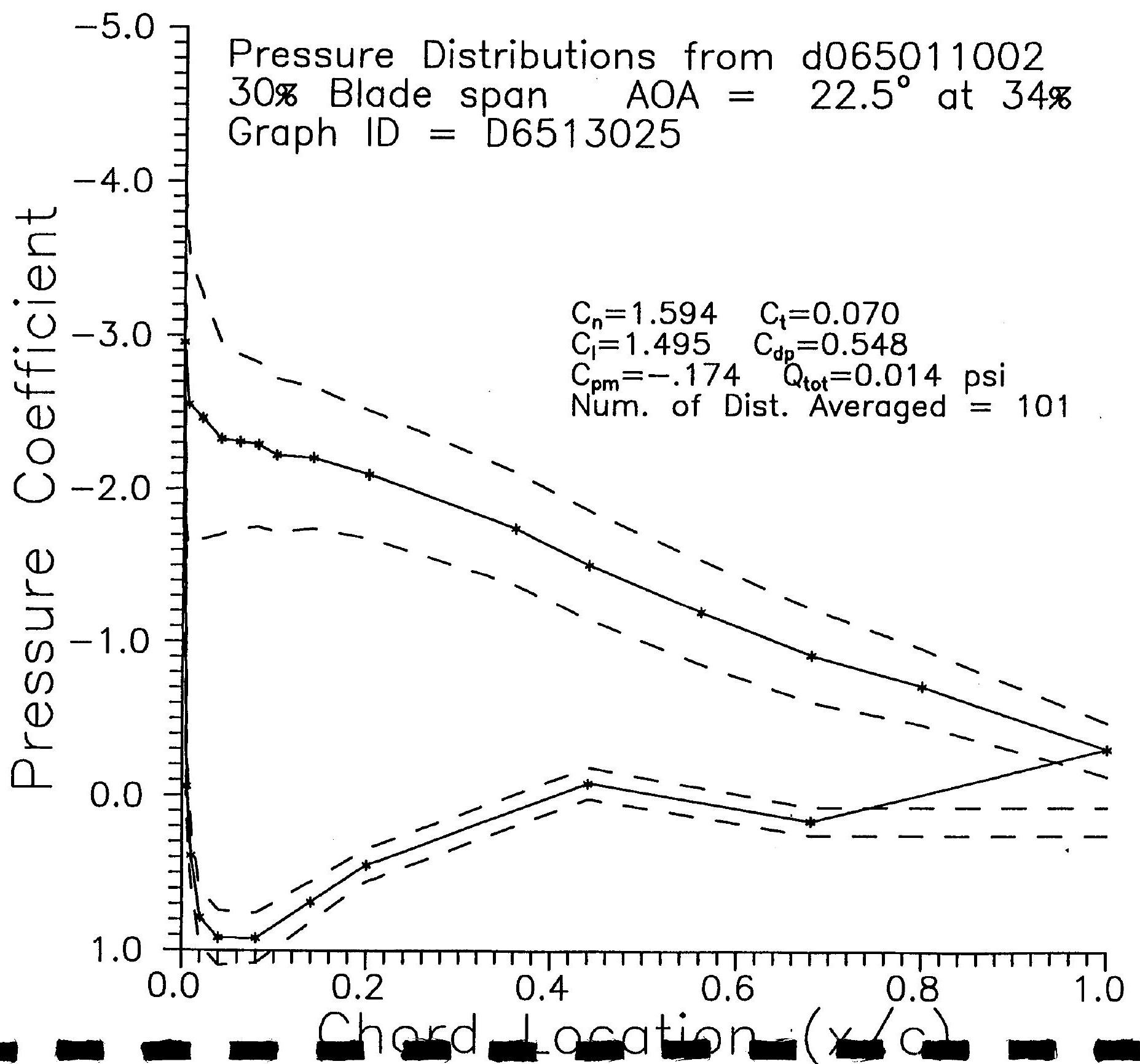




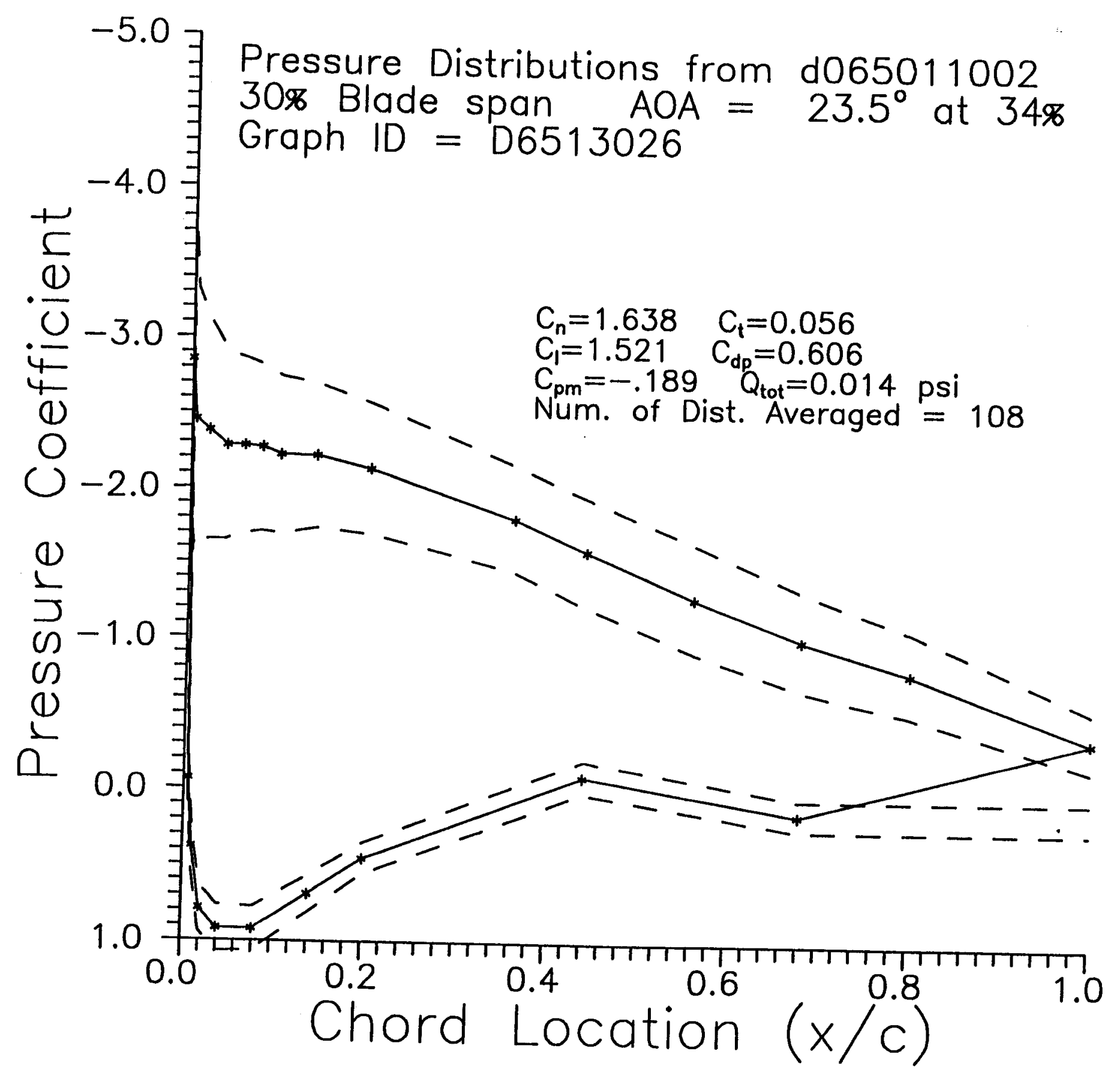




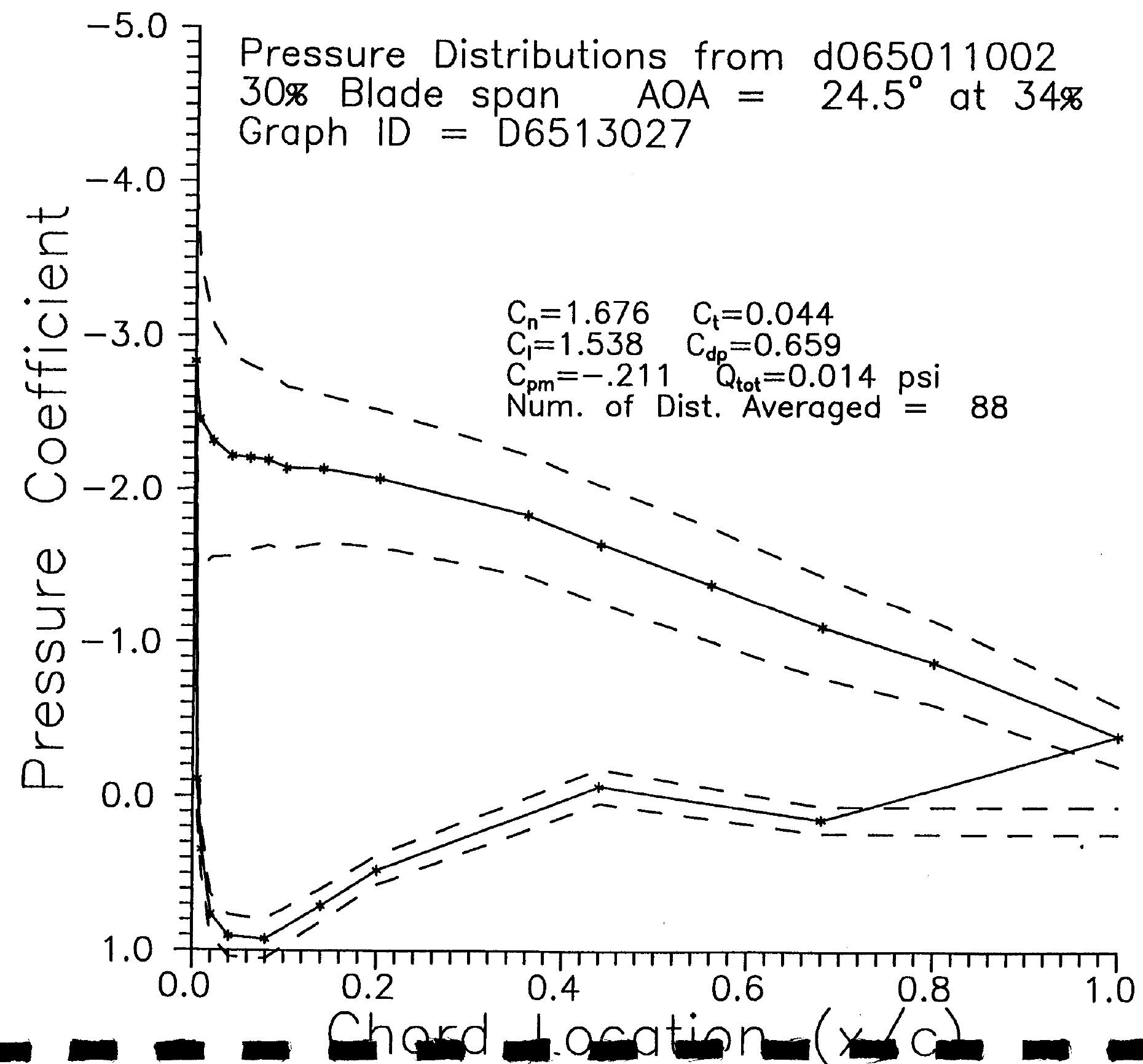




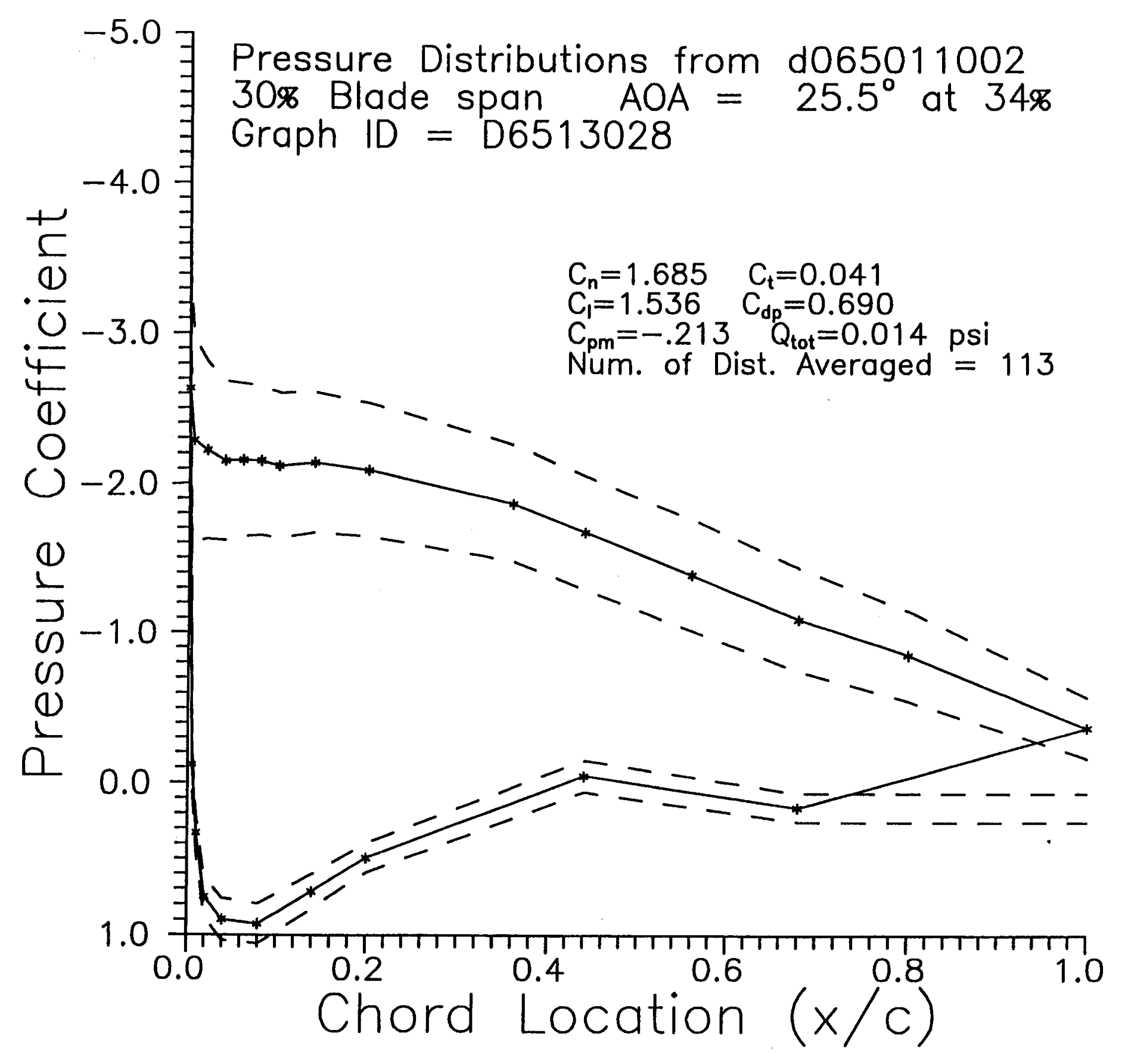




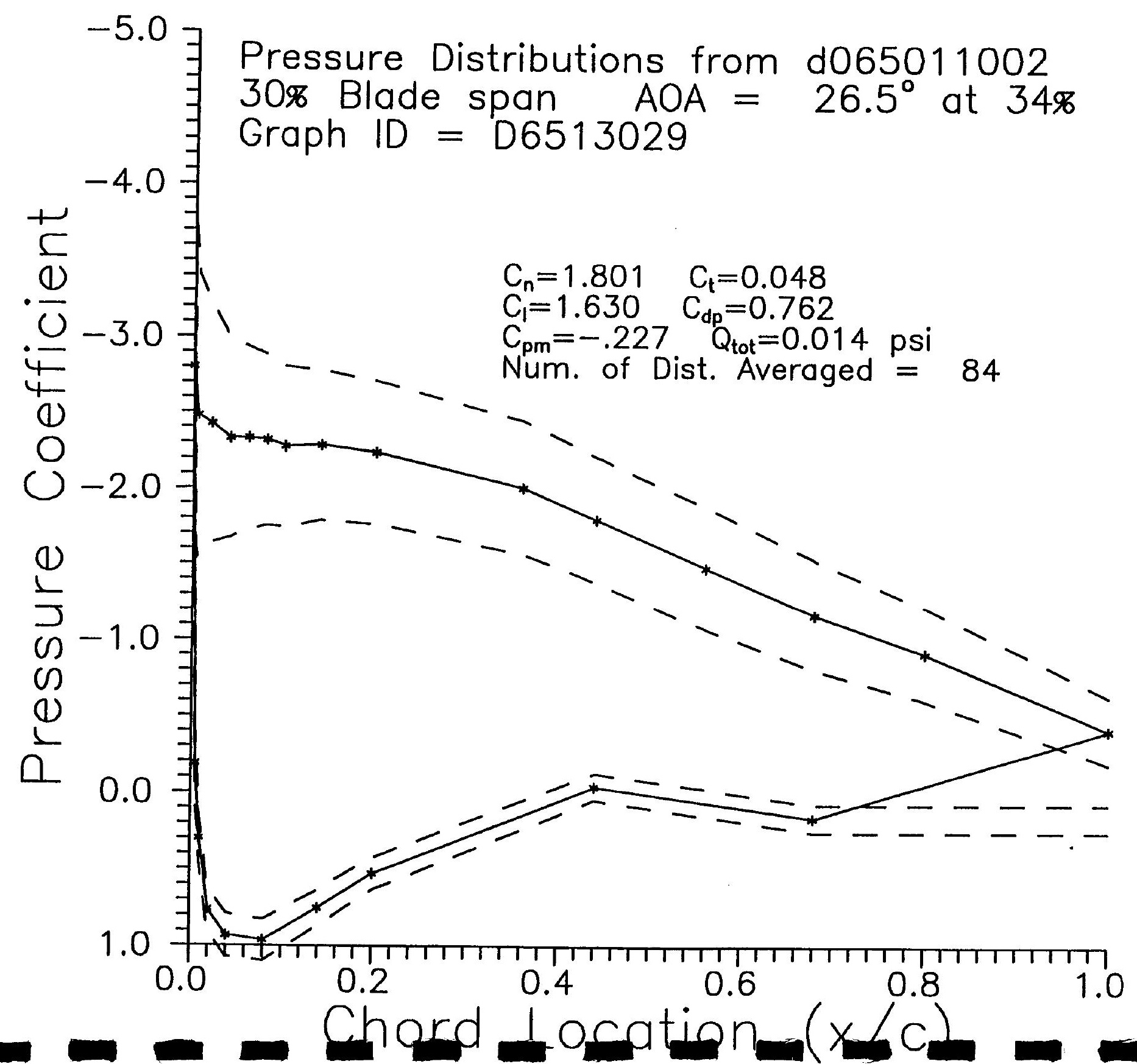




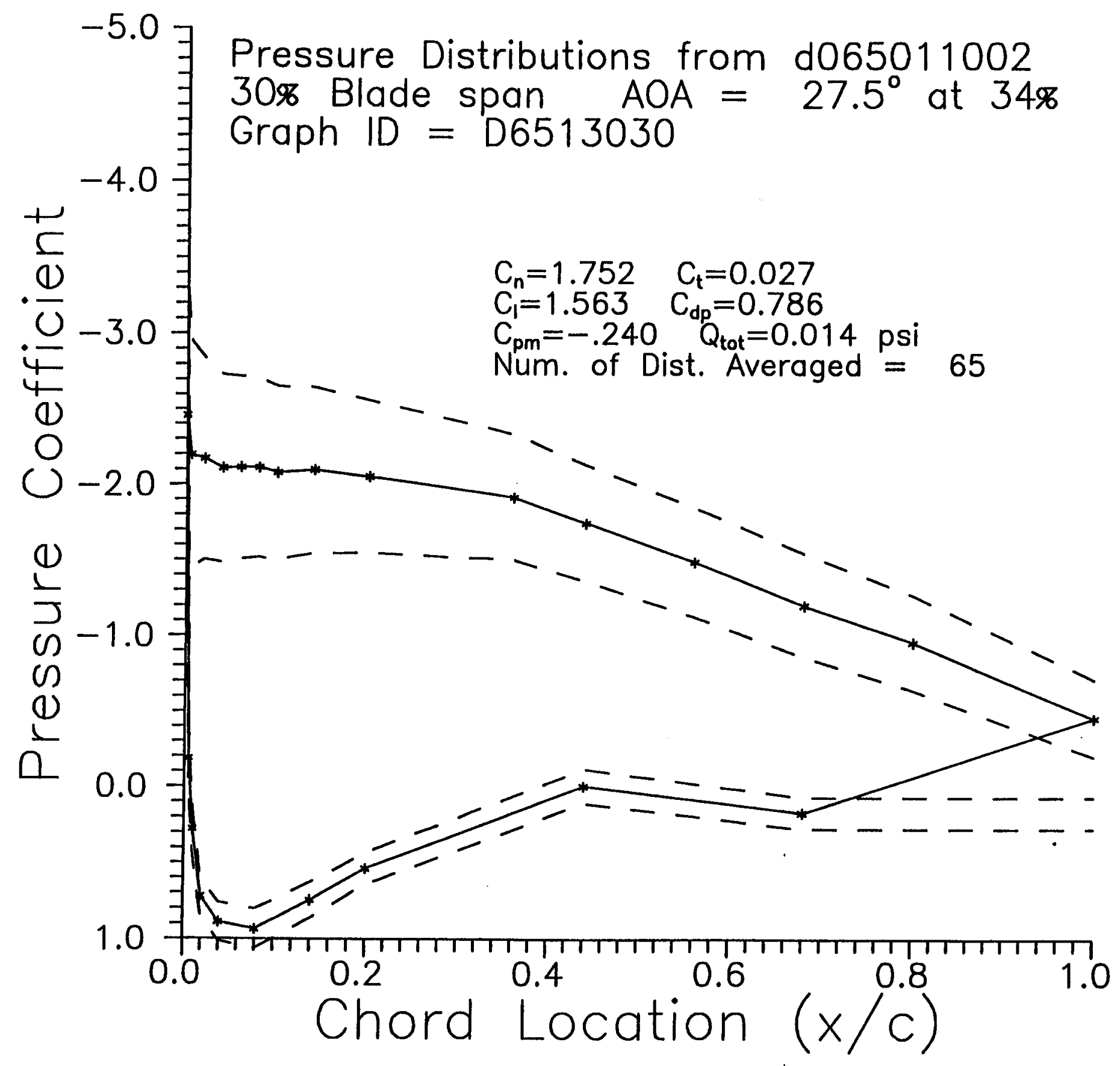




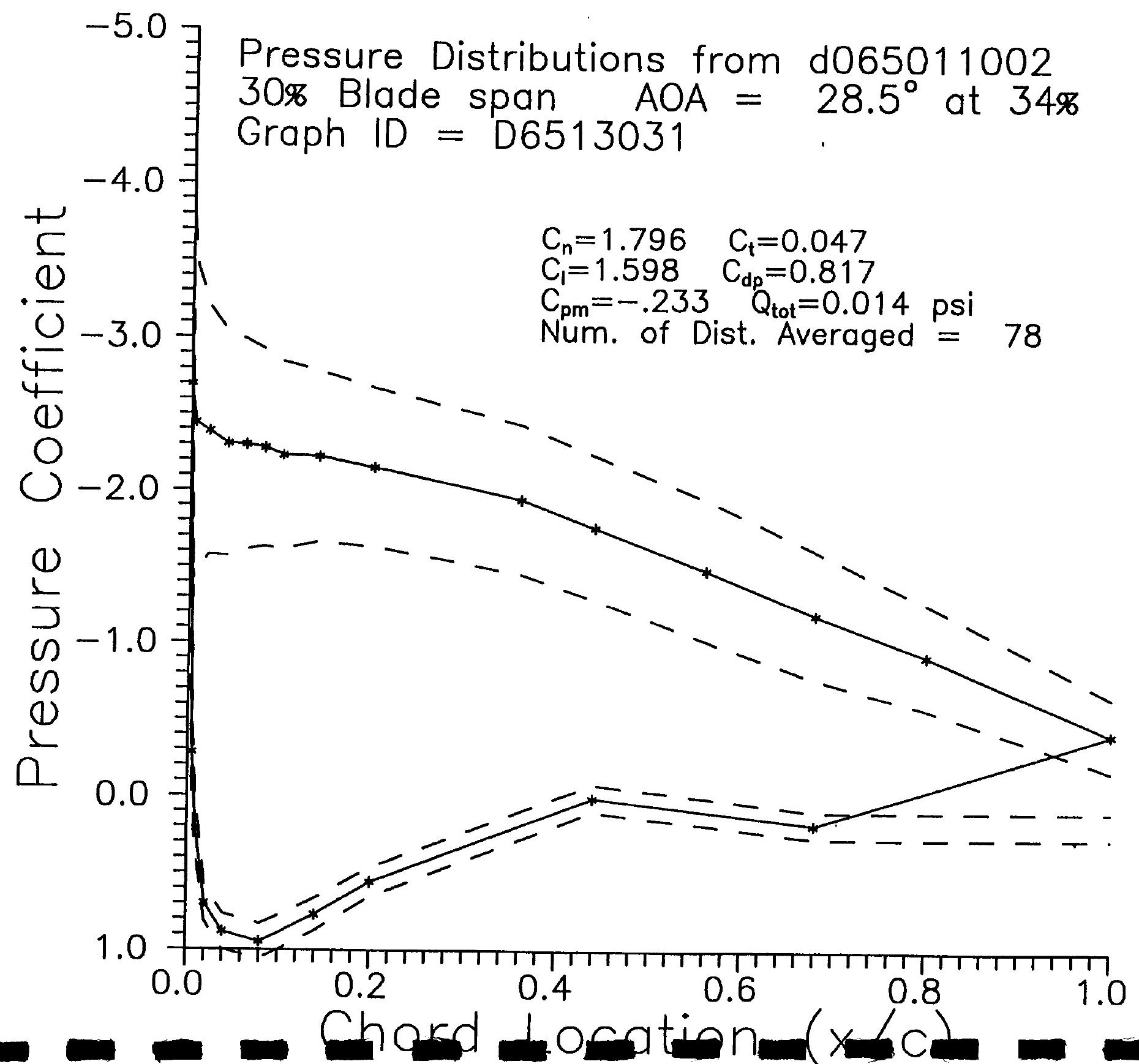




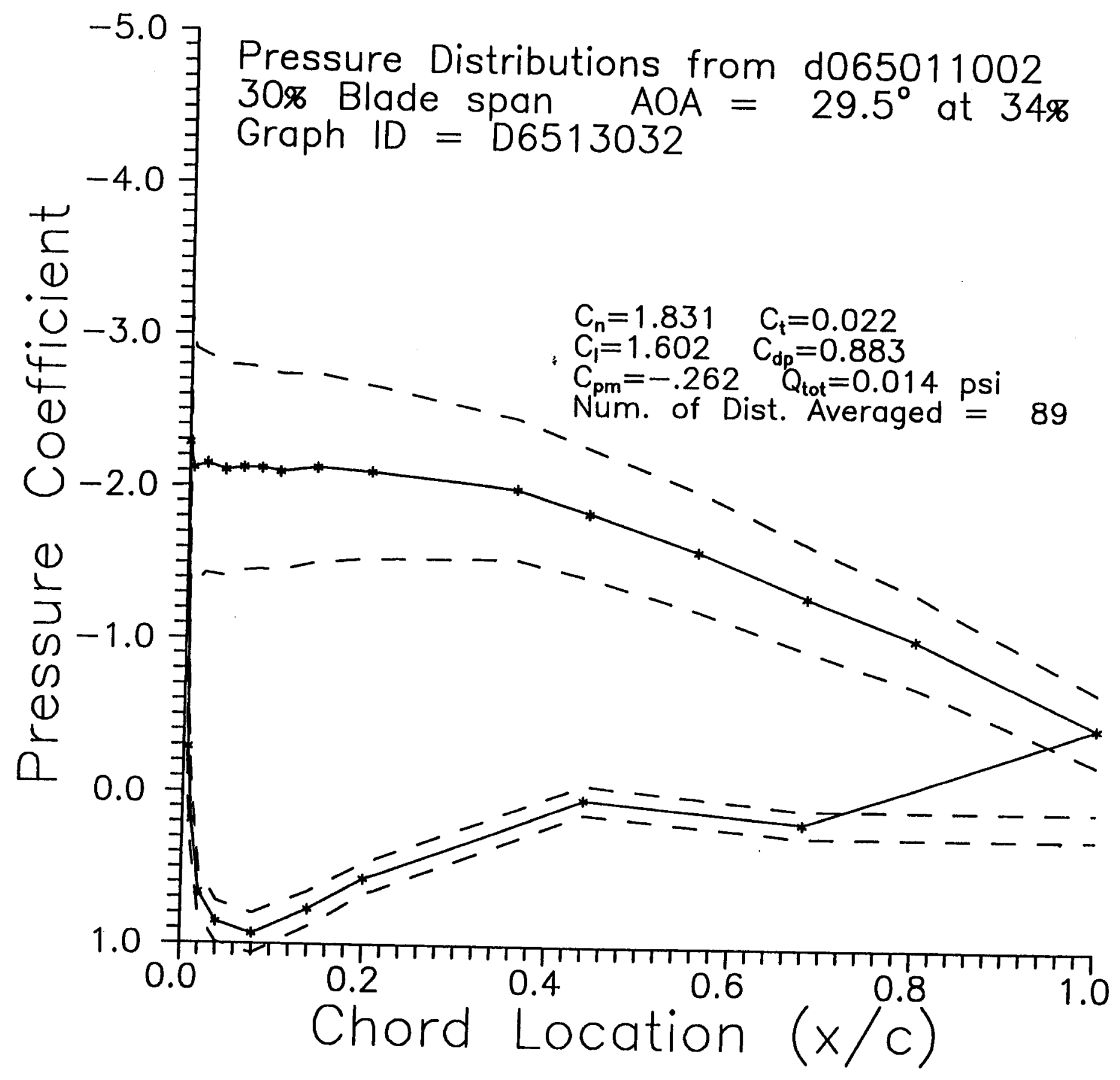




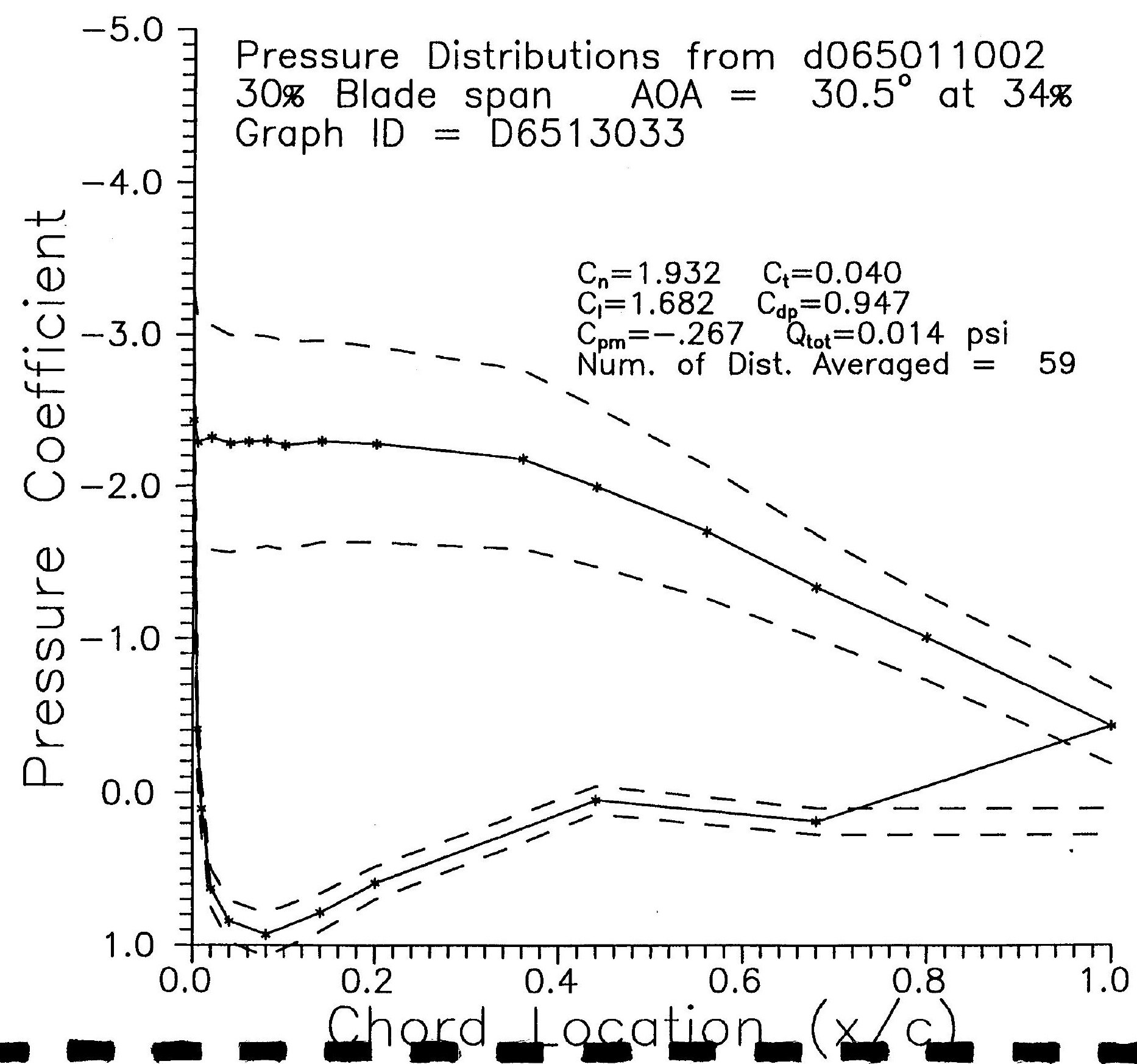




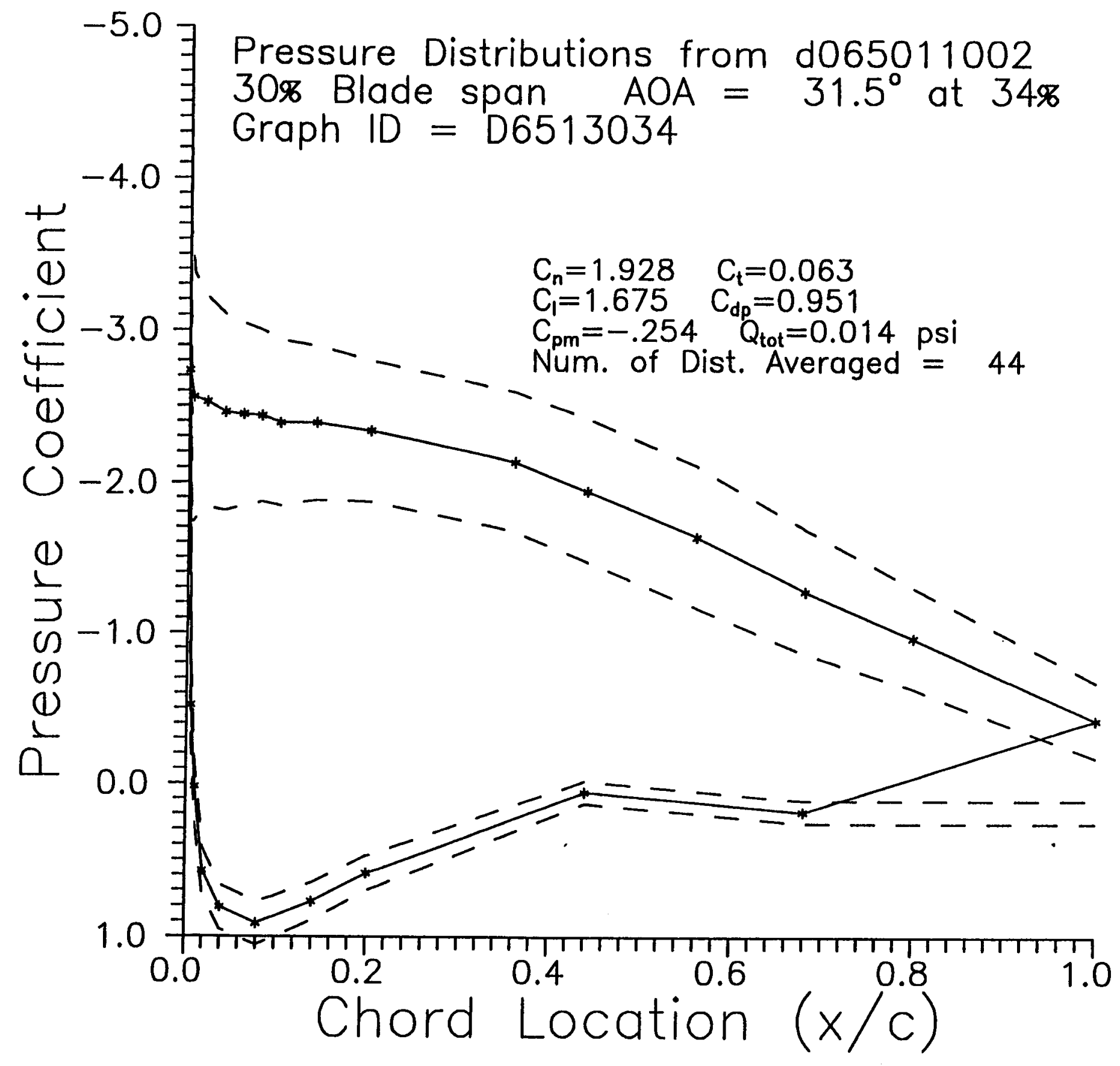




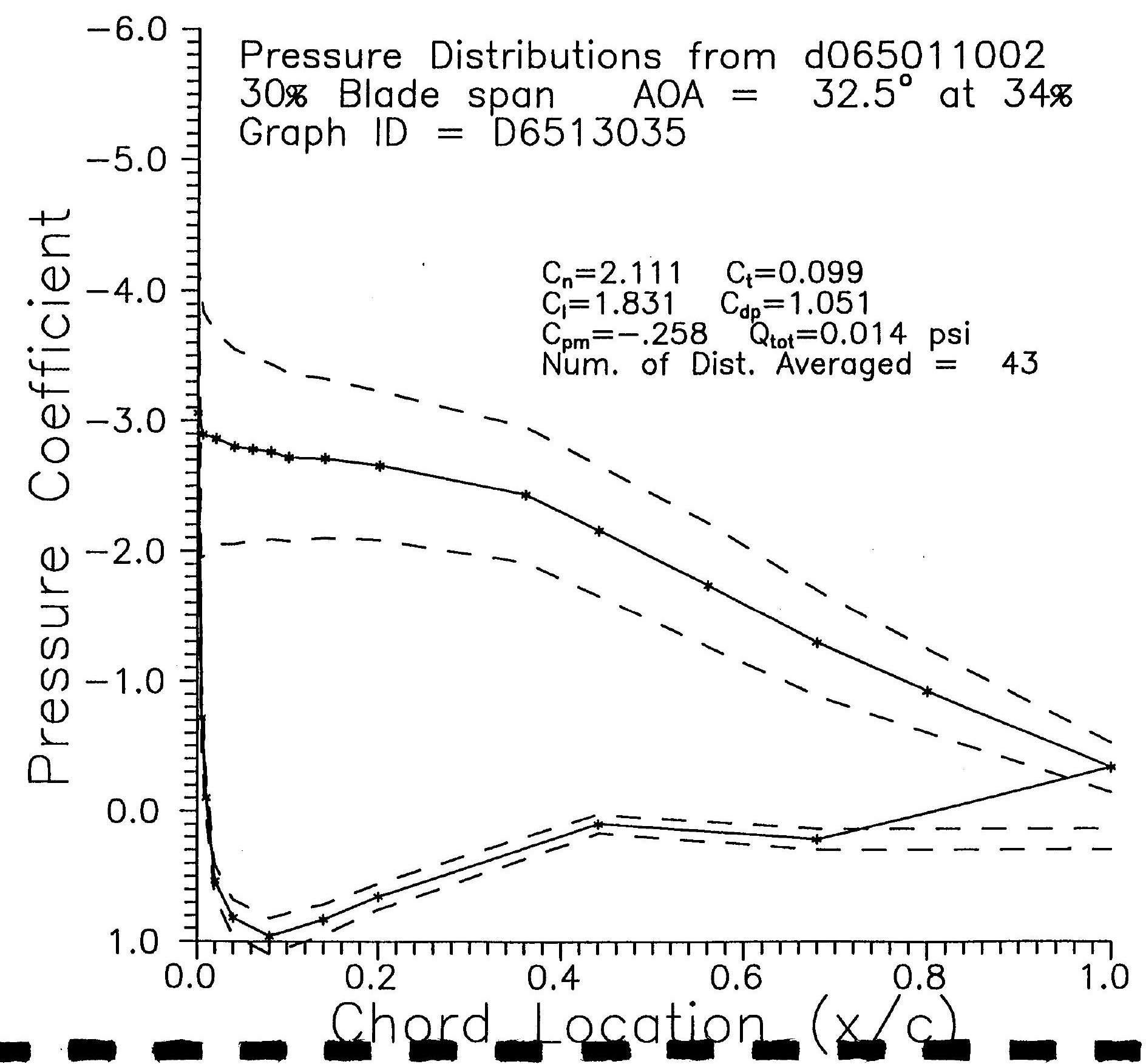




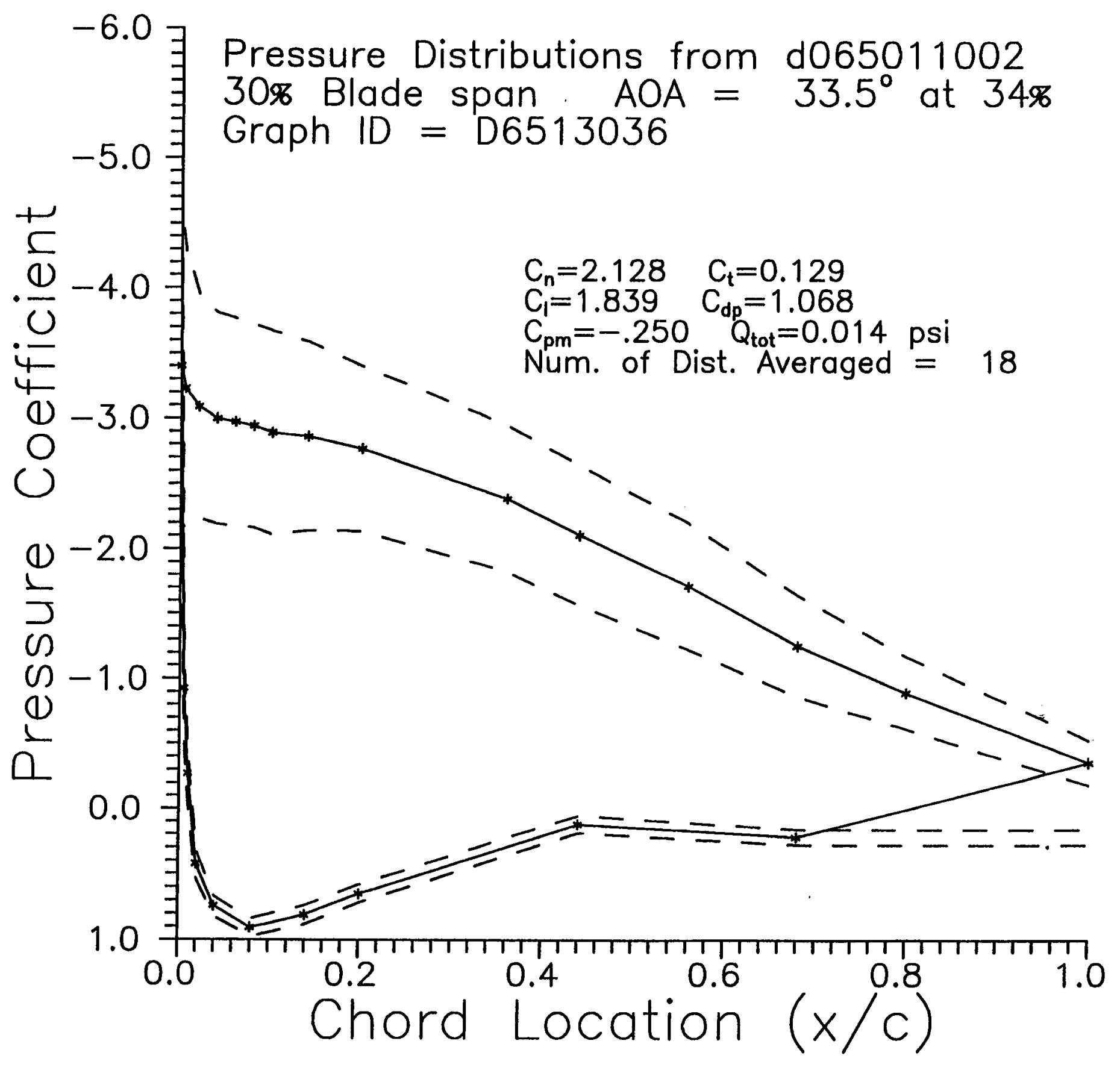




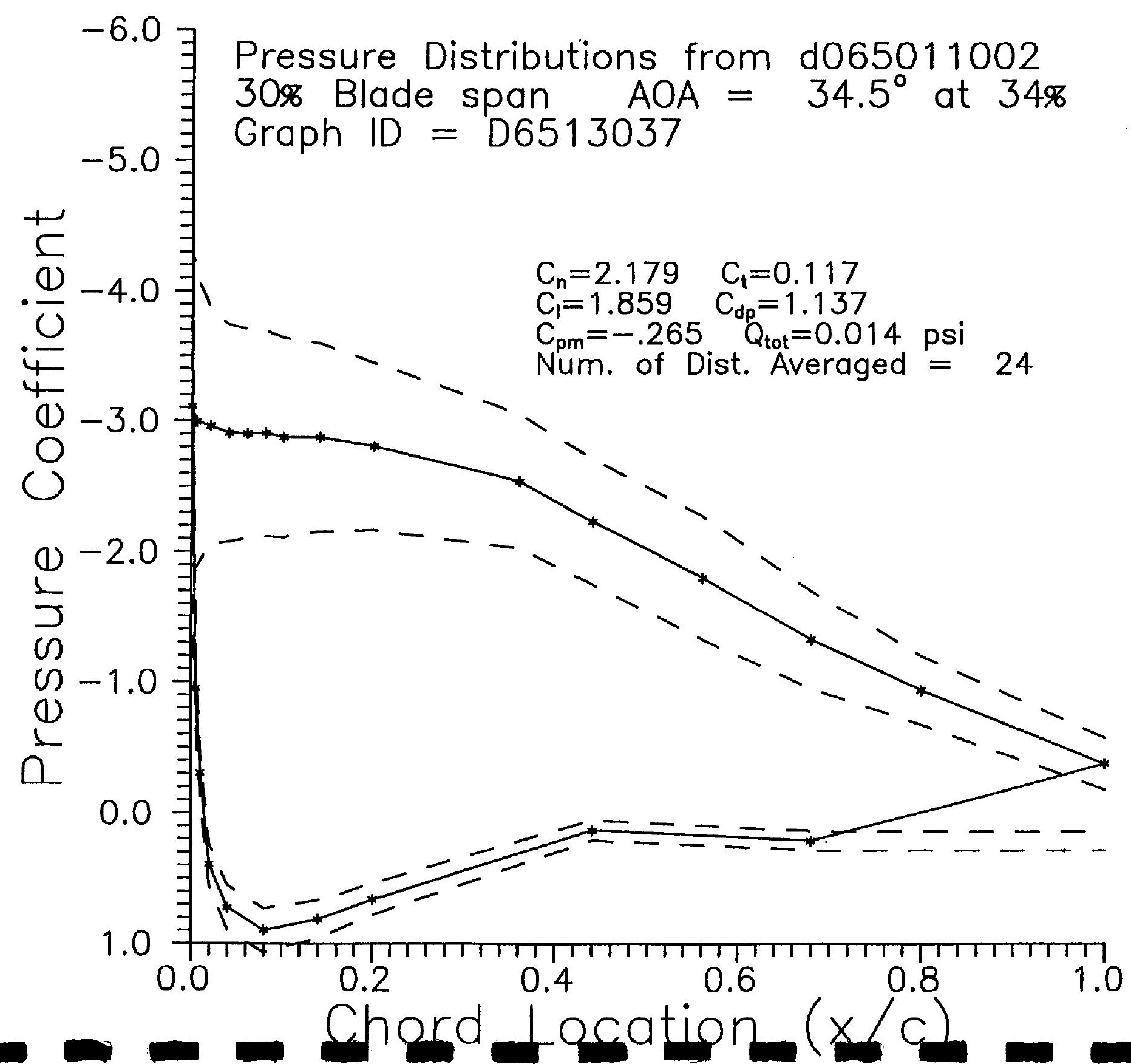




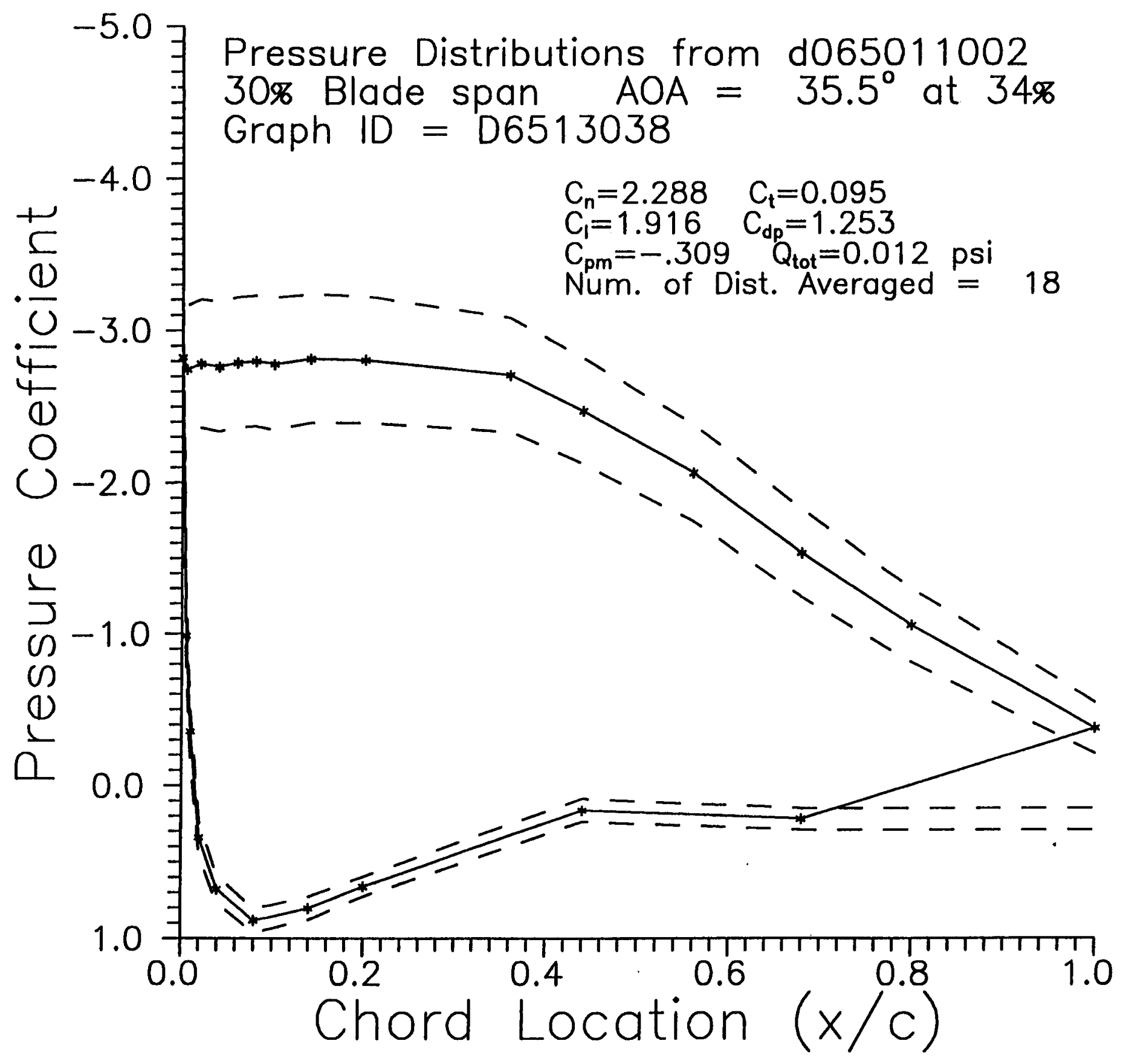




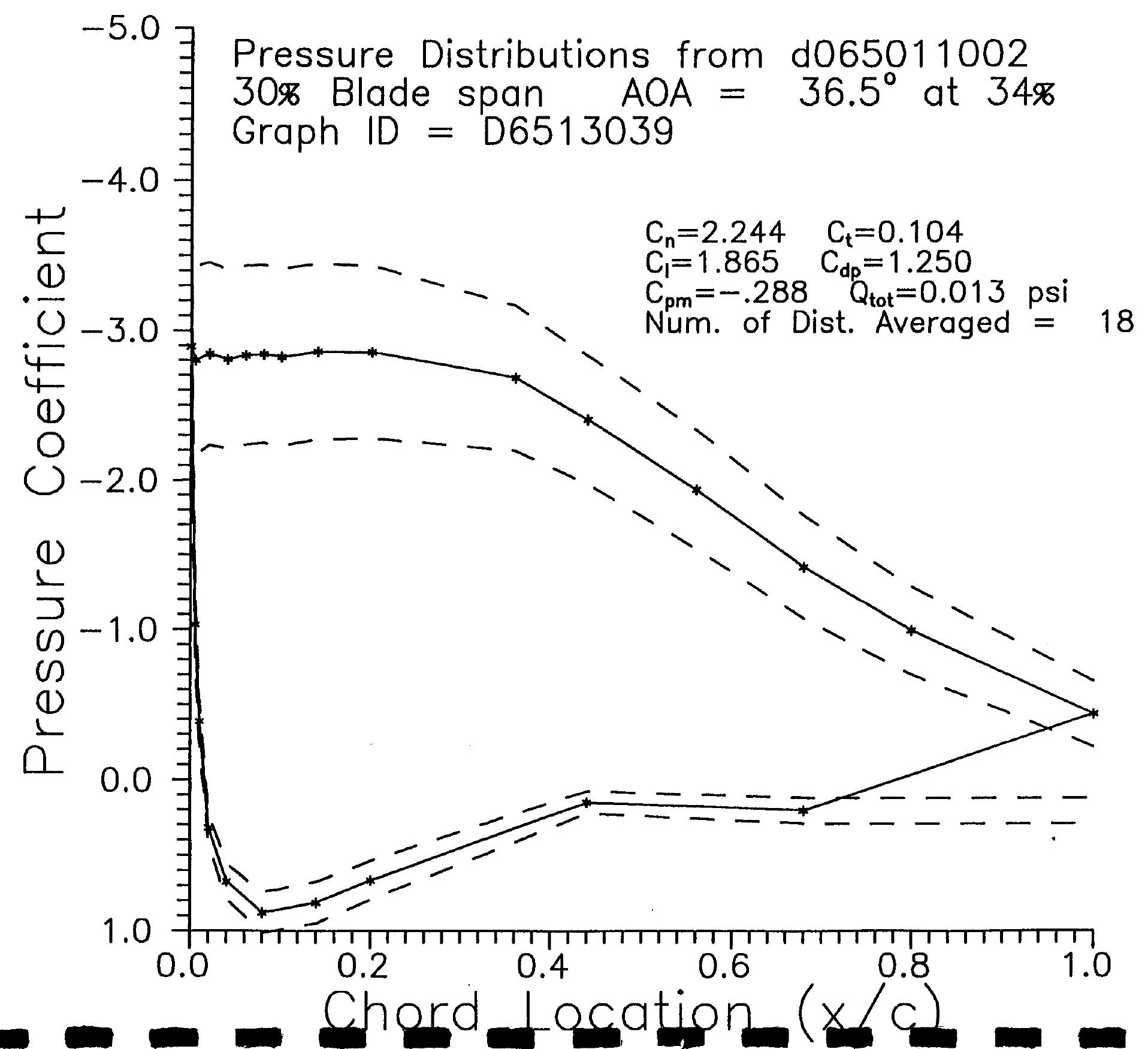




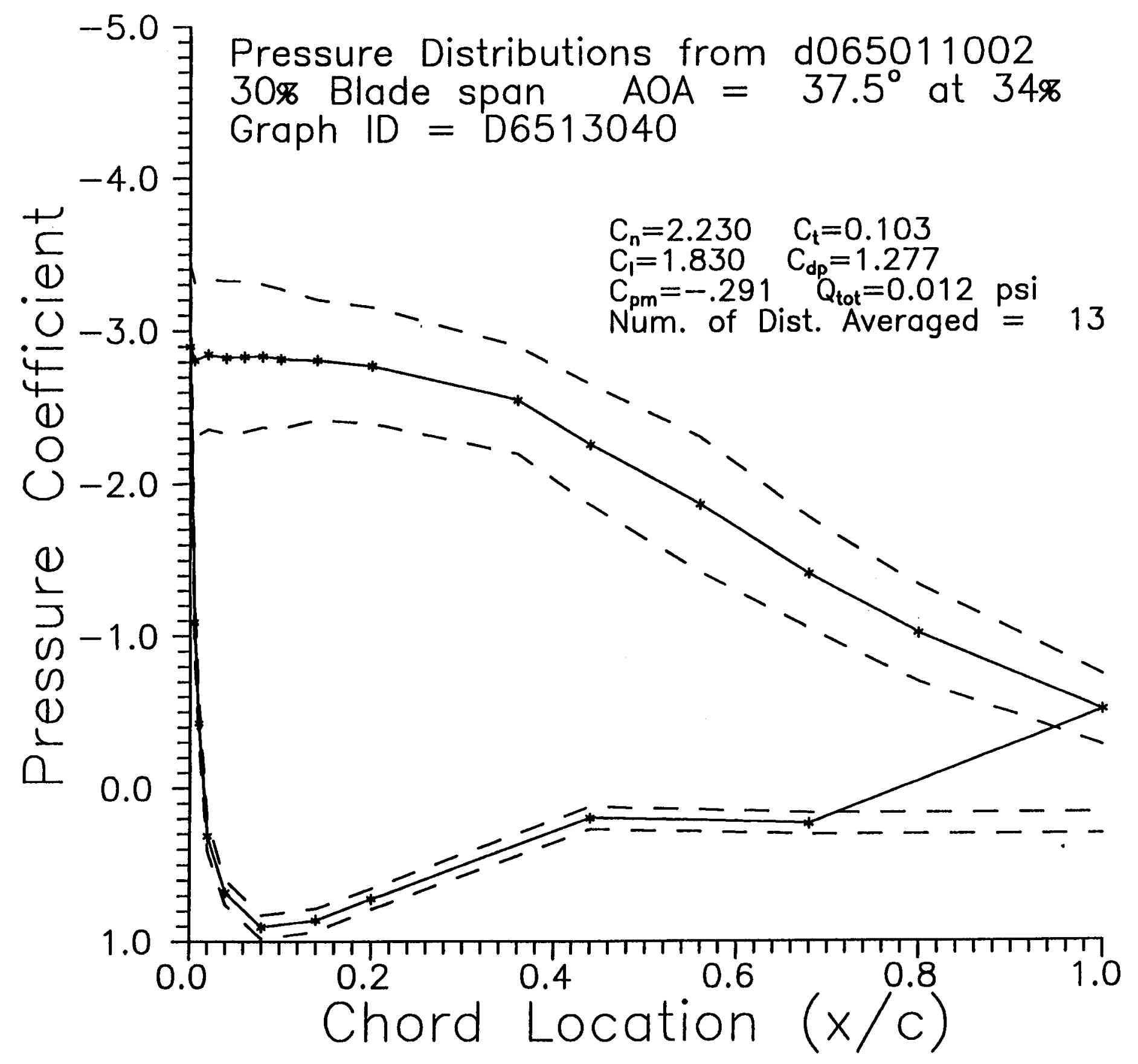


Appendix C.2

High Wind Speed Case 
Appendix C.2.1

Statistics for All Channels for Tape 68, Case 1 
COMBINED EXPERIMENT PHASE II - REDUCED DATA FILE SUMMARY

Processed EU binary real data read from file: corresponding header read from file:

Output version 1

Reduced EU binary data written to file:

Corresponding header written to file:

This sumary for printing written to file:

Data processed on: Wed Jul 18 11:32:48 1990

This data set contains:

99.84 usec data averaged by a factor of 52 to 0.0998 sec.

- 299.1206 seconds of data, from 135:17:07:28:499 to 135:17:12:27:619.

- 236 channels of engineering unit data values.

- 2996 data scans at $10.0160 \mathrm{~Hz}$.

\section{Channel Summary:}

\# Tag Channel Name

1(901): Pressure \#11 StaPT5, $36 \%$ chord, upper 2(902): Pressure \#18 StaPT5, 4\% chord, upper

$3(903)$ : Pressure \#1 StaPT7, 100\% chord, trailing

4(904): Pressure \#2 StaPT7, $92 \%$ chord, upper $5(905)$ : Pressure \#4 StaPT7, 80\% chord, upper 6(906): Pressure \#6 stapT7, 68\% chord, upper $7(907)$ : Pressure $\# 8$ stapT7, 56\% chord, upper 8(908): Pressure \#10 StapT7, $44 x$ chord, upper 9(909): Pressure \#11 StaPT7, 36\% chord, upper 10(910): Pressure \#12 StaPT7, $28 \%$ chord, upper 11(911): Pressure \#13 StaPT7, $20 \%$ chord, upper 12(912): Pressure \#14 StaPT7, $14 \mathrm{X}$ chord, upper 13(913): Pressure \#15 5taPT7, $10 \%$ chord, upper 14(914): Pressure \#16 StaPT7, 8\% chord, upper 15(915): Pressure \#17 staPT7, 6\% chord, upper 16(916): Pressure \#18 StaPT7, 4\% chord, upper $17(917)$ : Pressure \#19 StaPT7, 2\% chord, upper 18(918): Pressure \#20 StaPT7, 1\% chord, upper 19(919): Pressure "21 StaPT7, 0.5\% chord, upper 20(920): Pressure \#22 stapT7, 0\% chord, leading 21(921): Pressure \#23 stapT7, 0.5\% chord, lower 22(922): Pressure \#24 StaPT7, 17 chord, Lower 23(923): Pressure \#25 StaPT7, 2X chord, Lower 24(924): Pressure \#26 StaPT7, 4\% chord, lower 25(925): Pressure \#28 StaPT7, 87 chord, Lower 26(926): Pressure \#30 StaPT7, 14\% chord, lower $27(927)$ : Pressure $\# 32$ StaPT7, $28 \%$ chord, lower 28(928): Pressure \#34 StaPT7, $44 \%$ chord, lower 29(929): Pressure \#36 StaPT7, 68\% chord, Lower $30(930)$ : Pressure \#38 StaPT7, 92\% chord, Lower 31(931): Pressure \#18 StaPT6, 47 chord, upper 32(932): Pressure \#18 StaPT8, 4\% chord, upper 33(933): Pressure \#11 StaPT6, $36 \%$ chord, upper 34(934): Total Pressure Probe, 67.3\% span 35(935): Angle-of-Attack, 86\% span 36(936): Angle-of-Attack, 67.3\% span $37(937)$ : Angle-of-Attack. 50.6x span 38(938): Angle-of-Attack, $34 X$ span $39(201)$ : VPA Prop Vane Speed US-1 $(12: 00)$ 40(202): VPA Prop Vane Speed WS-2 (1:30) 41(203): VPA Prop Vane Speed US-3 (3:00) 42(204): VPA Prop Vane Speed WS-4 $(4: 30)$ 43(205): VPA Prop Vane speed US-5 $(6: 00)$ 44(206): VPA Prop Vane Speed US-6 $(7: 30)$ 45(207): VPA Prop Vane Speed wS-7 $(9: 00)$ 46(208): VPA Prop Vane Speed WS-8 (10:30) 47(209): UPA Prop Vane Speed US-9 Hub Height 48(210): VPA Prop Vane Direction wD-9 Hub Height 49(211): VPA Bi-Vane Speed WS-12 (3:00 a100\%) 50(212): VPA Bi-Vane Direction WD-12 (3:00 a100\%) 51(213): VPA Bi-Vane Angle WA-12 (3:00 a100\%) /usr/data5/d068011. dat
/usr/data5/d068011. hdr

/usr50/dave/p2/d068011001. dat /usr50/dave/D2/0068011001.hdr /usr50/dave/p2/d068011001.prt
Units Mean Std Min Max [coef] $-1.953 E+00(3.841 E-01)-4.237 E+00-7.717 E-01$ [coef] $-2.460 E-01$ (1.839E-01) $-8.666 E-01 \quad 3.100 E-01$ [coef] $-3.256 E-01(1.974 E-01)-1.005 E+001.874 E-01$ [coef] $-4.568 E-01(2.267 E-01)-1.248 E+00 \quad 3.226 E-02$ [coef] -5.499E-01 (2.467E-01) -1.383E+00 -4.767E-02 [coef] -6.511E-01 (2.503E-01) $-1.458 E+00-1.665 E-01$ [coef] -9.367E-01 (2.175E-01) - $1.816 E+00-3.683 E-01$ [coef] $-1.050 E+\infty 0$ (2.146E-01) -1.877E+00 -3.925E-01 [coef] $-1.185 E+\infty 0$ (2.196E-01) $-2.000 E+\infty 0-4.251 E-01$ [coef] $-1.342 E+\infty 0(2.406 E-01)-2.110 E+00-5.448 E-01$ [coef] $-1.470 E+00(2.964 E-01)-2.442 E+00-5.917 E-01$ [coef] $-1.550 E+00(3.315 E-01)-2.692 E+00-6.305 E-01$ [coef] $-1.614 E+00$ (3.796E-01) $-2.880 E+00-6.403 E-01$ [coef] $-1.742 E+00(4.525 E-01)-3.175 E+00-6.909 E-01$ [coef] $-1.849 E+00(5.439 E-01)-3.484 E+00-6.871 E-01$ [coef] $-2.190 E+00(8.305 E-01)-4.587 E+00-7.127 E-01$ [coef] $-2.438 E+00(1.080 E+\infty 0)-5.594 E+00-6.527 E-01$ [coef] $-2.726 E+\infty 0(1.331 E+\infty)-7.142 E+\infty 0-4.940 E-01$ [coef] $-3.110 E+00(1.644 E+00)-8.482 E+00 \quad 7.493 E-02$ [coef] $5.550 E-01$ (1.906E-01) $-2.453 E-01 \quad 1.544 E+00$ [coef] 7.886E-01 (1.259E-01) 2.011E-01 1.601E+00 [coef] 8.939E-01 (1.031E-01) 3.894E-01 1.571E+00 [coef] 9.108E-01 (9.999E-02) 4.294E-01 1.352E+00 [coef] 7.988E-01 (1.063E-01) 3.348E-01 1.144E+00 [coef] 5.758E-01 (1.125E-01) 1.134E-01 9.363E-01 [coef] 3.460E-03 (1.311E-01) -4.753E-01 4.873E-01 [coef] -2.894E-01 (1.168E-01) -7.953E-01 1.480E-01 [coef] 1.139E-01 (6.035E-02) -3.545E-01 4.413E-01 [coef] 1.019E-01 (8.357E-02) -4.023E-01 3.944E-01 [coef] $-2.203 E+\infty 0(4.251 E-01)-3.663 E+\infty 0-8.199 E-01$ [coef] $-2.068 E+00(4.342 E-01)-4.022 E+00-5.834 E-01$ [coef] $-1.041 E+00$ (2.645E-01) $-2.045 E+00-3.946 E-01$ [Dsi ] 5.809E-02 (6.787E-03) 2.865E-02 9.377E-02 [Degr] 1.015E+01 (3.385E+00) 1.169E+DO 2.178E+01 [Degr] 1.645E+01 (4.946E+00) 3.801E+00 3.169E+01 [Degr] $1.568 E+01(4.397 E+00) \quad 4.165 E+\infty 02.937 E+01$ [Degr] 3.476E+01 $(4.926 E+\infty 0) \quad 8.563 E+\infty 03.966 E+01$ $[\mathrm{w} / \mathrm{s}] \quad 1.438 \mathrm{E}+01(2.236 \mathrm{E}+00) 8.777 \mathrm{E}+001.985 \mathrm{E}+01$ [m/s ] 1.401E+01 (2.192E+00) 7.947E+00 1.939E+01 $[\mathrm{w} / \mathrm{s}] \quad 1.376 E+01(2.136 E+\infty 0) \quad 8.284 E+\infty 0 \quad 1.961 E+01$ $[\mathrm{m} / \mathrm{s}] \quad 1.353 \mathrm{E}+01(2.151 \mathrm{E}+\infty) \quad 7.248 \mathrm{E}+\infty \quad 1.989 \mathrm{E}+01$ $[\mathrm{m} / \mathrm{s}] 1.336 \mathrm{E}+01(2.185 \mathrm{E}+\infty) \quad 7.519 \mathrm{E}+00 \quad 1.972 \mathrm{E}+01$ [m/s ] 1.360E+01 $(2.228 E+\infty) \quad 7.901 E+\infty 0 \quad 2.011 E+01$ $[\mathrm{m} / \mathrm{s}] 1.395 \mathrm{E}+01(2.294 \mathrm{E}+\infty) \quad 7.962 \mathrm{E}+001.985 \mathrm{E}+01$ $[\mathrm{m} / \mathrm{s}] \quad 1.427 \mathrm{E}+01(2.274 E+00) \quad 8.552 E+00 \quad 1.997 \mathrm{E}+01$ $[\mathrm{m} / \mathrm{s}] \quad 1.401 \mathrm{E}+01(2.206 \mathrm{E}+\infty) \quad 8.657 \mathrm{E}+00 \quad 1.997 \mathrm{E}+01$ [degr] 2.806E+02 $(7.974 E+00) \quad 2.551 E+02 \quad 3.072 E+02$ $[\mathrm{m} / \mathrm{s}] \quad 1.350 E+01(2.120 E+\infty 0) \quad 7.519 E+\infty 01.922 E+01$ [deg ] 2.829E+02 $(8.641 E+\infty) \quad 2.596 E+02 \quad 3.118 E+02$ [deg ] $-5.528 E+\infty 0(3.847 E+\infty)-1.681 E+019.491 E+\infty 0$ 

53(215): VPA Bi-Vane Direction WD-13 (9:00 a100x) 54(216): VPA Bi-Vane Angle UA-13 (9:00 a100x) 55(301): VPA Prop Vane Speed US-10 (12:00 240\%) 56(302): VPA Prop Vane Speed US-11 (6:00 240\%) 57(303): Low Speed Shaft Azimuth Angle 58(304): Yaw moment

59(305): Tower Bending about East-West Mxis (X) $60(306)$ : Tower Bending about North-South Axis (Y) 61(307): Yaw Angle

62(308): Generator Power

63(310): TSI (South) $X$-Fil $X$ (NOT WORKINS) 64(311): TSI (South) X-FiLn $Y$ (NOT WORKING) 65(314): Sonic Anewoweter Channel $A$ (NOT NORKIMG) 66(315): Sonic Anemometer Channel B (NOT WORKING) $67(316)$ : Sonic Anewoweter Channel $C$ (wOT wORKING) 6B(401): Root Flap Bending "RTFBM- $A$ " 69(402): Root Flap Bending 'RTFBN-B' 70(403): Root Flap Bending -RTFBH-2* (Blade 2) 71(404): Root Flap Bending 'RTFBN-3' (Blade 3) 72(405): $20 \%$ Flap Bending "20FBm" 73(406): 40\% Flap Bending "40FBM" $74(407)$ : $50 x$ flap Bending $50 \mathrm{FBw}$ 75(409): $70 x$ flap Bending =70FBM76(413): 90\% Flap Bending -90FBN. 77(414): Root Edge Bending 'RTEBH-A" 78(415): $20 \%$ Edge Bending - 20Een: 79(416): 50\% Edge Bending -50EBM80(417): $70 \%$ Blade Torque "70TQ" 81(419): Root Torque (Link) "RTTQ" $82(420): 50 \%$ Torsion $50 \mathrm{TQ}$

83(421): $X-X$ LSS Bending "LSSX- $X$ " 84(422): $Y-Y$ LSS Bending 'LSSY-Y" 85(423): LSS Torque "LSSTA-A" 86(624): LSS Torque "LSSTO-B.

87(427): Pressure 1 Stapt10, $100 \%$ chord,trailing 88(428): Pressure 2 stapt10, 927 chord. upper 89(429): Pressure \$4 stapT10, 80z chord, upper $90(430)$ : Pressure 16 StaPT10, $68 \%$ chord, upper 91(431): Pressure $\$ 8$ StapT10, $56 \%$ chord, upper 92(432): Pressure \$10 StapT10, 44X chord, upper 93(433): Pressure \$11 StaPT10, 36x chord, upper 94(434): Pressure $\$ 12$ StaPT10, $28 \%$ chord, upper 95(435): Pressure $\$ 13$ StaPT10, $20 \%$ chord, upper 96(436): Pressure \#14 StaPT10, $14 \%$ chord, upper 97(437): Pressure \#15 staPT10, 10\% chord, upper 98(438): Pressure \$16 5tapT10, 8\% chord, upper 99(439): Pressure 17 stapt10, 6\% chord, upper $100(440)$ : Pressure $\$ 18$ StapT10, 4\% chord, upper 101(441): Pressure 119 stapt10, 27 chord, upper 102(442): Pressure $\$ 20$ StapT10, 1\% chord, upper 103(443): Pressure \#21 staPT10, 0.5X chord, upper 104(444): Pressure \$22 stapT10, Ox chord, leading 105(445): Pressure \#23 stapr10, 0.5\% chord, Lower 106(446): Pressure \$24 StapT10, 12 chord, Lower 107(447): Pressure \#25 5tapT70, 27 chord, Lower 108(448): Pressure \#26 StapT10, 4X chord, lower 109(44\%): Pressure \#28 StapT10, 8X chord, lower $110(450)$ : Pressure $\$ 30$ StaPT10, $14 \%$ chord, Lower 111(451): Pressure \$32 StaPT10, 287 chord, Lower 112(452): Pressure $\$ 34$ StapT10, 44X chord; lower 113(453): Pressure \$36 StaPT10, 68\% chord, lower 114(454): Pressure \$38 StaPT10, 92\% chord, lower $115(455)$ : Pressure \#18 StaPT9, 4X chord, upper 116(456): Pressure 111 StaPT9, 36\% chord, upper $117(457)$ : Pressure $\$ 11$ StapT8, 36\% chord, upper 118(458): Total Pressure Probe, 86\% span 119(459): Absolute Reference Pressure 120(461): Pitch Angle 121(804): Pressure \#1 StaPT1, 100\% chord, trailing 122(805): Pressure \#4 StaPTI, 80\% chord, upper $123(806)$ : Pressure 16 StaPT1, 68\% chord, upper 124(807): Pressure $\$ 8$ StaPT1, 56\% chord, upper $[\mathrm{m} / \mathrm{s}] \quad 1.367 \mathrm{E}+01(2.331 \mathrm{E}+00) \quad 7.500 E+\infty 0 \quad 1.991 E+01$ [deg ] $2.830 E+02(8.502 E+00) 2.590 E+02 \quad 3.105 E+02$ [deg ] -8.400E+00 (3.884E+00) -2.137E+01 1.404E+01 $[\mathrm{D} / \mathrm{s}] \quad 1.428 E+01(2.244 E+\infty 0) \quad 8.684 E+00 \quad 2.000 E+01$ $[\mathrm{m} / \mathrm{s}] \cdot 1.371 \mathrm{E}+01(2.184 \mathrm{E}+\infty \mathrm{O}) \quad 7.783 \mathrm{E}+001.993 \mathrm{E}+01$ [Degr] 1.800E+02 (9.148E+01) 1.775E+01 3.388E+02 [N-H ] 1.323E+02 (8.296E+00) 1.198E+02 1.451E+02 $[N-H]-2.272 E+04(2.086 E+03)-2.913 E+04-1.764 E+04$ $[N-n]-2.249 E+02(1.726 E+03)-4.662 E+035.654 E+03$ [Degr] 2.763E+02 (8.029E+00) 2.544E+02 2.937E+02 [KW ] : 1.123E+01 $(2.577 E+00) \quad 2.671 E+00 \quad 1.656 E+01$

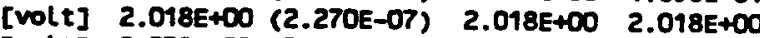
[volt] $2.039 E+00(5.005 E-07) \quad 2.039 E+00 \quad 2.039 E+\infty 0$ $[\varpi / s]$ 4.918E-01 (9.616E-03) 4.354E-01 5.648E-01 $[\mathrm{m} / \mathrm{s}] \quad 4.558 \mathrm{E}-01$ (9.993E-03) 3.952E-01 5.255E-01 $[\mathrm{m} / \mathrm{s}] \quad 6.299 \mathrm{E}-01$ (9.220E-03) 5.703E-01 7.038E-01 $[N T-N] \quad 6.918 E+02(4.938 E+02)-7.433 E+02 \quad 2.632 E+03$ [NT-M] 6.853E+02 (4.936E+02) -7.478E+02 2.625E+03 [NT-M] $6.642 E+02(4.532 E+02)-7.091 E+02 \quad 2.157 E+03$ [WT-N] 7.319E+02 (4.561E+02) -7.268E+02 2.139E+03 [NT-M] $6.340 E+02(3.798 E+02)-4.706 E+022.165 E+03$ [NT-N] 4.009E+02 (2.286E+02) $-2.632 E+02$ 1.350E+03 [NT-H] $3.092 E+02(1.628 E+02)-1.644 E+02 \quad 9.854 E+02$ [NT-H] $1.120 E+02(6.251 E+01)-6.583 E+013.742 E+02$

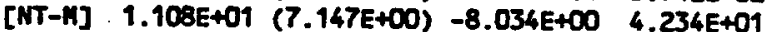
[NT-H] $3.647 E+02(6.867 E+02)-8.222 E+02 \quad 1.600 E+03$ [NT-H] $1.782 E+02(4.615 E+02)-6.326 E+02 \quad 1.036 E+03$ [NT-H] 1.446E+02 (1.800E+02) -2.014E+02 5.160E+02 [NT-N] $-2.793 E+00(1.306 E-01)-2.797 E+00 \quad 2.259 E+\infty 0$ [NT-K] 5.558E-01 (1.909E+01) -3.898E+01 6.480E+01 [MT-H] $-2.766 E+01$ (1.084E+01) $-5.122 E+01-3.106 E+00$ [NT-H] -1.716E+01 (4.445E+03) -8.490E+03 8.060E+03 [NT-H] 1.950E+02 (3.062E+03) $-5.491 E+03 \quad 5.976 E+03$ [NT-H] $1.783 E+03 \quad(3.888 E+02) \quad 5.297 E+02 \quad 2.637 E+03$ [Degr] 1.748E+03 (3.809E+02) $5.501 E+02 \quad 2.573 E+03$ [coef] -5.700E-02 (1.800E-01) -5.357E-01 3.084E-01 [coef] -1.168E-01 (1.640E-01) -6.869E-01 1.943E-01 [coef] -1.878E-01 (1.256E-01) -8.060E-01 2.514E-02 [coef] -2.900E-01 (1.061E-01) -9.282E-01 -3.943E-02 [coef] -5.010E-01 (1.510E-01) -1.013E+00 -9.570E-02 [coef] -8.595E-01 (2.117E-01) -1.673E+00 -3.164E-01 [coef] $-1.008 E+00$ (1.832E-01) -1.764E+00 -4.433E-01 [coef] $-1.098 E+\infty 0(1.820 E-01)-1.817 E+00-5.261 E-01$ [coef] $-1.221 E+00(2.069 E-01)-1.916 E+00-4.835 E-01$ [coef] -1.386E+00 (2.691E-01) -2.191E+00 -4.382E-01 [coef] -1.510E+C0 (3.180E-01) -2.393E+00 -3.817E-01 [coef] $-1.637 E+\infty 0(3.689 E-01)-2.636 E+00-3.740 E-01$ [coef] $-1.758 E+00(4.310 E-01)-2.924 E+00-3.183 E-01$ [coef] $-1.946 E+00$ (5.275E-01) $-3.370 E+\infty 0-2.132 E-01$ [coef] $-2.291 E+00(7.201 E-01)-4.218 E+00-8.941 E-02$ [coef] $-2.546 E+00(9.792 E-01)-4.942 E+00$ 1.611E-01 [coef] $-2.912 E+\infty 0(1.232 E+\infty 0)-6.579 E+\infty 0 \quad 3.924 E-01$ [coef] $-2.756 E+00(1.540 E+\infty)-7.239 E+\infty 0$ 9.794E-01 [coef] 7.620E-01 (1.945E-01) 1.875E-01 1.410E+00 [coef] $9.207 E-01$ (1.063E-01) 4.822E-01 $1.508 E+00$ [coef] 9.225E-01 (9.540E-02) 4.592E-01 $1.454 E+\infty 0$ [coef] 8.880E-01 (1.235E-01) 3.620E-01 1.319E+00 [coef] 6.833E-01 (1.436E-01) 1.282E-01 1.007E+00 [coef] 3.977E-01 (1.472E-01) -1.499E-01 7.185E-01 [coef] -1.186E-01 (1.433E-01) -6.724E-01 2.264E-01 [coef] -3.931E-01 (1.131E-01) -8.076E-01 -9.725E-02 [coef] 8.274E-02 (4.599E-02) -2.641E-01 2.004E-01 [coef] 1.993E-01 (?.488E-02) -1.539E-01 4.151E-01 [coef] $-2.004 E+00$ (4.710E-01) -3.612E+00 -3.278E-01 [coef] -1.010E+00 (2.280E-01) -1.896E+00 -3.966E-01 [coef] $-9.859 E-01(2.340 E-01)-1.827 E+00-2.848 E-01$ [PSI ] 8.937E-02 (8.114E-03) 6.140E-02 1.319E-01 [ab ] 8.070E+02 (2.447E-01) 8.065E+02 8.121E+02 [Degr] 1.206E+01 (2.553E-01) $1.149 E+01 \quad 1.266 E+01$ [coef] $-6.694 E-01$ (2.976E-01) $-2.468 E+\infty 01.007 E-01$ [coef] $-1.130 E+00(3.166 E-01)-2.959 E+00-1.859 E-01$ [coef] $-1.465 E+\infty 0(3.757 E-01)-3.259 E+\infty 0-3.194 E-01$ [coef] $-1.920 E+00(4.902 E-01)-3.755 E+\infty 0-5.513 E-01$ 
167 125(808): Pressure \#10 StapT1, 44\% chord, upper 148 126(809): Pressure \$11 StapT1, 36X chord, upper $149127(810)$ : Pressure 113 StaPT1, 20X chord, upper 150 128(811): Pressure \#14 StaPT1, 14X chord, upper 151 129(812): Pressure 115 StaPT1, 10\% chord, upper 152 130(813): Pressure 116 StaPT1, 8\% chord, upper 153 131(814): Pressure 117 StapT, 6\% chord, upper 154 132(815): Pressure "18 stapT1, 4X chord, upper 155 133(816): Pressure 19 stapT1. 2\% chord, upper 156 134(817): Pressure 21 . Stapt1, 0.5x chord, upper 157 135(818): Pressure $\$ 22$ stapT1, ox chord, Leading 158 136(819): Pressure 23 stapt, 0.5x chord, lower 159 137(820): Pressure 26 stapt1. 12 chord, lover 160 138(821): Pressure "25 StaPT1, 2X chord, lower 161 139(822): Pressure "26 StaPT1, 4\% chord, Lower 162 140(823): Pressure $\$ 28$ StaPT1, 8\% chord, Lover 163 141(826): Pressure \$30 StaPT1, 14X chord, Lower 164 142(825): Pressure \$31 StaPT1, 20\% chord, lower 165 143(826): Pressure 34 StaPT1, 44\% chord, lower 166 144(827): Pressure 336 StaPT1, 68X chord, lower 167 145(828): Pressure 338 stapT1. 92\% chord, lower 168 146(829): Pressure "18 stapT2, 4X chord, upper

169 147(830): Pressure \#11 StaPT2, 36\% chord, upper 170 148(831): Pressure \#11 StaPT3, 36X chord, upper $-171$

172

173

176

175

176

177

178

179

181

182

183

184

185

186

187

188

189

190

191

192

193

194

195

196

197

198

199

200

$-201$

202

203

204

205

206

207

208

209

210

211

212

213

214

215

216

217

218

219
149(832): Total Pressure Probe, 34X span

150(833): Pressure \#1 StaPT4, $100 \%$ chord, trailing 151(834): Pressure "12 StaPT4, 92X chord, upper 152(835): Pressure 14 StaPT4, 80\% chord, upper 153(836): Pressure 16 StaPT4, 68\% chord, upper 154(837): Pressure 18 StaPT4, 56\% chord, upper 155(838): Pressure 110 StapT4, $44 \%$ chord, upper 156(839): Pressure $\$ 11$ stapT4. 36\% chord, upper 157(840): Pressure \$12 StaPT4, $28 \%$ chord, upper 158(841): Pressure "13 StaPT4, 20X chord, upper 159(842): Pressure \$14 StaPT4, $14 Z$ chord, upper 160(843): Pressure 115 StaPT4, 10\% chord, upper 161(844): Pressure \$16 StaPT4, 8x chord, upper 162(845): Pressure 17 StapT4, 6\% chord, upper 163(846): Pressure \#18 StaPT4, 4\% chord, upper 164(847): Pressure \#19 StaPT4, 2X chord, upper 165(848): Pressure 120 StaPT4, 12 chord, upper 166(849): Pressure "21 StaPT4, 0.5\% chord, upper 167(850): Pressure \#22 StaPT4, 0x chord, leading 168(851): Pressure 23 StaPT4, 0.5x chord, lower 169(852): Pressure "25 StaPT4, 2x chord, Lower 170(853): Pressure \#26 StaPT4, 4X chord, lover 171(854): Pressure \$27 StaPT4, 6x chord, lower 172(855): Pressure "128 StaPT4, 8\% chord, Lower 173(856): Pressure 130 StapT4, 16\% chord, lower 174(857): Pressure $\$ 32$ StaPT4, $28 \%$ chord, Lower 175(858): Pressure \$34 StaPT4, 44X chord, lower 176(859): Pressure $\$ 36$ StaPT4, 68X chord, lower 177(860): Pressure 138 StaPT4, $92 \%$ chord, Lower 178(861): Pressure \#18 StaPT3, 4\% chord, upper 179(862): Total Pressure Probe, 50.6\% span 180(105): 5 M WD 181(106): 5 in US 182(107.): $10 \%$ WD 183(108): 10 n US 184(109): 20 in WD 185(110): 20 in wS 186(111): $50 \mathrm{n}$ wo 187(112): 50 in US 188(113): 5 i Air Temperature 189(114): Delta Temperature T50 - T05 190(116): Baro Pressure 191(701): Time 192(702): Time 193(703): Time 194(704): Time 195(705): Time 196(706): Lift Cl(80\%) StaPT10 197(707): Lift Cl(63\%) StapT7 [coef] $-2.312 E+\infty 0(5.720 E-01)-4.147 E+00-7.056 E-01$ [coef] $-2.538 E+\infty 0(6.332 E-01)-4.423 E+00-7.068 E-01$ [coef] $-2.692 E+00(7.011 E-01)-5.245 E+00-6.742 E-01$ [coef] $-2.737 E+00(7.079 E-01)-5.580 E+00-7.209 E-01$ [coef] $-2.695 E+00(7.167 E-01)-5.838 E+00-7.145 E-01$ [coef] $-2.741 E+\infty 0(7.164 E-01)-5.871 E+\infty 0-6.526 E-01$ [coef] $-2.753 E+00(7.218 E-01)-5.794 E+00-6.846 E-01$ [coef] $-2.717 E+00(7.273 E-01)-5.744 E+00-6.902 E-01$ [coef] $-2.769 E+00(7.499 E-01)-6.061 E+00-7.048 E-01$ [coef] $-2.786 E+00$ (8.264E-01) $-6.749 E+00-6.770 E-01$ [coef] $-2.873 E+00(8.977 E-01)-7.689 E+00-3.362 E-01$ [coef] -9.818E-01 (4.666E-01) -2.663E+00 8.482E-01 [coef] -3.590E-01 (3.762E-01) - $1.995 E+001.005 E+\infty 0$

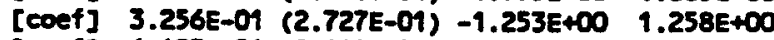
[coef] 6.493E-01 (2.149E-01) -8.688E-01 1.386E+00 [coef] 8.544E-01 (1.733E-01) -6.280E-01 1.490E+00 [coef] 7.977E-01 (1.523E-01) -6.623E-01 1.275E+00 [coef] 6.742E-01 (1.527E-01) -7.701E-01 1.101E+C0 [coef] 8.994E-02 (1.752E-01) -1.394E+00 5.610E-01 [coef] 1.368E-01 (1.119E-01) -1.399E+00 4.932E-01 [coef] -1.947E-02 (1.229E-01) -1.614E+00 2.906E-01 [coef] -2.283E+00 (8.897E-01) -6.276E+00 -8.209E-01 [coef] $-1.691 E+00(4.505 E-01)-3.592 E+00-5.050 E-01$ [coef] $-1.366 E+00$ (2.980E-01) $-2.832 E+00$-5.990E-01 [Dsi ] 2.219E-02 (4.971E-03) -1.350E-02 3.947E-02 [coef] -7.027E-01 (2.086E-01) $-1.529 E+00$ 1.510E-01 [coef] -7.522E-01 (2.031E-01) -1.572E+00 7.988E-02 [coef] -8.203E-01 (2.048E-01) -1.685E+00 -1.352E-02 [coef] -8.677E-01 (2.037E-01) -1.852E+00 -1.292E-01 [coef] -8.952E-01 (2.011E-01) -1.966E+00 -2.629E-01 [coef] -9.708E-01 (1.829E-01) -1.991E+00 -3.928E-01 [coef] $-1.005 E+00$ (1.841E-01) $-2.096 E+00-3.618 E-01$ [coef] $-9.037 E+00(1.943 E-01)-2.282 E+00-4.137 E-01$ [coef] $-1.109 E+\infty 0(2.214 E-01)-2.401 E+00-4.357 E-01$ [coef] $-1.154 E+00(2.545 E-01)-2.642 E+00-5.705 E-01$ [coef] $-1.191 E+00(2.878 E-01)-2.993 E+00-5.974 E-01$ [coef] $-1.214 E+\infty 0(3.170 E-01)-3.302 E+00-6.113 E-01$ [coef] $-1.220 E+00(3.463 E-01)-3.591 E+00-6.110 E-01$ [coef] $-1.267 E+00(4.237 E-01)-4.039 E+00-6.297 E-01$ [coef] $-1.329 E+00(5.474 E-01)-4.857 E+00-6.506 E-01$ [coef] $-1.368 E+\infty 0(6.544 E-01)-5.830 E+00-6.607 E-01$ [coef] $-1.413 E+00$ (7.600E-01) $-6.806 E+00-6.042 E-01$ [coef] $-1.611 E+00(8.641 E-01)-7.878 E+00-5.066 E-01$ [coef] . 1.614E-01 (2.411E-01) -1.197E+00 1.044E+00 [coef] 8.105E-01 (1.565E-01) -1.476E-02 1.424E+00 [coef] 9.005E-01 (1.235E-01) 7.205E-02 1.429E+00 [coef] 8.925E-01 (1.115E-01) 5.579E-02 1.371E+00 [coef] 8.496E-01 (1.070E-01) 1.251E-02 1.188E+00 [coef] : $6.399 E-01$ (1.162E-01) -2.097E-01 9.848E-01 [coef] : 1.246E-01 (1.594E-01) -7.277E-01 5.397E-01 [coef] -2.878E-01 (1.646E-01) -1.142E+00 2.365E-01 [coef] 1.964E-02 (8.938E-02) -8.104E-01 3.056E-01 [coef] -1.057E-01 (1.076E-01) -9.337E-01 2.926E-01 [coef] $-1.503 E+00(4.382 E-01)-3.915 E+00-6.978 E-01$ [Dsi $] \quad 3.770 E-02$ (5.849E-03) 6.678E-03 6.198E-02 [Deg ] 3.548E+02 $(1.788 E+00) \quad 2.621 E+02 \quad 3.549 E+02$ $[\mathrm{m} / \mathrm{s}] \mathrm{8.589E+00}(2.167 \mathrm{E}+00) \quad 3.917 \mathrm{E}+00 \quad 1.547 \mathrm{E}+01$ [Deg ] 3.597E+02 (6.960E-02) 3.563E+02 3.597E+02 [a/s ] 2.926E-01 (1.655E+00) -4.666E-02 8.164E+01 [Deg ] 2.916E+02 (1.003E+01) 8.607E+01 3.394E+02 $[\mathrm{a} / \mathrm{s}] \quad 1.260 \mathrm{E}+01(3.146 \mathrm{E}+00) \quad 7.335 E+\infty 0 \quad 9.483 E+01$

[Deg ] 2.908E+02 (8.688E+00) 1.770E+02 3.182E+02

$[a / s] 1.389 E+01(2.129 E+\infty 0) \quad 1.021 E+01 \quad 2.250 E+01$

[Deg ] 1.409E+01 (2.352E-01) 1.382E+01 2.502E+01 [Deg ] -8.861E-01 (2.465E-02) -9.447E-01 -8.218E-01

[ $m b] \cdot 8.065 E+02(6.315 E-01) \quad 7.749 E+02 \quad 8.067 E+02$ [Day ] $1.350 E+02(0.000 E+00) 1.350 E+02$ 1.350E+02 [Hour] 1.700E+01 $(0.000 E+00)$ 1.700E+01 1.700E+01 [Hinu] $9.474 E+00(1.496 E+00) 7.000 E+001.200 E+01$ [Seco] $2.950 E+01(1.732 E+01) \quad 0.000 E+00 \quad 5.900 E+01$ [Mill] 5.000E+02 (2.603E+02) 4.900E+01 9.508E+02 [coef] $8.441 E-01$ (1.578E-01) 1.959E-01 1.336E+00 [coef] 9.914E-01 (1.528E-01) 4.499E-01 $1.514 E+\infty 0$ 


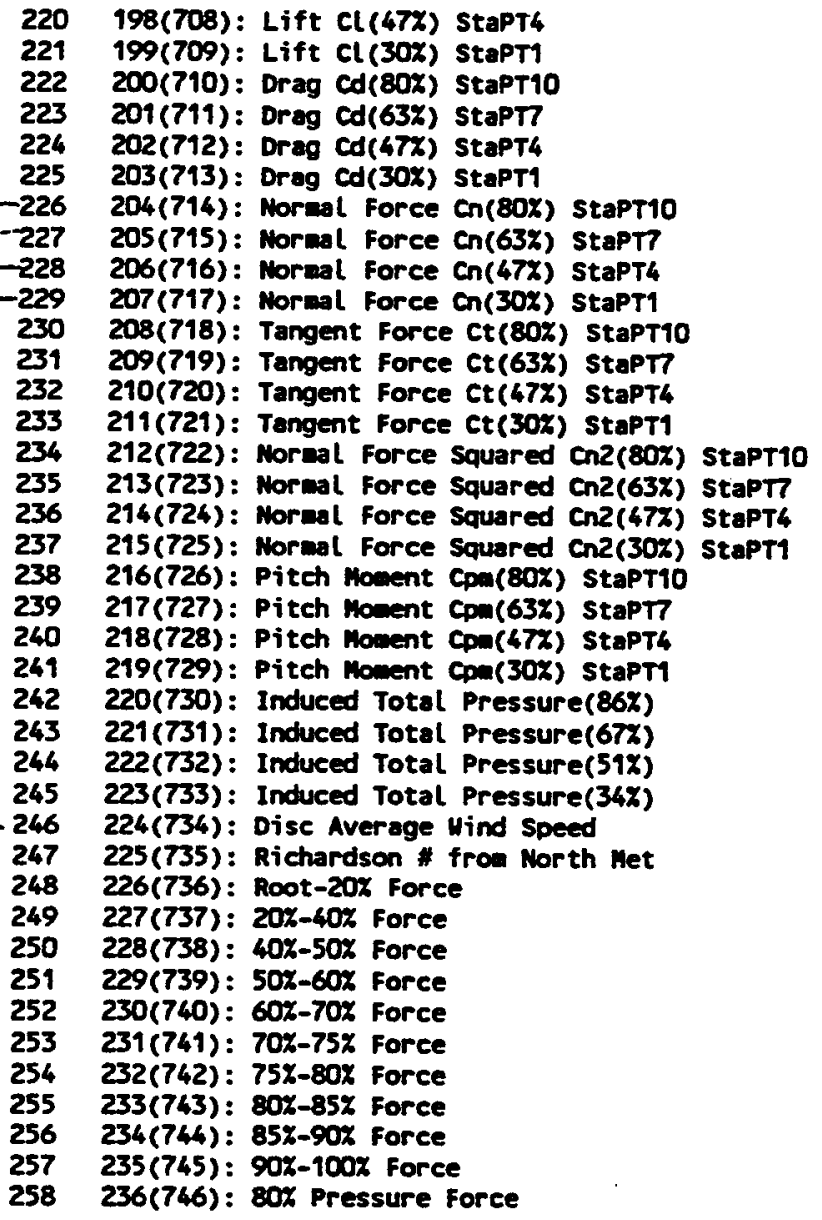

\begin{tabular}{|c|c|c|c|c|}
\hline bet & $\begin{array}{l}9.765 E-01 \\
1.812 E+00 \\
4.838 E-02 \\
2.148 E-01 \\
3.024 E-01 \\
1.186 E+00 \\
8.393 E-01 \\
1.020 E+00 \\
1.028 E+00 \\
2.175 E+00 \\
1.089 E-01 \\
8.448 E-02 \\
2.228 E-02 \\
7.599 E-02 \\
7.340 E-01 \\
1.080 E+00 \\
1.092 E+00 \\
4.932 E+00 \\
2.658 E-02\end{array}$ & 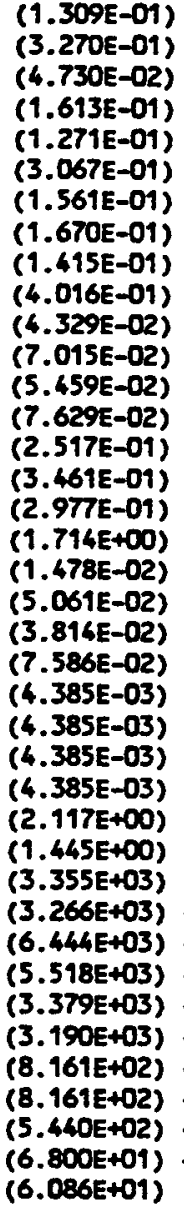 & $\begin{array}{r}7 . \\
-2 . \\
-6 . \\
-6 . \\
1 . \\
4 . \\
5 . \\
9 . \\
-2 . \\
-6 . \\
-9 . \\
-8 . \\
4 . \\
2 . \\
3 . \\
8 . \\
-1 . \\
-2 . \\
-2 . \\
-4 . \\
8 . \\
5 . \\
3 . \\
1 . \\
8 . \\
-5.0 \\
-2.0 \\
-3.2 \\
-3.9 \\
-5.7 \\
-2.0 \\
-3.4 \\
-4.8 \\
-9.1 \\
-3.2 \\
-7.6\end{array}$ & $\begin{array}{l}+00 \\
+00 \\
.00 \\
-01 \\
-01 \\
.01 \\
.01 \\
100 \\
.00 \\
.00 \\
.01 \\
.03 \\
.03 \\
.03 \\
.02 \\
.01 \\
.02 \\
.02 \\
.02 \\
01 \\
.04 \\
03 \\
04 \\
03 \\
04 \\
04 \\
04 \\
04 \\
02 \\
03 \\
02\end{array}$ \\
\hline
\end{tabular}


Appendix C.2.2

Pressure Distributions for $80 \%$ Span 


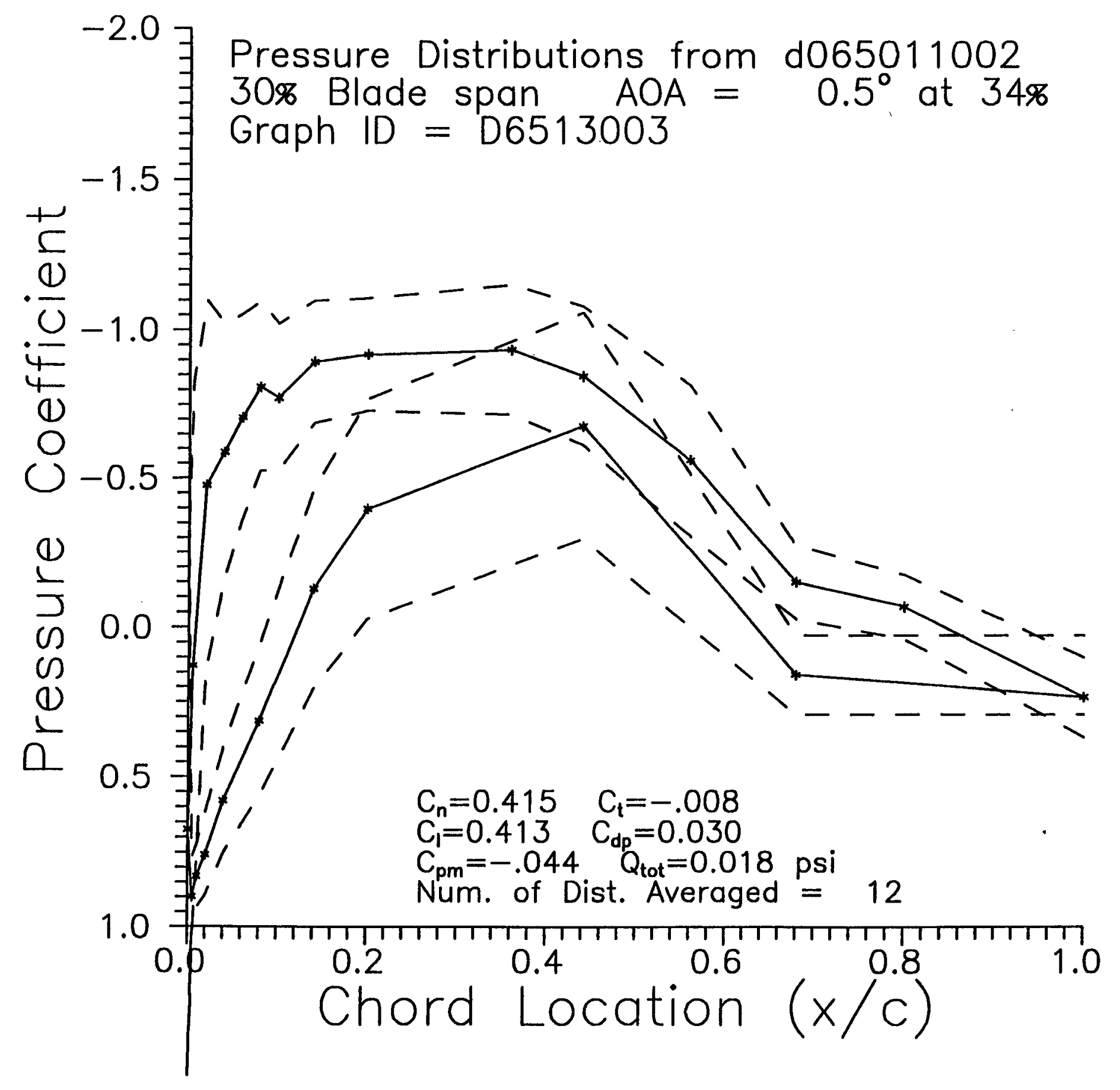




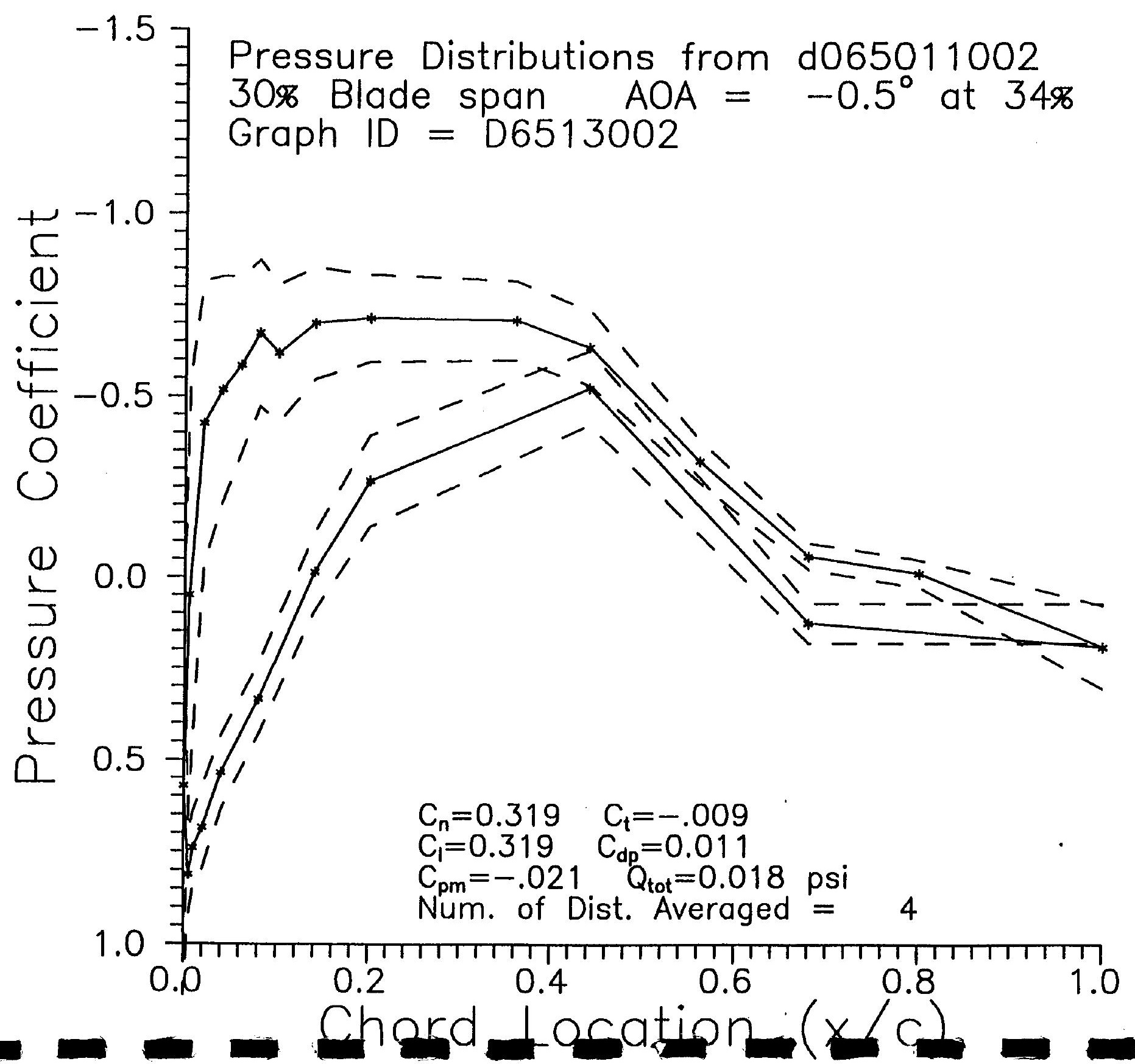




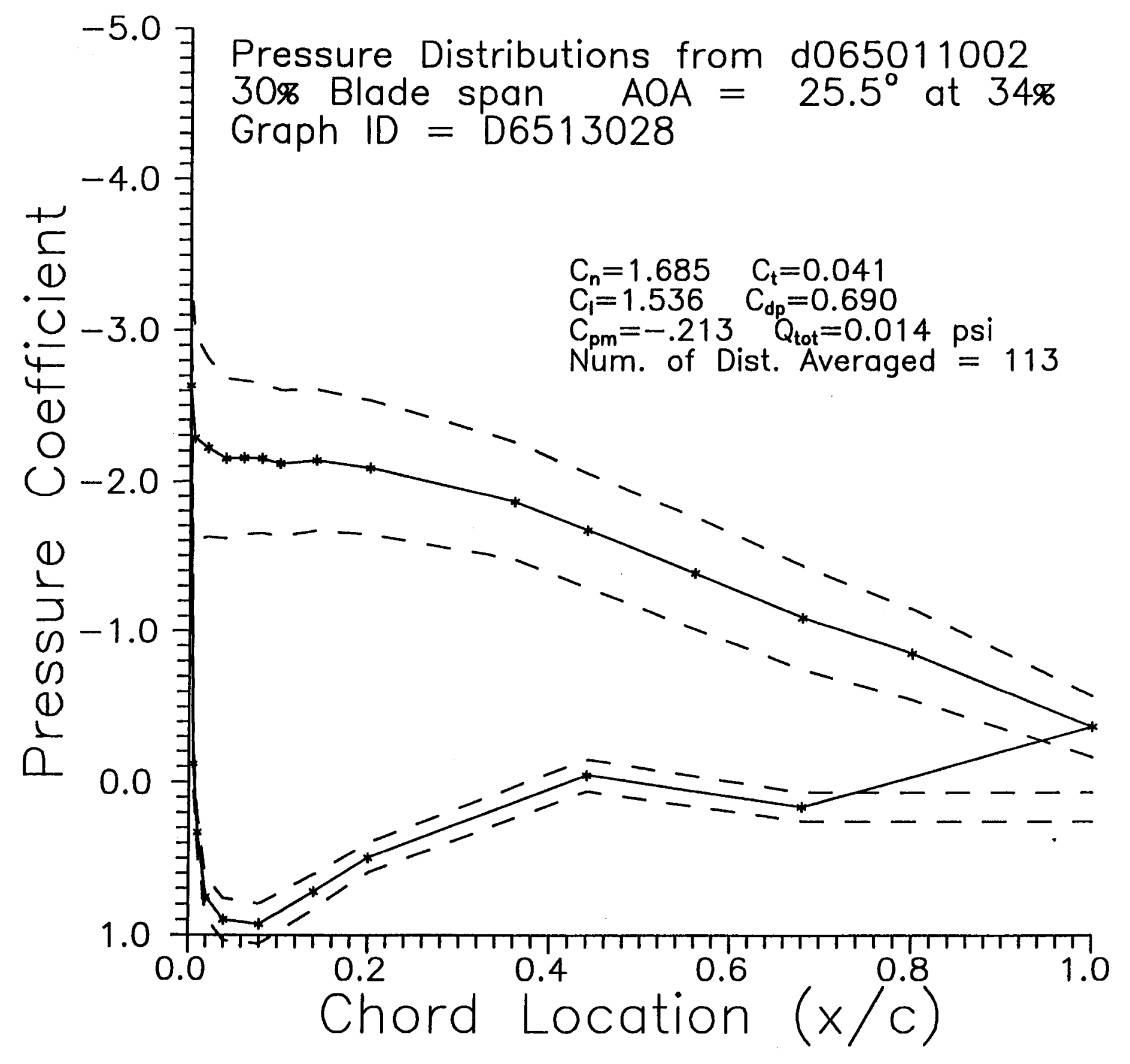




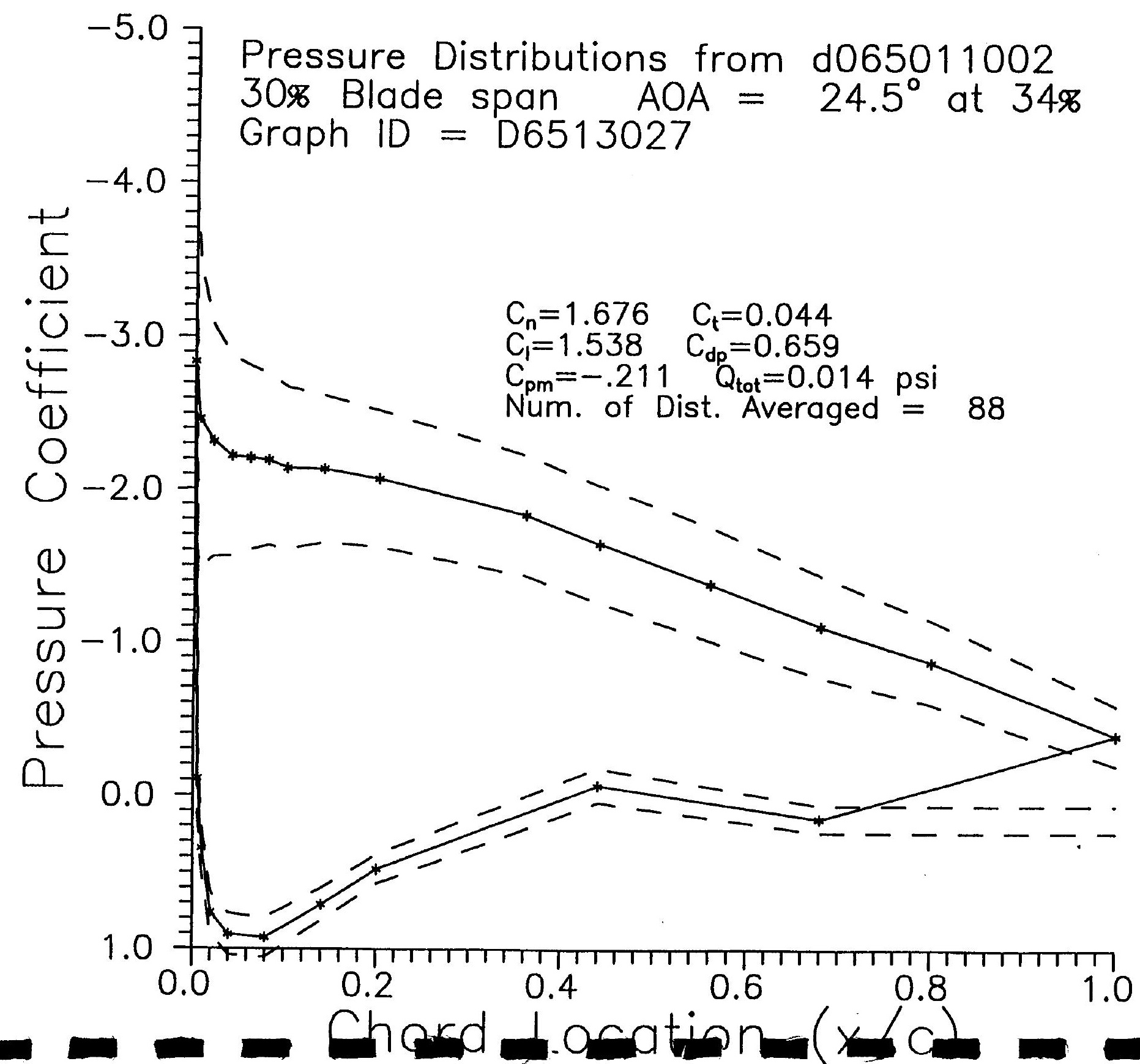




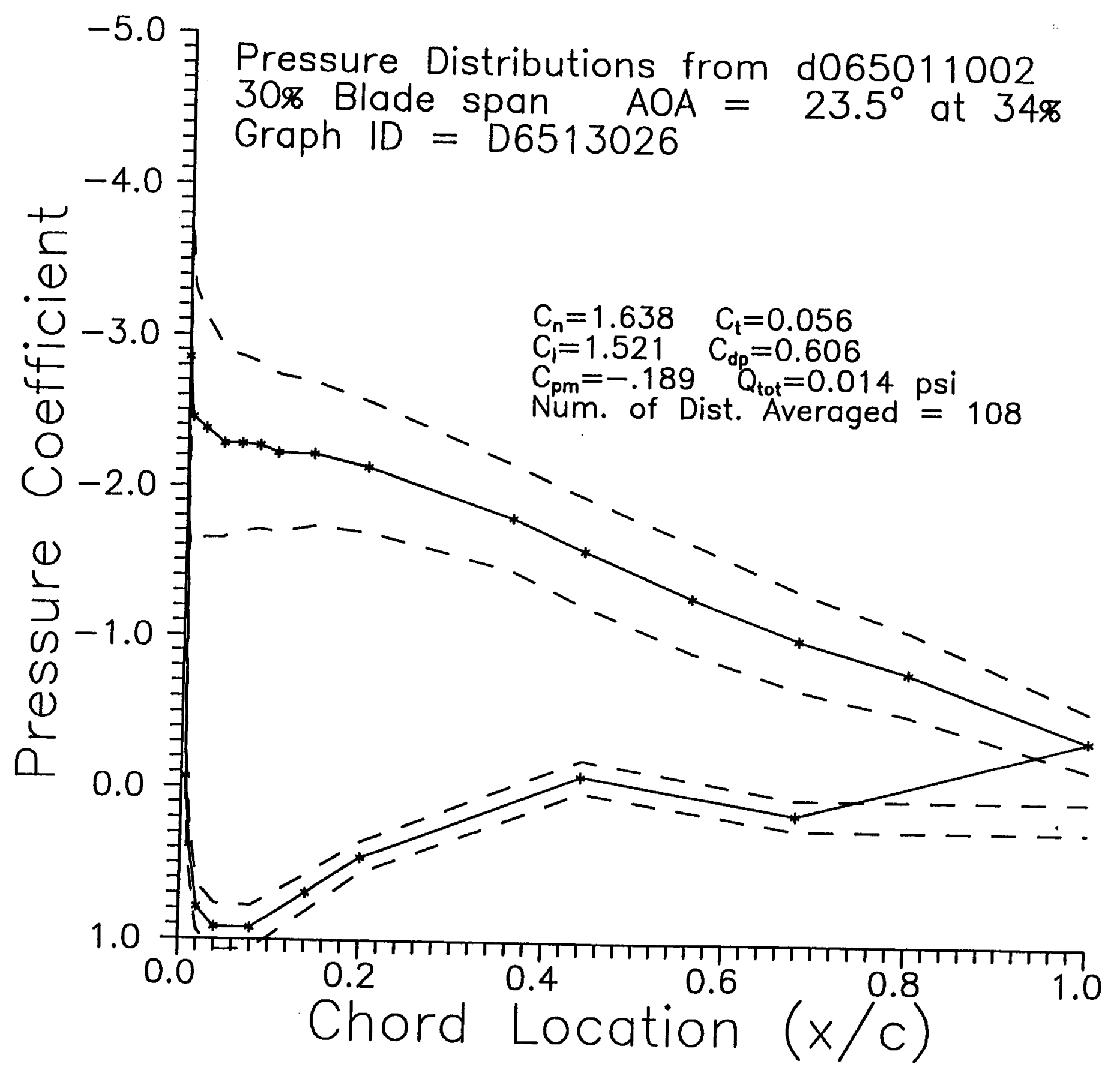




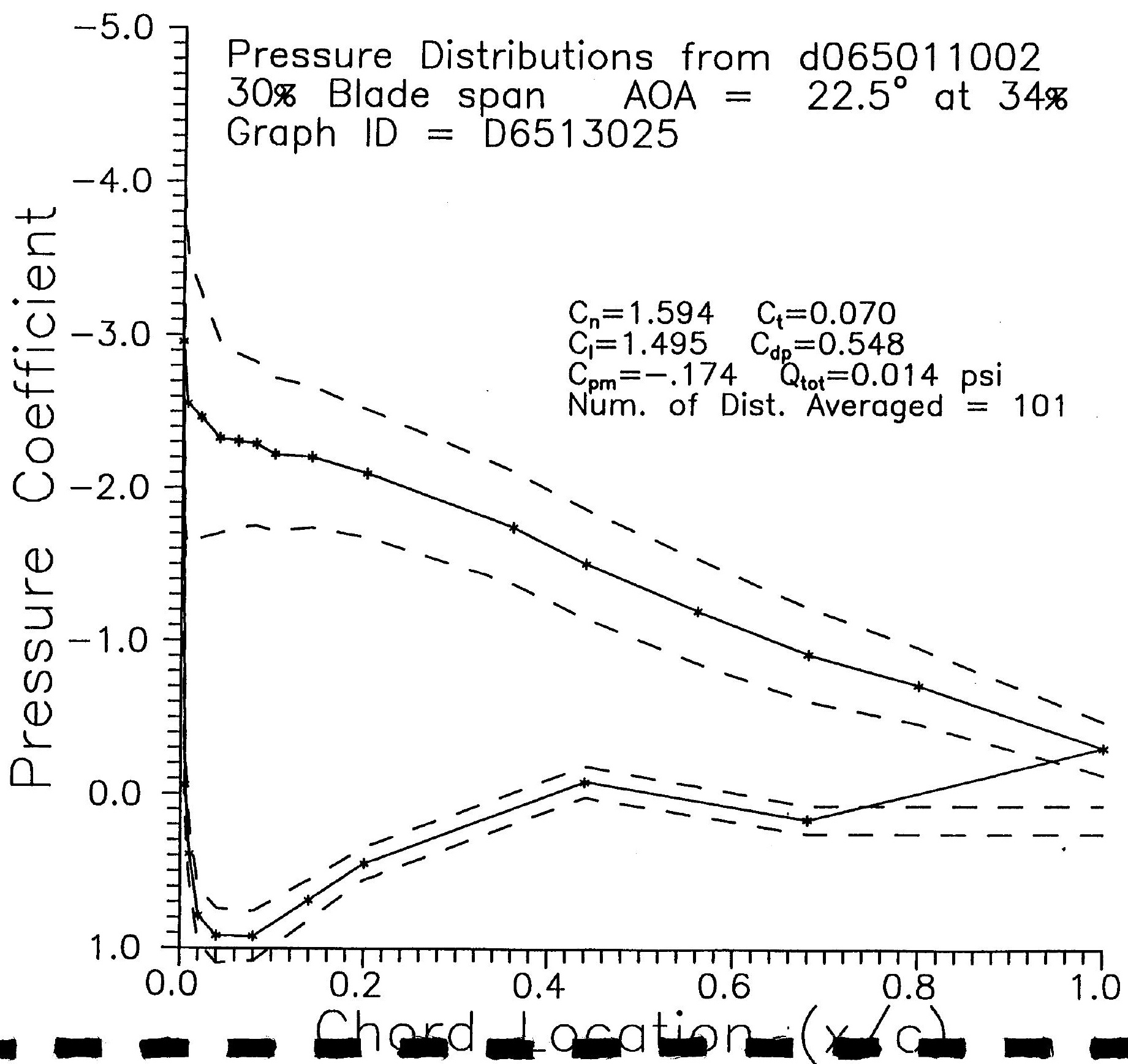




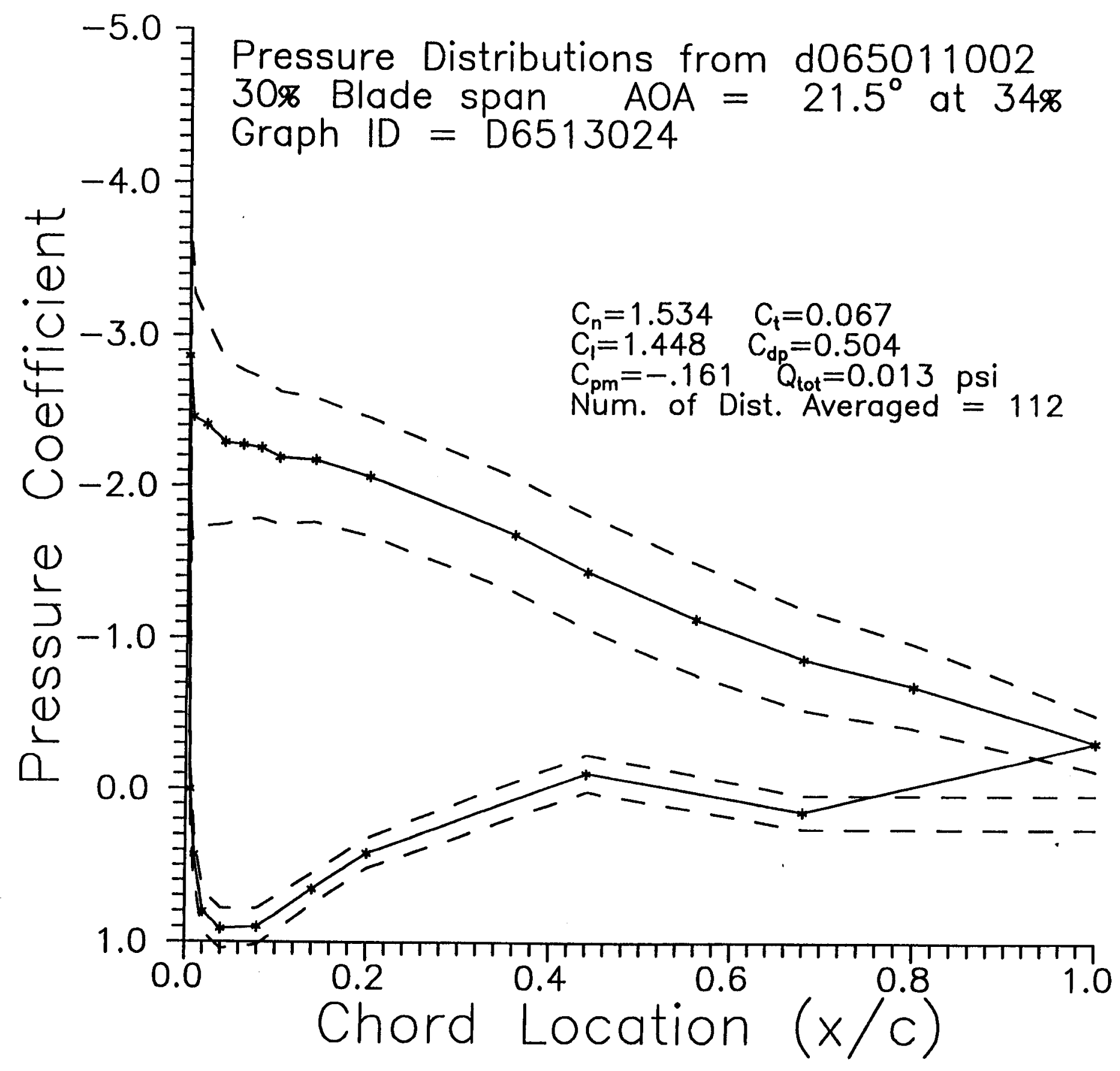




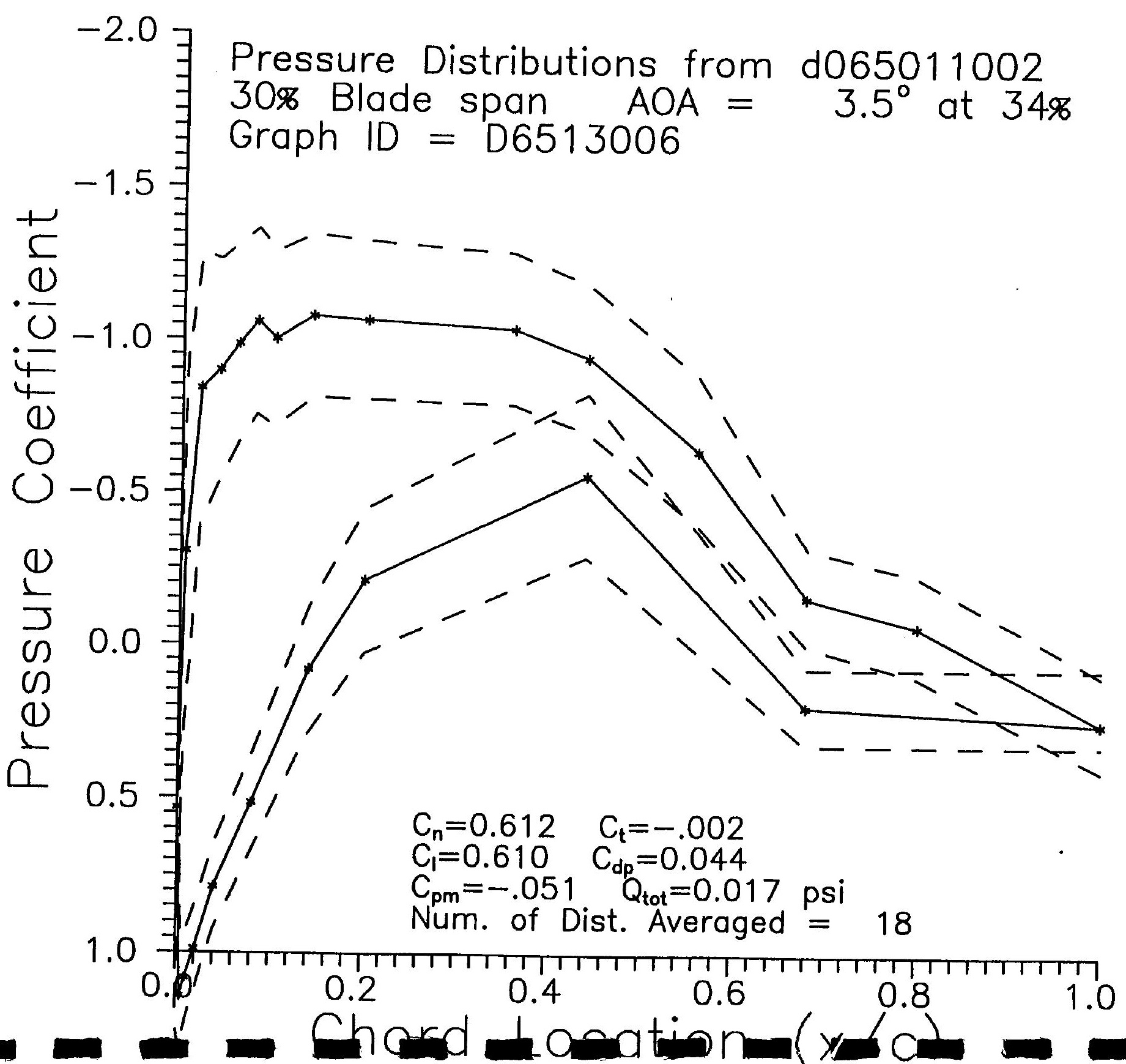




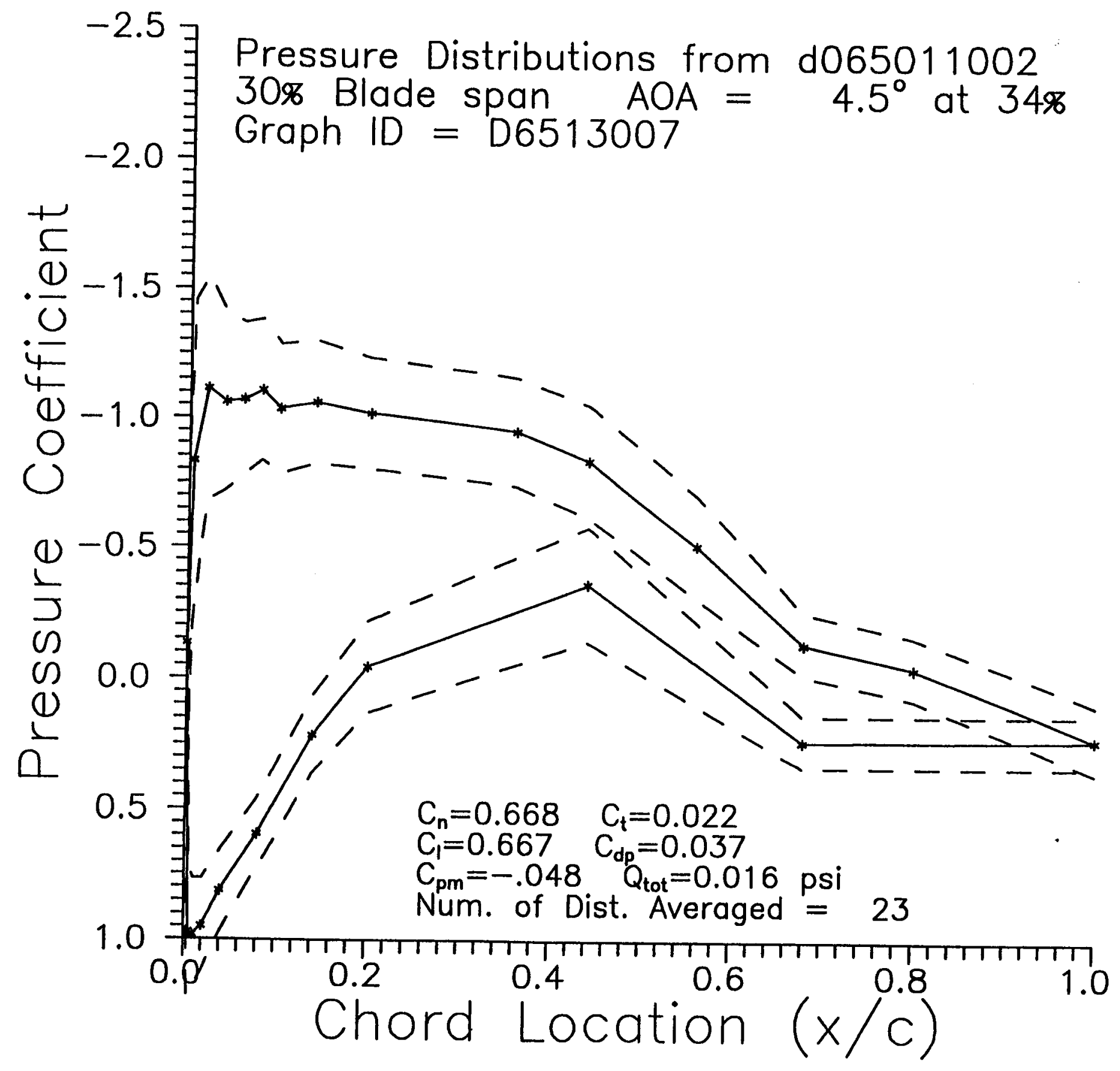




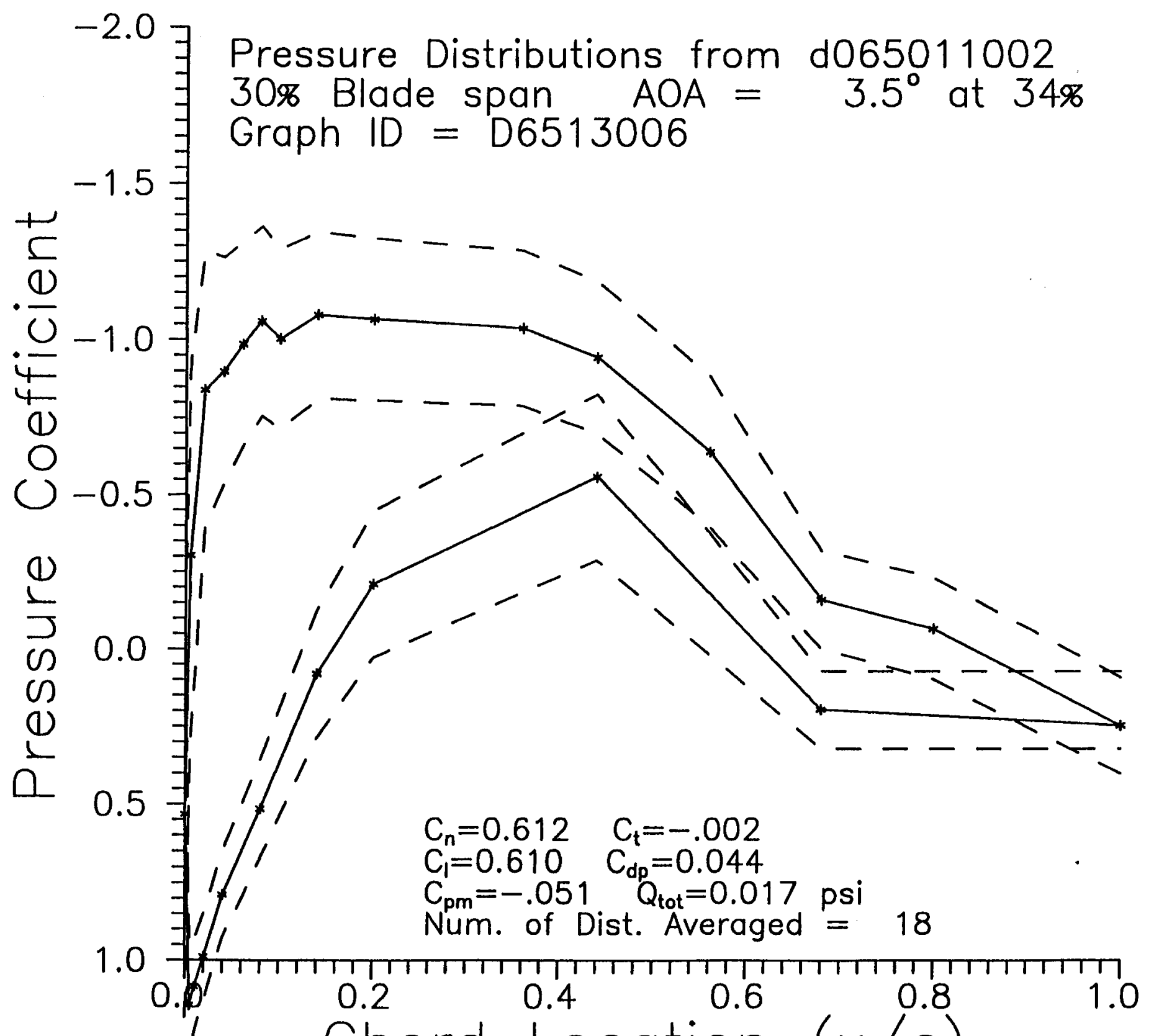

chord doation (x) 


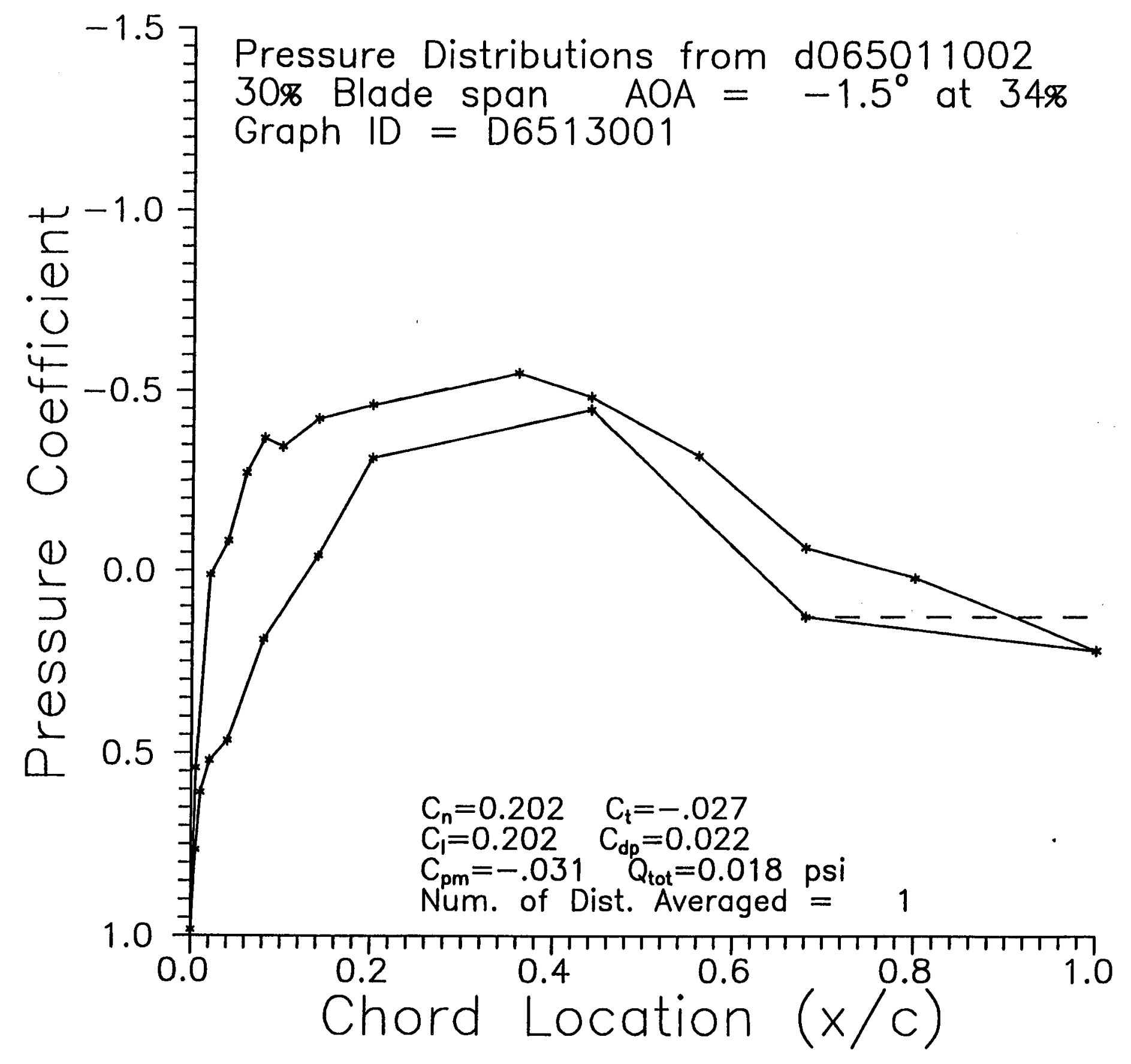




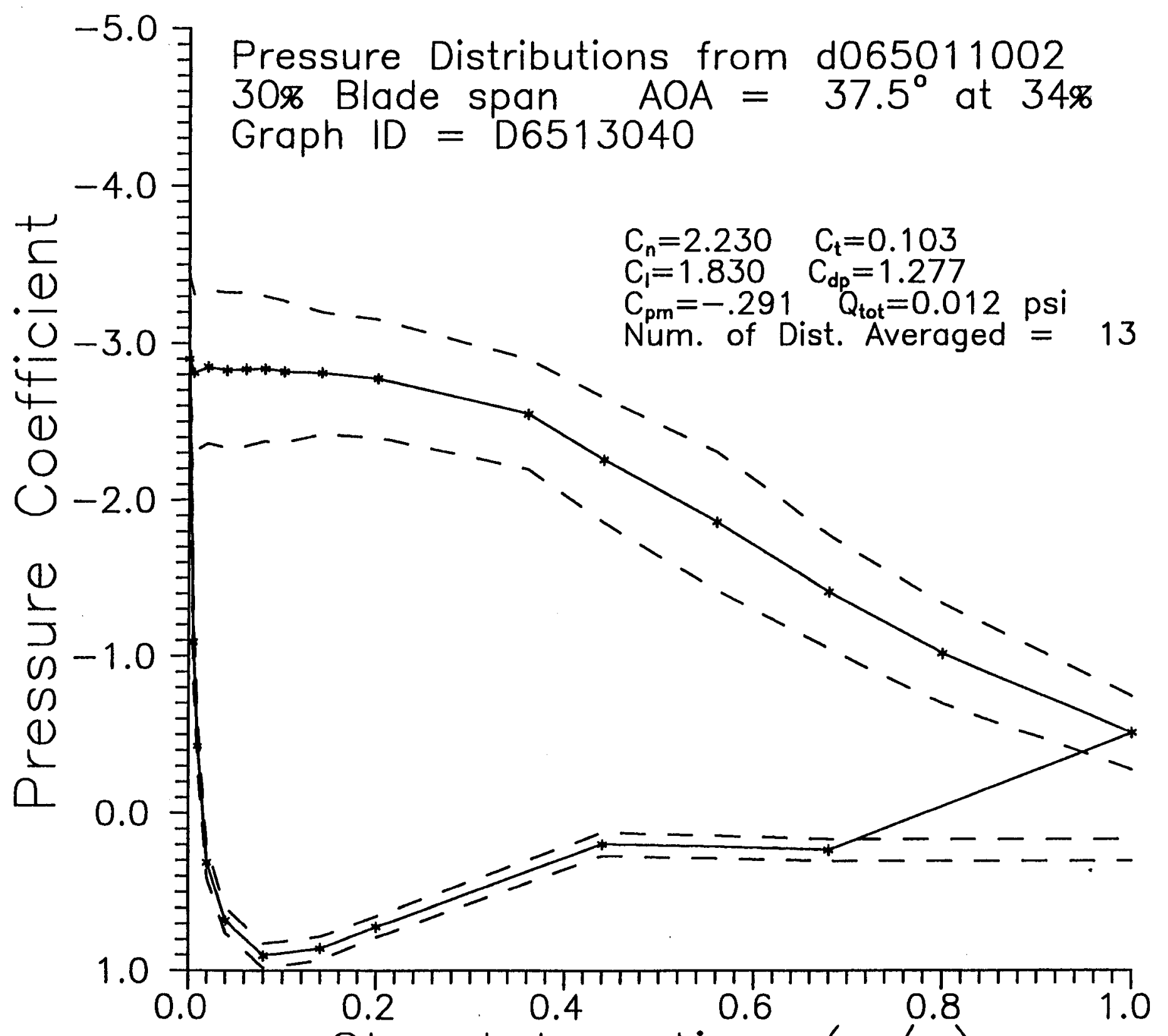

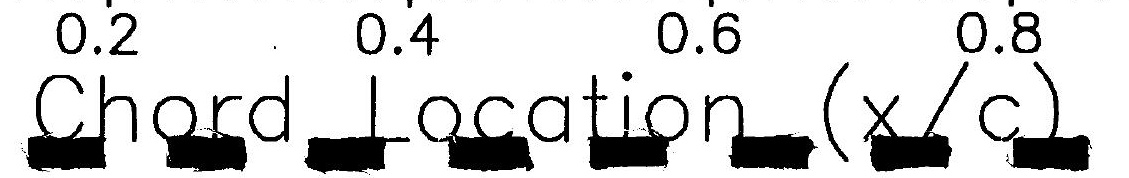




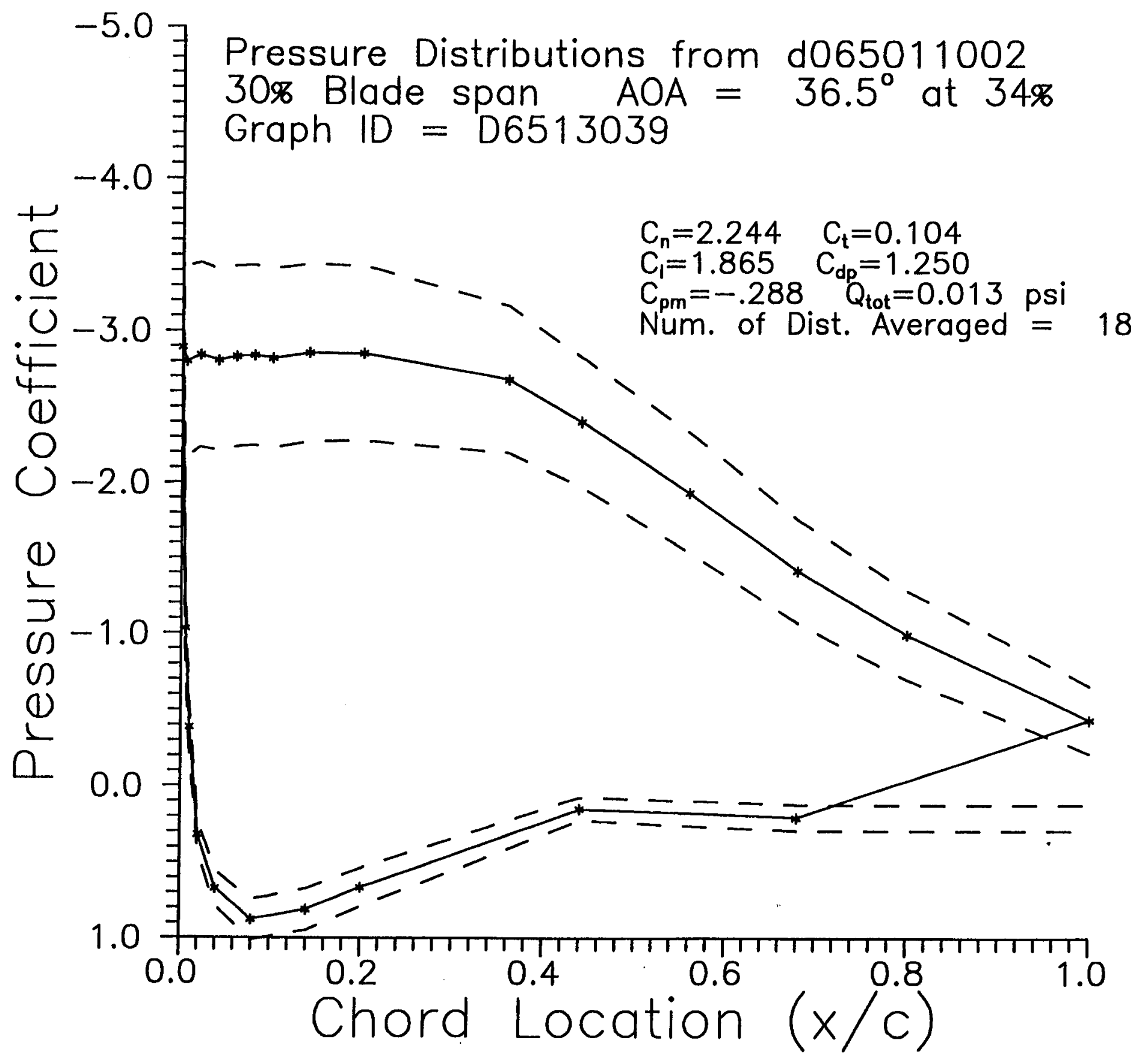




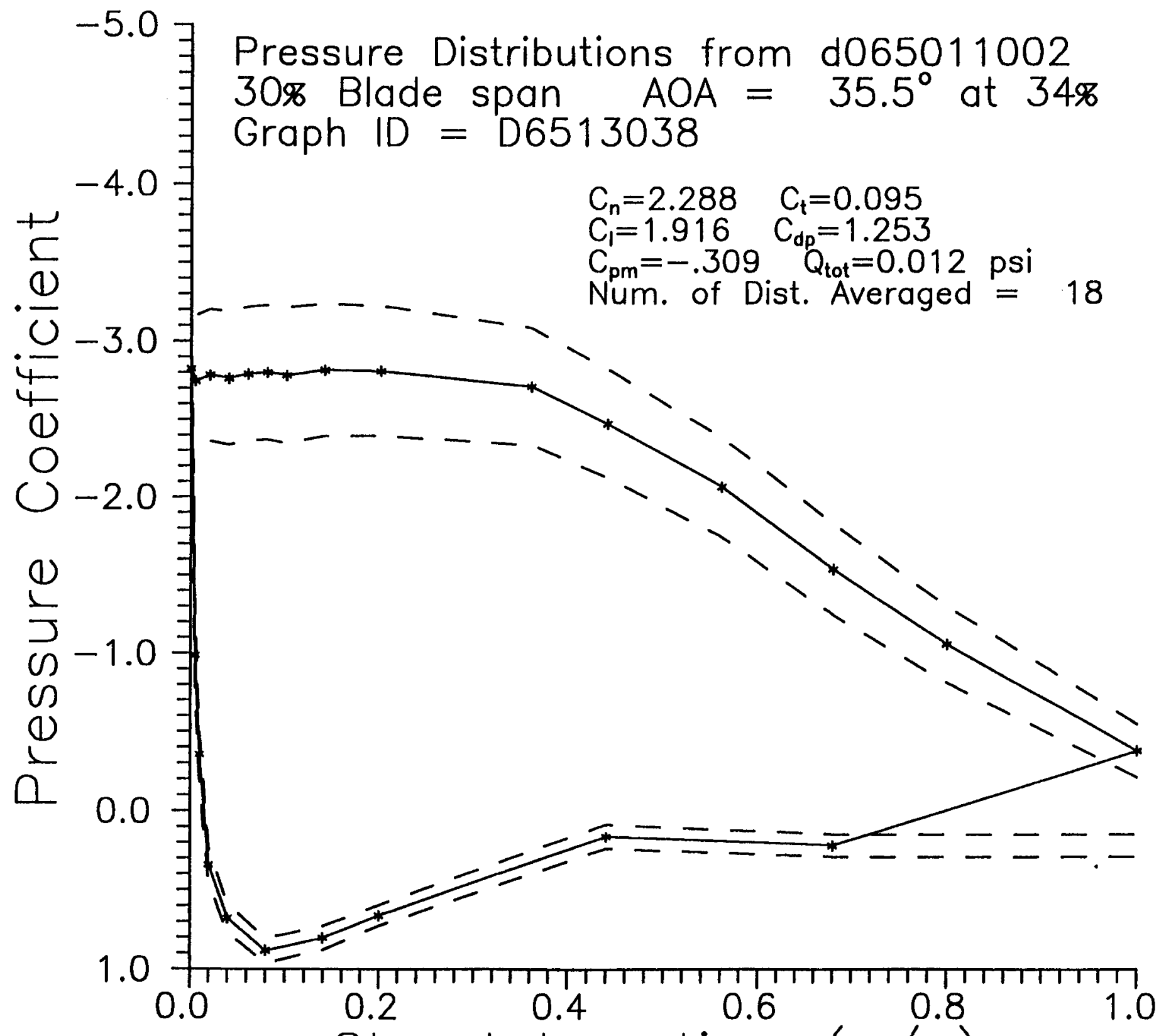

$\begin{array}{lllll}0.2 & 0.4 & 0.6 & 0.8 & 1.0\end{array}$ 


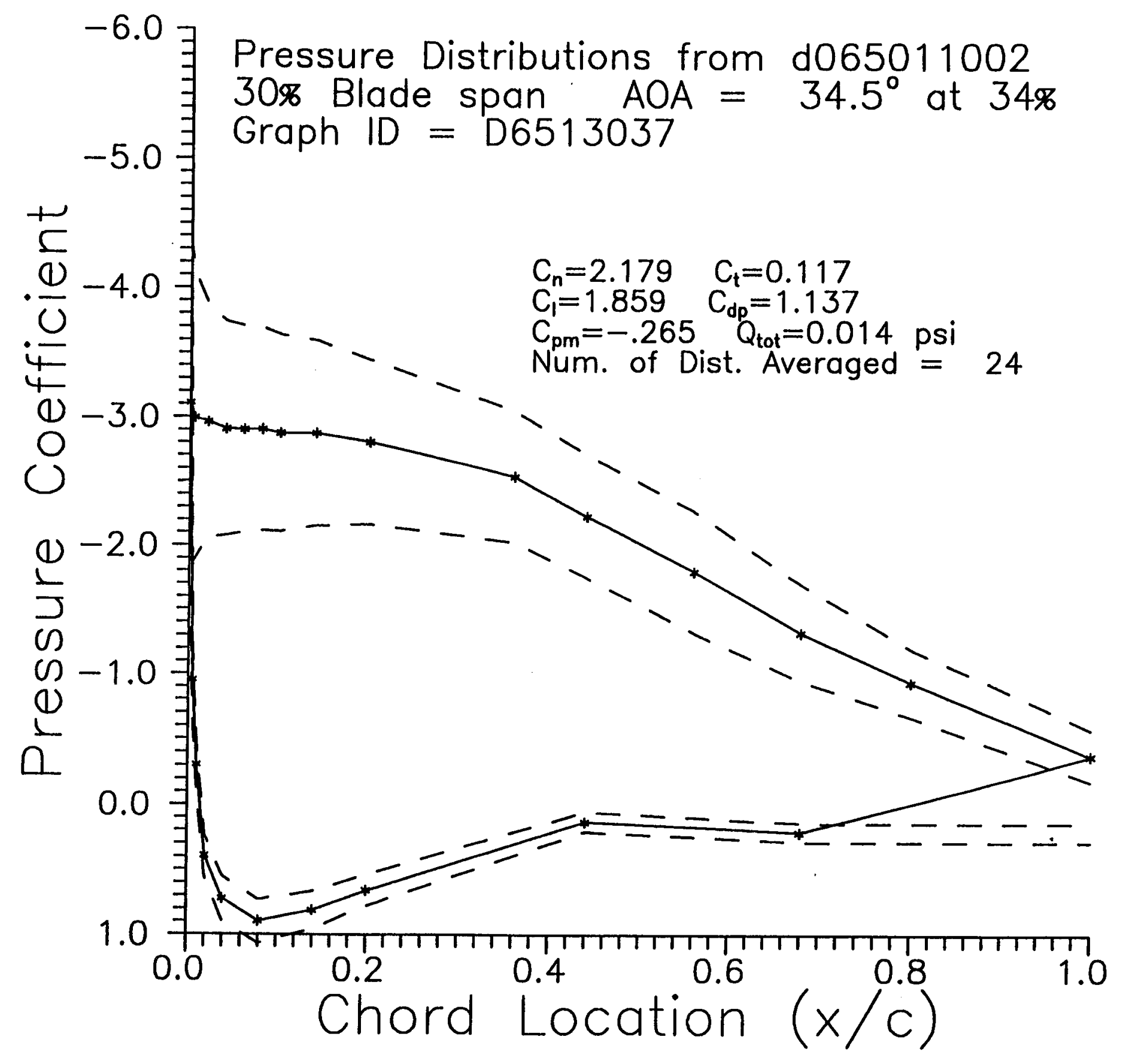




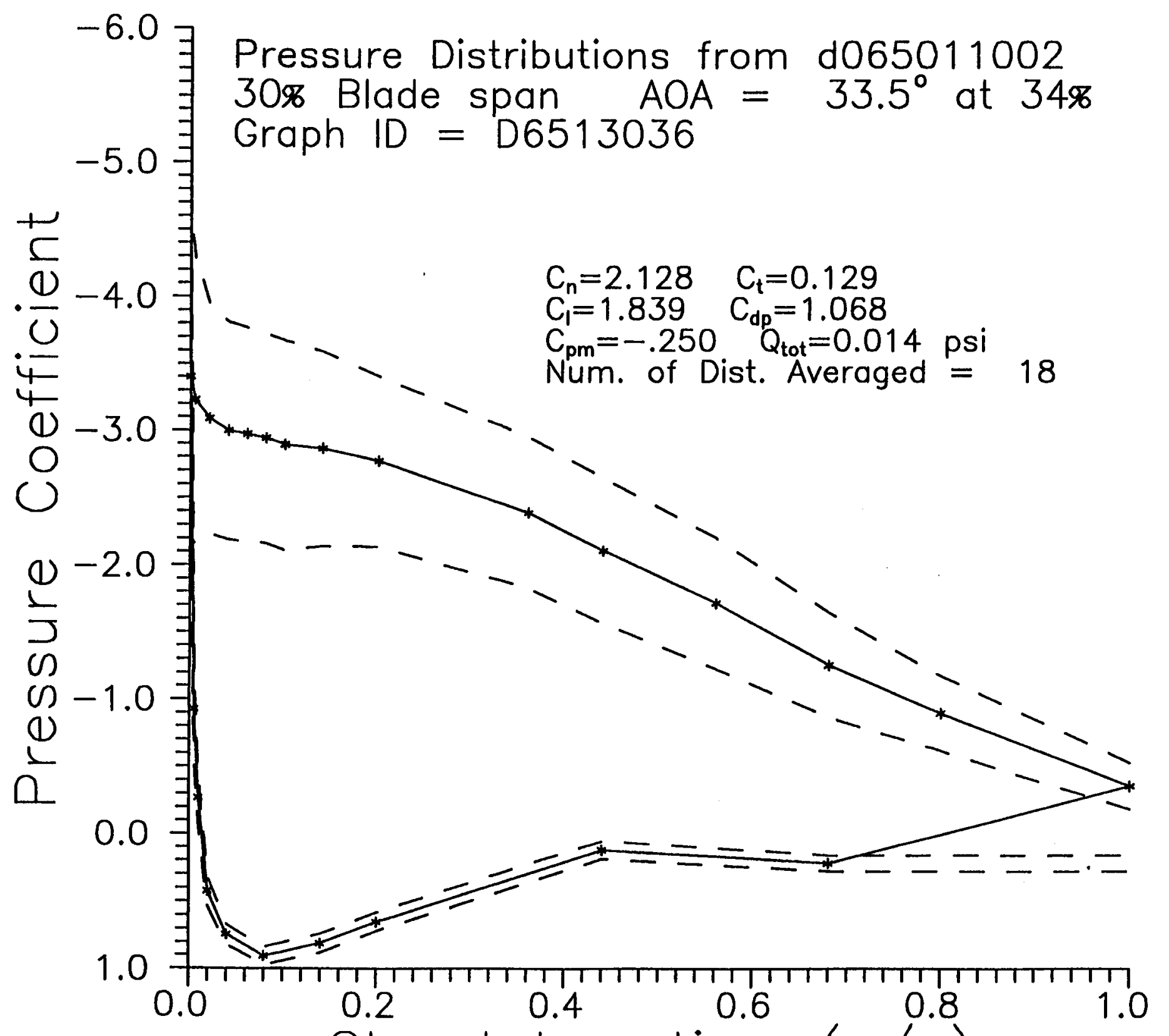

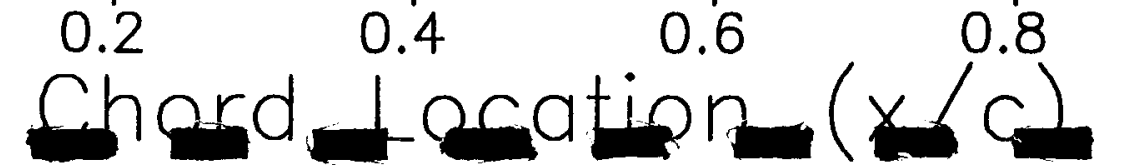




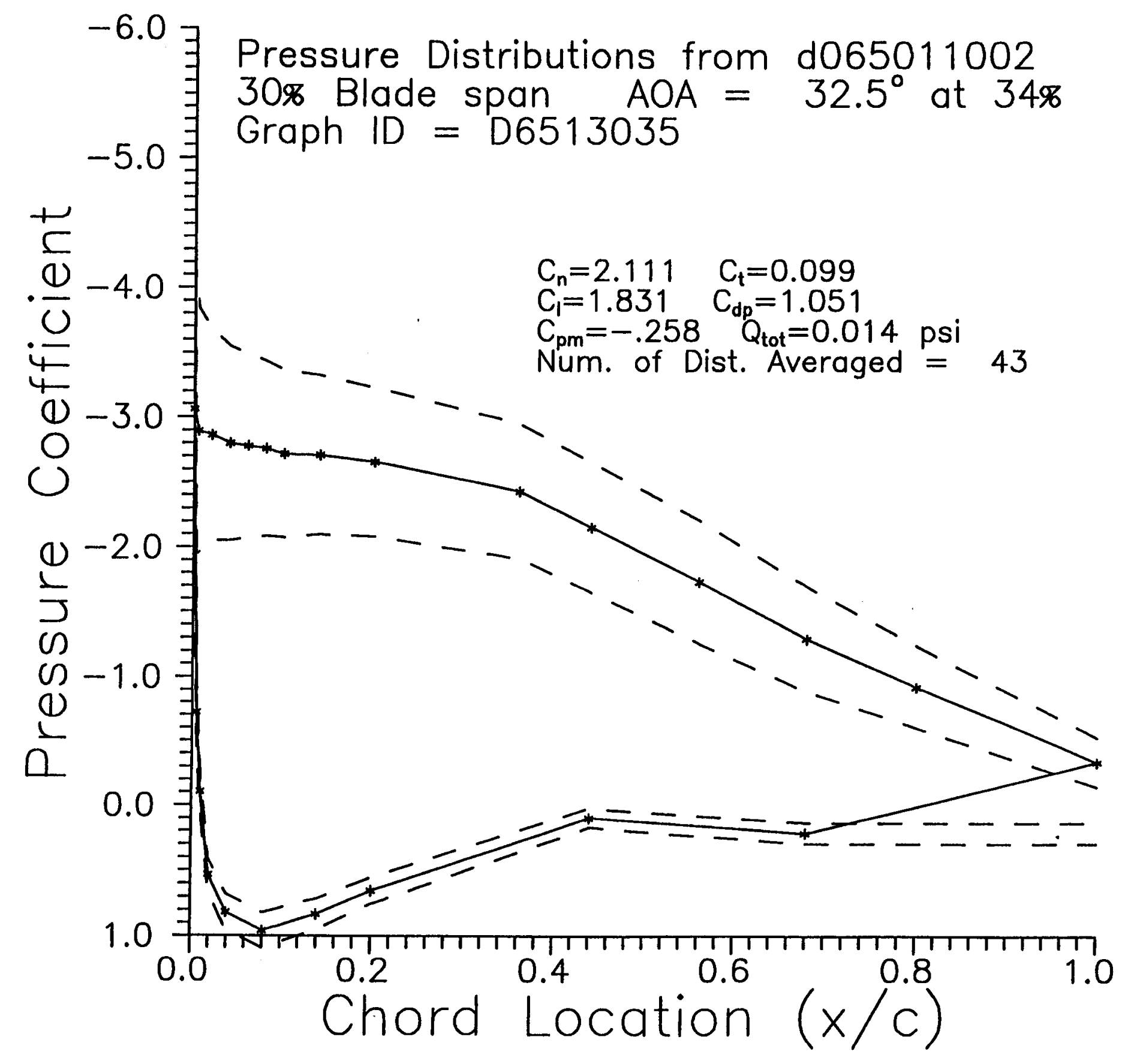




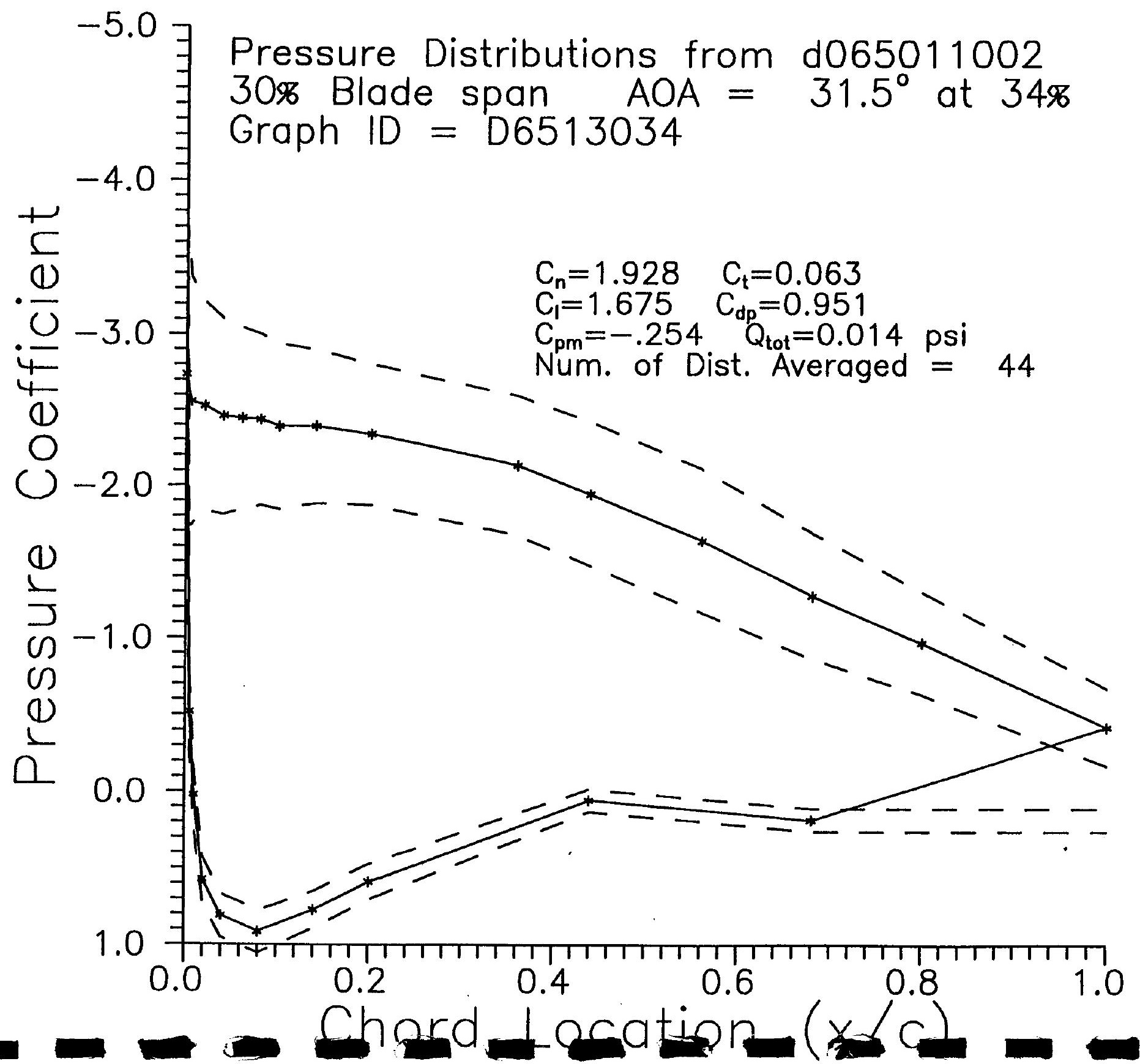




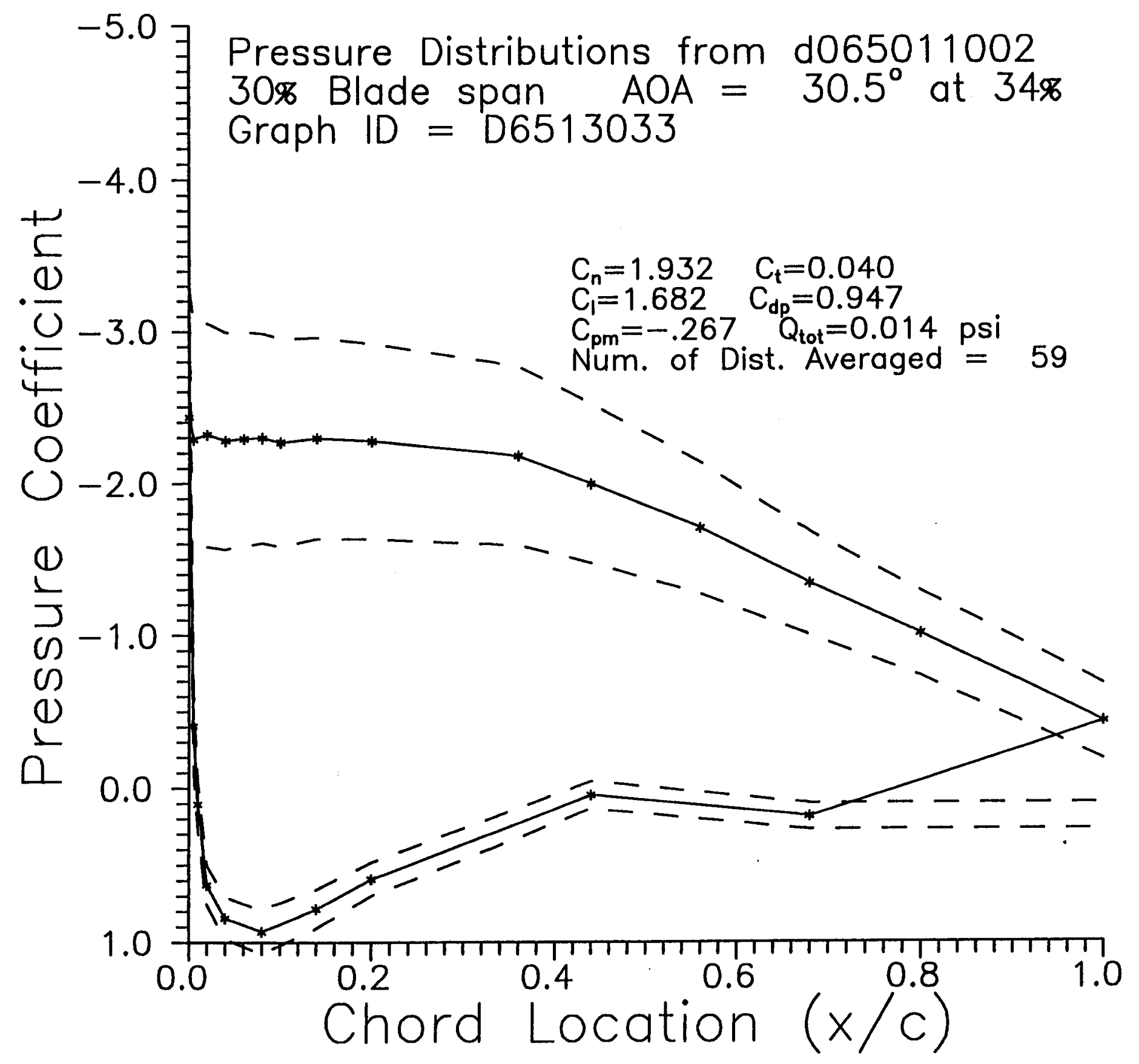




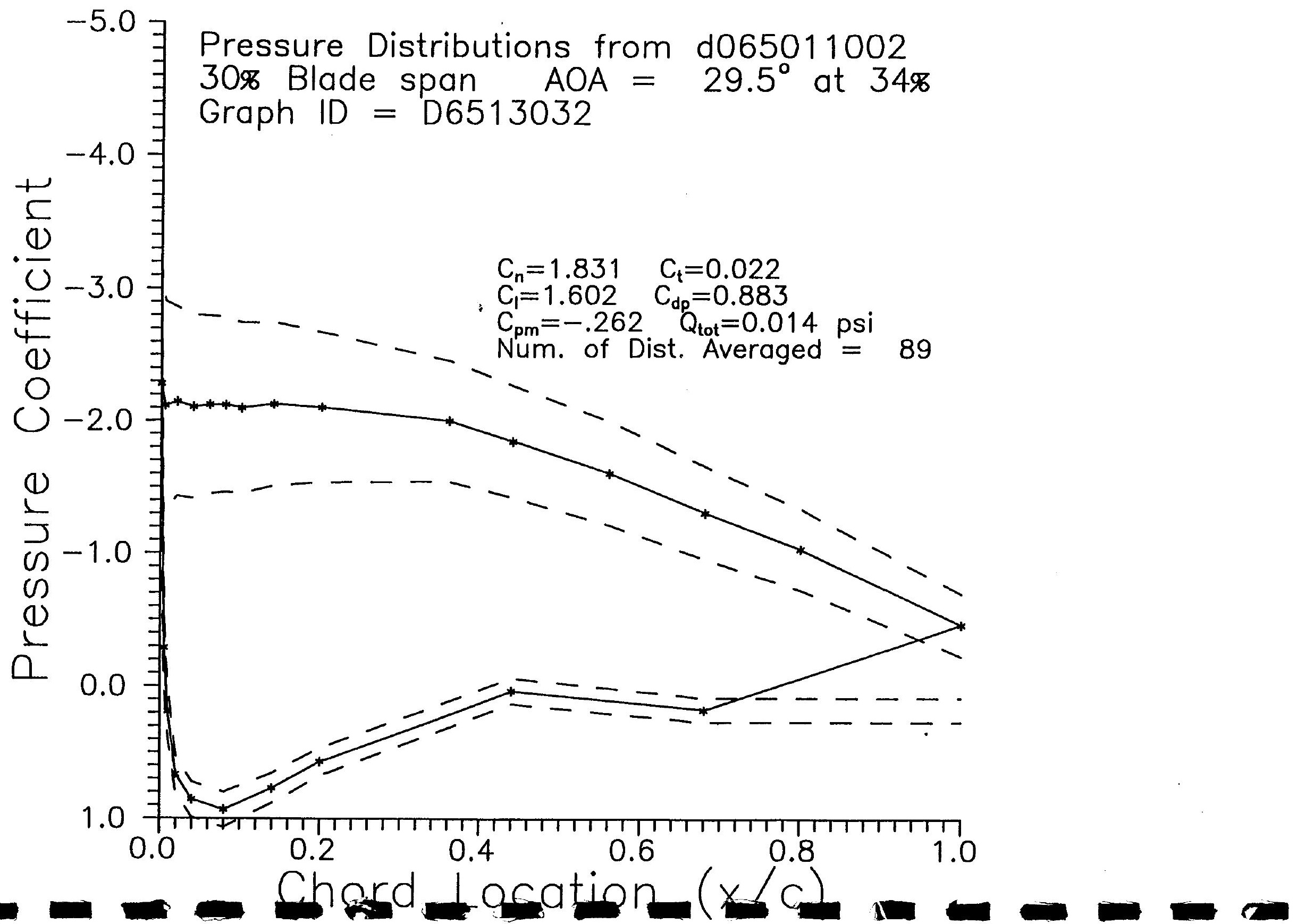




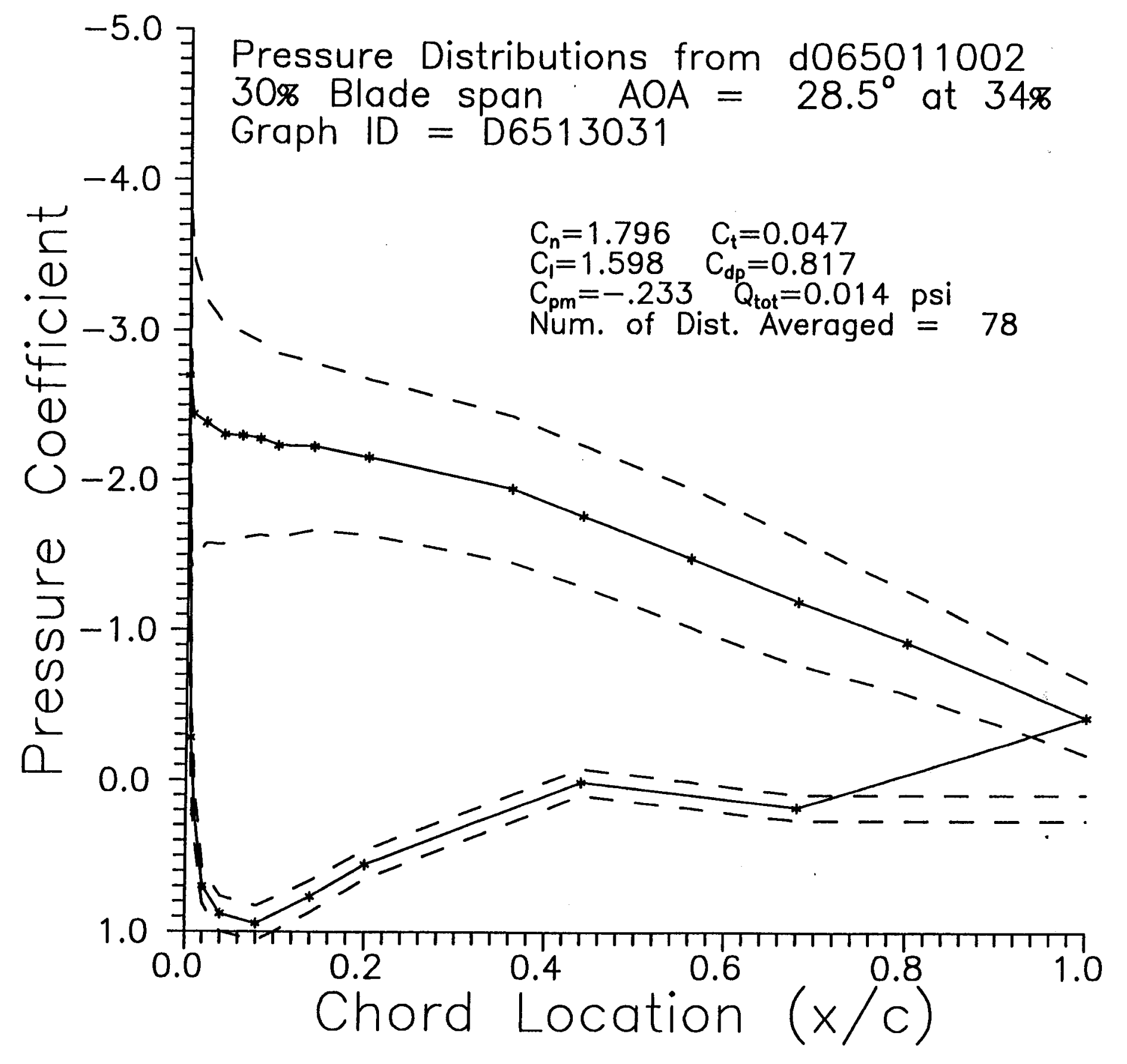




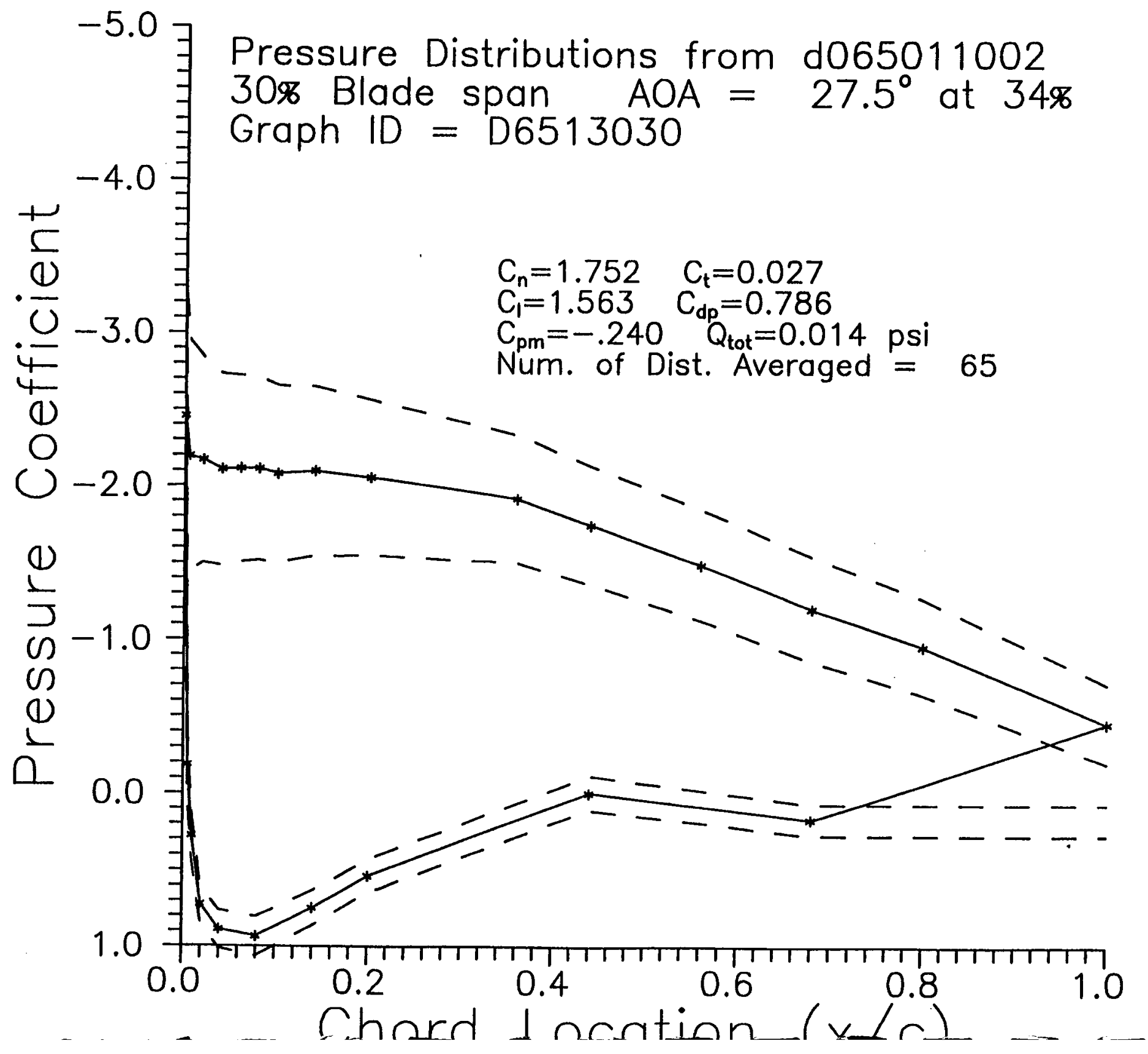




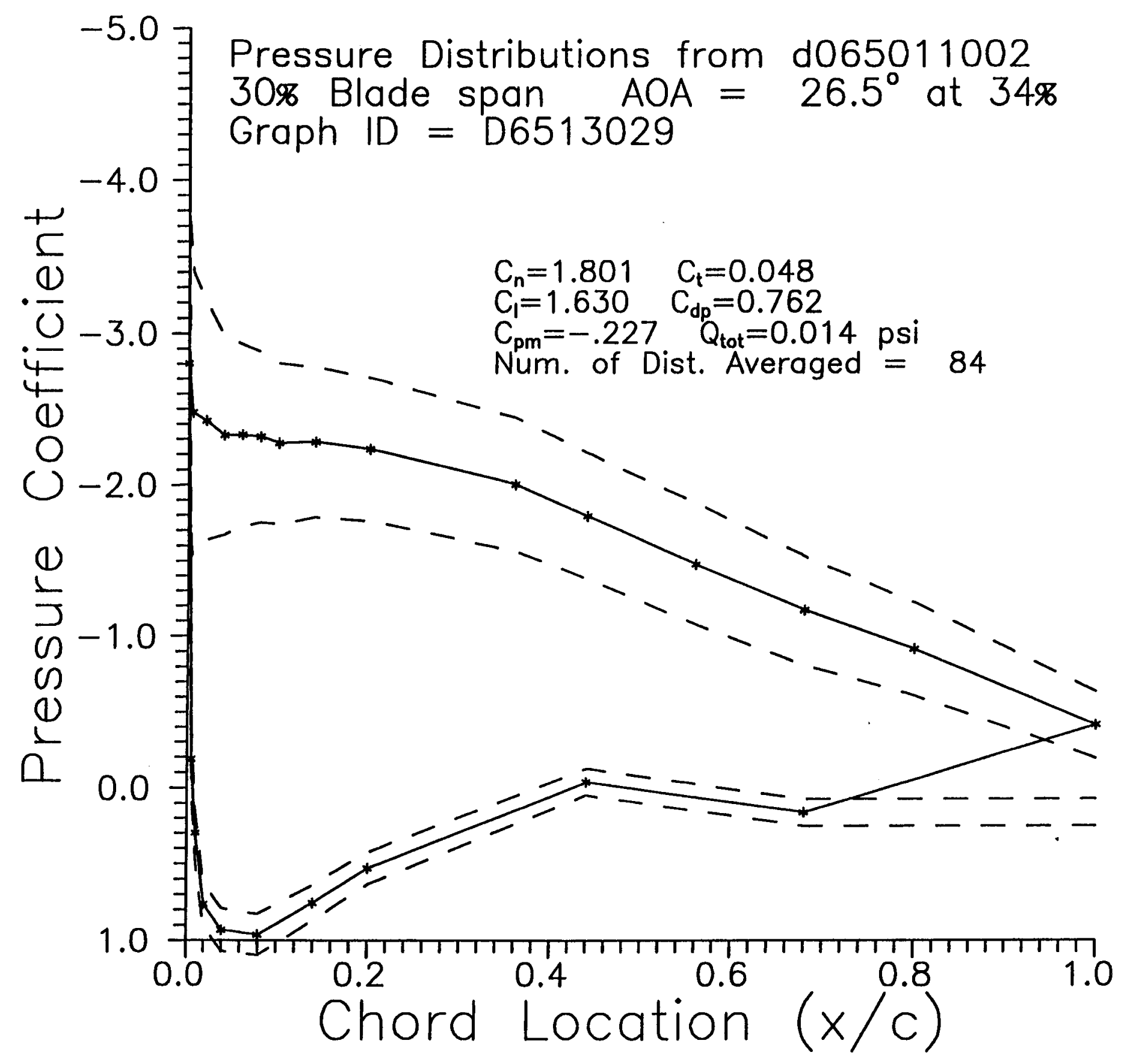




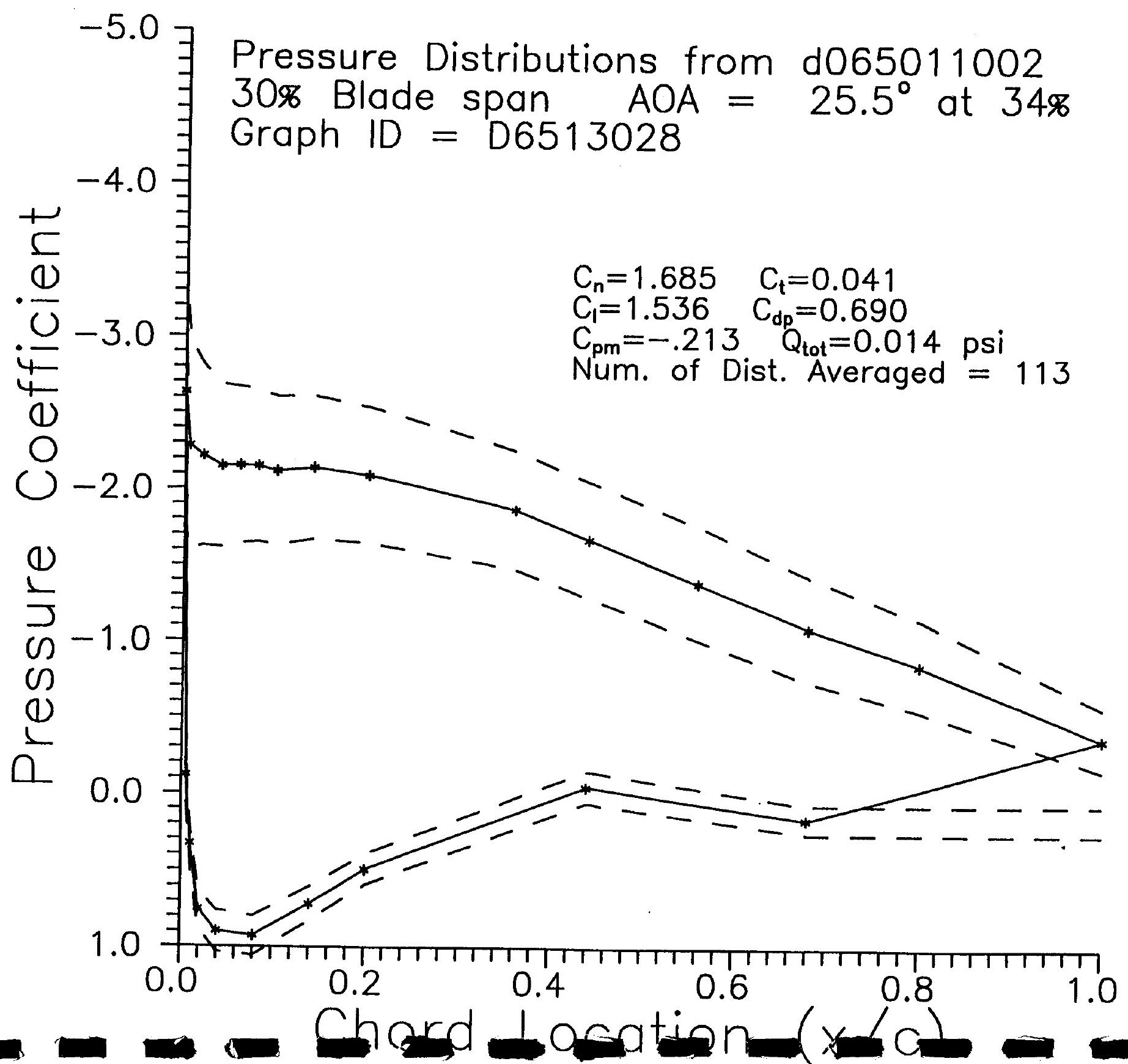




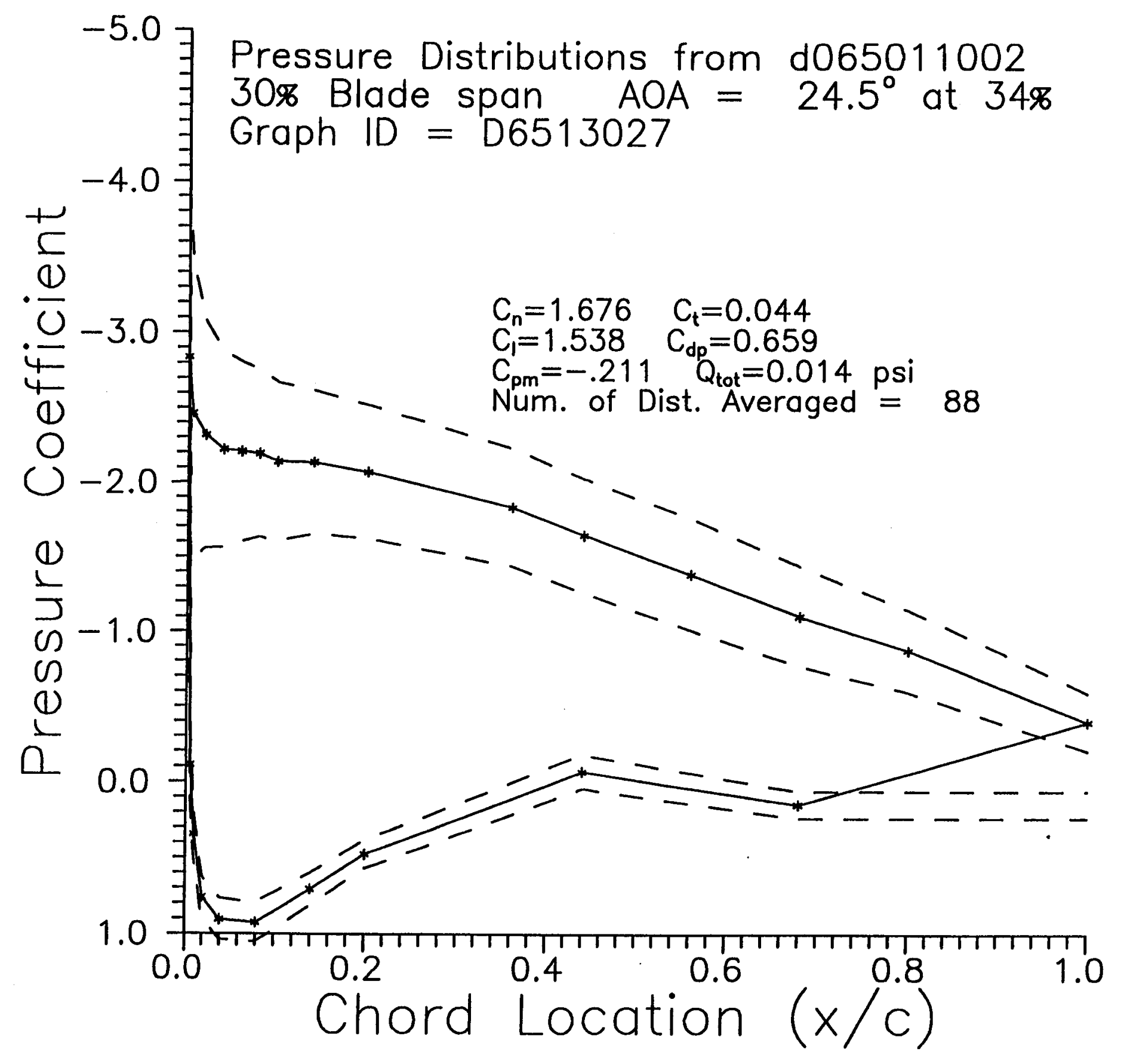




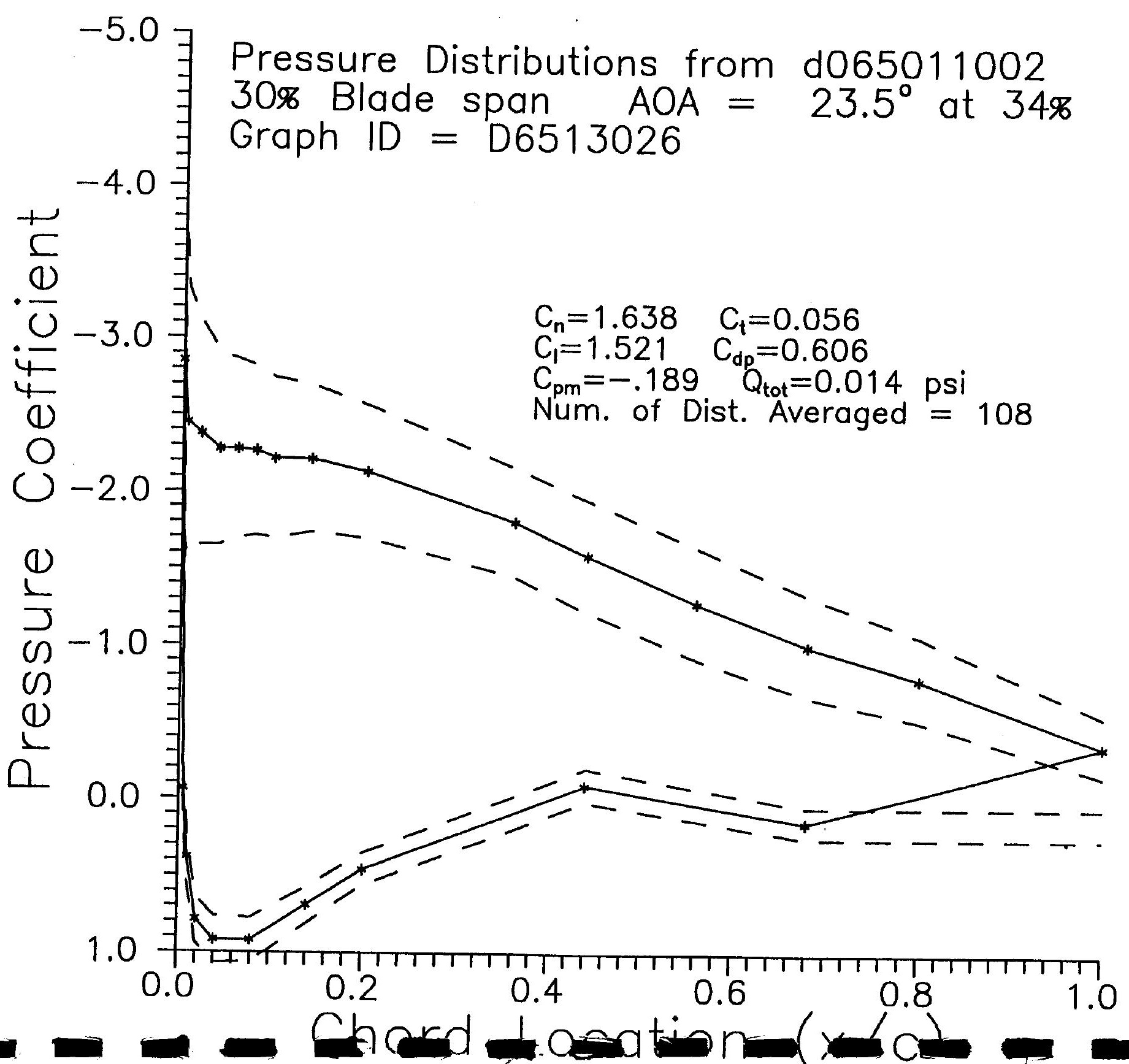




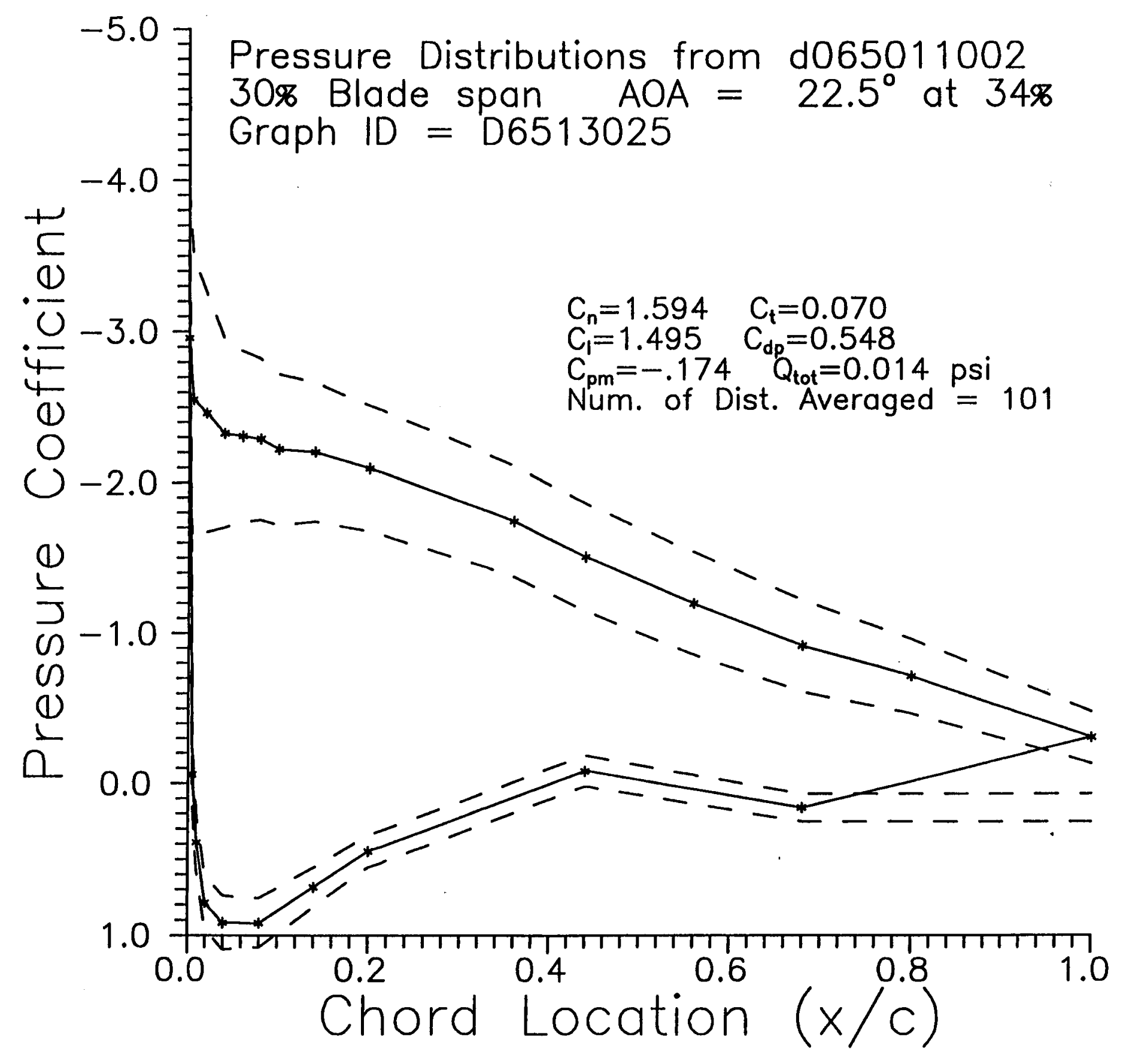




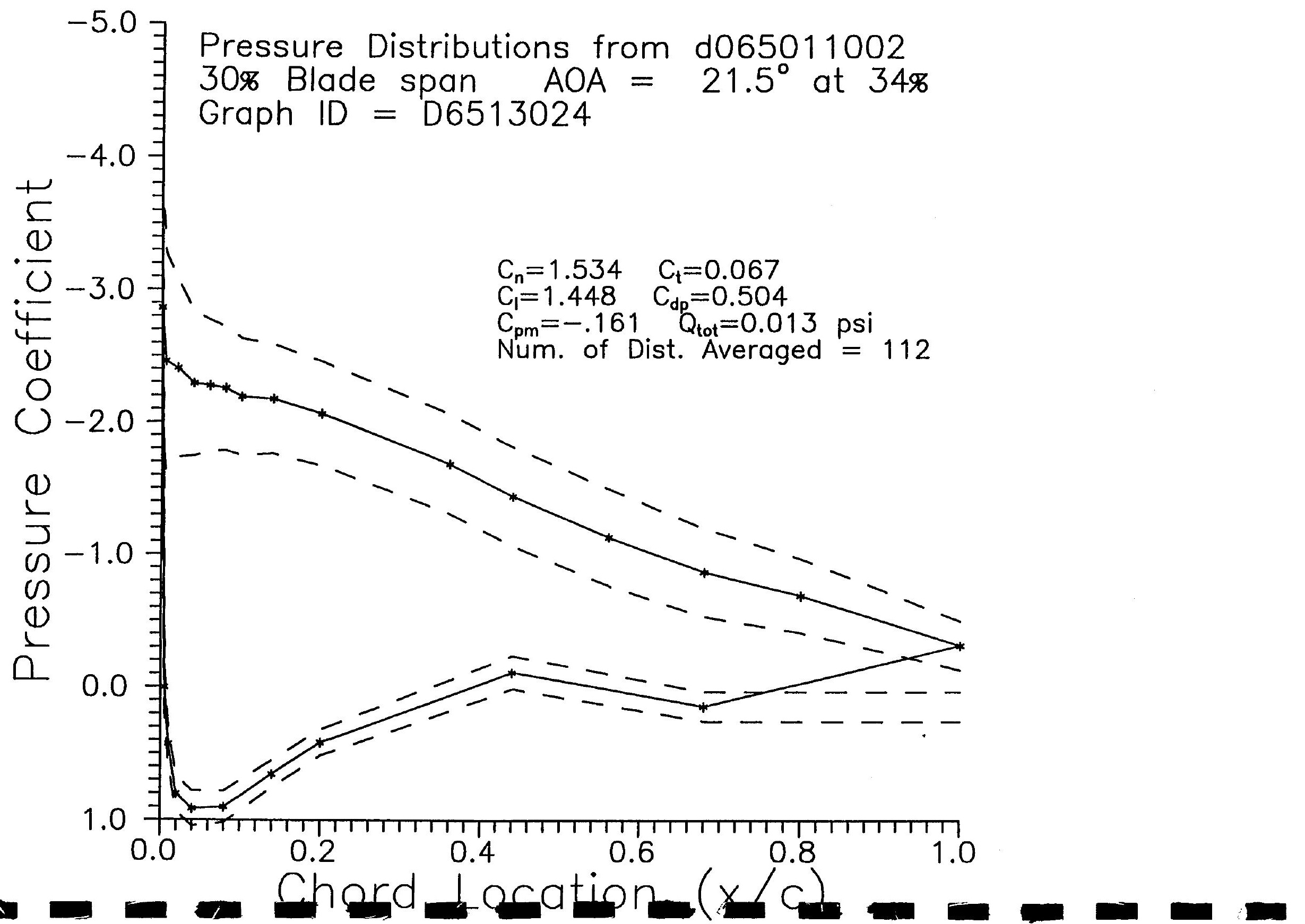




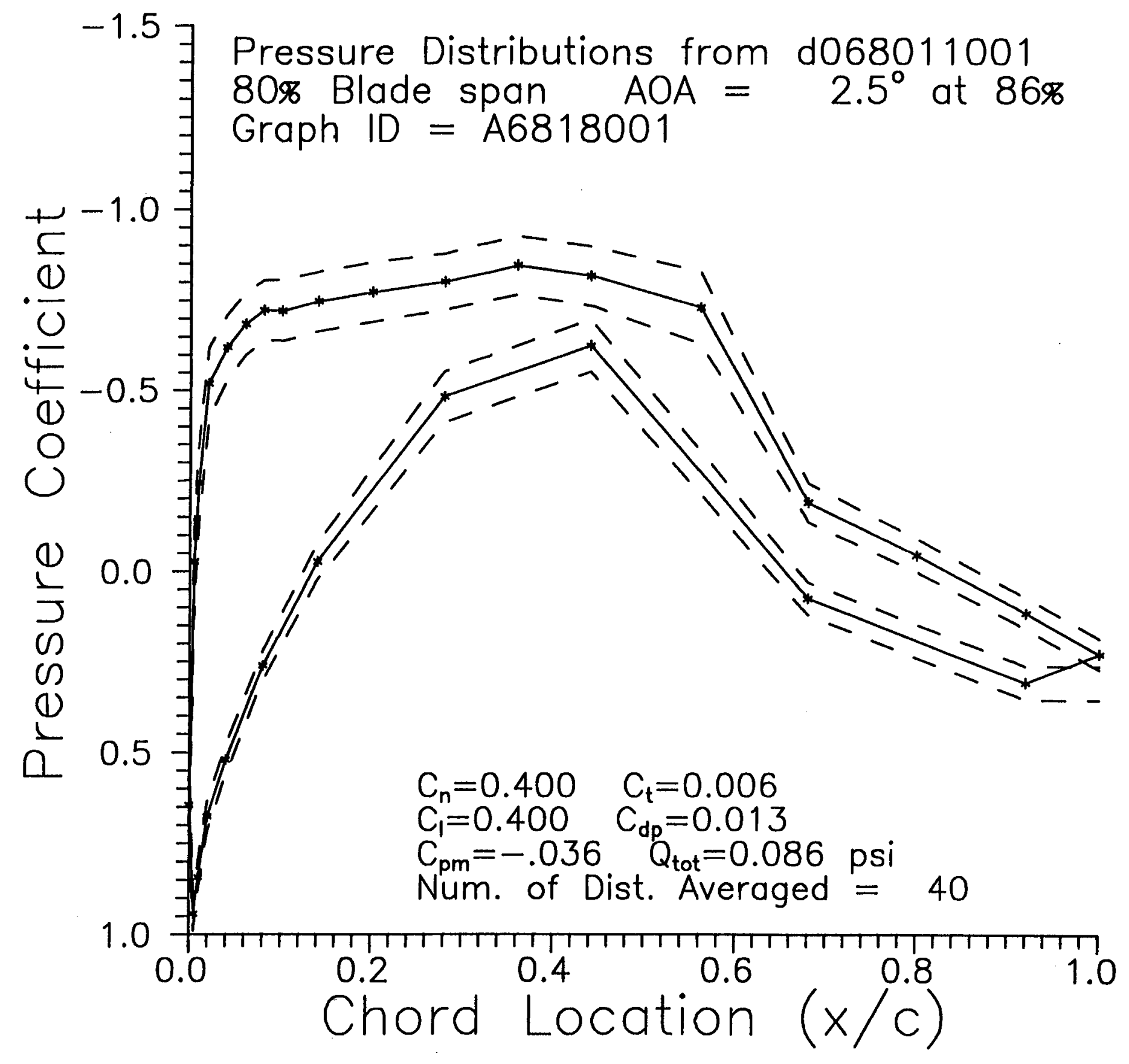




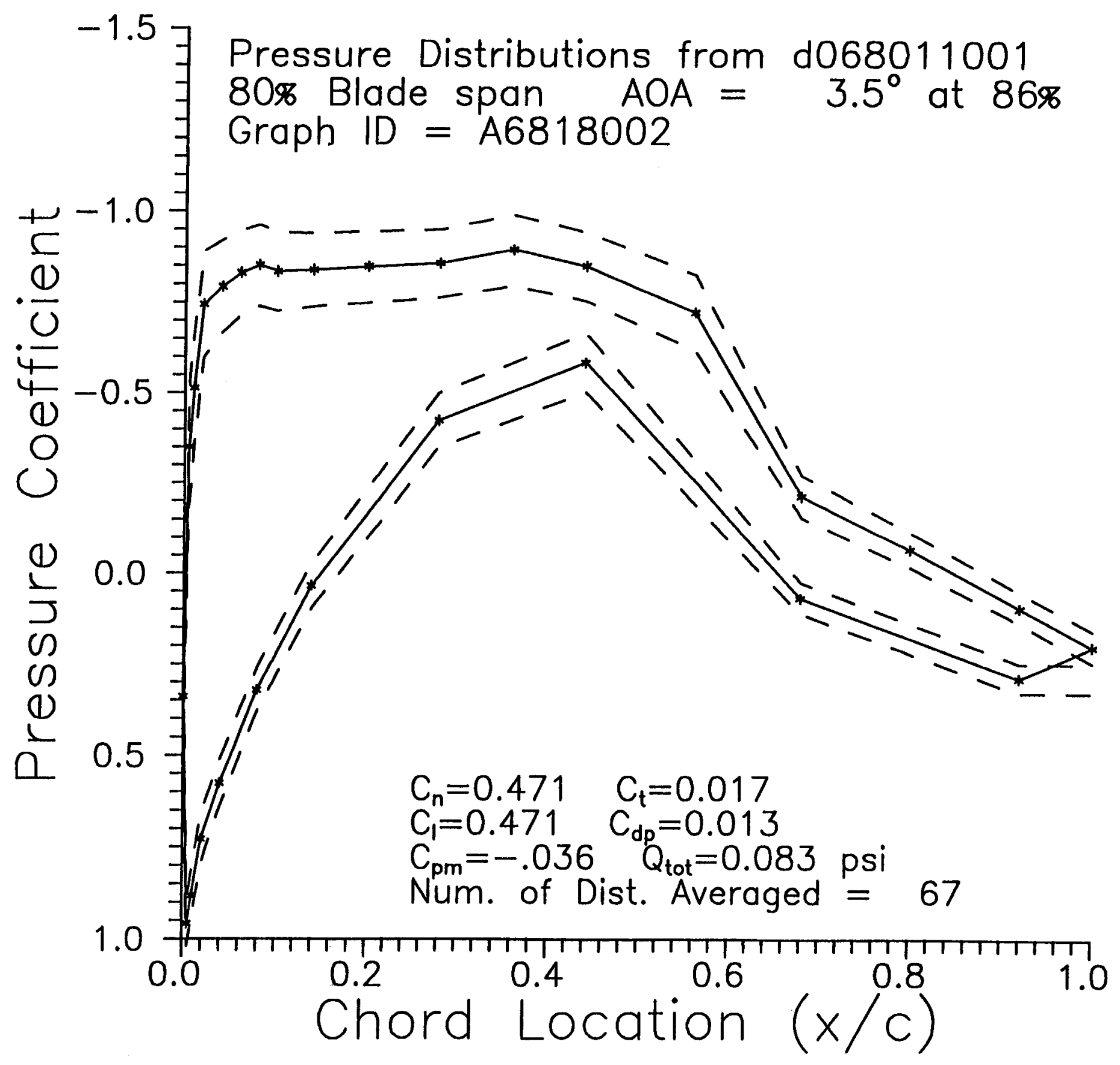




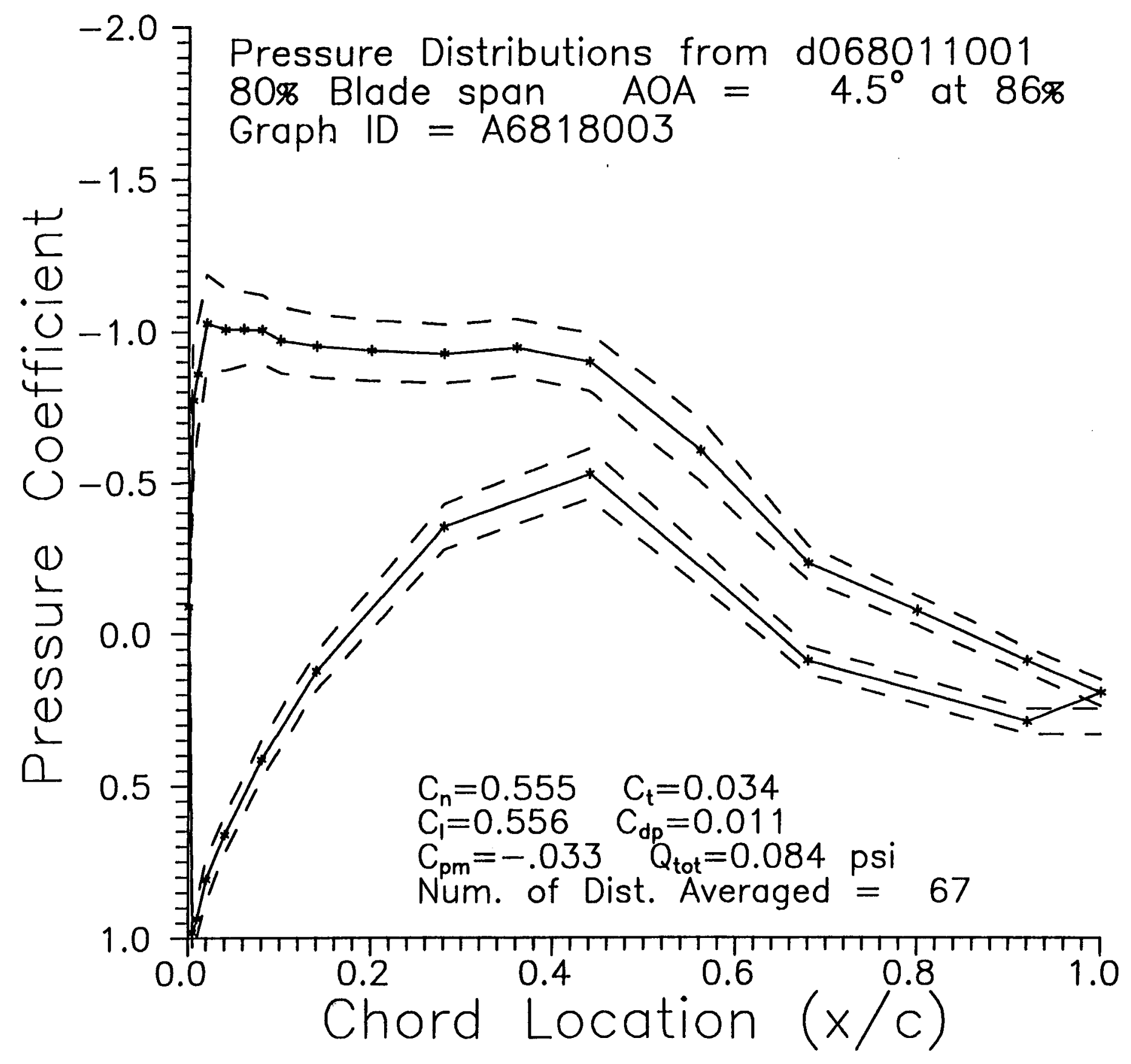




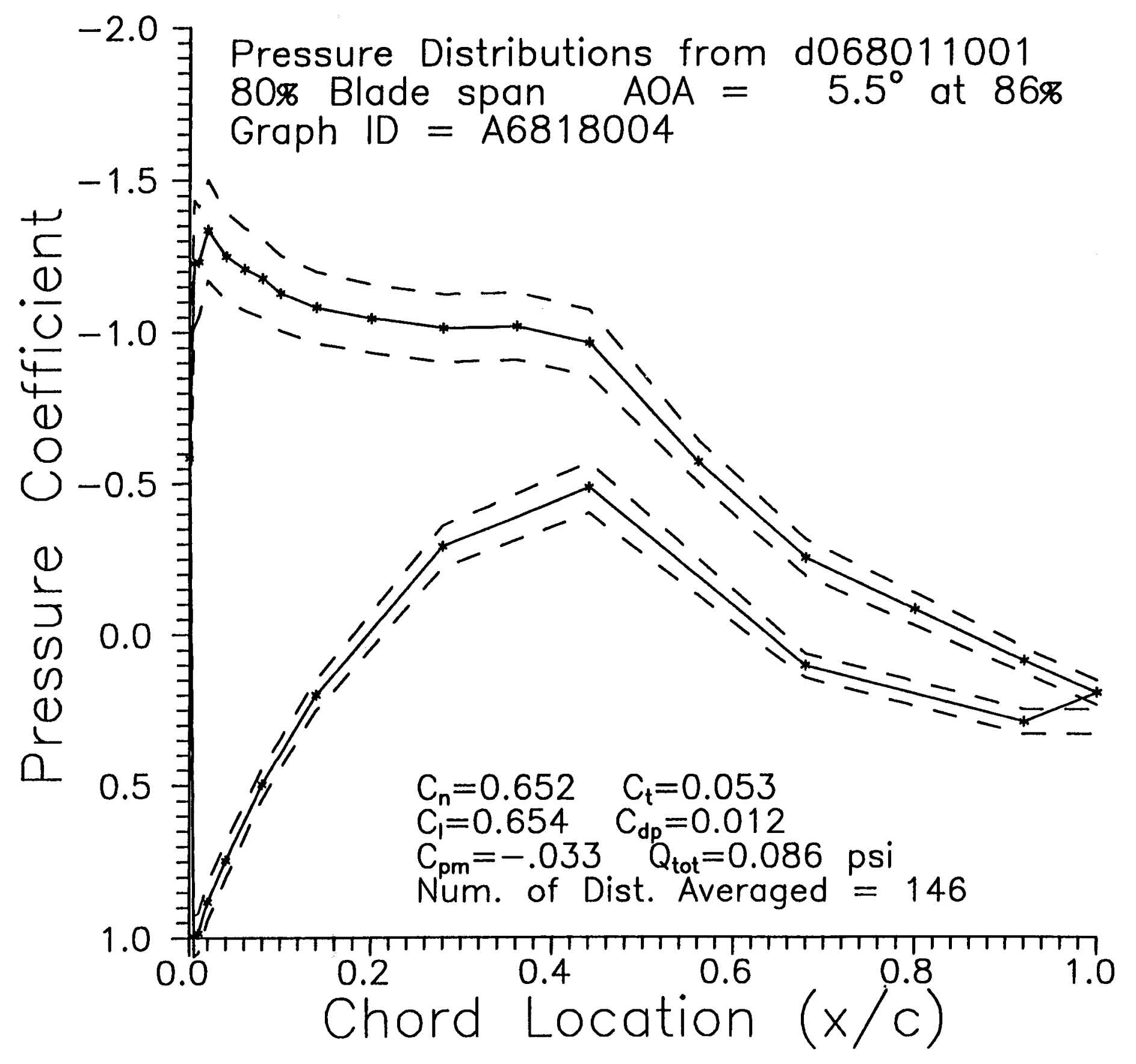




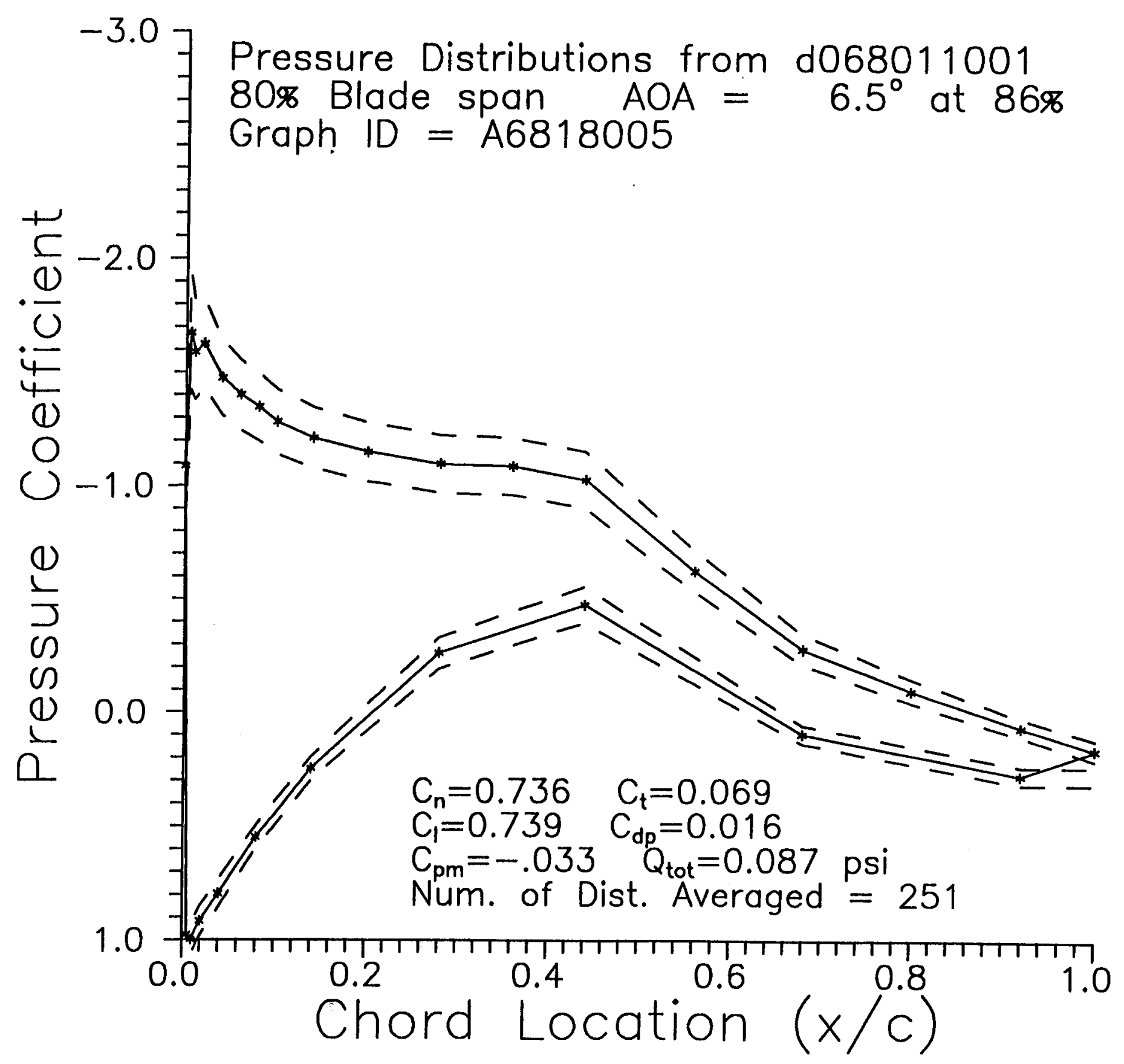




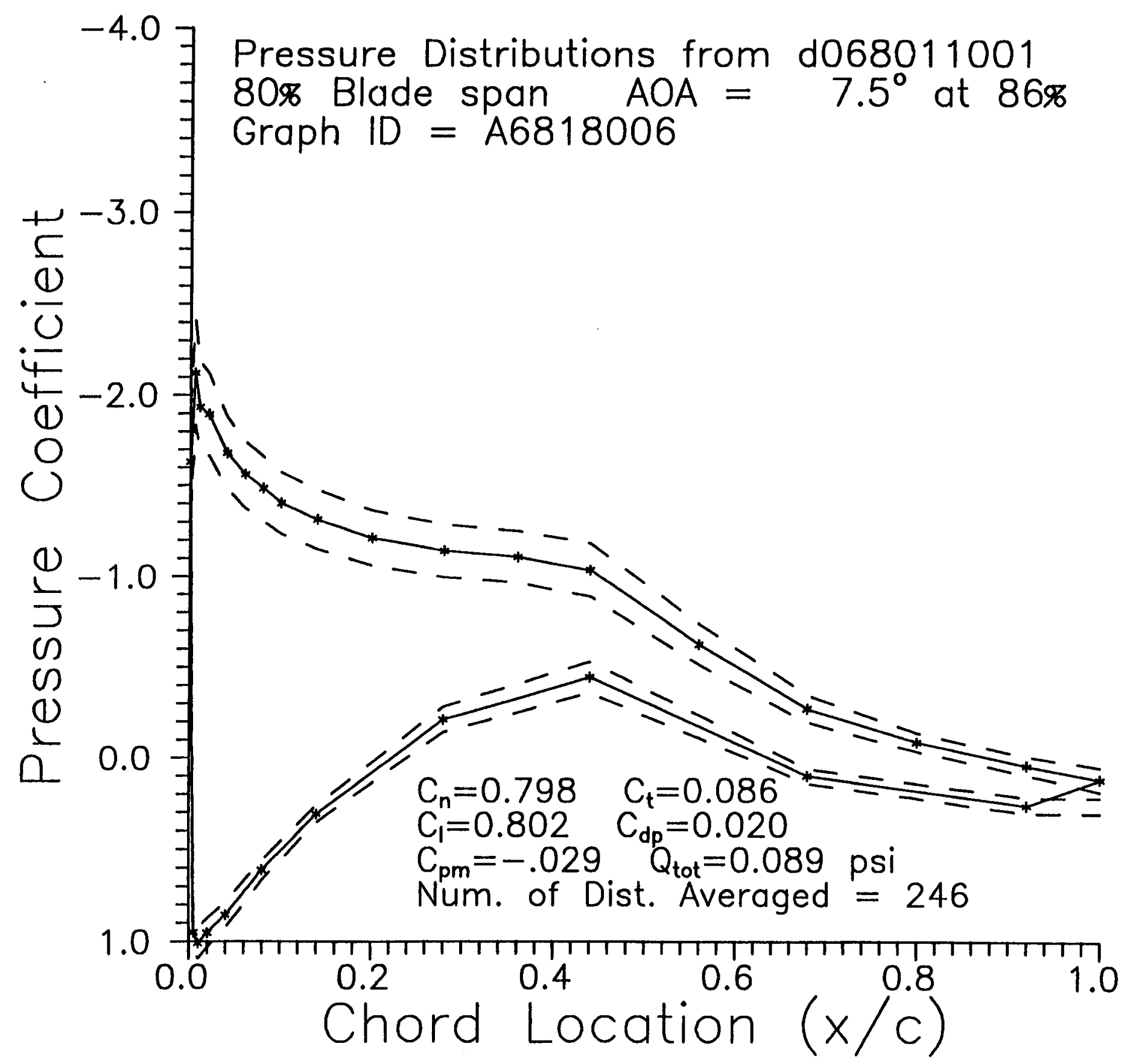




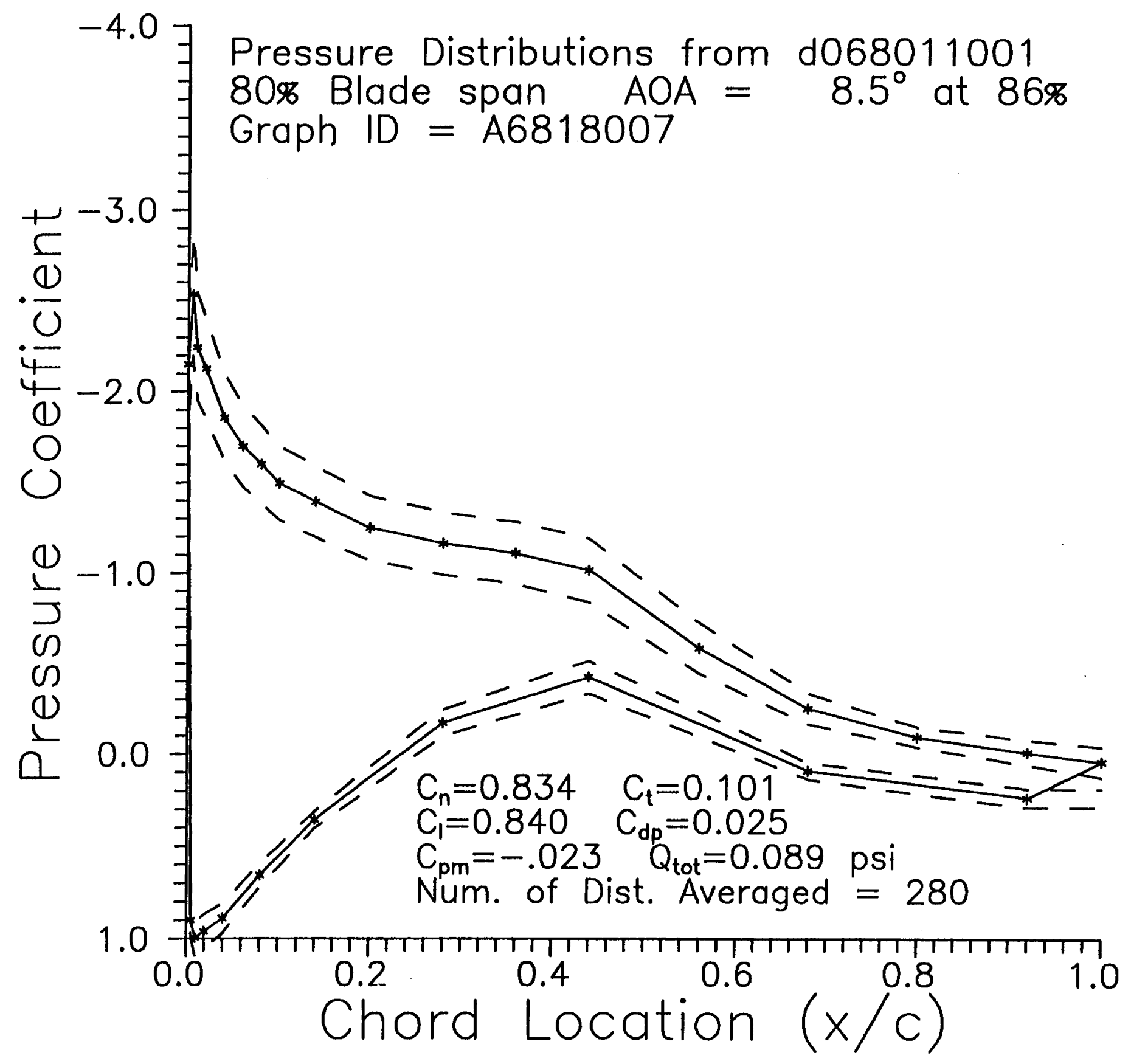




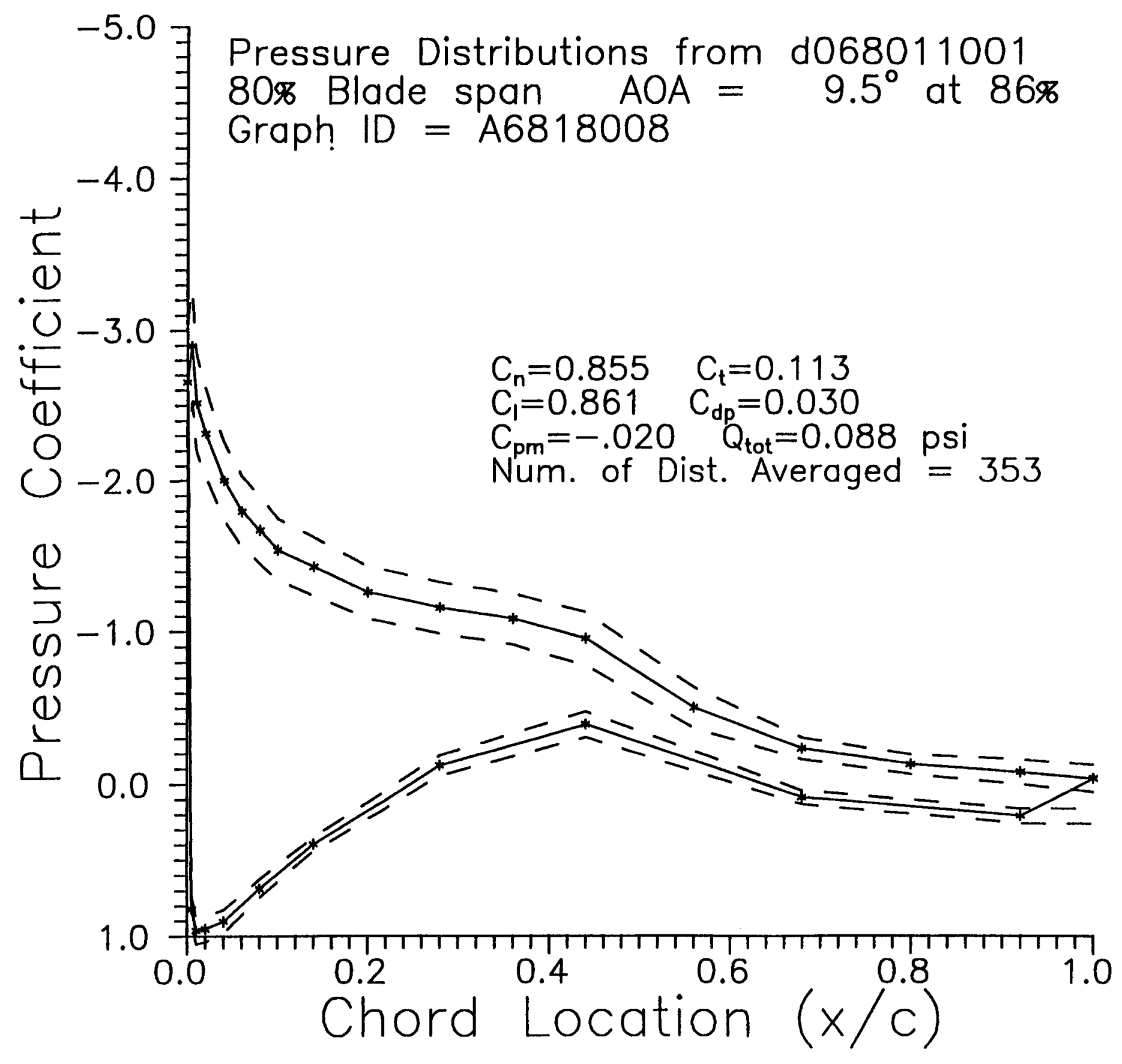




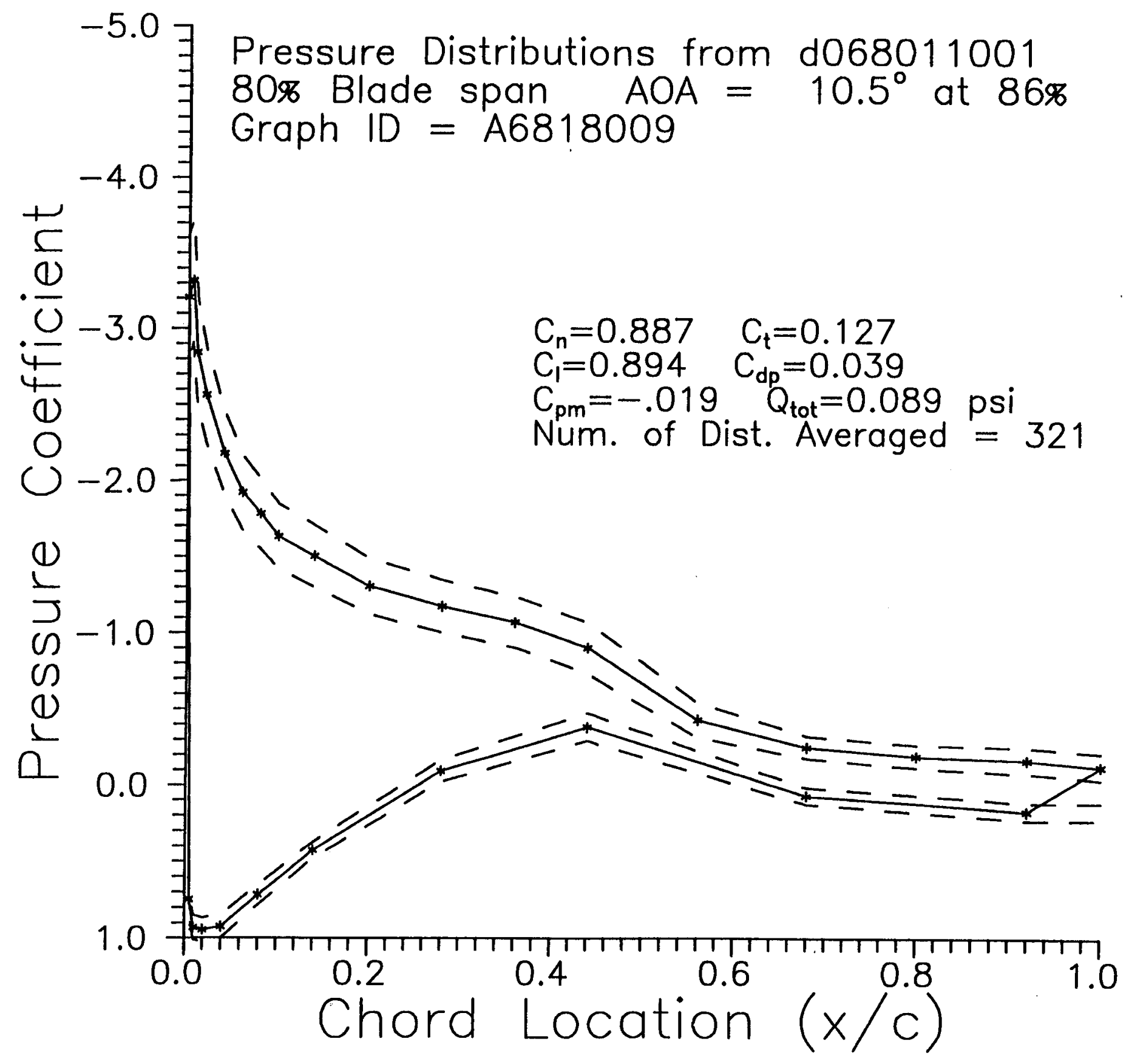




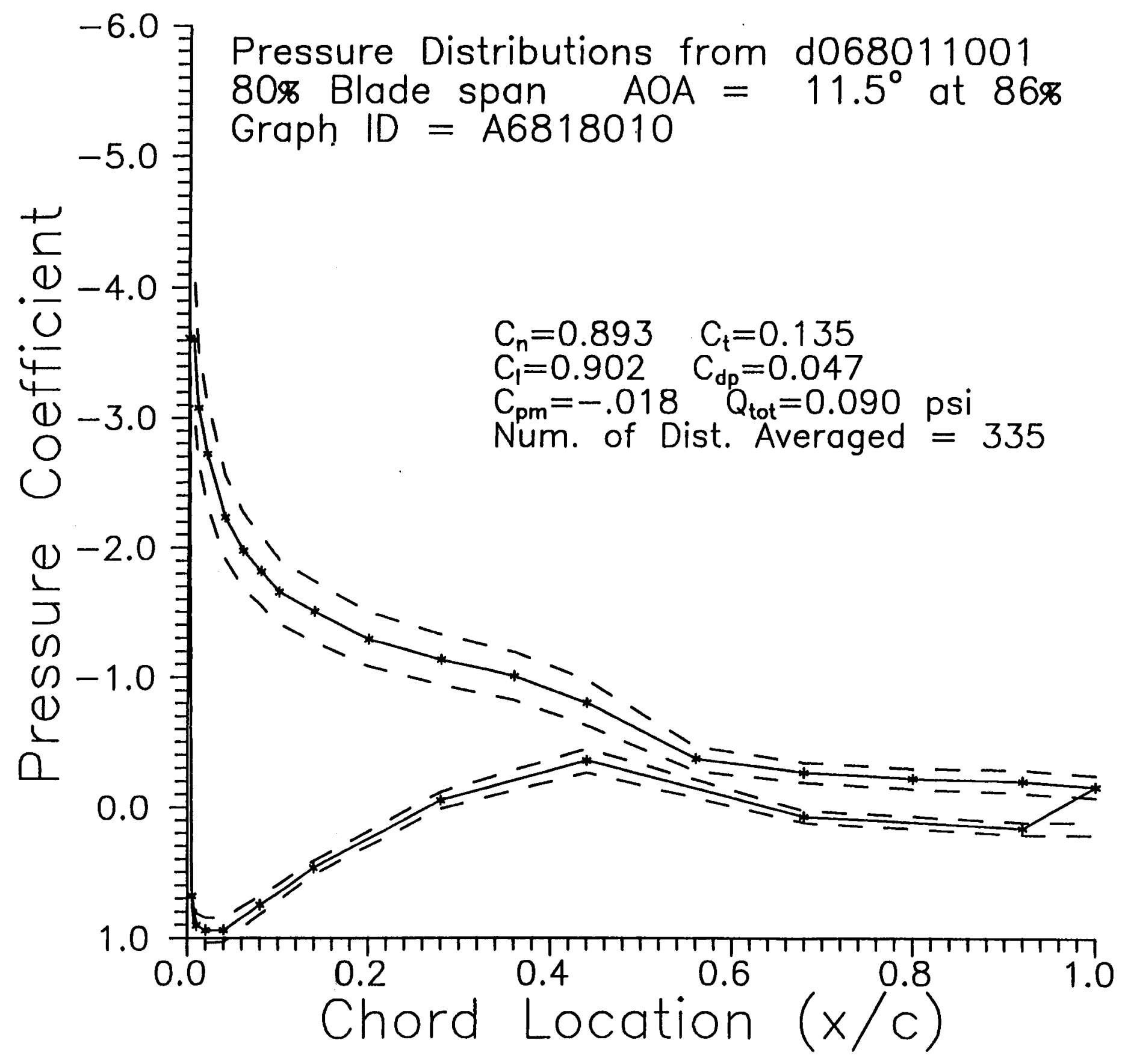




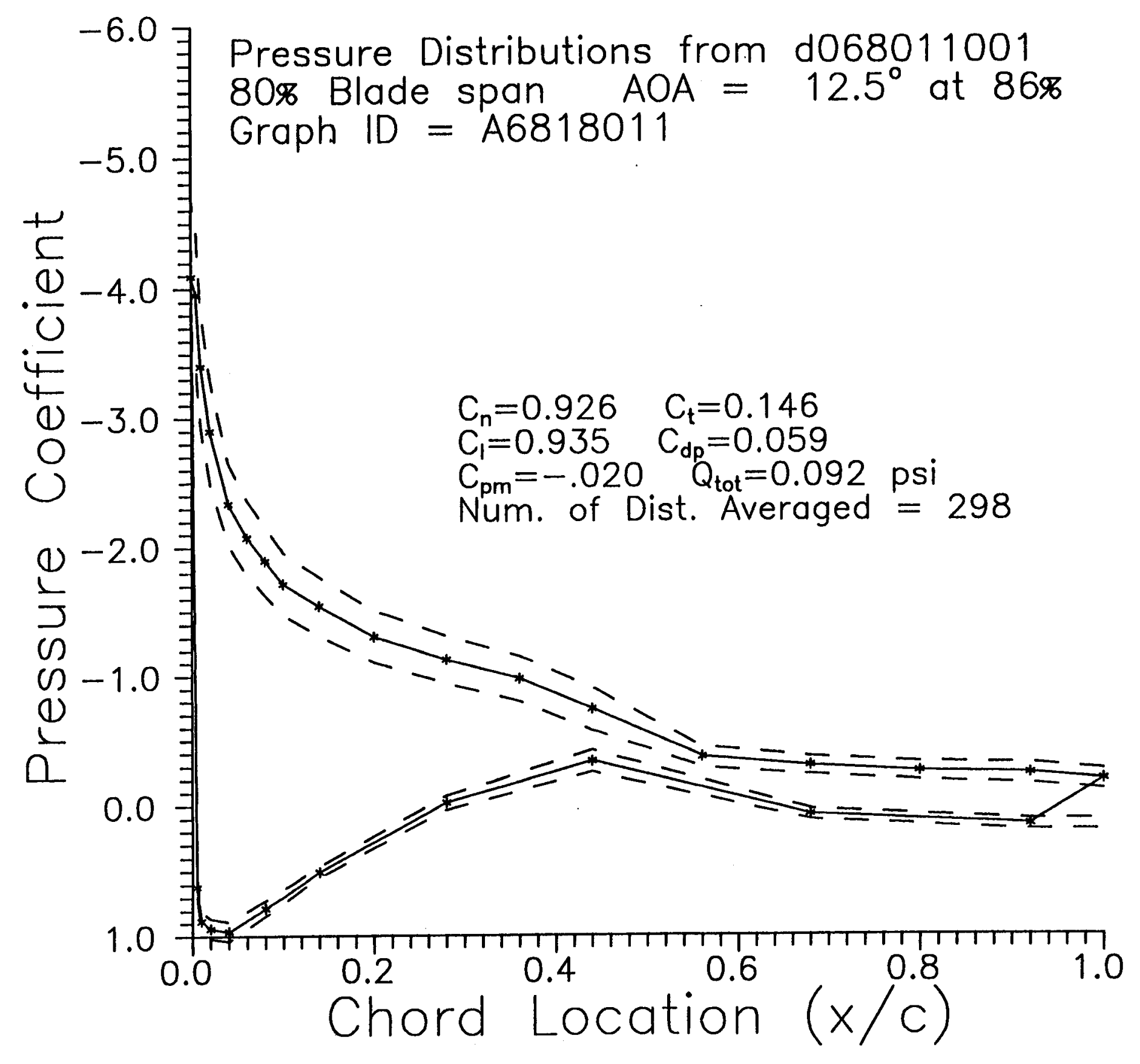




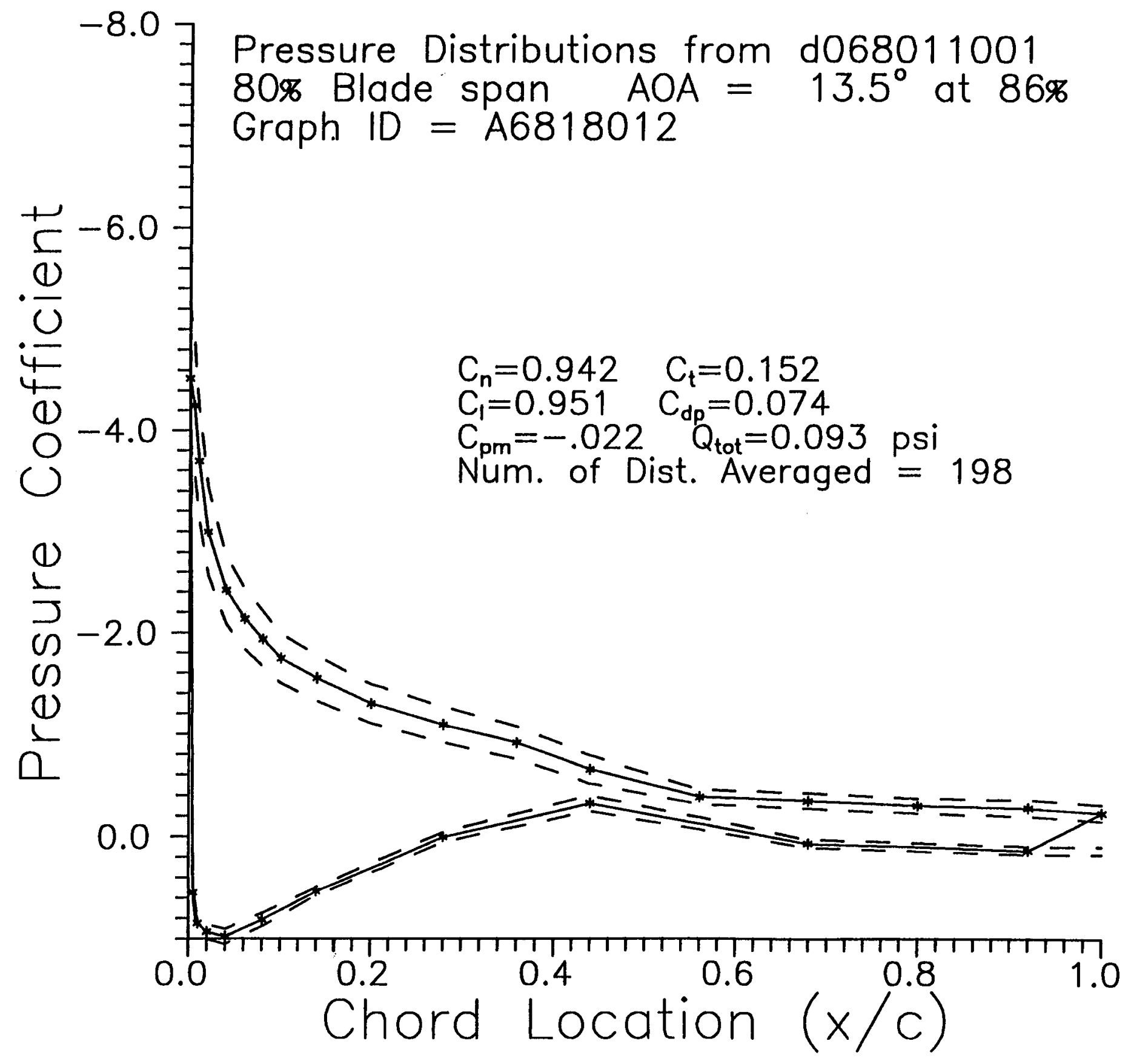




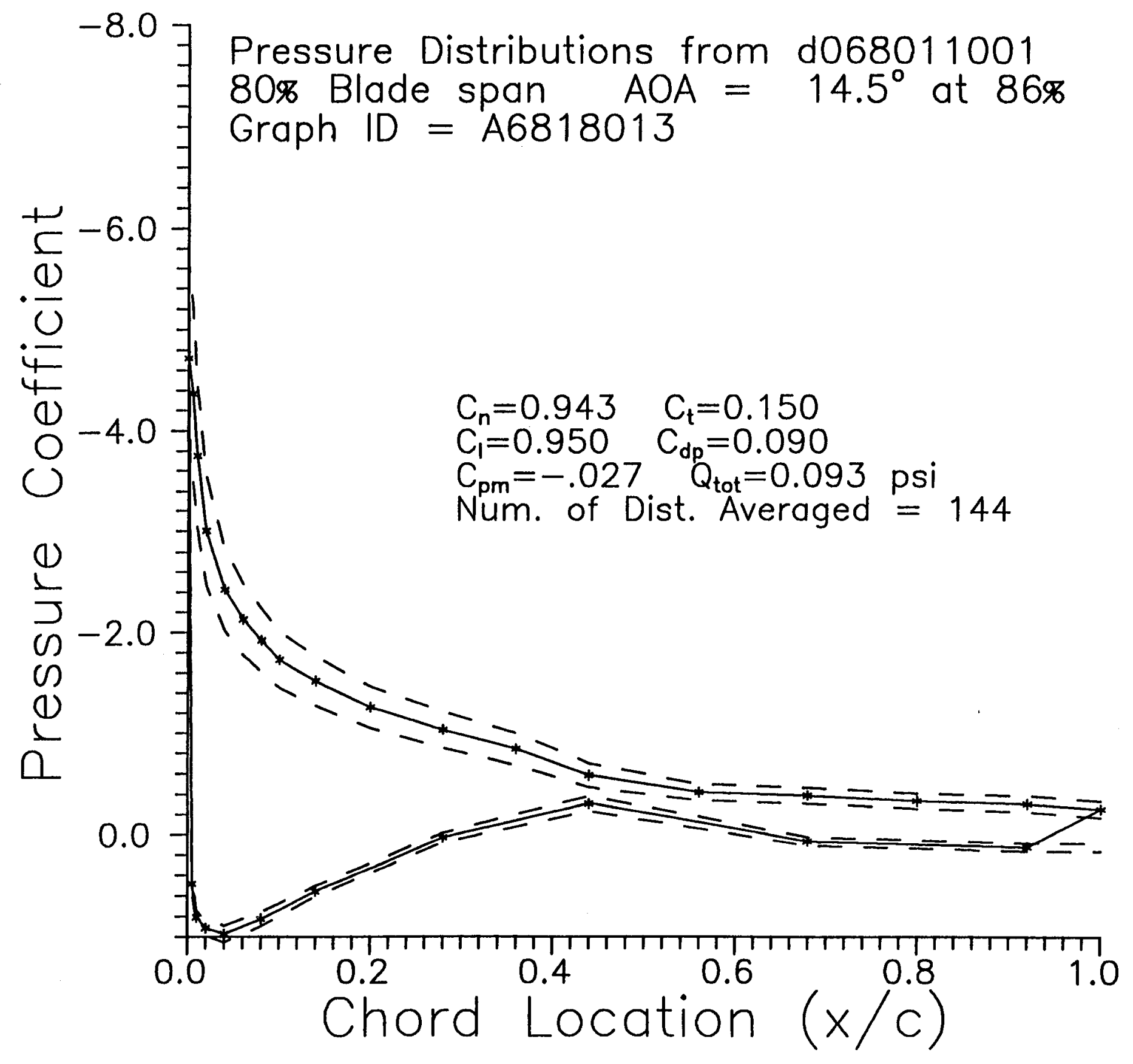




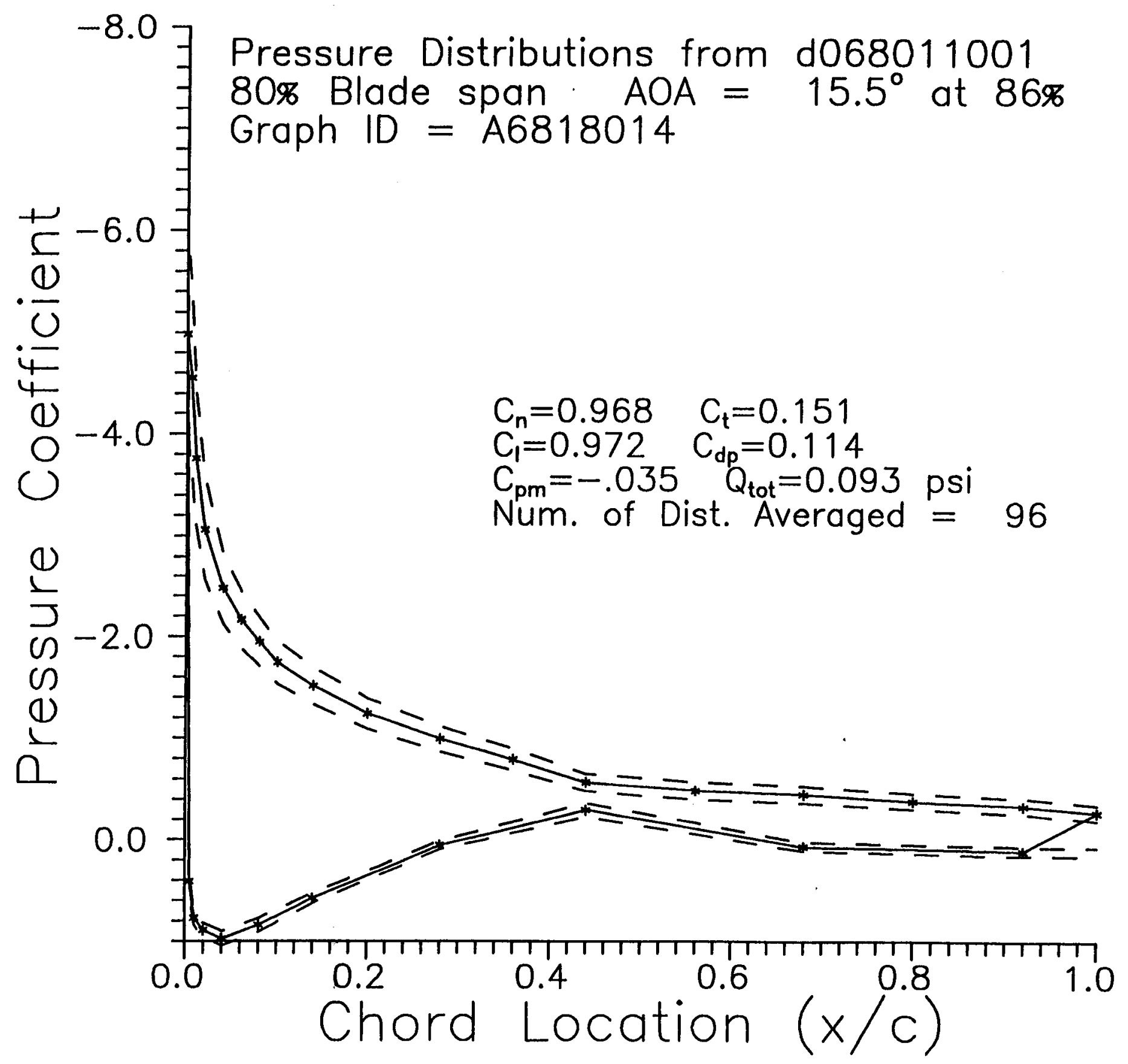




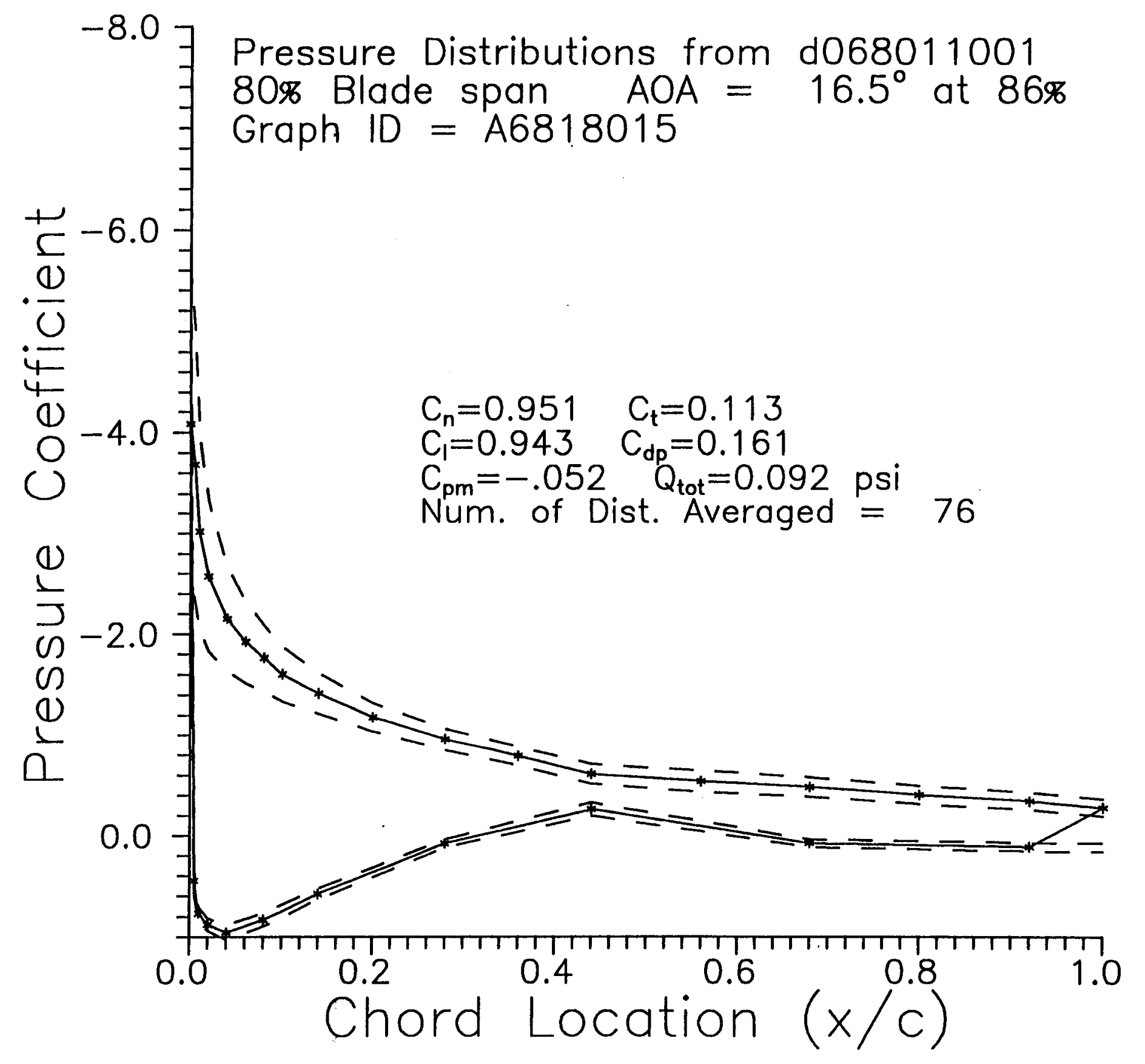




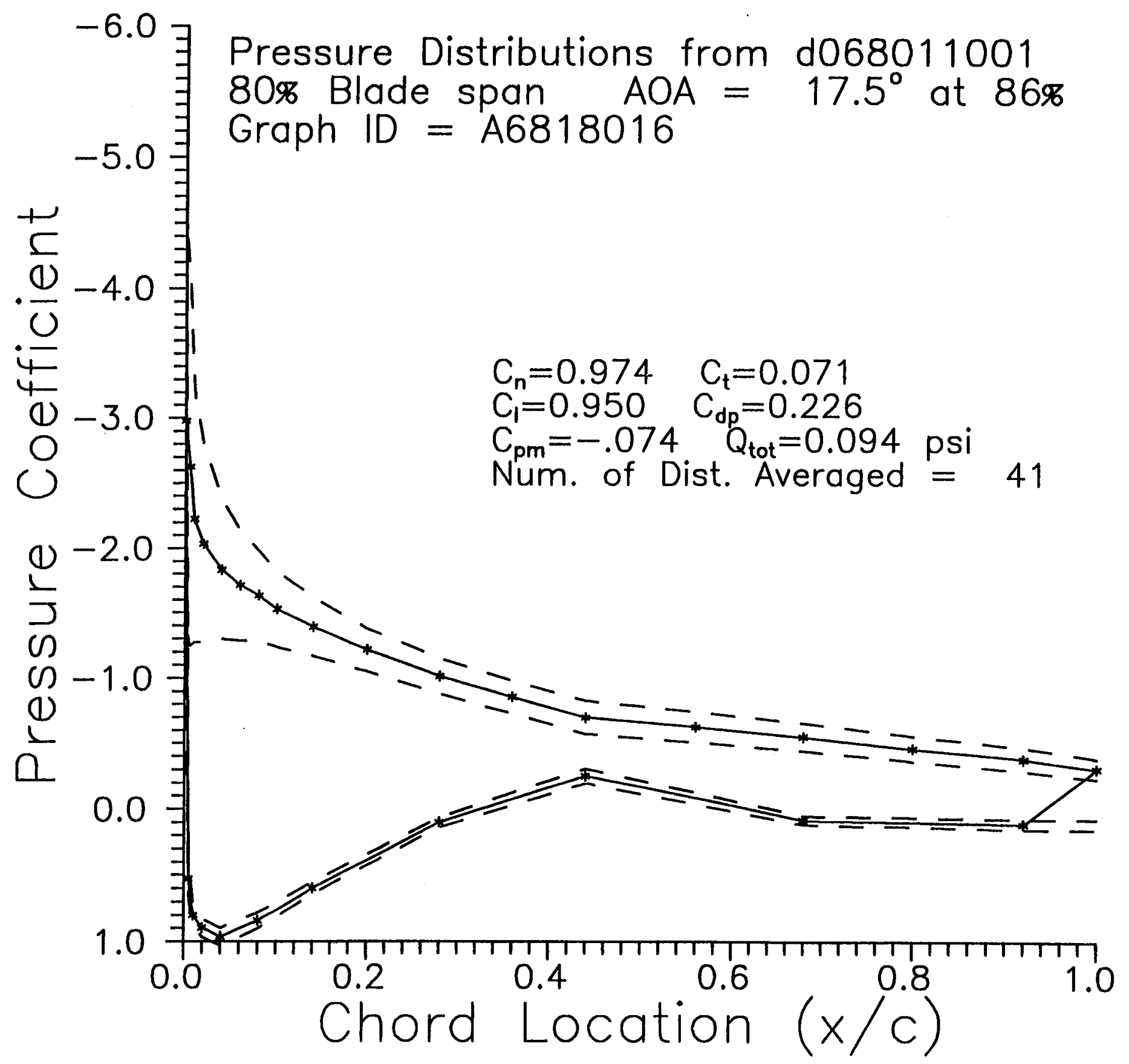




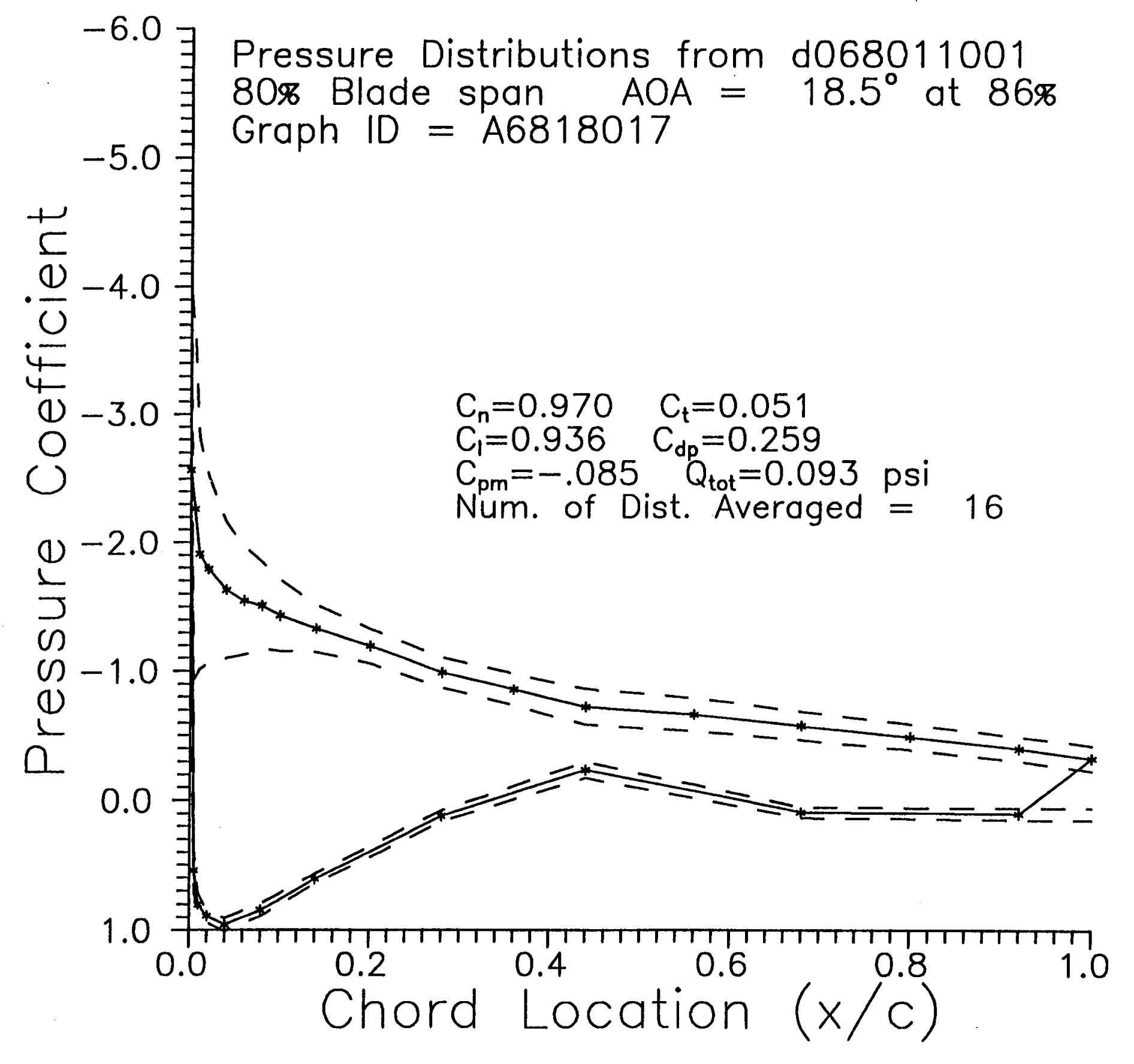




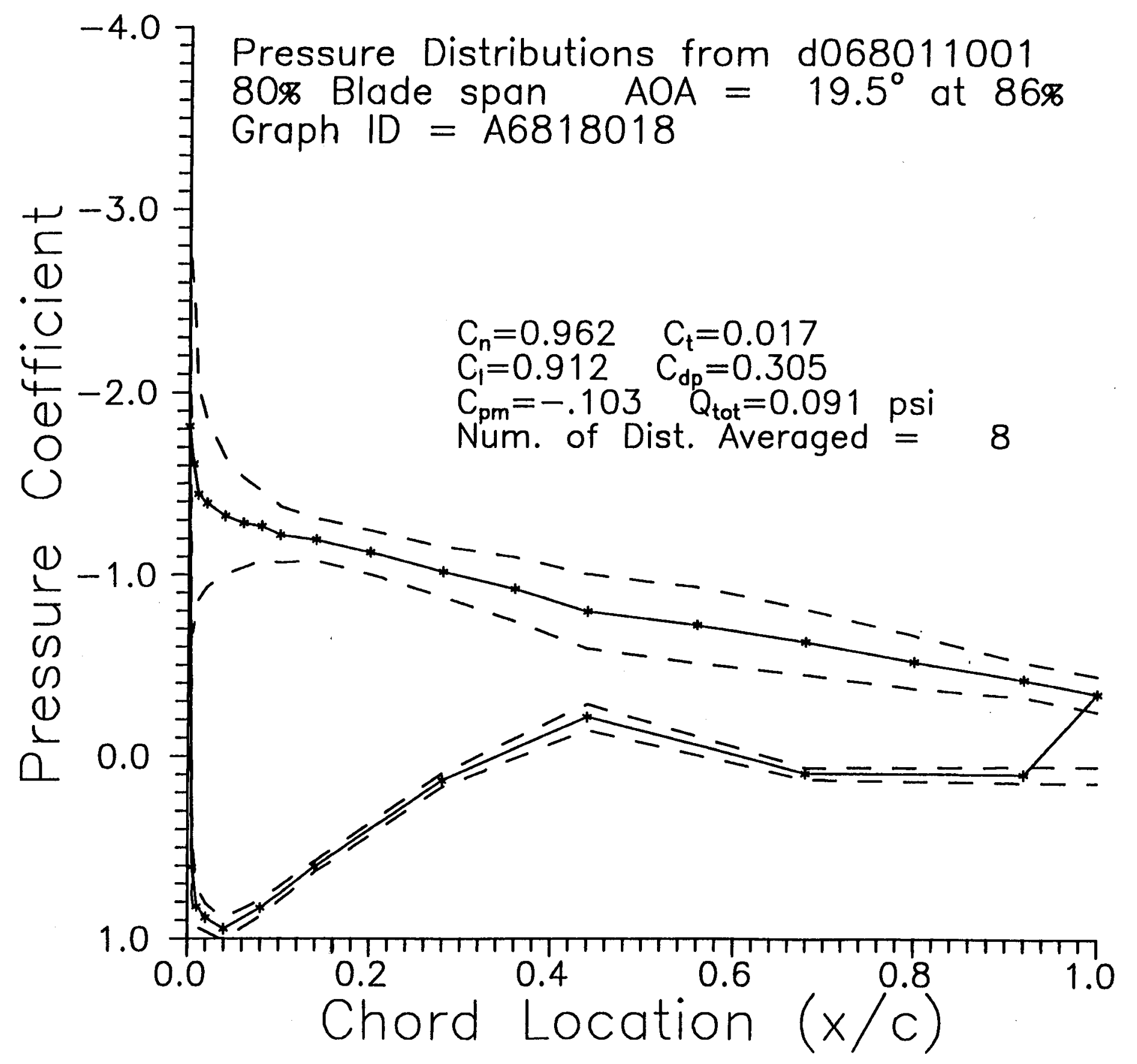




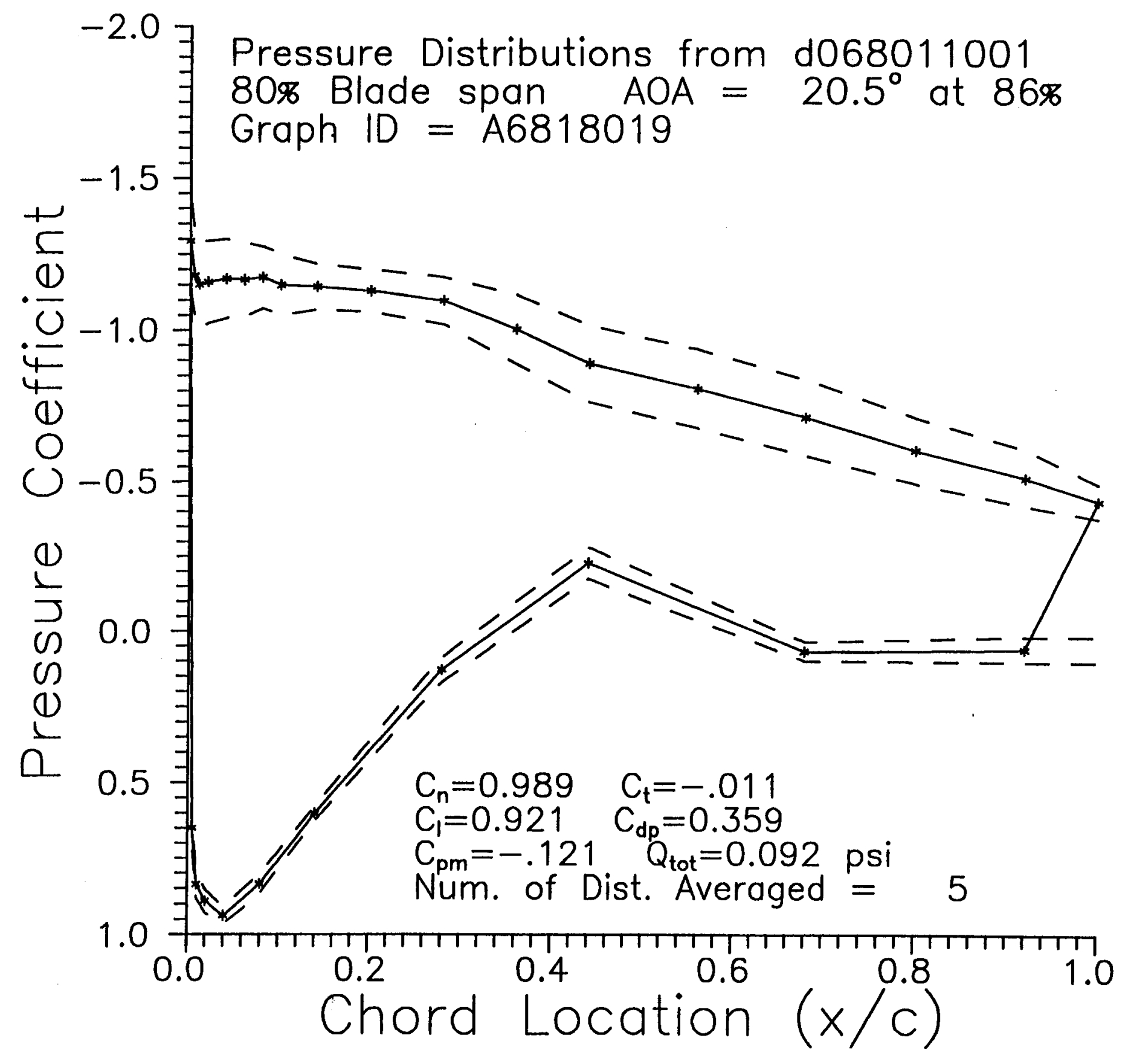




\section{Appendix C.2.3}

Pressure Distributions for 63\% Span 


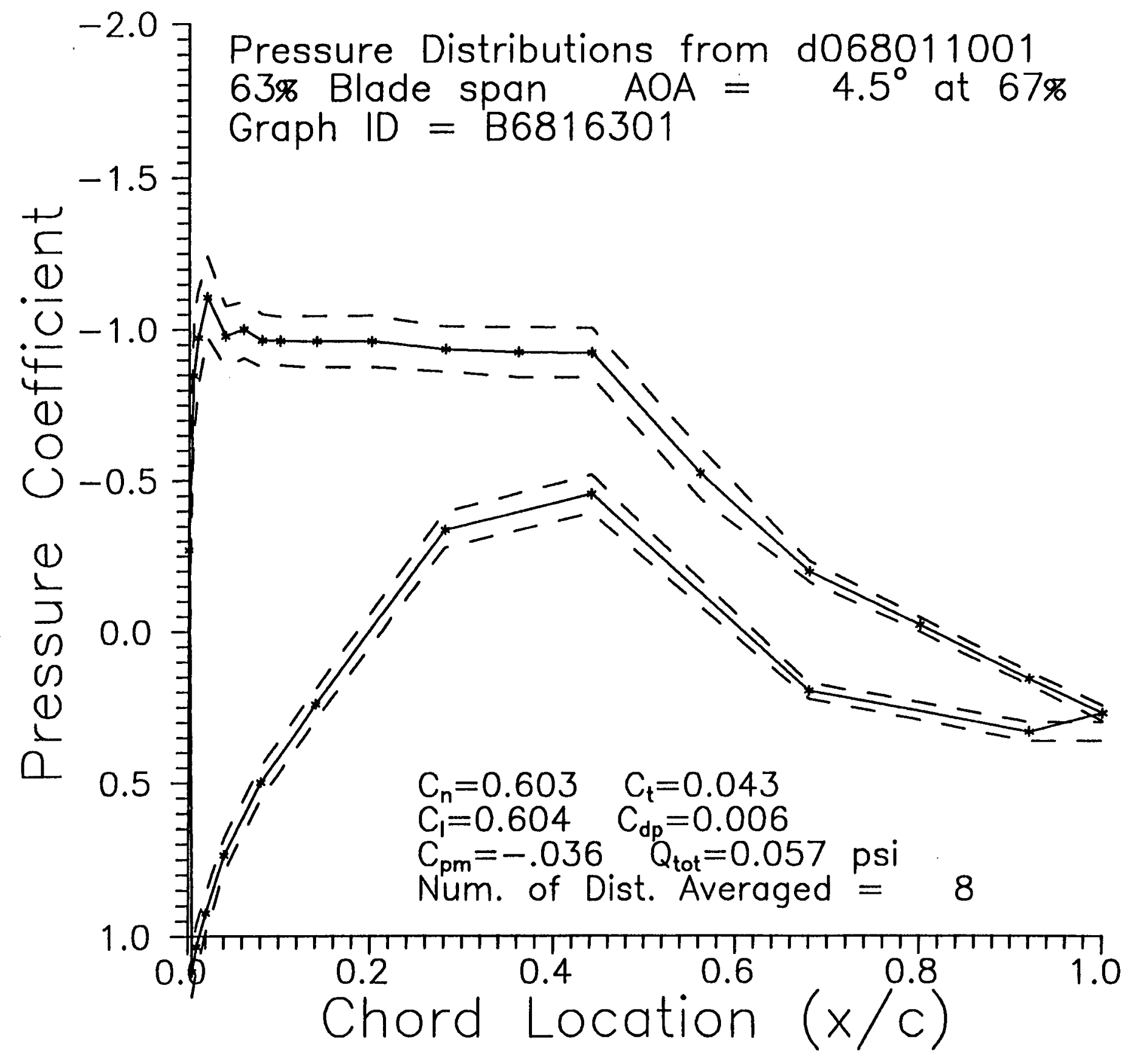




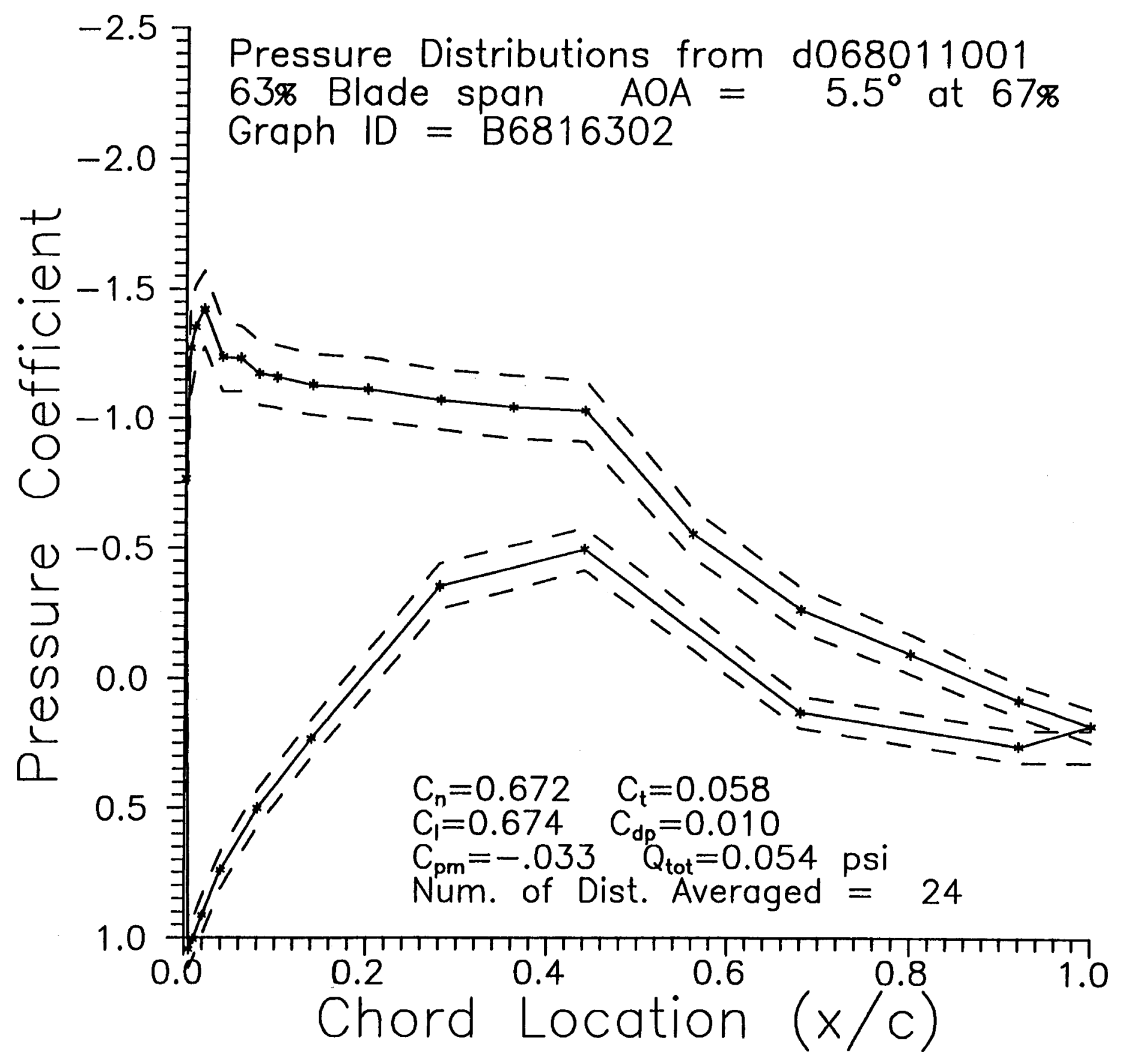




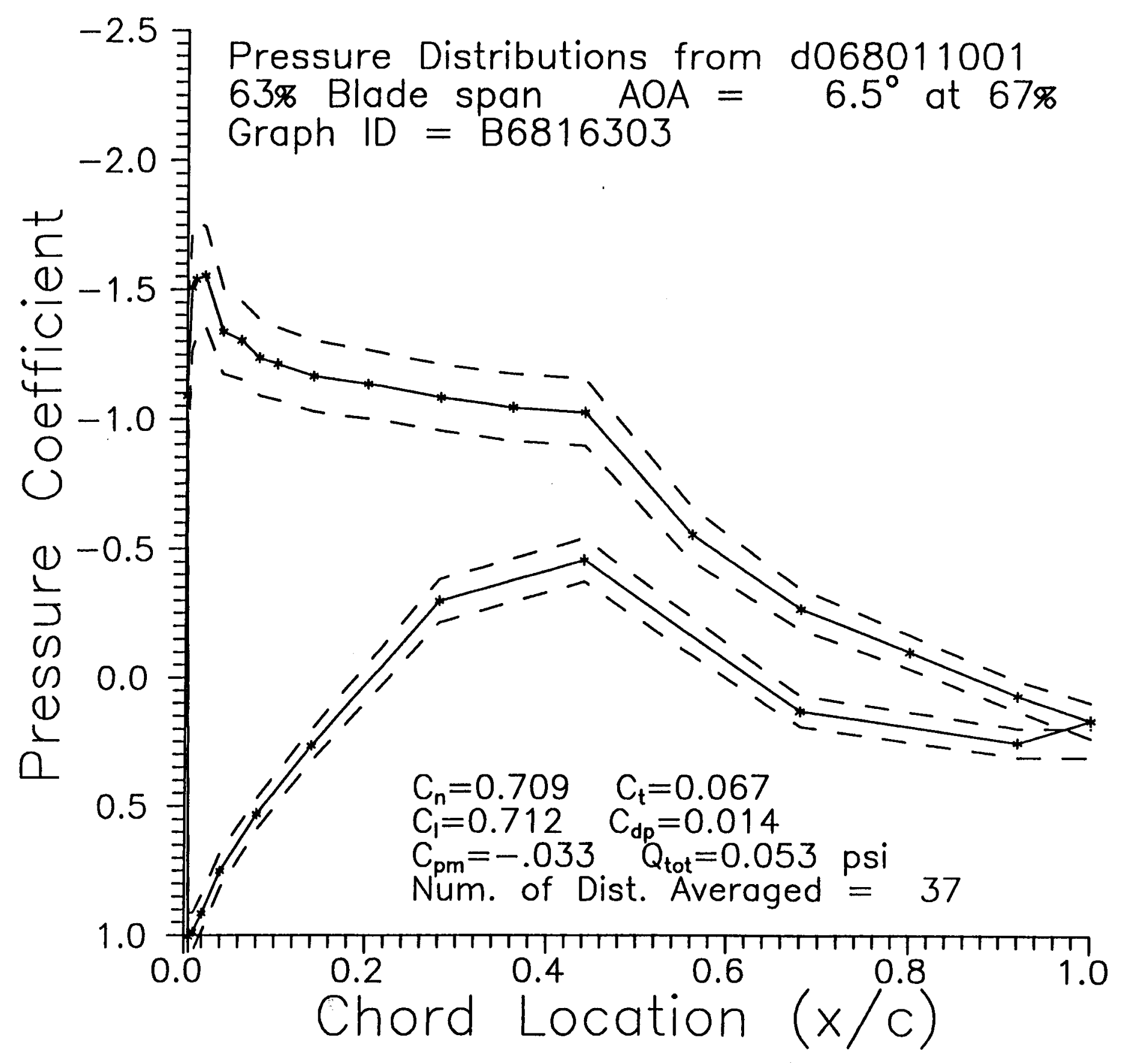




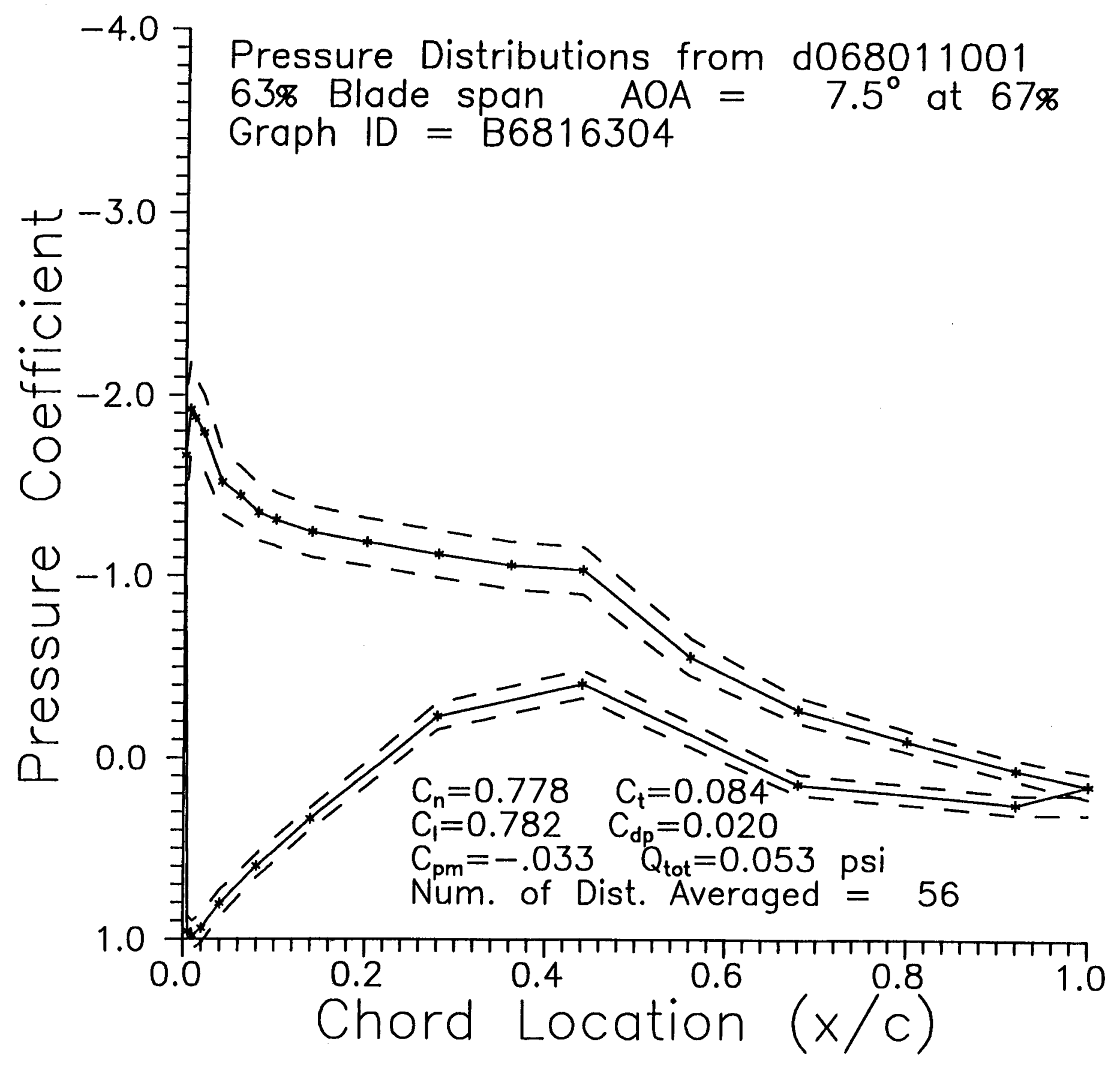




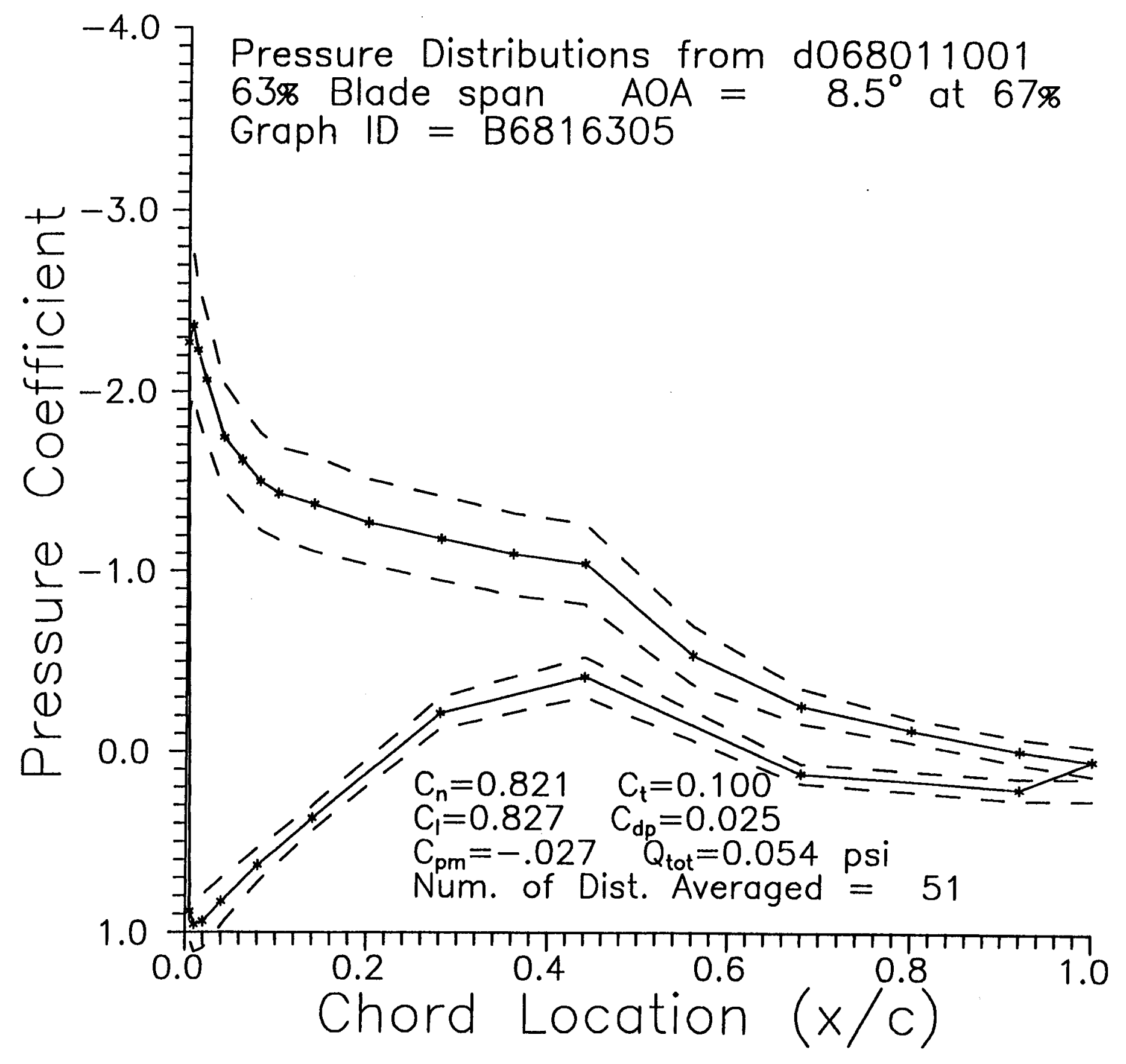




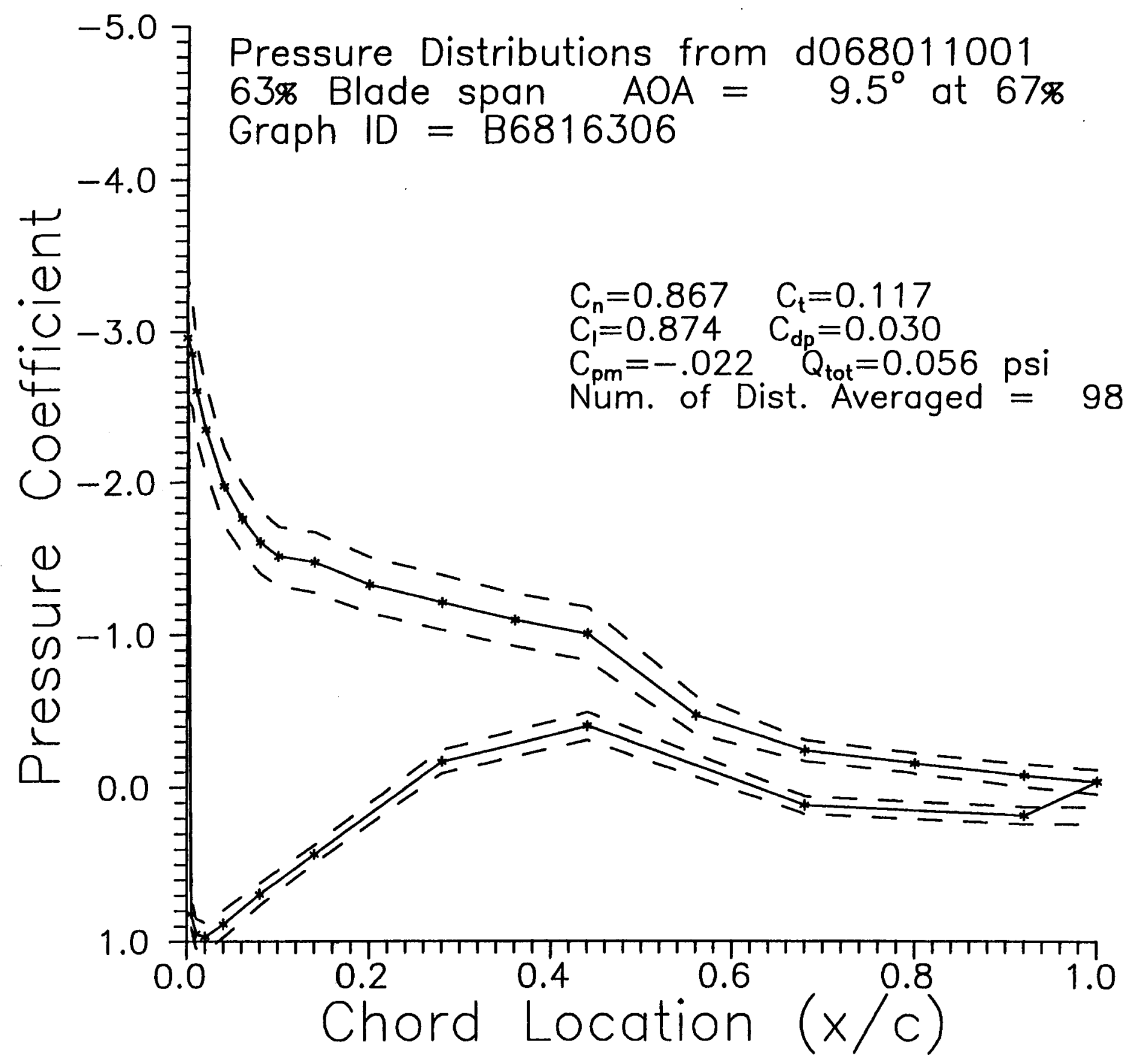




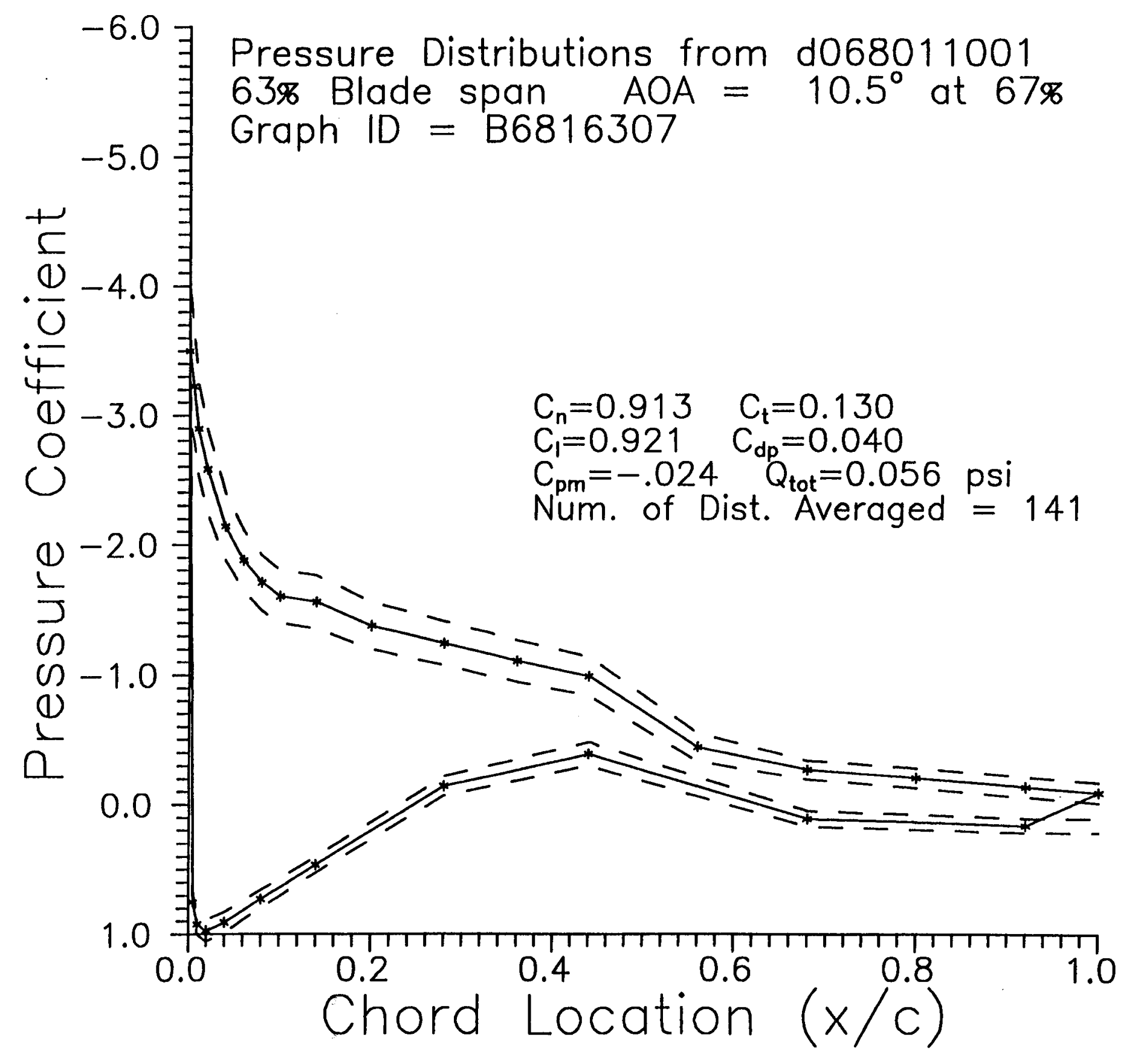




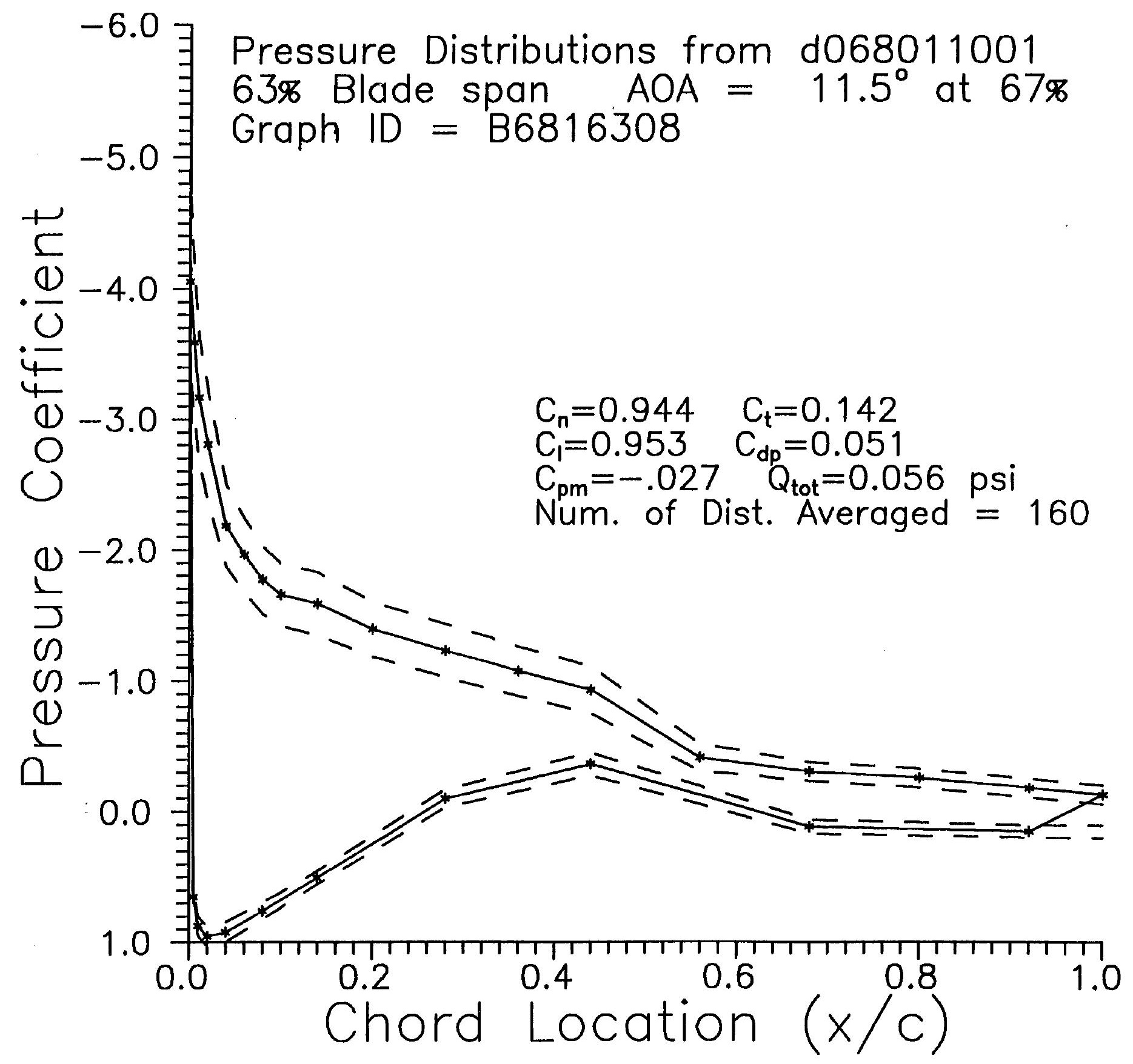




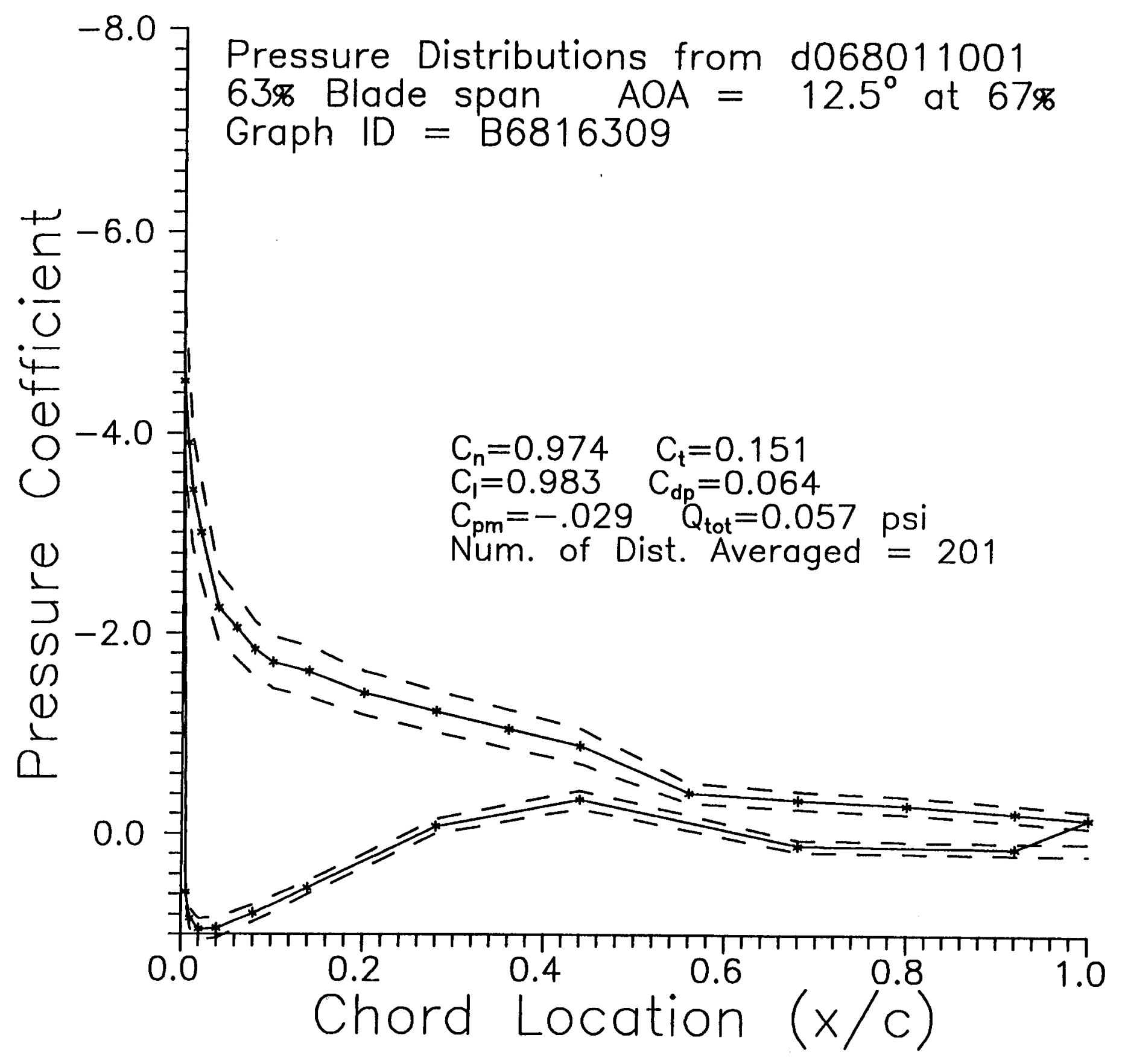




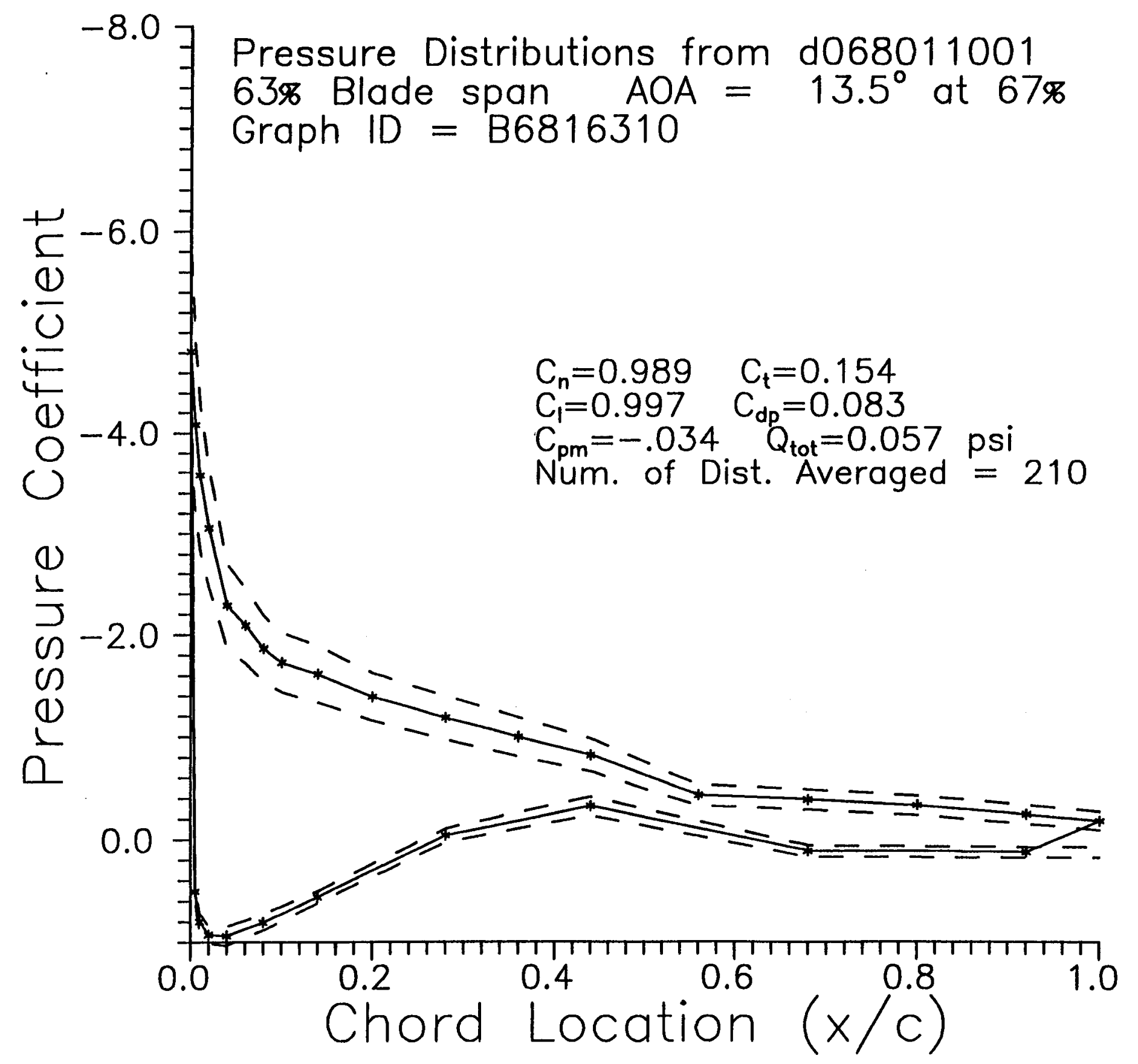




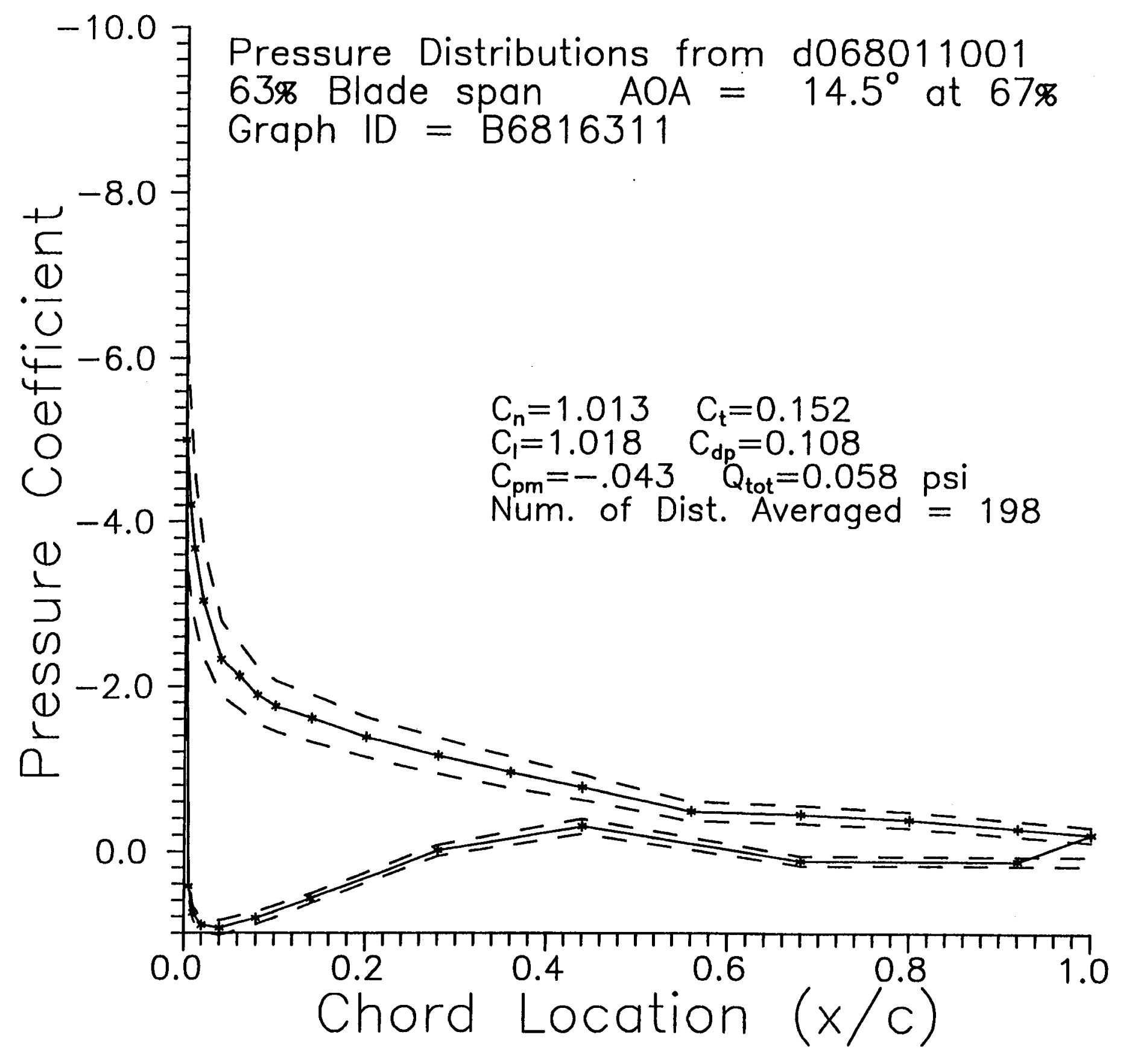




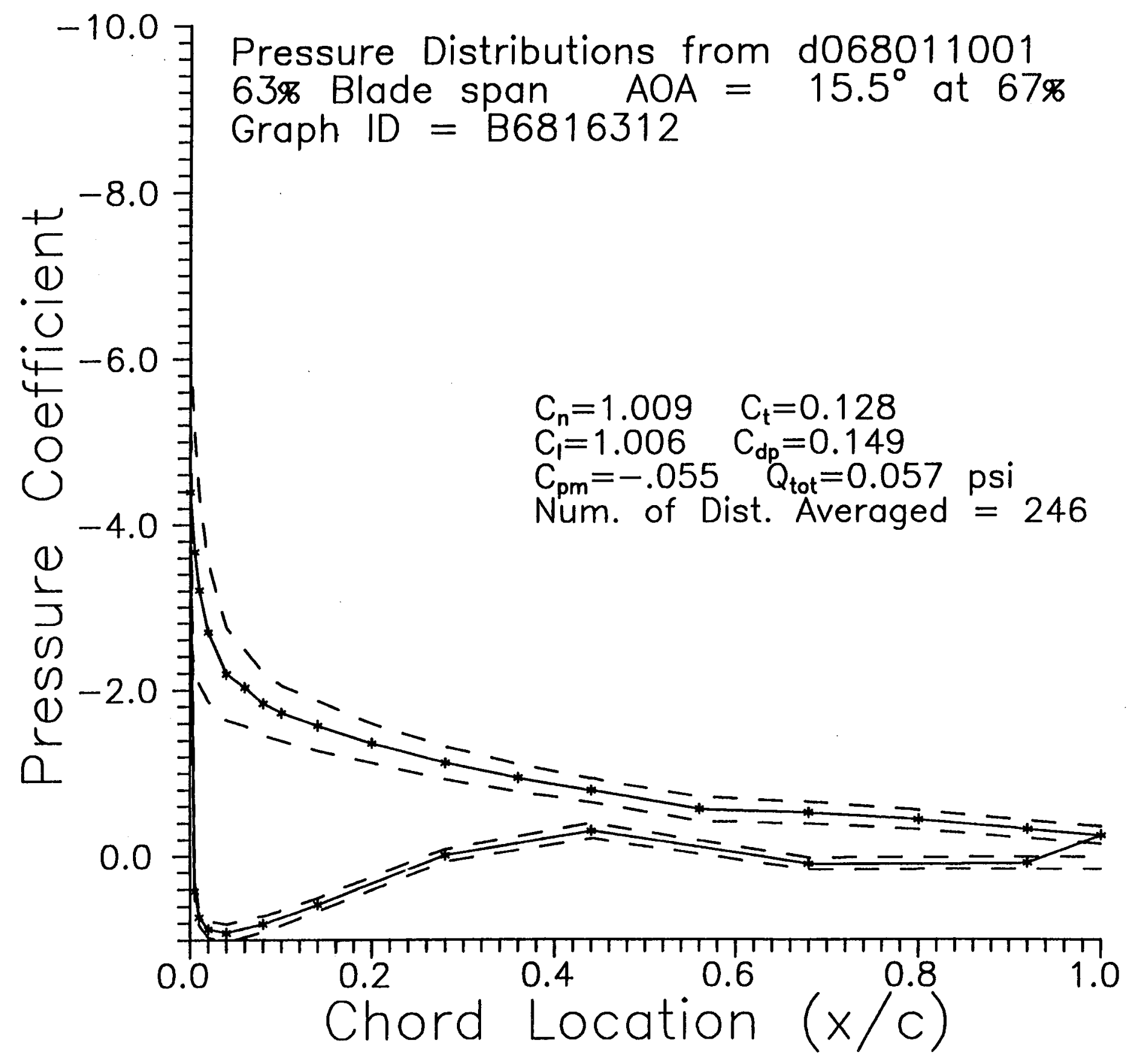




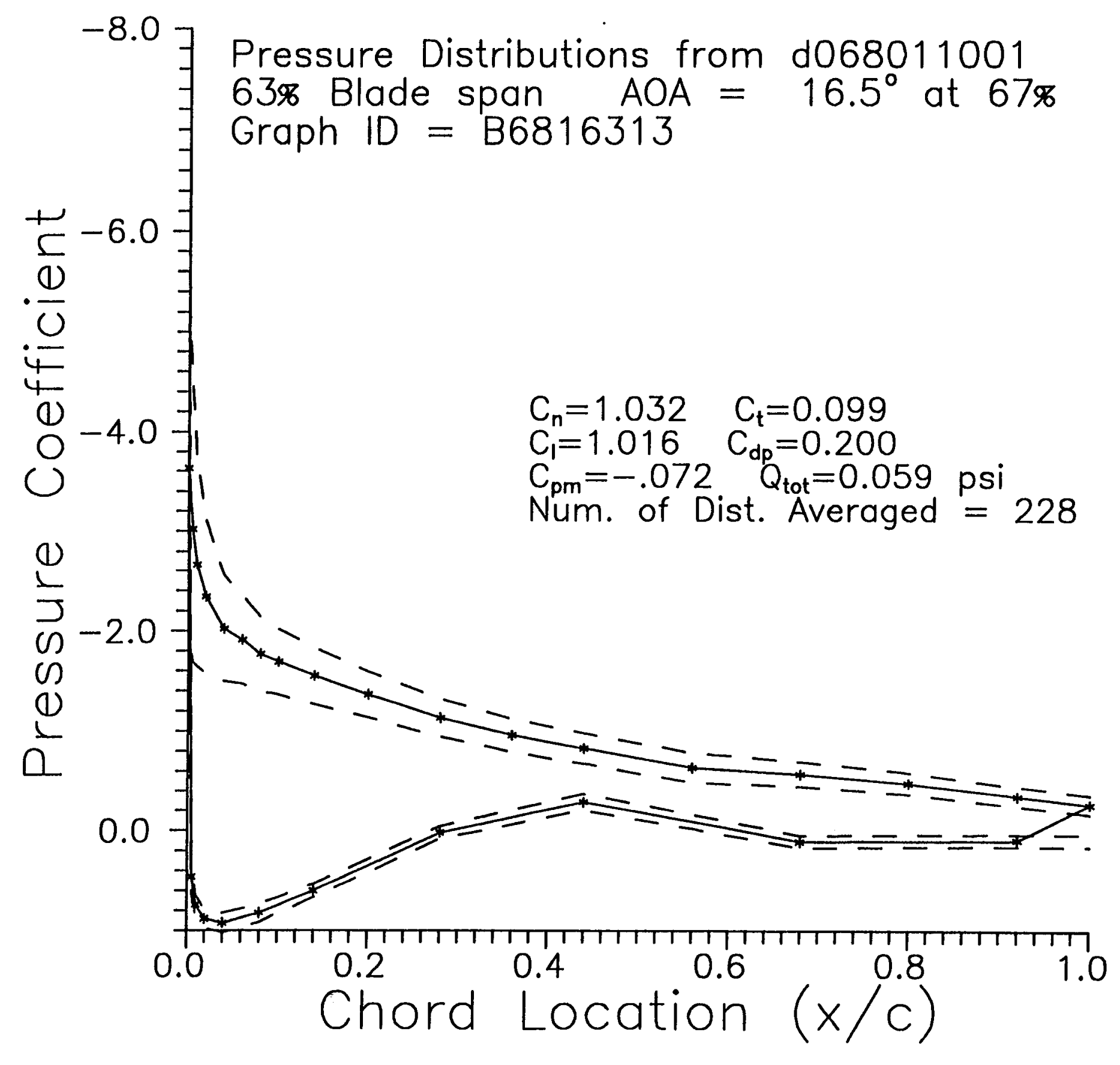




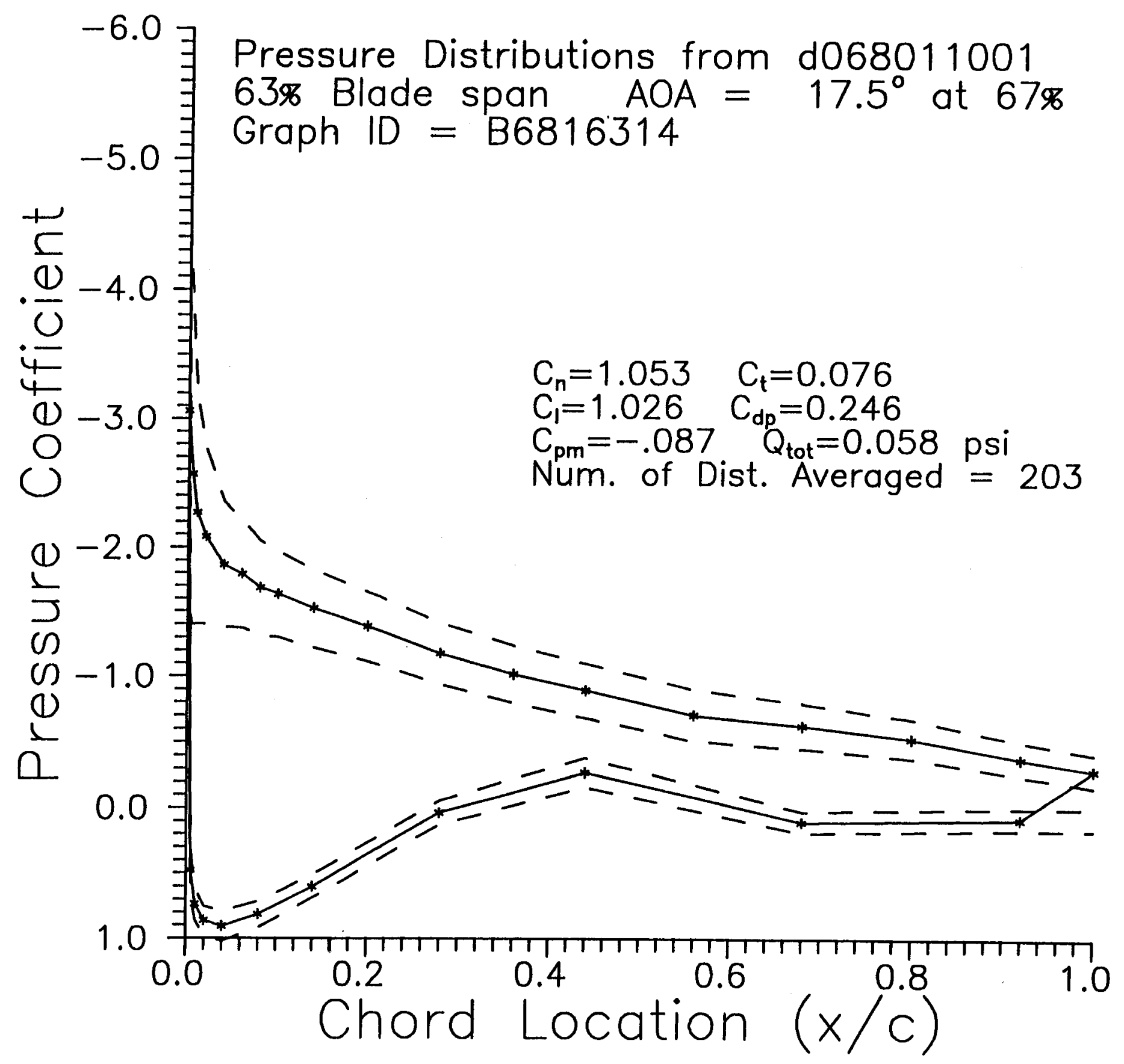




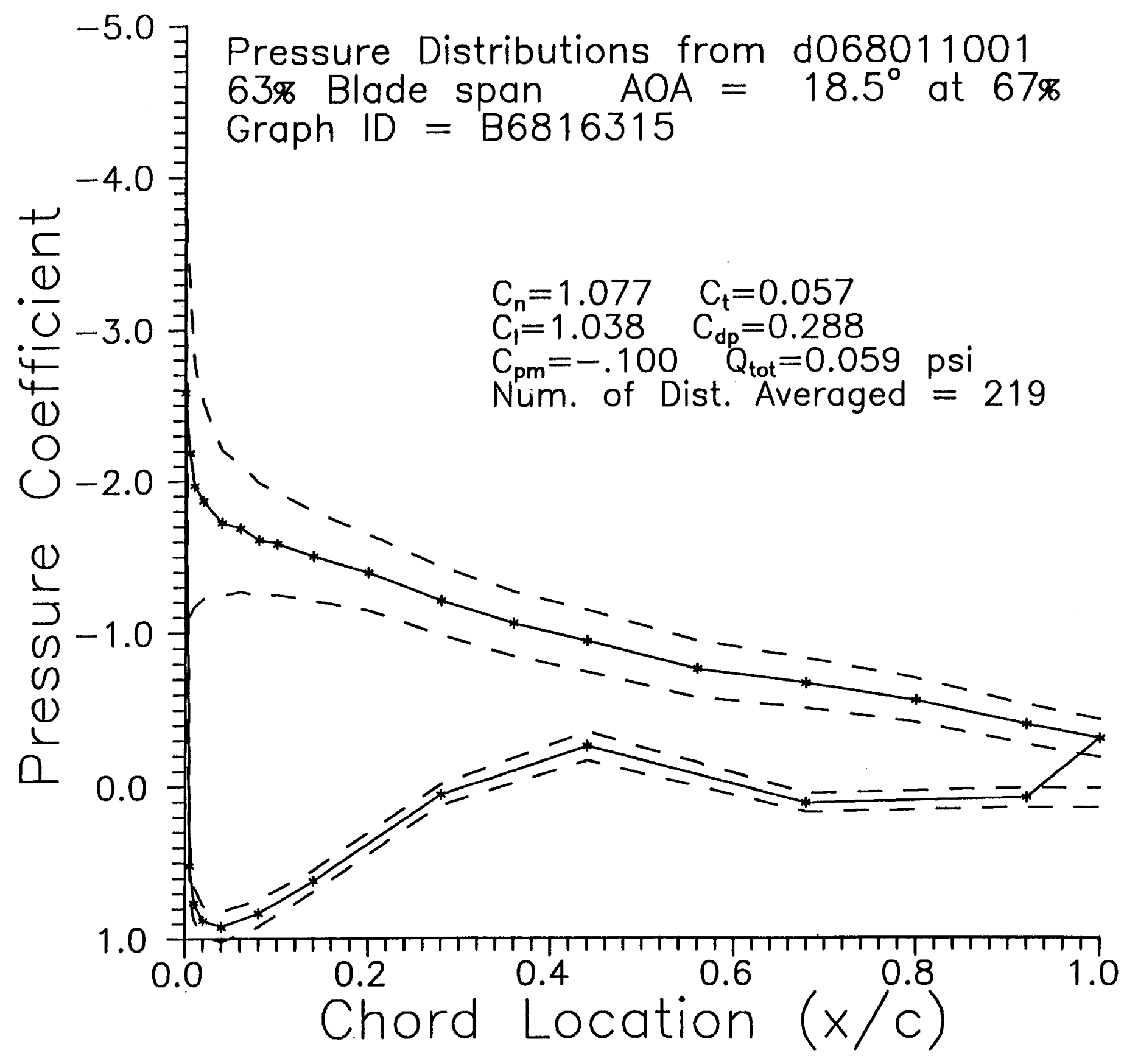




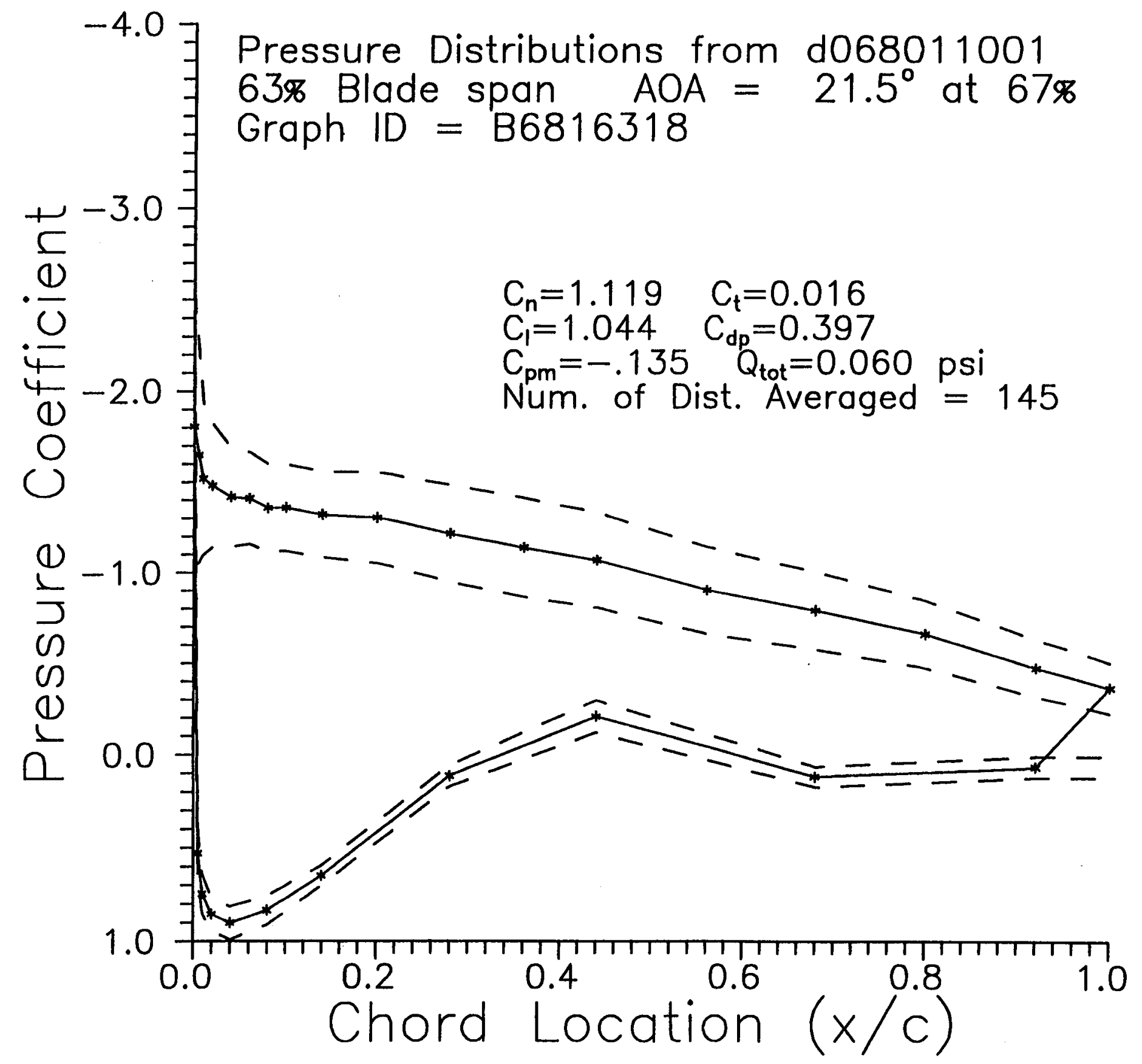




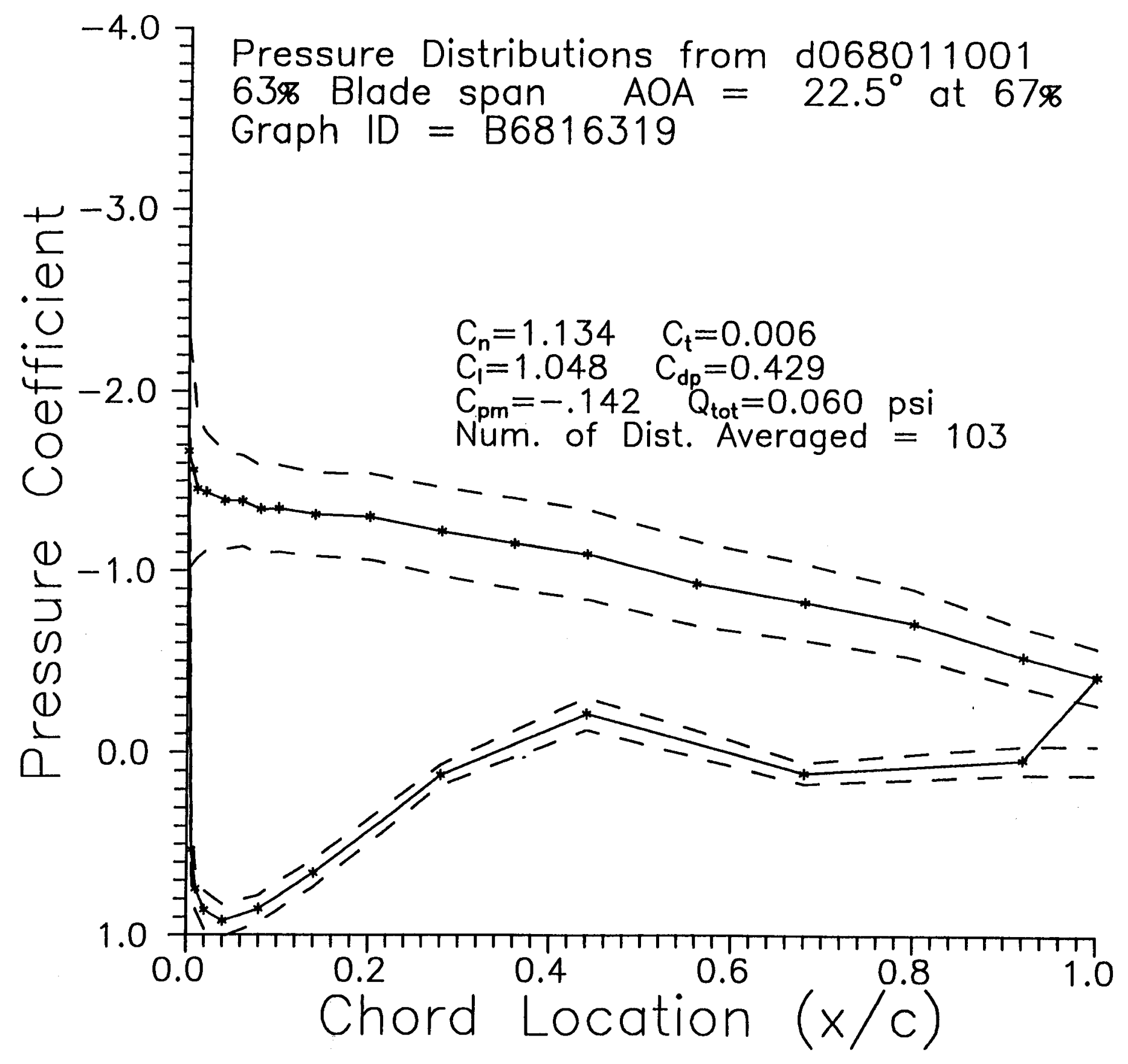




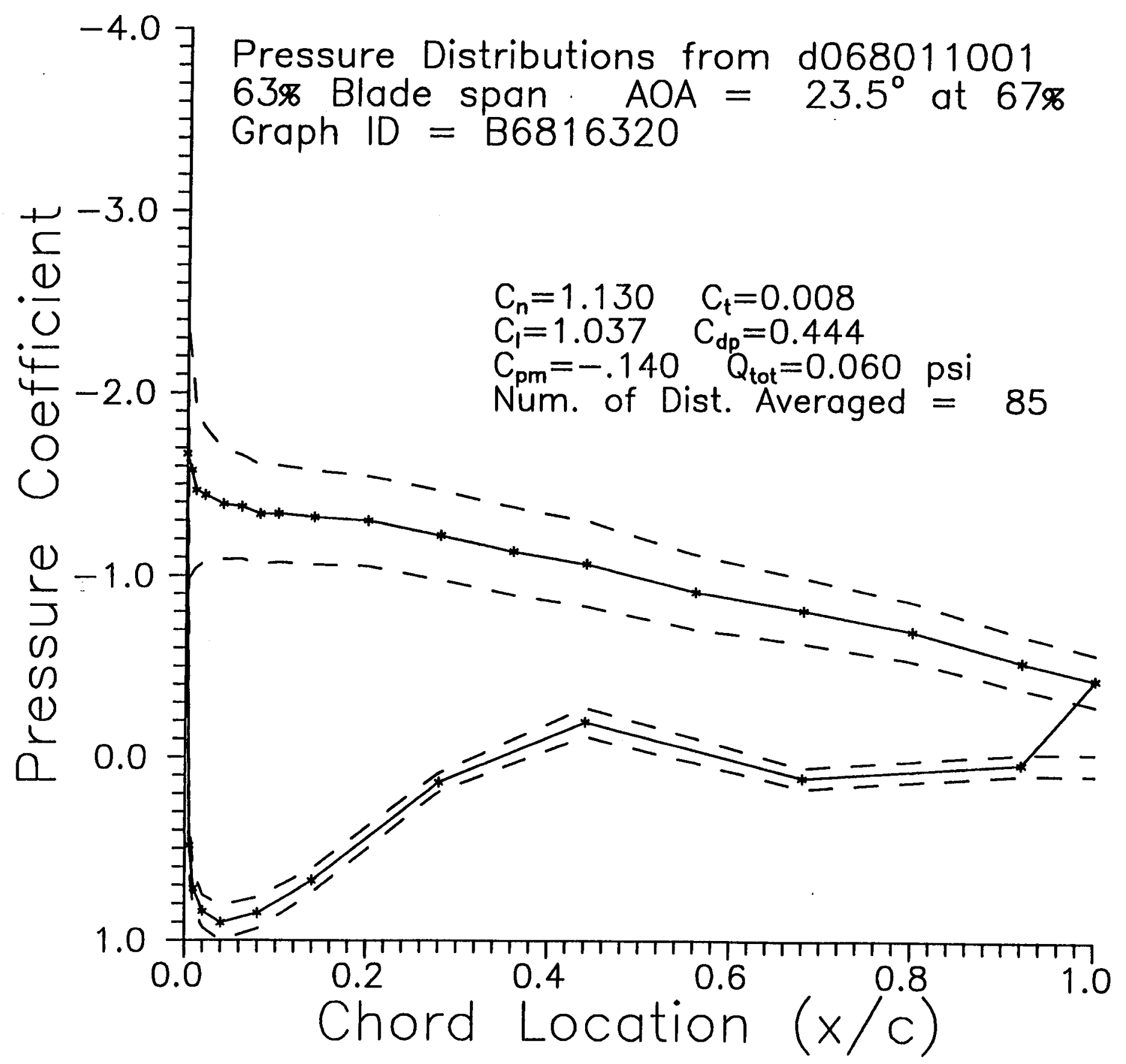




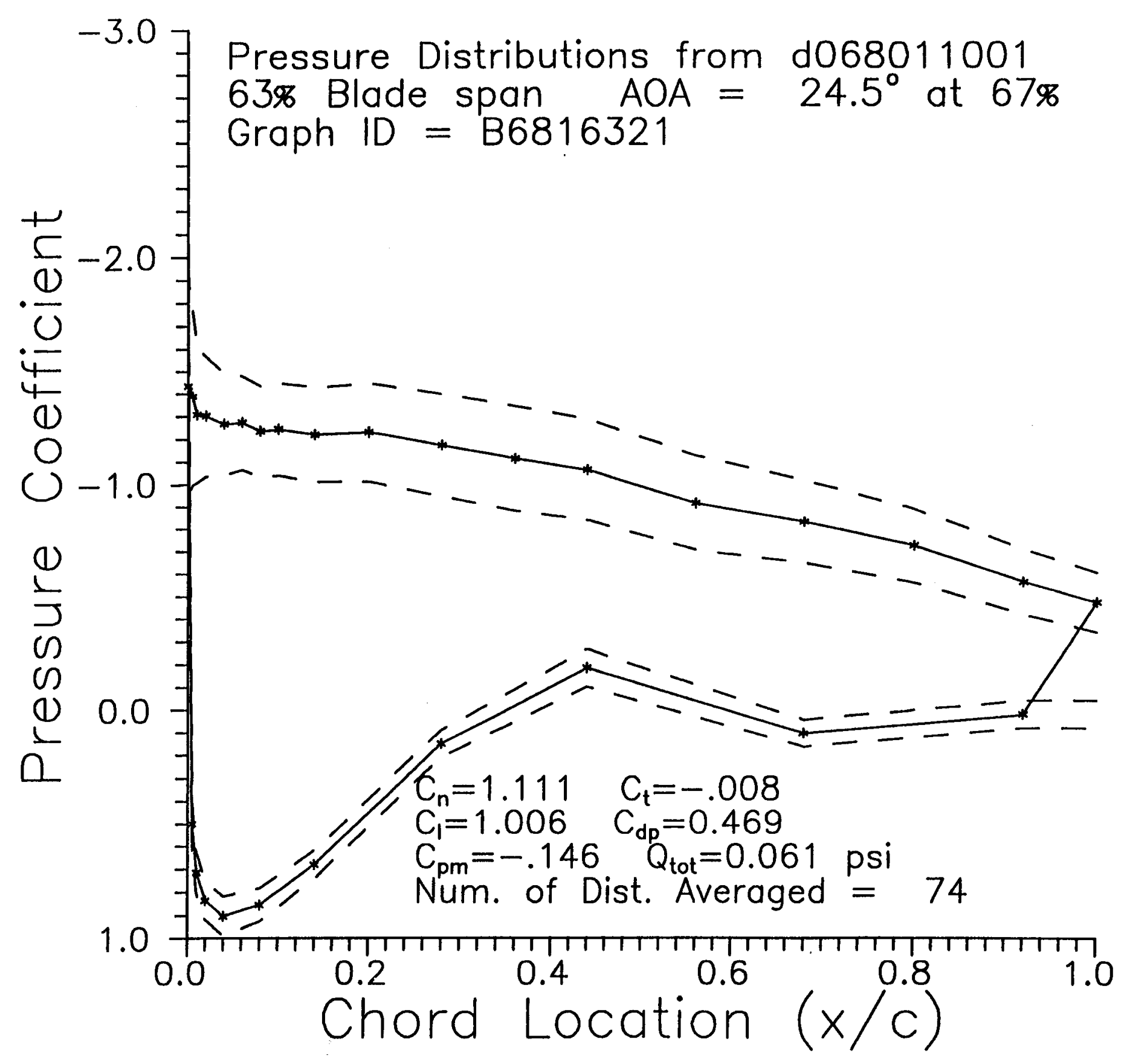




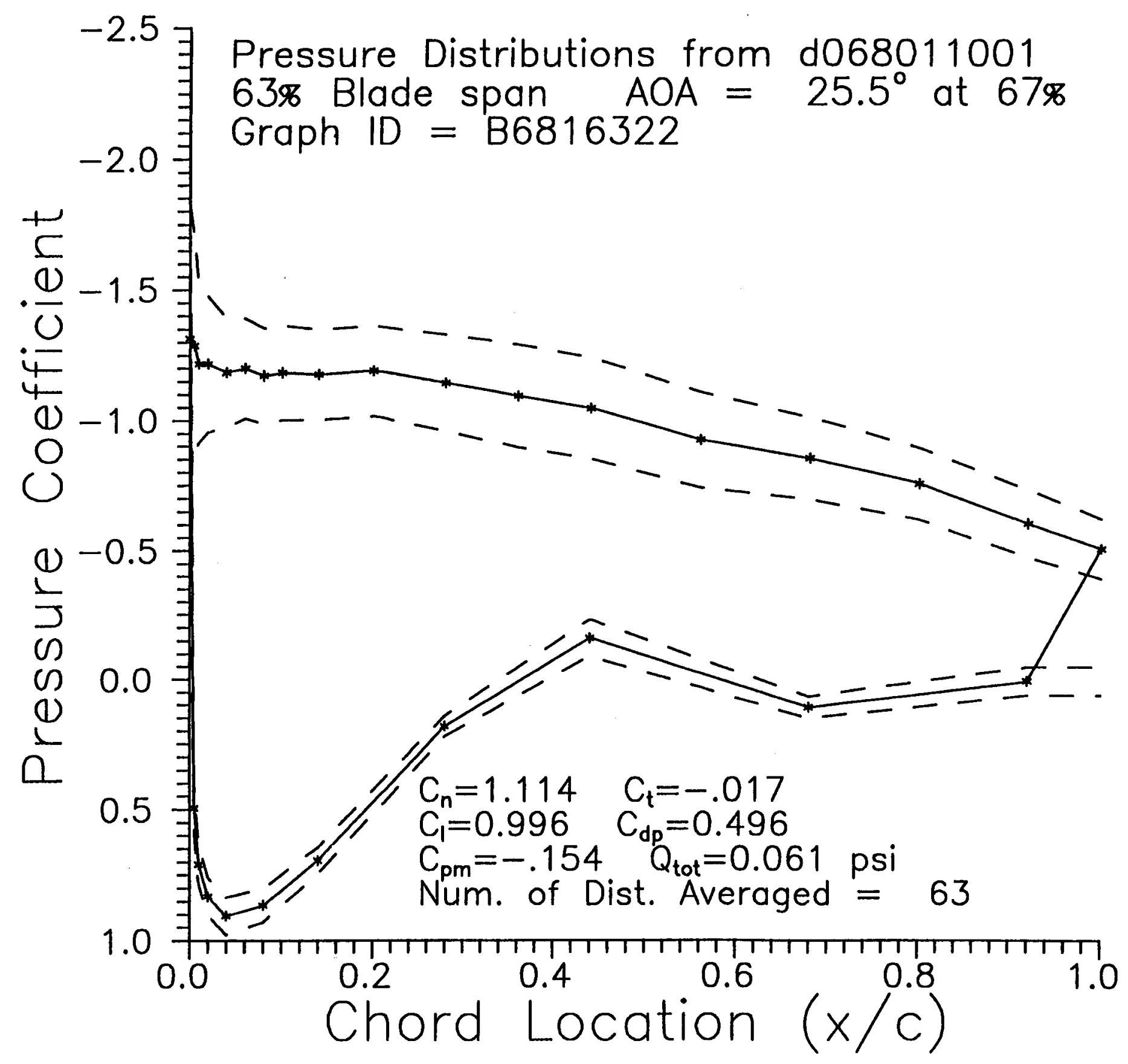




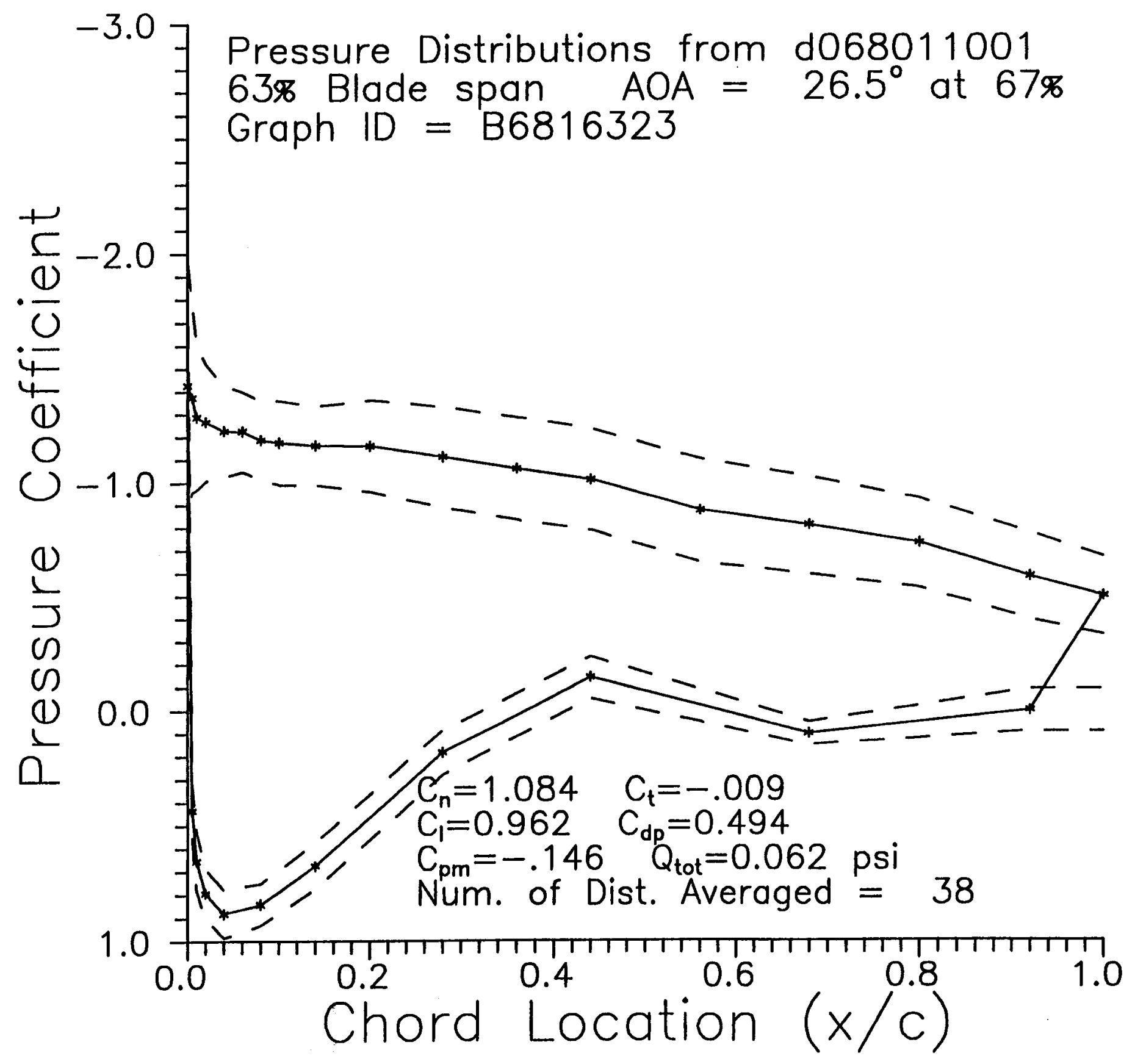


Appendix C.2.4

Pressure Distributions for 30\% Span 


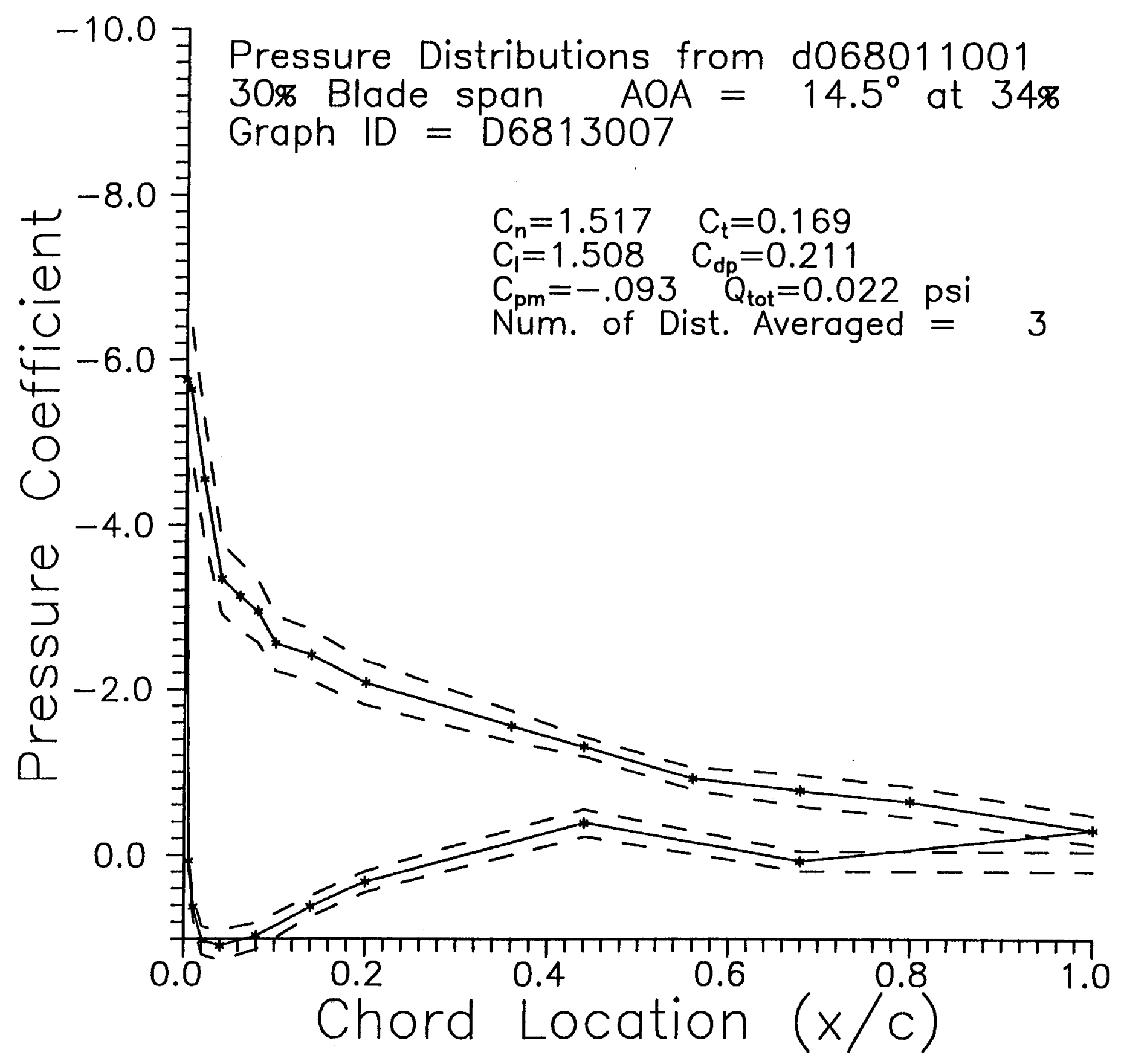




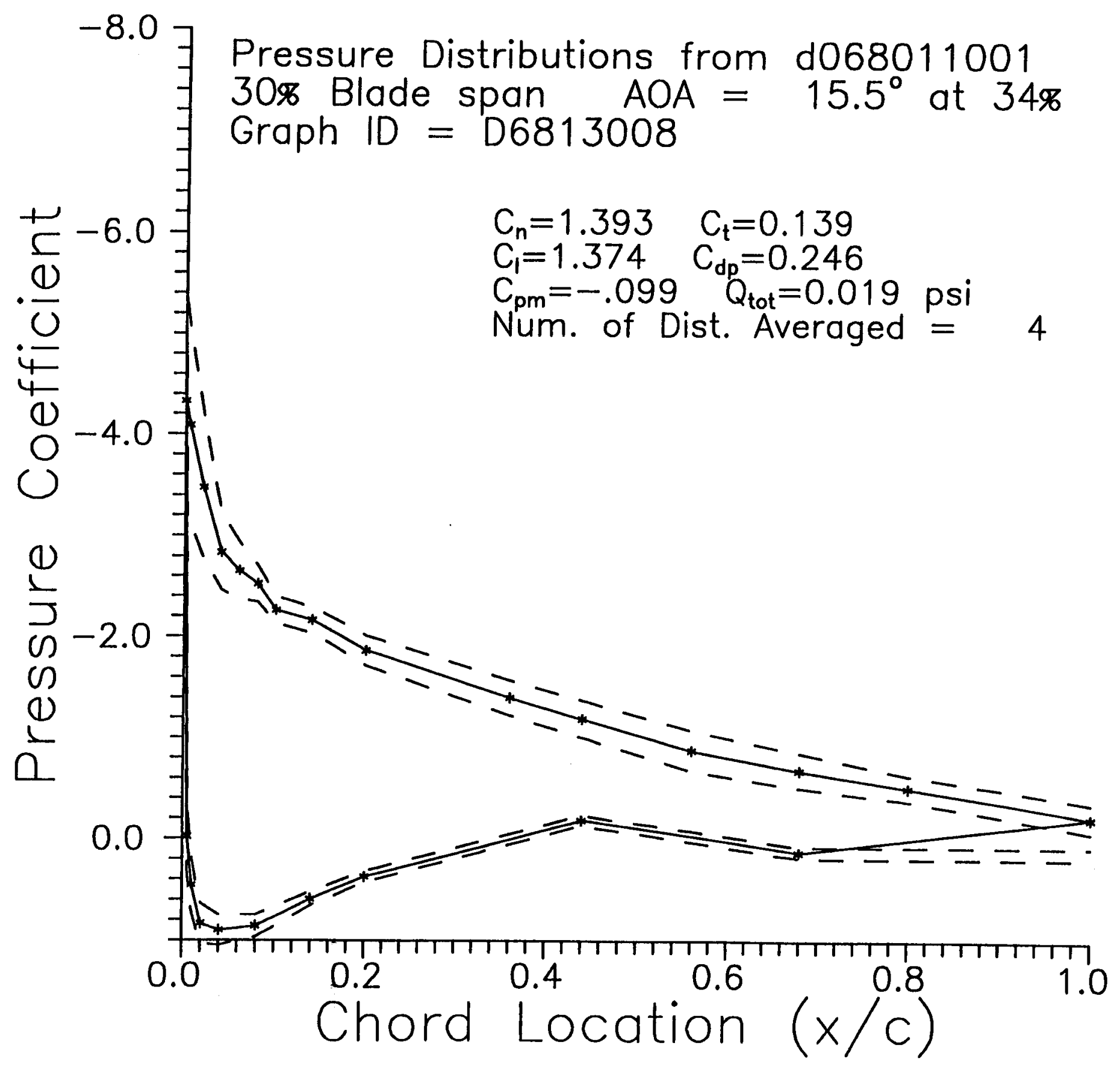




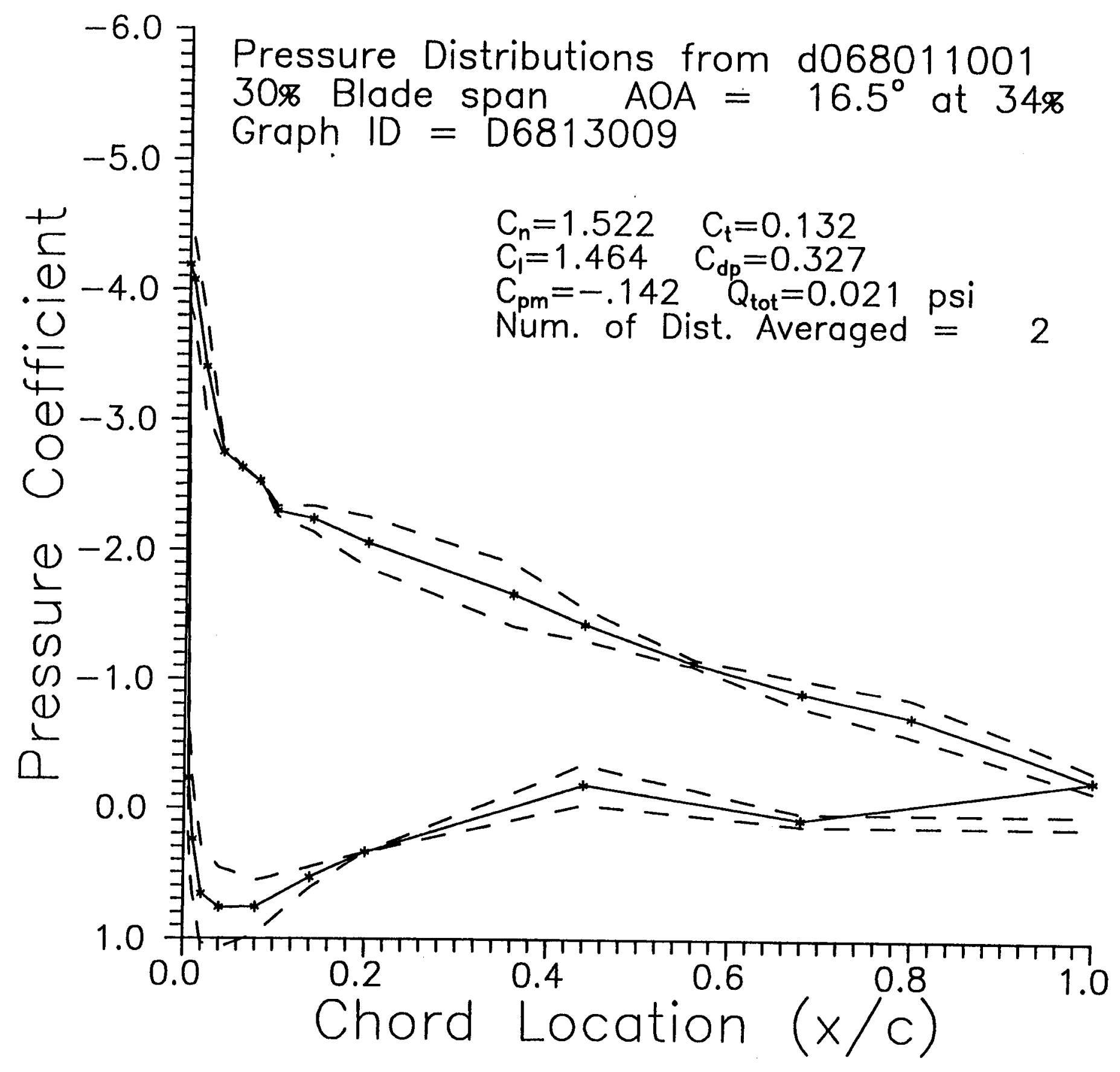




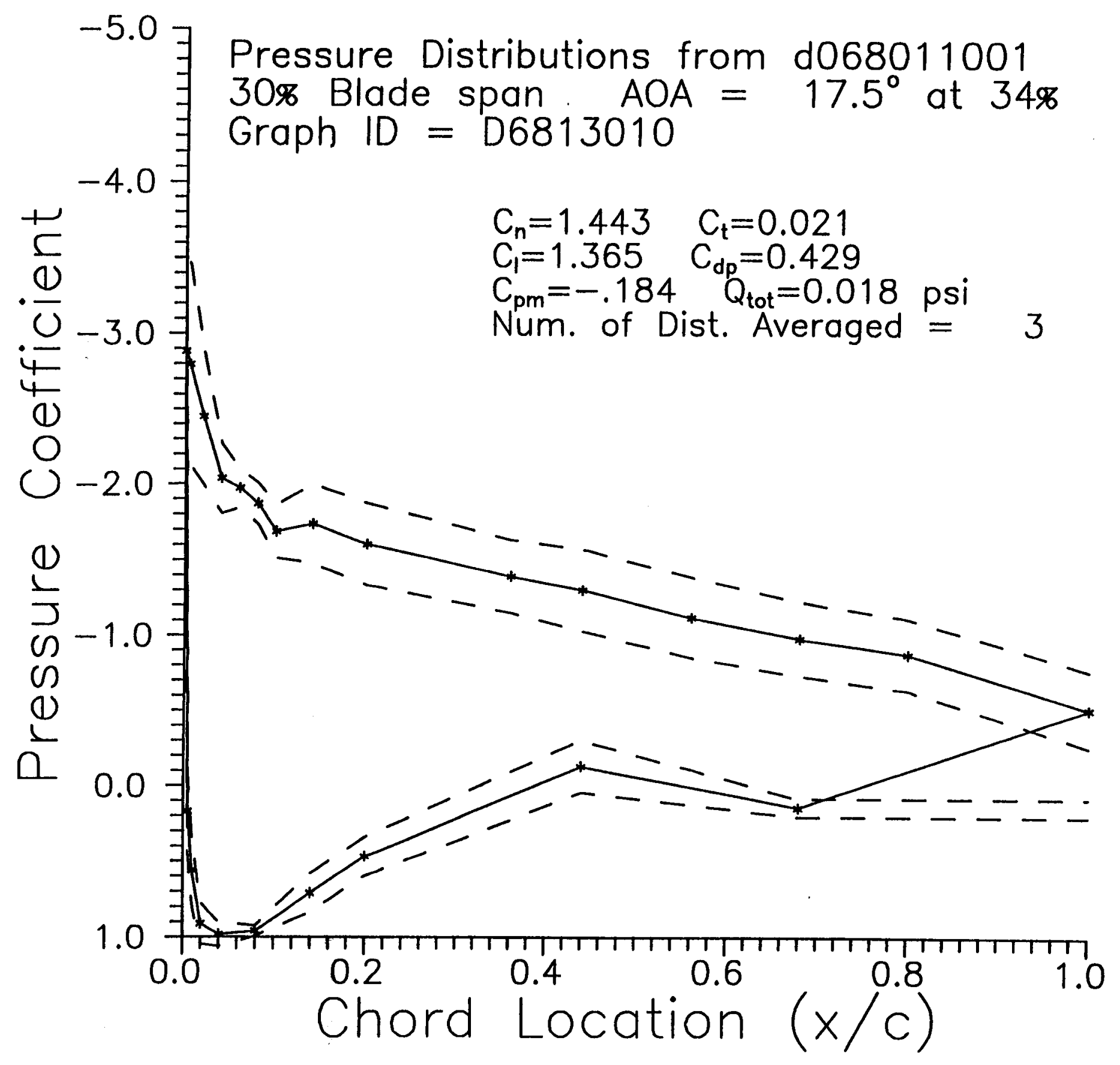




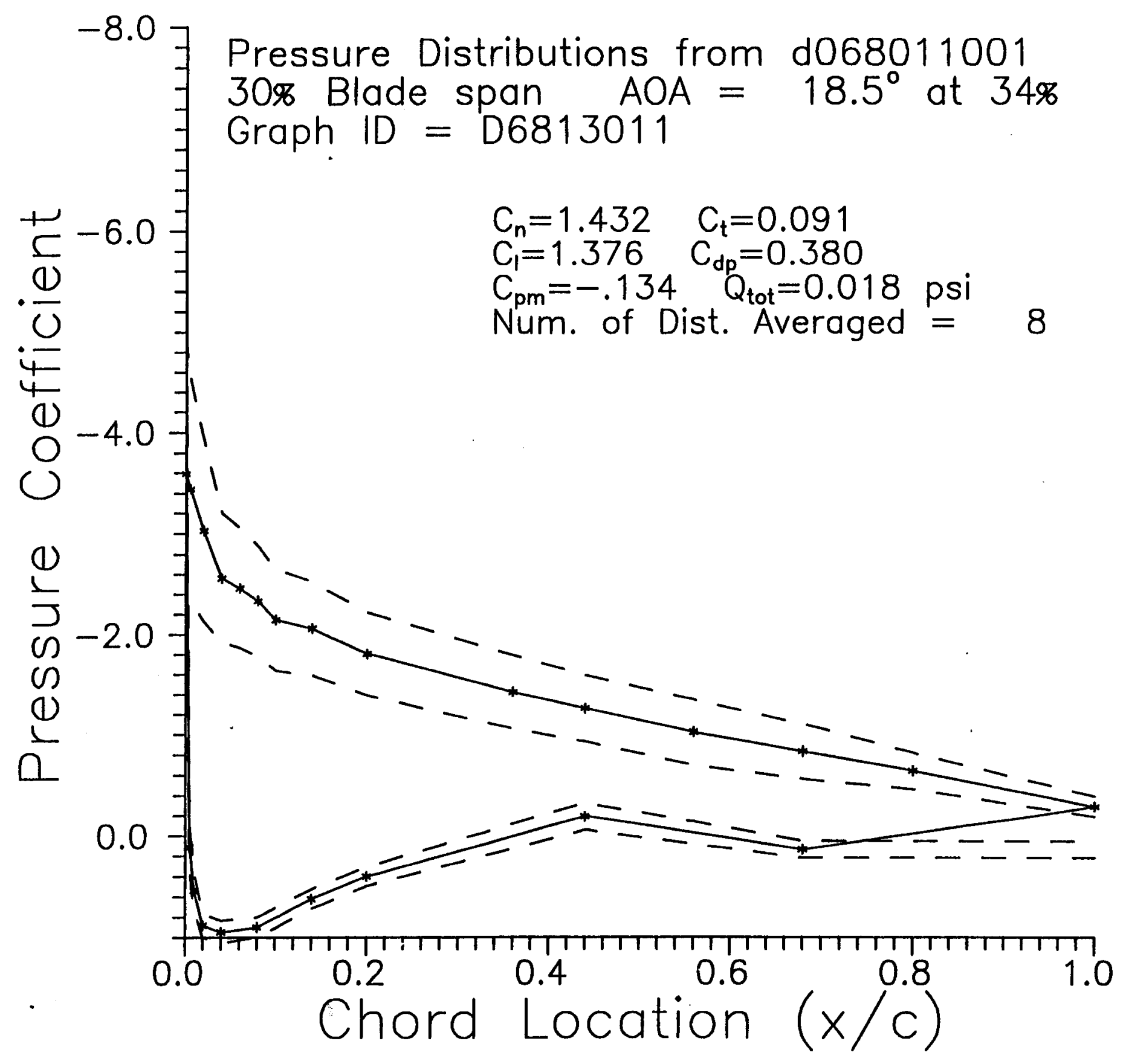




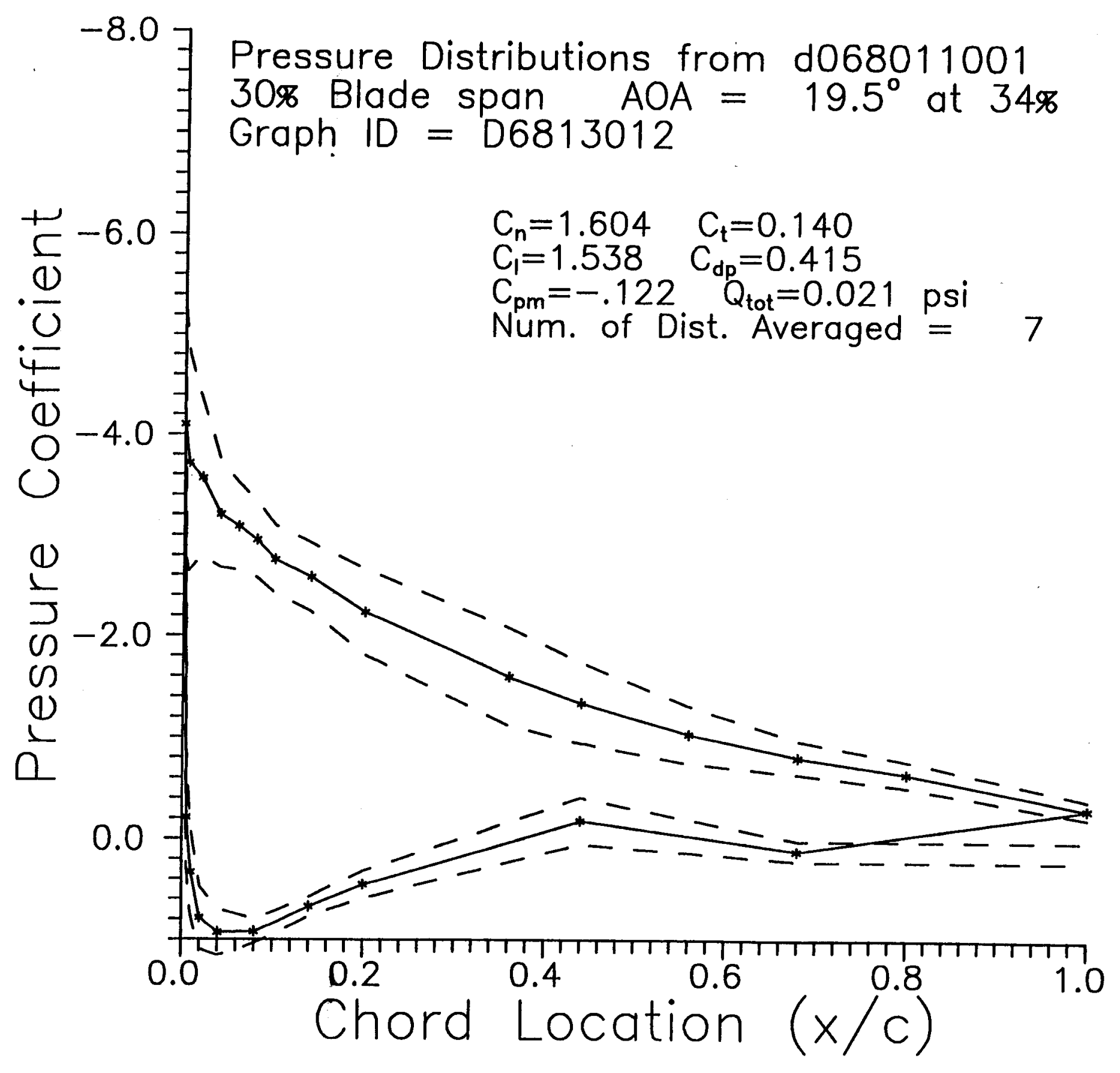




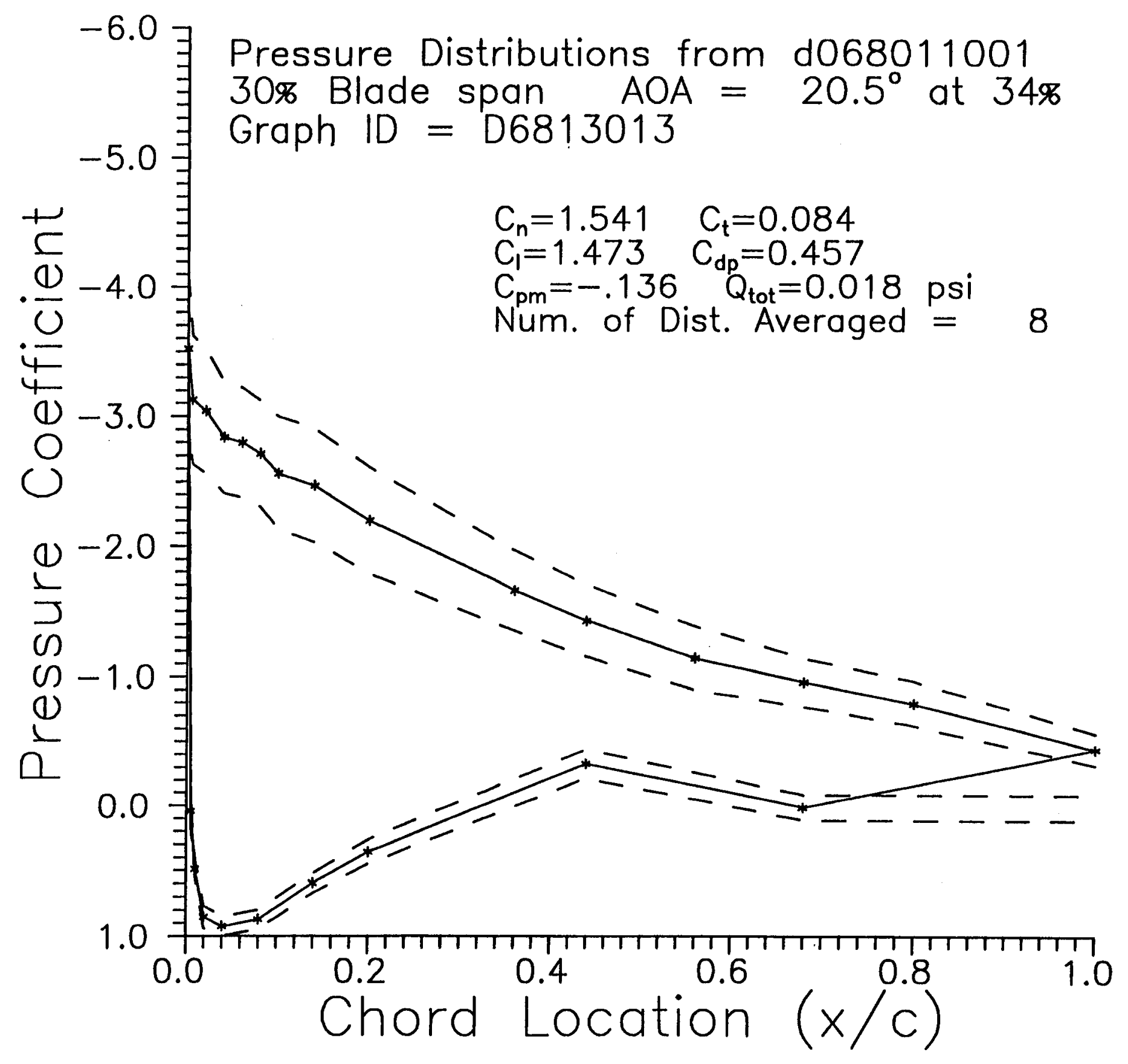




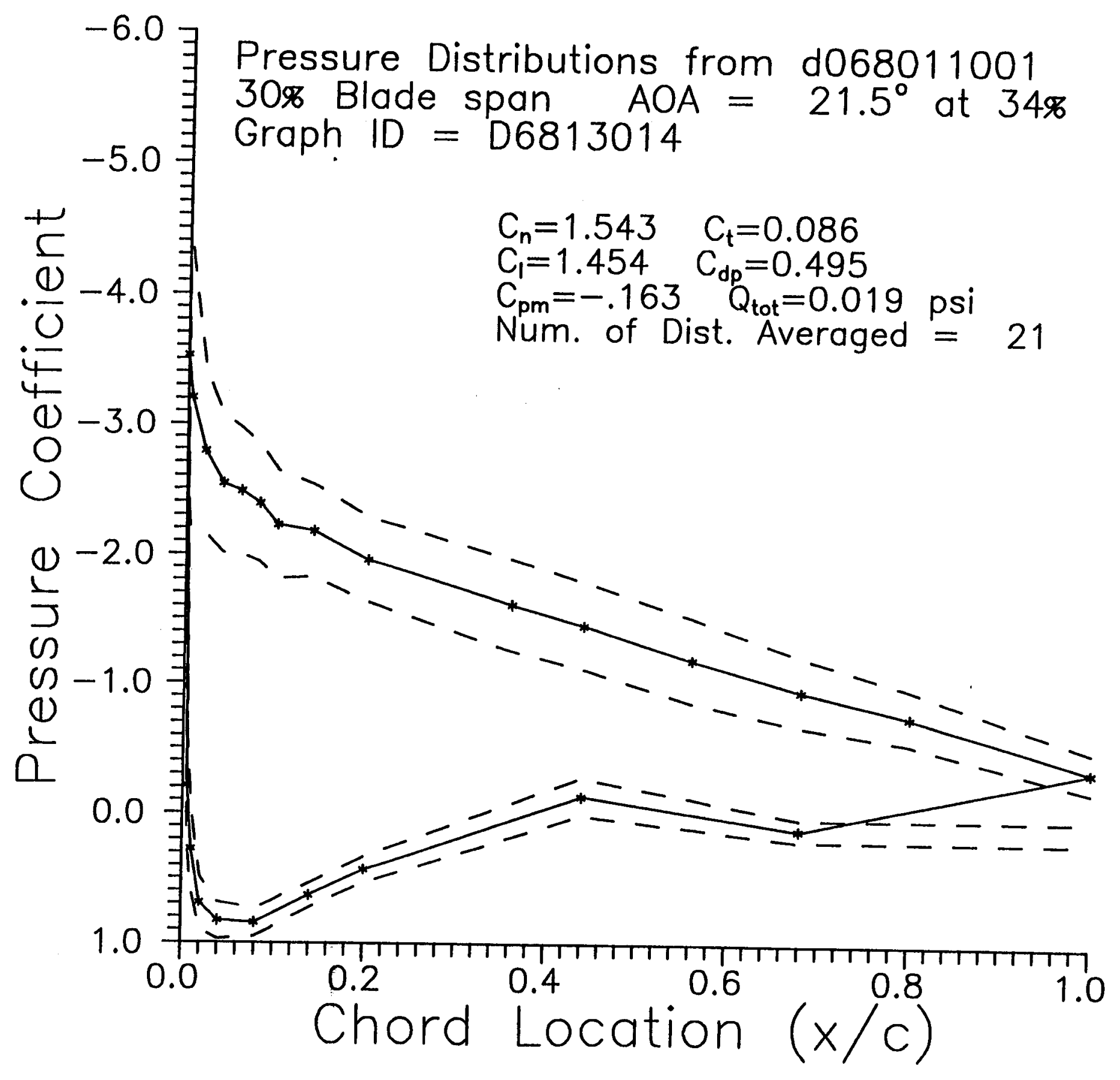




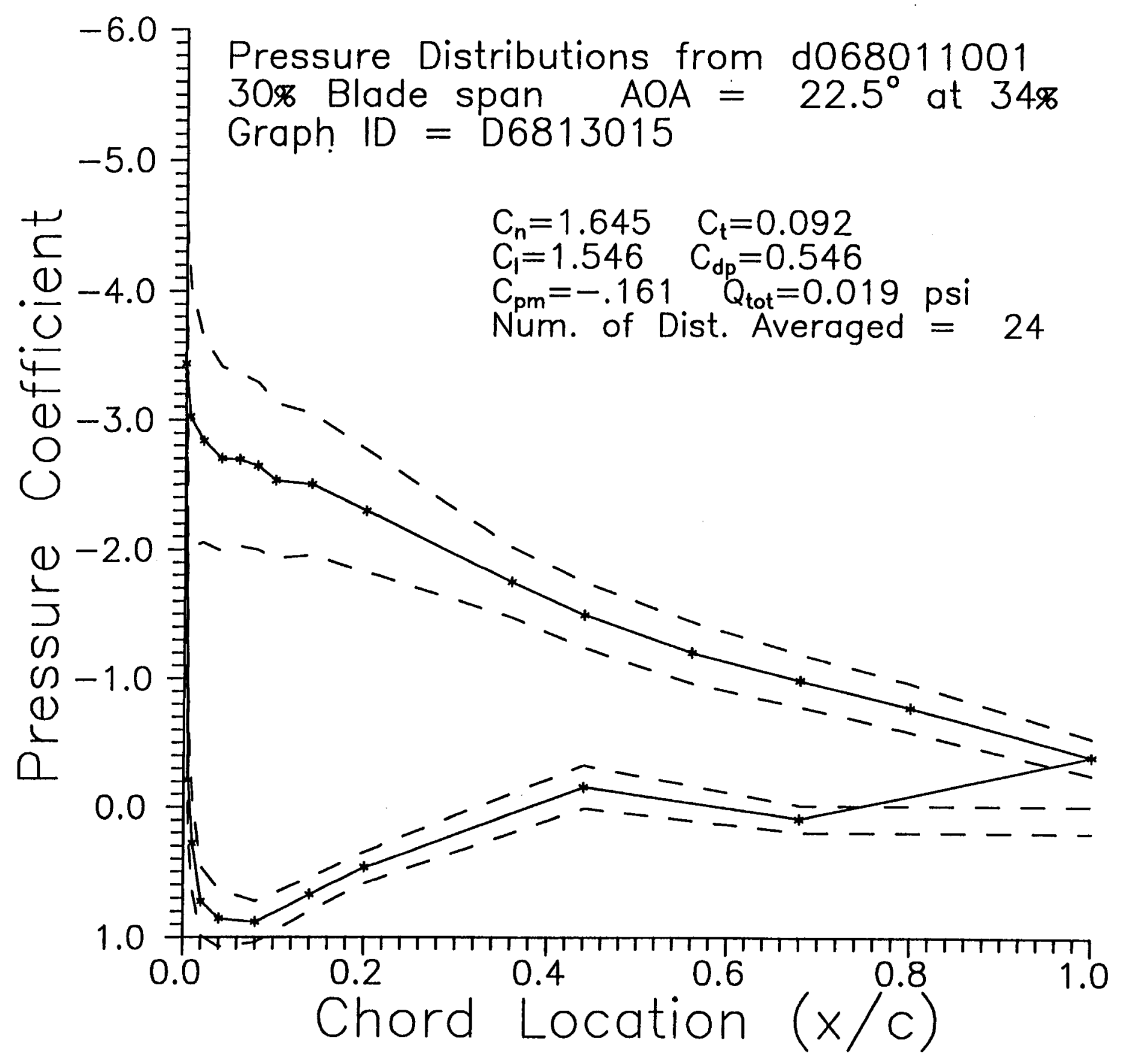




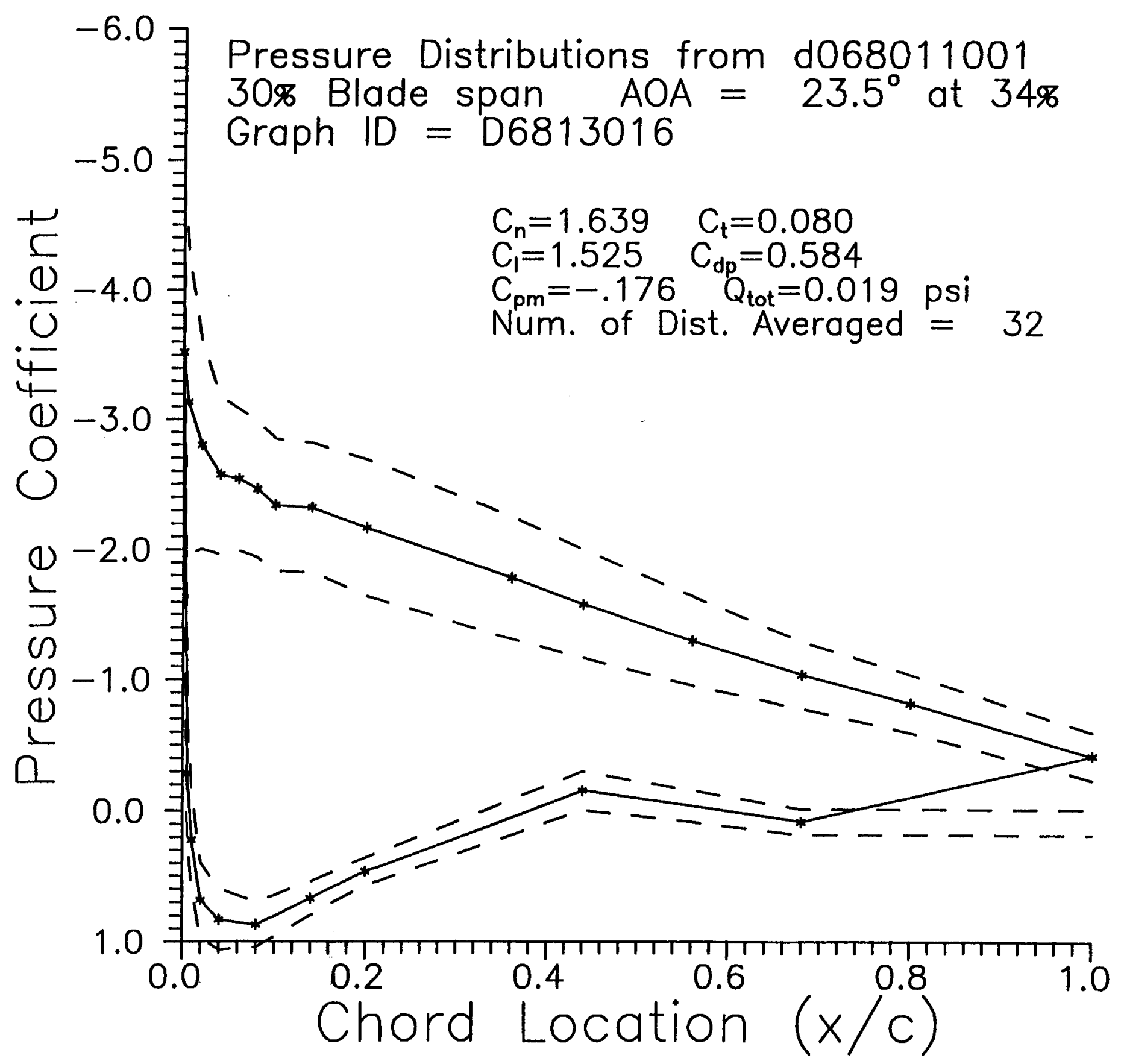




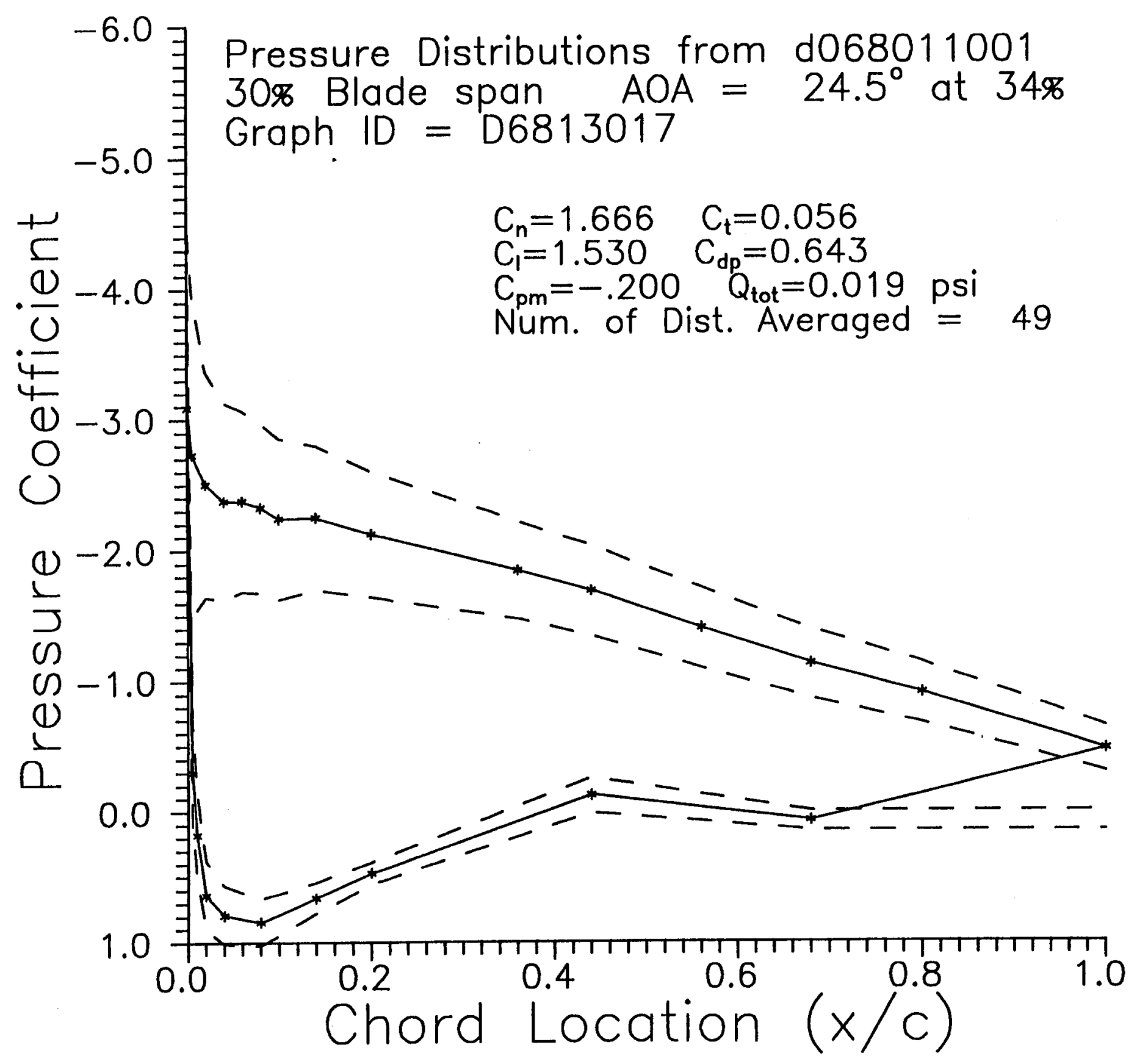




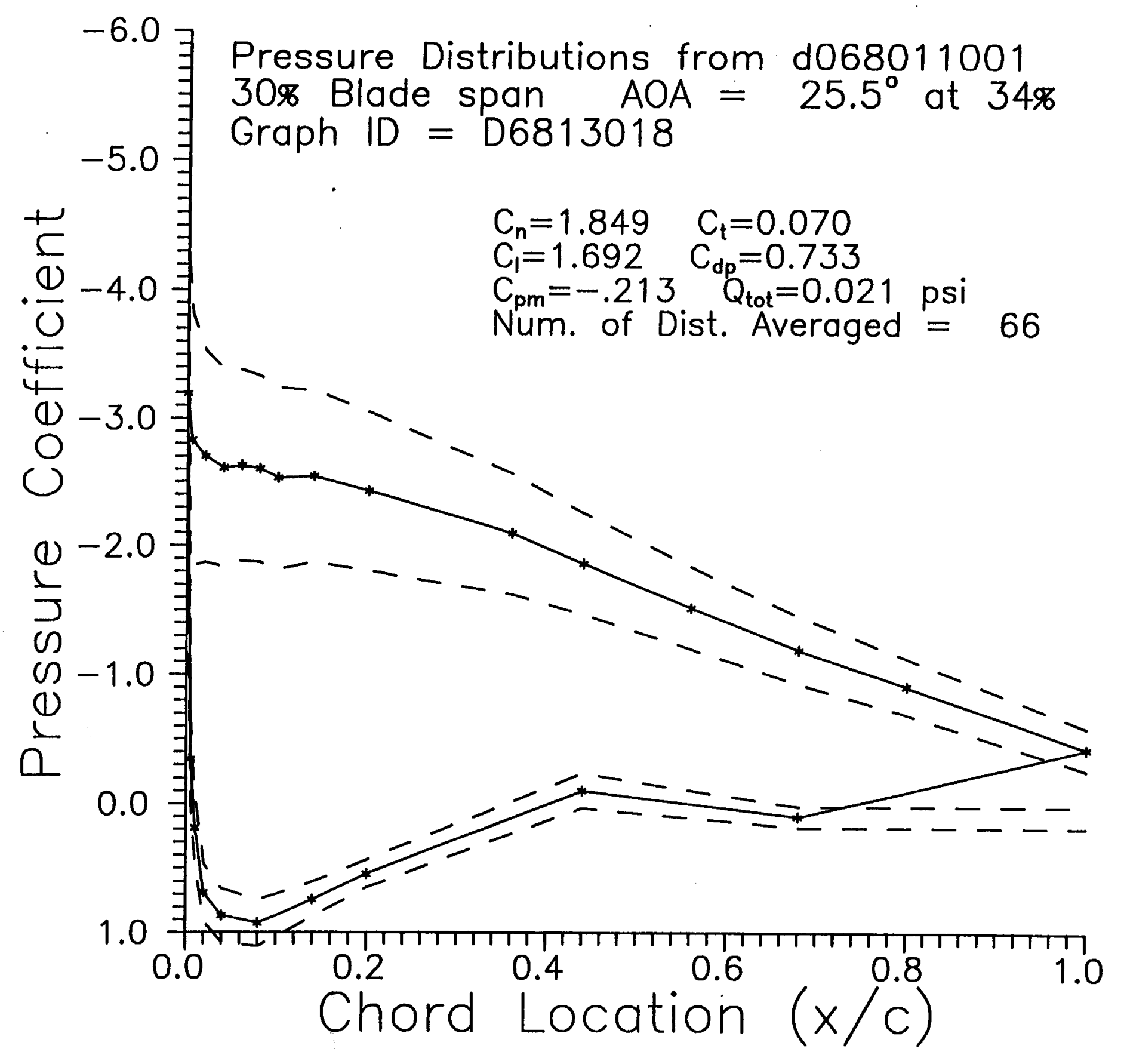




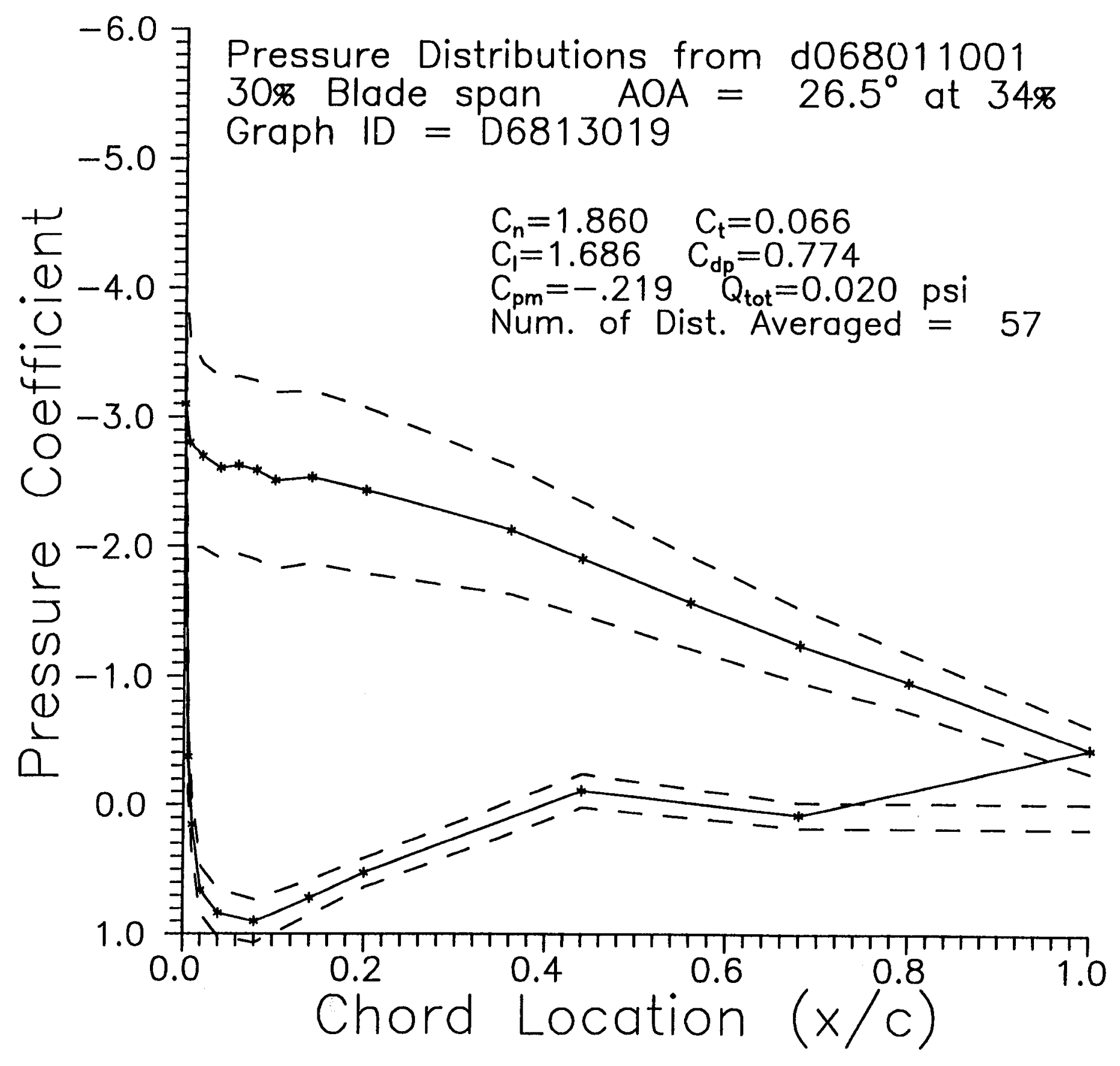




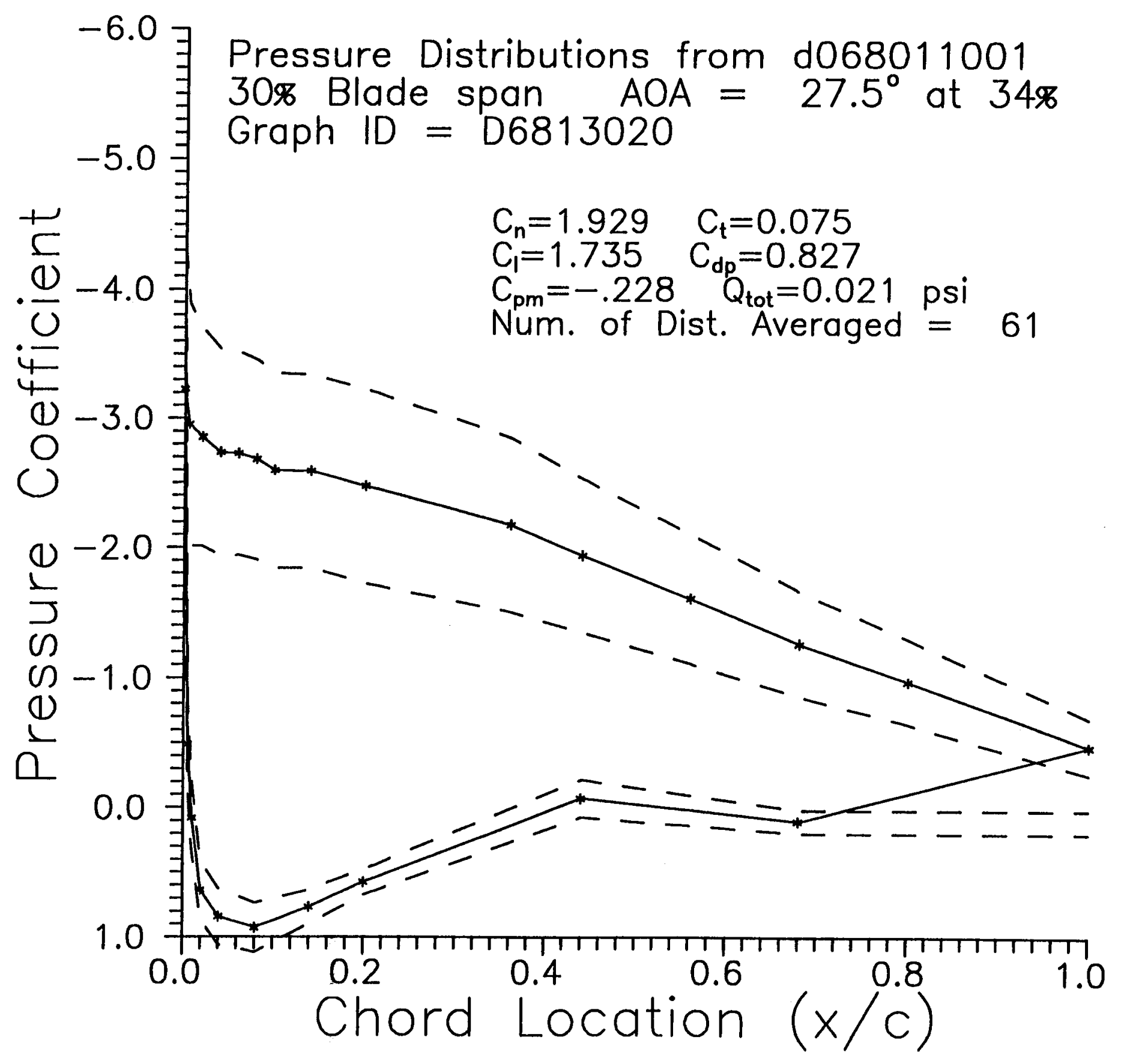




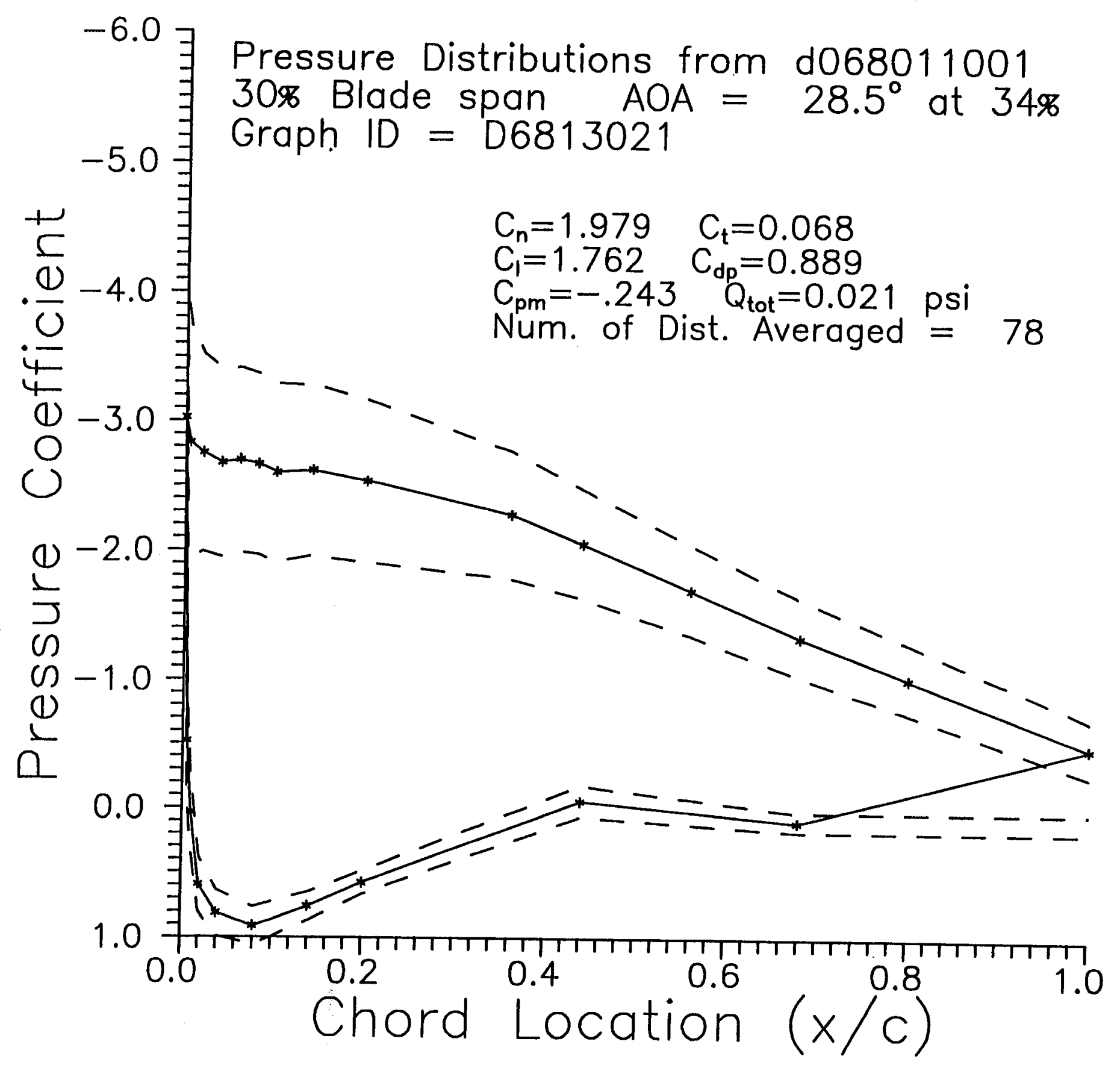




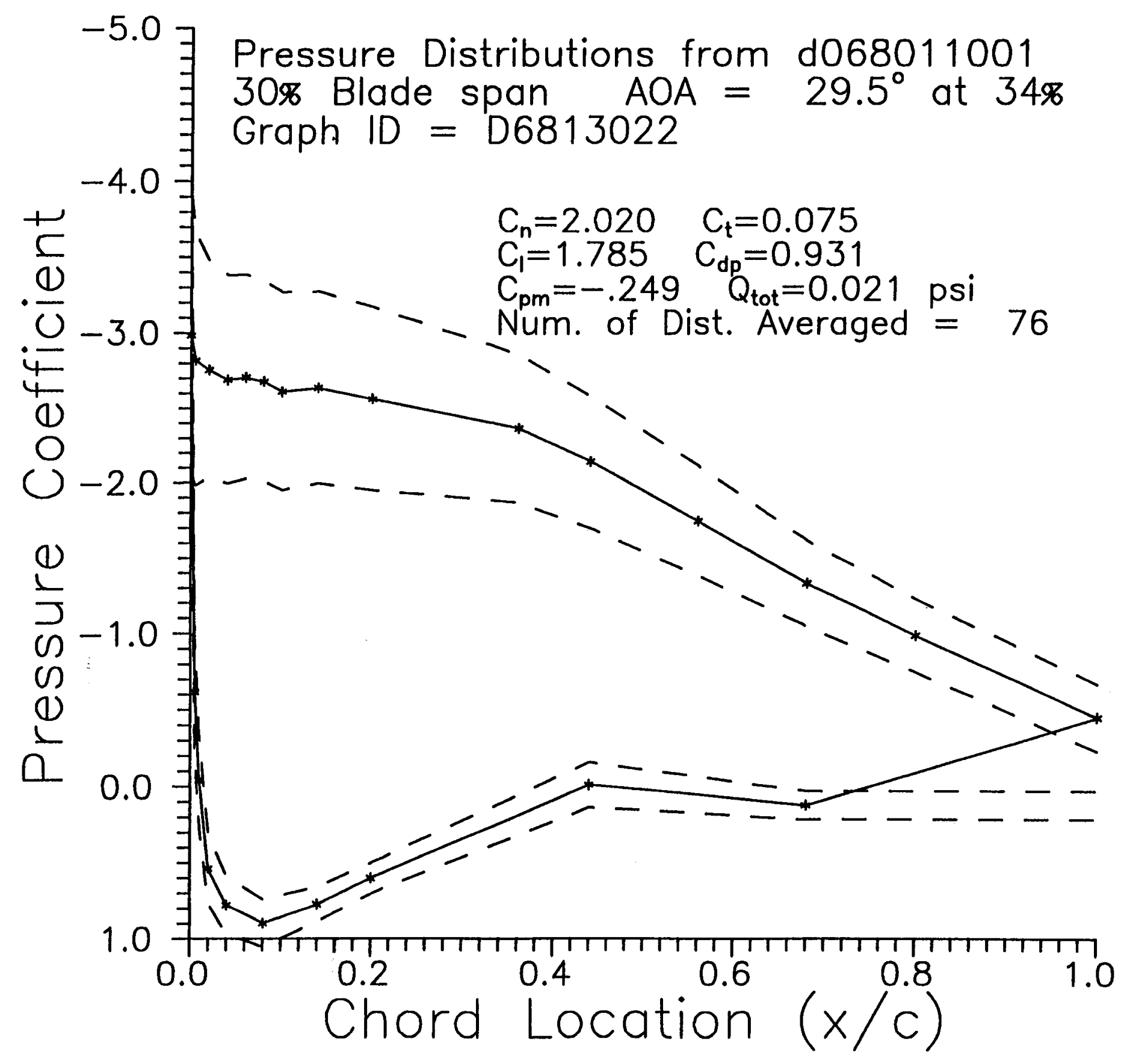




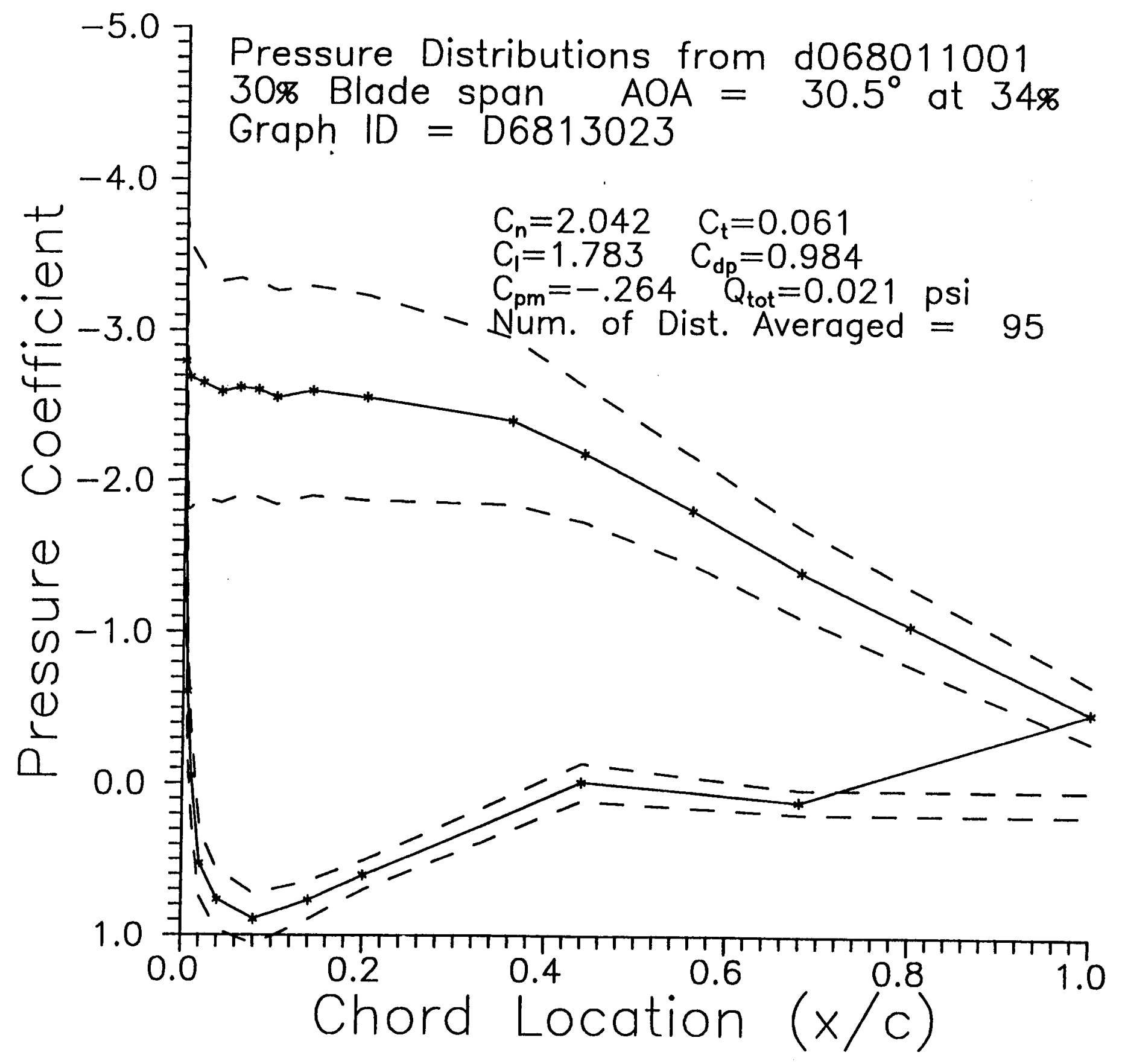




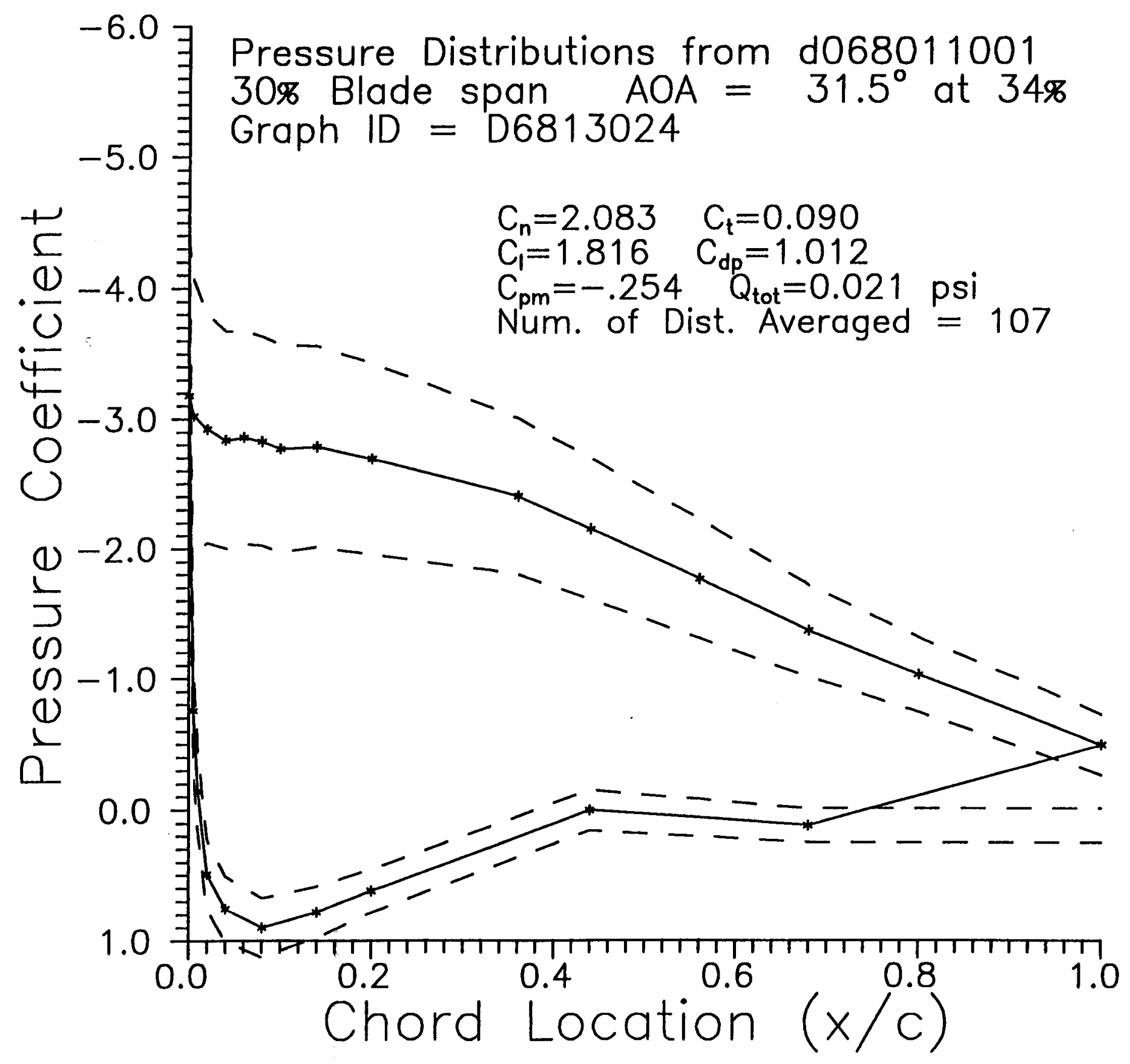




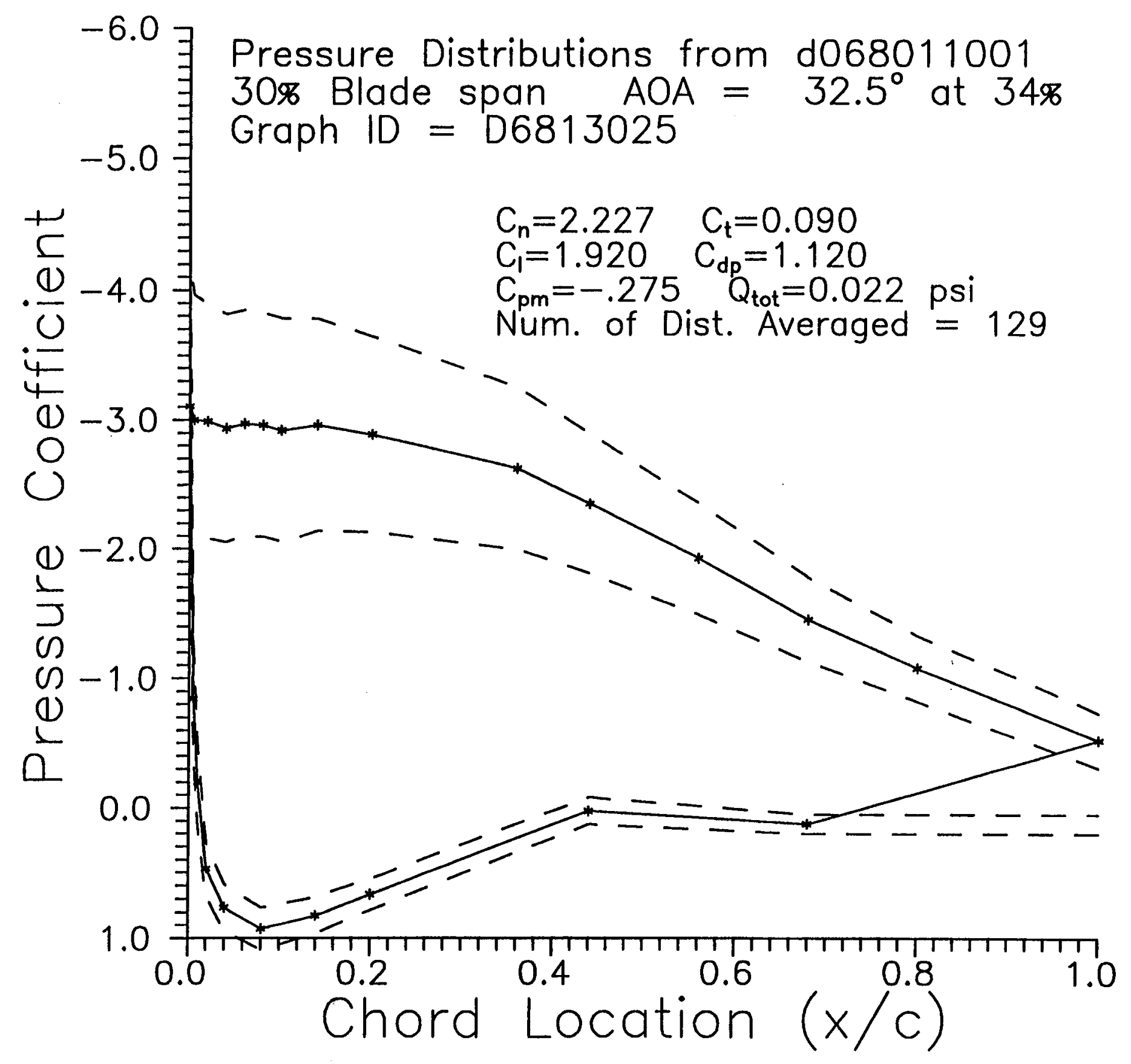




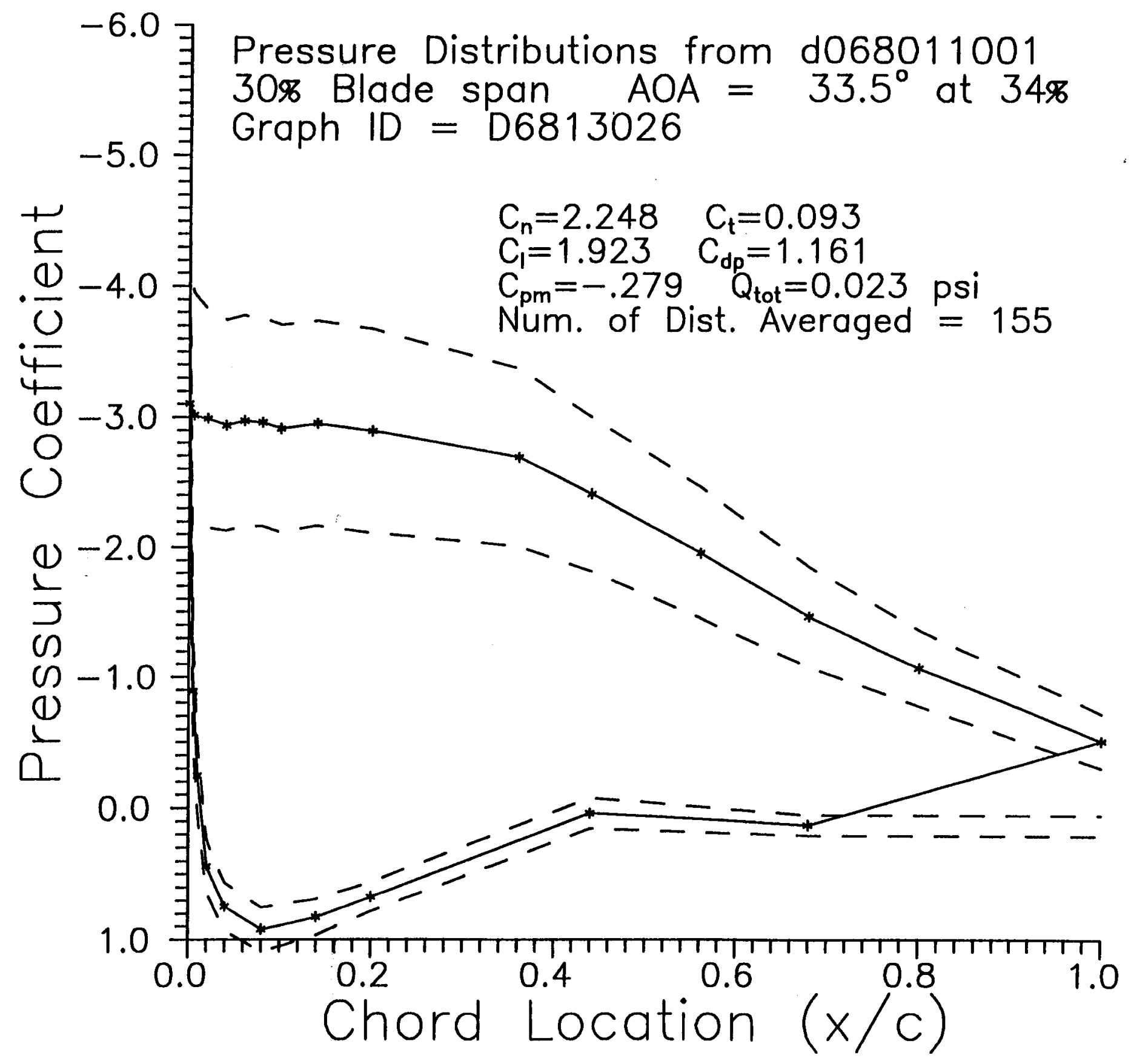




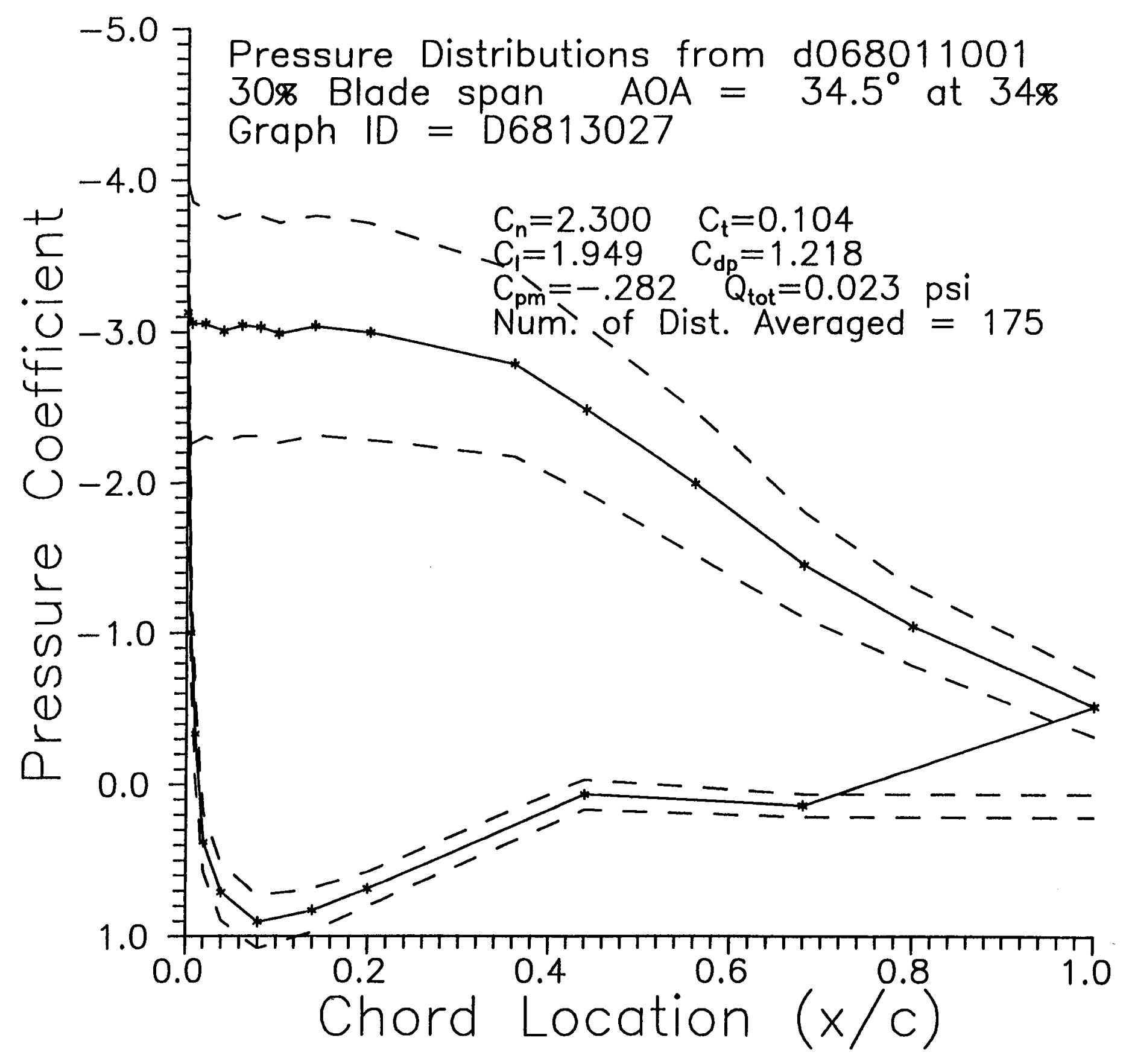




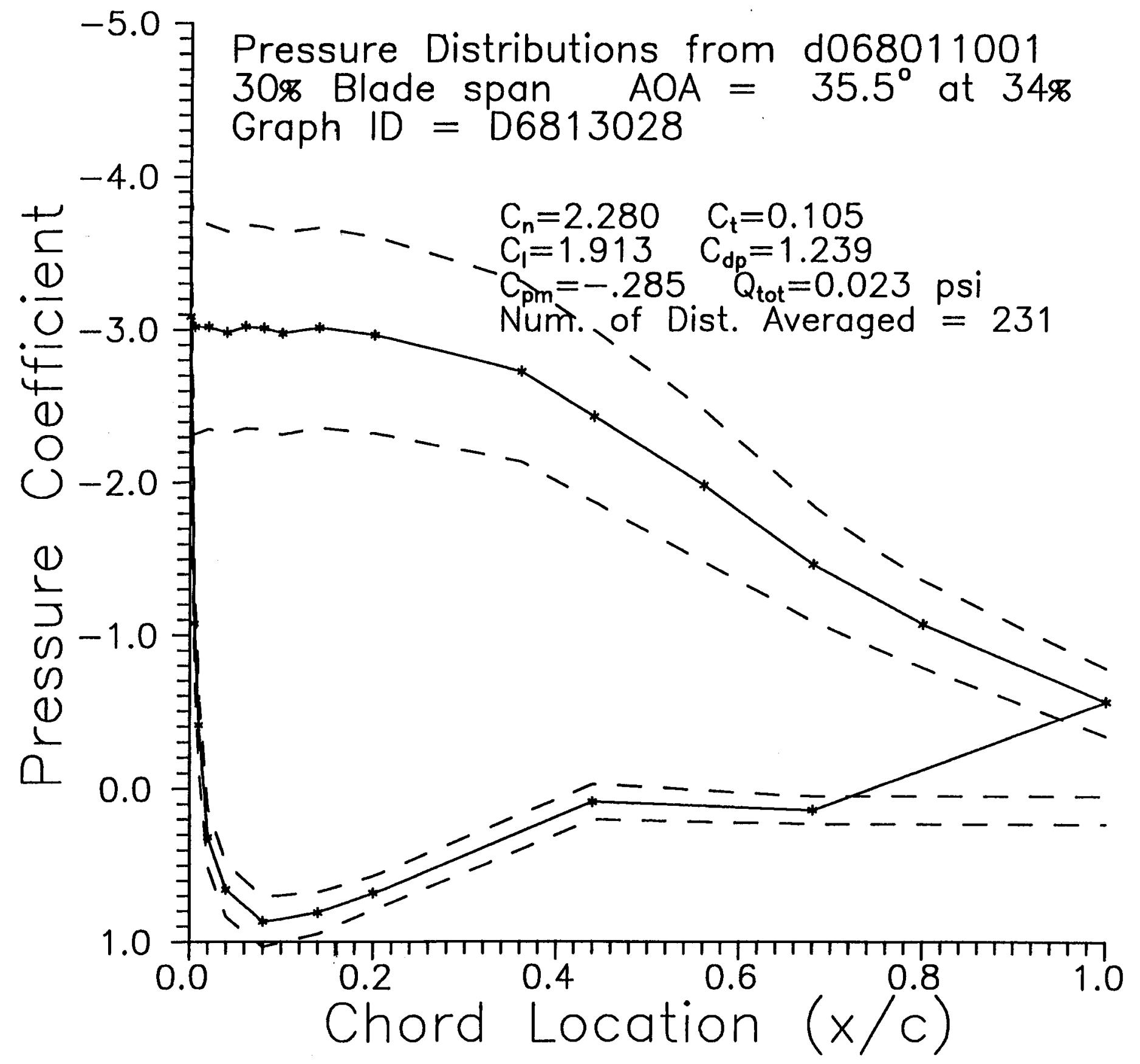




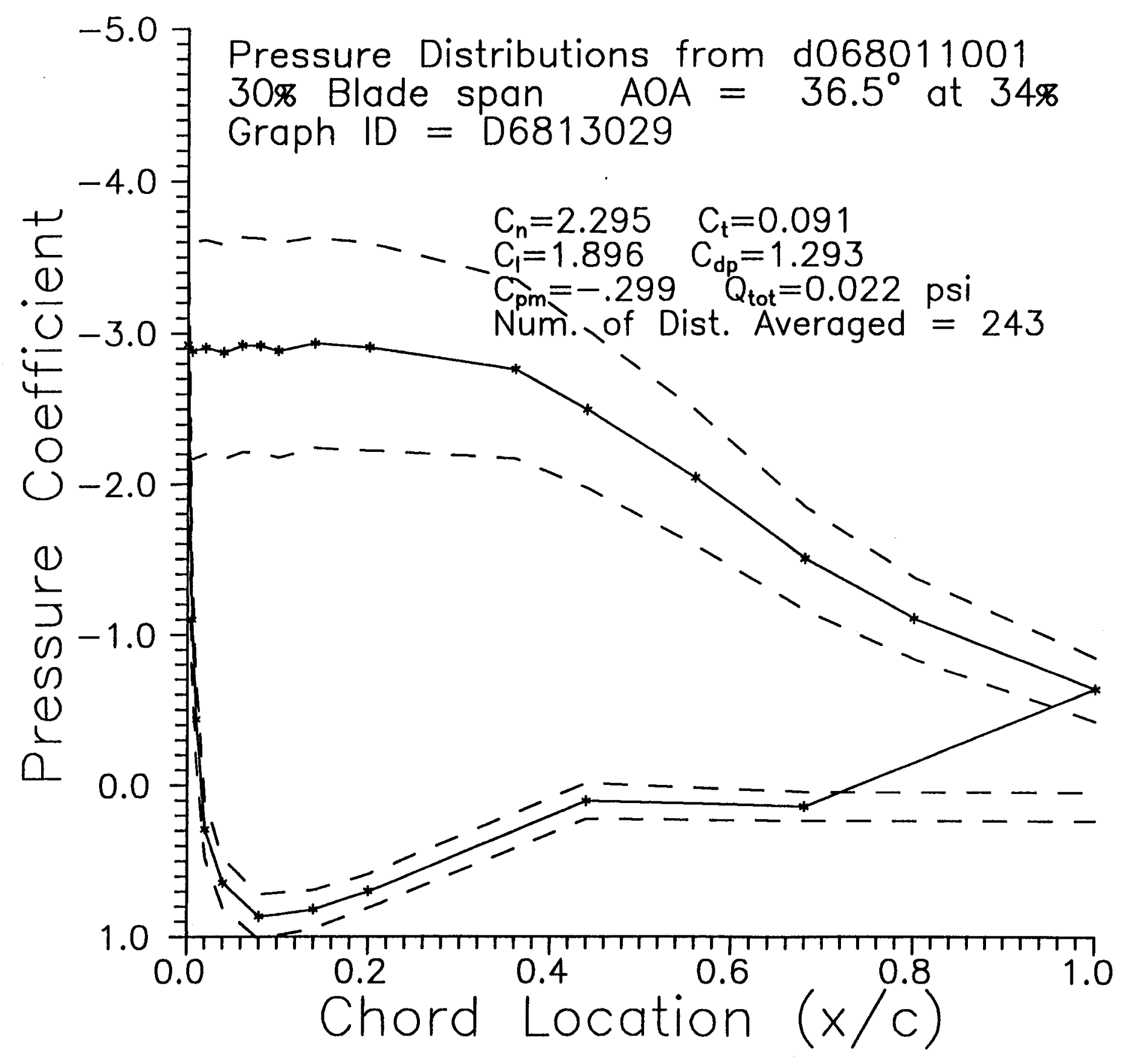




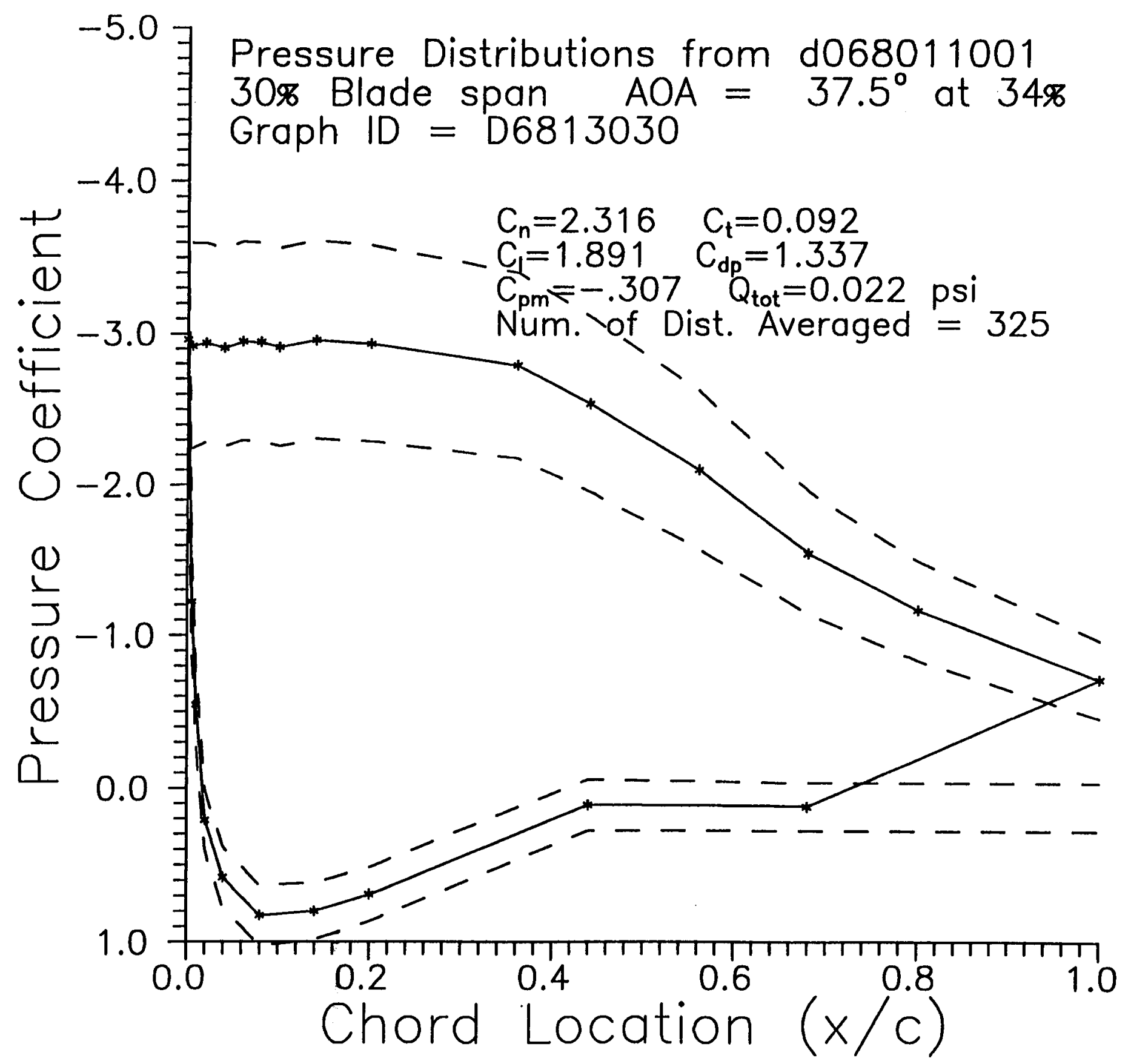




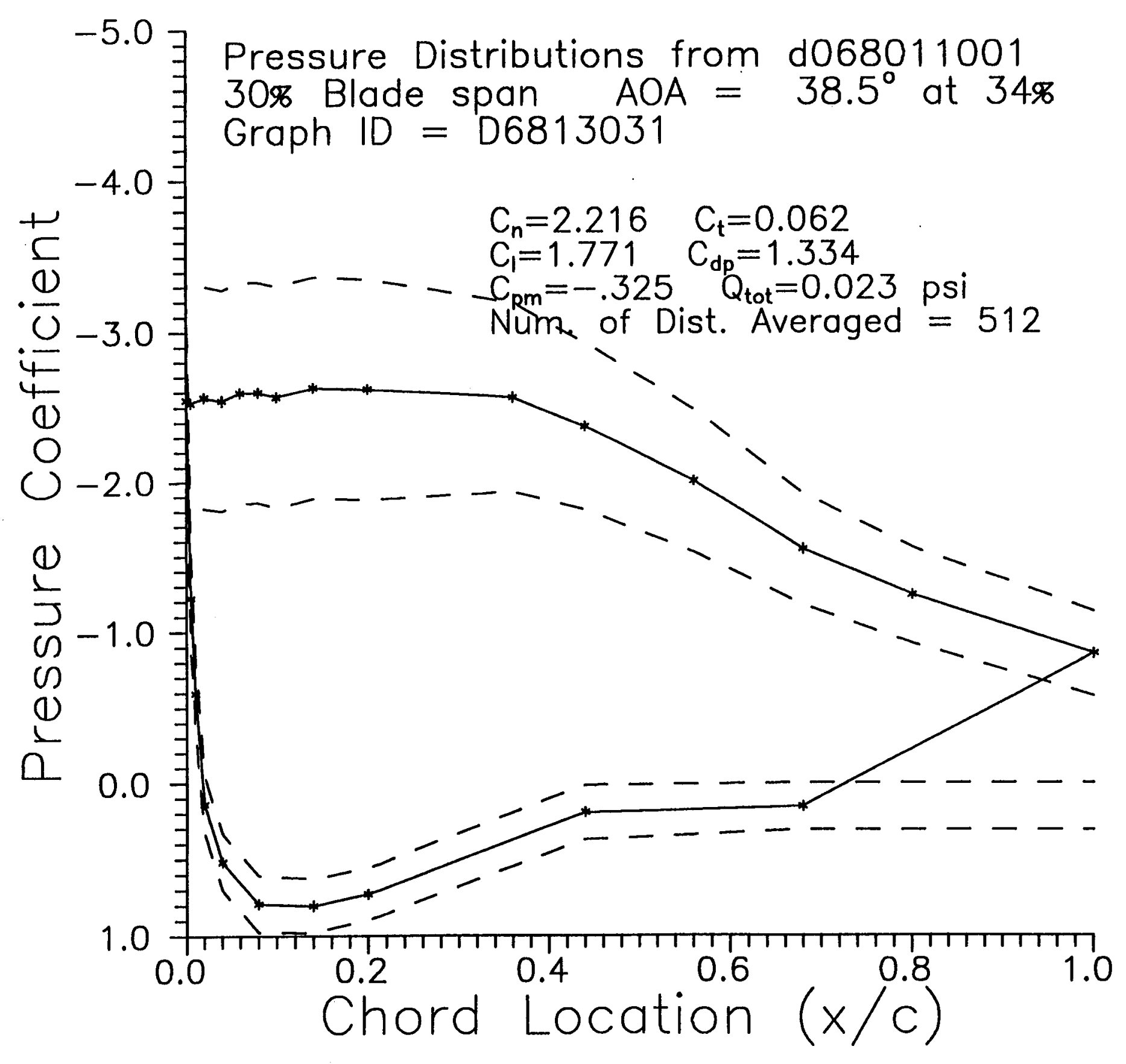




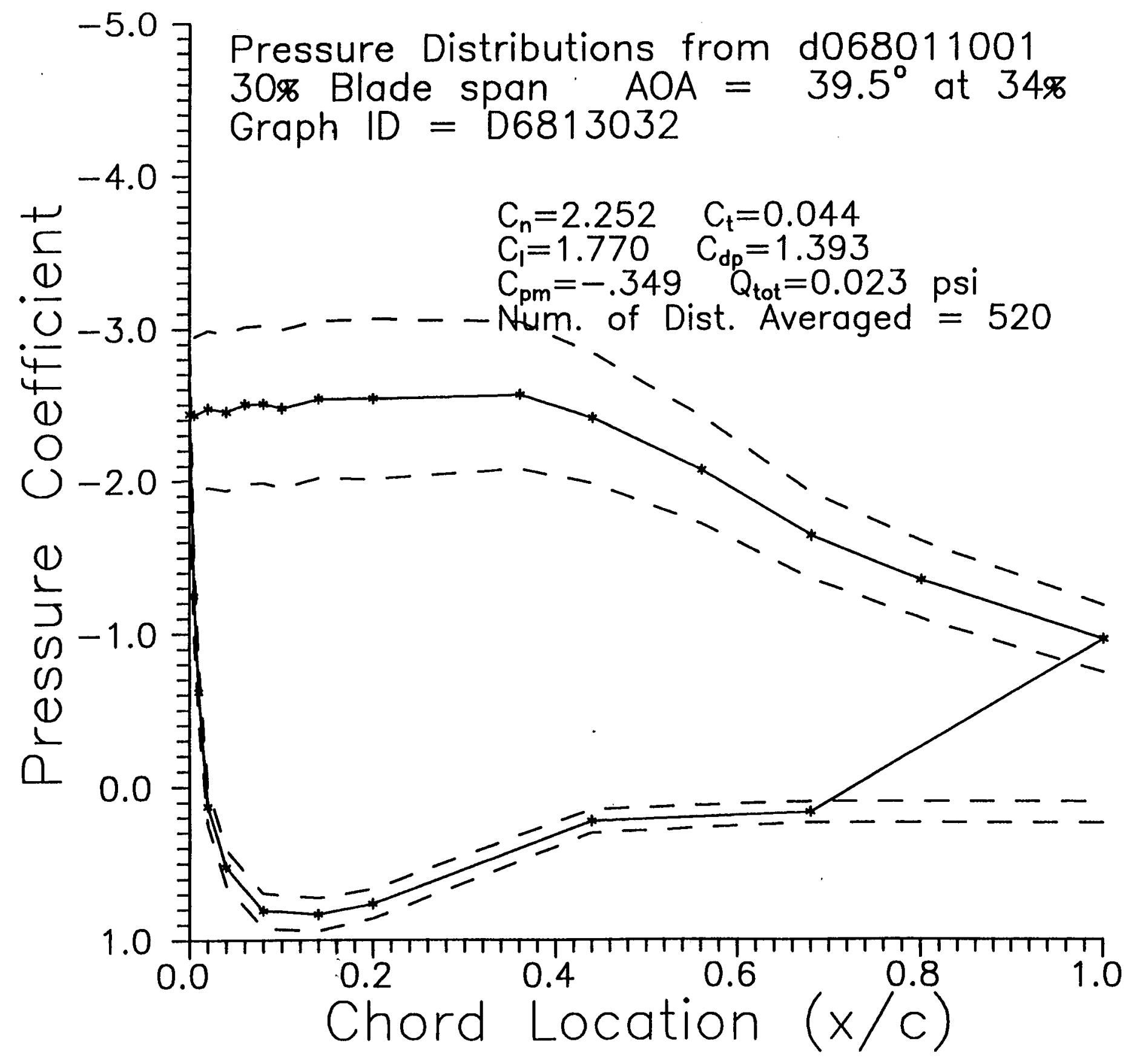


Appendix D
Pressure Distributions Based on Measured Wind Speed 


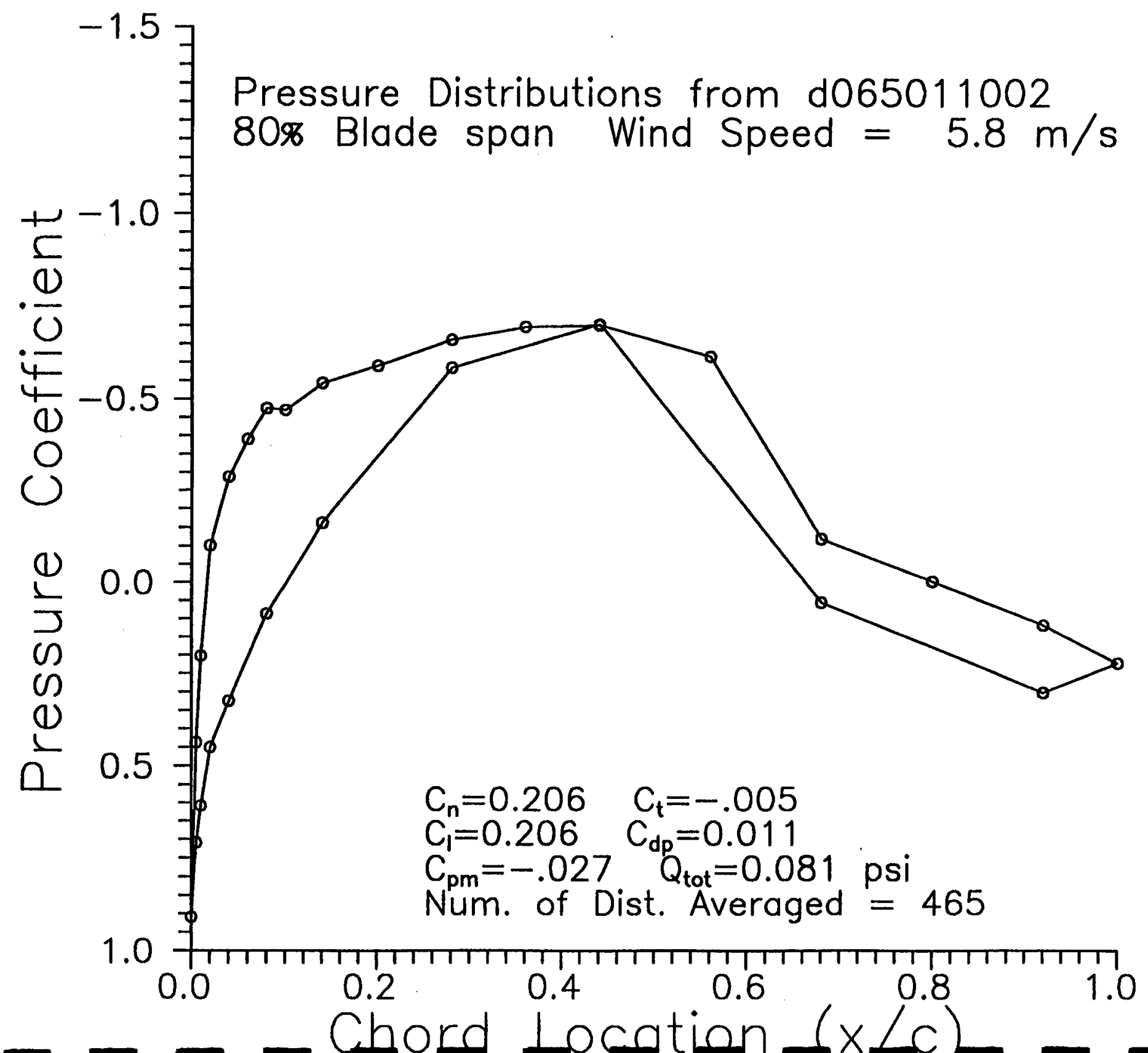




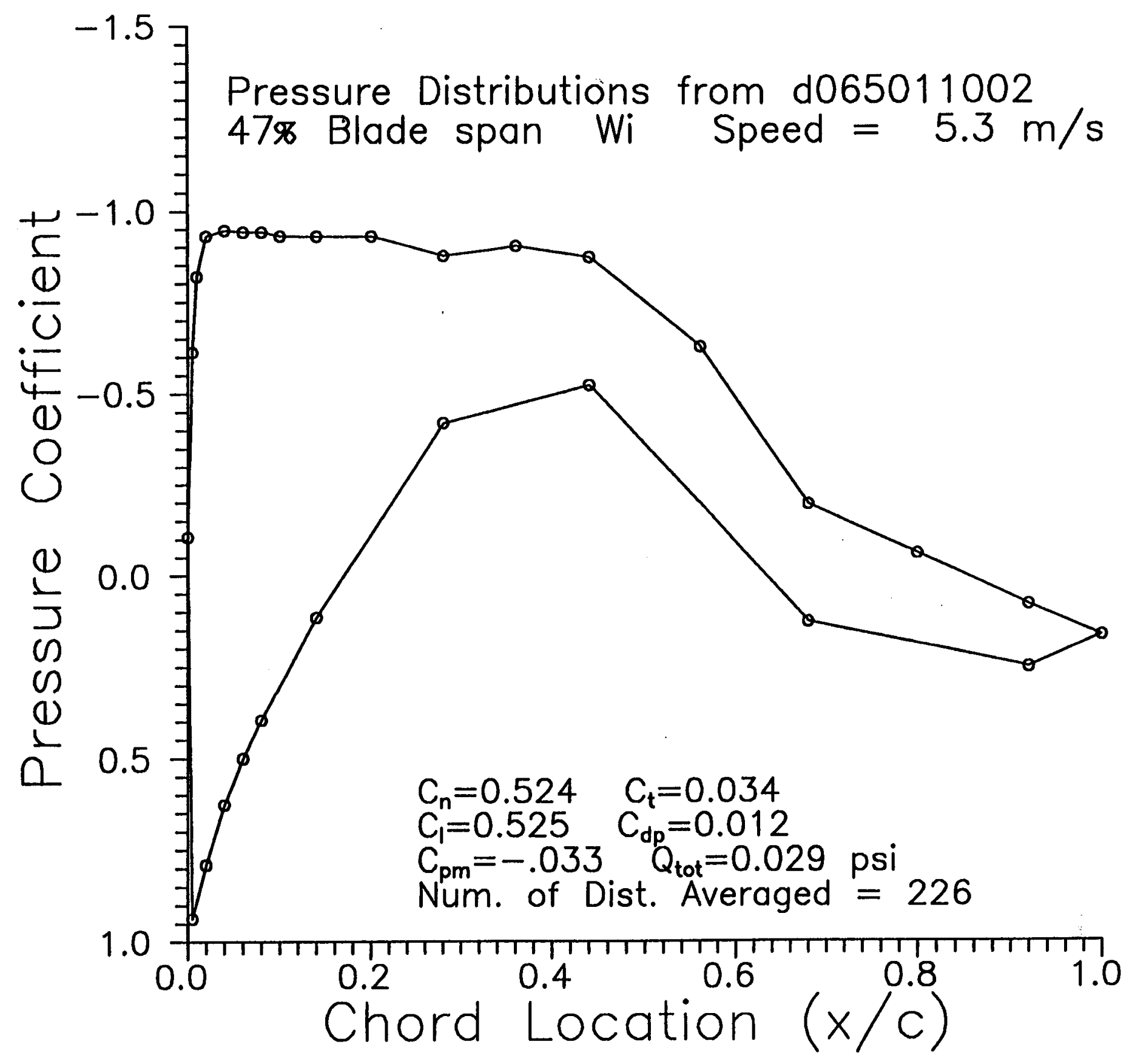




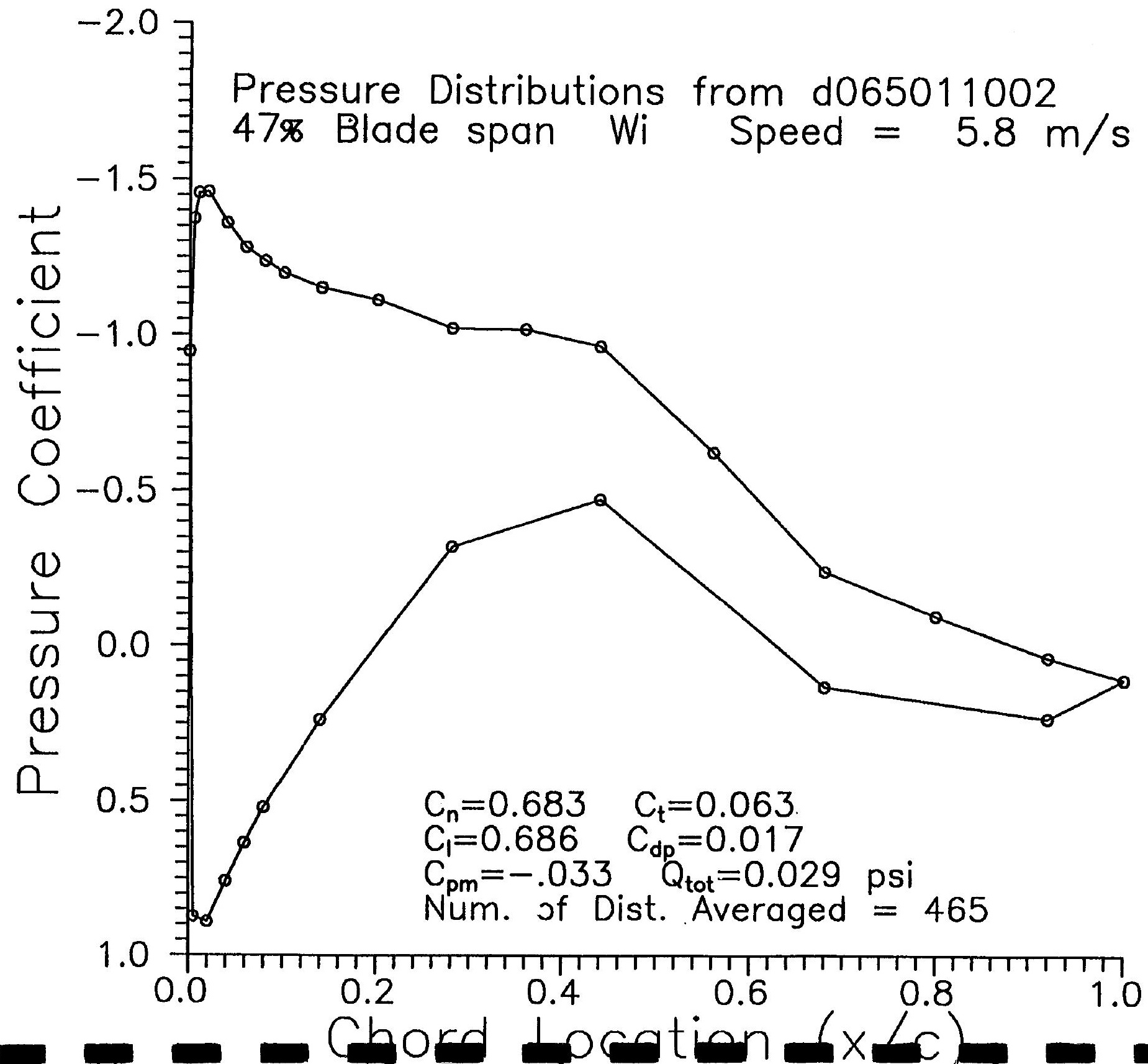




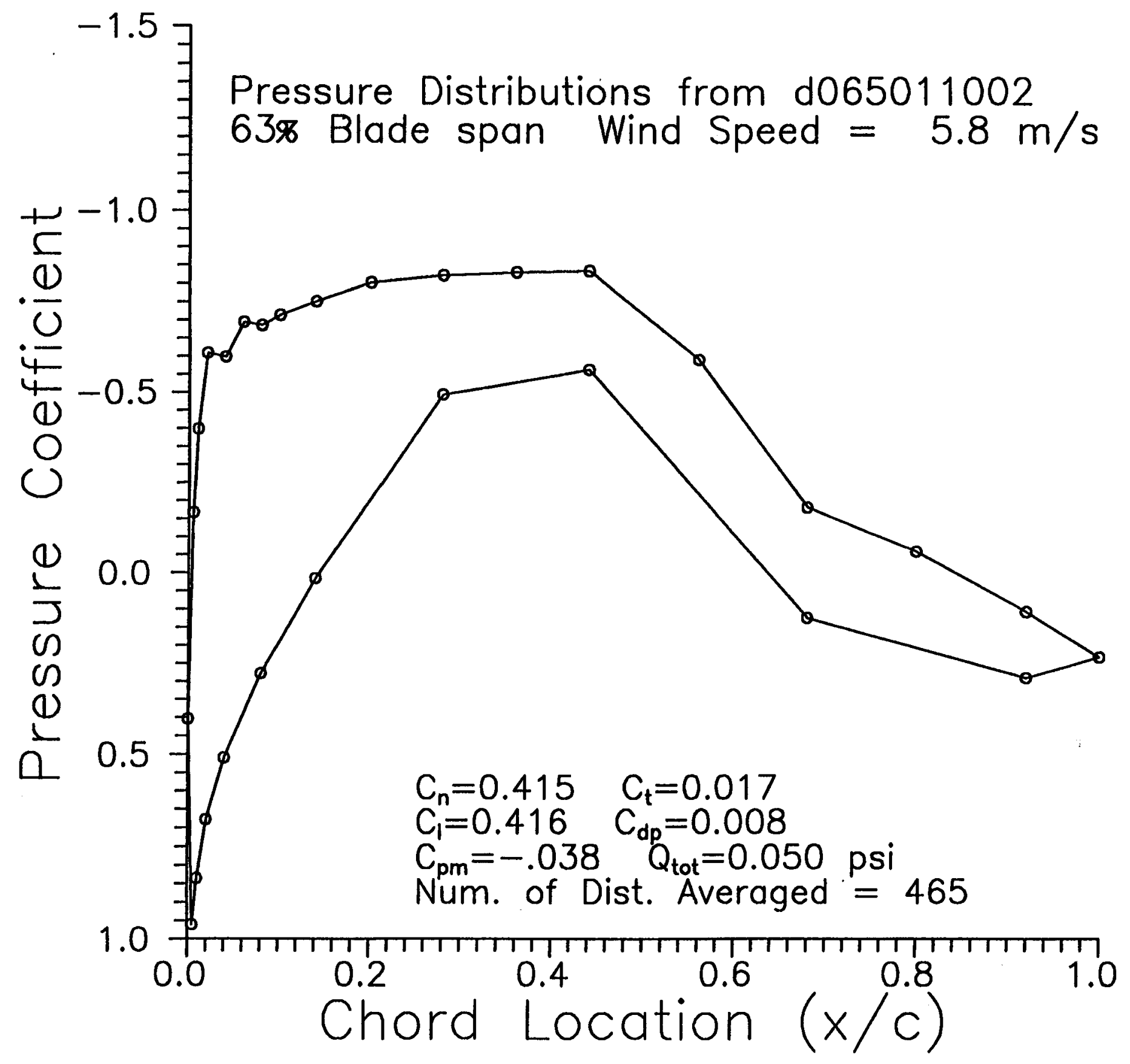




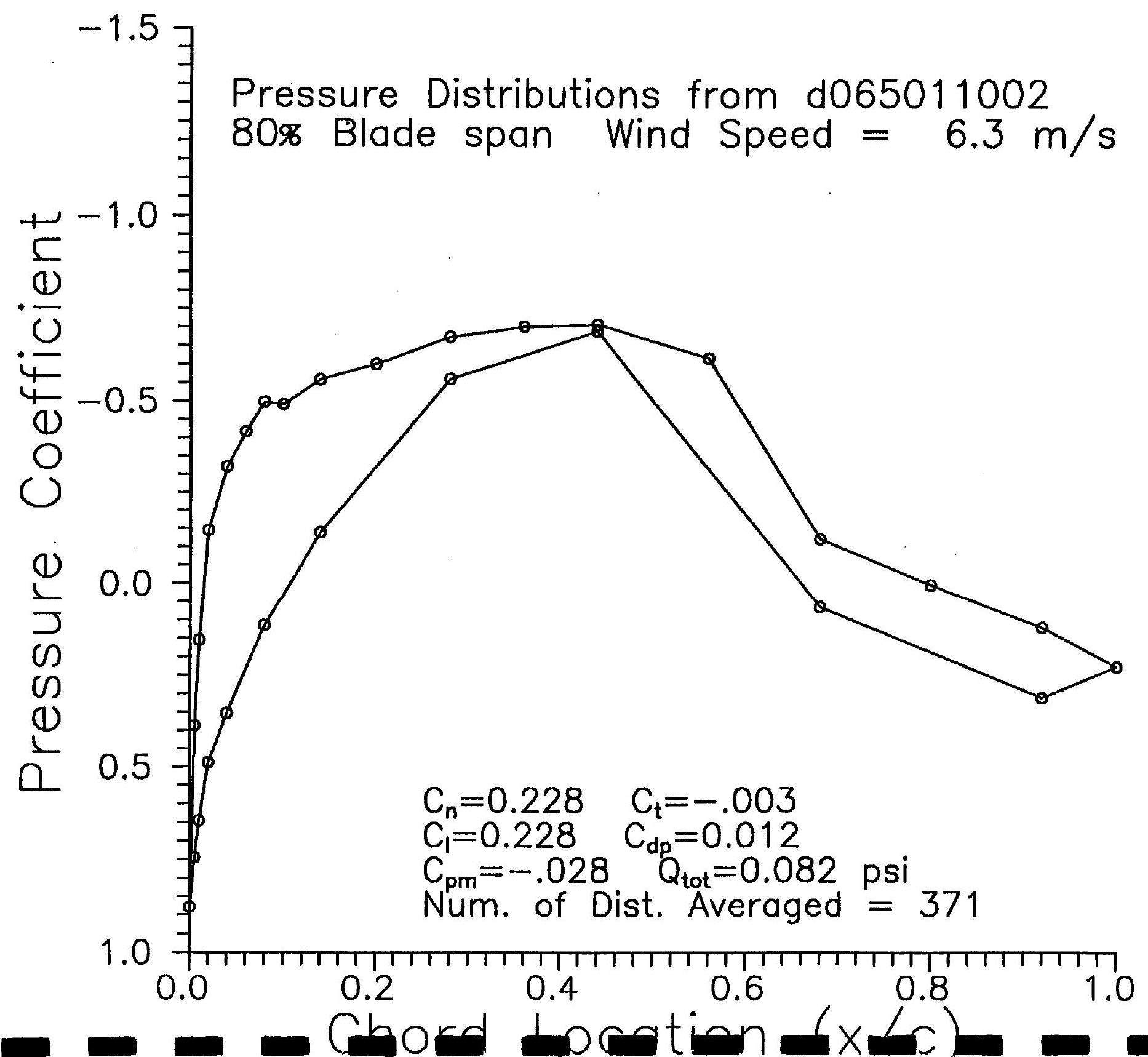




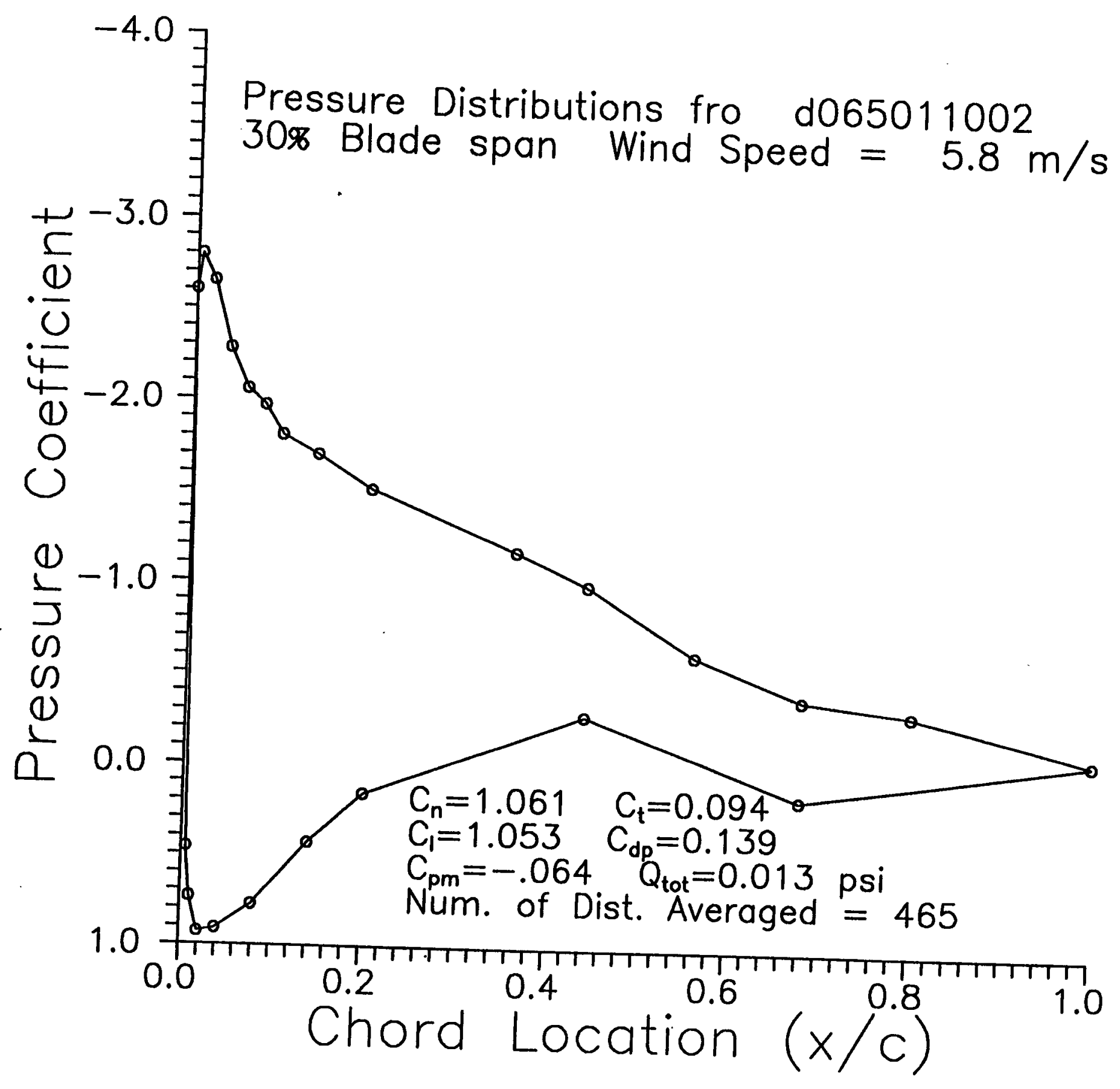




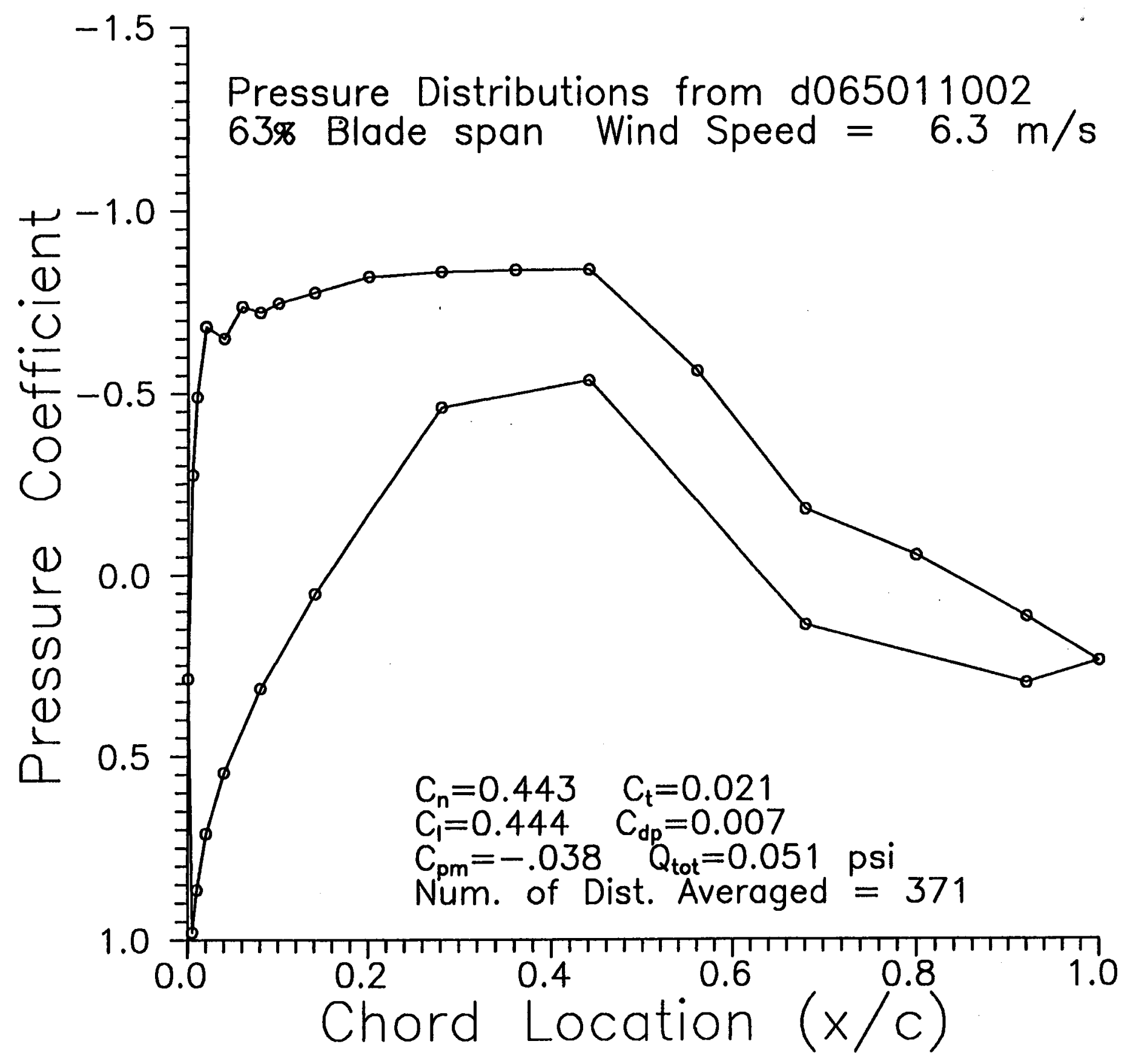




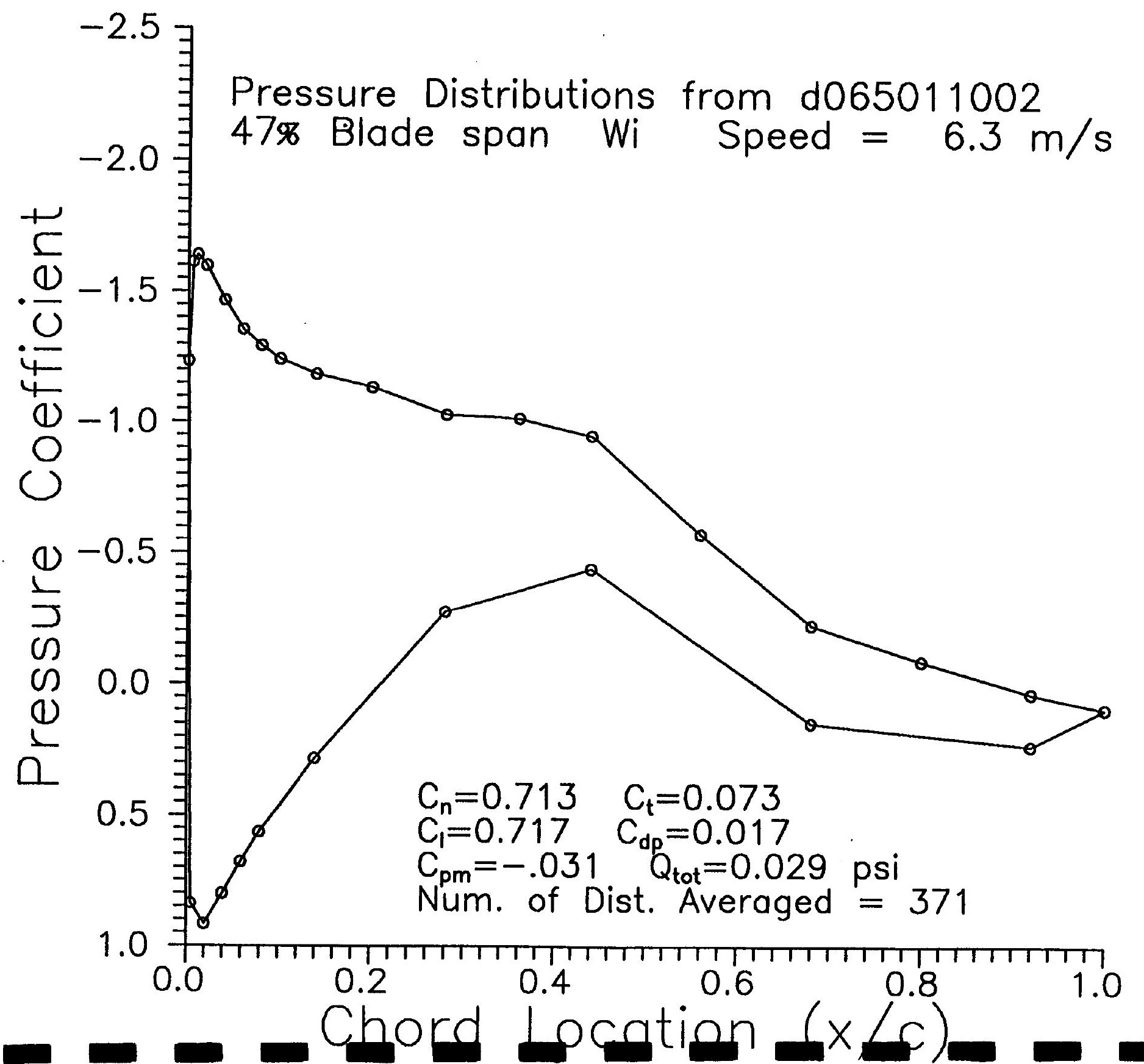




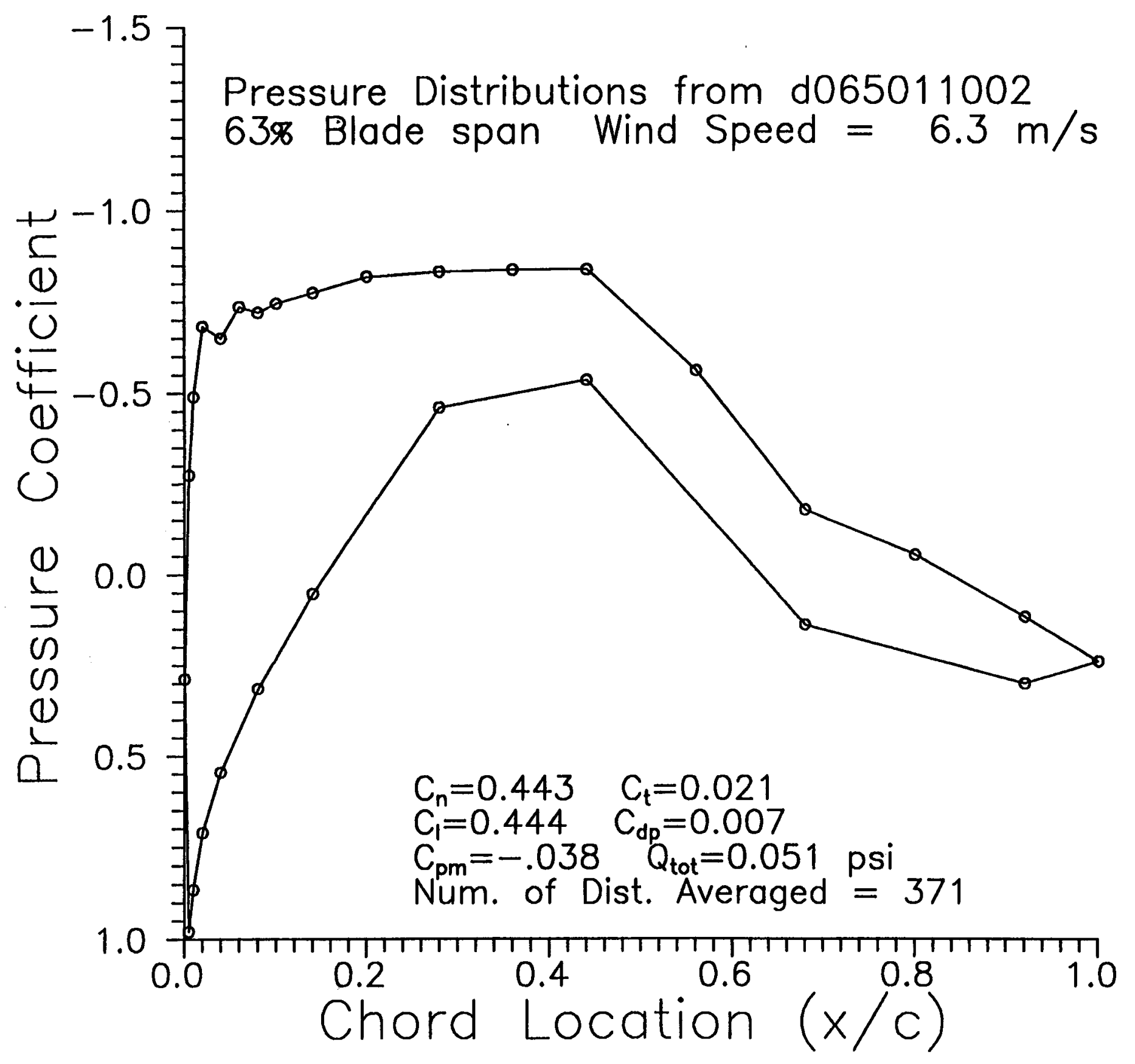




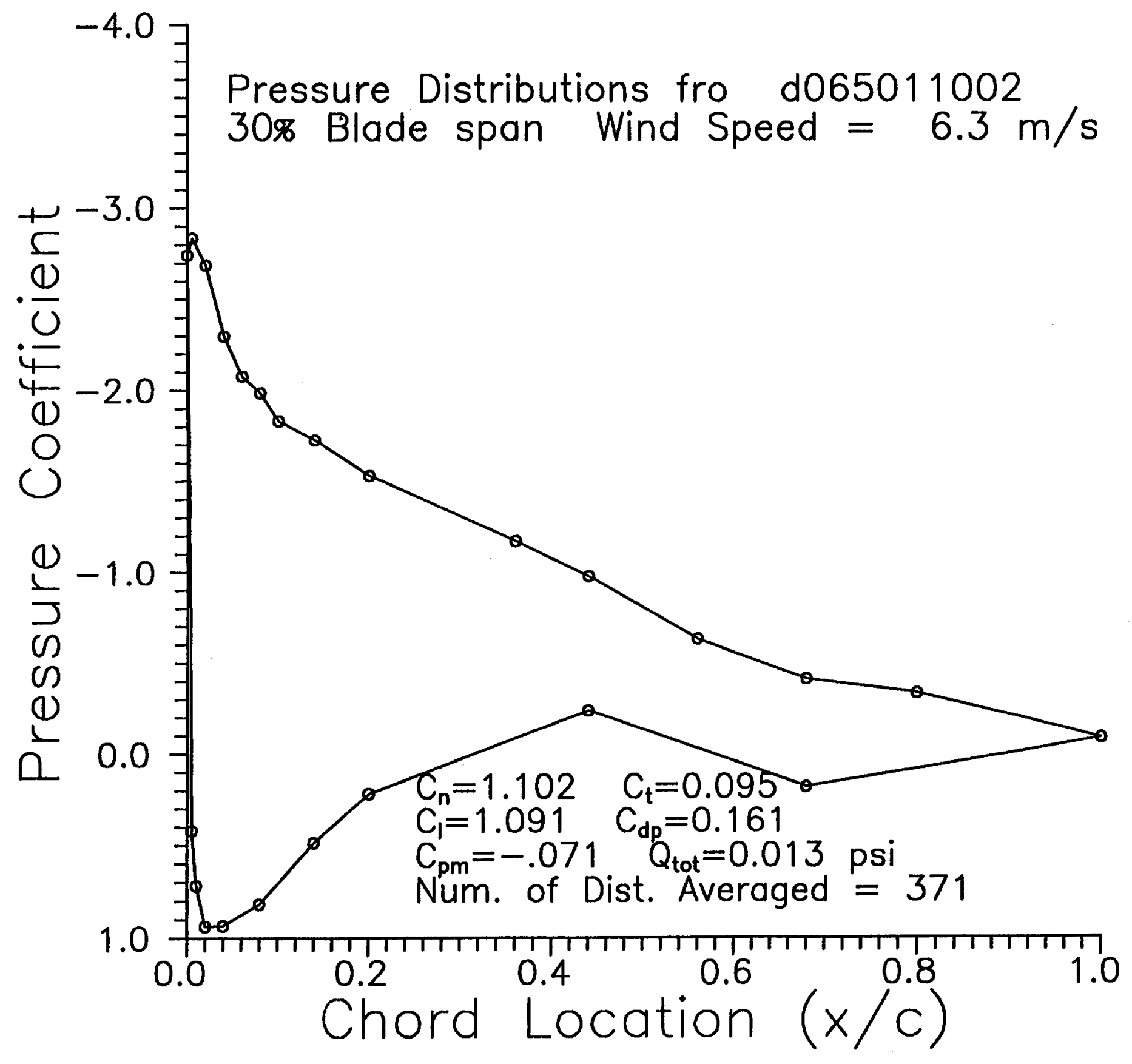




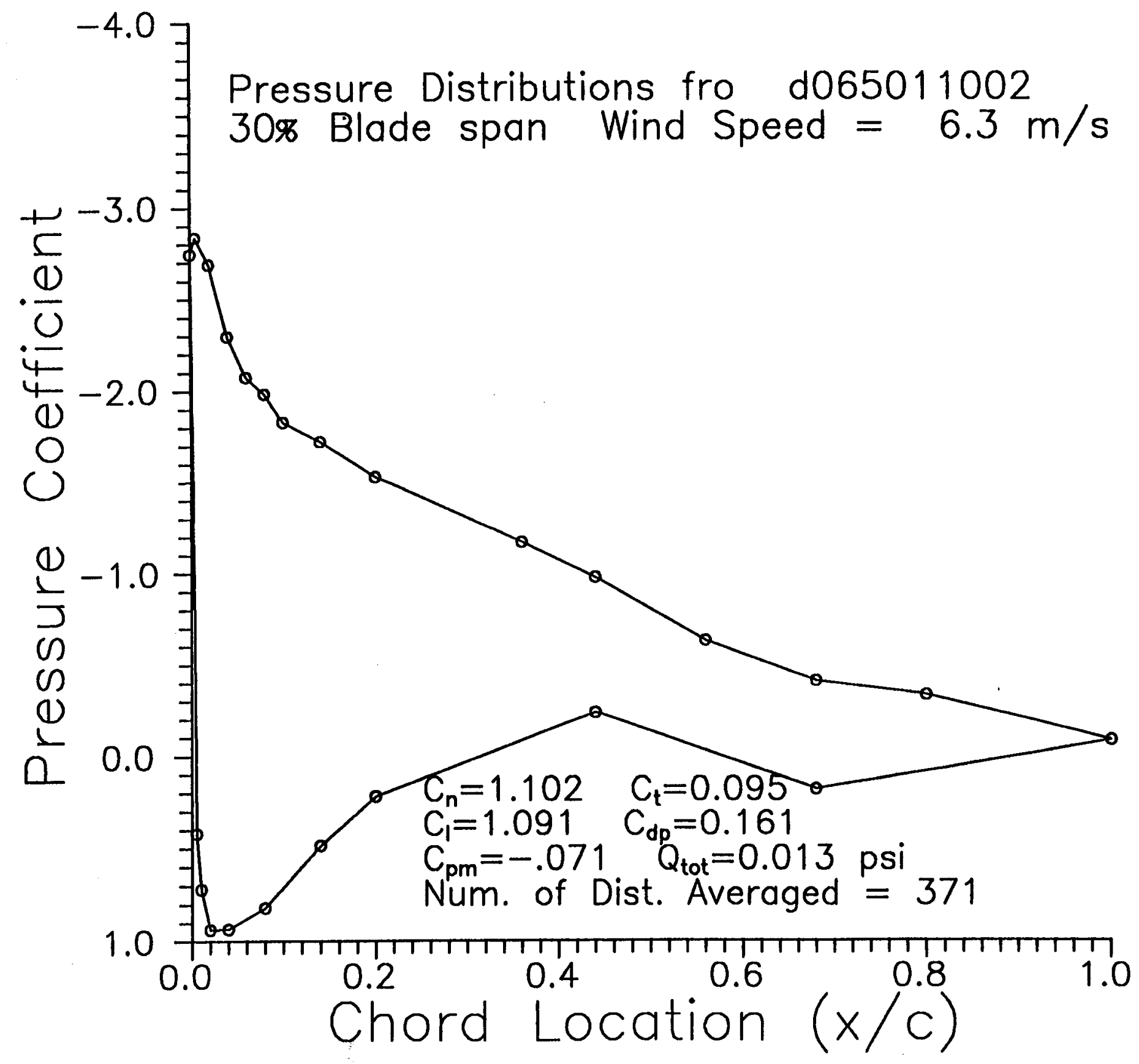




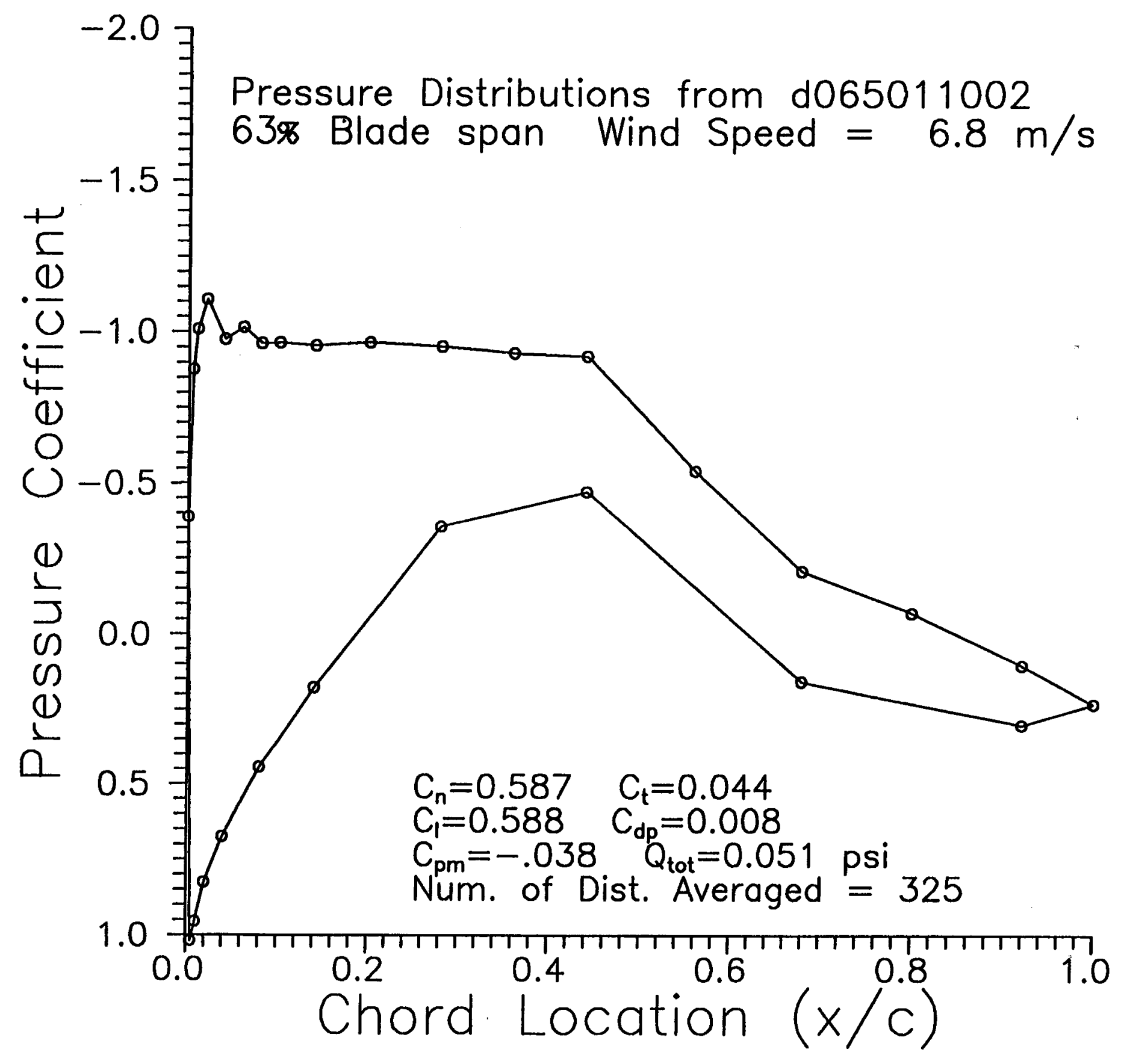




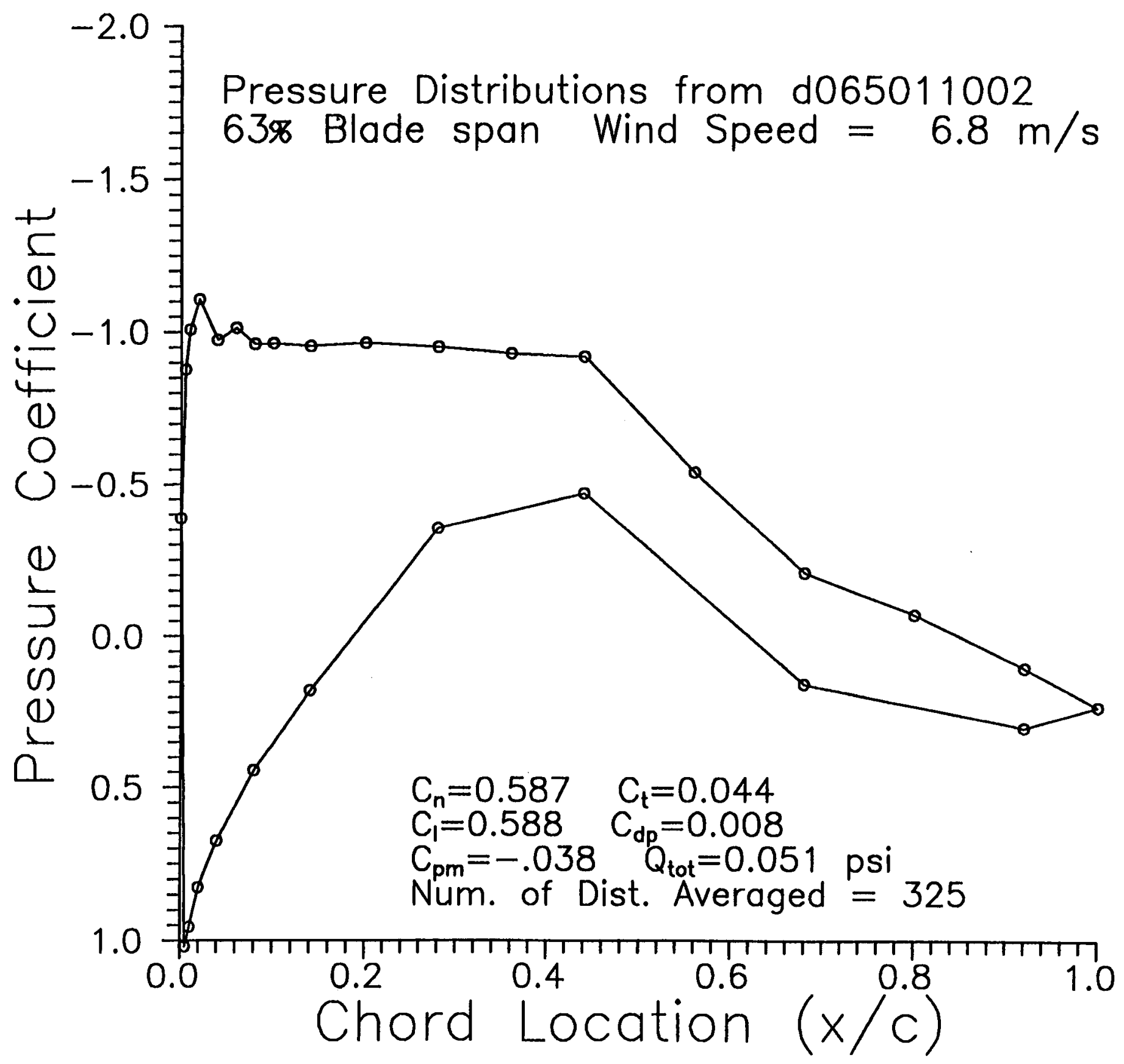




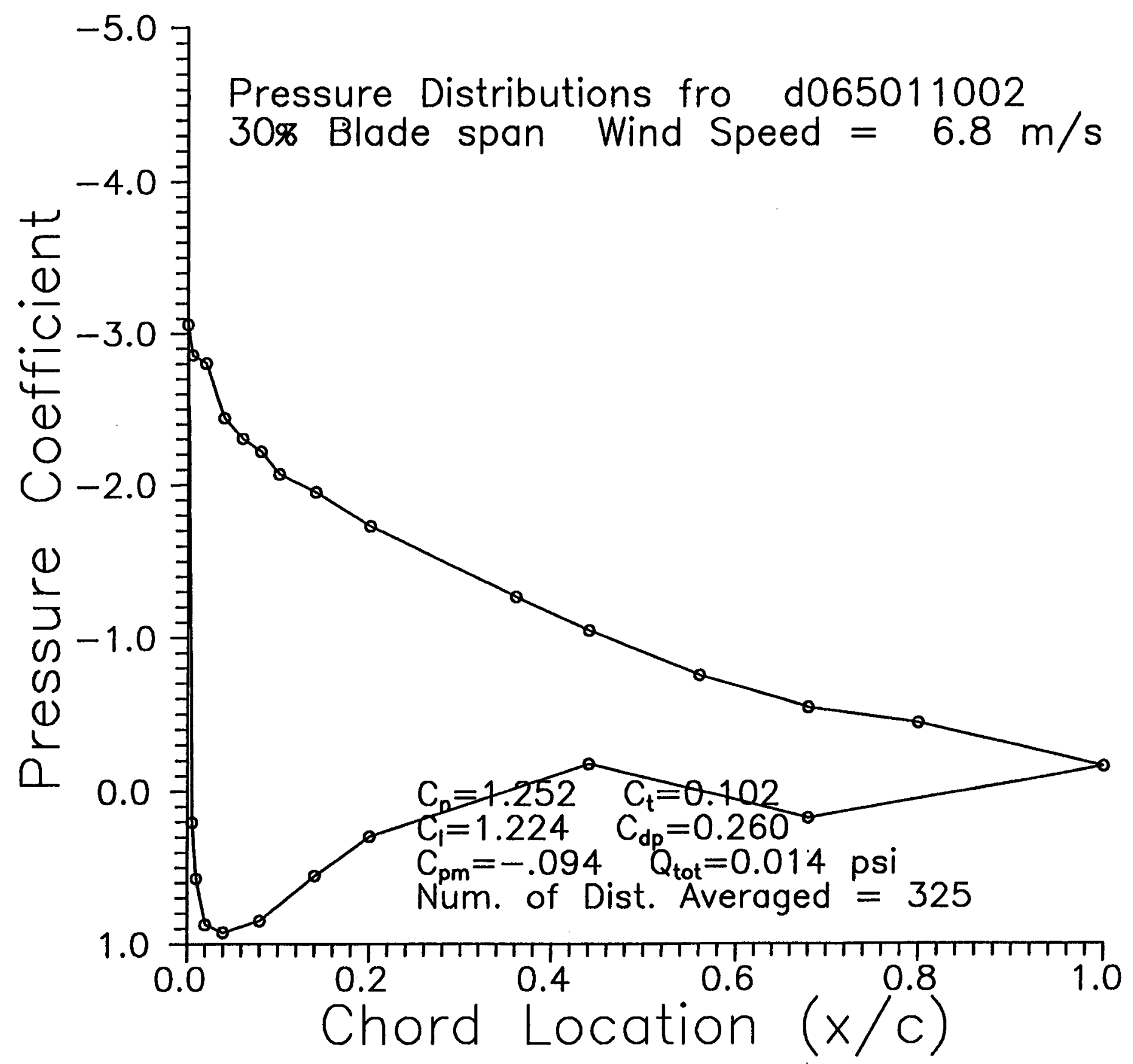




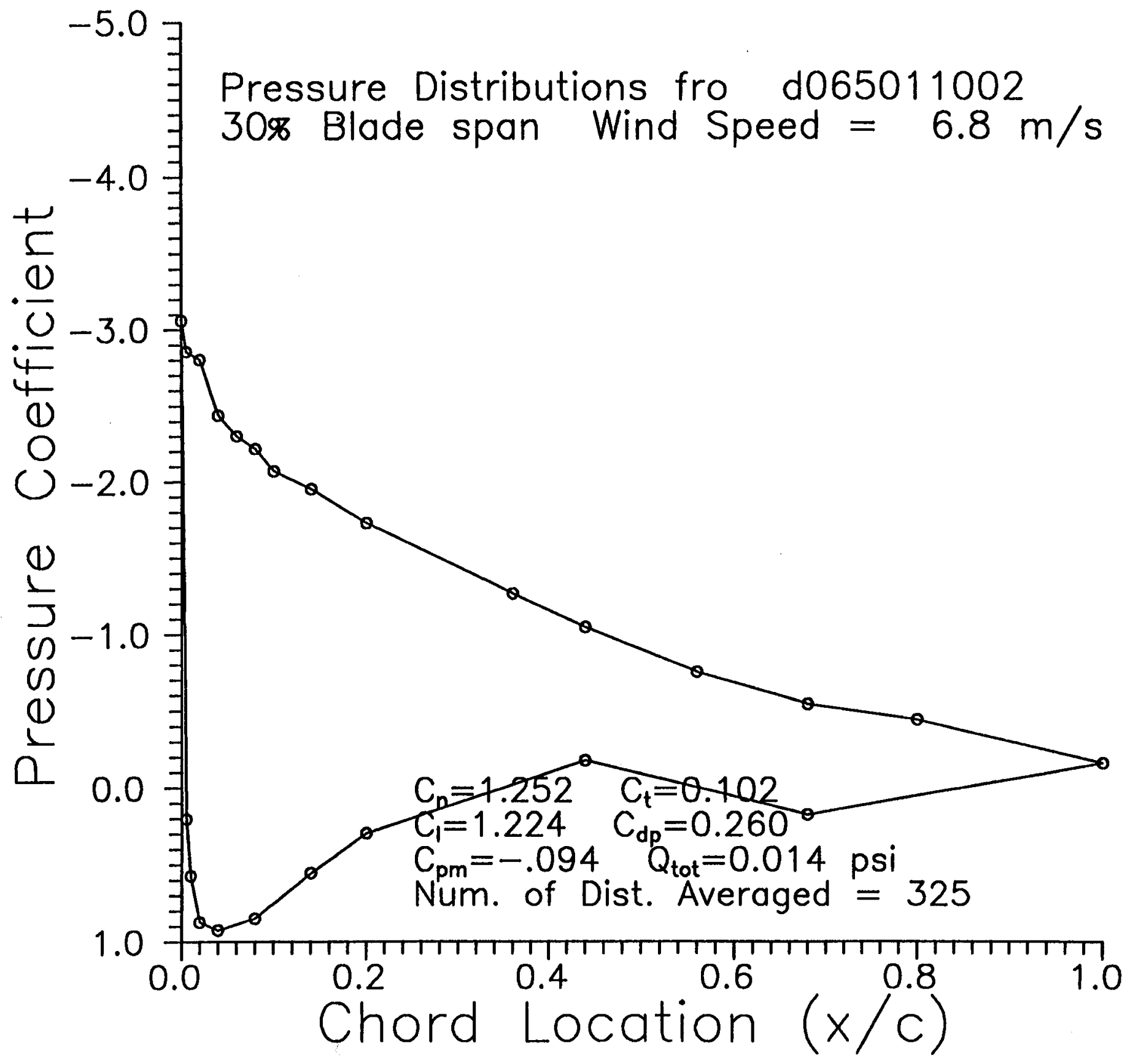




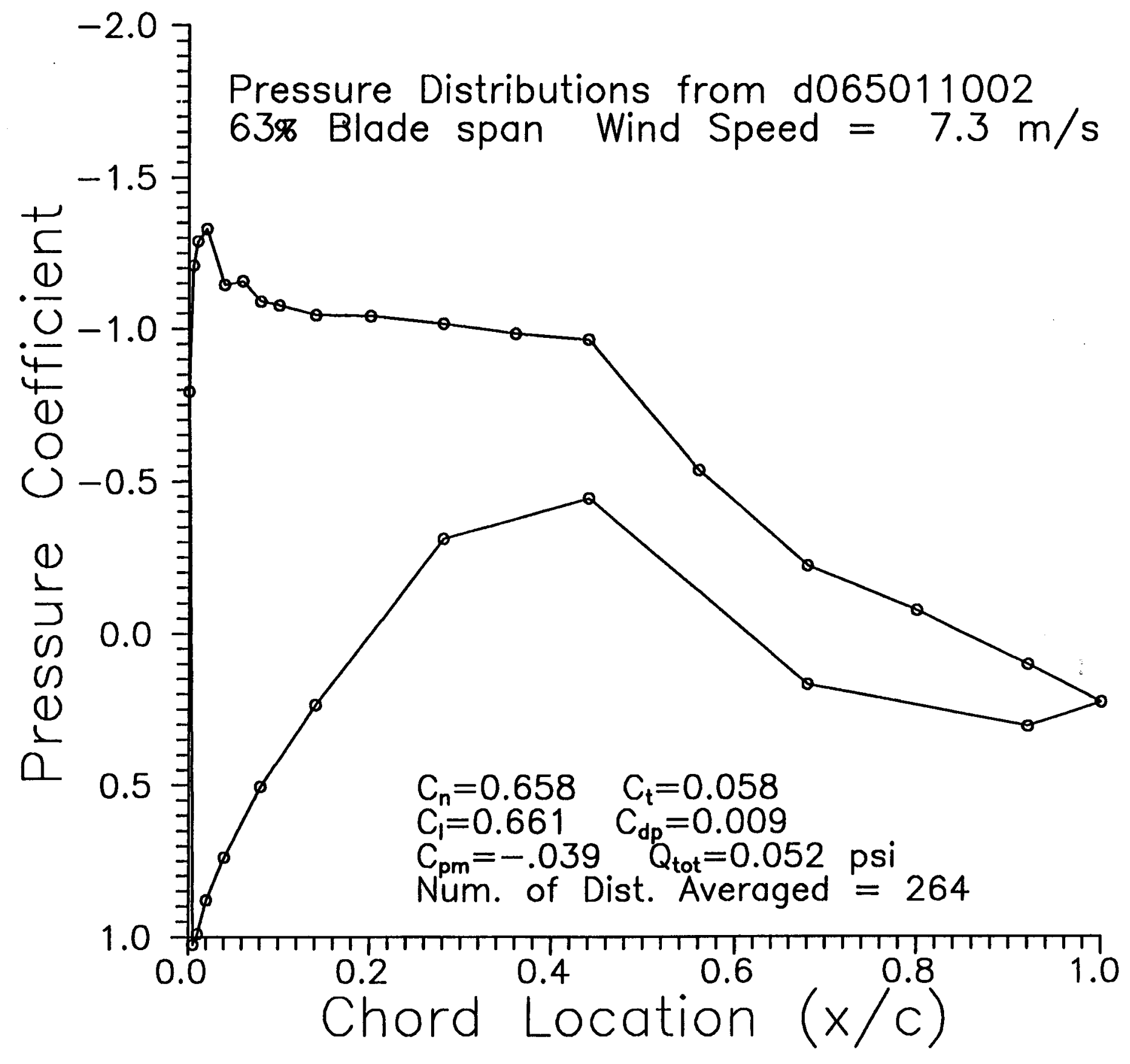




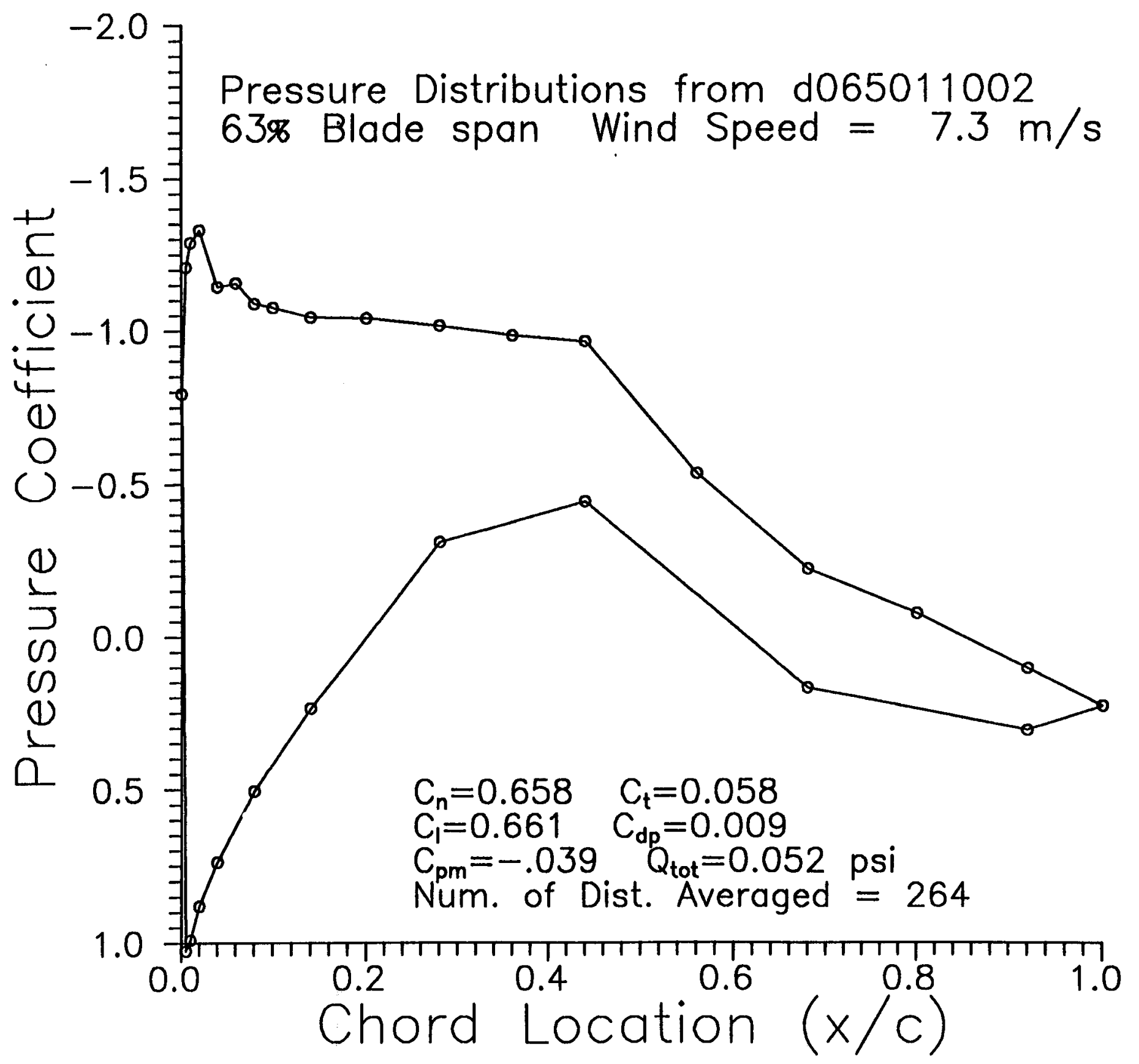




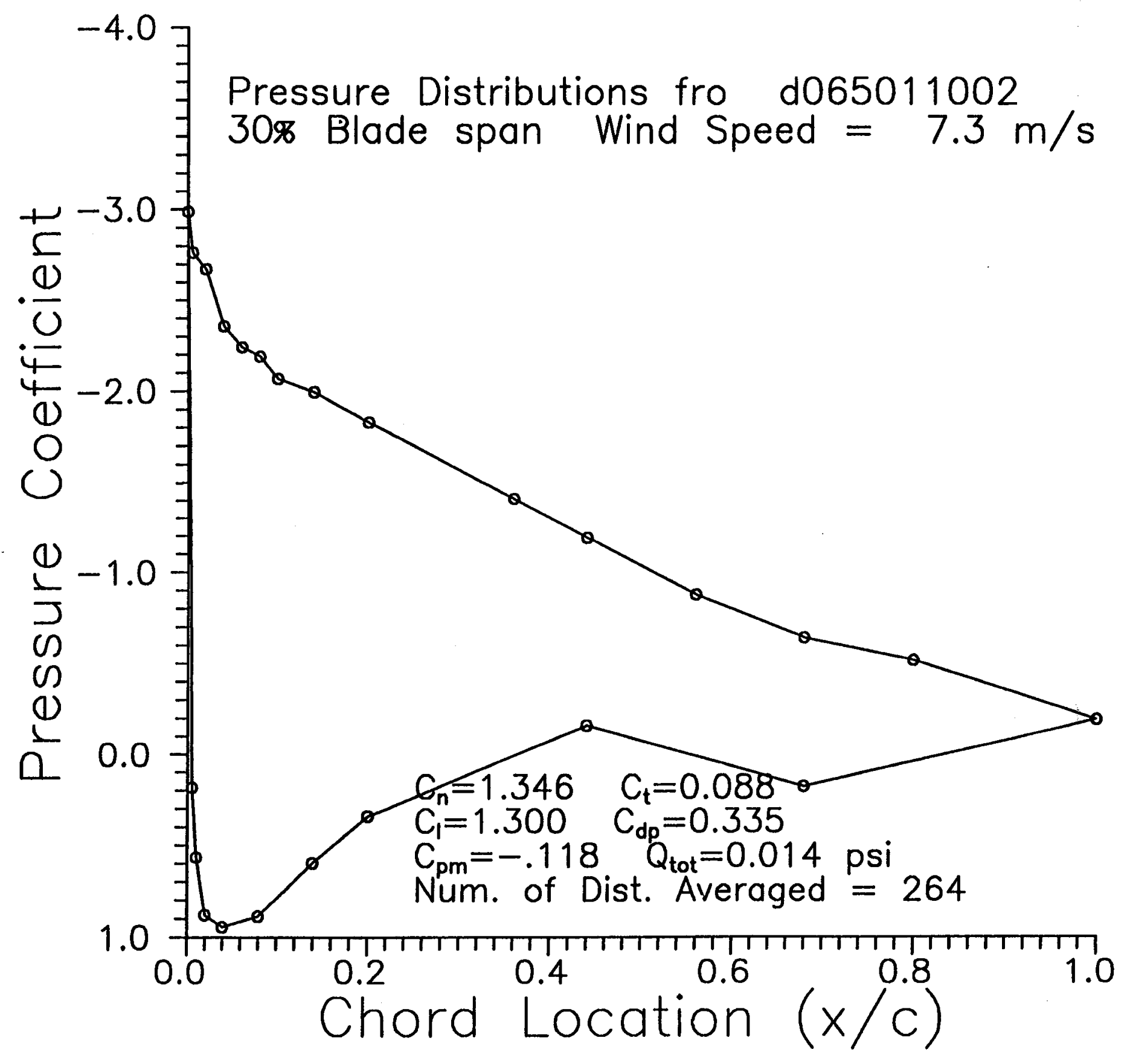




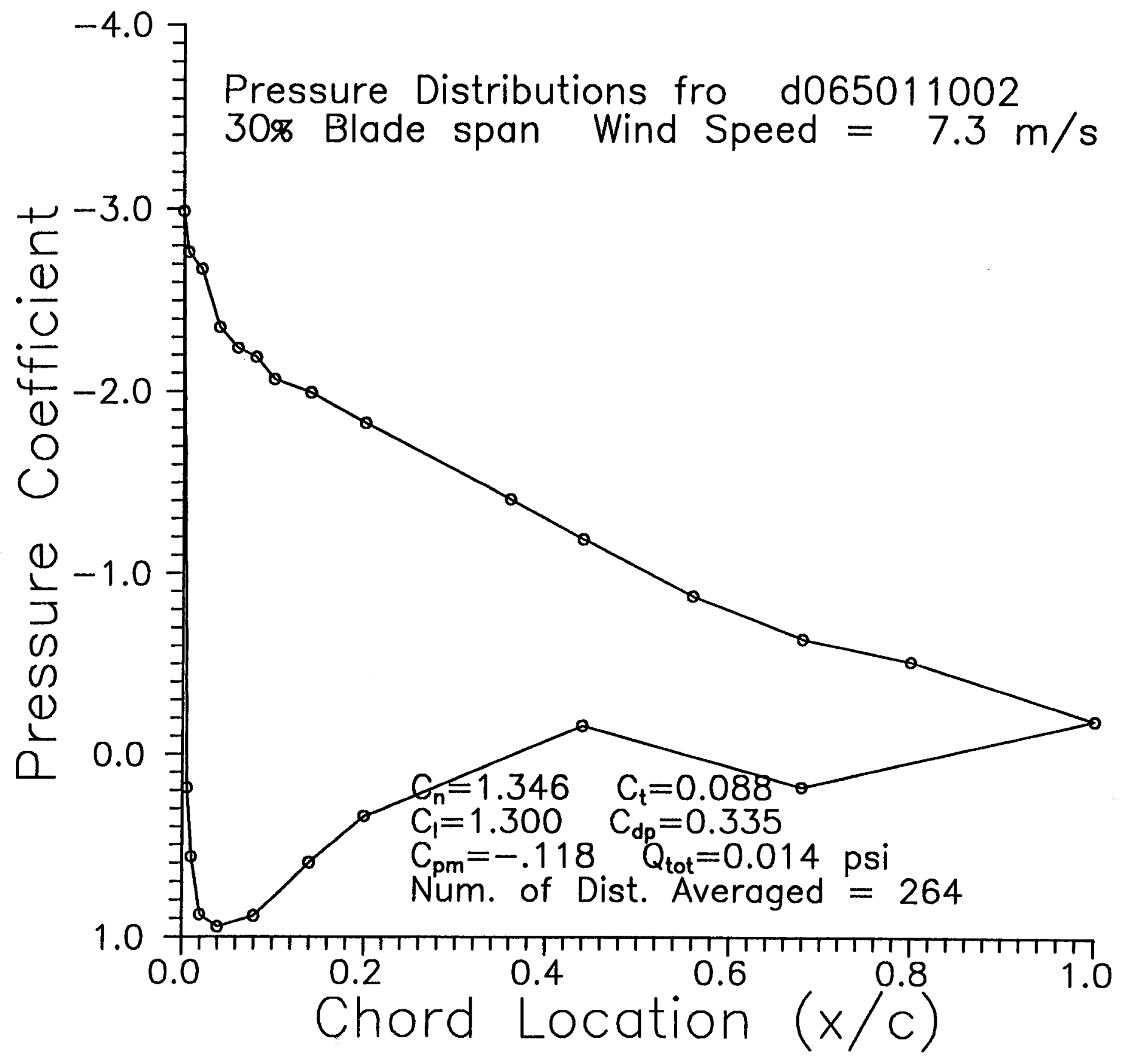




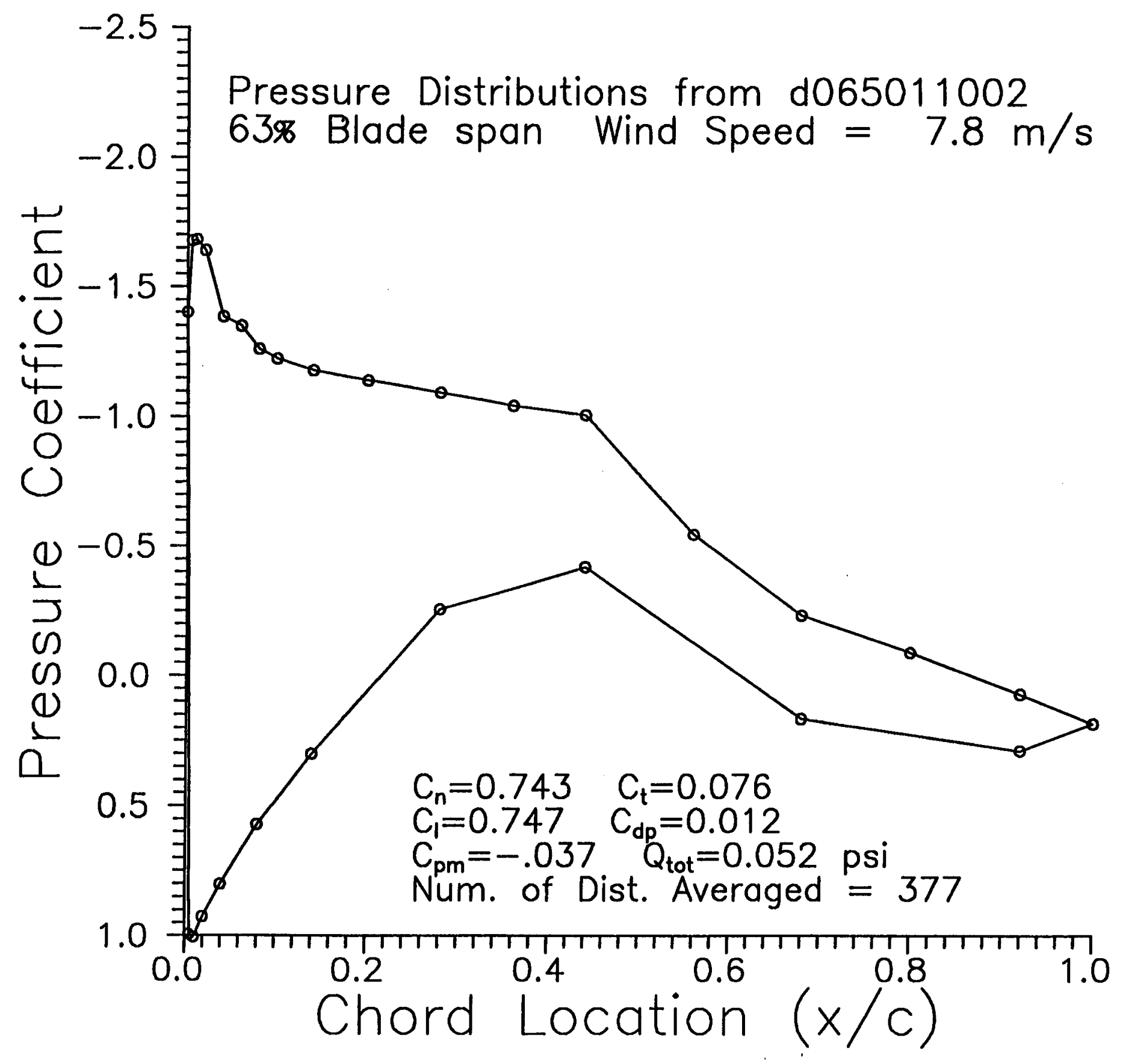




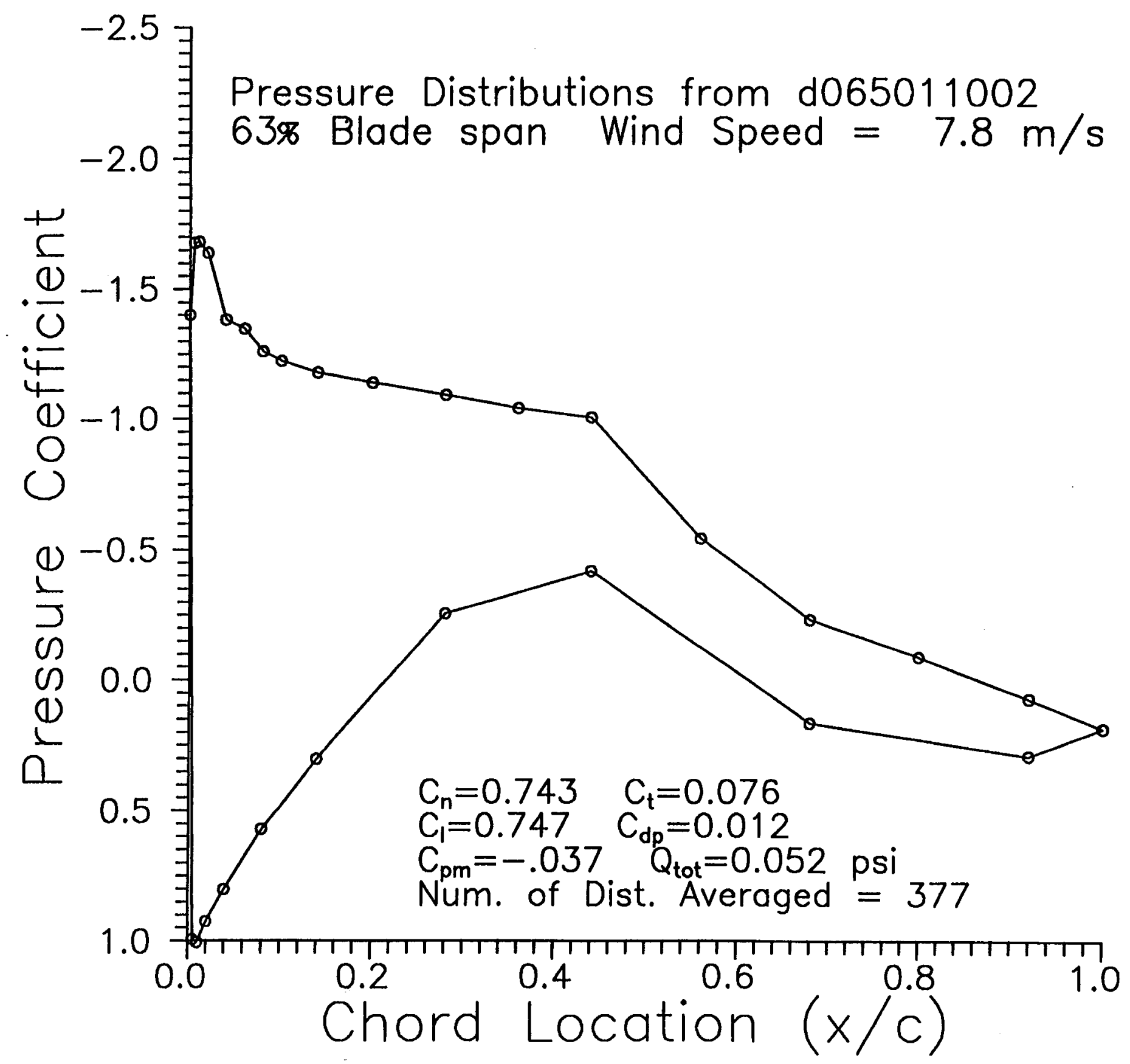




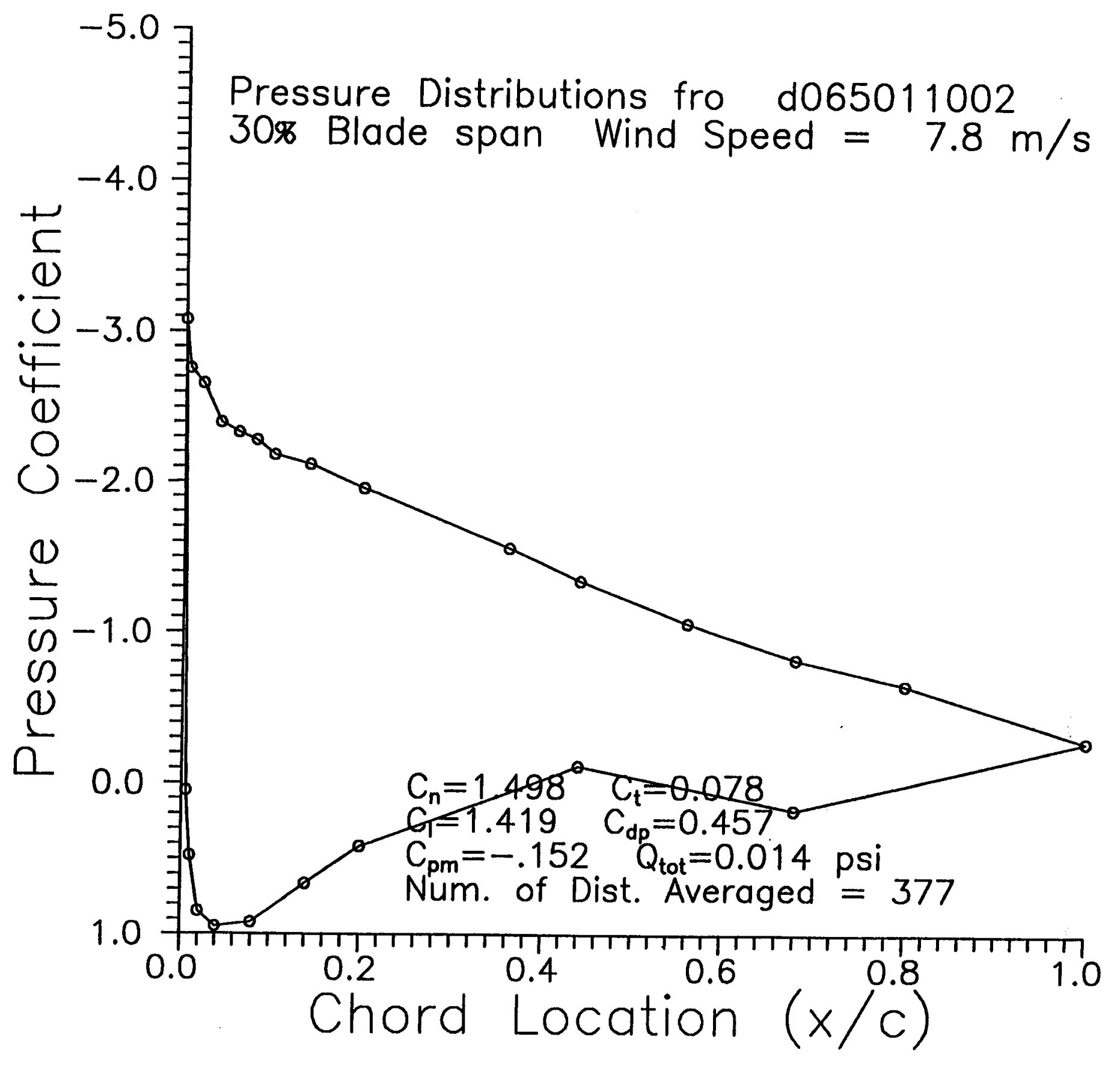




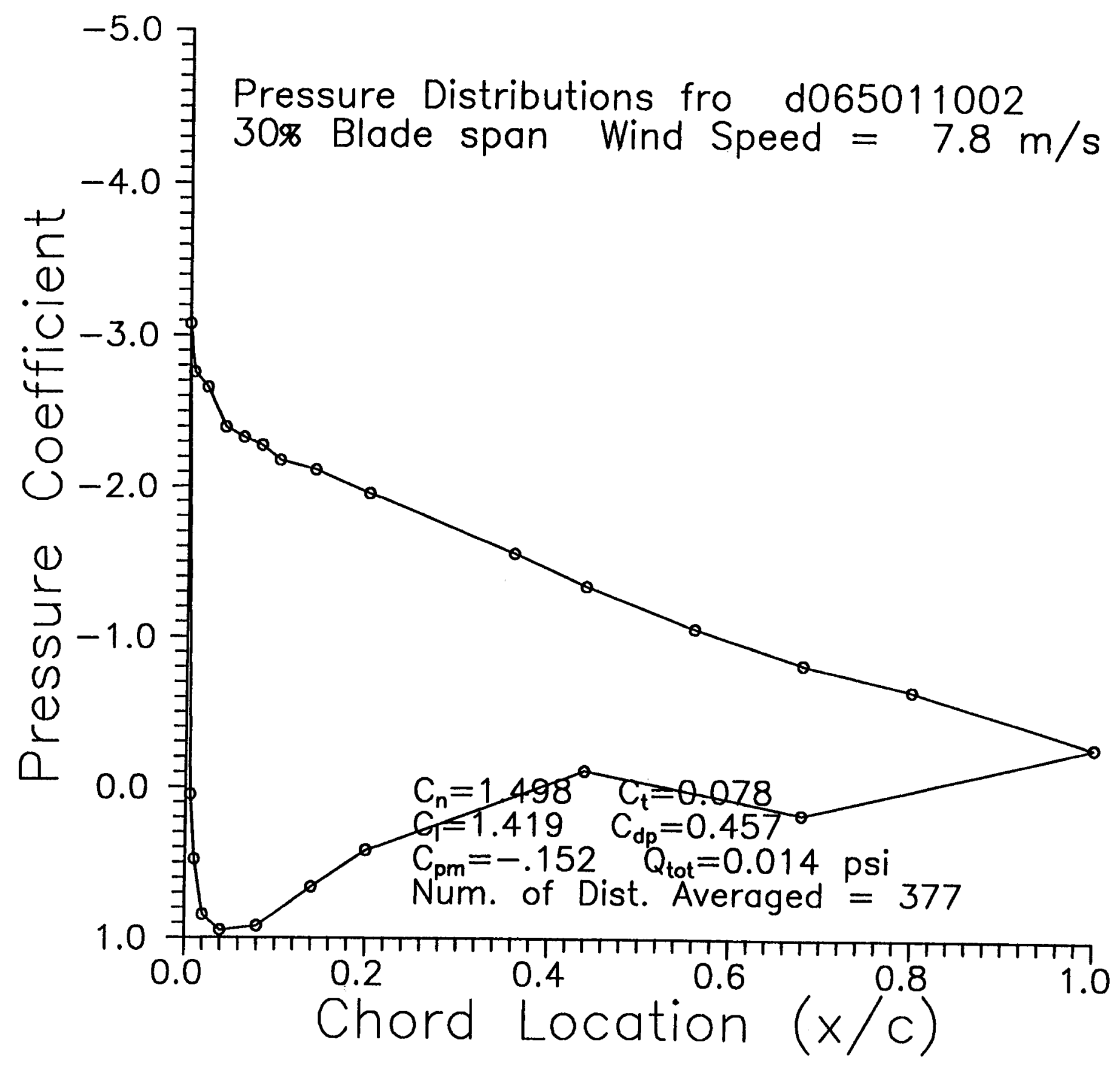




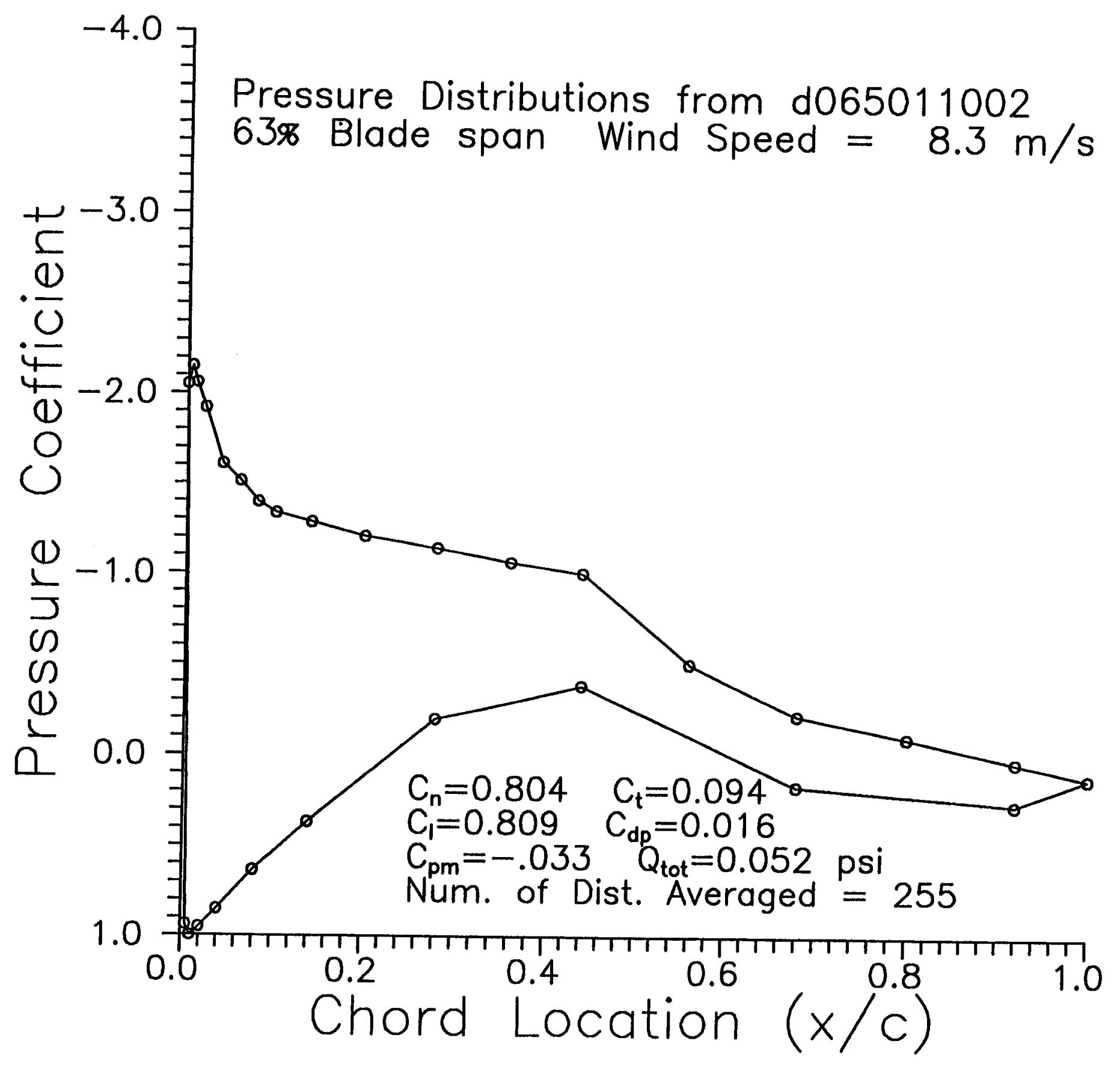




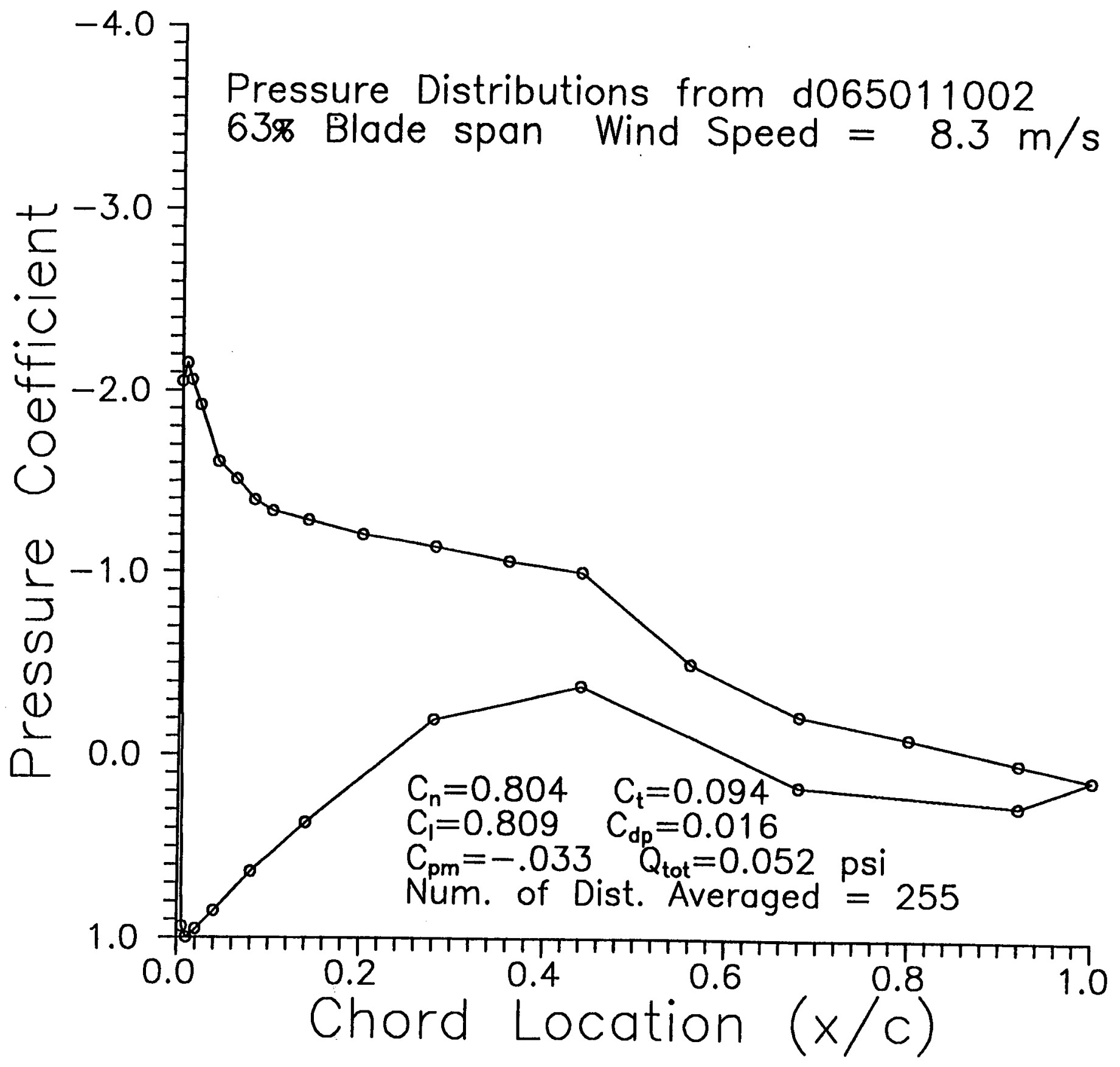




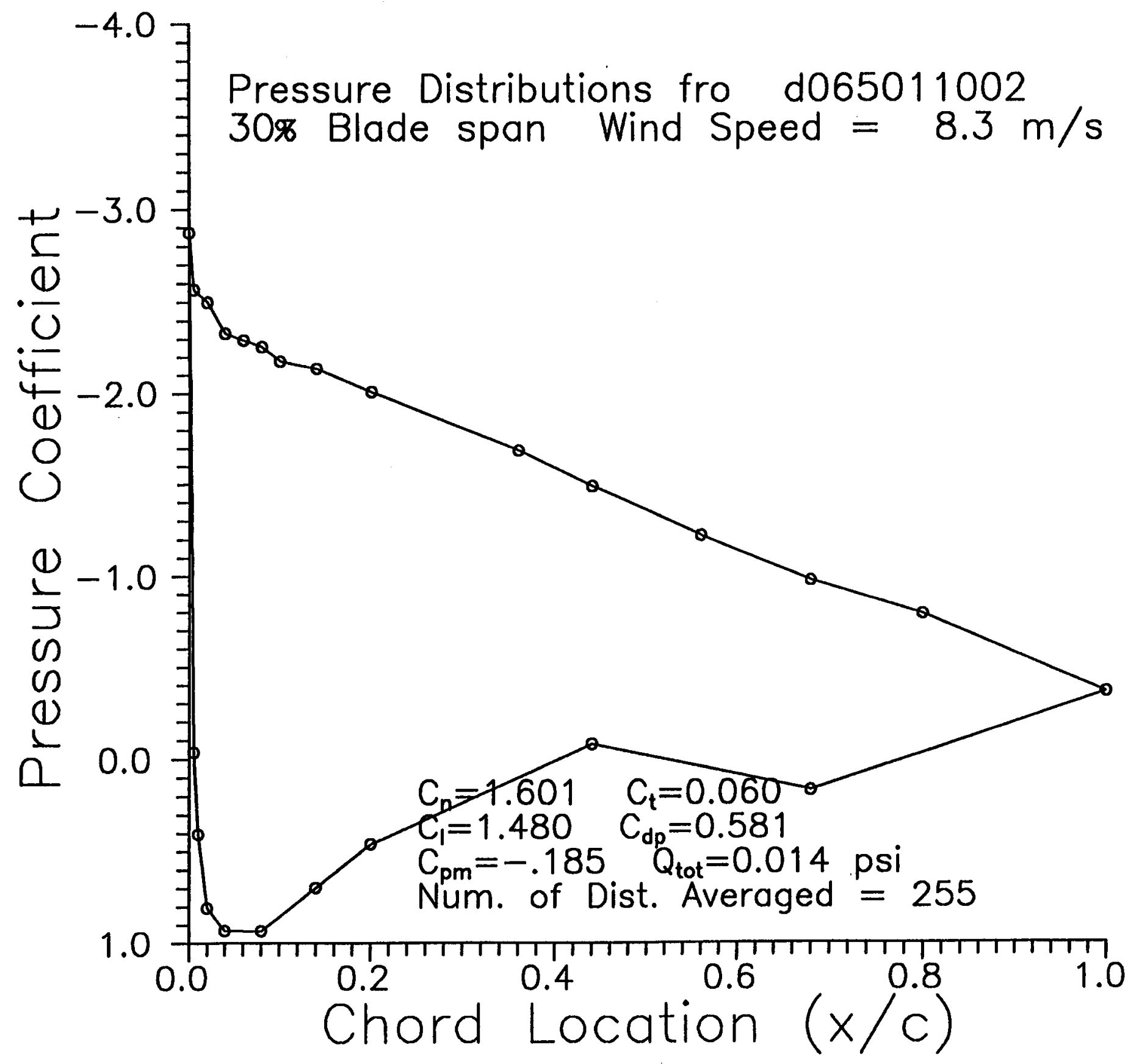




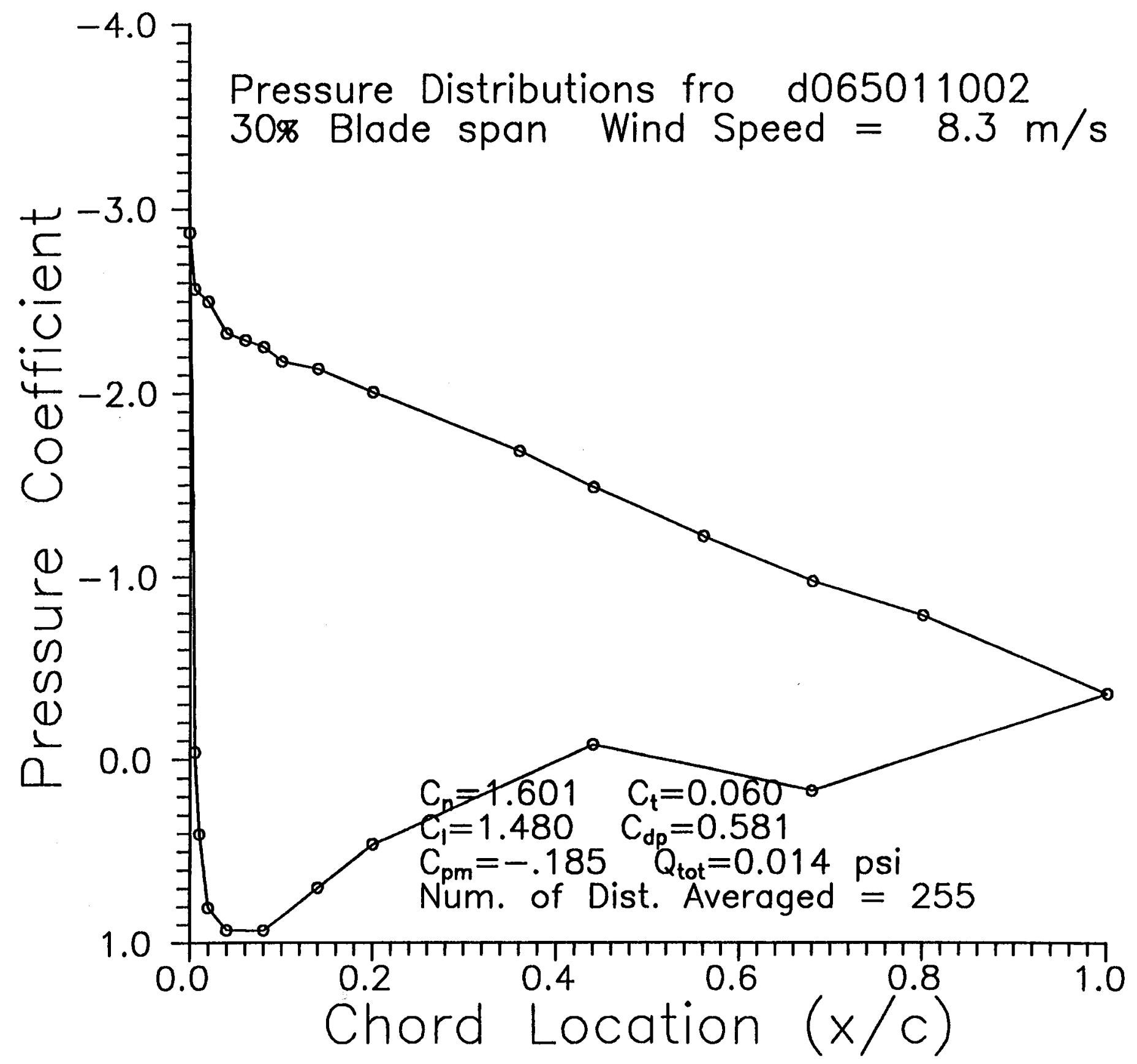




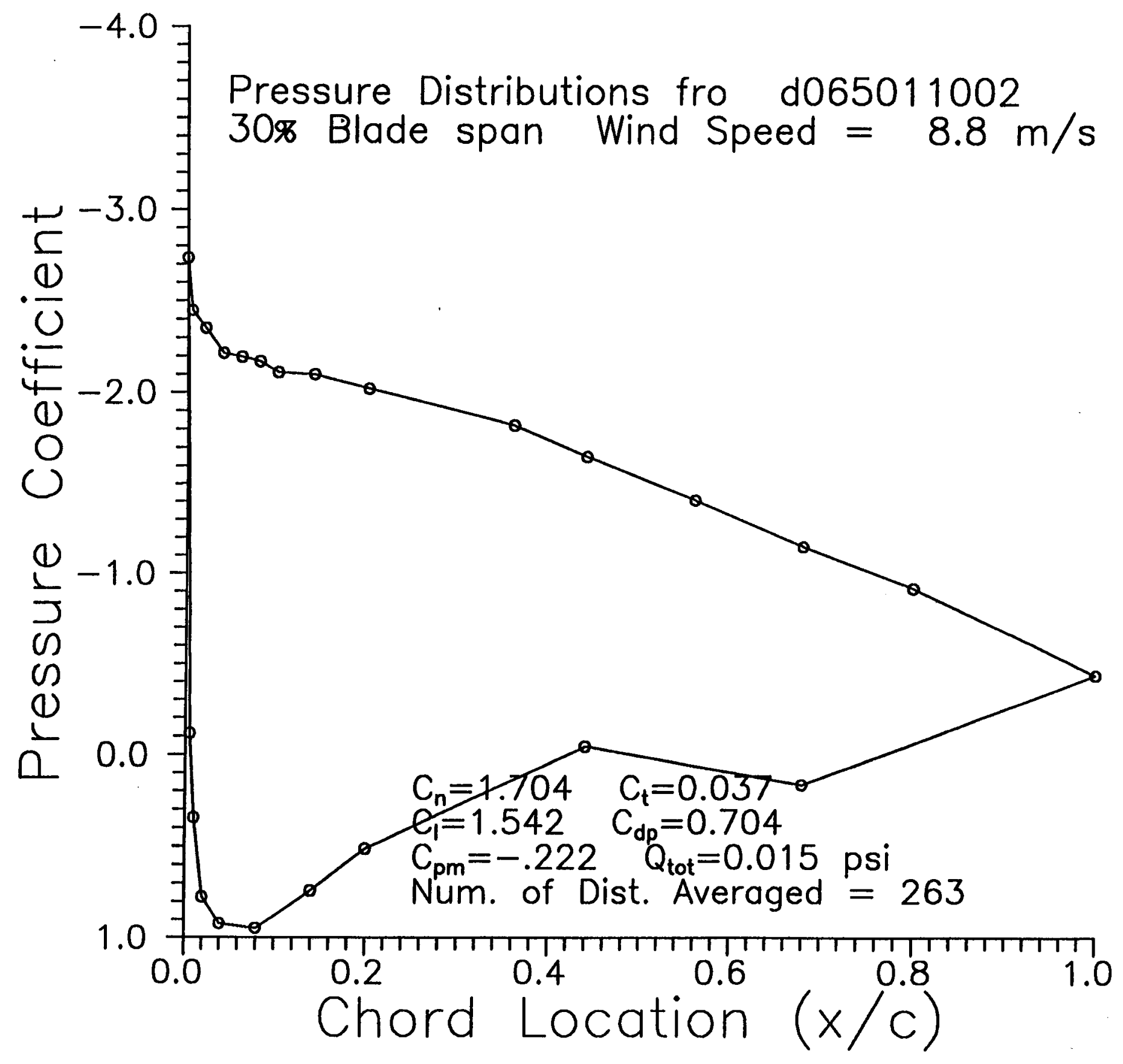




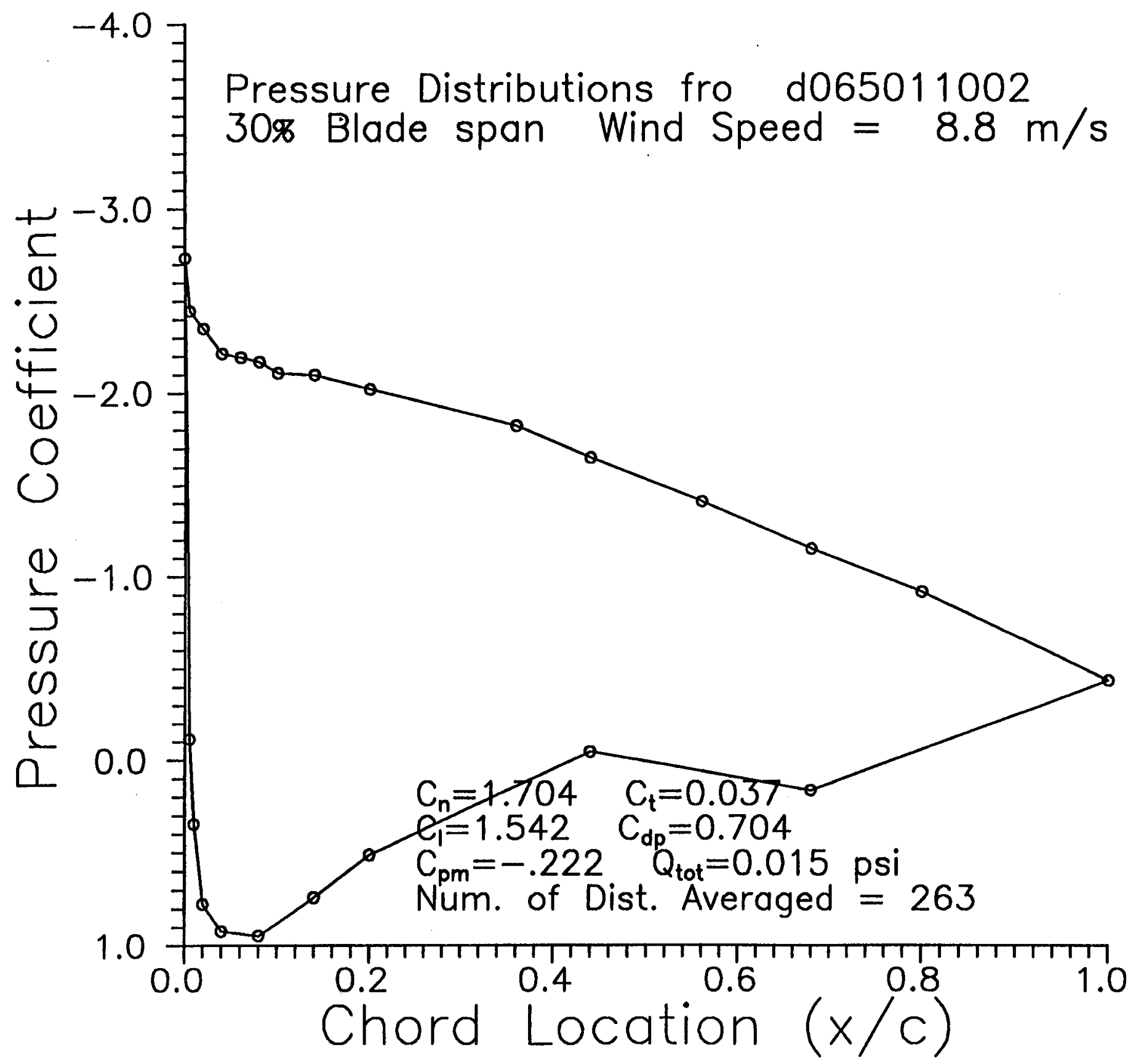




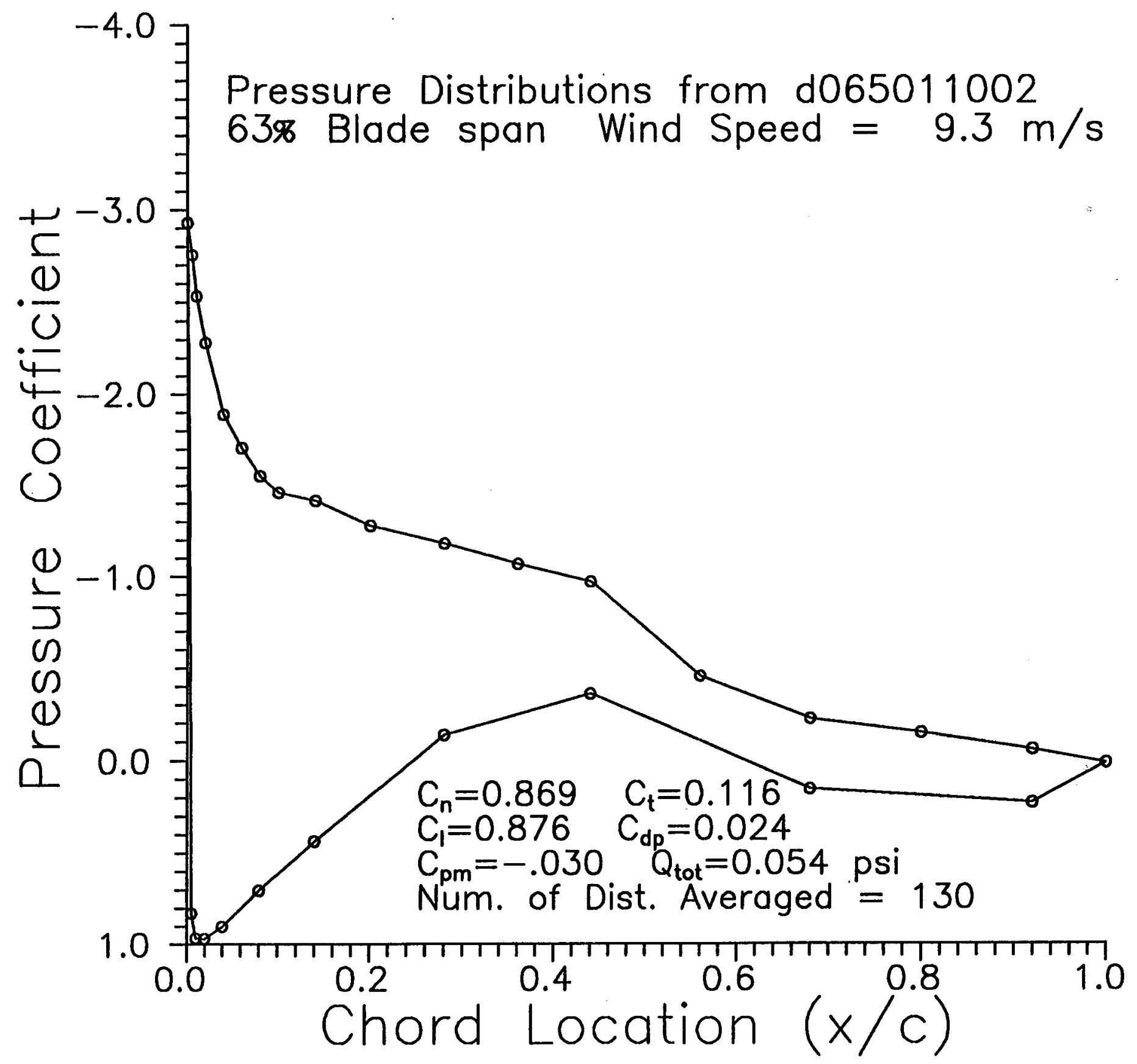




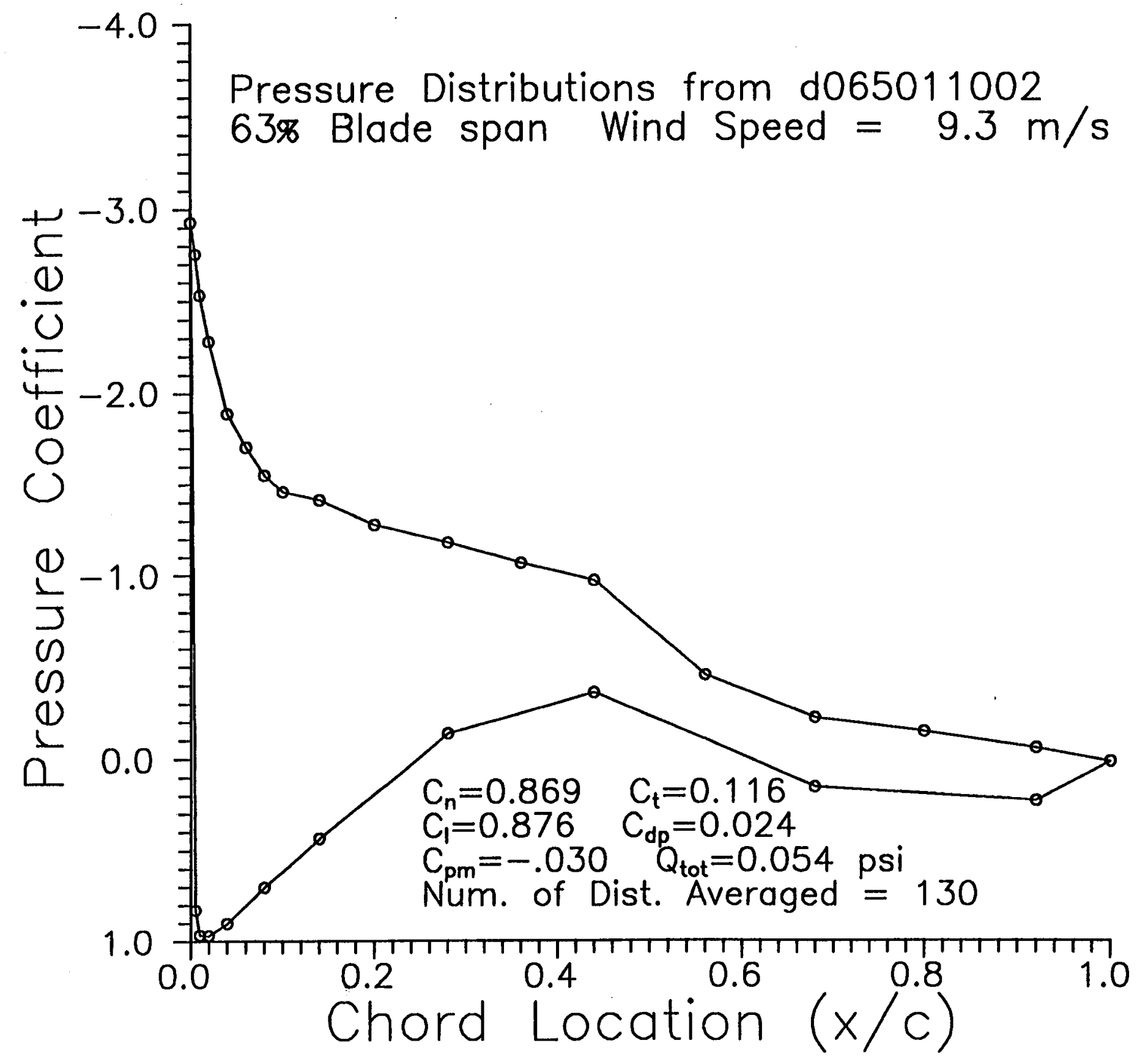




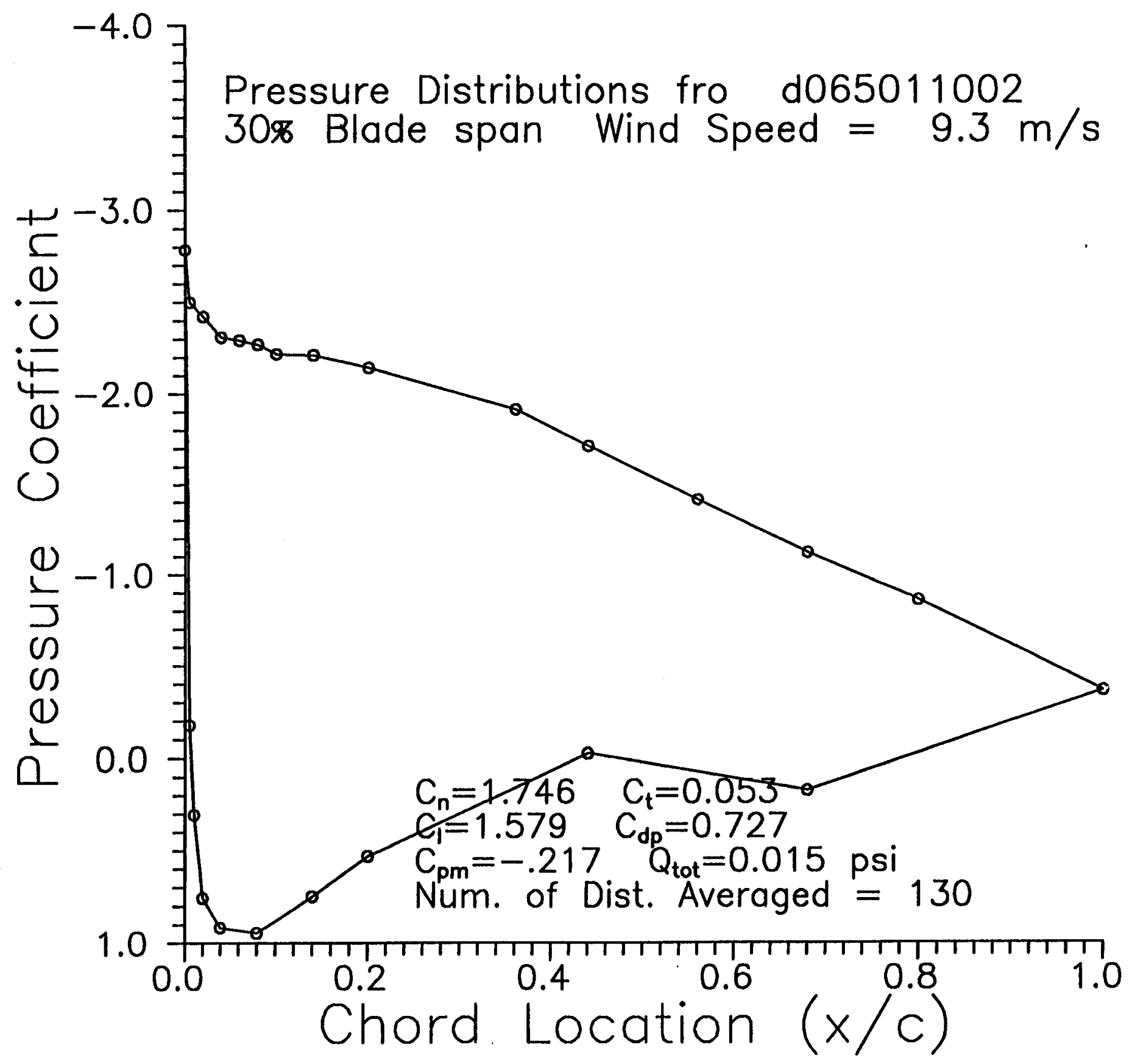




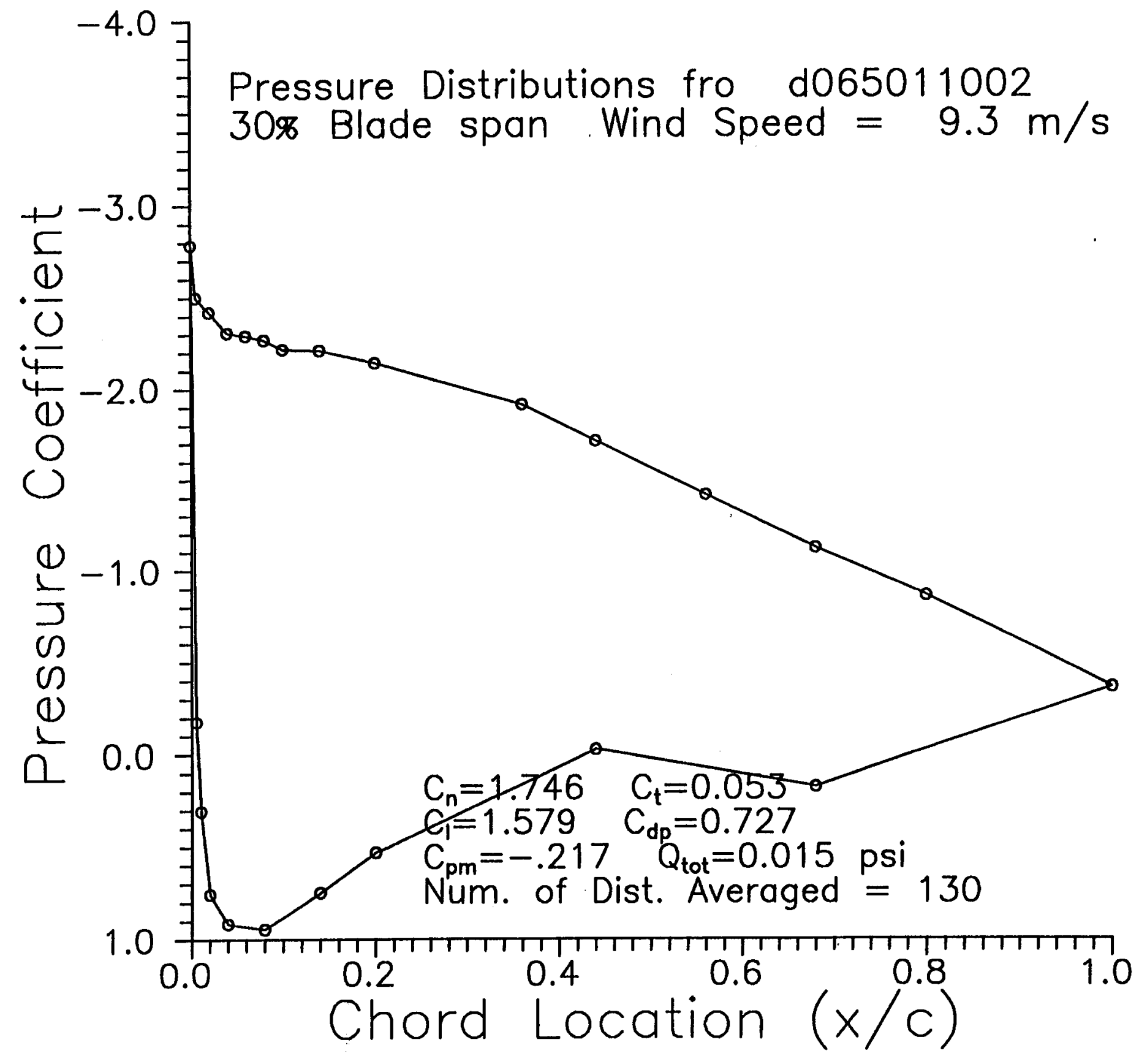




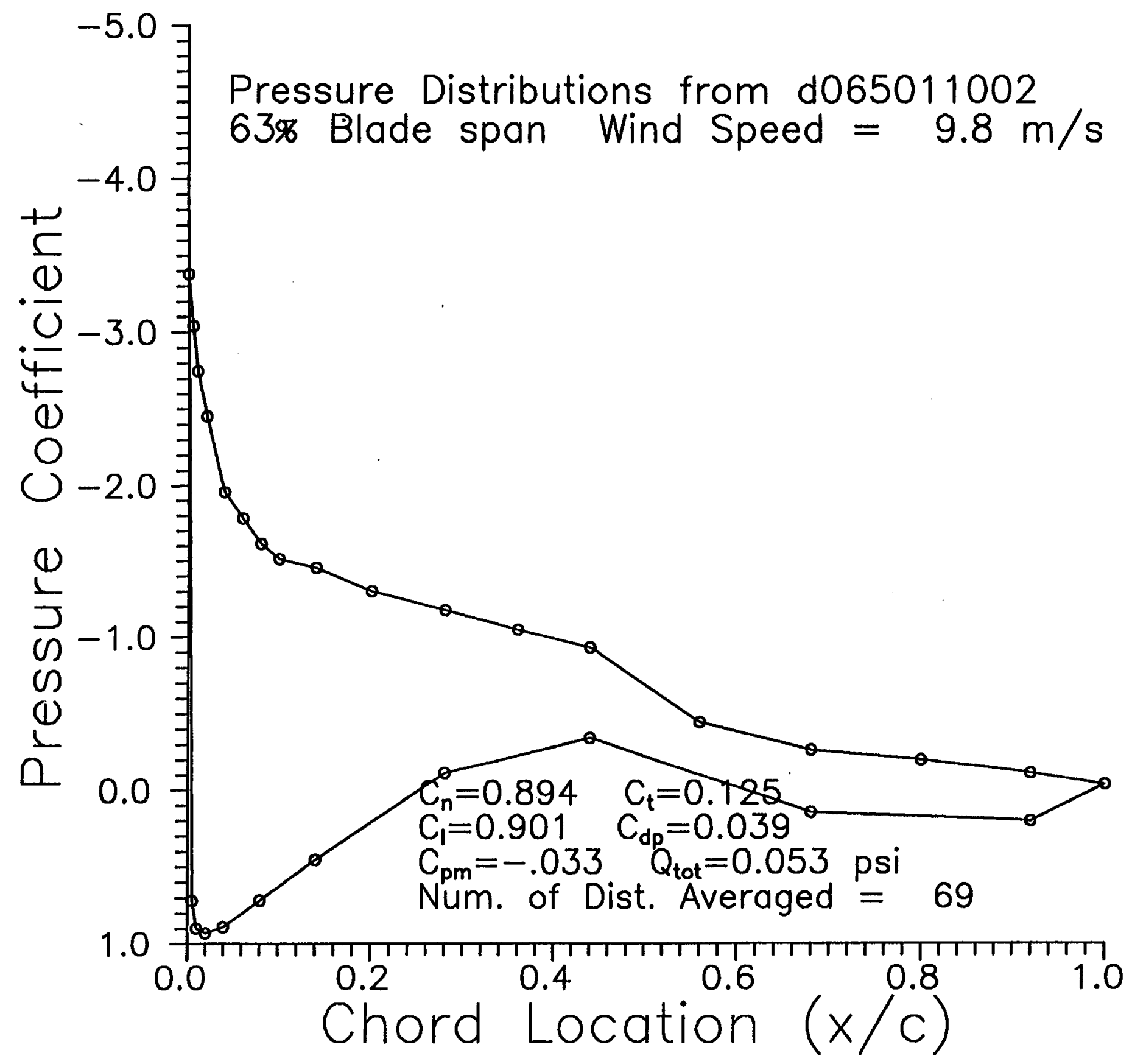




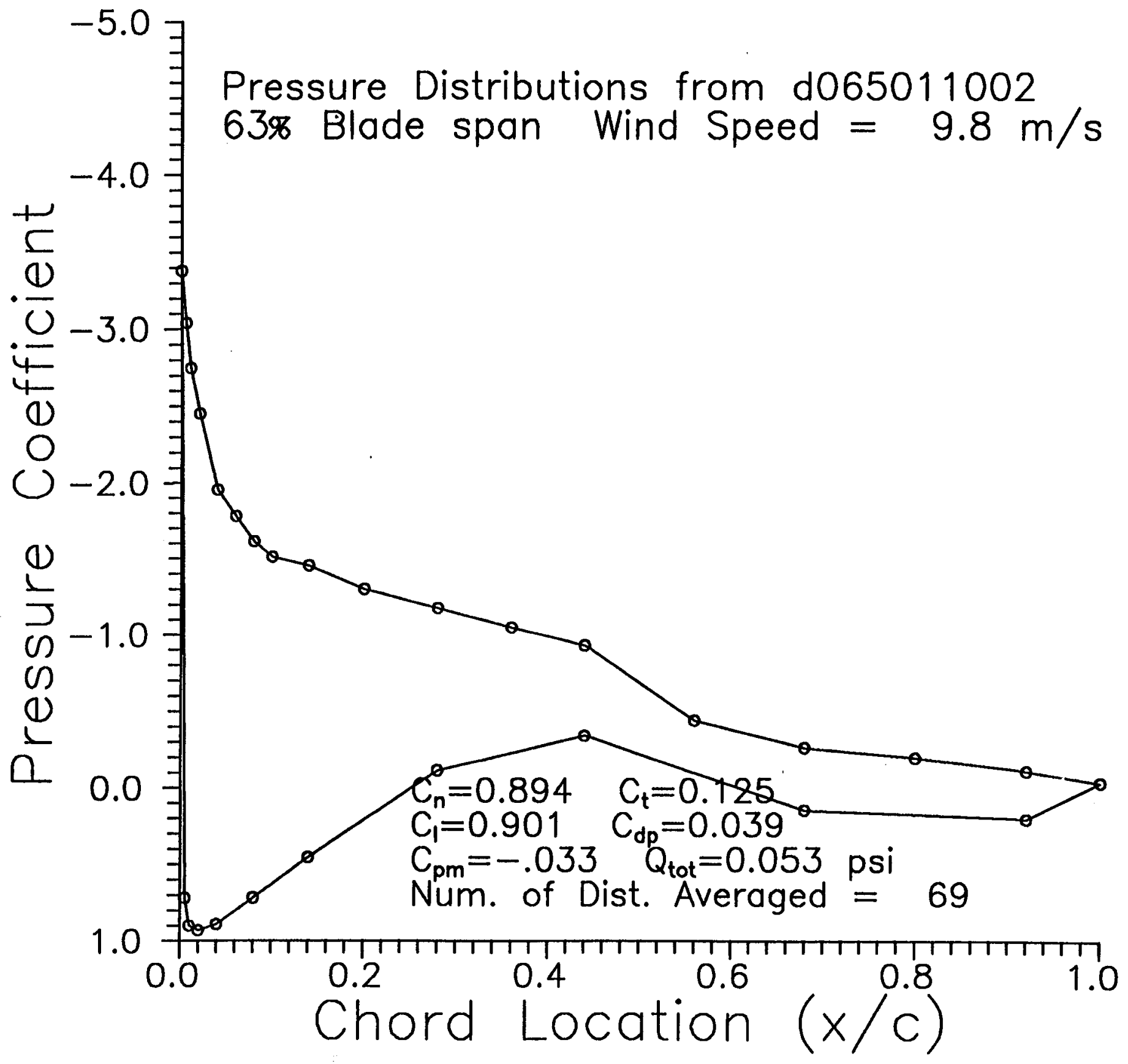




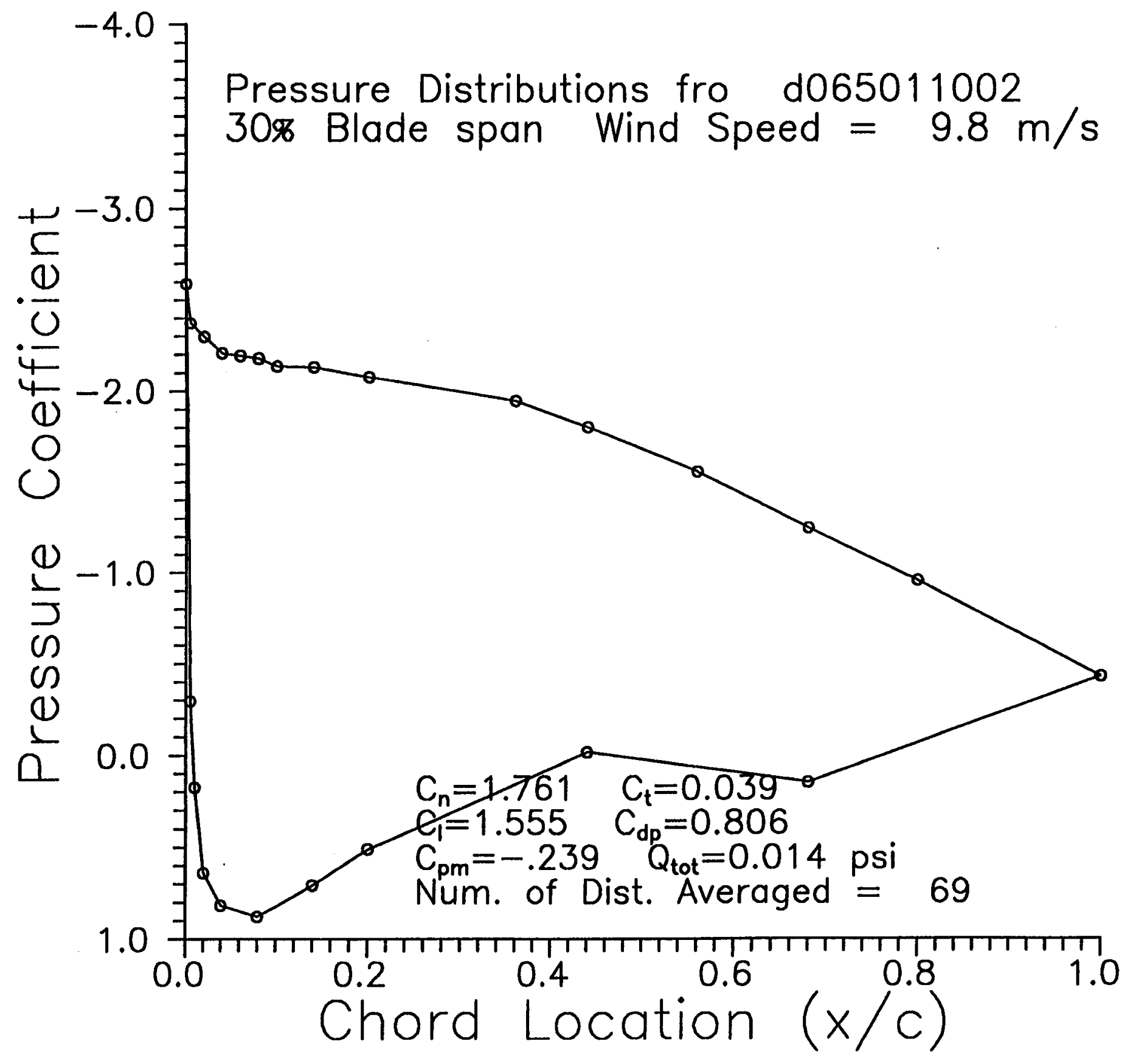




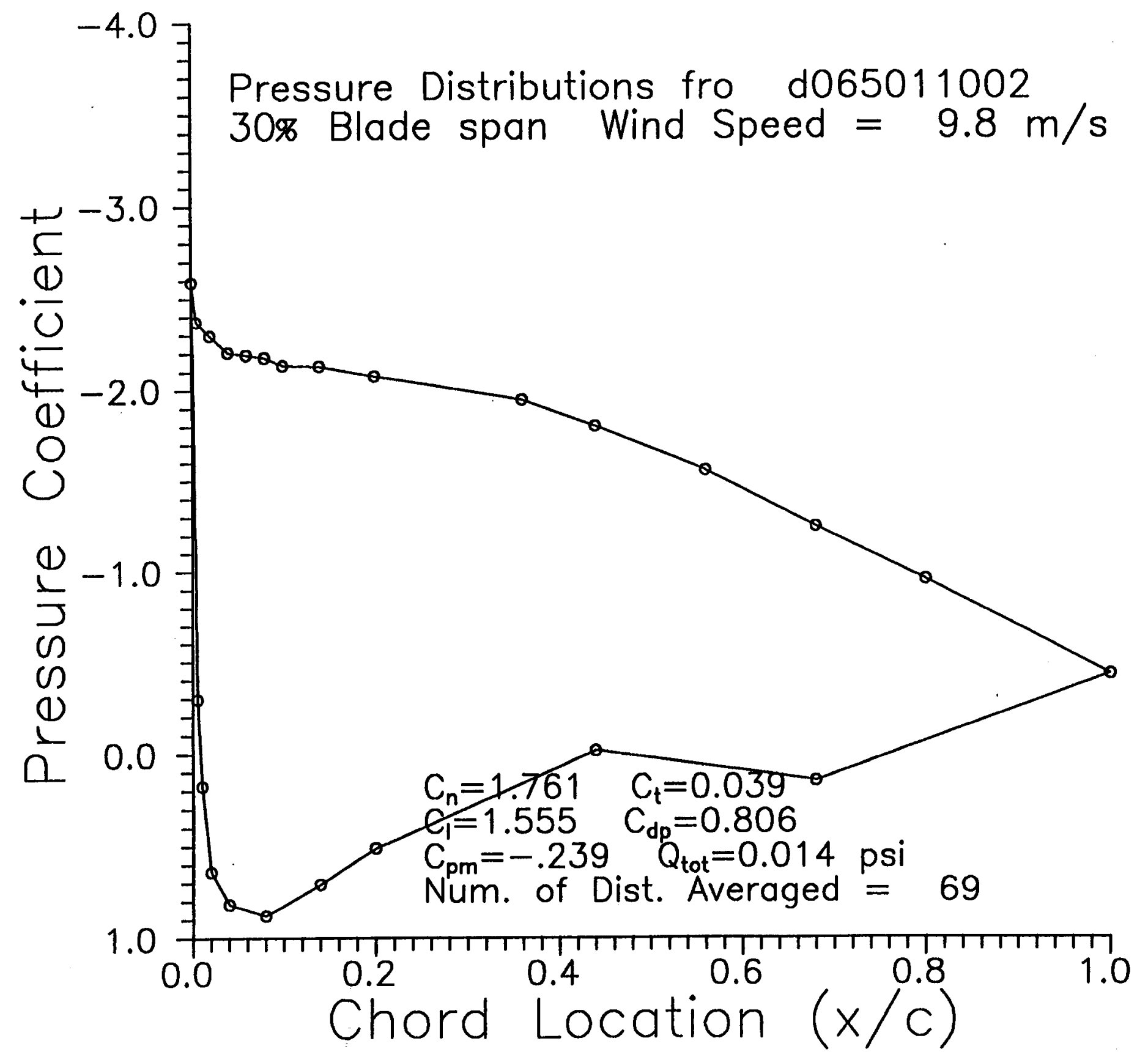




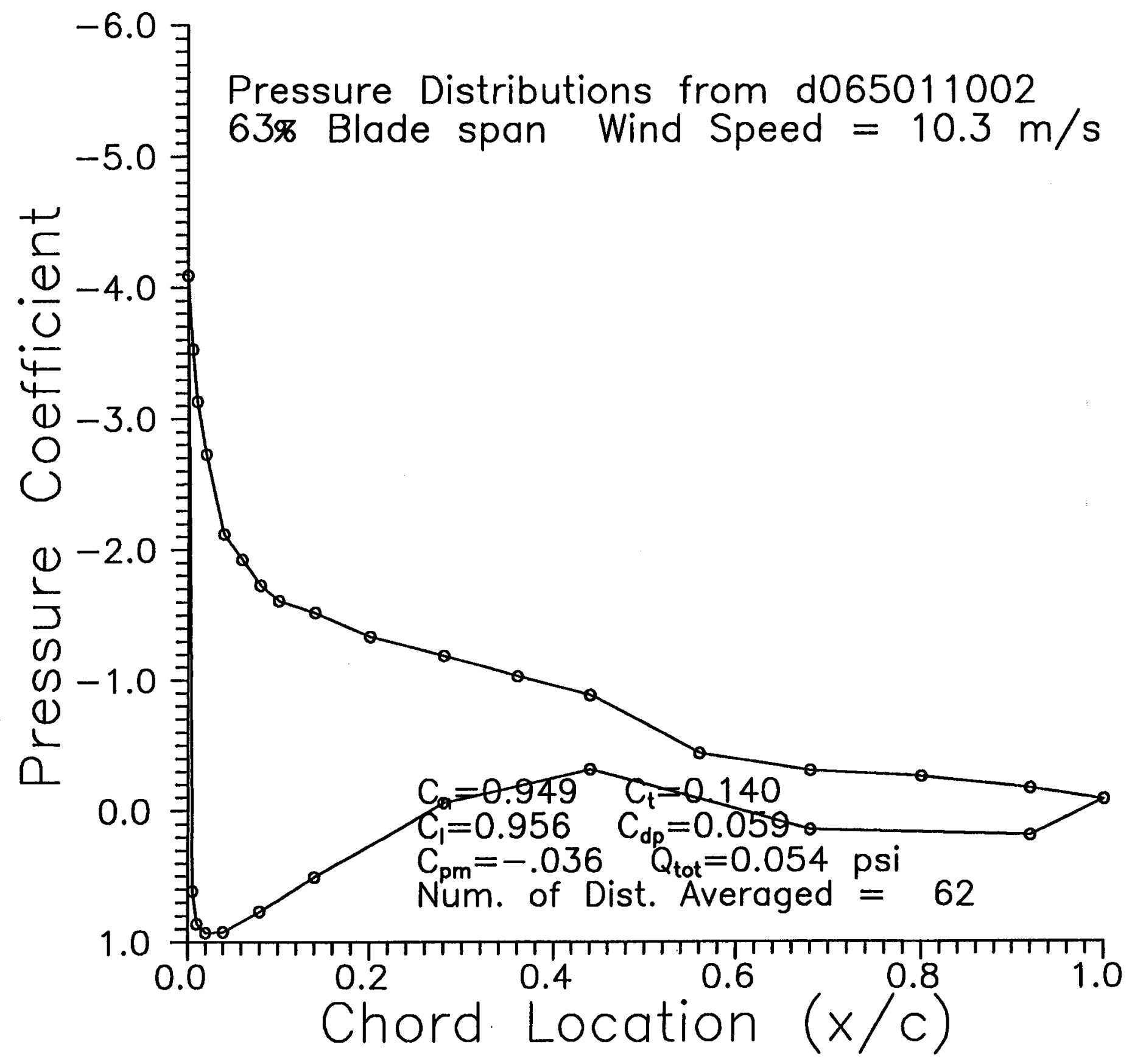




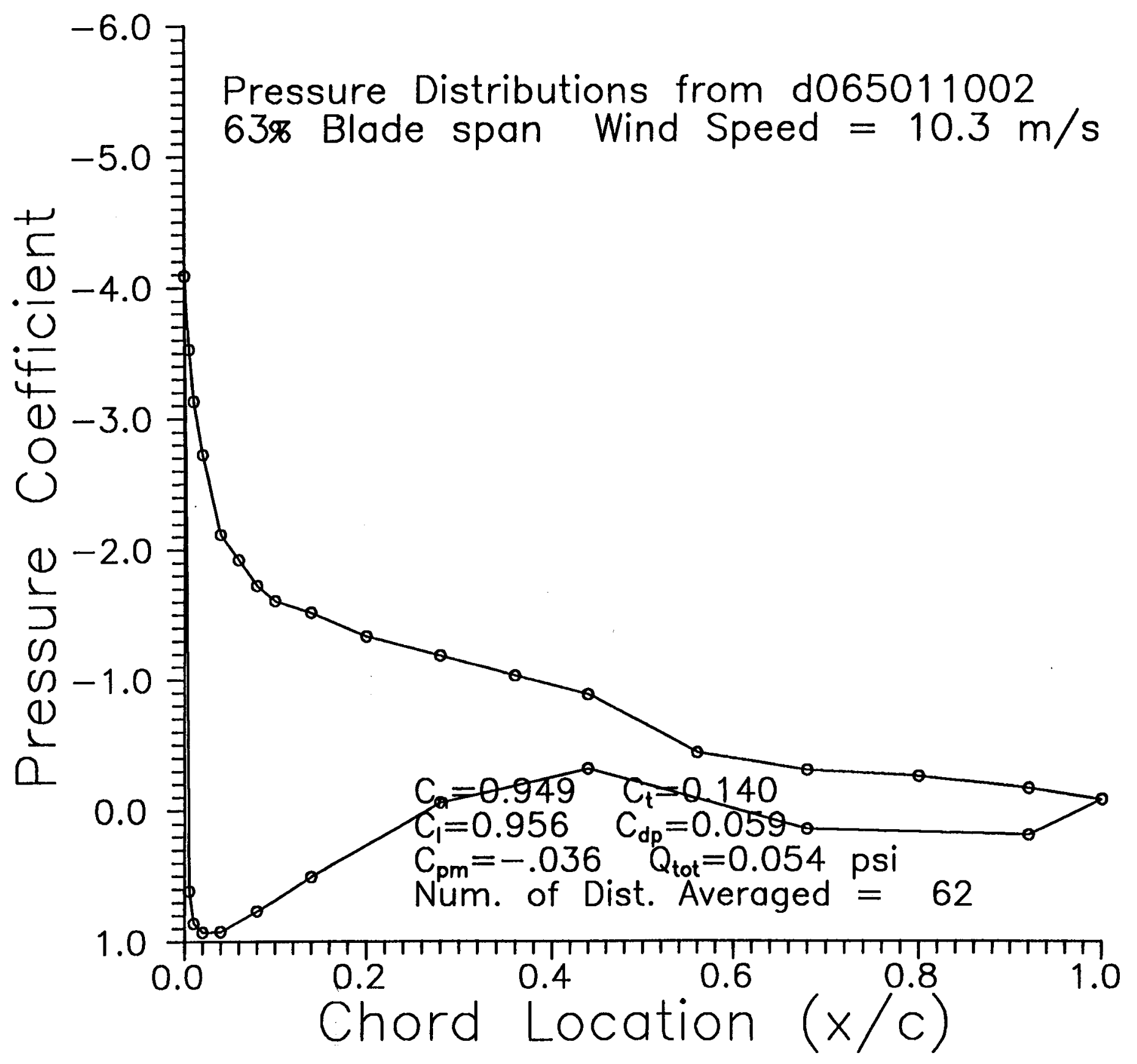




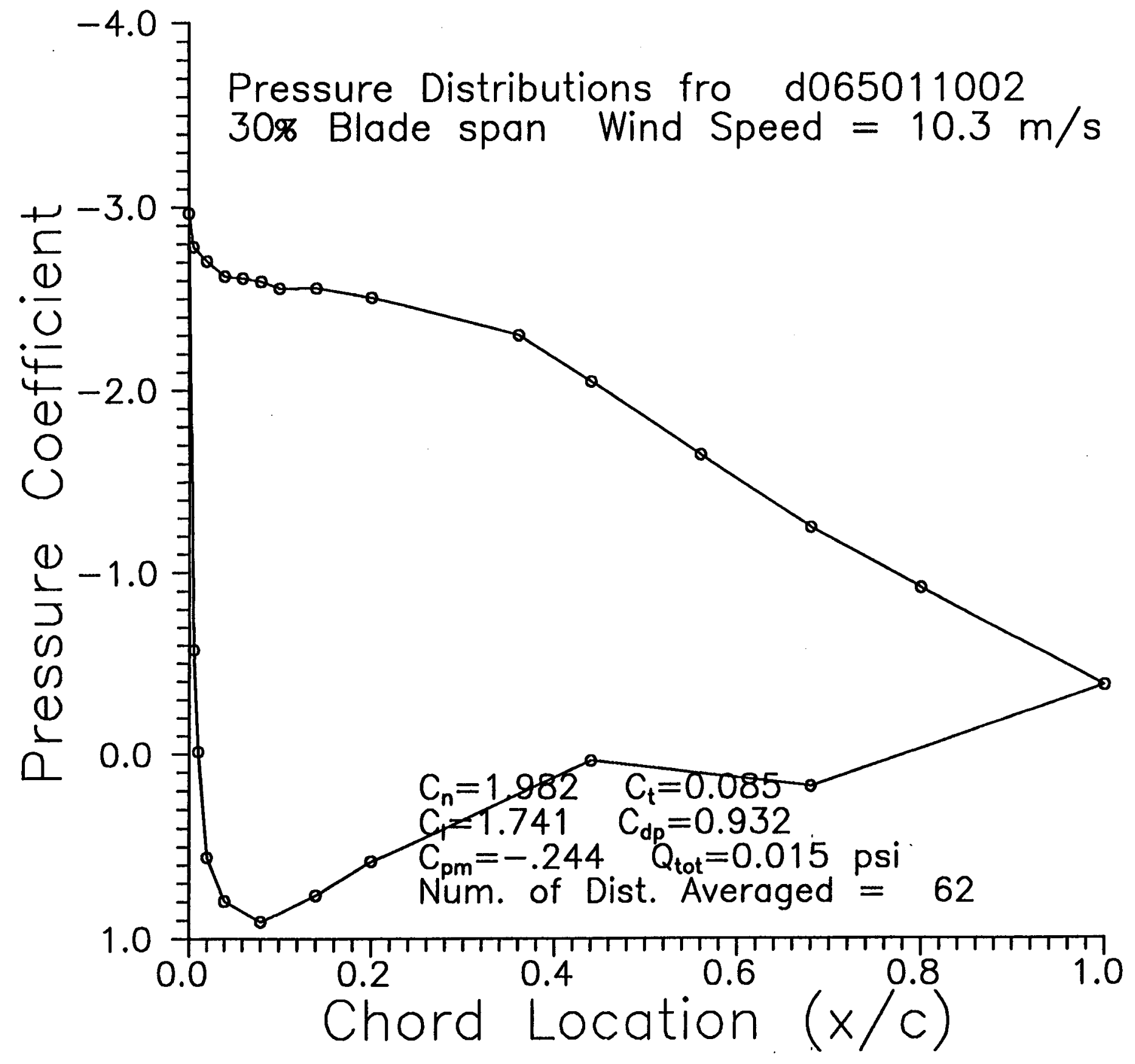




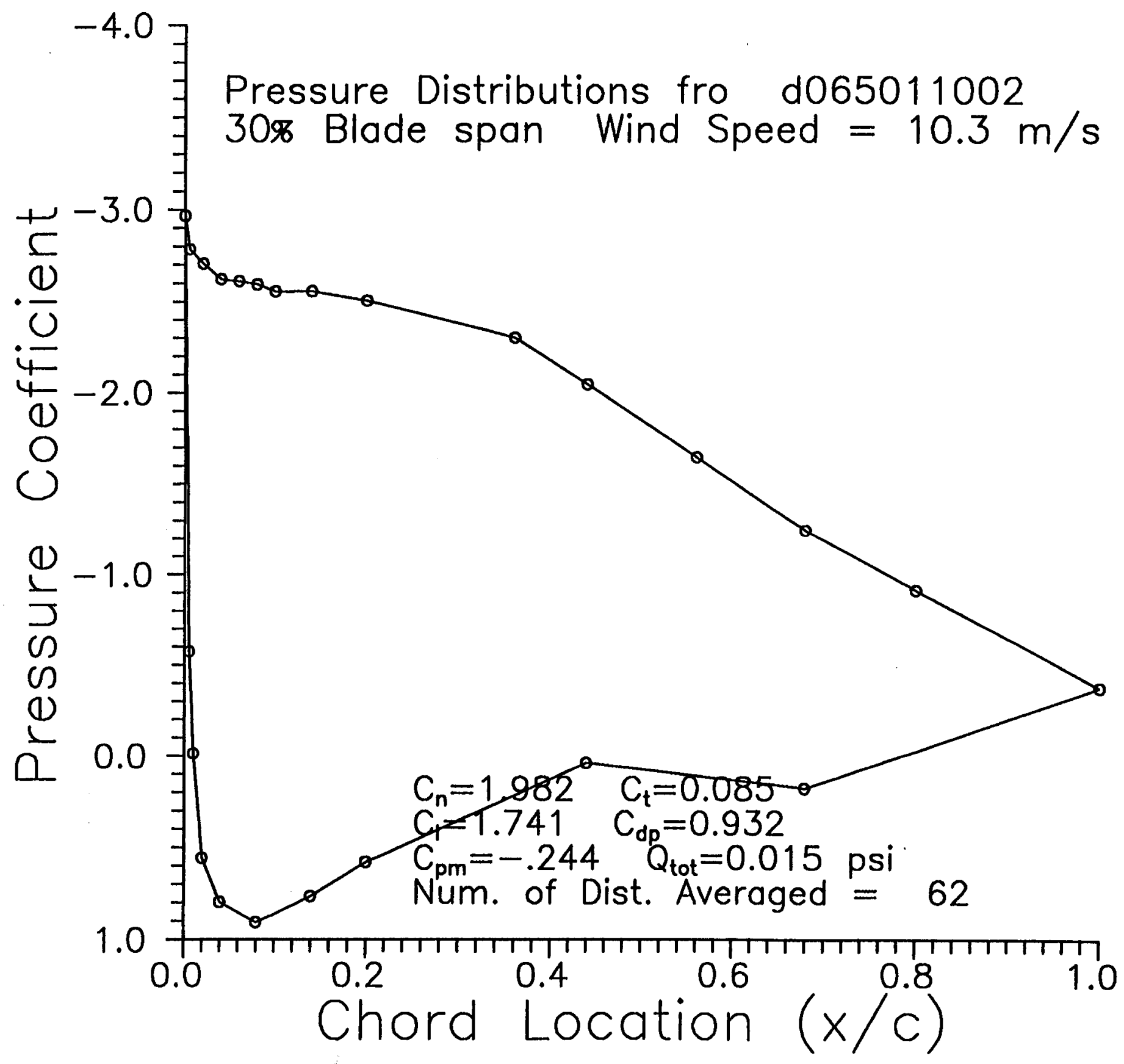




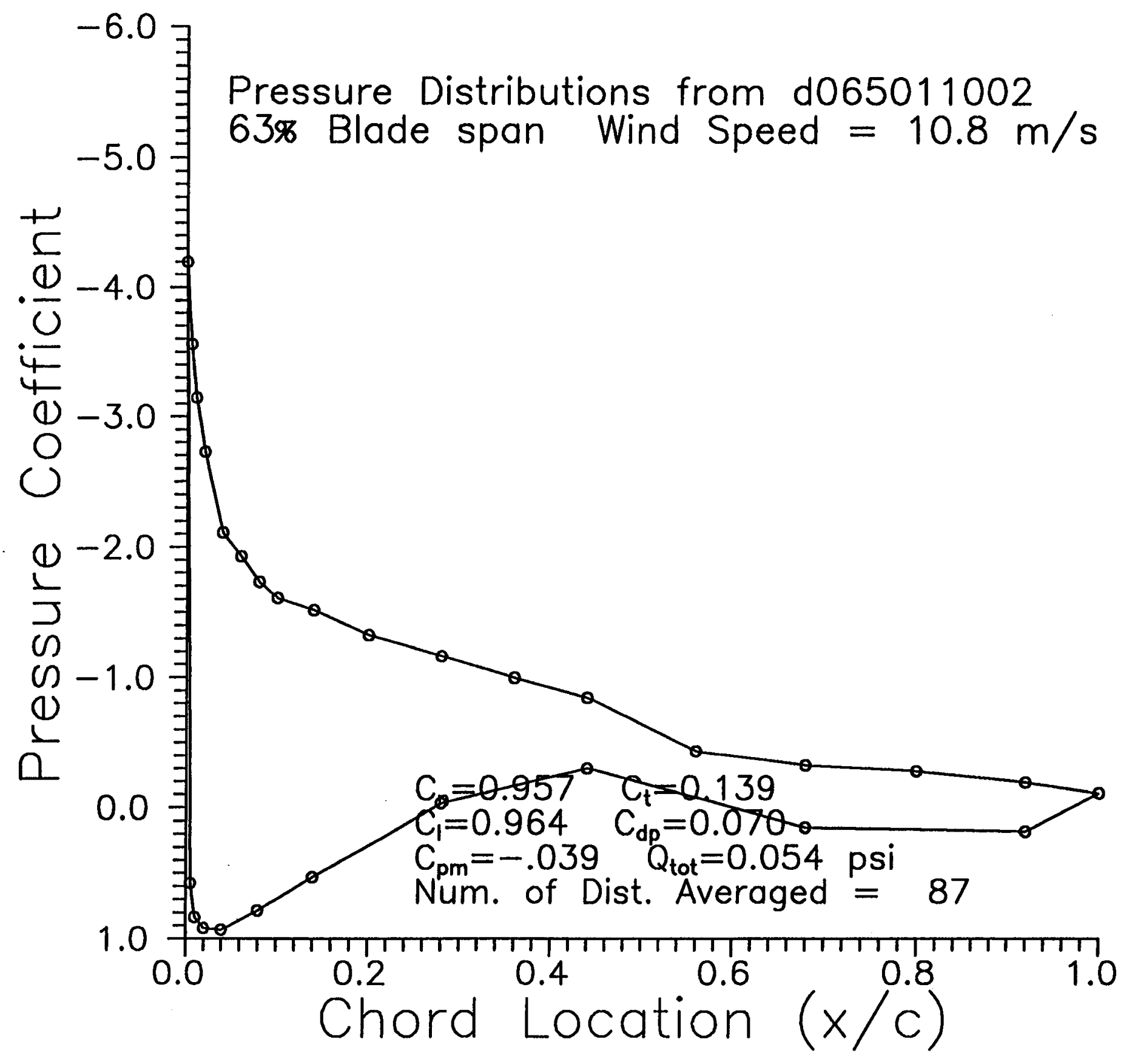




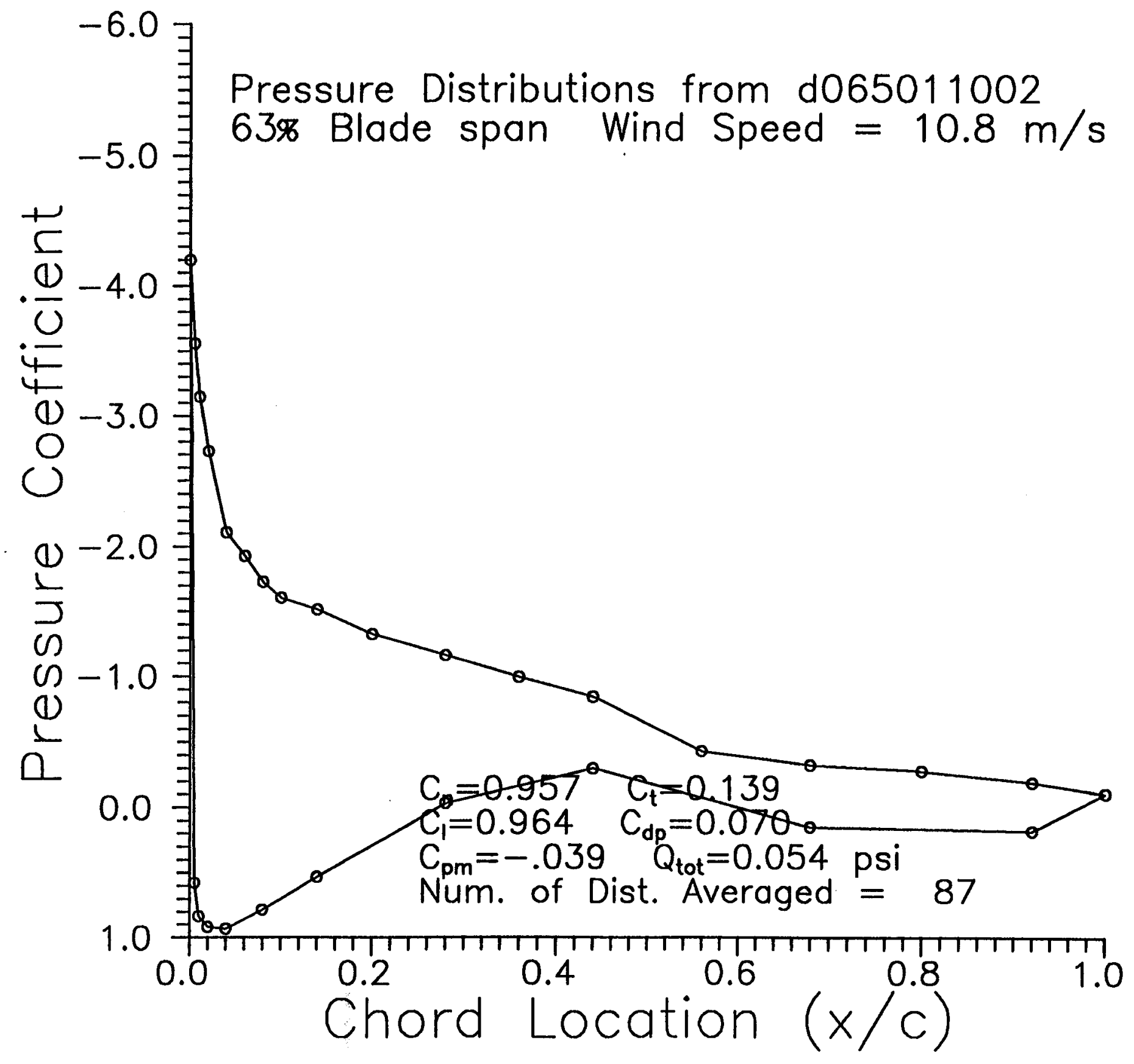




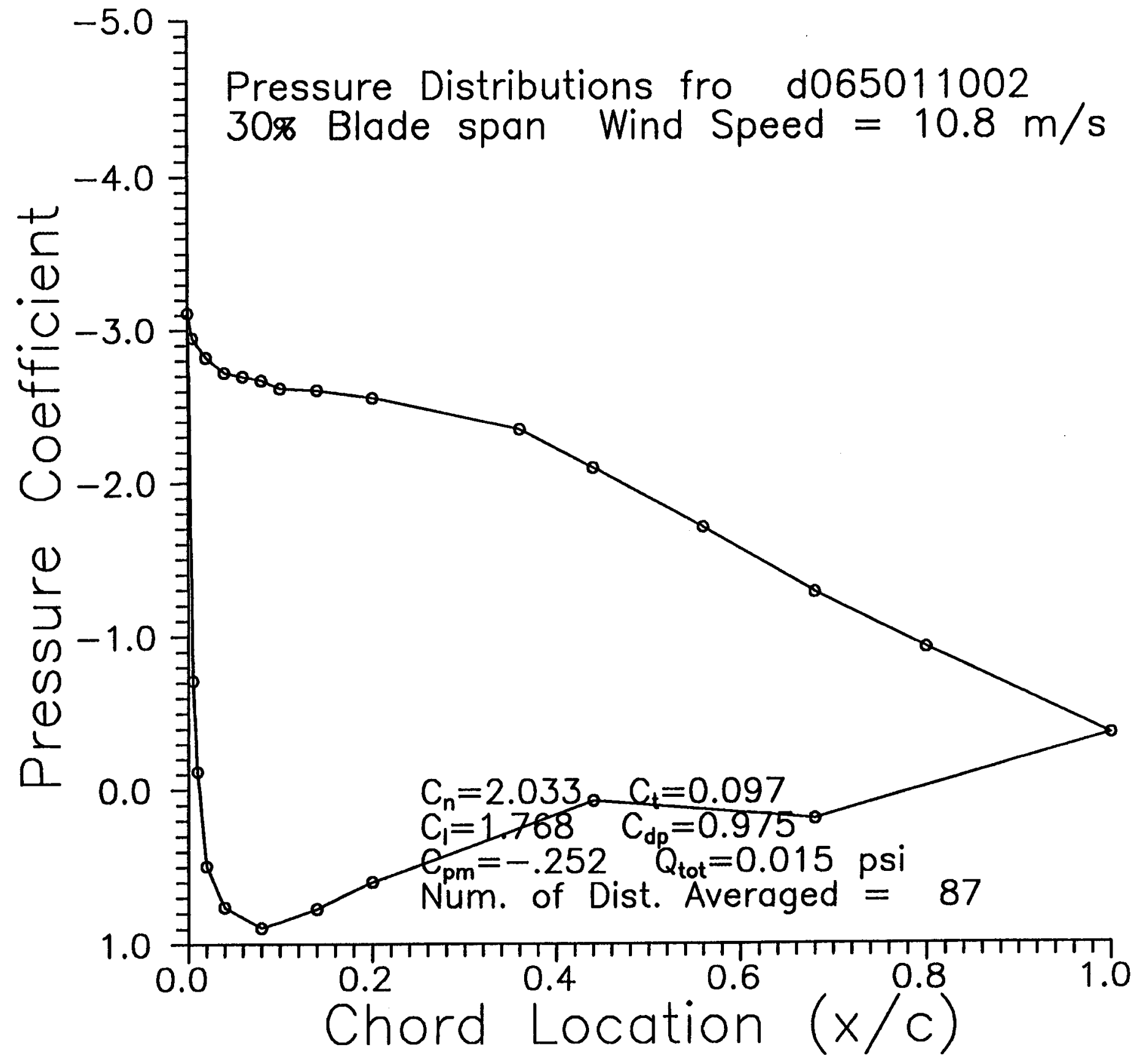




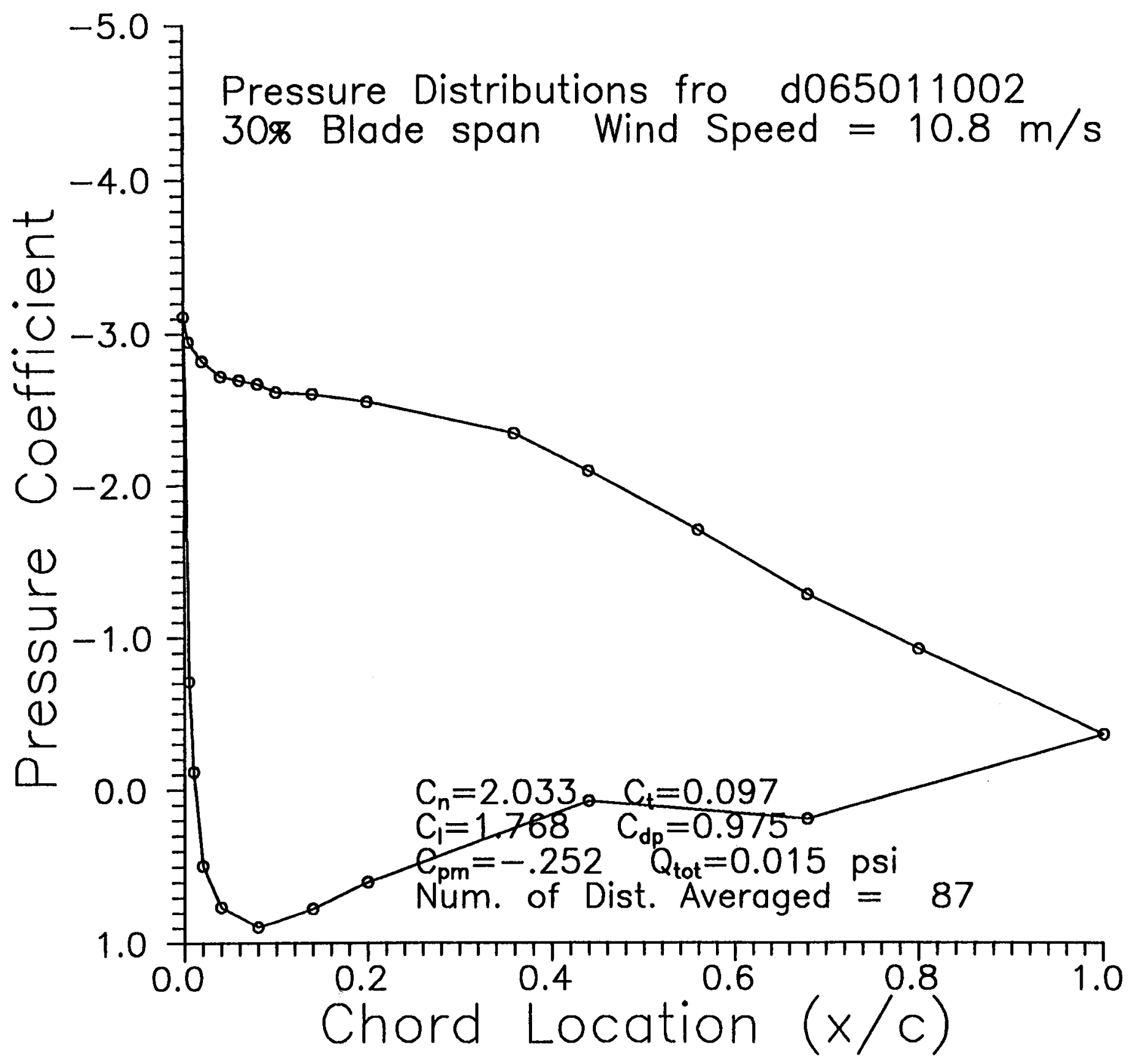




\section{Appendix E \\ Bin-Averaged Blade Loads \\ Table of Coefficients}


Tape 65 Data

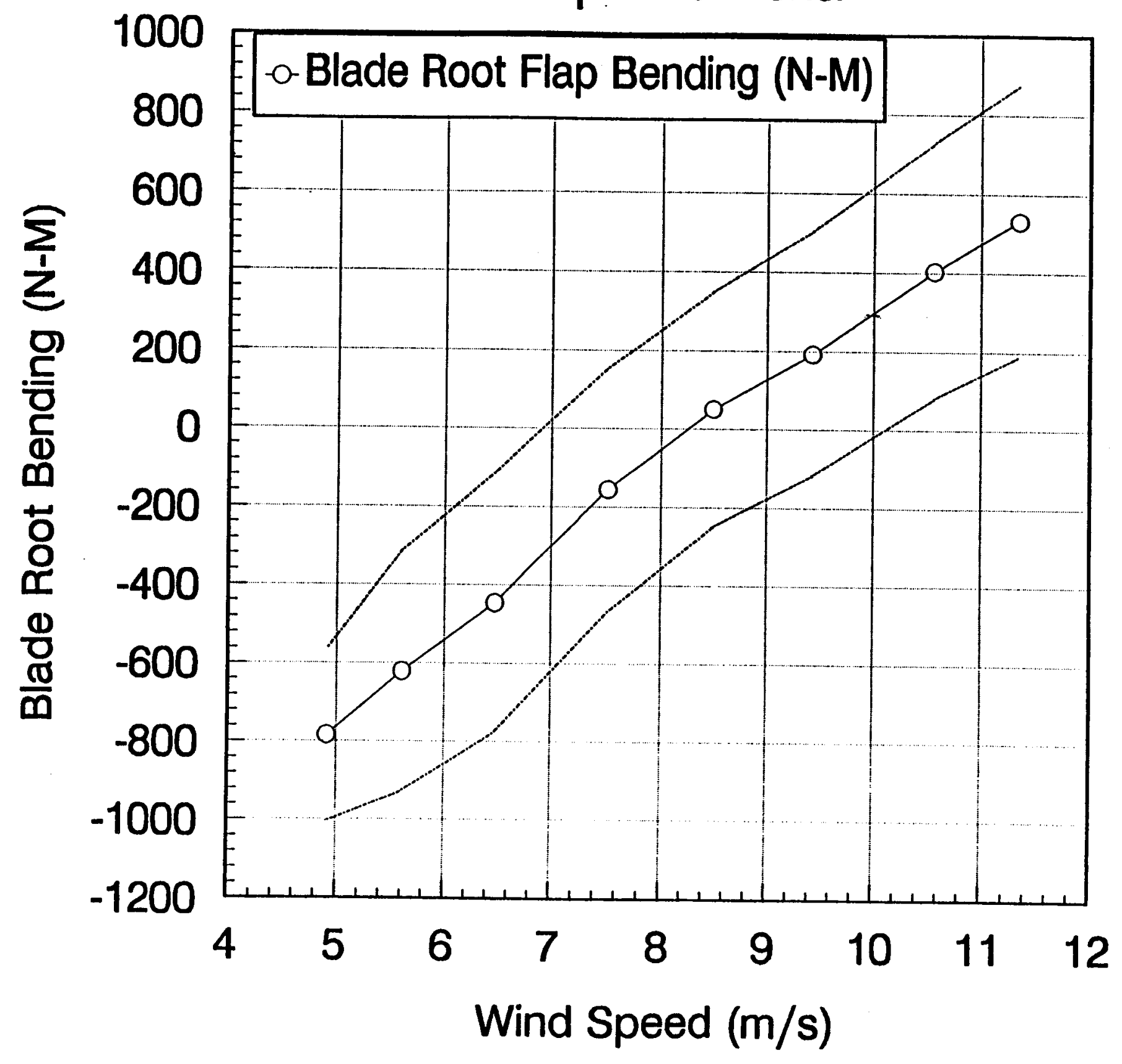




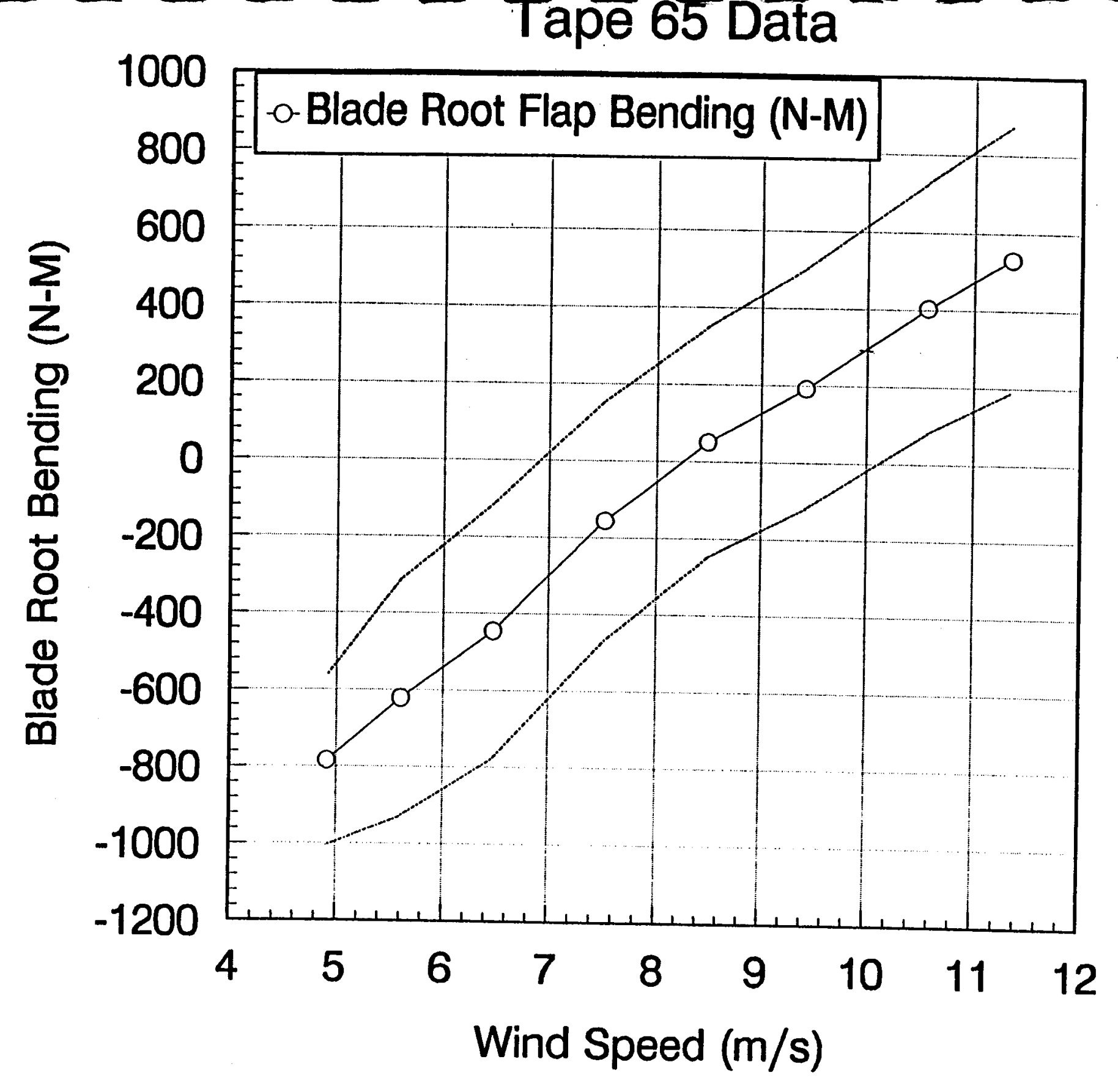


Tape 65 Data

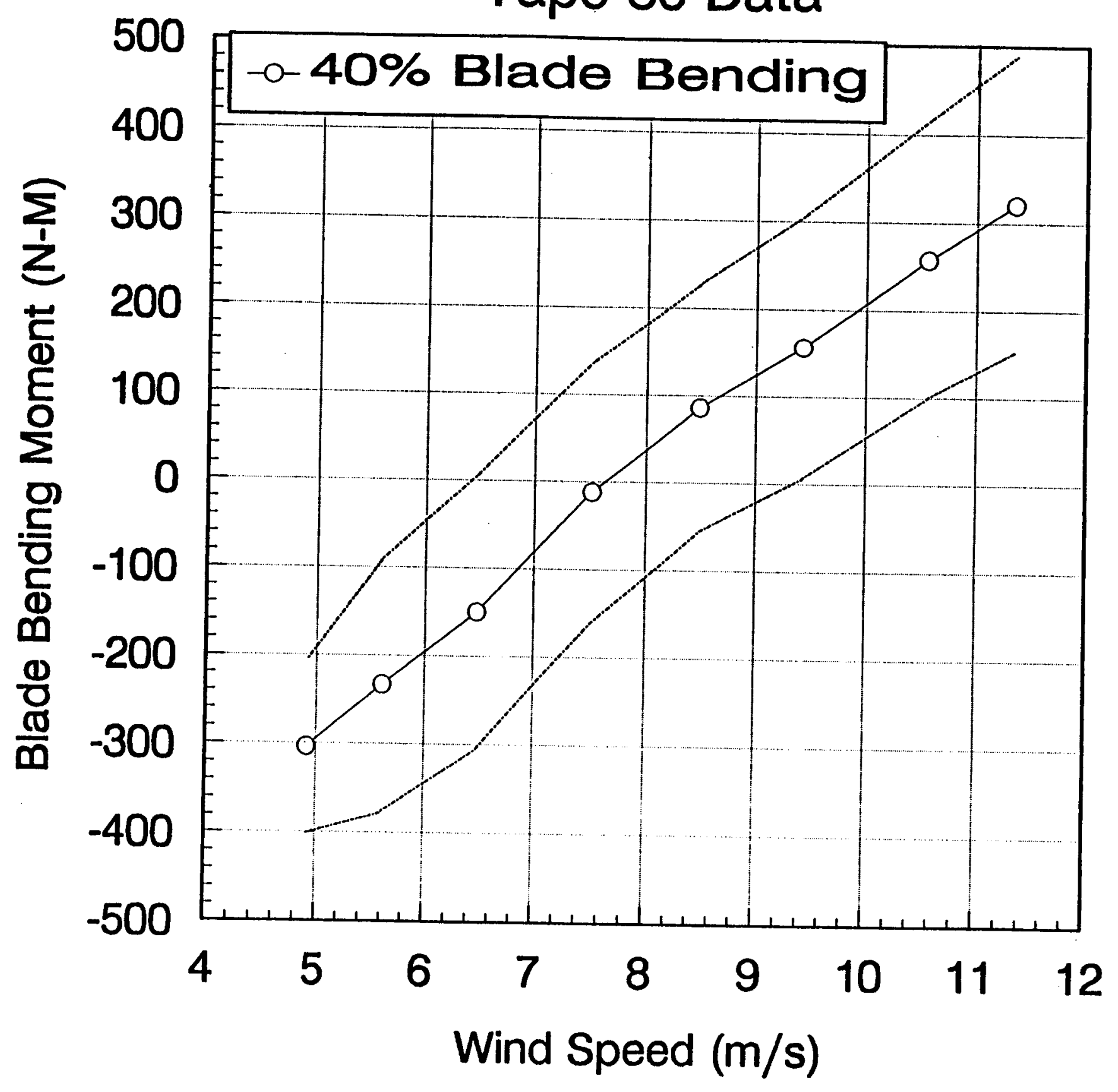




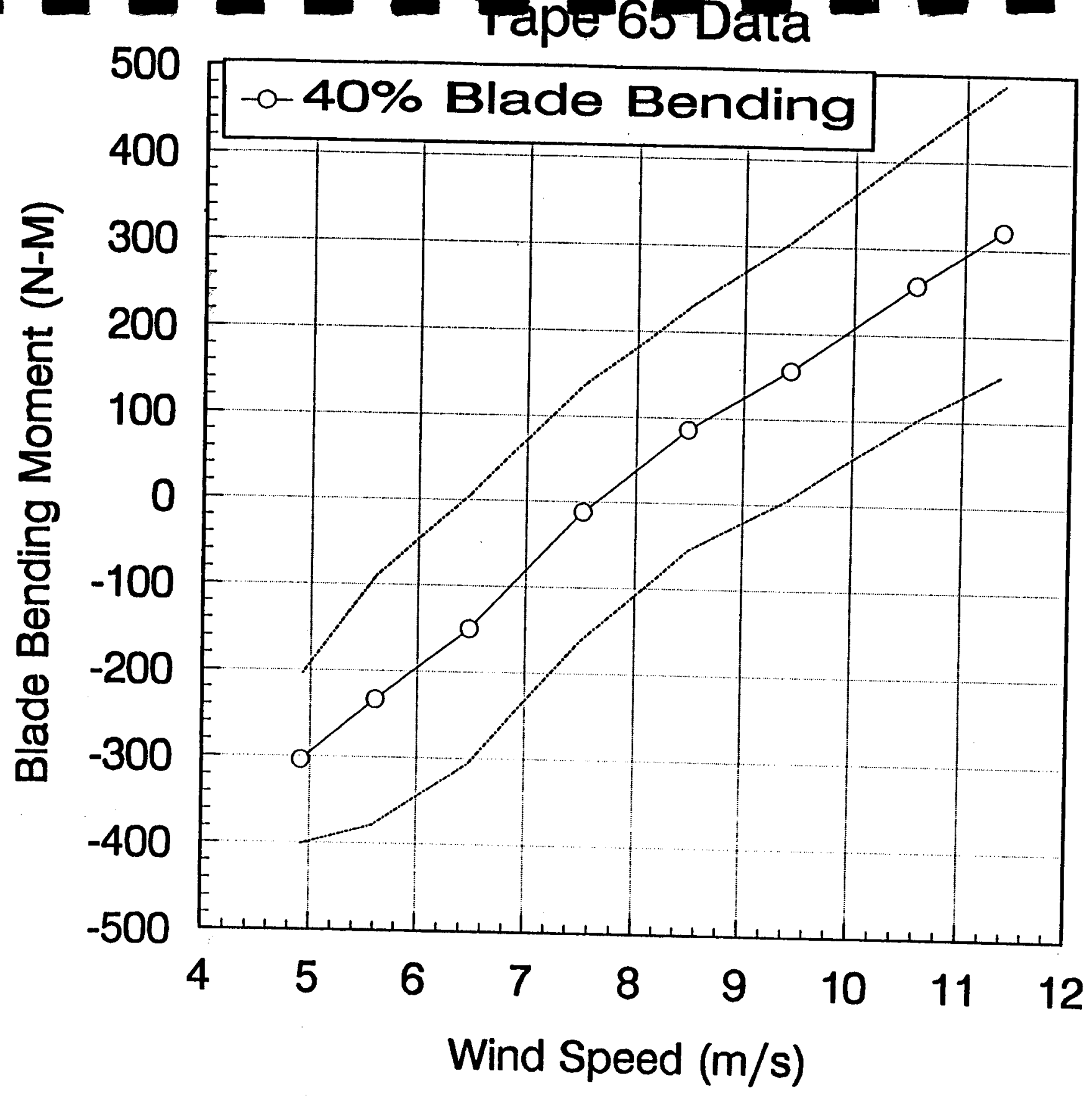




\section{Tape 68 Data}

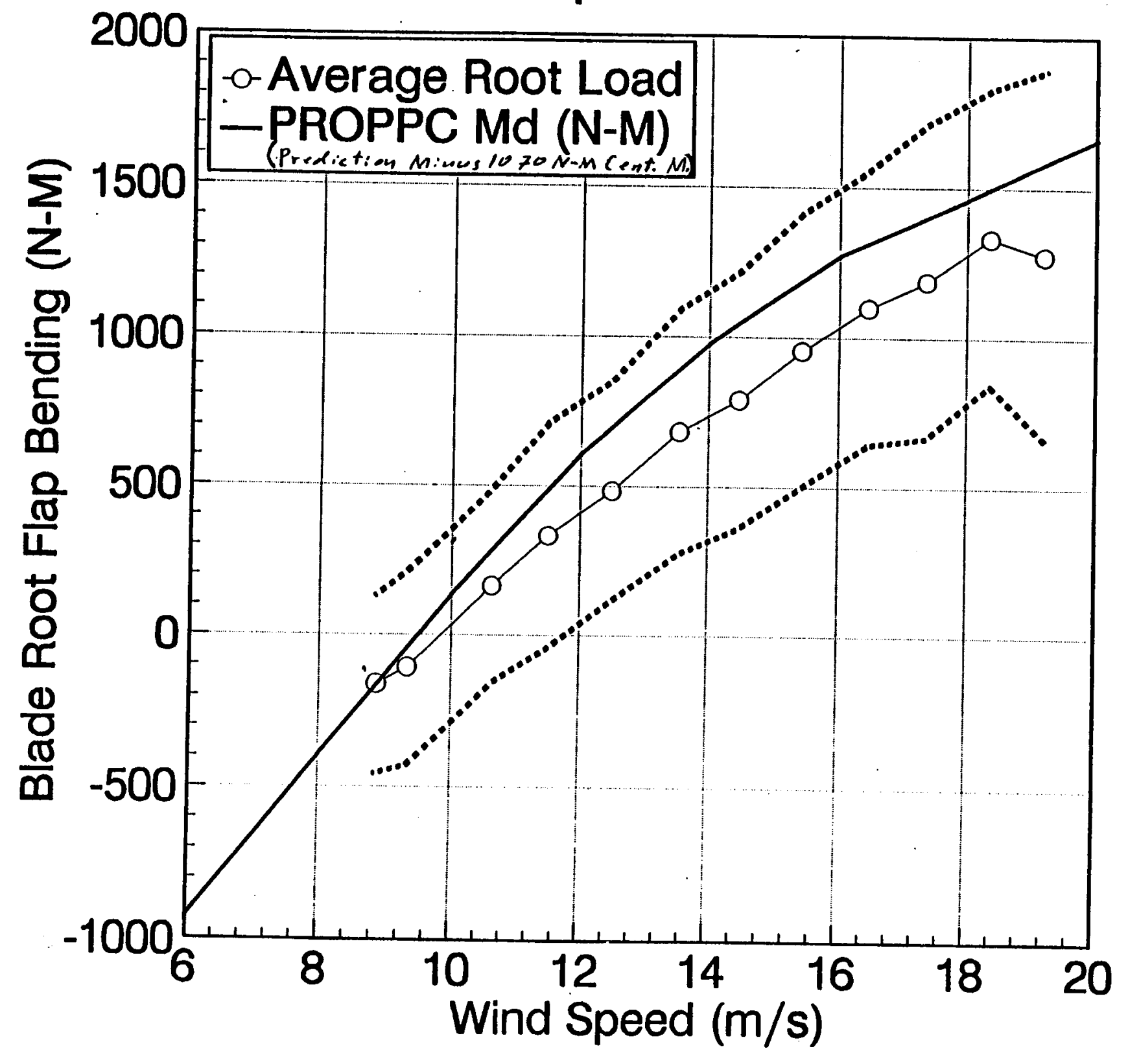


Tape 68 Data

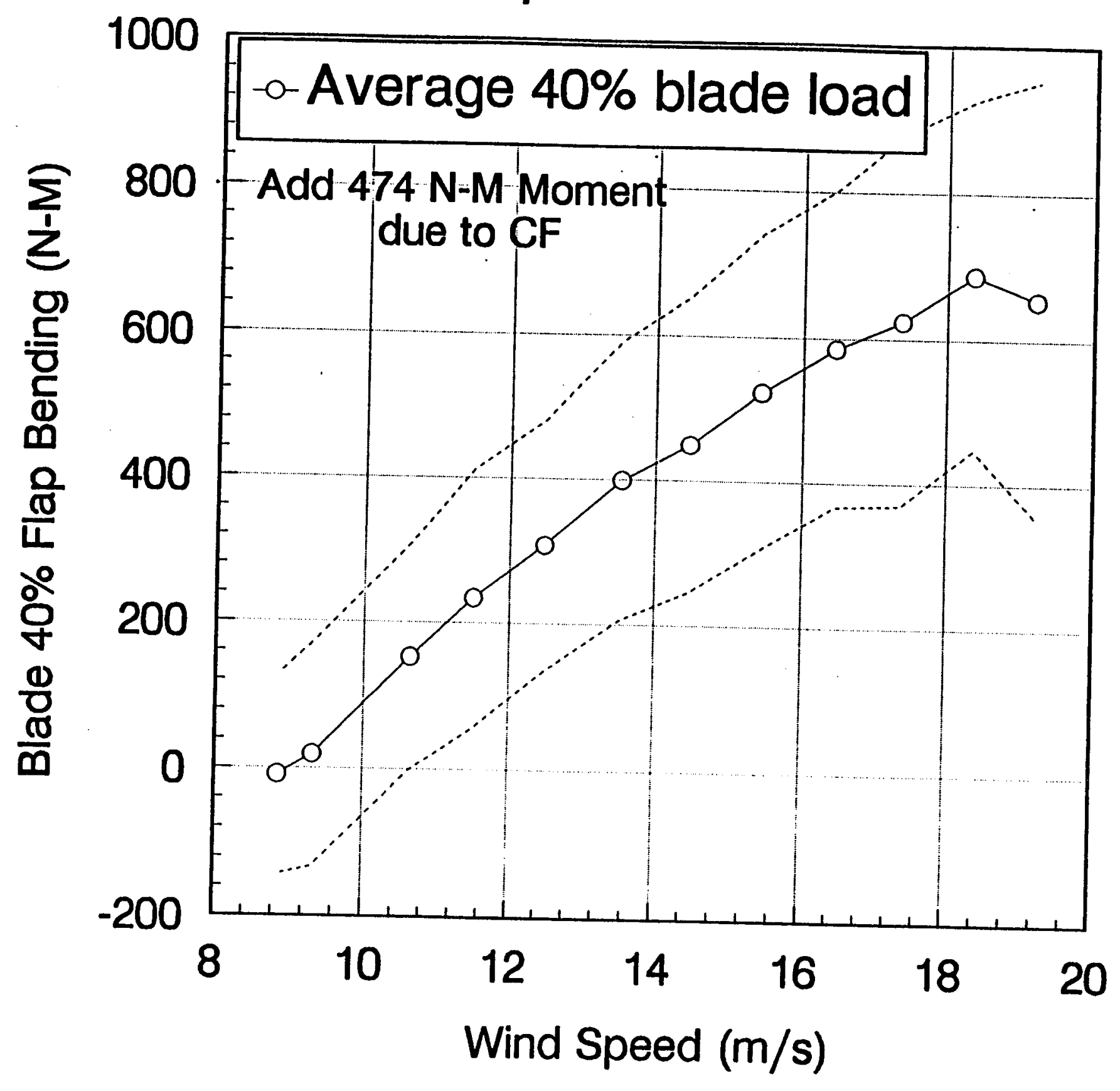




\section{Tape 68 Data}

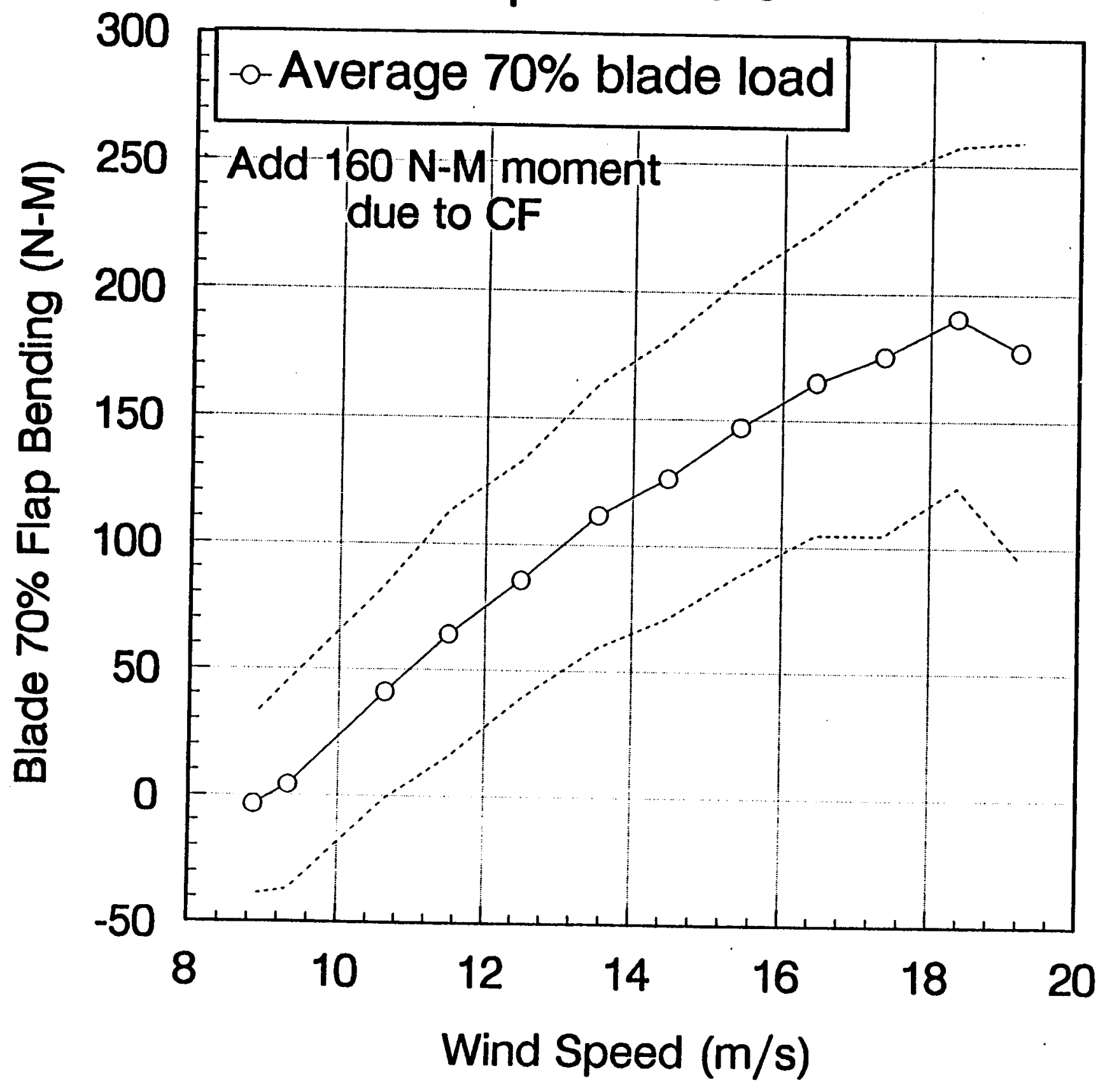




\section{Tape 68 Data}

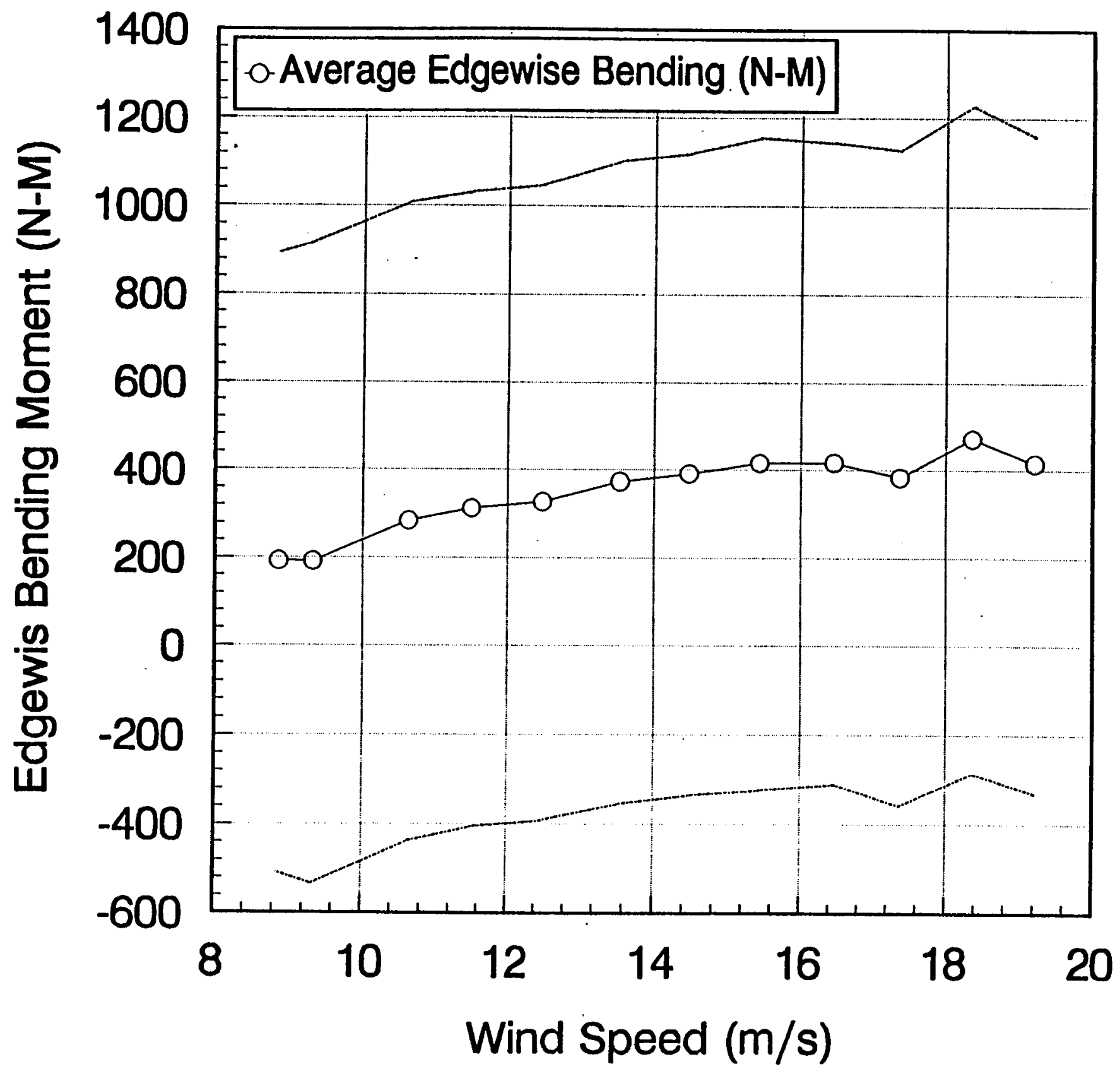




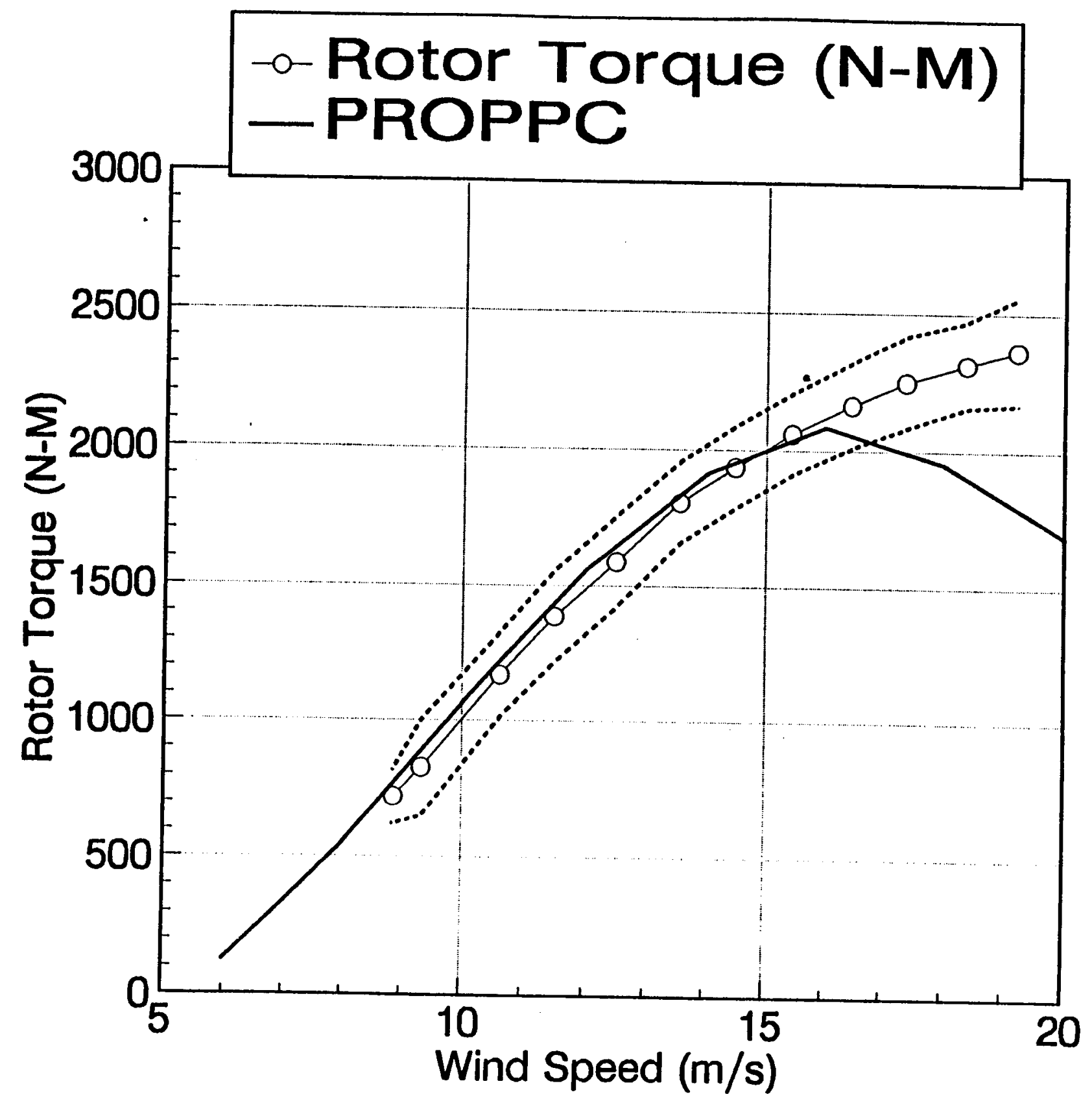




\section{Tape 68 Data}

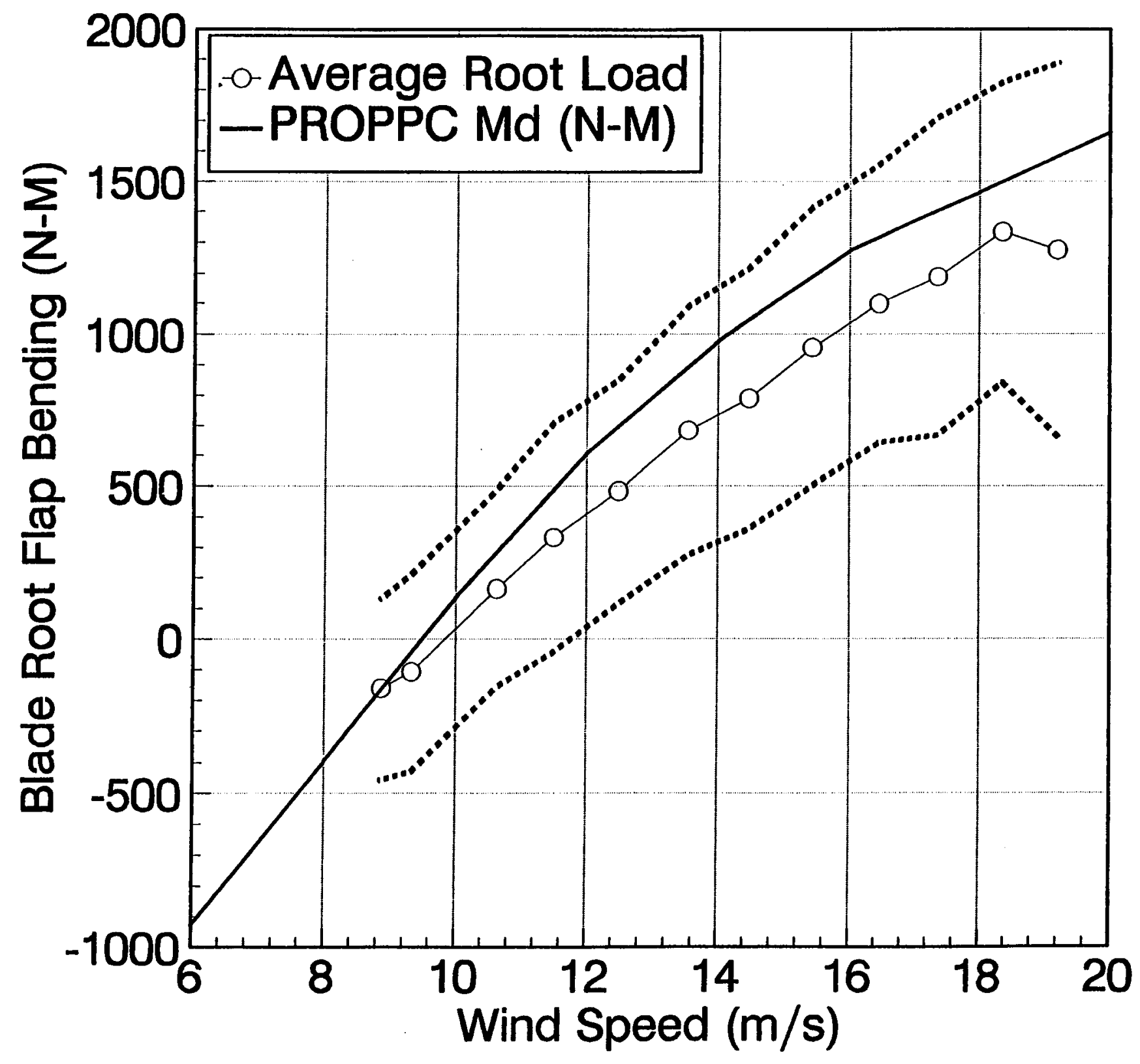




\section{Tape 68 Data}

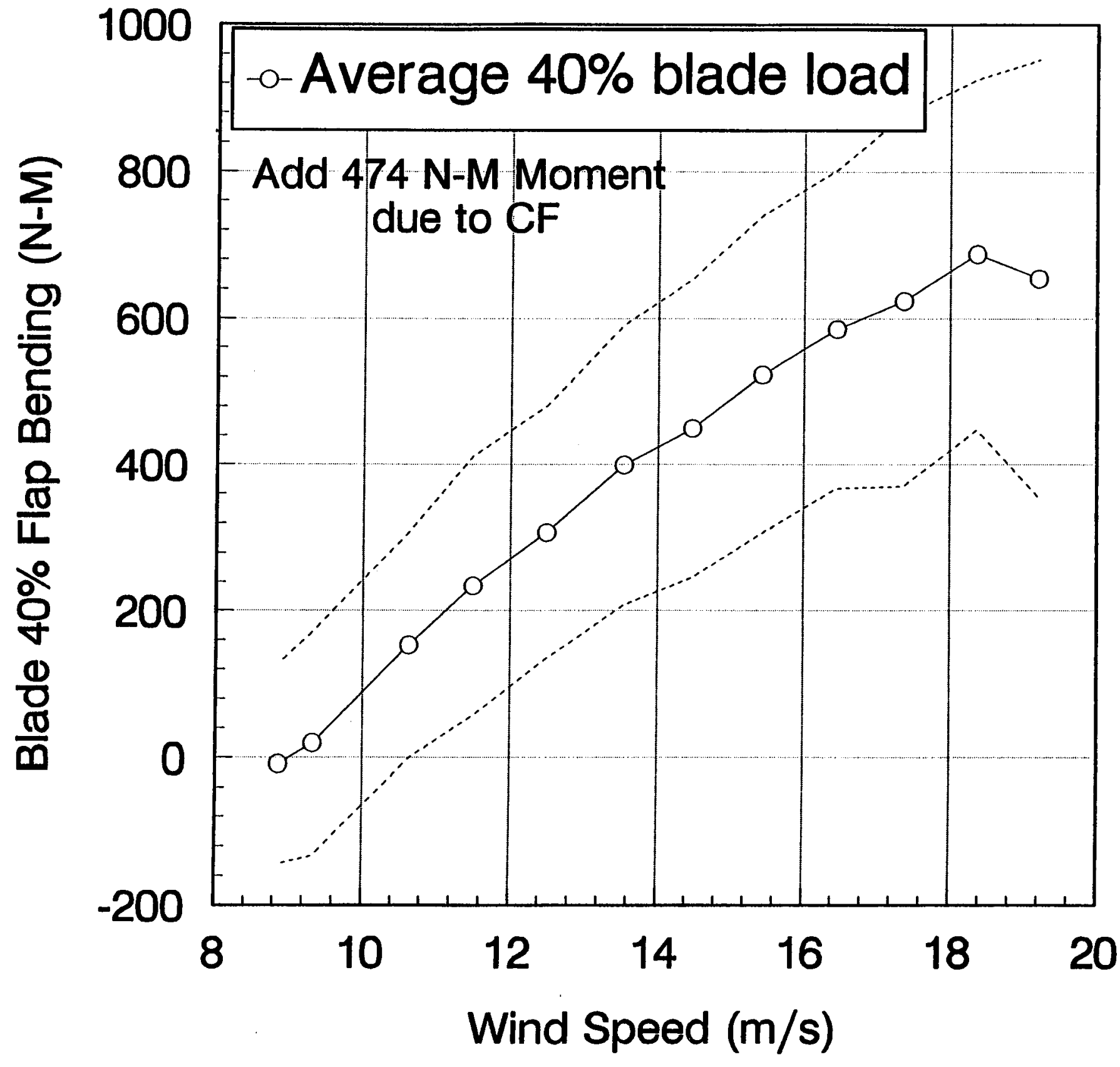


Tape 68 Data

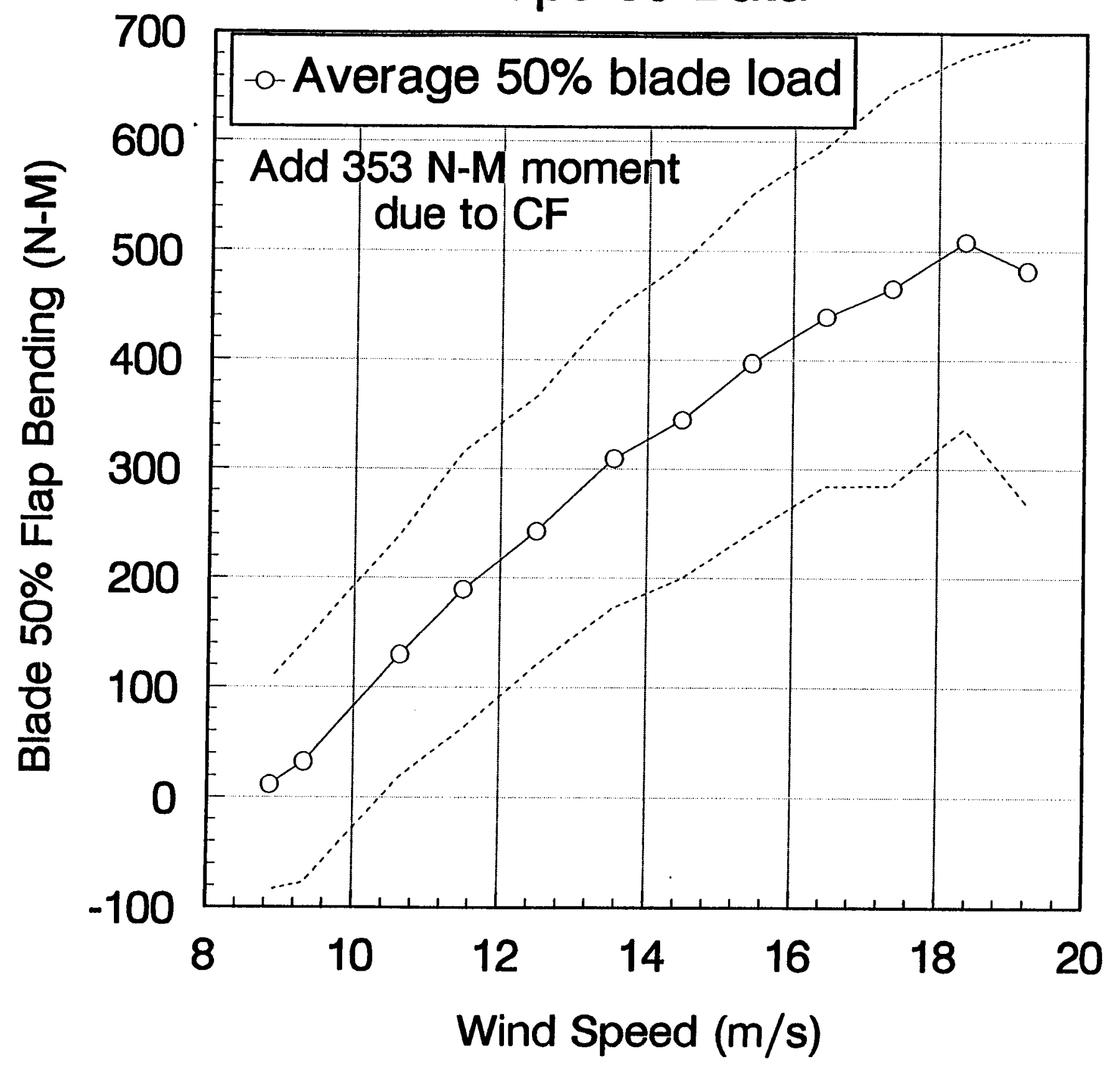


Tape 68 Data

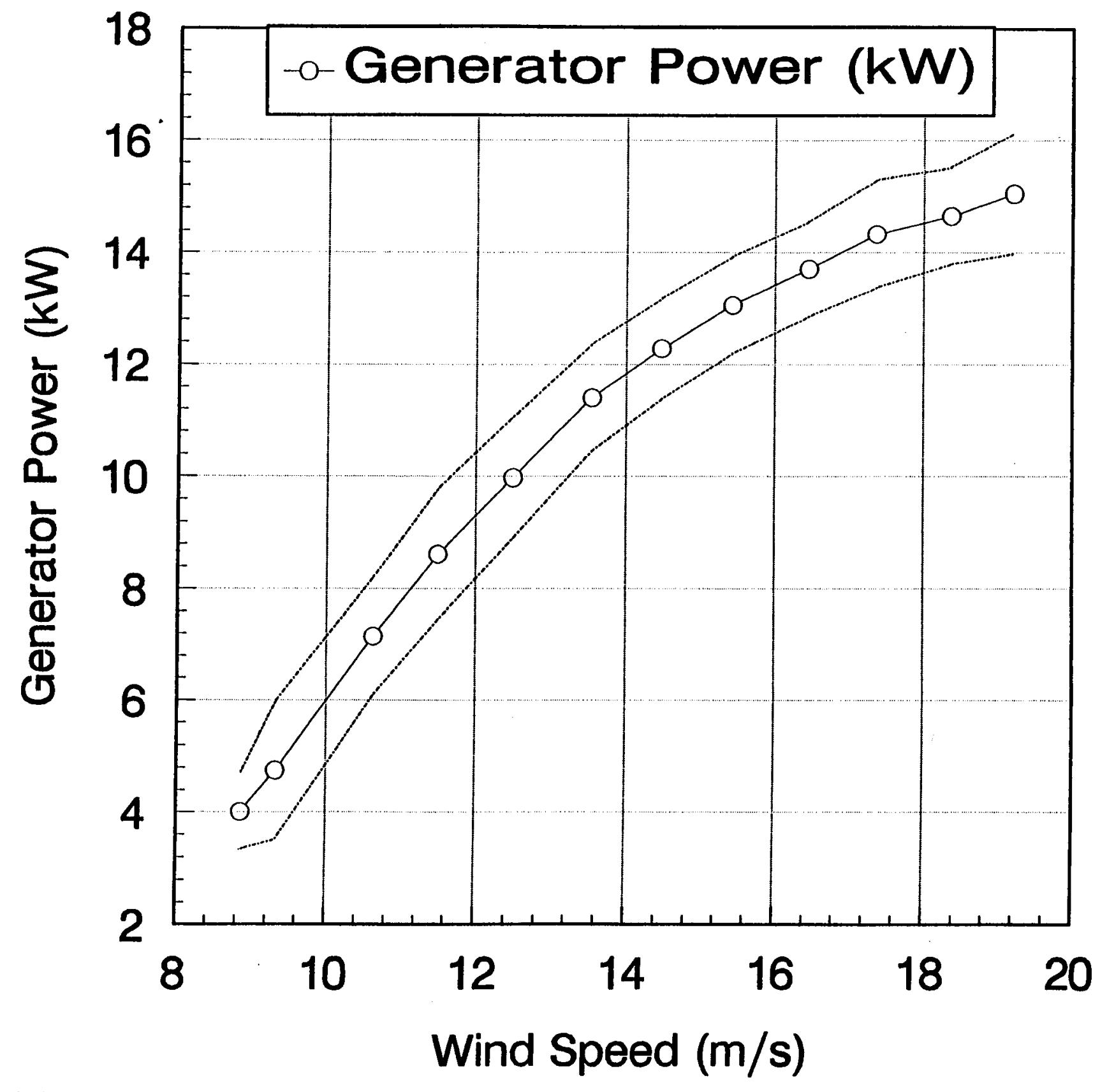




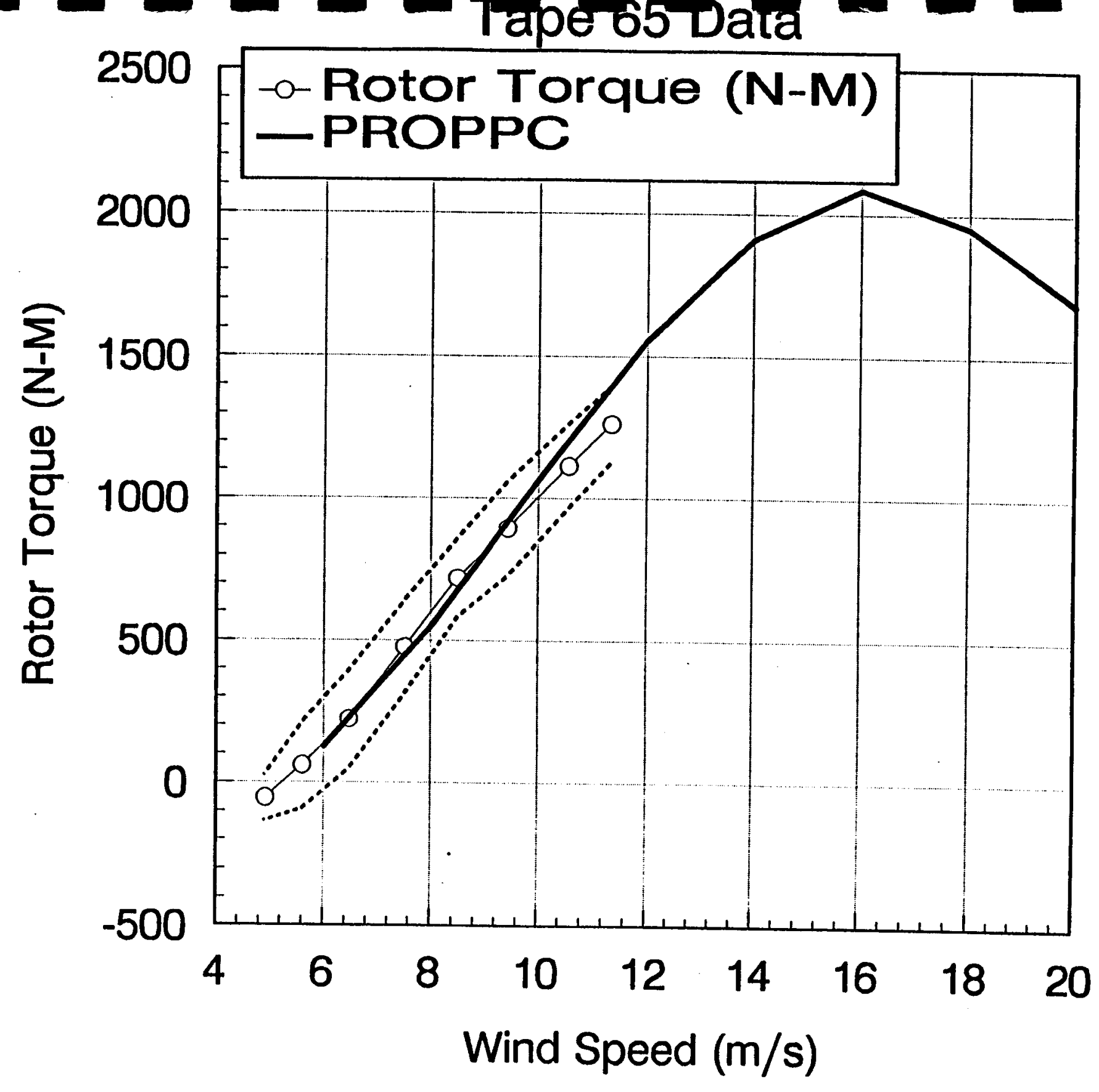




\section{Tape 68 Data}

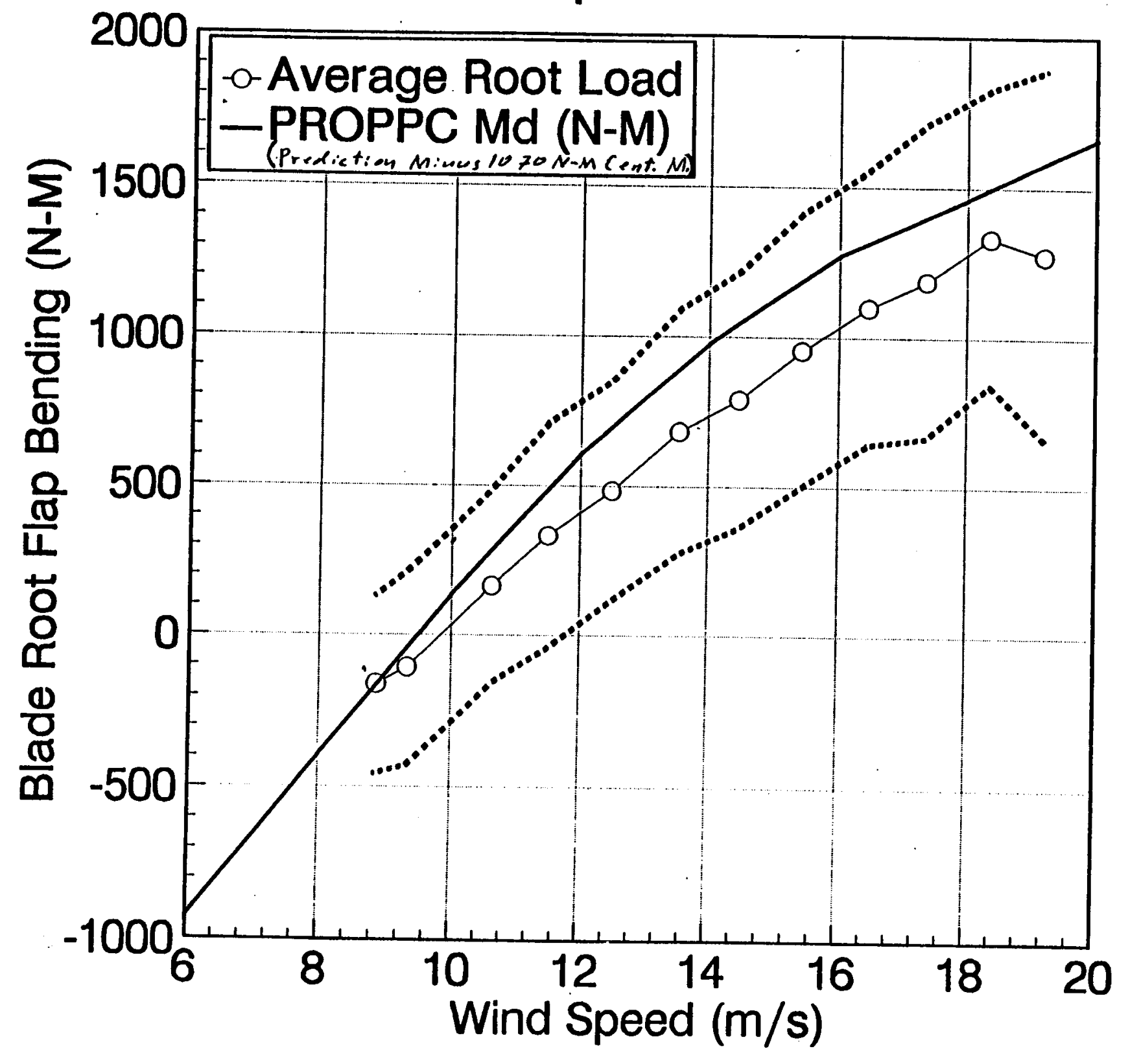




\section{Tape 68 Data}

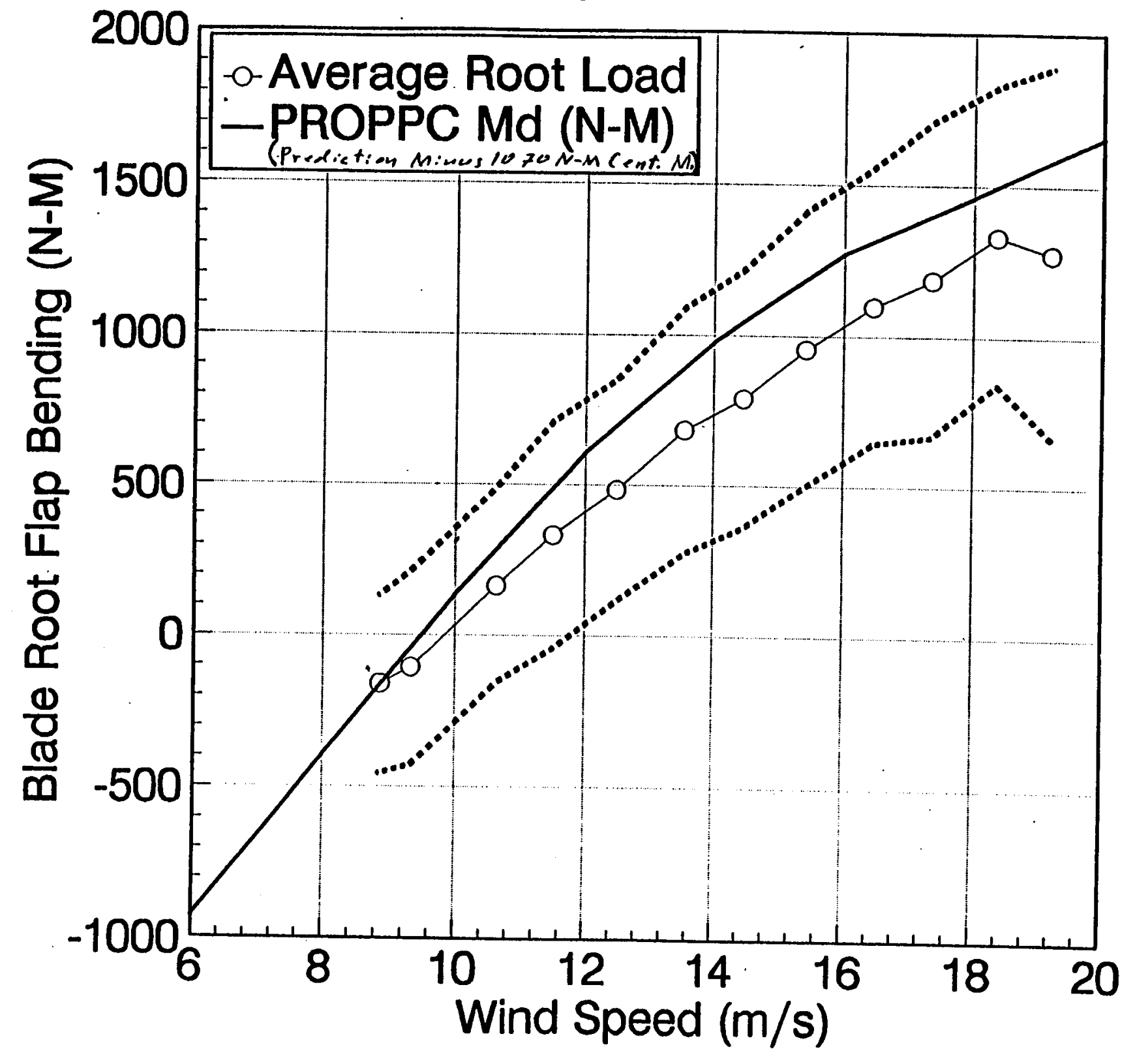


Tape 68 Data

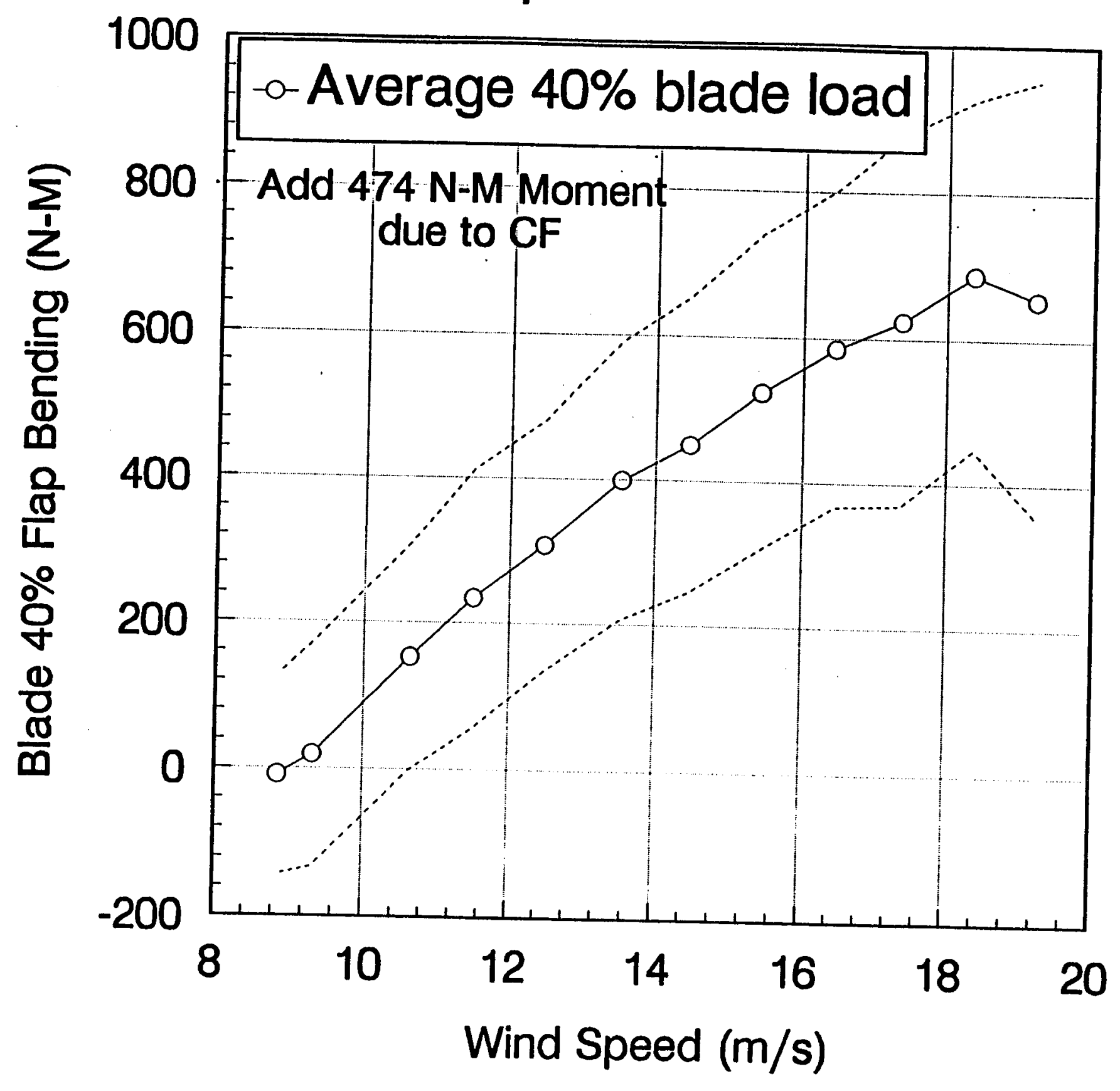




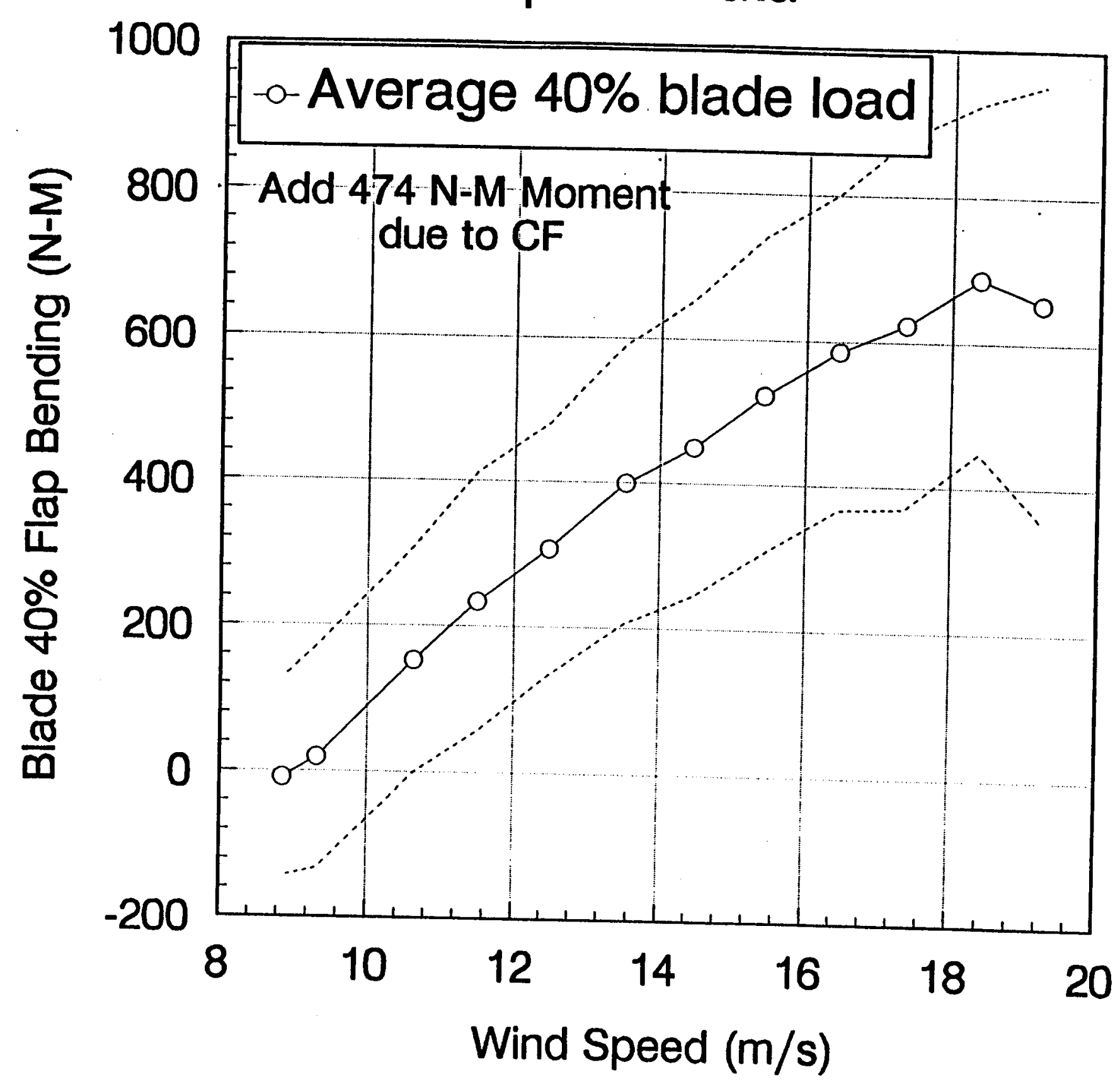




\section{Tape 68 Data}

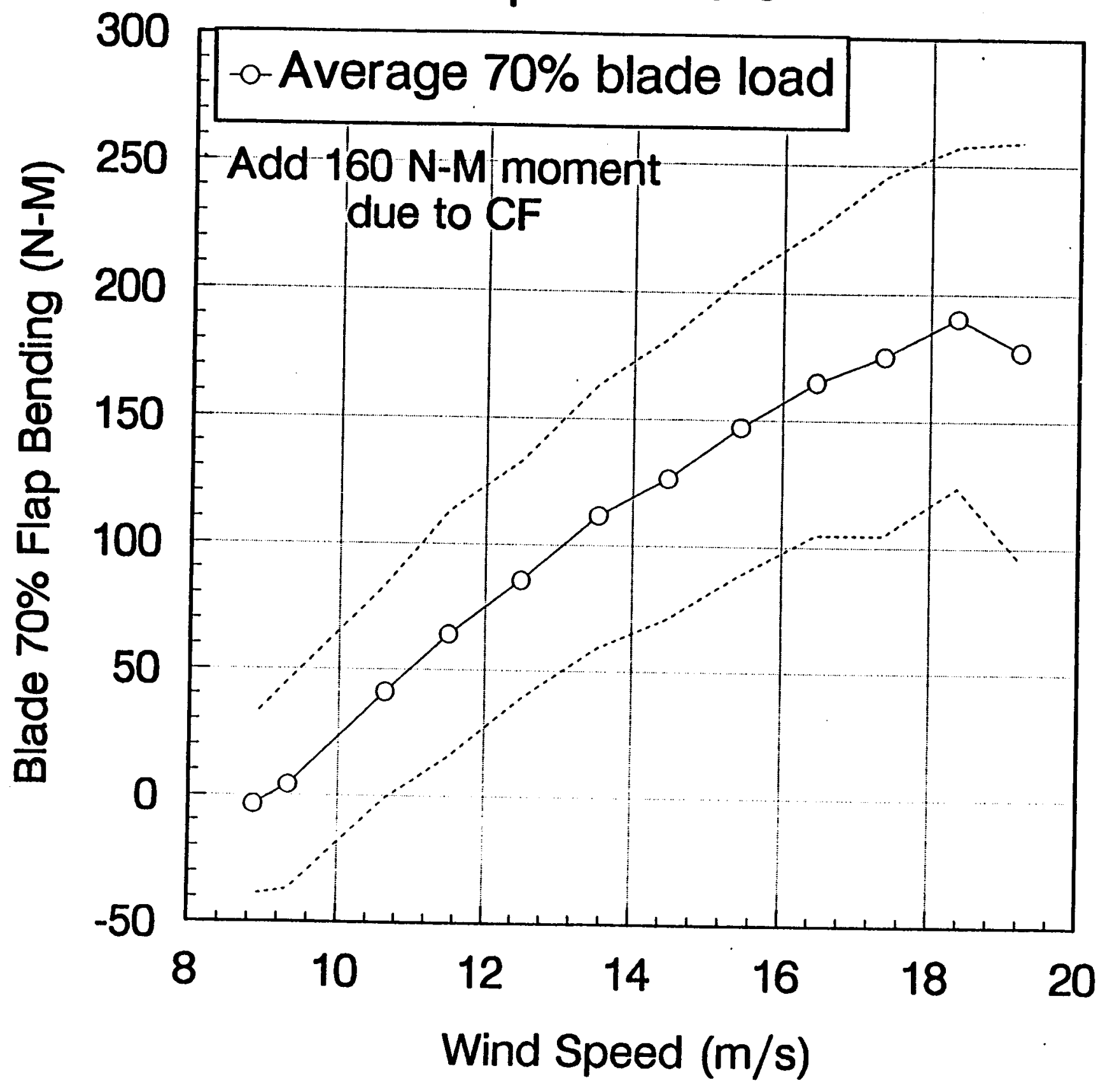


Tape 68 Data

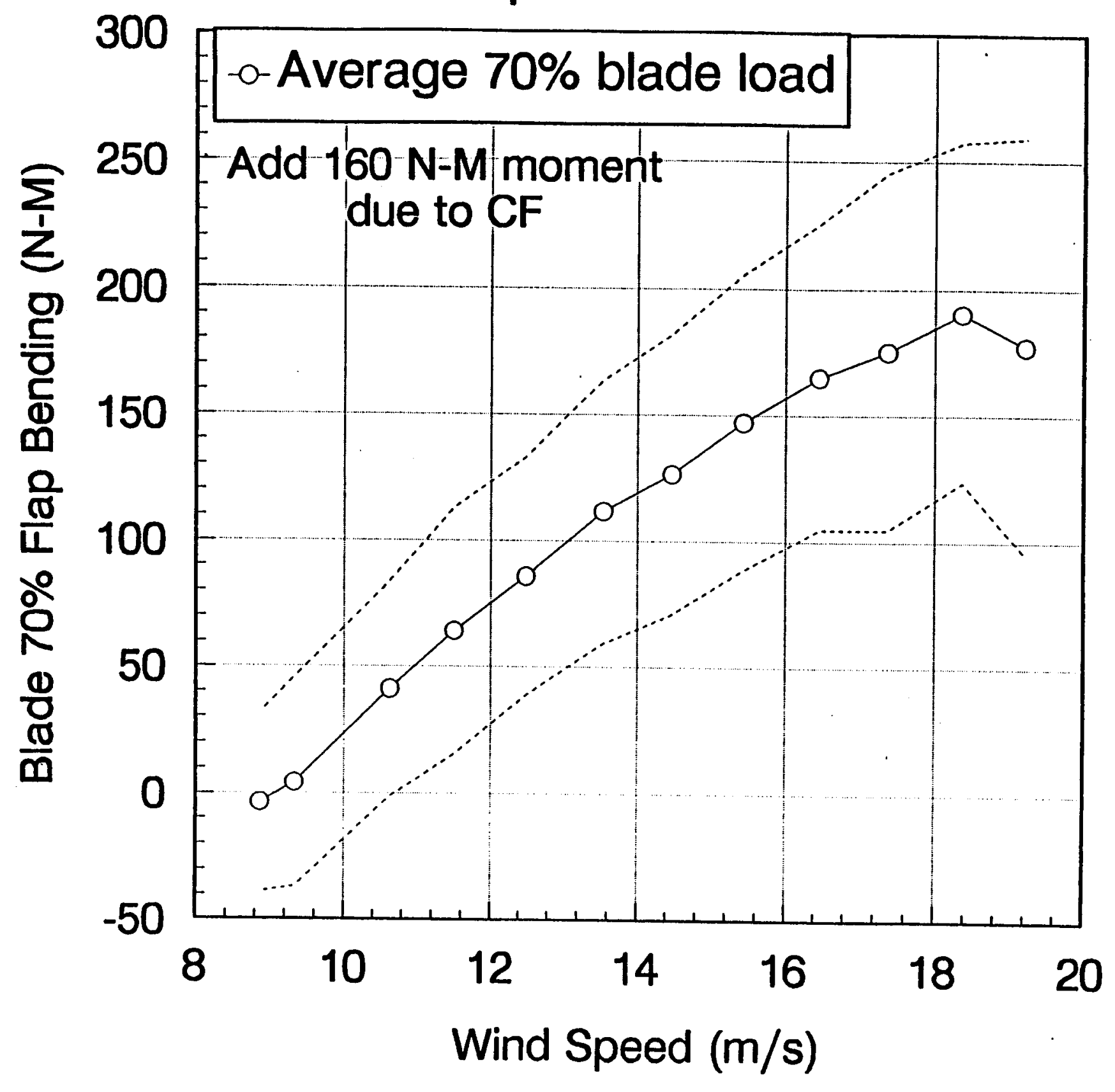




\section{Tape 68 Data}

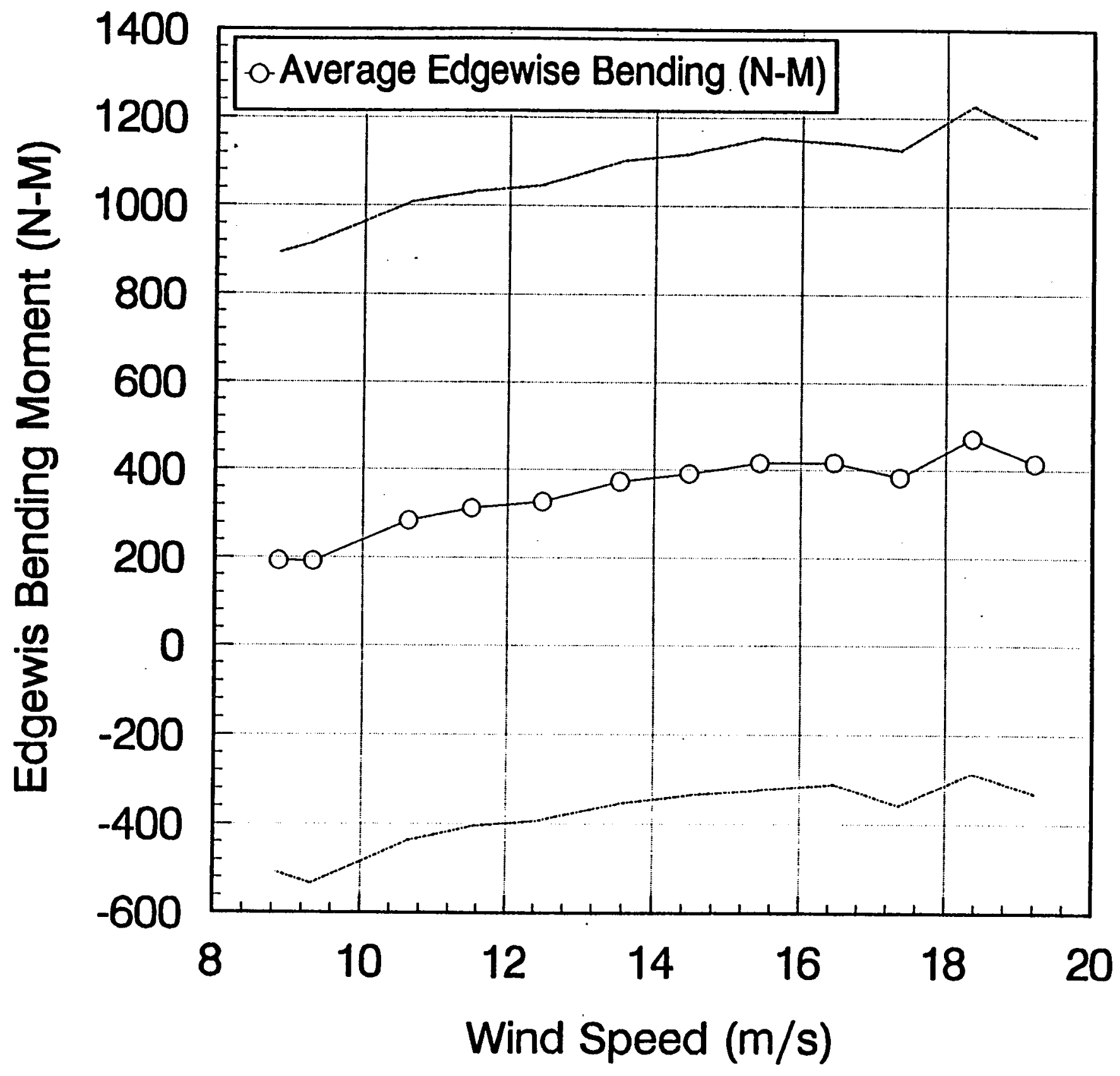


Tape 68 Data

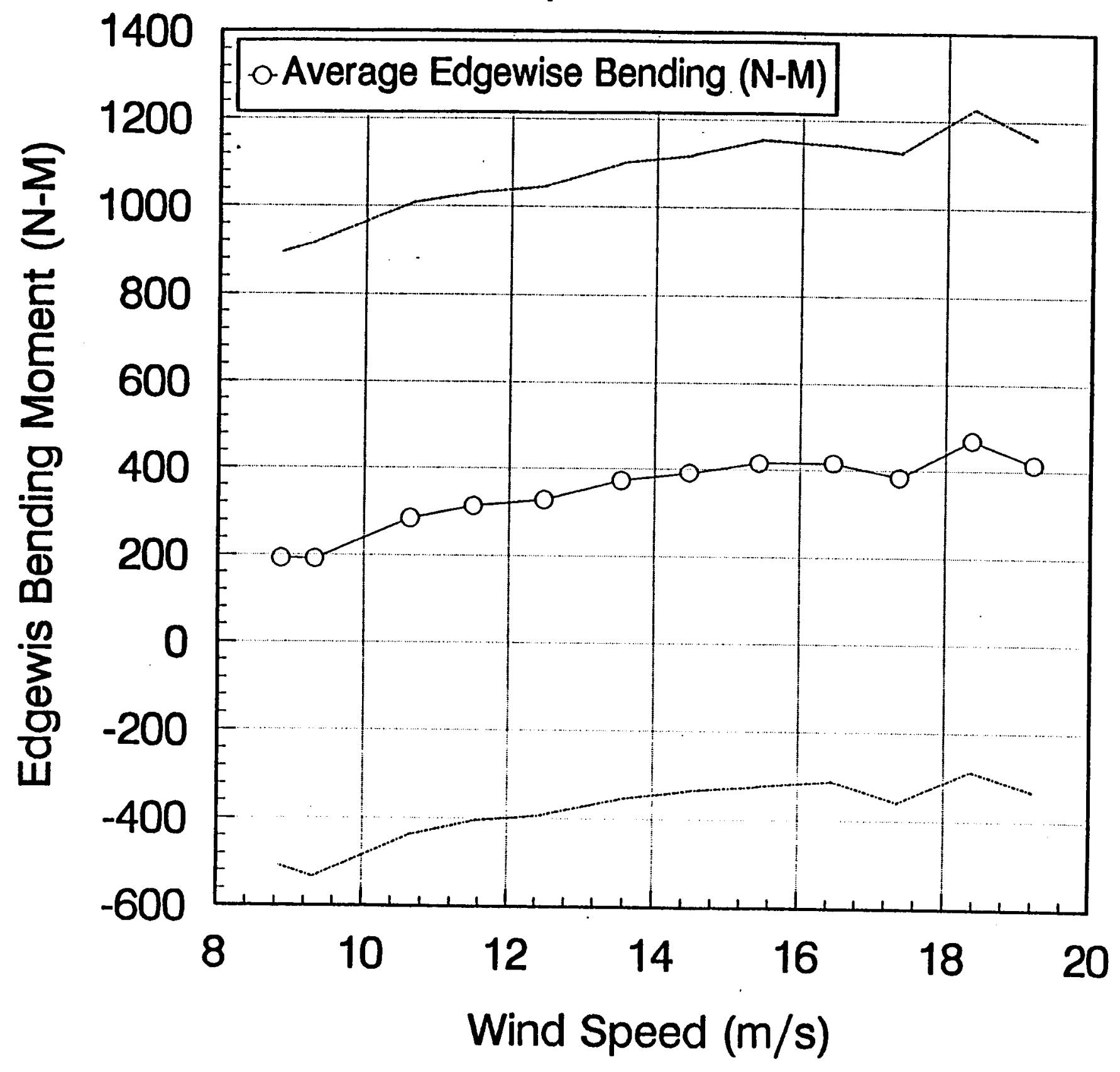




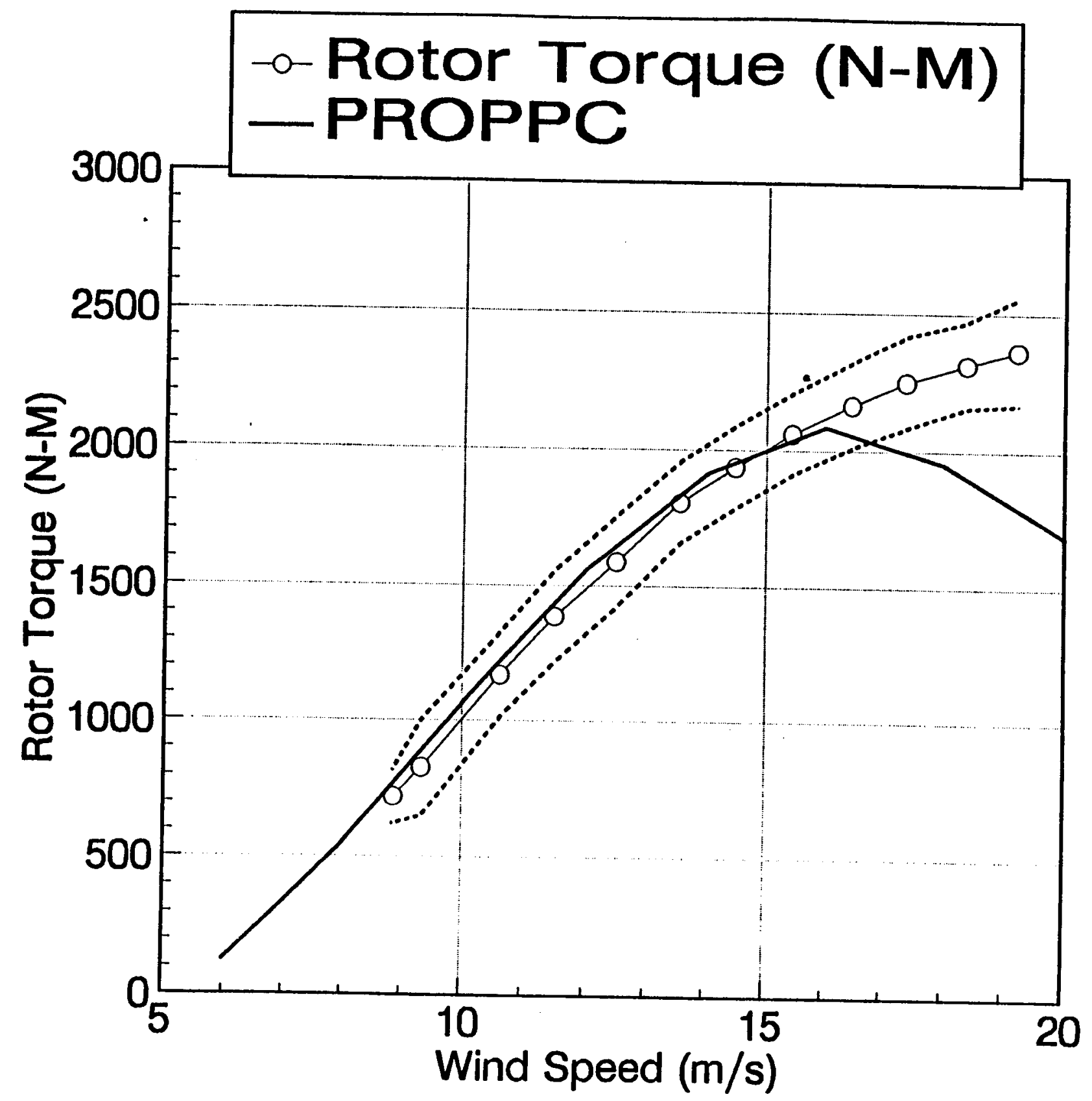




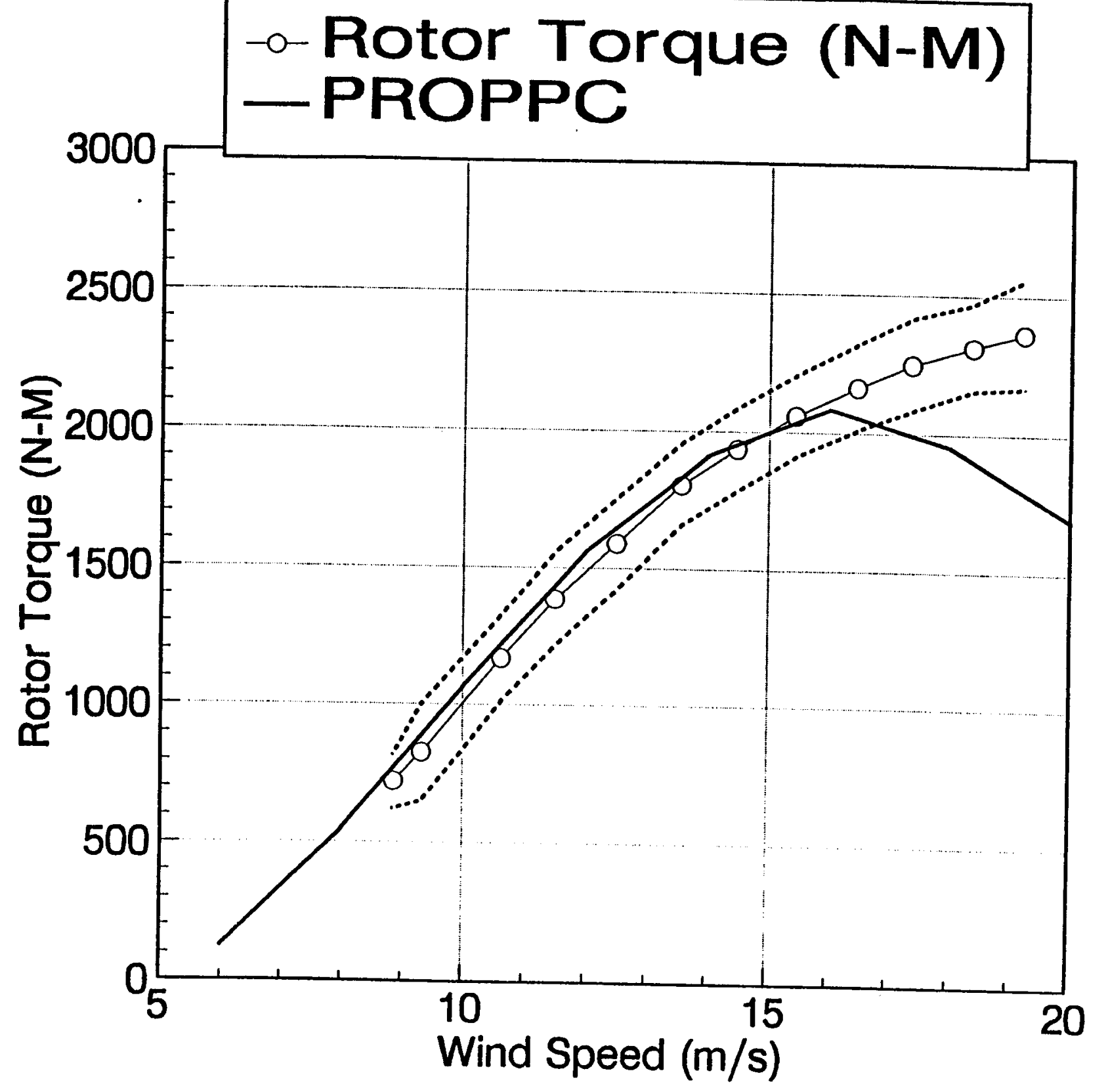




\section{Tape 68 Data}

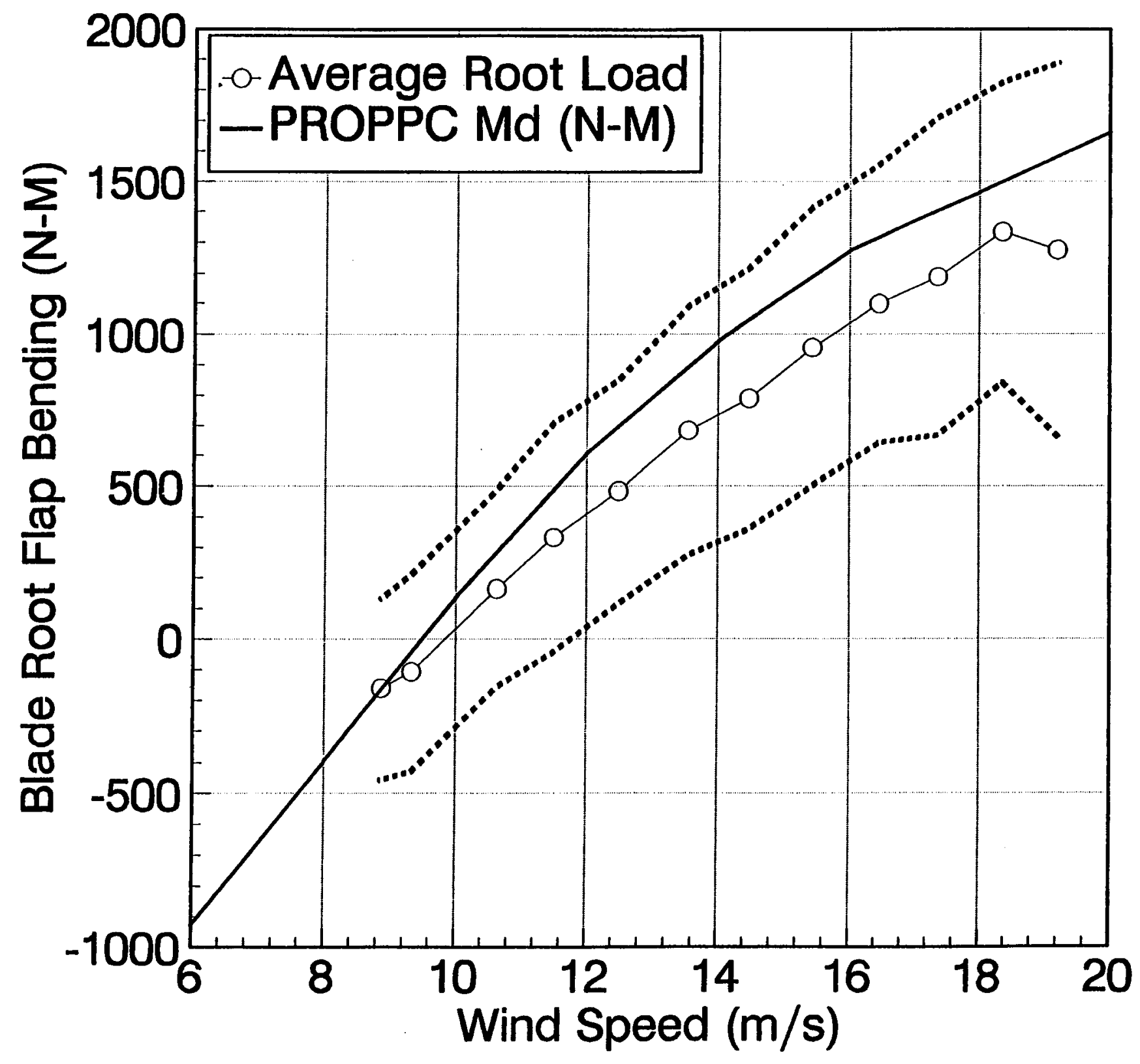




\section{Tape 68 Data}

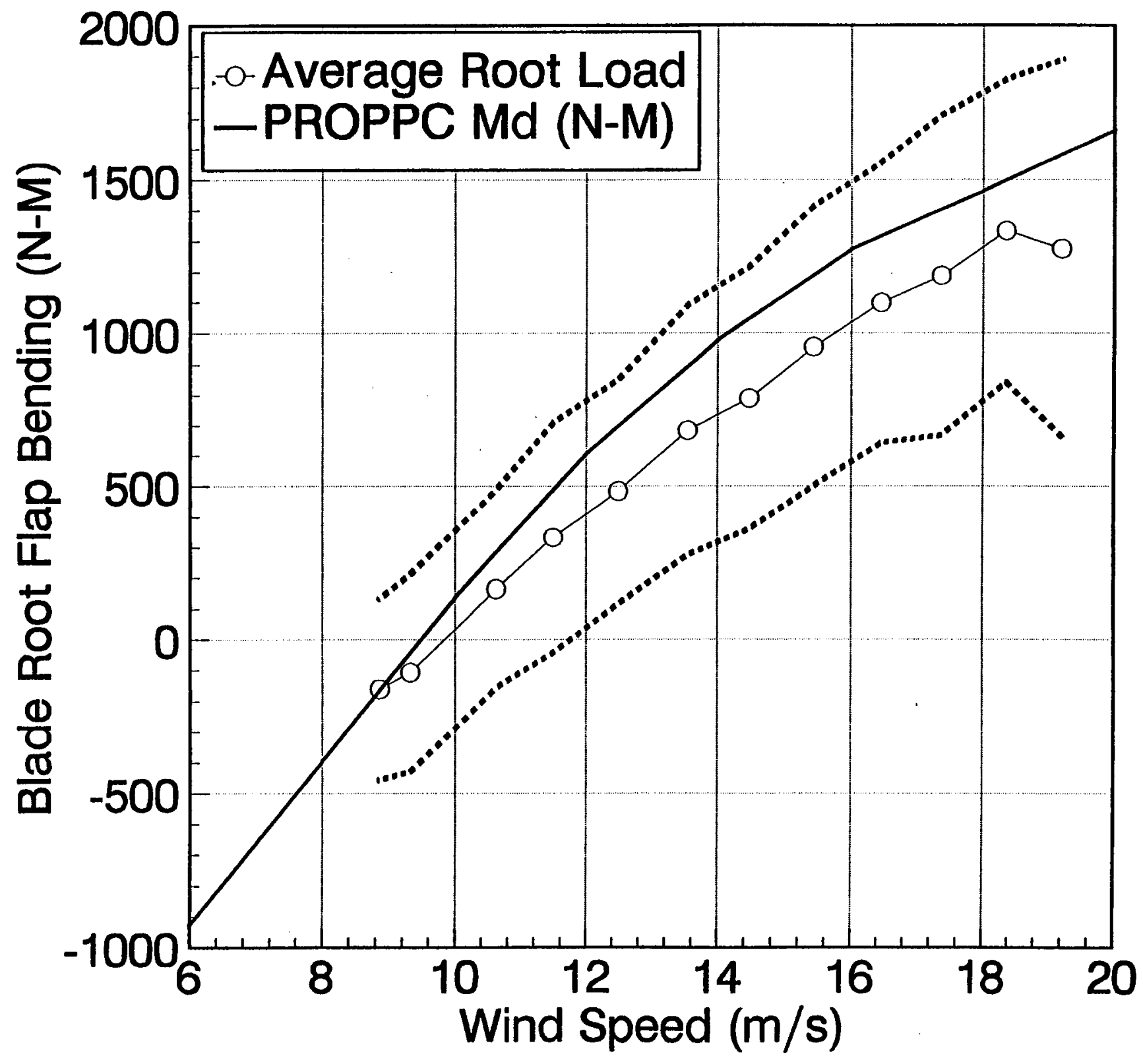




\section{Tape 68 Data}

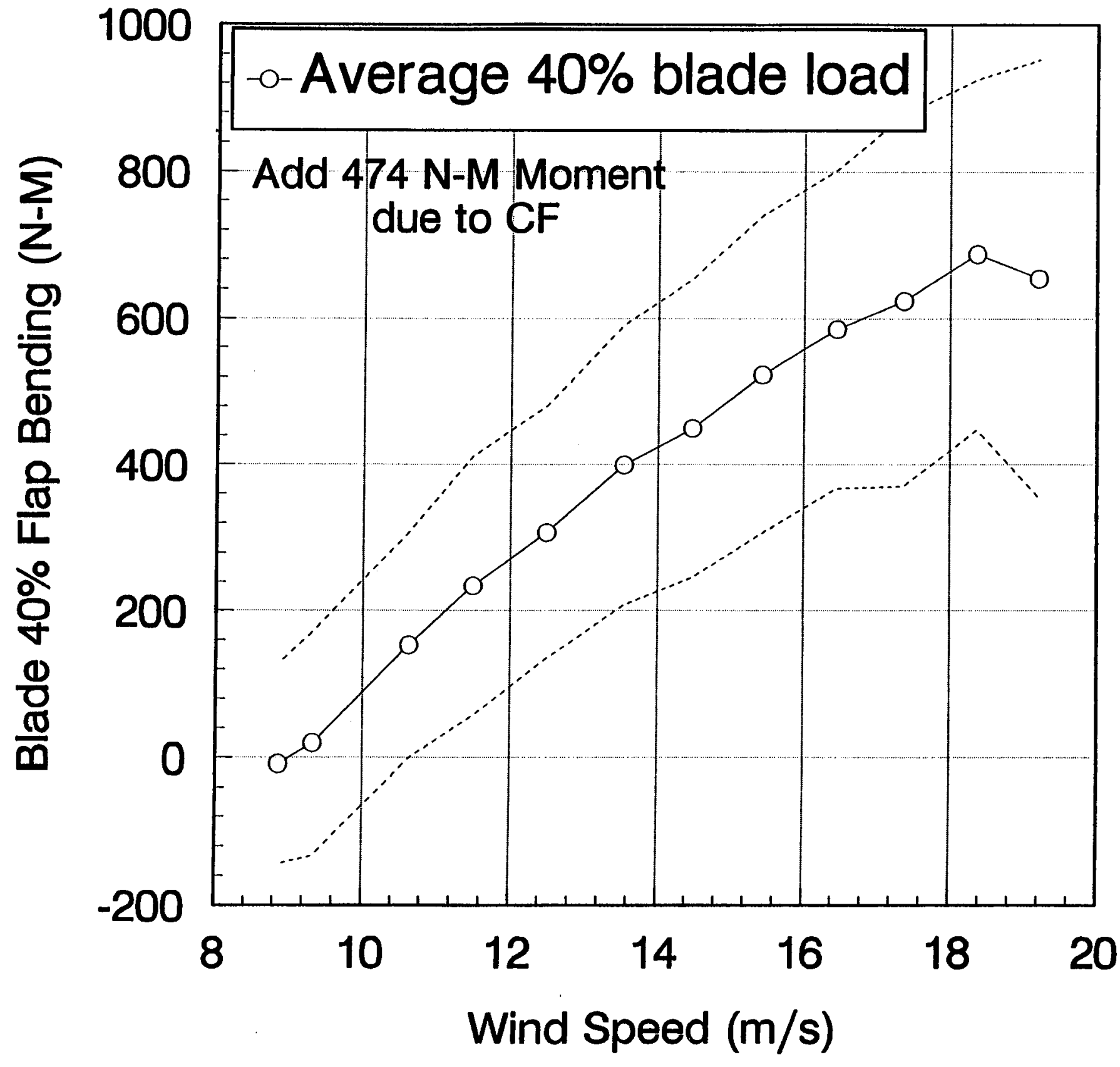


Tape 68 Data

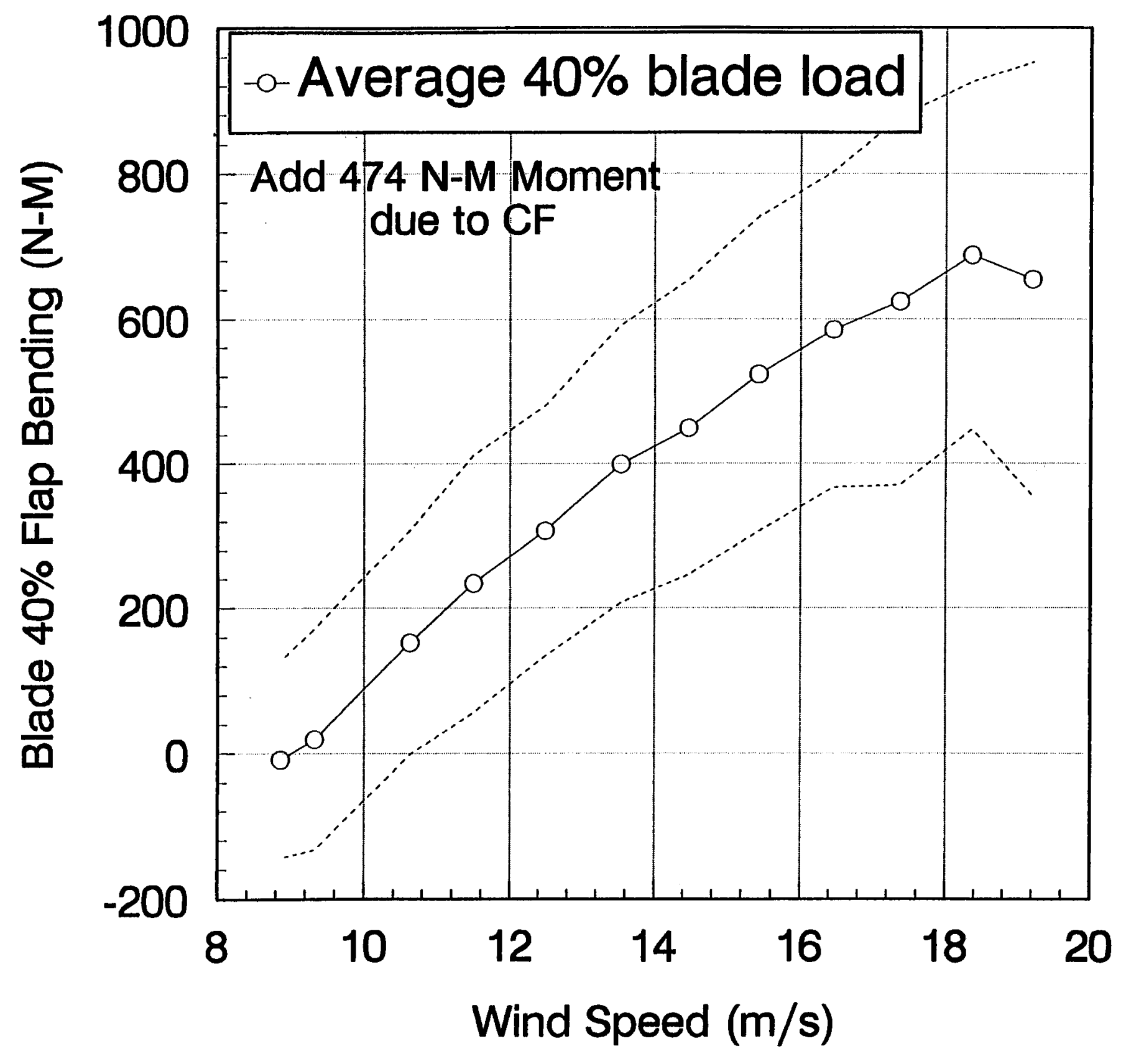


Tape 68 Data

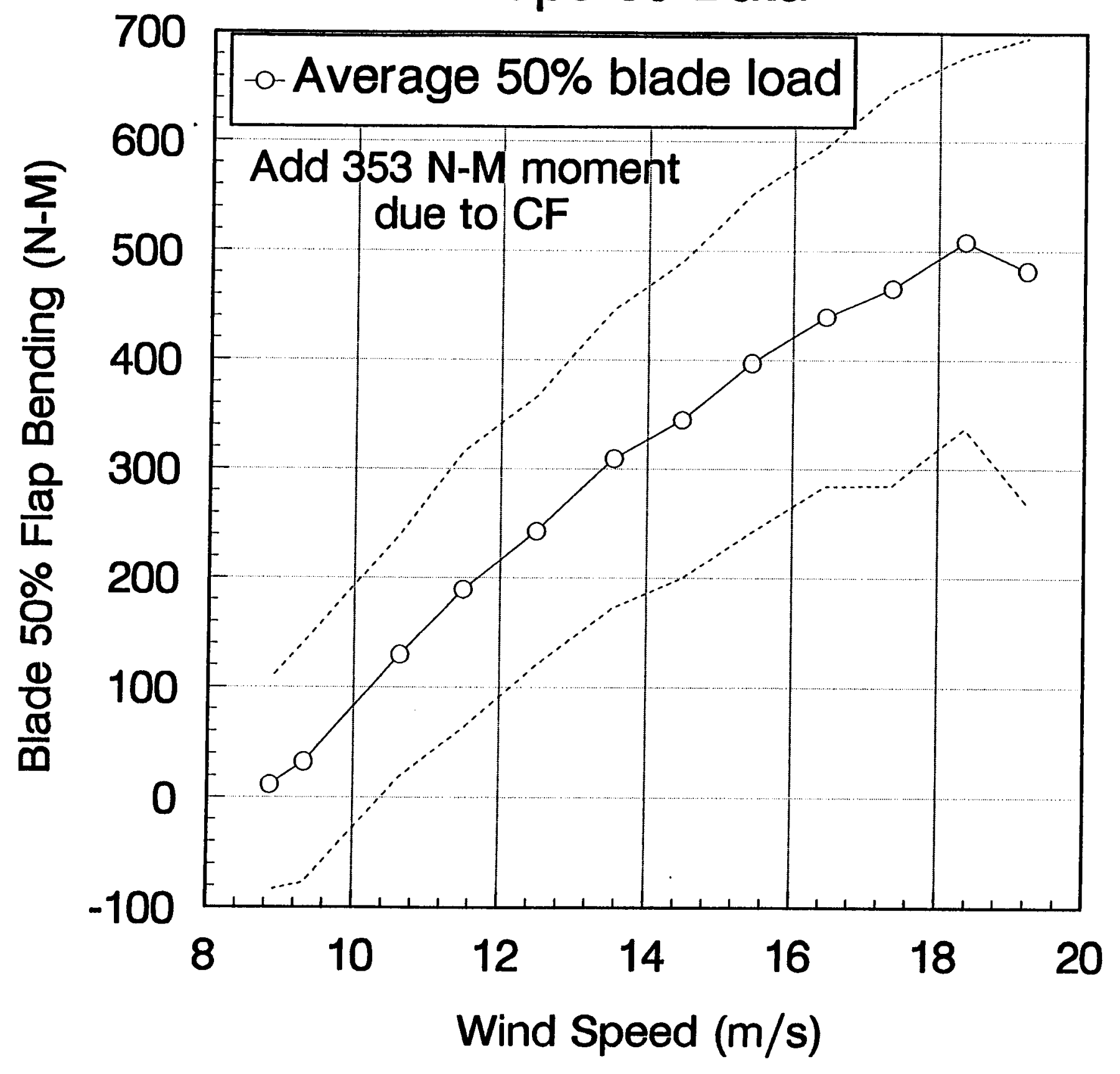


Tape 68 Data

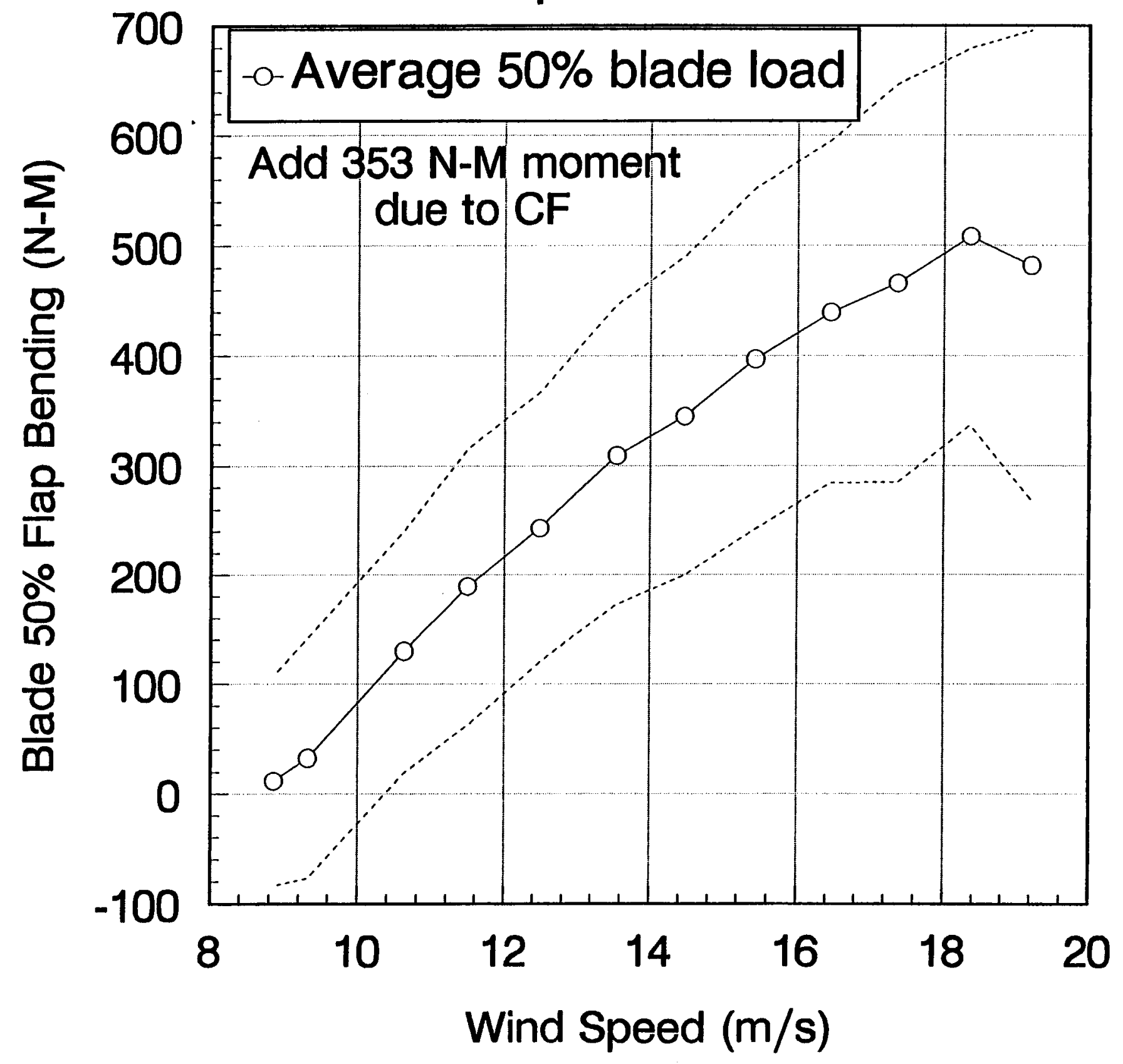


Tape 68 Data

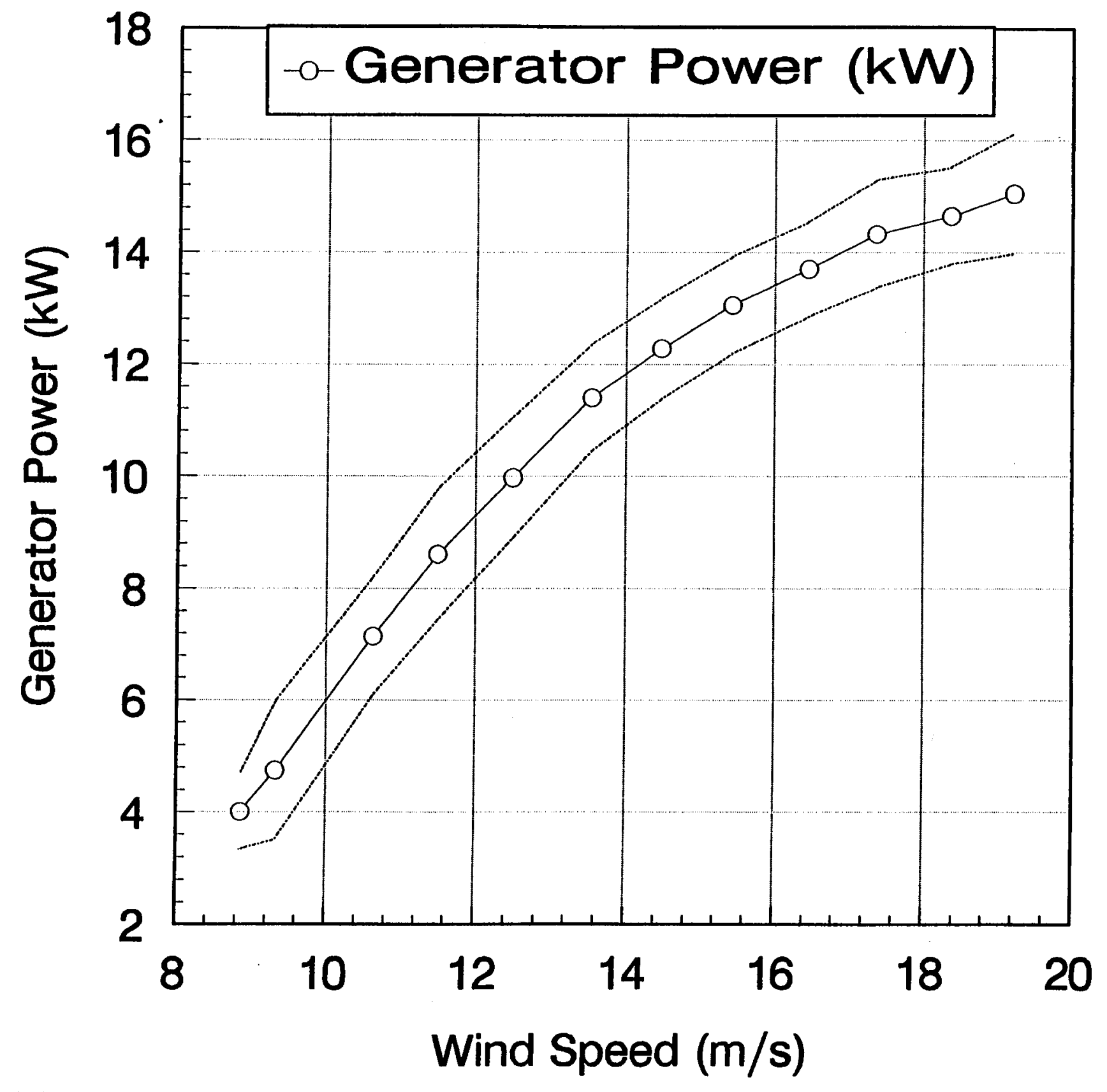


Tape 68 Data

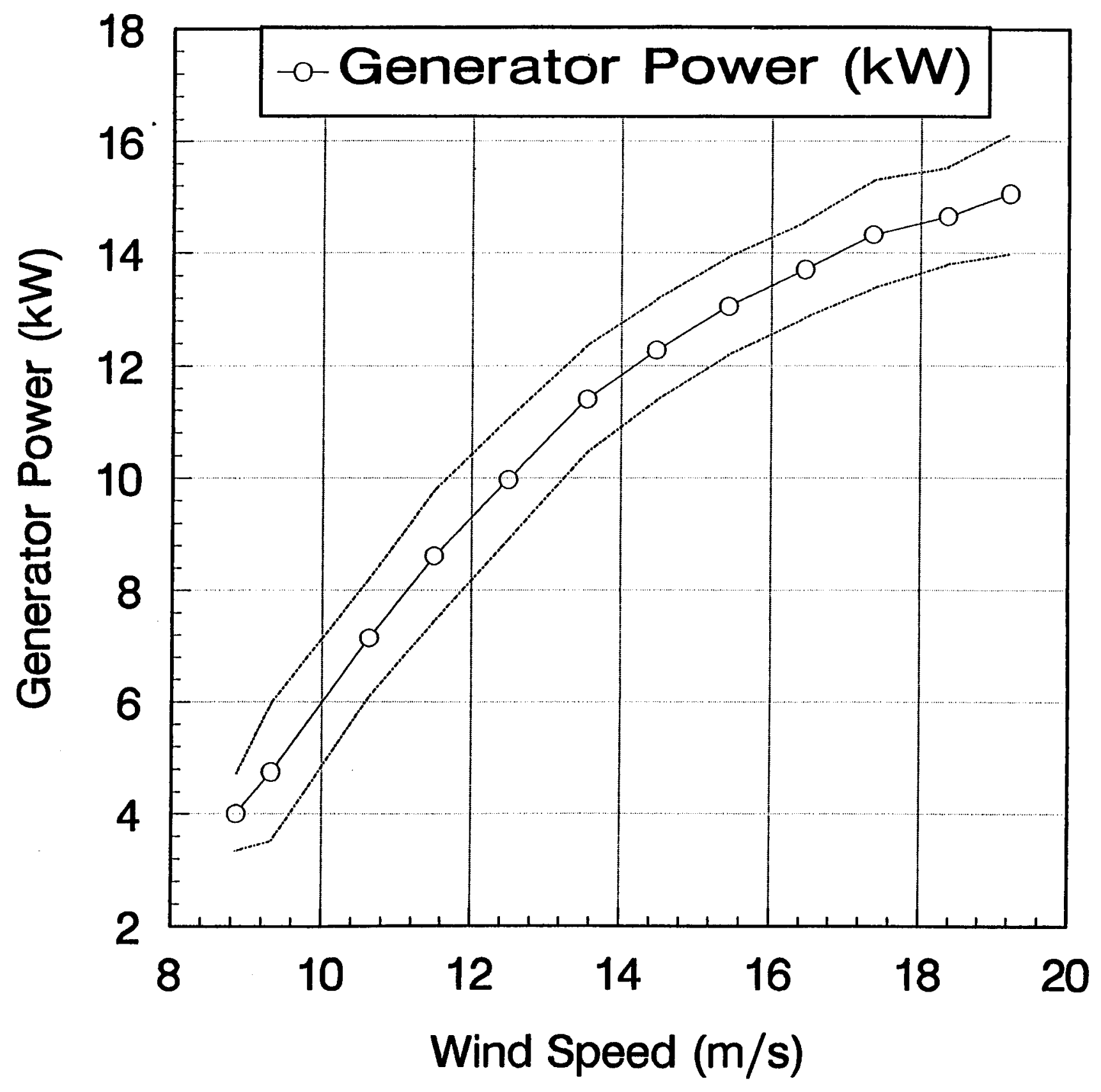




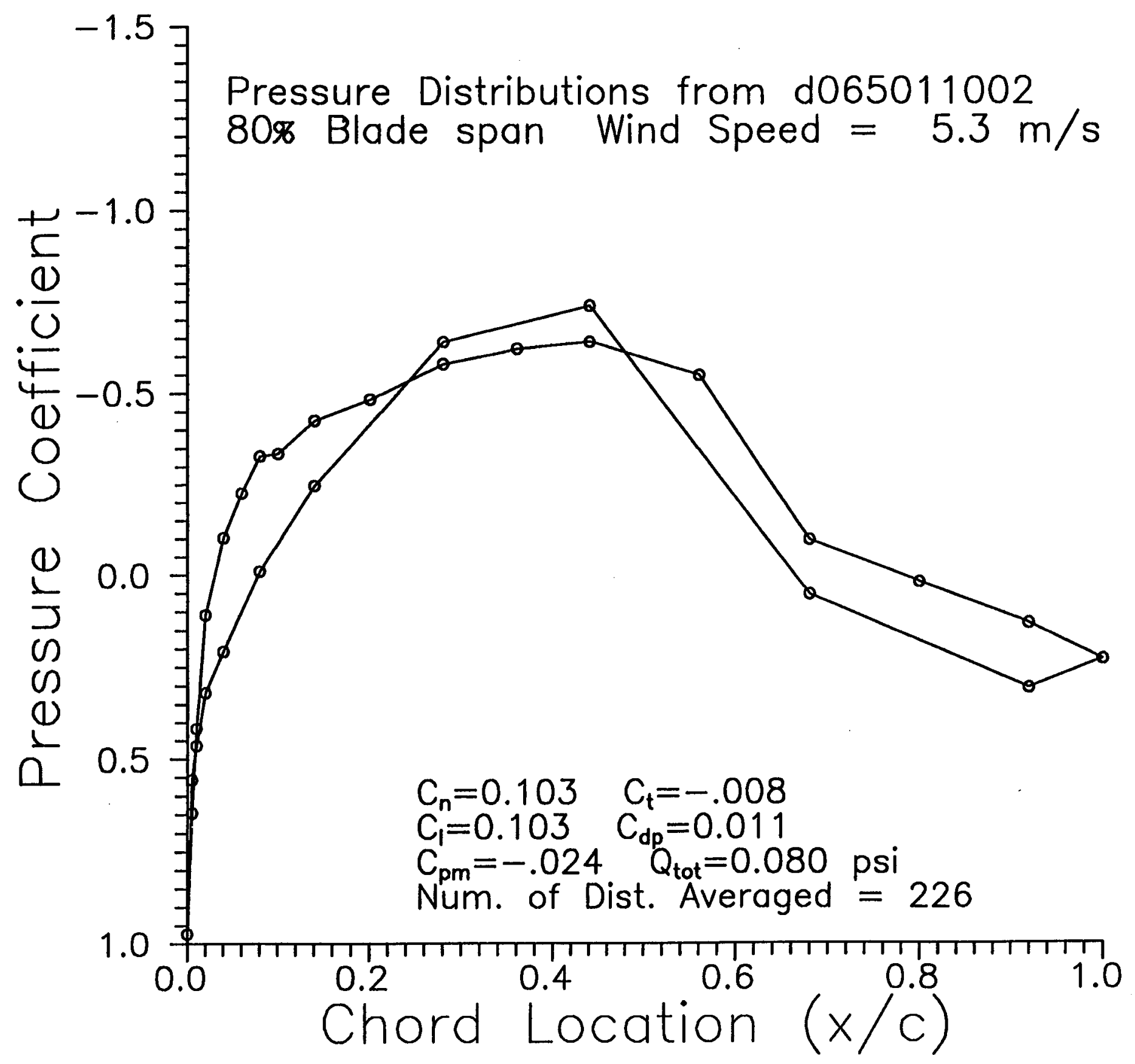

